\title{
Palladium-katalysierte Domino-Reaktionen zum Aufbau bi- und tricyclischer Systeme
}

\author{
Dissertation \\ zur Erlangung des Doktorgrades \\ der Mathemathisch-Naturwissenschaftlichen Fakultäten \\ der Georg-August-Universität zu Göttingen
}

\author{
vorgelegt von \\ Stefanie Körbe \\ aus \\ Bad Hersfeld
}

Göttingen 2001 
D 7

Referent: Prof. Dr. A. de Meijere

Korreferent: Prof. Dr. H. Lackner

Tag der mündlichen Prüfung: 26.06.2001 
Die vorliegende Arbeit wurde in der Zeit von August 1998 bis April 2001 im Institut für Organische Chemie der Georg-August-Universität Göttingen durchgeführt.

Für die Überlassung des Themas, die hilfreichen Diskussionen und Anregungen sowie die ständige Unterstützung während der Arbeit möchte ich meinem Lehrer, Herrn Prof. Dr. A. de Meijere, ganz herzlich danken. 
Meiner Familie 


\section{Inhaltsverzeichnis}

$\begin{array}{ll}\text { A. Einleitung } & 1\end{array}$

$\begin{array}{ll}\text { B. Hauptteil } & 10\end{array}$

1. Untersuchungen von Domino-Prozessen aus intramolekularer 10

Palladium-katalysierter Kreuzkupplung und intermolekularer DielsAlder-Reaktion

1.1. Aufbau von Hexahydroindengerüsten durch Enin-Cycloisomerisierung und Diels-Alder-Reaktion

1.1.1. Darstellung der Cyclisierungsvorläufer und Cyclisierung 14

1.1.2. Untersuchungen zum Einfluß von Substituenten an Position 4 des Hexahydroindengerüstes

1.1.3. Variation der Dienophile und Untersuchung des Einflusses auf die Diastereoselektivität und Regioselektivität der Reaktion

1.2. Synthese von Tricyclen durch einen Domino-Prozeß aus Cycloisomerisierung, Diels-Alder-Reaktion und Lactonisierung

1.3. Untersuchung von Heck-Diels-Alder-Domino-Reaktionen unter Zusatz von Lewis-Säuren

1.3.1. Verwendung von Cyclopentenon als Dienophil

2. Synthese von Heterocyclen durch Einsatz heteroatomhaltiger Vorläufer

2.1. Aufbau von Hexahydroindol- und Hexahydroisoindolgerüsten

2.2. Verwendung von Sulfonamiden als Cyclisierungsvorläufer

2.3. Darstellung eines sauerstoffhaltigen Cyclisierungsvorläufers und Versuche zu dessen Cyclisierung

3. Aufbau von Heterocyclen durch Kombination von Palladiumkatalysierter Cyclisierung und Diels-Alder-Reaktion mit Heterodienophilen 
3.1. Vorüberlegungen 42

3.1.1. Einsatz von in situ erzeugten Iminiumionen als Heterodienophile in Heck-Diels-Alder-Reaktionssequenzen

3.1.2. Versuch einer Domino-Cycloisomerisierung-Hetero-Diels-AlderReaktion

3.2. Versuch zur Verwendung von Trimethylsilylaldiminen als Heterodienophile

3.3. Versuch zum Aufbau von sauerstoffhaltigen Heterocyclen

4. Heck-Reaktionen an Bromeninen mit terminaler Dreifachbindung

5. Darstellung eines Cyclisierungsvorläufers zum Aufbau eines Spinosyn-Gerüstes und dessen Umsetzung

5.1. Vorüberlegungen

5.2. Versuche zur Synthese eines Vorläufers mit Silylenolether-Funktion

5.3. Synthese eines Vorläufers mit Vinylchlorid-Terminus

6. Palladium-katalysierte Reaktionen von 1-Chlor-1-ethenylcyclopropan-Derivaten

6.1. Vorbemerkungen

6.2. Synthese der Ausgangsverbindungen 68

6.2.1. Verwendung von Natriumformiat als Hydridquelle 70

6.2.2. Verwendung von $n$-Butylzinkchlorid als Hydridquelle 71

6.3. Versuch zur Durchführung von Folgereaktionen des Allylsilans 72 (E)-224

6.3.1. Versuche einer Sakurai-Reaktion

6.3.2. Deprotonierung des Allylsilans (E)-224 und Versuch der Verwendung als Nucleophil

\section{Experimenteller Teil}


2. Darstellung der Verbindungen 77

2.1. Synthese der Enin-Cycloisomerisierungsvorläufer 77

2.2. Darstellung von Hexahydroindengerüsten durch Enin-Cyclo- 81 isomerisierung

2.3. Heck-Diels-Alder-Reaktionen unter Zusatz von Lewis-Säure 86

2.4. Darstellung heteroatomhaltiger Cyclisierungsvorläufer 89

2.5. Cyclisierung der heteroatomhaltigen Vorläufer 96

2.6. Verwendung von stickstoffhaltigen Heterodienophilen 104

2.7. Heck-Reaktionen an Bromeninen 112

2.8. Synthese eines Spinosyn-Vorläufers 115

2.9. Palladium-katalysierte nucleophile Substitutionen 128

$\begin{array}{ll}\text { D. Zusammenfassung } & 133\end{array}$

E. Literatur und Anmerkungen 136

$\begin{array}{ll}\text { F. Spektrenanhang } & 145\end{array}$

1. ${ }^{1} \mathrm{H}-\mathrm{NMR}-$ Spektren 146

2. ${ }^{13}$ C-NMR-Spektren 157

3. 2D-NMR-Spektren 168 


\section{A. Einleitung}

Schon seit frühester Zeit hat sich der Mensch die Chemie zunutze gemacht. Die ältesten bekannten chemischen Verfahren sind wohl Kochen, Backen und Braten, womit pflanzliche und tierische Nahrungsmittel in für den menschlichen Körper leichter verwertbare Formen überführt werden. Auch Farbstoffe waren seit alters bekannt, sei es für Höhlenmalereien oder dann später zum Färben von Kleidung. Doch anfangs entwickelte sich die Chemie nur durch Beobachtungen der Natur oder durch Zufälle. So wurde der Farbstoff Purpur von den Phöniziern, wie die Legende berichtet, nur durch Zufall entdeckt, als ein Hund eine Purpurschnecke zerbissen hatte, woraufhin sich nach einiger Zeit seine Lefzen violett verfärbten. Bis es den Menschen jedoch möglich war, solche Vorgänge zu verstehen und zu erklären, verstrich viel Zeit. Das Brennen von Tonen war zur Herstellung von Wasserkrügen schon frühzeitig bekannt, bis man aber wußte, daß dabei eine Polykondensation von Aluminiumsilicaten stattfindet, vergingen Jahrtausende. Als man jedoch einfache Vorgänge zu verstehen begann, und auf andere Prozesse übertragen konnte, setzte eine explosionsartige Entwicklung ein. Waren Mitte des 19. Jahrhunderts einige hundert chemische Substanzen bekannt, so gibt es heute einige Millionen von chemischen Verbindungen.[1] Aufgrund dieser Entwicklungen wachsen in der heutigen Zeit die Anforderungen an die organische Synthesechemie ständig, so daß neue Technologien notwendig sind, damit auch der Ablauf komplexer Vorgänge leichter verstanden und somit planbar gemacht werden kann. Das eigentliche Ziel ist es dabei, möglichst effiziente und kostengünstige Synthesen zu entwickeln. Die Effizienz einer Reaktion wird heute u. a. mit dem von Trost eingeführten Begriff der Atomökonomie beschrieben.[2] Während die Diels-Alder-Reaktion[3] unter diesem Aspekt zu den leistungsfähigsten klassischen Methoden zählt, macht sich die am schnellsten wachsende moderne Methodik die Übergangsmetall-katalysierten Reaktionen zunutze. Oftmals dienen Übergangsmetall-katalysierte C-C-Verknüpfungsreaktionen als Schlüsselschritt in der Totalsynthese biologisch aktiver Naturstoffe, da sie häufig auch in Anwesenheit vielfältiger funktioneller Gruppen anwendbar sind. Reaktionen, die bei selektiver C-CBindungsbildung von großer Bedeutung sind, sind z. B. die Pauson-Khand-Reaktion, bei der $\mathrm{Co}(\mathrm{CO})_{8}$-vermittelt Cyclopentenon-Gerüste aufgebaut werden ${ }^{[4]}$ oder Metathesereaktionen unter Verwendung Ruthenium-haltiger Katalysatoren. ${ }^{[5]}$ Nennenswert sind ebenfalls 
Chrom-haltige Fischer-Carbenkomplexe, die in vielfältiger Weise zum Aufbau von Naturstoffen oder naturstoffähnlichen Verbindungen genutzt werden.[6] Eine weitere effiziente Reaktion ist die Titan-vermittelte Kulinkovich-Reaktion zum Aufbau von Cyclopropanolen aus Estern und Cyclopropylaminen aus Amiden.[7]

Das jedoch bei weitem am häufigsten verwendete und untersuchte Übergangsmetall ist Palladium. Anfangs hauptsächlich bei Oxidationsreaktionen verwendet, $[8]$ erweiterte sich das Einsatzspektrum vor allem durch die von Heck[9] und unabhängig davon von Mizoroki[9c] Ende der sechziger Jahren entdeckten Palladium-katalysierten Arylierung und Alkenyleriung von Alkenen. Mittlerweile ist die Heck-Reaktion zu einer der wichtigsten Palladium-katalysierten $\mathrm{C}-\mathrm{C}$-Verknüpfungsreaktionen geworden. Auch in der asymmetrischen Synthese lassen sich mit der Heck-Reaktion durch die Verwendung chiraler Liganden hohe Enantiomerenüberschüsse erzielen. [10]

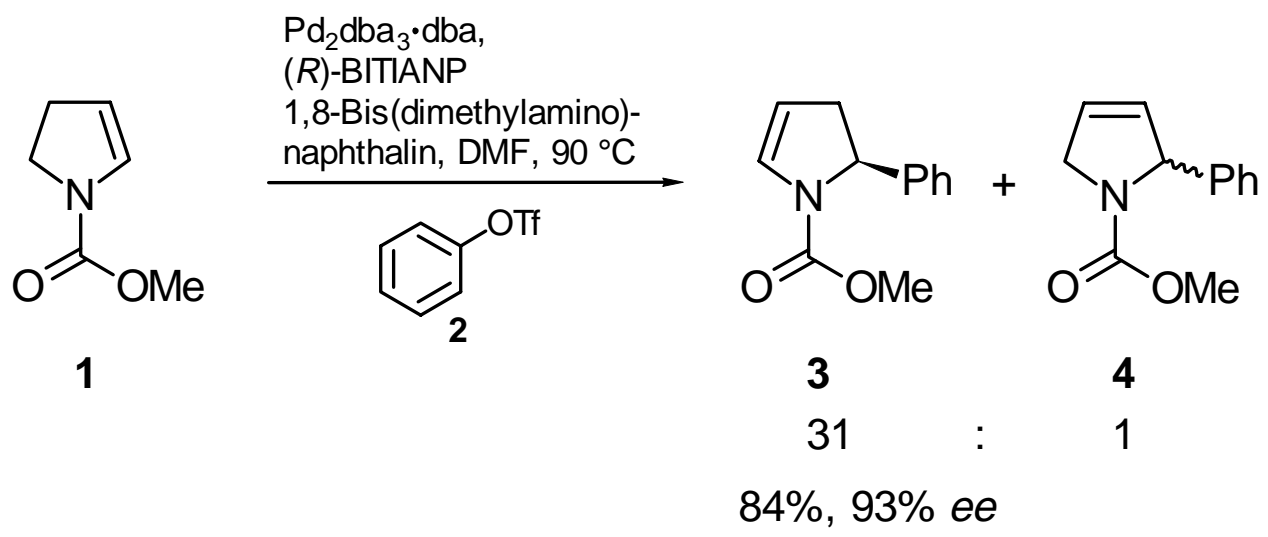

Schema 1. BITIANP = 2,2-Bis(diphenylphosphino)-3,3'-dibenzo $[b]$ thiophen.

Desweiteren kann die Alkenkomponente durch ein metalliertes Alken oder Aren ersetzt werden, wobei eine Transmetallierung auf den Palladiumkomplex der zentrale Schritt ist. Herausragende Bedeutung haben die Kreuzkupplung unter Verwendung von Zinnorganylen (Stille-Reaktion), ${ }^{[11]}$ Boronsäurederivaten (Suzuki-Kupplung) ${ }^{[12]}$ und Grignard-Verbindungen (Kumada-Reaktion) ${ }^{[13]}$ erlangt. Auch Kupplungen an Alkine sind unter Cokatalyse von Kupfer möglich (Sonogashira-Reaktion). [14] 
Der Mechanismus der Heck-Reaktion ist noch nicht vollständig geklärt, jedoch existiert eine brauchbare Arbeitshypothese.[9]

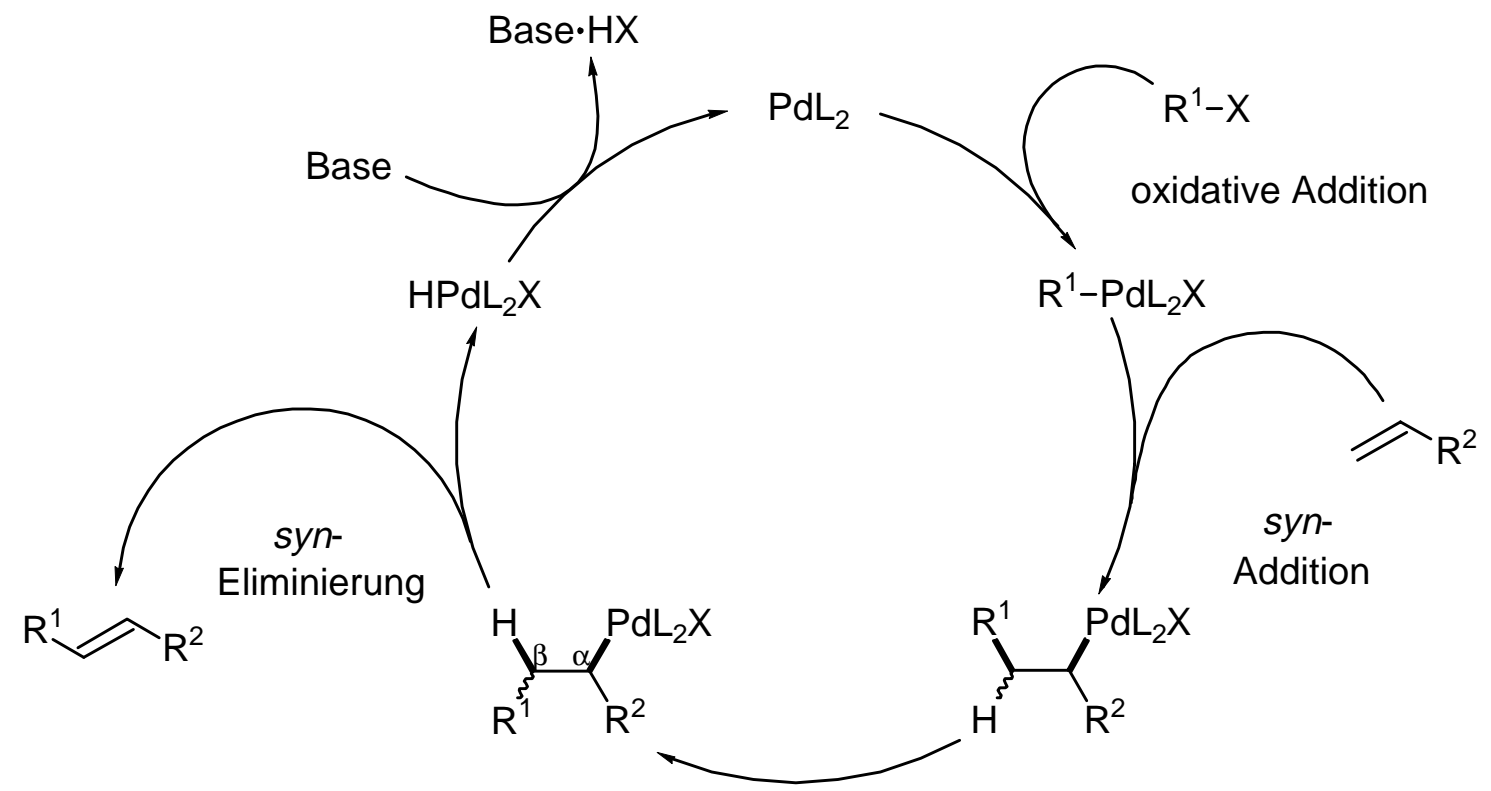

innere Rotation

Schema 2. Mechanismus der Heck-Reaktion.

Ein koordinativ ungesättigter $\operatorname{Pd}(0)$-Komplex wirkt dabei als katalytisch aktive Spezies, an die $\mathrm{R}^{1}-\mathrm{X}(\mathrm{X}=$ Halogen, Perfluoralkansulfonat) oxidativ addiert wird. Im zweiten Schritt erfolgt eine syn-Addition an ein Alken zu einem Alkylpalladiumkomplex. Die Addition findet mit hoher Regioselektivität statt, das Palladium wird bevorzugt an das höher substituierte Kohlenstoffatom geknüpft. Aus diesem Komplex kann durch eine $\beta$-Eliminierung Hydridopalladiumhalogenid abgespalten werden. Dies ist jedoch erst nach innerer Rotation möglich, da die Eliminierung ein $\beta$-Wasserstoffatom in synperiplanarer Stellung zum Metallzentrum benötigt. Die Regenerierung des Katalysators erfolgt durch die Abspaltung von Halogenwasserstoff mit Hilfe der Base.

In neuere Untersuchungen von Jutand und Amatore konnte gezeigt werden, daß bei Verwendung von Palladiumacetat und Triphenylphosphan ein anionischer Palladium(0)Komplex der Form $\mathrm{Pd}^{0}\left(\mathrm{PPh}_{3}\right)_{2}(\mathrm{OAc})^{-}$die reaktive Spezies darstellt.[91] 
Nacheinander ablaufende Transformationen unter Bildung reaktiver Intermediate, die Folgereaktionen eingehen, bezeichnet man als Domino-Reaktionen.[15] Mit Hilfe der Domino-Reaktionen die sowohl intra- als auch intermolekular ablaufen können, lassen sich in einer einzigen Reaktion komplexe Systeme aufbauen.

Domino-Heck-Reaktionen können stattfinden, wenn die $\beta$-Hydrideliminierung vermieden werden kann, z. B. durch Bildung von Neopentylpalladium-Intermediaten oder durch Carbopalladierung eines Alkins, wobei ein Alkenylpalladiumkomplex entsteht. Dabei ist es möglich, daß die Organopalladiumhalogenid-Spezies an weitere $\mathrm{C}-\mathrm{C}-$ Mehrfachbindungen addiert. Befindet sich in $\beta$-Stellung zum Palladium ein Wasserstoffatom, ist jedoch die Rotation eingeschränkt, so daß Wasserstoff und Palladium keine synperiplanare Anordnung einnehmen können, ist ebenfalls eine Weiterreaktion des Palladiumkomplexes realisierbar.[16]

In Gegenwart von $\mathrm{CO}$ kann eine Insertion in die Metall-Kohlenstoff- $\sigma$-Bindung unter Bildung eines $\sigma$-Acylpalladiumkomplexes stattfinden. Diese Komplexe können dann erneut eine Transmetallierung eingehen, wobei ein weiterer $\sigma$-Acylpalladiumkomplex entsteht, der dann durch reduktive Eliminierung zum Keton abreagiert. Anstelle der Transmetallierung kann der Komplex auch durch Nucleophile gespalten werden. Erfolgt diese Spaltung intramolekular durch Amine oder Alkohole, erhält man Lactame oder Lactone. Negishi et al. untersuchten eine Kaskade aus vierfacher Addition an Dreifachbindungen, CO-Insertion und intramolekularer Spaltung der Palladium-Spezies, wobei der Quintacyclus 6 in einer Ausbeute von 66\% isoliert werden konnte.[17]

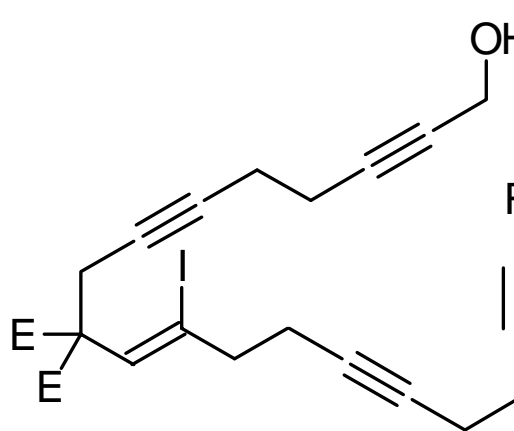

5<smiles>[R]C1=C2CCC3=C4CCC5=CCC(F)(F)CC5=C4CCC3=C2COC1=O</smiles>

6

Schema 3. $\mathrm{R}=t \mathrm{Bu}, \mathrm{E}=\mathrm{CO}_{2} \mathrm{Me}$. 
Eine große Klasse natürlich vorkommender oligocyclischer Verbindungen sind die Steroide. Die Biosynthese aller tierischen Steroide erfolgt durch die Cyclisierung von Squalen zum Lanosterin, im Pflanzenreich tritt an die Stelle des Lanosterins das Cycloartenol.

Zum Aufbau tricyclischer Verbindungen wurde von de Meijere und Meyer ein DominoProzeß aus zweifacher Heck-Reaktion und anschließender 6 6 -Elektrocyclisierung entwickelt.[9e,18] Während zunächst nur 5-6-5-Tricyclen dargestellt wurden, konnten durch weiterführende Untersuchungen von Henniges, Schweizer und Thies auch 6-6-5-, 7-6-5und 5-6-6-Tricyclen synthetisiert werden.[19]

Schweizer gelang es ebenfalls bei der Umsetzung von 7 einen 6-6-6-Tricyclus in guten Ausbeuten darzustellen. Dabei findet zweimal ein 6-exo-dig-Ringschluß und anschließend ein 6-endo-trig-Ringschluß statt,[20] der nach reduktiver Eliminierung zum Produkt 8 führt.[19c]

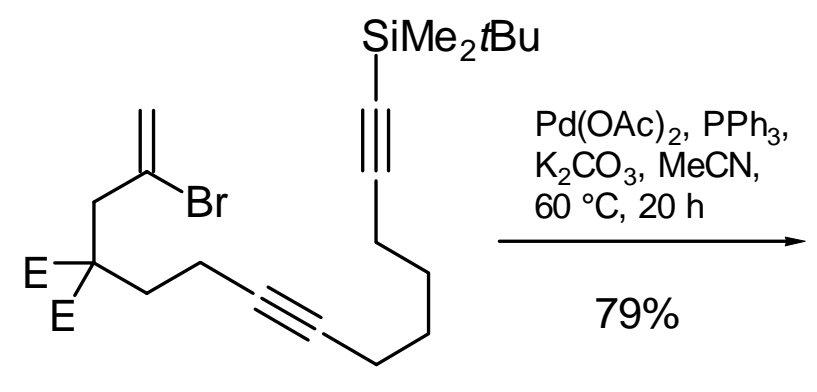

7<smiles>CSc1cc2c(c3c1CCCC3)CCCC(C(F)(F)F)(C(F)(F)F)C2</smiles>

8

Schema 4. $\mathrm{E}=\mathrm{CO}_{2} \mathrm{Me}$.

Vielfältige Variationen sind möglich, wenn man Palladium-katalysierte Kupplungen mit anderen Reaktionstypen im Sinne von Hetero-Domino-Reaktionen kombiniert. Intensiv untersucht wurden Domino-Prozesse aus Heck-Reaktionen und anschließender intra- oder intermolekularer Diels-Alder-Reaktion. Diese Sequenzen zeichnen sich vor allem dadurch aus, daß die Möglichkeit eines Eintopf-Verfahrens, bei dem sich das Dienophil von Beginn an im Reaktionsgemisch befindet, gegeben ist. Vergleiche zwischen Eintopf- und Zweischritt-Verfahren (das Dienophil wird erst nach beendeter Heck-Reaktion zugegeben) haben ergeben, daß die Ausbeuten deutlich höher sind, wenn auf eine Isolierung des intermediär gebildeten exocyclischen Diens verzichtet wird.[21] 
Grigg et al. zeigten, daß ein Domino-Prozeß aus intermolekularer Heck-Reaktion von 3-Iodpyridin (9) mit Dimethylallen (10) zu einem exocyclischen 1,3-Dien reagiert, welches in einer anschließenden Diels-Alder-Reaktion zum Produkt 13 umgesetzt werden kann, ohne $\mathbf{1 1}$ zu isolieren.[22]

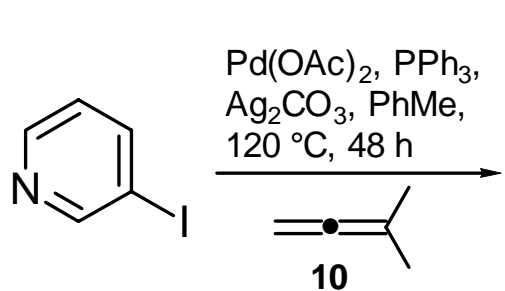

9<smiles>C=C(C)C(=C)c1cccnc1</smiles>

11<smiles>CC1=C(c2cccnc2)CC2C(=O)N(C)C(=O)C2C1</smiles>

13

Schema 5.

Eine der Heck-Reaktion sehr ähnliche C-C-Verknüpfungsreaktion ist die von Trost et al. entwickelte Palladium-katalysierte Enin-Cycloisomerisierung, die vor allem zum Aufbau fünfgliedriger Ringe geeignet ist.[23] Für den mechanistischen Ablauf der Reaktion existieren zwei Hypothesen, die je nach verwendetem Katalysatorsystem in Betracht kommen.[20a,b]

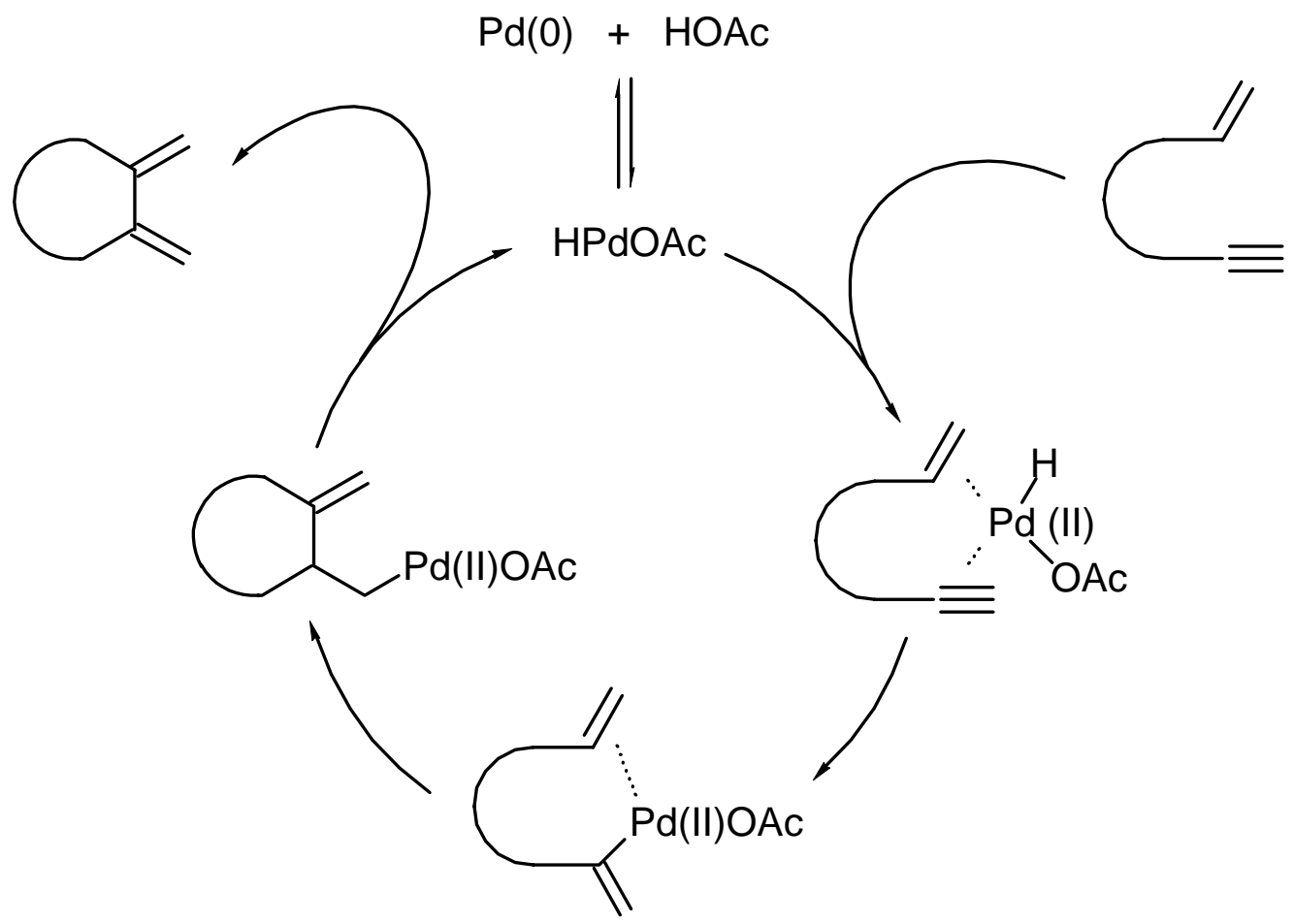

Schema 6. Mechanismus der Enin-Cycloisomerisierung bei Verwendung von $\operatorname{Pd}(0)$ und Essigsäure. 
Bei Verwendung einer $\operatorname{Pd}(0)-S p e z i e s$, wie z. B. $\operatorname{Pd}(\mathrm{dba})_{2}$ in Gegenwart einer schwachen Säure, meistens Essigsäure, wird zuerst die katalytisch aktive Spezies HPd(OAc) gebildet, die sich dann an die Alkineinheit addiert. Nach Koordination des Palladium-Komplexes an die Doppelbindung erfolgt anschließend Insertion zu einem Palladium- $\sigma$-Komplex. Nach einer formalen reduktiven Eliminierung wird der aktive Katalysator regeneriert.

Verwendet man eine Palladium(II)-Spezies, meist $\mathrm{Pd}(\mathrm{OAc})_{2}$, in Abwesenheit von Säure als Katalysator, ist ein $\mathrm{Pd}(\mathrm{II}) / \mathrm{Pd}(\mathrm{IV})-$ Mechanismus der wahrscheinlichere. Die $\mathrm{Pd}(\mathrm{II}) \mathrm{L}_{n^{-}}$ Spezies koordiniert gleichzeitg an Alkin- und Alkeneinheit, woraus dann nach Insertion ein Pallada(IV)cyclopenten resultiert. Dieser Komplex lagert sich zu einem HPd(IV)-Komplex um, der nach Dehydropalladierung das entsprechende 1,3-Dien und die katalytisch aktive Spezies zurückbildet.

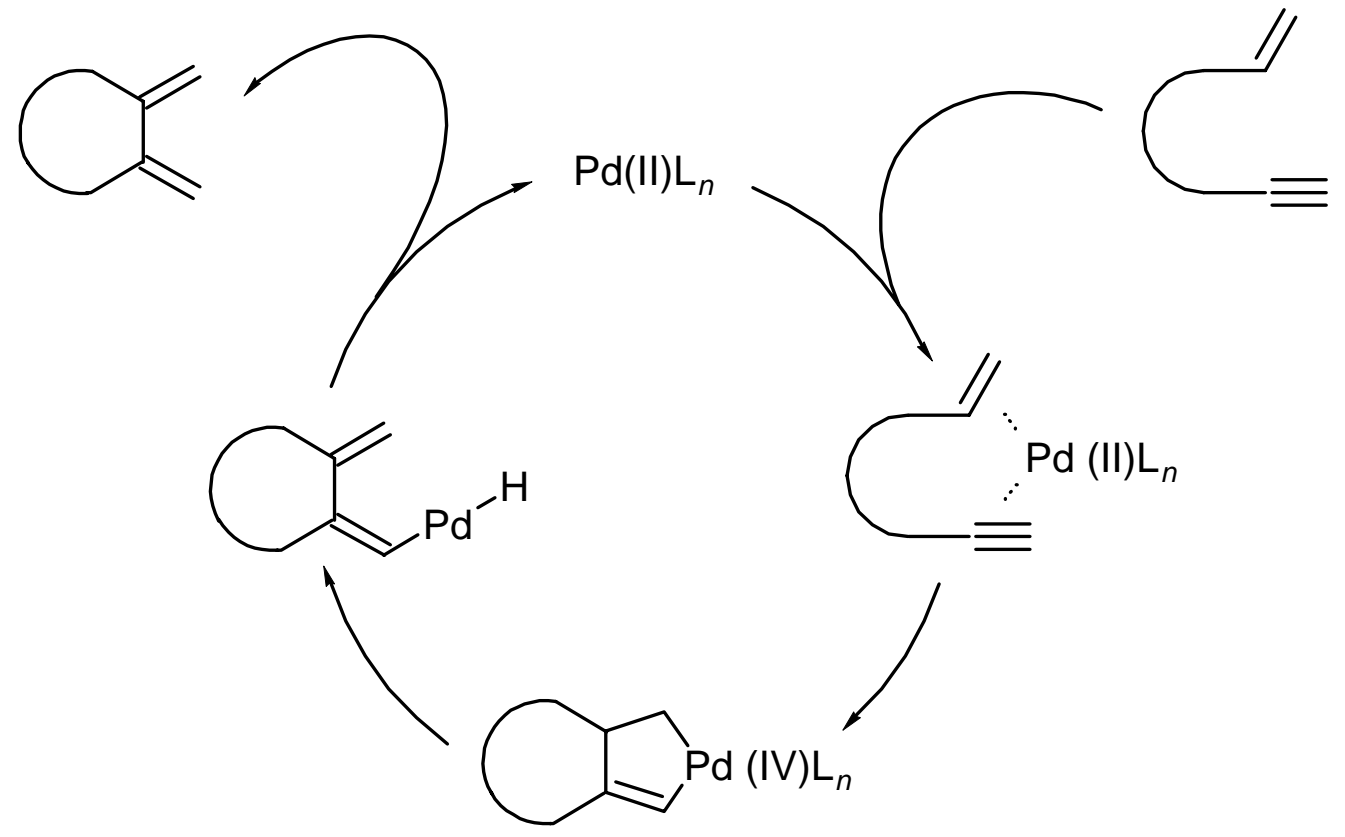

Schema 7. Mechanismus der Enin-Cycloisomerisierung bei Verwendung von $\mathrm{Pd}(\mathrm{II})$ in Abwesenheit von Säure.

Im Vergleich mit der Heck-Reaktion sind die Vorteile der Enin-Cycloisomerisierung die größere Atomökonomie, da keine Base benötigt wird, und die einfachere Synthese der Cyclisierungsvorläufer. Wie bei der Heck-Reaktion werden viele funktionelle Gruppen toleriert. Jedoch sind manche Substrate, an denen die Heck-Reaktion gelingt, für die Enin- 
Cycloisomerisierung nicht geeignet. Die Darstellung verschiedener Naturstoffe konnte mittels der Enin-Cycloisomerisierung realisiert werden. So konnten Trost et al. ausgehend von $R$-Carvon (14) in wenigen Schritten Picrotoxan-Sesquiterpene vom Typ 16 darstellen.[24]

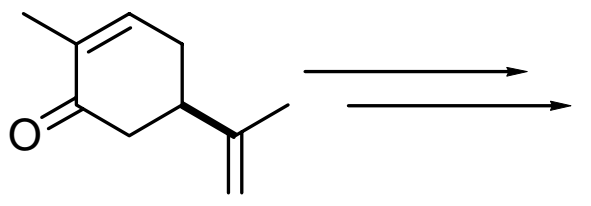

14

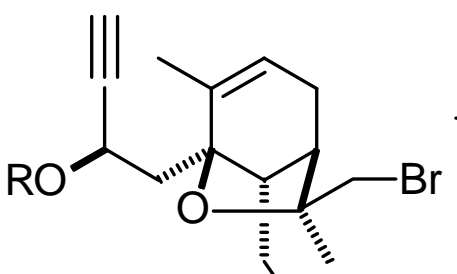

15
1) $\mathrm{Pd}(\mathrm{OAc})_{2}$, dpba, dbpp, DCE, $84^{\circ} \mathrm{C}$

2) TBAF, THF, RT

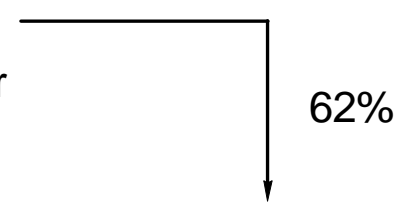

$62 \%$

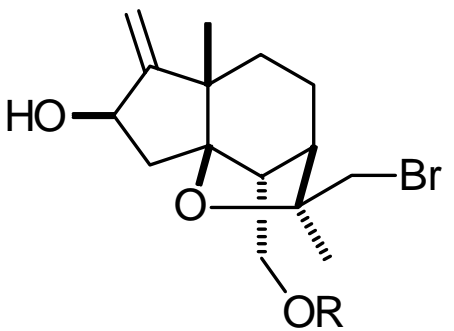

17 16

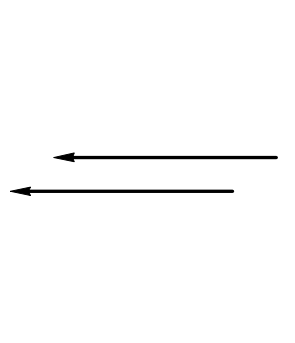

Picrotoxinin

Schema 8. $\mathrm{dpba}=2$-(Diphenylphosphino)benzoesäure, $\mathrm{dbpp}=1,3$-Bis(dibenzophospholyl)propan, DCE = 1,2-Dichlorethan, TBAF = Tetrabutylammoniumfluorid.

Von großem Interesse in der pharmazeutischen Forschung sind biologisch aktive Naturstoffe oder daraus abgeleitete Derivate für die Entwicklung neuer Medikamente. Dazu sind jedoch Totalsynthesen der Naturstoffe notwendig, damit der Medizin ausreichende Mengen zur Verfügung stehen, um mit genügend Material breite biologische Untersuchungen abzuschließen. Viele biologisch aktive Substanzen bestehen aus stickstoffhaltigen Heterocyclen. Daher untersuchten de Meijere et al. das Cyclisierungsverhalten stickstoffhaltiger Bromdiene vom Typ 18 und isolierten in mäßig bis guten Ausbeuten die entsprechenden Domino-Heck-Diels-Alder-Addukte 20.[25] 


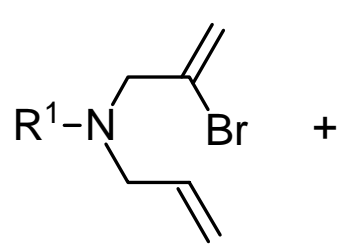

18<smiles>[R]C([R])=C([P])P</smiles>

19

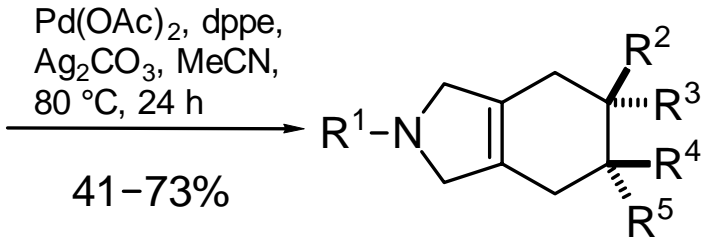

20

Schema 9.

Durch den Aufbau weiterer, geeigneter Vorläufer und deren Palladium-katalysierte Transformationen, sollten weitere Heterocyclen dargestellt werden, wobei die Heteroatome auch an anderen Positionen eingeführt werden sollten. Desweiteren sollte auch die Verwendung von Heterodienophilen in der Diels-Alder-Reaktion näher untersucht werden.

Die Aufgabenstellung dieser Arbeit ergibt sich damit wie folgt:

- Vergleich von Heck-Reaktion und Enin-Cycloisomerisierung in Abhängigkeit von Ausbeute, Nebenreaktionen, Einfachkeit der Darstellung der Ausgangsverbindungen und der Ringgröße.

- Einbau von Heteroatomen durch Verwendung heteroatomhaltiger Cyclisierungsvorläufer.

- Einbau von Heteroatomen durch Verwendung von Heterodienophilen.

- Untersuchungen der Ausbeuten und Selektivität der Diels-Alder-Reaktion bei Verwendung von Lewis-Säuren.

- Cyclisierungen von Bromeninen mit terminaler Dreifachbindung. 


\section{B. Hauptteil}

\section{Untersuchungen von Domino-Prozessen aus intramolekularer Palladium- katalysierter Kreuzkupplung und intermolekularer Diels-Alder-Reaktion}

Domino-Reaktionen, die eine Palladium-katalysierte C-C-Verknüpfung beinhalten, zählen zu den am intensivsten untersuchten Reaktionssequenzen der organischen Chemie. Führt man die Verknüpfung intramolekular durch, so können cyclische Systeme dargestellt werden. Die Ringgröße wird dabei von der Kettenlänge und Struktur des Cyclisierungsvorläufers bestimmt. Während die Darstellung von fünf- und sechsgliedrigen Ringen mittels Heck-Reaktion eingehend untersucht wurde[9] und in guten Ausbeuten realisierbar ist, sind Cyclisierungen zu mittleren Ringgrößen (8-12-gliedrig) bisher nur unter Ausbeuteverlusten möglich.[26] Die Bildung von großen Ringen (bis 21-gliedrig) ist in hochverdünnten Lösungen wieder leichter möglich.[27] Die Bildung eines Cyclobutans durch einen 4-exo-trig-Ringschluß gelang erstmals Larock in einer Heck-Monocyclisierung (Schema 10). [28]

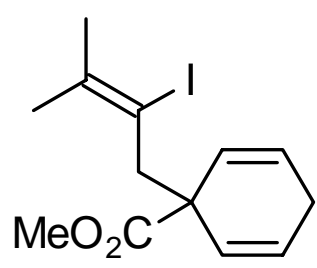

21
$10 \mathrm{~mol} \% \mathrm{Pd}(\mathrm{dba})_{2}$,

1.1 Äquiv. $n \mathrm{Bu}_{4} \mathrm{NCl}$,

2.5 Äquiv. $\mathrm{K}_{2} \mathrm{CO}_{3}$,

2 Äquiv. $\mathrm{H}_{2} \mathrm{C}\left(\mathrm{CO}_{2} \mathrm{Et}\right)_{2}$

DMSO, $100^{\circ} \mathrm{C}, 16 \mathrm{~h}$ $70 \%$

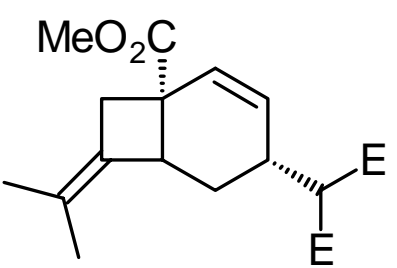

22

Schema 10. $\mathrm{E}=\mathrm{CO}_{2} \mathrm{Et}$.

Während die Bildungen kleiner Ringe i. a. als exo-Ringschlüsse verlaufen, finden bei der Cyclisierung von längerkettigen Vorläufern hauptsächlich endo-Ringschlüsse statt. Doch auch die exo-Cyclisierungen unterscheiden sich untereinander. So ist der 5-exo-trigRingschluß gegenüber dem 6-exo-trig-Ringschluß kinetisch bevorzugt, der seinerseits gegenüber der 7-trig-Cyclisierung favorisiert ist.[20]

Doch nicht nur die unterschiedliche Cyclisierungsneigung ist verantwortlich für die vielfältigen möglichen Reaktionsprodukte. Schon geringfügige Änderungen der Ketten- 
länge und der Substituenten können zu völlig unterschiedlichen $\mathrm{C}-\mathrm{C}$-Verknüpfungen führen. Einen weiteren entscheidenden Einfluß auf die Cyclisierung hat das Katalysatorgemisch. Je nach Wahl von Palladium-Katalysator, Ligand und zugesetztem Salz, können einzelne Schritte im Katalysecyclus verlangsamt oder beschleunigt werden.

Ein anderer effizienter Zugang zu cyclischen Systemen ist durch Palladiumkatalysierte Enin-Cycloisomerisierung möglich. Auch hierbei ist die Bildung von sechsgliedrigen Ringen gegenüber der von Cyclopentanderivaten deutlich benachteiligt. Trost et al. erklärten die geringere Cyclisierungsneigung zu Cyclohexanderivaten mit einer höheren Cyclisierungsentropie.[23b,c]

Frühere Untersuchungen von Steinig zeigten, daß der Aufbau von Hexahydroindengerüsten durch eine Sequenz aus intramolekularer Cyclisierung und intermolekularer Diels-AlderReaktion leicht zu verwirklichen ist (Schema 11).[29]<smiles>C=CCC(F)(F)CC(=C)Br</smiles>

23

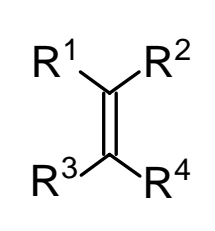

24

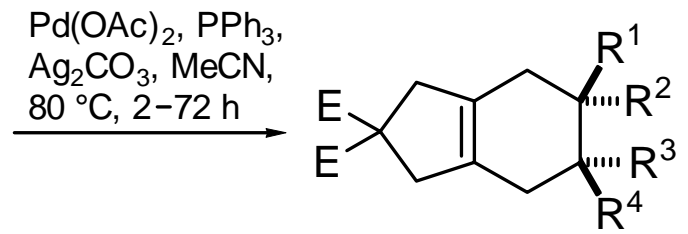

25

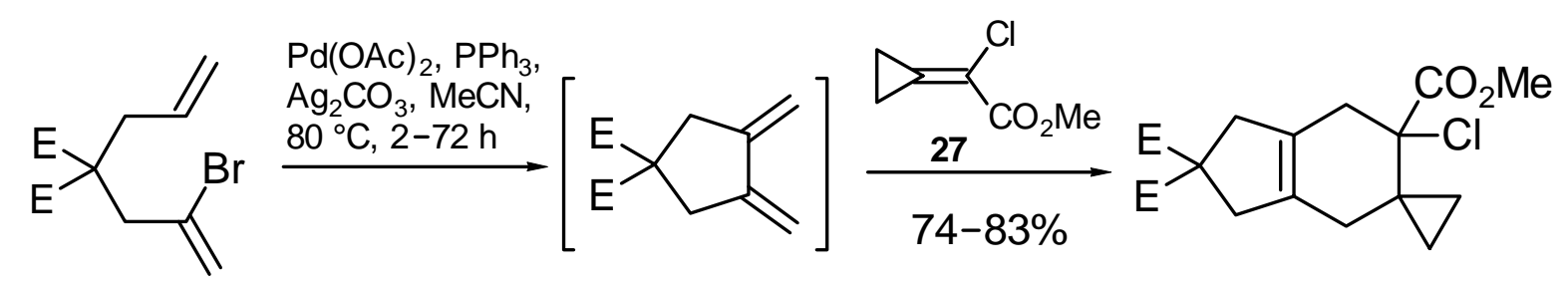

Schema 11. $\mathrm{E}=\mathrm{CO}_{2} \mathrm{Me}$. 
1.1. Aufbau von Hexahydroindengerüsten durch Enin-Cycloisomerisierung und DielsAlder-Reaktion

In einer im Laufe der eigenen Diplomarbeit durchgeführten Untersuchung wurden die Enine 32 und 34 dargestellt (Schema 12).[21c] Die Addition von tert-Butyldimethylsilylgeschütztem Propargylalkohol als Lithiumacetylid an 2,2-Dimethyl-4-penten-1-al (31) ergab den Cyclisierungsvorläufer 32, die Kupplung von 31 mit ungeschütztem Propargylalkohol führte zu 33, nach anschließender Schützung mit Pivaloylchlorid erhielt man den Vorläufer 34.

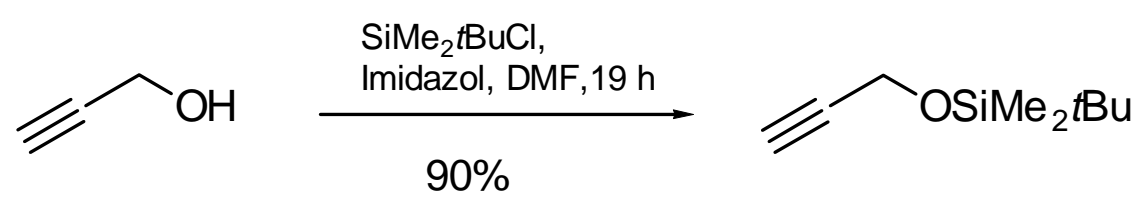

29

30
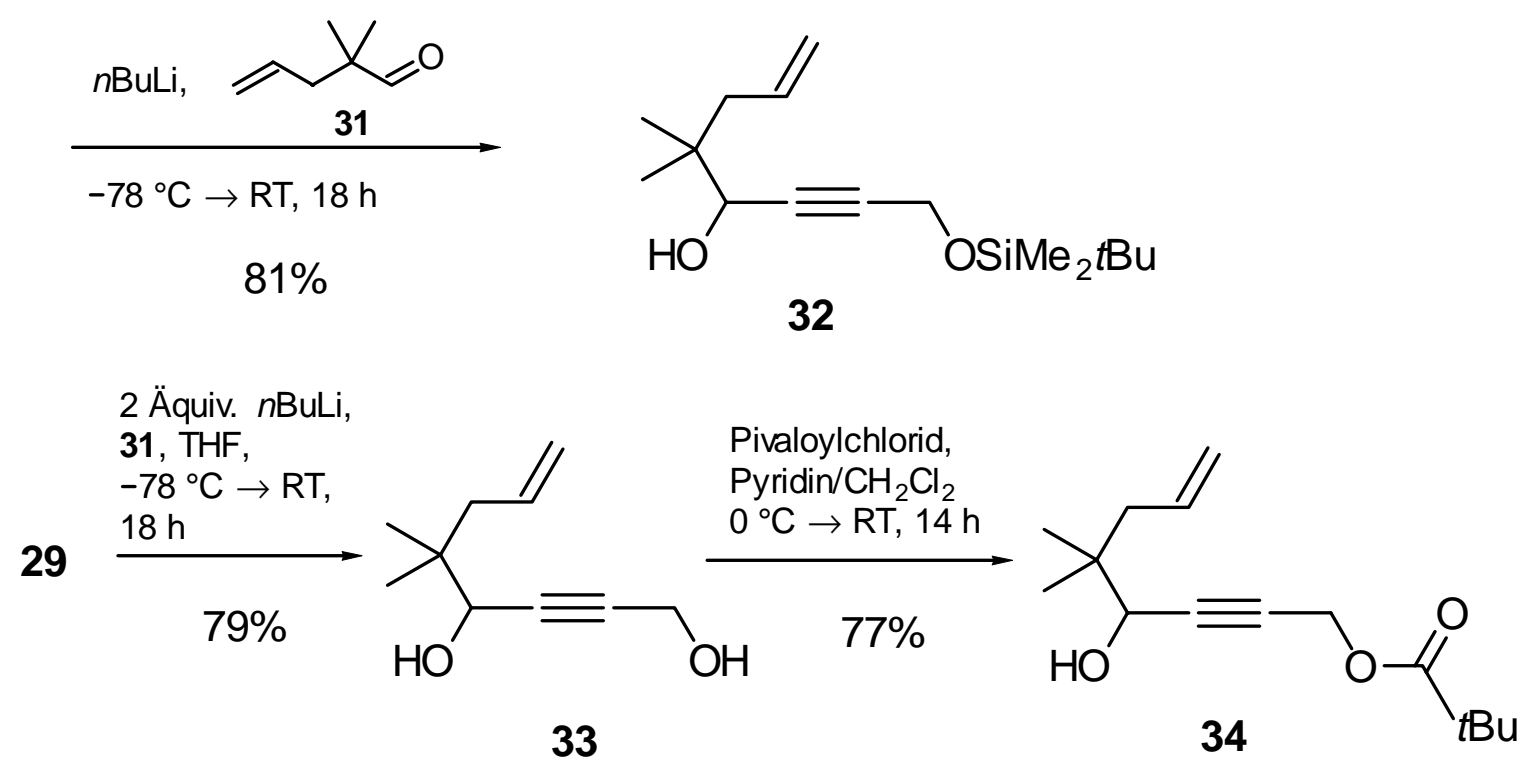

Schema 12.

Die Umsetzung von 32 und 34 mit Palladiumacetat in Gegenwart von Methylacrylat (35) ergab die Hexahydroindene $\mathbf{3 6}$ und $\mathbf{3 7}$ in Form zweier diastereomerenreiner Regioisomere in guten Ausbeuten (Schema 13, Tabelle 1). Während die Cyclisierung der Enine 32 und 34 in Gegenwart von $5 \mathrm{~mol} \% \quad \mathrm{Pd}(\mathrm{OAc})_{2}, \quad 10 \mathrm{~mol} \% \quad N, N^{\prime}$-Bisbenzylidenethylendiamin 
(BBEDA) und 3 Äquivalenten Methylacrylat bereits untersucht wurde (Einträge 1 und 5), sollten noch weitere verschiedene Katalysatorsysteme getestet werden.

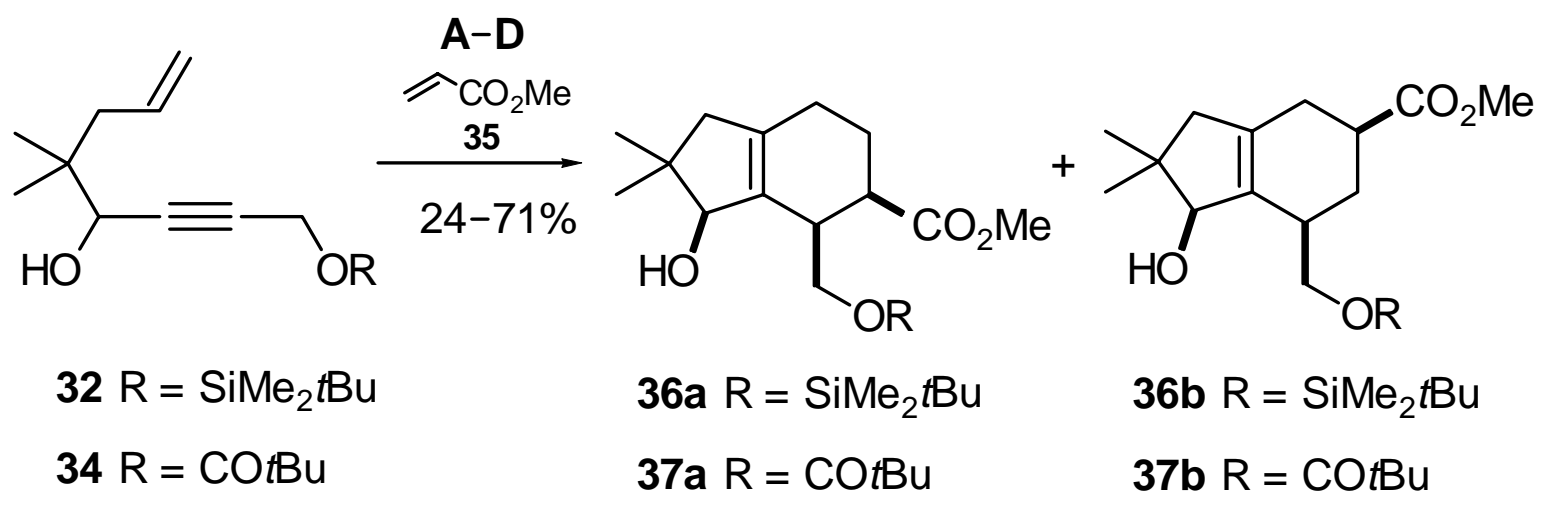

Schema 13.

Tabelle 1. Palladium-katalysierte Cycloisomerisierung von $\mathbf{3 2}$ und $\mathbf{3 4}$ unter verschiedenen Bedingungen.[a]

\begin{tabular}{|c|c|c|c|c|}
\hline Eintrag & Enin & Bedingungen ${ }^{[a]}$ & Produkt & Ausbeute $(\%)^{[b]}$ \\
\hline $1^{[21 c]}$ & 32 & A & 36a,b & 44 \\
\hline 2 & 32 & B & 36a,b & 35 \\
\hline 3 & 32 & C & - & $-[c]$ \\
\hline 4 & 32 & D & - & $-[c]$ \\
\hline $5[21 \mathrm{c}]$ & 34 & A & $37 \mathbf{a}, \mathbf{b}$ & 71 \\
\hline 6 & 34 & D & $37 \mathbf{a}, \mathbf{b}$ & 24 \\
\hline \multicolumn{5}{|c|}{ 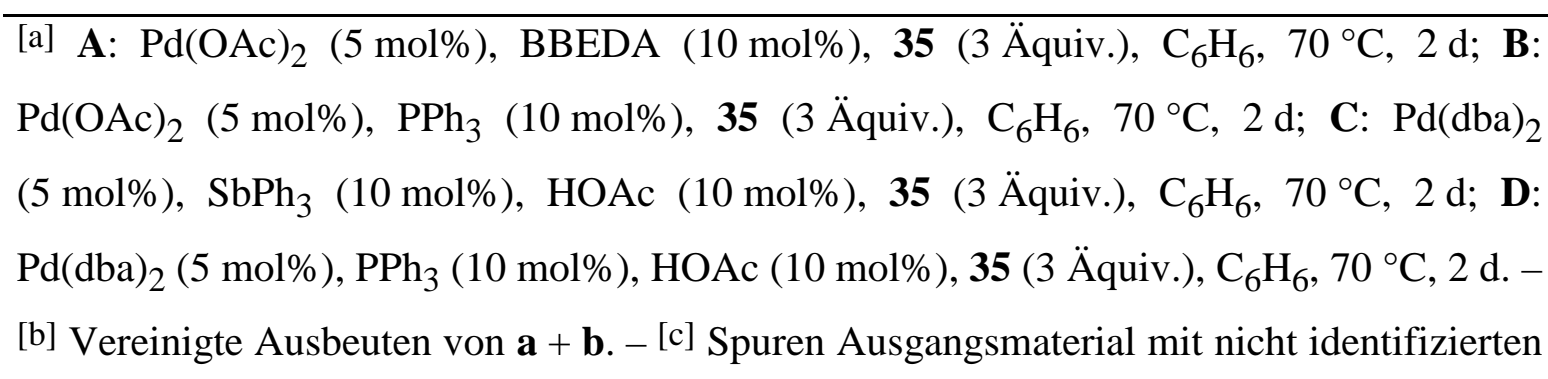 } \\
\hline
\end{tabular}


Setzte man 32 mit einem Katalysatorgemisch aus $\mathrm{Pd}(\mathrm{OAc})_{2}$ und Triphenylphosphan um, so sank die Ausbeute von 44\% (bei Verwendung von BBEDA als Ligand) auf 35\% (Eintrag 2). Bei der Cyclisierung von Enin 32 mit 5 mol\% Pd(dba), 10 mol\% Triphenylphosphan, 10 mol\% Essigsäure und 3 Äquivalenten Methylacrylat erhielt man keinerlei Produkt 36 (Eintrag 4). Auch bei Verwendung von Triphenylantimon anstelle von Triphenylphosphan, was nach Beobachtungen von Trost et al. bei disubstituierten Dreifachbindungen besser geeignet sein sollte,[23f] konnte keinerlei Cyclisierungsprodukt isoliert werden (Eintrag 3).

Setzte man hingegen das Enin 34 mit 5 mol\% Pd(dba), 10 mol\% Triphenylphosphan, 10 mol\% Essigsäure und 3 Äquivalenten Methylacrylat um, so konnten in 24\% Ausbeute die Hexahydroindene 37a und 37b isoliert werden (Eintrag 6). Die Ausbeute lag jedoch deutlich unter der bei Verwendung des Katalysatorsystems $\mathrm{Pd}(\mathrm{OAc})_{2}$ und BBEDA erhaltenen Ausbeute von 71\% (Eintrag 5).

Generell läßt sich sagen, daß die Ausbeuten für carbocyclische Systeme mit beidseitig substituierter Dreifachbindung bei Verwendung von Palladium(II)-Katalysator und Ligand ohne Zusatz von Säure deutlich höher sind. Dabei wird wahrscheinlich der in Schema 7 dargestellte Mechanismus durchlaufen. Bei Einsatz einer Palladium(0)-Spezies, Ligand und Säure wird ein anderer Mechanismus durchlaufen (Schema 6).[23a,b]

Aufgrund der einfacheren Synthesesequenz wurden die propargylischen $\mathrm{OH}-\mathrm{Gruppen}$ in Kauf genommen. Dies stellt jedoch ein strukturelles Problem dar, da so bereits im Cyclisierungsvorläufer ein stereogenes Zentrum sowie ein prochirales Zentrum vorhanden sind.

Daher sollten in weiterführenden Studien verschieden substituierte Enine dargestellt und das unterschiedliche Cyclisierungsverhalten untersucht werden.

\subsubsection{Darstellung der Cyclisierungsvorläufer und Cyclisierung}

Zunächst sollte die Enin-Cycloisomerisierung an einem Vorläufer ohne propargylische OH-Gruppe untersucht werden. Dazu wurde das Enin 41 mit geminalen Methylgruppen, die die Cyclisierung durch den Thorpe-Ingold-Effekt [30] unterstützen sollten, gewählt. 
Ausgehend von Isophoronoxid (38) wurde das Alkin 39 durch Epoxyketon-AlkinonFragmentierung nach Eschenmoser in 63\% Ausbeute dargestellt.[31] Dazu stellte man aus dem Keton 38 bei $0{ }^{\circ} \mathrm{C}$ das entsprechende Tosylhydrazon dar, das dann beim Erwärmen auf Raumtemperatur unter Abspaltung von Stickstoff zum Alkinon 39 fragmentierte. Anschließende Hydrierung mit Lindlar-Katalysator, der mit Chinolin vergiftet wurde, ergab das Alken 40 in 90\% Ausbeute. Das Alken wurde dann mit Diethylchlorophosphat in das kinetische Enolphosphat überführt, welches nach Zusatz von 2 Äquivalenten Lithiumdiisopropylamid eine $\beta$-Eliminierung zum Alkin 41 durchlief, das man in einer nur geringen Ausbeute von $22 \%$ erhielt (Schema 14). Negishi et al. konnten bei der Umsetzung von 2-Octanon unter den hier beschriebenen Bedingungen das entsprechende Alkin ebenfalls nur in 23\% Ausbeute isolieren. [32] Die Gründe dafür könnten einerseits in der Bildung des thermodynamisch bevorzugten Enolats liegen, andererseits könnte der $\beta$-Eliminierungsschritt für die schlechten Ausbeuten verantwortlich sein. Negishi et al. isolierten als Nebenprodukt das entsprechende Allen, was die letztere Vermutung noch bestärkt. Die Verwendung von sterisch anspruchsvolleren Basen, wie z. B. Lithiumtetramethylpiperidid, könnte Abhilfe schaffen.

Die anschließende Cyclisierung in Gegenwart von $5 \mathrm{~mol} \% \mathrm{Pd}(\mathrm{dba})_{2}, 10 \mathrm{~mol} \%$ Triphenylphosphan und 10 mol\% Essigsäure ergab das Hexahydroinden 42 in guter Ausbeute von $60 \%$ (Schema 14).

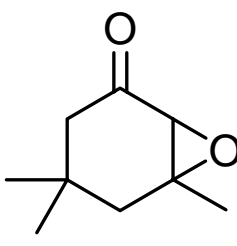

38

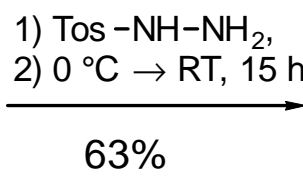

39

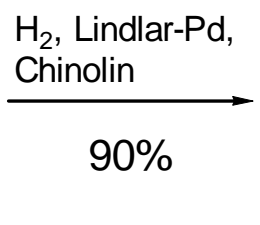<smiles>C=CCC(C)(C)CC(C)=O</smiles>

40

1) LDA, THF, $-78^{\circ} \mathrm{C}, 1 \mathrm{~h}$

2) $(\mathrm{EtO}){ }_{2} \mathrm{POCl}$

3) 2 Äquiv. LDA,

$-78^{\circ} \mathrm{C} \rightarrow \mathrm{RT}, 15 \mathrm{~h}$

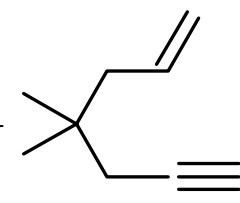

41<smiles>COC(=O)C1CCC2=C(C1)CC(C)(C)C2</smiles>
$60 \%$
42

Schema 14. 
Verwendete man als Katalysatorsystem $5 \mathrm{~mol} \% \mathrm{Pd}(\mathrm{OAc})_{2}$ und $10 \mathrm{~mol} \%$ BBEDA, so verringerte sich die Ausbeute auf $16 \%$. Im Vergleich mit früheren Untersuchungen zeigte sich, daß bei der Cyclisierung von Vorläufern mit terminaler Dreifachbindung unter Verwendung einer Palladium(0)-Spezies, Ligand und Säure zu carbocyclischen Systemen die Ausbeuten deutlich höher lagen als bei Verwendung von Palladium(II)-Katalysator und BBEDA ohne Zusatz von Säure. Umgekehrte Ergebnisse hatte man bei der Cyclisierung von Vorläufern mit beidseitig substituierter Dreifachbindung $\mathrm{zu}$ carbocycylischen Systemen erhalten (Tabelle 1).

Ebenfalls näher untersucht werden sollten die Cyclisierungen von Vorläufern vom Typ 44. Die Darstellung erfolgte ausgehend von einseitig Trimethylsilyl-geschützem Acetylen 43, welches mit $n$-Butyllithium deprotoniert wurde. Das so erhaltene Litiumacetylid wurde mit 2,2-Dimethylpent-4-en-1-al (31) versetzt, wobei man 44-SiMe 3 in 93\% Ausbeute isolierte (Schema 15). Zur Abspaltung der Trimethylsilylgruppe löste man 44-SiMe 3 in Methanol, fügte Kaliumcarbonat hinzu und rührte zwei Stunden bei Raumtemperatur. Nach Reinigung erhielt man das Enin 44-H mit terminaler Dreifachbindung in einer Ausbeute von $82 \%$.

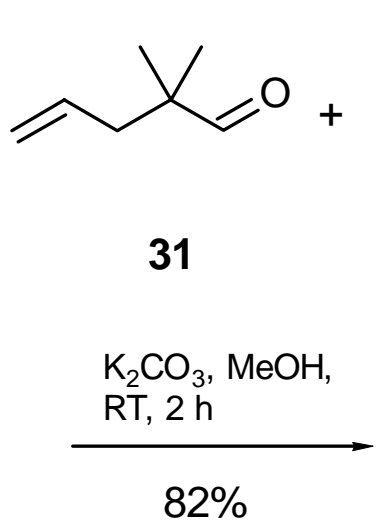<smiles>C#C[AsH3]</smiles>

43 $n B$ BuLi, THF, $-78^{\circ} \mathrm{C} \rightarrow \mathrm{RT}, 18 \mathrm{~h}$ $93 \%$

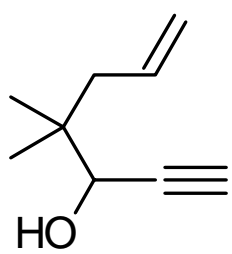

44-H

Schema 15.

Setzte man das Enin 44-SiMe 3 in Gegenwart von $5 \mathrm{~mol} \% \mathrm{Pd}(\mathrm{OAc})_{2}$ und $10 \mathrm{~mol} \%$ BBEDA mit Methylacrylat (35) in Benzol um, konnten die Hexahydroindene 45a-SiMe 3 und 45b-SiMe 3 in einer Ausbeute von $48 \%$ als säulenchromatographisch nicht trennbare 
Regioisomere im Verhältnis 1.3: 1 isoliert werden. Die erhaltenen Produkte bestanden aus jeweils nur einem Diastereomer (Schema 16).

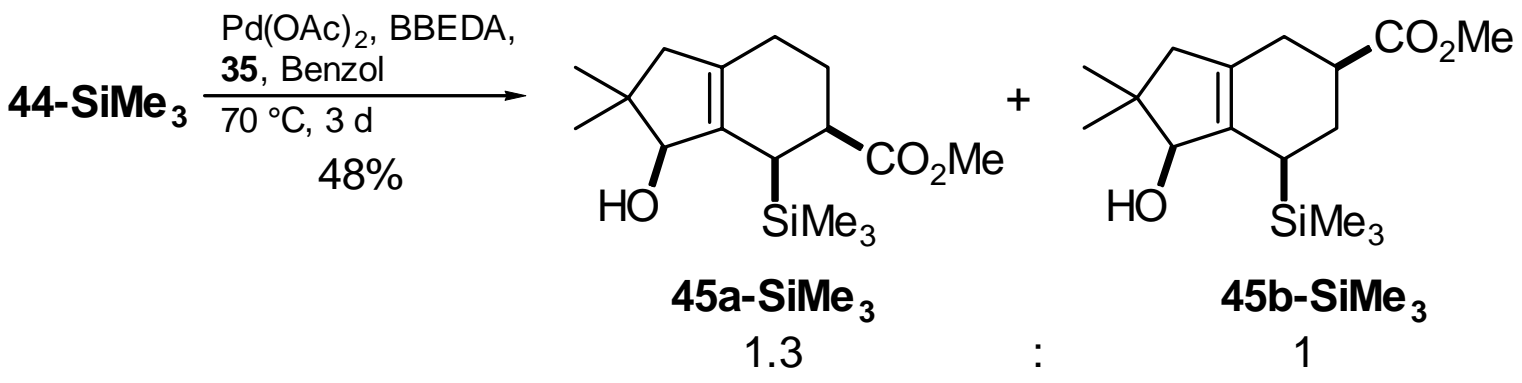

Schema 16.

Unter identischen Bedingungen wurde das Enin 44-H cyclisiert, dabei erhielt man als Produkt zwei jeweils aus zwei Diastereomeren bestehende Regioisomere in einer Ausbeute von 24\%. Das Verhältnis von 45a-H zu 45b-H betrug 1.2 : 1 (Schema 17).

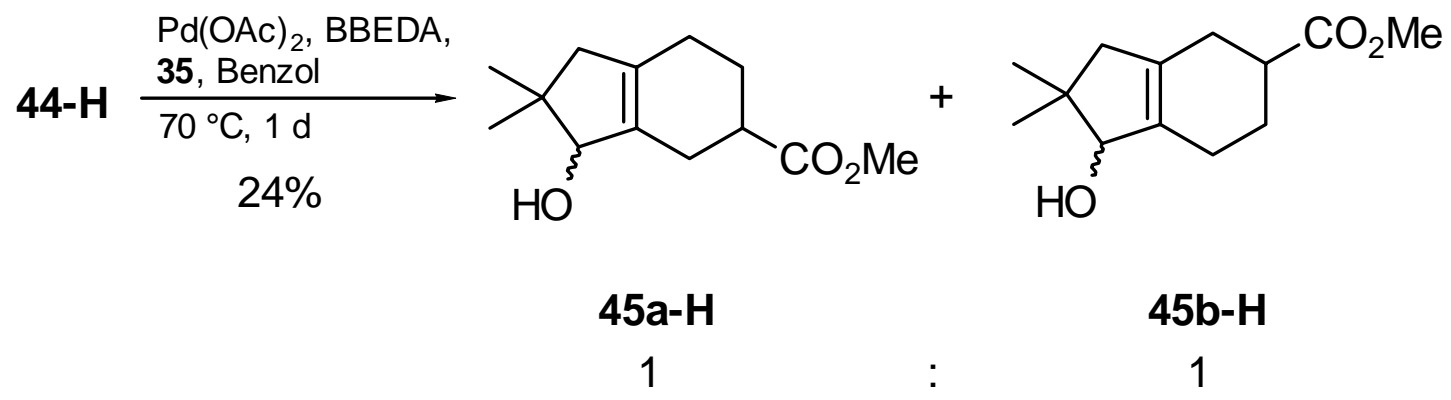

Schema 17.

1.1.2. Untersuchungen zum Einflu $\beta$ von Substituenten an Position 4 des Hexahydroindengerüstes

Für die Reaktivität und Regioselektivität der Diels-Alder-Reaktion sind vor allem die Grenzorbital-Wechselwirkungen zwischen dem LUMO (lowest unoccupied molecular orbital) des Dienophils und dem HOMO (highest occupied molecular orbital) des Diens verantwortlich. Die Reaktivität ist umso höher je geringer die Differenz zwischen HOMO und LUMO ist. Dies ist der Fall, wenn man ein elektronenreiches Dien mit einem 
elektronenarmen Dienophil zur Reaktion bringt. Trägt ein Dien elektronenspendende Substituenten oder Alkylgruppen, so wird die Elektronendichte erhöht und damit auch die Energie des HOMOs, was eine Reaktivitätssteigerung zur Folge hat.[33]

Die Regioselektivität einer Diels-Alder-Reaktion läßt sich mit Hilfe der Grenzorbitalkoeffizienten erklären. Dabei binden sich die Atome mit den jeweils kleineren Grenzorbitalkoeffizienten aneinander und ebenfalls die Atome mit den größeren Orbitalkoeffizienten. Alkylsubstituenten und elektronenspendende Gruppen in 1-Stellung eines Diens verringern den Grenzorbitalkoeffizienten an C-1, der Einfluß auf den Koeffizienten an C-4 ist vernachlässigbar.[34]

Betrachtet man die Verhältnisse der Regioisomere bei den Cyclisierungen von 32, 34 und 44-R, so beobachtet man je nach Substituent R die in Tabelle 2 dargestellten Selektivitäten.

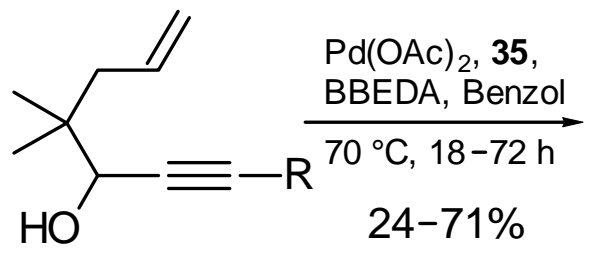

44-R

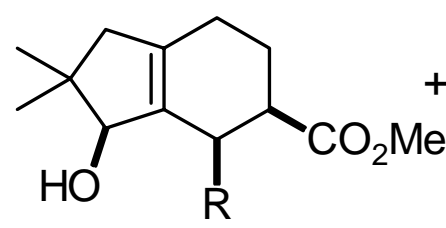

45a-R<smiles>COC(C)C1CC2=C(C(P)C(O)C2(C)[18F])C(C)(C)C1</smiles>

45b-R

Schema 18.

Tabelle 2. Verhältnisse der Regioisomeren 45a-R : 45b-R in Abhängigkeit vom Substituenten R.

\begin{tabular}{cccc}
\hline Eintrag & $\mathrm{R}$ & 45a-R : 45b-R & Ausbeute (\%) \\
\hline $1[21 \mathrm{c}]$ & $\mathrm{CH}_{2} \mathrm{OSiMe}_{2} t \mathrm{Bu}(\equiv \mathbf{3 2})$ & $1.4: 1$ & 44 \\
$2[21 \mathrm{c}]$ & $\mathrm{CH}_{2} \mathrm{OCO} t \mathrm{Bu}(\equiv \mathbf{3 4})$ & $2.9: 1$ & 71 \\
3 & $\mathrm{SiMe}_{3}$ & $1.3: 1$ & 48 \\
4 & $\mathrm{H}$ & $1.2: 1[\mathrm{a}]$ & 24 \\
\hline
\end{tabular}

[a] Die Regioisomere liegen als Gemisch von jeweils zwei Diastereomeren vor.

Die Ergebnisse zeigen, daß ein Substituent an der terminalen Dreifachbindung sowohl einen merklichen Einfluß auf die Regioselektivität als auch auf die Diastereoselektivität hat. Befindet sich an dieser Position ein Substituent, verläuft die Reaktion diastereoselektiv 
und es werden nur die all-cis Diastereomer gebildet (s. Tabelle 2). Der Einfluß ist mit dem Rest $\mathrm{R}=\mathrm{CH}_{2} \mathrm{OCO} t \mathrm{Bu}$ am größten (Eintrag 2). Sowohl bei dem Trimethylsilylrest als auch bei $\mathrm{R}=\mathrm{CH}_{2} \mathrm{OSiMe}_{2} t \mathrm{Bu}$ ist die Regioselektivität nur sehr gering (Einträge 1 und 3). Die sterischen Wechselwirkungen zwischen Dienophil und Substituent können die beobachtete Regioselektivität nicht erklären, da sonst die Produkte 45b-R bevorzugt gebildet werden sollten. Betrachtet man jedoch die Grenzorbitalkoeffizienten, entspricht diese Selektivität den theoretischen Vorhersagen. Wie bereits erwähnt, verringert ein Alkylrest den Grenzorbitalkoeffizienten an dem Kohlenstoffatom, an dem sich der Substituent befindet.

Ist $\mathrm{R}=\mathrm{H}$, ist die Regioselektivität noch geringer, die Regioisomere liegen zudem noch als Diastereomere vor. Auch die Ausbeute war mit 24\% deutlich geringer als bei den anderen untersuchten Systemen. Die Diastereomerenbildung entsprach jedoch den Erwartungen, da an einem dem Vorläufer 44-H entsprechenden Bromdien bereits Heck-Reaktionen durchgeführt wurden, die zu einem analogen Ergebnis führten. [21c]

Zusammenfassend lässt sich sagen, daß ein Substituent an Position 4 des Hexahydroindengerüstes notwendig ist, um die Bildung von Diastereomeren zu vermeiden. Verwendet man dabei als Substituent $\mathrm{R}=\mathrm{CH}_{2} \mathrm{OCO} t \mathrm{Bu}$, so erhält man neben höchster Regioselektivität auch die besten Ausbeuten.

Auf Untersuchungen von Systemen mit $\mathrm{R}=\mathrm{CH}_{2} \mathrm{OH}$ wird in Kapitel 1.2. näher eingegangen.

\subsubsection{Variation der Dienophile und Untersuchung des Einflusses auf die Diastereoselektivität und Regioselektivität der Reaktion}

Bereits in einer vorangegangenen Arbeit ${ }^{21 c]}$ wurde die Cyclisierung des Enins 34 unter Verwendung von Methylacrylat (35) und 2-Chlor-2-cyclopropylidenacetat (27) eingehend untersucht. Dabei beobachtete man, daß beim Einsatz von Methylacrylat als Dienophil zwei Regioisomere gebildet wurden, während bei Verwendung von 27 als Dienophil nur ein einziges Regioisomer entstand. Die Reaktion verlief jedoch in beiden Fällen diastereoselektiv. Um genauere Aussagen über den Einfluß des Dienophils auf die Regioselektivität machen zu können, wurde die gleiche Reaktion nun auch mit $\alpha$-Chlormethylacrylat (46) durchgeführt. Dabei löste man das Enin 34 in Benzol und versetzte mit $5 \mathrm{~mol} \% \mathrm{Pd}(\mathrm{OAc})_{2}, 10 \mathrm{~mol} \% \mathrm{BBEDA}$ und 3 Äquivalenten 46. Nach 18 Stunden bei $70{ }^{\circ} \mathrm{C}$ 
konnten die Hexahydroindene 47a und 47b im Verhältnis $4.5: 1$ in einer Gesamtausbeute von $45 \%$ isoliert werden (Schema 19).

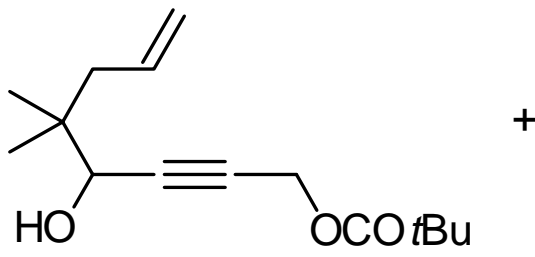

34<smiles>C=C(Cl)C(=O)OC</smiles>

46

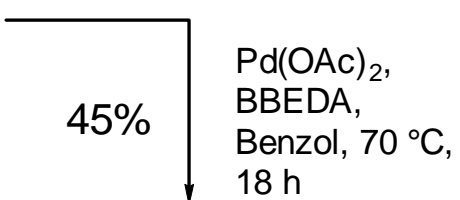
$18 \mathrm{~h}$
$47 a$

4.5<smiles>CCCCOCC1C[C@@](Cl)(C(C)=O)CC2=C1C(O)C(C)(C)C2</smiles>

47b

Schema 19.

Der Grenzorbitalkoeffizient eines Dienophils wird an dem Kohlenstoffatom verringert, das einen elektronenziehenden Substituenten trägt. Gleichermaßen erhöht sich der Grenzorbitalkoeffizient an dem anderen Kohlenstoffatom. [35]

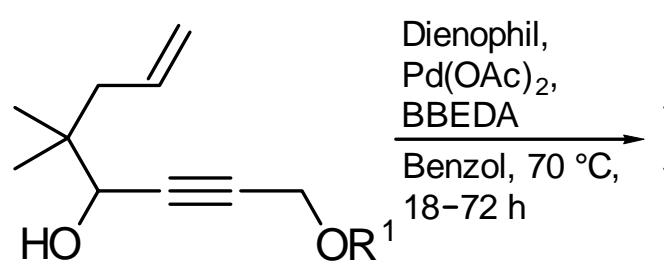

34<smiles>[R7]C[C@H]1C2=C(CC(C)(C)[C@@H]2O)CC([R7])([Z7])[C@]1([R7])F</smiles>

a<smiles>[R7]C[C@H]1C2=C(C[C@]([2H])(C)[C@@H]2O)C[C@]([R7])(F)[C@@]1([R7])F</smiles>

b

Schema 20. $\mathrm{R}^{1}=\mathrm{CO} t \mathrm{Bu}, \mathrm{E}=\mathrm{CO}_{2} \mathrm{Me}$. 
Tabelle 3. Regioisomerenverhältnis durch Enincycloisomerisierung mit nachfolgender Diels-Alder-Reaktion gebildeter Hexahydroindene in Abhängigkeit vom Dienophil.

\begin{tabular}{cccccccc}
\hline Eintrag & Dienophil & Produkt & $\mathrm{R}^{2}$ & $\mathrm{R}^{3}$ & $\mathrm{R}^{4}$ & Verhältnis a : b & Ausbeute (\%) \\
\hline $1[21 \mathrm{c}]$ & $\mathbf{3 5}$ & $\mathbf{3 7}$ & $\mathrm{H}$ & $\mathrm{H}$ & $\mathrm{H}$ & $2.9: 1$ & 71 \\
2 & $\mathbf{4 6}$ & $\mathbf{4 7}$ & $\mathrm{Cl}$ & $\mathrm{H}$ & $\mathrm{H}$ & $4.5: 1$ & 45 \\
$3[21 \mathrm{c}]$ & $\mathbf{2 7}$ & $\mathbf{4 8}$ & $\mathrm{Cl}$ & $\mathrm{CH}_{2}-\mathrm{CH}_{2}$ & $100: 0$ & 56 \\
4 & $\mathbf{4 9}$ & $\mathbf{5 0}$ & $\mathrm{Cl}$ & $\mathrm{CH}_{2}-\mathrm{CH}_{2}$ & - & $-[\mathrm{a}]$ \\
\hline
\end{tabular}

[a] $87 \% 49$ konnte zurückisoliert werden.

Im Vergleich zu der Reaktion mit Methylacrylat (35) erhöhte sich die Regioselektivität bei der Reaktion mit $\alpha$-Chlormethylacrylat (47) auf 4.5 : 1 (Schema 20, Tabelle 3). Dies läßt sich dadurch erklären, daß der zusätzliche $\alpha$-Chlorsubstituent durch einen elektronenziehenden Effekt die Grenzorbitalkoeffizienten in der gleichen Richtung beeinflusst wie die Methylgruppen im Methylacrylat. Bezogen auf die Regioselektivitäten erhielt man das beste Ergebnis bei Verwendung des 2-Chlor-2-cyclopropylidenacetats (27) als Dienophil, wobei nur ein einziges Regioisomer 48a gebildet wurde. Das Gerüst von 48a hat Ähnlichkeit mit den Sesquiterpenen Illudin-S und -M,[36] bei denen der dreigliedrige Ring sich in Nachbarschaft zu einer Methylgruppe befindet. Bei 48a befindet sich Dreiring jedoch in quasi-meta Stellung zum $\mathrm{CH}_{2} \mathrm{OCO} t \mathrm{Bu}$-Rest. Um das andere Regioisomer darzustellen, wurde tert-Butyl-2-chlor-2-cyclopropylidenacetat (49) eingesetzt. Man hoffte, daß die vergrößerten sterischen Ansprüche für eine Inversion der Regioselektivität führen. Dieser Versuch schlug jedoch fehl, man konnte nach einer Reaktionsdauer von drei Tagen 87\% des Dienophils 49 zurückgewinnen. Offenbar fand keine Diels-Alder-Reaktion statt, da die stereoelektronischen Einflüsse eine Umkehr der Reaktion nicht ermöglichten. Desweiteren ist das intermediär gebildete exocyclische 1,3-Dien nicht über längere Zeit stabil, nach einer Reaktionsdauer von drei Tagen konnte nur noch Zersetzung festgestellt werden. 
1.2. Synthese von Tricyclen durch einen Domino-Proze $\beta$ aus Cycloisomerisierung, Diels-Alder-Reaktion und Lactonisierung

In weiteren Untersuchungen wurde überprüft, ob die freie Hydroxymethylgruppe im Enin 33 die Regioselektivität der [4 + 2]-Cycloaddition ebenfalls beeinflussen kann. Daher wurde das ungeschützte Diol unter den in Kapitel 1.1.2. beschriebenen Bedingungen der Enin-Cycloisomerisierung umgesetzt. Dabei erhielt man nicht die erwarteten bicyclischen Methylester, sondern das tricycische Lacton 52. Nach Cycloisomerisierung und DielsAlder-Reaktion wird das quasi-ortho Produkt 51 gebildet, das dann umgehend eine intramolekulare Umesterung zu 52 eingeht (Schema 21).

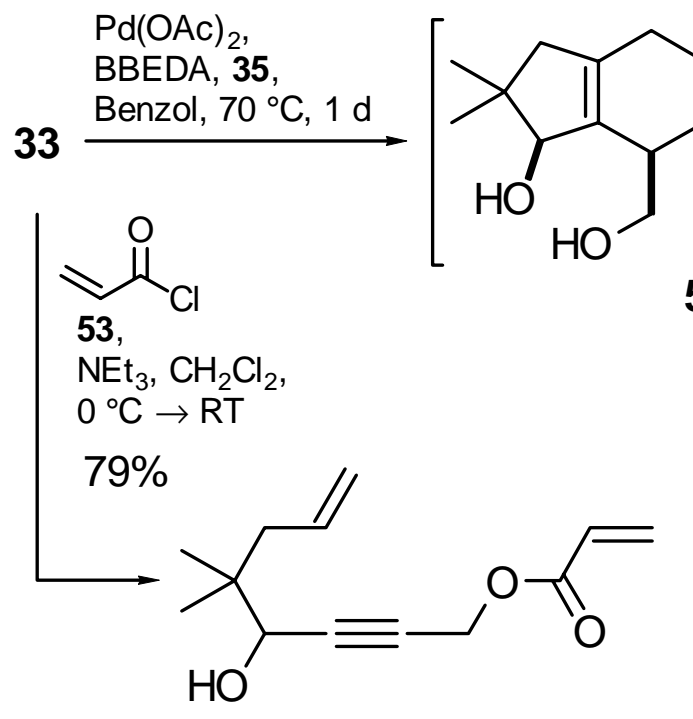

54

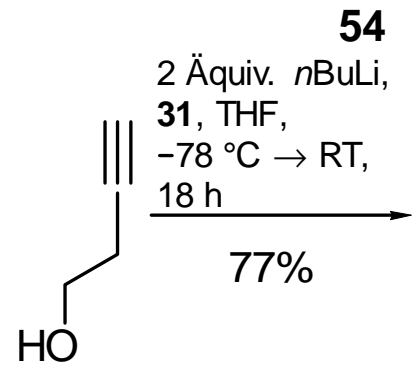

56

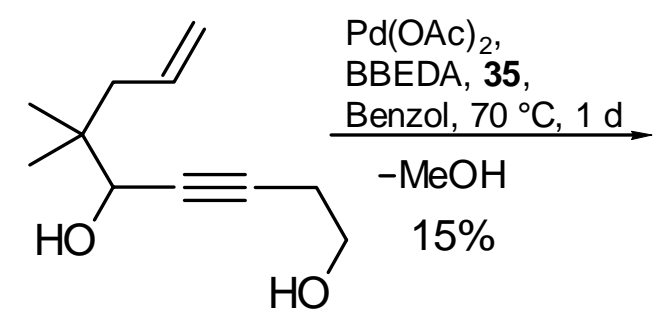

57<smiles>CC1(C)CC2=C(C1O)[C@H]1COC(=O)[C@@H]1CC2</smiles>

52

51

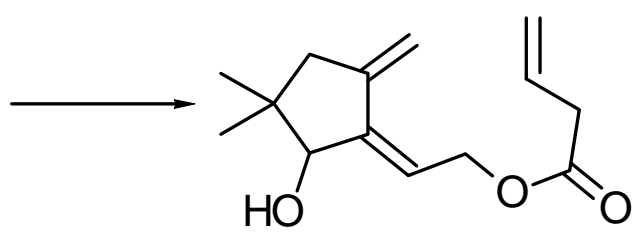

55<smiles>CC1(C)CC2=C(C1O)[C@H]1CCOC(=O)[C@@H]1CC2</smiles>

58

Schema 21. 
Ausgehend von 3-Butin-1-ol (56) konnte durch Deprotonierung mit $n$-Butyllithium und Alkylierung mit 31 das homologe Diol 57 in 77\% Ausbeute dargestellt werden. Eine Kaskade aus Cycloisomerisierung, Diels-Alder-Reaktion und Lactonisierung ergab das Sechsringlacton 58 in einer geringen Ausbeute von nur 15\% (Schema 21). Mit Hilfe von NOESY-Spektren (Nuclear Overhauser Effect SpectroscopY) konnte eindeutig ermittelt werden, daß der Lactonring cis-verknüpft wurde.

Bei den erreichten mäßigen Ausbeuten kann keine eindeutige Aussage über die Regioselektivität getroffen werden. Es wurden zwar nur die Lactone 52 und $\mathbf{5 8}$ isoliert, von einer ausschließlichen Bildung der Produkte zu sprechen, ist bei diesen geringen Ausbeuten aber nicht möglich.

Um die Ausbeute zu erhöhen, führte man die Reaktion in Gegenwart von 10 mol\% Titantetraisopropylat durch, das bekanntermaßen Umesterungen mit Alkoholen katalysiert.[37] Dabei könnte in situ das Acrylat 54 gebildet werden, das dann in einer Lactonisierung reagieren sollte. Dabei konnte jedoch kein $\mathbf{5 2}$ isoliert werden, vielmehr entstanden nur nicht identifizierte Oligomere neben Zersetzungsprodukten. Fraglich ist, ob das zugesetzte Titantetraisopropylat diese Polymerisation katalysierte, oder ob das in situ gebildete Acrylat 54 selbst zur Polymerisation neigte. Um dies näher untersuchen zu können, wurde das Enin 33 mit Acryloylchlorid (53) umgesetzt und das Acrylat 54 in $79 \%$ Ausbeute isoliert (Schema 21). Die Umsetzung von 54 unter den Bedingungen der Cycloisomerisierung ergab ebenfalls nur oligomeres Material und Zersetzung. Dabei ist nicht auszuschließen, daß eine Cycloisomerisierung stattfand und das gebildete Trien $\mathbf{5 5}$ polymerisierte. 
1.3. Untersuchung von Heck-Diels-Alder-Domino-Reaktionen unter Zusatz von Lewis-Säuren

Zur Erhöhung von Ausbeute und Selektivität bei Diels-Alder-Reaktionen ist der Einsatz von Lewis-Säuren in katalytischen bis stöchiometrischen Mengen lange bekannt und bereits eingehend untersucht worden. Die am häufigsten verwendeten Lewis-Säuren sind dabei $\mathrm{BF}_{3} \cdot \mathrm{OEt}_{2}, \mathrm{TiCl}_{4}, \mathrm{AlCl}_{3}$ und $\mathrm{ZnCl}_{2}$. Aber auch verschiedenste alkyl- und alkoxysubstituierte Aluminiumchloride und Titanderivate wurden mit unterschiedlichem Erfolg eingesetzt.[38] Auch die asymmetrische Induktion bei der Diels-Alder-Reaktion mit der bis zu vier neue Chiralitätszentren entstehen, ist vielfach bearbeitet worden. Ein Vorteil des Zusatzes von Lewis-Säuren bei einer Diels-Alder-Reaktion liegt darin, daß die Reaktion schon unter sehr milden Bedingungen (bei Temperaturen um $0{ }^{\circ} \mathrm{C}$ ) stattfindet. Bei Verwendung von chiralen Lewis-Säuren lassen sich hohe Enantiomerenüberschüsse erzielen.[3b]

Durch Koordination der Lewis-Säure an das Dienophil wird die Energie des LUMOs abgesenkt, was eine Reaktivitätserhöhung zur Folge hat. Der Grenzorbitalkoeffizient am $\alpha$-Kohlenstoffatom wird verkleinert, der Koeffizient am $\beta$-Kohlenstoffatom vergrößert (Abb. 1). Diese erhöhte Polarisierung führt zu einer Steigerung der Regioselektivität. [39]
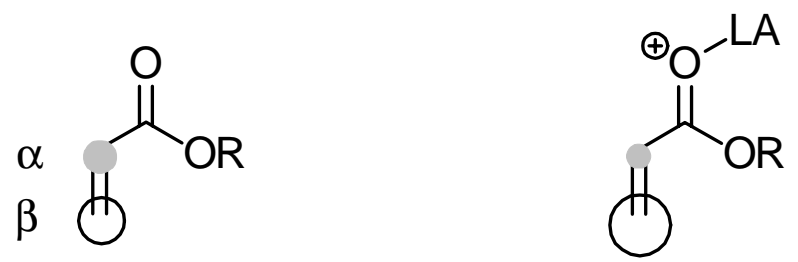

Abbildung 1. LA = Lewis-Säure. Einfluß von Lewis-Säure auf die Grenzorbitalkoeffizienten eines Dienophils.

Da die Heck-Diels-Alder-Reaktionssequenz des Bromdiens 59 mit verschiedenen Dienophilen unter rein thermischen Bedingungen immer Gemische aus Diastereomeren und Regioisomeren ergab, wurde diese Reaktion auch unter Zusatz einer Lewis-Säure durchgeführt.

Dazu versetzte man eine Lösung von 59 in Acetonitril mit 5 mol\% $\mathrm{Pd}(\mathrm{OAc})_{2}, 10 \mathrm{~mol} \%$ $\mathrm{PPh}_{3}$ und einem Äquivalent Kaliumcarbonat. Nach 18 Stunden bei $80^{\circ} \mathrm{C}$ filtrierte man das 
Katalysatorgemisch über Celite ab. Zu 3 Äquivalenten Methylacrylat in Acetonitril wurden vorsichtig 3 Äquivalente einer $1 \mathrm{M}$ Diethylaluminiumchlorid-Lösung in Hexan gegeben. Die Lösung färbte sich intensiv gelb und wurde 10-15 min bis zur vollständigen Komplexierung gerührt. $\mathrm{Zu}$ der erhaltenen Lösung wurde die Lösung des exocyclischen Diens 60 gegeben. Nachdem man einen Tag bei Raumtemperatur gerührt hatte, konnte das Tetrahydroinden $\mathbf{6 1}$ in 26\% Ausbeute als einziges Isomer neben Zersetzungsprodukten und Edukt 59 isoliert werden.

Diethylaluminiumchlorid wird vermutlich sowohl an das Dienophil als auch an die $\mathrm{OH}-$ Gruppe des exocyclischen Diens 60 koordiniert und bildet den Komplex 62, der dann in einer [4+2]-Cycloaddition zu $\mathbf{6 3}$ reagiert. Die Lewis-Säure bedingt auch eine anschließende Wassereliminierung zum konjugierten Dien 61 (Schema 22). Daher ist es auch nicht möglich, die Reaktion mit katalytischen Mengen Diethylaluminiumchlorid durchzuführen, da ein Äquivalent zur Eliminierung verbraucht wird.<smiles>C=CC(O)C(C)(C)CC(=C)Br</smiles>

59

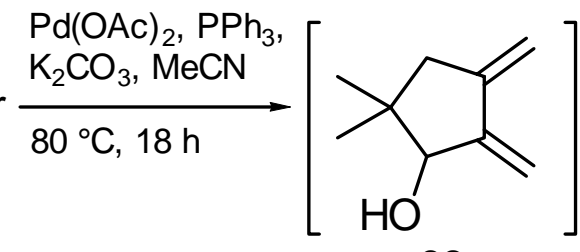

60

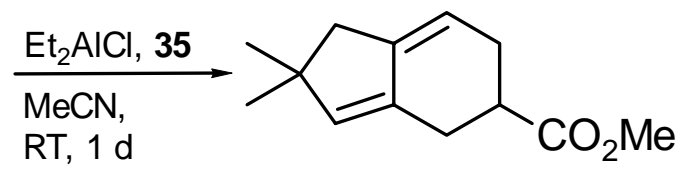

$26 \%$

61

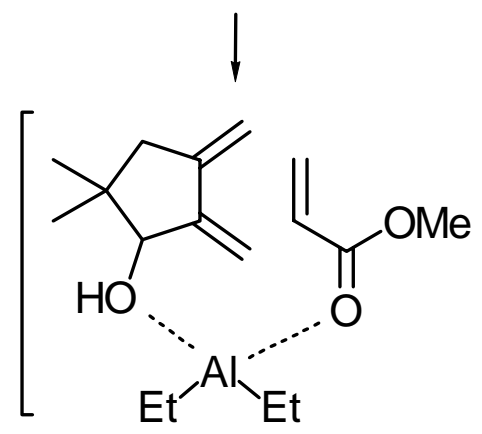

62

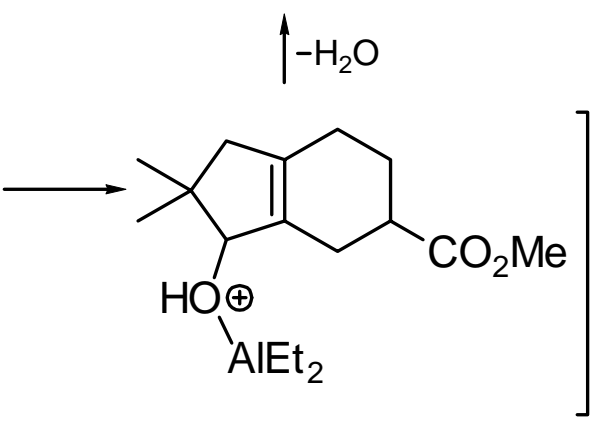

63

Schema 22. 


\subsubsection{Verwendung von Cyclopentenon als Dienophil}

Bei Benutzung von cyclischen Dienophilen wie Cyclopentenon in der beschriebenen Reaktionssequenz sollte der Aufbau von linear anellierten Tricyclen möglich sein. Dabei könnten Octahydro-s-indacenderivate vom Typ 65 gebildet werden. Cycloalkenone reagieren unter thermischen Bedingungen nur langsam mit Dienen, durch Zusatz einer Lewis-Säure kann die Reaktivität aber deutlich gesteigert werden.[40]

Deshalb wurden Heck-Diels-Alder-Domino-Sequenzen mit Cyclopentenon als Dienophil an dem schon bekannten System 59 unter Zusatz verschiedener Lewis-Säuren untersucht. Ebenfalls untersucht wurde das Reaktionsverhalten ohne Zusatz von Lewis-Säure bei hohem Druck von 10 kbar.

Brachte man das Bromdien 59 unter den in Kapitel 1.3. beschriebenen Bedingungen mit 5 Äquivalenten Cyclopentenon (64) unter Zugabe verschiedener Lewis-Säuren zur Reaktion, so konnte in bis zu 25\% Ausbeute das Octahydro-s-indacen 65 als einziges Isomer isoliert werden (Schema 23, Tabelle 4). Mit Hilfe von Korrelationsspektren konnte die Struktur von 65 eindeutig zugeordnet werden. Führte man die Reaktion ohne LewisSäure, aber bei $10 \mathrm{kbar}$ durch, so erhielt man die beiden Regioisomere 66a und $6 \mathbf{6 b}$ als Diastereomerengemisch in $31 \%$ Ausbeute.

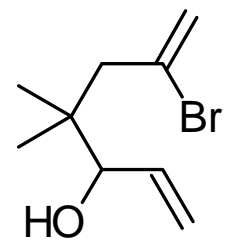

59

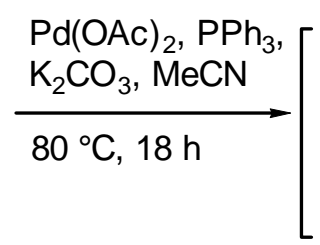<smiles>C=C1CC(C)(C)C(O)C1=C</smiles>

60

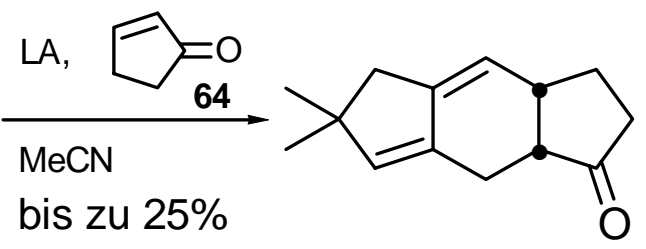

65<smiles>CC1(C)CC2=C(CC3C(=O)CC[C@@H]3C2)[C@@H]1O</smiles>

$66 a$
64, 10 kbar RT, $4 \mathrm{~d}$<smiles>CC1(C)CC2=C(CC3CCC(=O)[C@H]3C2)[C@@H]1O</smiles>

$66 b$

Schema 23. LA = Lewis-Säure 
Tabelle 4. Heck-Diels-Alder-Reaktion von 59 mit 64 unter Zusatz verschiedener Lewis-Säuren.

\begin{tabular}{cccc}
\hline Eintrag & Lewis-Säure (Äquiv.), Dauer $[\mathrm{h}]$ & Produkt & Ausbeute (\%) \\
\hline 1 & $\mathrm{LiBF}_{4}(3) 336$ & $\mathbf{6 5}$ & 25 \\
2 & $\mathrm{Et}_{2} \mathrm{AlCl}(3), 18$ & $\mathbf{6 5}$ & 22 \\
3 & $\mathrm{Et}_{2} \mathrm{AlCl}(1.5), 18$ & $\mathbf{6 5}$ & $6[\mathrm{a}]$ \\
4 & $\mathrm{Me}_{3} \mathrm{Al}(3), 72$ & $\mathbf{6 0}$ & $-[\mathrm{b}]$ \\
5 & $\mathrm{Ti}\left(\mathrm{O} \mathrm{Pr}_{4}(3), 72\right.$ & $\mathbf{6 0}$ & $-[\mathrm{b}]$ \\
6 & $\mathrm{BF}_{3} \cdot \mathrm{Et}_{2} \mathrm{O}(3), 336$ & $\mathbf{6 9}$ & 13 \\
7 & $336[\mathrm{c}]$ & - & - \\
8 & $10 \mathrm{kbar}, 96[\mathrm{c}]$ & $\mathbf{6 6 a}, \mathbf{b}$ & 31
\end{tabular}

[a] $17 \%$ des Diens 60 wurden ebenfalls isoliert - [b] Nicht isoliert, NMR-spektroskopisch nachgewiesen. - [c] Ohne Zusatz von Lewis-Säure.

Der Zusatz einer Lewis-Säure bewirkt eine Beschleunigung und vollständige Regioselektivität der Reaktion. Die beste Ausbeute erzielte man bei Zugabe von Lithiumtetrafluoroborat.[41] Sie ist mit $25 \%$ jedoch immer noch relativ gering. Ein Grund hierfür könnte die lange Reaktionsdauer von 14 Tagen sein, da das nach Heck-Reaktion gebildete exocyclische 1,3-Dien 60 bei längerer Reaktionszeit zur Polymerisation oder zur Zersetzung neigt (Eintrag 1). Verwendete man Diethylaluminiumchlorid als Lewis-Säure, betrug die Ausbeute 22\% (Eintrag 2), die Reaktionszeit verkürzte sich jedoch auf 18 Stunden. Neben diesem Produkt wurden dünnschichtchromatographisch noch weitere Komponenten detektiert, doch konnte kein weiteres identifizierbares Produkt erhalten werden. Vermutlich ist Diethylaluminiumchlorid als Lewis-Säure so stark, daß viele Nebenreaktionen auftreten. Daher führte man die Reaktion mit der schwächeren Lewis-Säure Trimethylaluminium erneut durch. Nach drei Tagen wurde die Reaktion abgebrochen, da keinerlei Octahydro-s-indacen 65 gebildet worden war (Eintrag 4). Bei Verwendung von Titantetraisopropylat erhielt man dasselbe Ergebnis (Eintrag 5). 
Interessanterweise wurde bei Zugabe von Bortrifluorid-Etherat nicht der Tricyclus 65 isoliert, sondern das 2,3,5,5-Tetramethylcyclopent-2-en-1-on (69) (Schema 24). Das Bortrifluorid wird wahrscheinlich an die Hydroxygruppe des exocyclischen Diens 60 koordiniert, das sich dann durch Säurekatalysierte Doppelbindungsverschiebung zum Enol 67 umlagert. Das Enol wiederum lagert sich zum Keton 68 um, das dann unter Säurekatalyse zum konjugierten System isomerisiert.

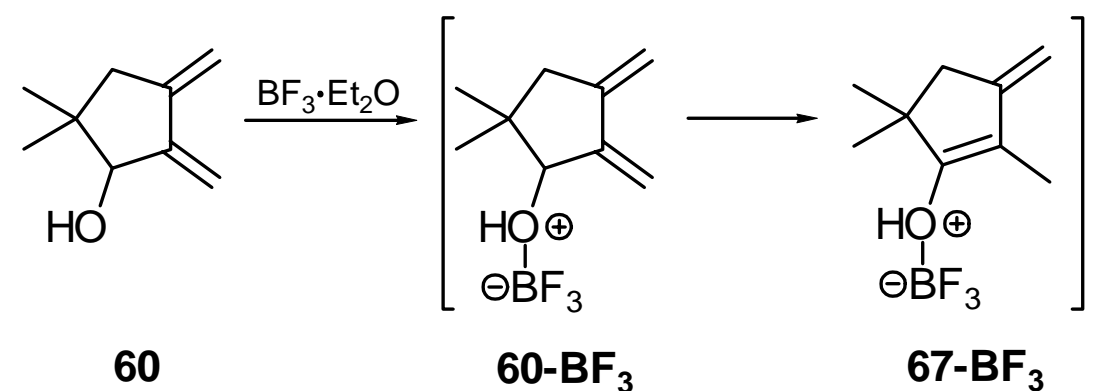

60

$60-\mathrm{BF}_{3}$

$67-\mathrm{BF}_{3}$

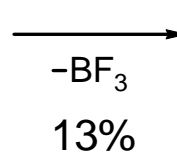

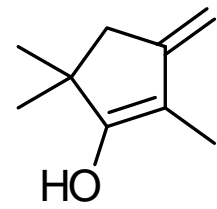

67

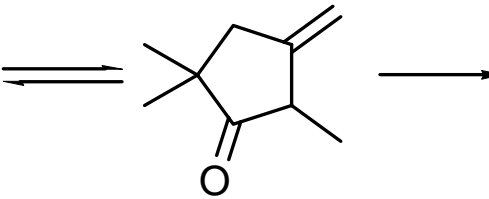

68

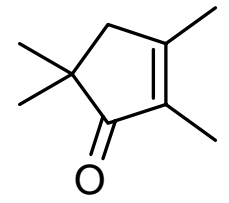

69

Schema 24. Möglicher Mechanismus für die Bildung von des Tetramethylcyclopentenons 69 aus dem 2,3-Dimethylen-5,5-dimethylcyclopentan-1-ol (60). 


\section{Synthese von Heterocyclen durch Einsatz heteroatomhaltiger Vorläufer}

\subsection{Aufbau von Hexahydroindol- und Hexahydroisoindolgerüsten}

Natürlich vorkommende Heterocyclen sind noch vielfältiger als carbocyclische Verbindungen. Die größte Gruppe der Heterocyclen ist die der sogenannten Alkaloide, die als Heteroatom Stickstoff enthalten. Neben den Isochinolin-Alkaloiden sind die IndolAlkaloide am weitesten verbreitet.[42] Viele verfügen über pharmakologische Wirkungen und werden therapeutisch genutzt, als Grundkörper liegt ihnen das Indol zugrunde.

Entsprechend dem beschriebenen Aufbau carbocyclischer Systeme wurde daher versucht auch heterocyclische Systeme, insbesondere Hexahydroindol- und HexahydroisoindolGerüste aufzubauen.

Zur Darstellung des stickstoffhaltigen Enins $\mathbf{7 3}$ alkylierte man das mit Natriumhydrid deprotonierte $N$-Allyl- $N$-( $p$-tosyl)amin (70) mit Propargylbromid (71) und gewann den Cyclisierungsvorläufer $\mathbf{7 3}$ in 49\% Ausbeute.[43] Dabei entstand als Nebenprodukt das Allen 72 in 19\% Ausbeute. Das Enin 73 wurde anschließend unter den Bedingungen der Enin-Cycloisomerisierung umgesetzt. Das Hexahydroisoindol $\mathbf{7 4}$ konnte je nach Wahl des Katalysatorsystems in 55-60\% Ausbeute isoliert werden (Schema 25, Tabelle 5). Bei Verwendung von $\mathrm{Pd}(\mathrm{OAc})_{2}$ und BBEDA betrug die Ausbeute 55\%, bei $\mathrm{Pd}(\mathrm{OAc})_{2}$ und $\mathrm{PPh}_{3}$ war sie mit $60 \%$ nur unwesentlich besser. Auch bei Verwendung von $\mathrm{Pd}(\mathrm{dba})_{2}, \mathrm{PPh}_{3}$ und Essigsäure konnte die Ausbeute nicht verbessert werden, sie betrug ebenfalls $60 \%$. Damit ist keine gültige Aussage darüber möglich, welche Katalysatorzusammensetzung zur Cycliserung von stickstoffhaltigen Eninen am besten geeignet ist.

Im Vergleich zu der Heck-Diels-Alder-Reaktion von stickstoffhaltigen Vorläufern ist die Enin-Cycloisomerisierung mit anschließender [4+2]-Cycloaddition zum Aufbau von Hexahydroisoindolen besser geeignet, da bei der Cyclisierung kein formaler 6-endo-trigRingschluß stattfand.[29] 


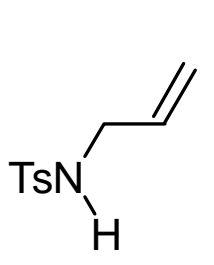

70

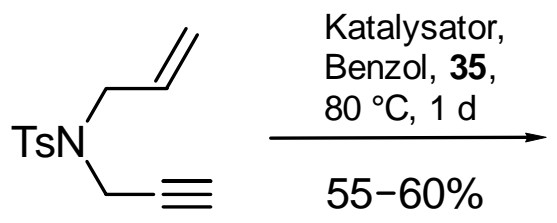

73
1) $\mathrm{NaH}, \mathrm{DME}$,
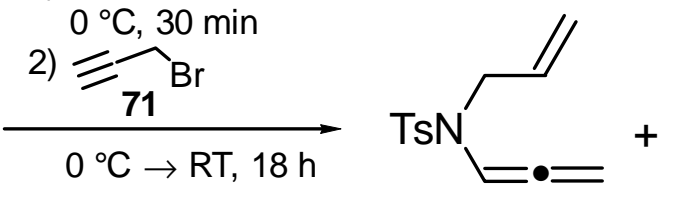

$72(19 \%)$

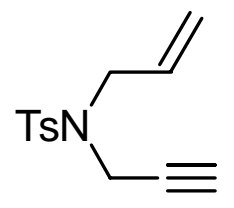

$73(49 \%)$<smiles>CC(=O)C1CCC2=C(C1)CN([As])C2</smiles>

74

Schema 25. Katalysator siehe Tabelle 5.

Tabelle 5. Enin-Cycloisomerisierung und Diels-Alder-Reaktion von $\mathbf{7 3}$ unter verschiedenen Bedingungen.

\begin{tabular}{ccc}
\hline Eintrag & Bedingungen & Ausbeute (\%) \\
\hline 1 & $5 \mathrm{~mol} \% \mathrm{Pd}(\mathrm{OAc})_{2}, 10 \mathrm{~mol} \% \mathrm{BBEDA}$ & 55 \\
2 & $5 \mathrm{~mol} \% \mathrm{Pd}(\mathrm{OAc})_{2}, 10 \mathrm{~mol}^{2} \mathrm{PPh}_{3}$ & 60 \\
3 & $5 \mathrm{~mol} \% \mathrm{Pd}(\mathrm{dba})_{2}, 10 \mathrm{~mol}_{2} \mathrm{PPh}_{3}, 10 \mathrm{~mol} \% \mathrm{HOAc}$ & 60 \\
\hline
\end{tabular}

Steinig und Bhat setzten dem Enin 73 entsprechende Bromdiene vom Typ 75-R in HeckReaktionen um. Die Ausbeuten lagen bei dem Bromdien 75-pNbs mit 48\% unter denen in der Cycloisomerisierung erzielten, des weiteren trat als Nebenreaktion die Bildung des Produktes 77-pNbs in $12 \%$ Ausbeute auf (Schema 26). [29]

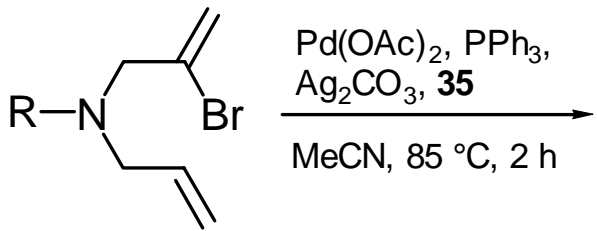

75-R<smiles>[R]N1CC2=C(CC(C(C)=[18O])CC2)C1</smiles>

76-R (48\%)
$77-\mathbf{R}(12 \%)$

Schema 26. $\mathrm{R}=\mathrm{pNbs}=4-\mathrm{NO}_{2}-\mathrm{C}_{6} \mathrm{H}_{4} \mathrm{SO}_{2}$. 
Zur Darstellung von Hexahydroindolgerüsten sollte ein Enamin als Vorläufer synthetisiert werden. Man gab zu Benzylamin (78) nach einer Vorschrift von Normant et al. und Buchwald et al. 2,4-Dibrombuten (79), jeweils 2 Äquivalente $\mathrm{NaI}$ und $\mathrm{K}_{2} \mathrm{CO}_{3}$ und erwärmte in DMF auf $100{ }^{\circ} \mathrm{C}$ (Schema 27).[44] Hierbei fand in situ ein Brom-IodAustausch statt. Mit dem so gebildeten reaktiveren Iodid gelang die Alkylierung in mäßigen Ausbeuten. Die direkte Alkylierung von Benzylamin mit $\mathbf{7 9}$ war nicht durchführbar, da die Homoallylposition für eine Substitution nicht genügend aktiviert ist. Die Umsetzung von 80 mit Propiolsäuremethylester (81) ergab in nahezu quantitativer Ausbeute das (E)-konfigurierte Enamin 82.
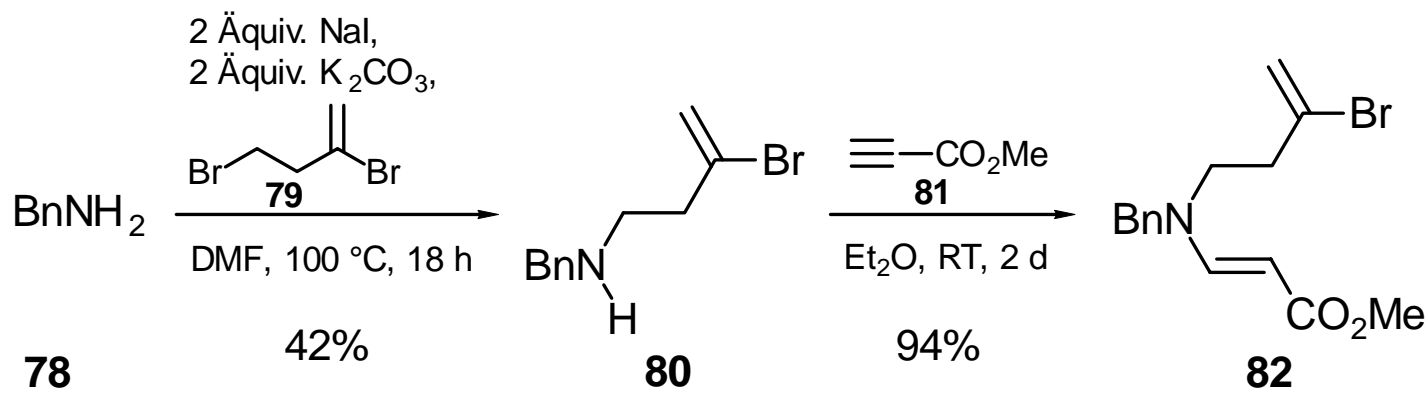

80

82

Schema 27.

Die Umsetzung von $\mathbf{8 2}$ unter den für die Heck-Diels-Alder-Reaktion üblichen Bedingungen (5 mol\% $\mathrm{Pd}(\mathrm{OAc})_{2}, 10 \mathrm{~mol} \% \mathrm{PPh}_{3}, 1$ Äquivalent Kaliumcarbonat) mit 3 Äquivalenten Methylacrylat (35) ergab nicht das Hexahydroindolderivat 83, sondern das Trienin 86 (Schema 28). Dabei muß eine Dehydrobromierung des Bromvinylenamins 82 zu 85 stattgefunden haben, welches dann Palladium-katalysiert über 84 an 82 gekuppelt worden sein muß.

Bei Verwendung von Natriumacetat bzw. Cäsiumcarbonat als Base trat keine Reaktion ein, man konnte neben Zersetzungsprodukten nur das Edukt zurückgewinnen. Auch bei Verwendung von nur 0.5 Äquivalenten Kaliumcarbonat konnte das Hexahydroindol 83 nicht einmal in Spuren gefunden werden. Ein formales 6-endo-Produkt wurde ebenfalls nicht isoliert. 


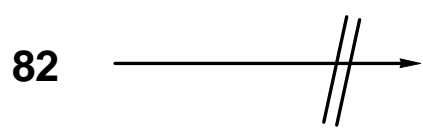

82, $\mathrm{Pd}(\mathrm{OAc})_{2}$,

$\mathrm{PPh}_{3}, \mathrm{~K}_{2} \mathrm{CO}_{3}$

$\mathrm{MeCN}, 80^{\circ} \mathrm{C}, 1 \mathrm{~d}$<smiles>COC(=O)C1CC2=C(C(C(C)=O)CCN2Cc2ccccc2)C1C(=O)OC</smiles>

83

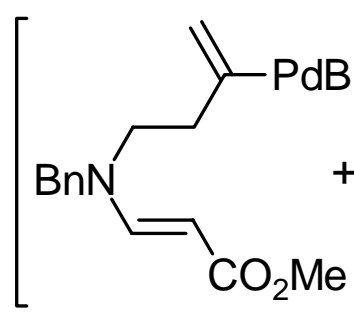

84

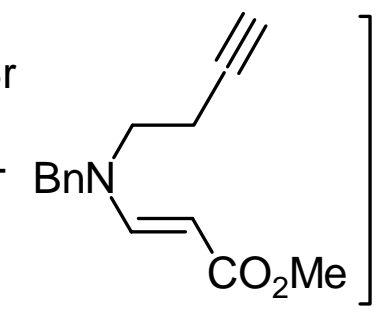

85

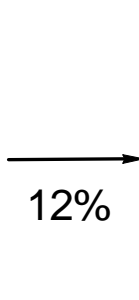

$\overbrace{\mathrm{CO}_{2} \mathrm{Me}}^{\mathrm{NBn}}$

86

Schema 28.

Auch das Reaktionsverhalten des Enins 85 unter den Bedingungen der EninCycloisomerisierung wurde untersucht. Dazu wurde Benzylamin (78) mit 4-Brom-1-butin (87) in 25\% Ausbeute zu 88 alkyliert (Schema 29). Dieses reagierte mit Methylpropargylat (81) in 93\% Ausbeute zum Enaminoester 85. Versetzte man 85 mit 5 mol\% $\mathrm{Pd}(\mathrm{OAc})_{2}$ und 10 mol\% BBEDA, so konnte selbst nach drei Tagen bei $80{ }^{\circ} \mathrm{C}$ nur das Edukt detektiert werden. Verwendete man $\operatorname{Pd}(\mathrm{dba})_{2}$ als $\mathrm{Pd}(0)$-Spezies, $10 \mathrm{~mol} \%$ Triphenylphosphan und 10 mol\% Essigsäure, erhielt man ebenfalls kein Hexahydroindolderivat. Auch eine Erhöhung der Temperatur auf $100{ }^{\circ} \mathrm{C}$ führte nicht zum gewünschten Produkt 83.

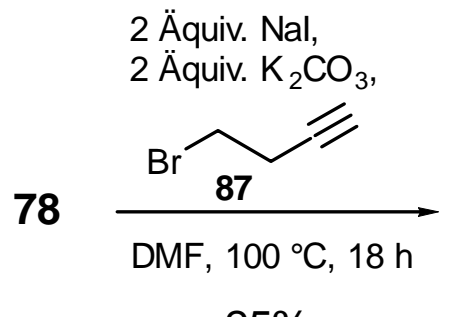

$25 \%$

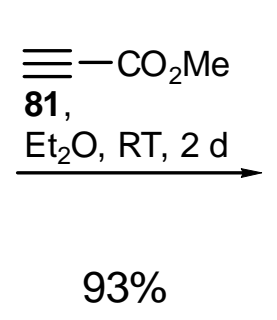

$93 \%$

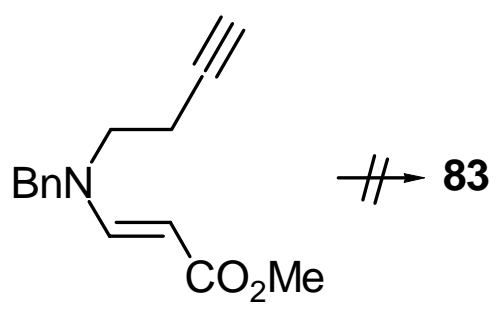

85

Schema 29. 


\subsection{Verwendung von Sulfonamiden als Cyclisierungsvorläufer}

Eine weitere interessante Klasse von Heterocyclen sind solche, die sowohl Schwefel als auch Stickstoff beinhalten. Dazu zählen u. a. Thiazole, Isothiazole und Thiazine. Isothiazole werden vor allem in Pharmazeutika und Pflanzenschutzmittel als Fungizide, Algizide und Schleimbekämpfungsmittel verwendet. Thiazole und Thiazinderivate findet man auch in Farbstoffen wieder.[45] Auch Saccharin, ein häufig benutzter Süßstoff, ist ein Schwefel-Stickstoff-Heterocyclus. Im Vergleich zu Saccharose ist die Süßungskraft von Saccharin 550 mal höher, es schmeckt zudem in einer Verdünnung von $1: 200.000$ noch süß. Daneben besitzt Saccharin keinen physiologischen Brennwert.[46] In der Technik findet Saccharin als Härtebildner für Dispersionsschichten in galvanischen Nickelbädern Verwendung.

Zur möglichen Synthese von Isothiazolin-Derivaten wurde die Reaktion von Sulfonamiden in Domino-Prozessen aus Palladium-katalysierter Cyclisierung und Diels-Alder-Reaktion näher untersucht. Dazu wurde Benzylamin (78) in Gegenwart von Triethylamin mit 2,3Dibrompropen (89) alkyliert. Während die Alkylierung von 78 in der Literatur mit Kaliumcarbonat als Base in DMF bei Temperaturen um $100{ }^{\circ} \mathrm{C}$ beschrieben ist, ${ }^{[47]}$ konnte hier bei Verwendung von Triethylamin die Reaktion bei Raumtemperatur durchgeführt werden (Schema 30). Man erhielt das Amin 90 in $72 \%$ Ausbeute.

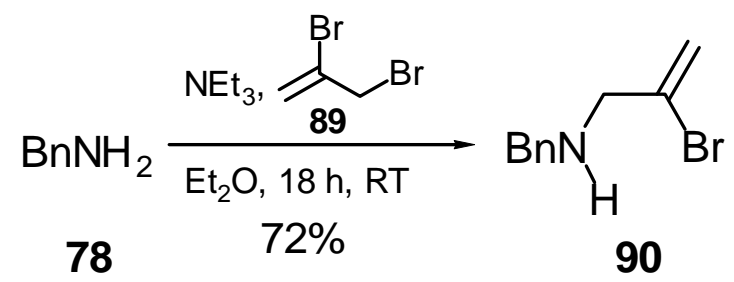

Schema 30.

Die Sulfoneinheit wurde ausgehend von Chlorethylsulfonylchlorid (91) eingeführt.[48] Dazu versetzte man 91 bei $-60^{\circ} \mathrm{C}$ mit Triethylamin, wobei unter $\mathrm{HCl}$-Eliminierung in situ das Vinylsulfonylchlorid (92) erhalten wurde. Diese Lösung wurde sofort mit dem alkylierten Amin 90 umgesetzt. Das Sulfonamid 93 konnte so in 87\% Ausbeute dargestellt 
werden (Schema 31). Das dem Bromdien 93 entsprechende Enin 95 wurde unter identischen Bedingungen bei Verwendung des Benzylamins 94 in 82\% synthetisiert.

Des weiteren wurde das Sulfonamid 99 mit Brom an der Vinylsulfonyleinheit synthetisiert. Dazu wurde Chlorethylsulfonylchlorid (91) in einem ersten Schritt zum Vinylsulfonylchlorid (92) umgesetzt. An dessen Doppelbindung ließ sich nach einer Vorschrift von Rondestvedt bei $0{ }^{\circ} \mathrm{C}$ Brom zu Dibromsulfonylchlorid (96) addieren.[49] Anschließende Dehydrobromierung zum Bromvinylsulfonylchlorid (97) und Umsetzung mit Allylbenzylamin (98) ergab das Sulfonamid 99 in einer Gesamtausbeute von 27\% Ausbeute (Schema 31).

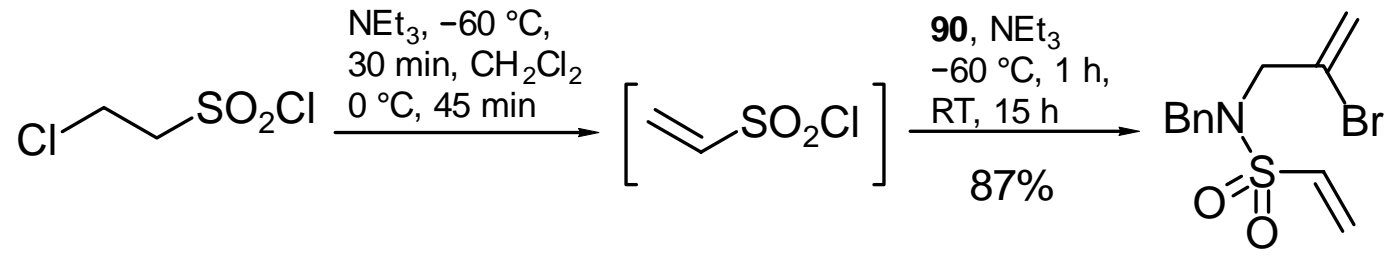

91

92

93

$$
\text { 92, } \mathrm{NEt}_{3}
$$

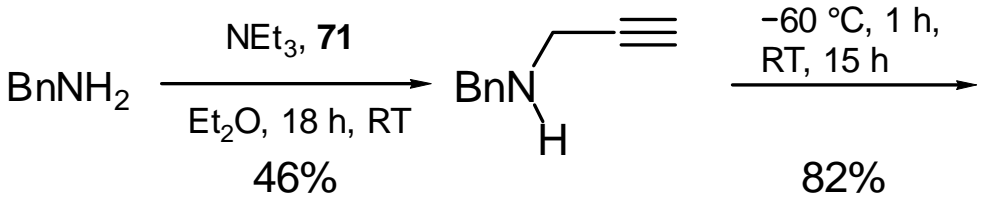

78

94
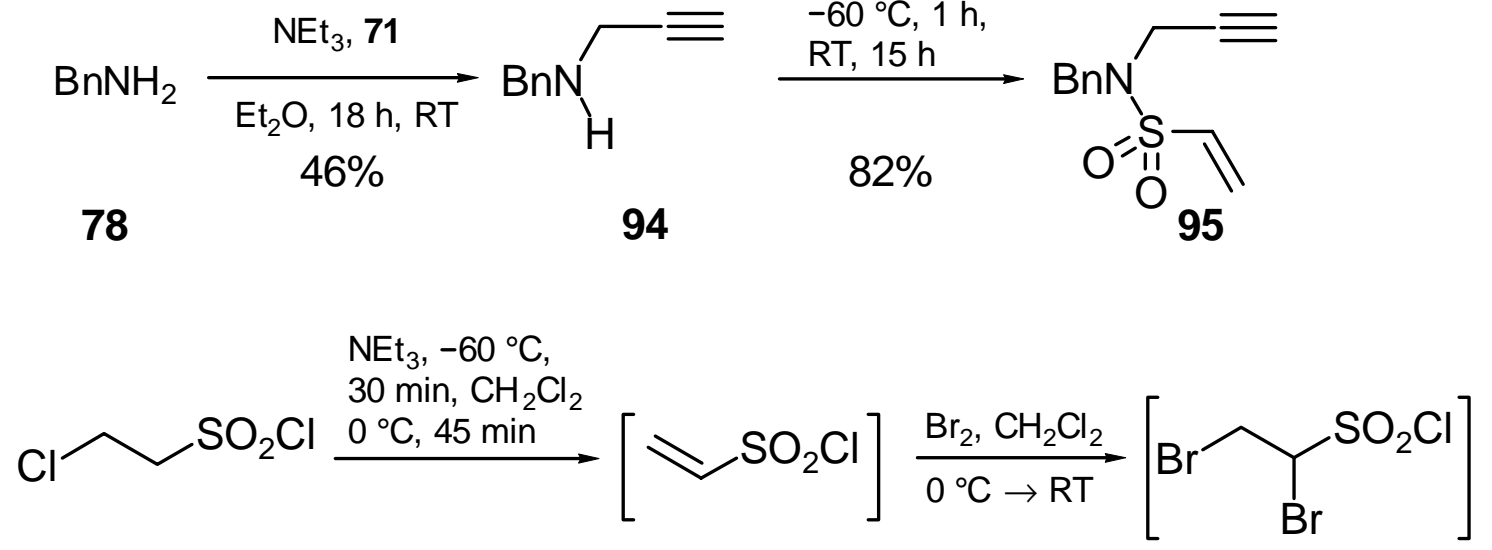

91

92

96<smiles>CC=C(Br)S(=O)(=O)Cl</smiles>

97

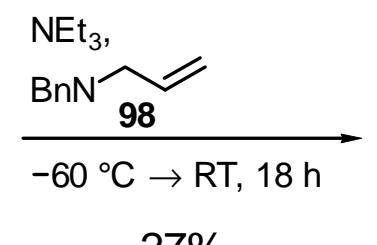

$27 \%$<smiles>C=CCN(Br)S(=O)(=O)C(=C)Br</smiles>

99

Schema 31. 
Bei der Behandlung der Sulfonamide 93 und 99 mit $\mathrm{Pd}(\mathrm{OAc})_{2}$ in Gegenwart von Triphenylphosphan als Ligand konnten je nach verwendeter Base die Bicyclen 100a,b als Regioisomerengemisch im Verhältnis 1.9:1 und die formalen 6-endo-Produkte 101, 102 und 103 isoliert werden (Schema 32, Tabelle 6). Das Tetrahydrothiazin 101 wurde nur bei Verwendung von Kaliumcarbonat als Base erhalten (5\% Ausbeute, ohne Zusatz von 35 erhöhte sich die Ausbeute auf 20\%), das Produkt 100a,b wurde nicht gebildet. Setzte man hingegen Cäsiumcarbonat als Base ein, konnten die Hexahydrobenzoisothiazole 100a,b in 29\% Ausbeute als Regioisomerengemisch im Verhältnis 1.9 : 1 synthetisiert werden.

Ausgehend vom Sulfonamid 99 konnten in Gegenwart von Kaliumcarbonat bereits nach zwei Stunden die beiden Hydrothiazine 102 und 103 in insgesamt 20\% Ausbeute (18\% 102 und 2\% 103) isoliert werden. Verwendete man Cäsiumcarbonat, erhielt man ebenfalls $\mathbf{1 0 2}$ und 103, das Mengenverhältnis kehrte sich jedoch um (3\% 102 und 12\% 103). Die Reaktionsdauer verlängerte sich bei Benutzung von Cäsiumcarbonat auf drei Tage. Führte man die Reaktion bei, wie sonst üblich, $80{ }^{\circ} \mathrm{C}$ aus, erhielt man kein Produkt. Eine Reaktion trat erst bei Erhöhung der Temperatur auf $100{ }^{\circ} \mathrm{C}$ ein. Die Reaktionsführung bei dieser Temperatur war nur durch die Verwendung eines dickwandigen Pyrex-Gefäßes möglich, da man Acetonitril (Siedepunkt $80-81{ }^{\circ} \mathrm{C}$ ) als Lösungsmittel benutzte. Bei allen diesen Cyclisierungen wurde neben den genannten Produkten noch polymeres Material gebildet.

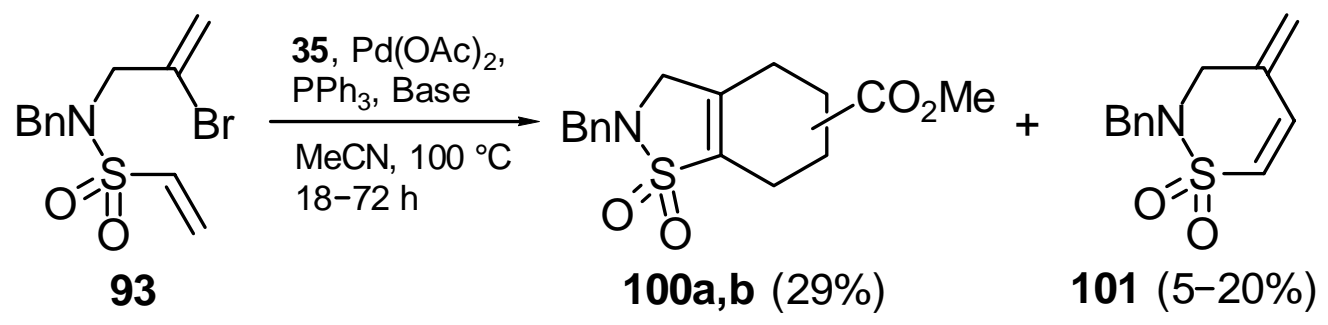

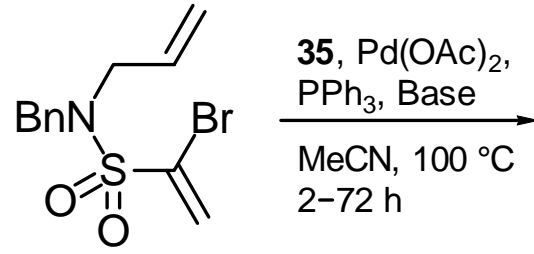

99<smiles>C=C1C=CCN(Br)S1(=O)=O</smiles>

$102(2-18 \%) \quad 103(2-12 \%)$

Schema 32. 
Tabelle 6. Cyclisierung von 93 und 99 unter Verwendung verschiedener Basen.

\begin{tabular}{cccccccc}
\hline Eintrag & Sulfonamid & Base & $\begin{array}{c}\text { Dauer } \\
{[\mathrm{h}]}\end{array}$ & Produkt & $\begin{array}{c}\text { Ausbeute } \\
(\%)\end{array}$ & $\begin{array}{c}\text { weiteres } \\
\text { Produkt }\end{array}$ & $\begin{array}{c}\text { Ausbeute } \\
(\%)\end{array}$ \\
\hline 1 & $\mathbf{9 3}$ & $\mathrm{K}_{2} \mathrm{CO}_{3}$ & 18 & $\mathbf{1 0 1}$ & $5[\mathrm{a}]$ & - & - \\
2 & $\mathbf{9 3}$ & $\mathrm{Cs}_{2} \mathrm{CO}_{3}$ & 72 & $\mathbf{1 0 0}$ & $29[\mathrm{~b}]$ & - & - \\
3 & $\mathbf{9 9}$ & $\mathrm{K}_{2} \mathrm{CO}_{3}$ & 2 & $\mathbf{1 0 2}$ & 18 & $\mathbf{1 0 3}$ & 2 \\
4 & $\mathbf{9 9}$ & $\mathrm{Cs}_{2} \mathrm{CO}_{3}$ & 72 & $\mathbf{1 0 3}$ & 12 & $\mathbf{1 0 2}$ & 2 \\
5 & $\mathbf{9 9}$ & $\mathrm{Cs}_{2} \mathrm{CO}_{3}$ & 2 & $\mathbf{1 0 2}$ & $<5$ & $\mathbf{9 9}$ & 45 \\
6 & $\mathbf{9 9}$ & $\mathrm{K}_{2} \mathrm{CO}_{3}$ & 72 & - & $-[\mathrm{c}]$ & - & - \\
\hline
\end{tabular}

[a] Ohne Zusatz von 35 erhöhte sich die Ausbeute auf 20\%. - [b] Als Regioisomerengemisch im Verhältnis $1.9: 1$. - [c] Zersetzung.

Die Bildung von 103 muß durch Doppelbindungsverschiebung aus 102 erfolgen. Diese Isomerisierung ist begünstigt, da ein Dien mit einer höher substituierten Doppelbindung entsteht, die exocyclische Methylengruppe verschwindet. Die Isomerisierung muß durch Readdition/Eliminierung von Hydridopalladiumbromid erfolgen.[29,50] Eine thermische 1,5-H-Verschiebung kann in einem 3-substituierten und starr s-trans-angeordnetem 1,3Diensystem wie 102 nicht stattfinden.[51] Betrachtet man die Palladium-katalysierte Isomerisierung, so wird an das durch $\beta$-Hydrideliminierung gebildete Tetrahydrothiazin 102 in umgekehrter Regioselektivität die Hydridopalladiumbromid-Spezies readdiert. Dabei wird der $\eta^{3}$-Komplex 104 gebildet, aus dem durch erneute $\beta$-Hydrideliminierung das Dihydrothiazin 103 entsteht (Schema 33). Das isomerisierte Dien 103 wurde hauptsächlich bei Verwendung von Cäsiumcarbonat und einer Reaktionsdauer von drei Tagen isoliert. In Gegenwart von Kaliumcarbonat fand die Isomerisierung bei der Cyclisierung von 99 bei einer Reaktionsdauer von zwei Stunden in nur geringem Maße statt. Verkürzte man die Reaktionsdauer von 99 in Anwesenheit von Cäsiumcarbonat auf zwei Stunden, wurde neben $45 \%$ unumgesetztem Ausgangsmaterial das Tetrahydrothiazin 102 (Ausbeute $<5 \%$ ) gebildet. Eine Verlängerung der Reaktionsdauer auf drei Tage bei Verwendung von Kaliumcarbonat führte nur zur Zersetzung. 


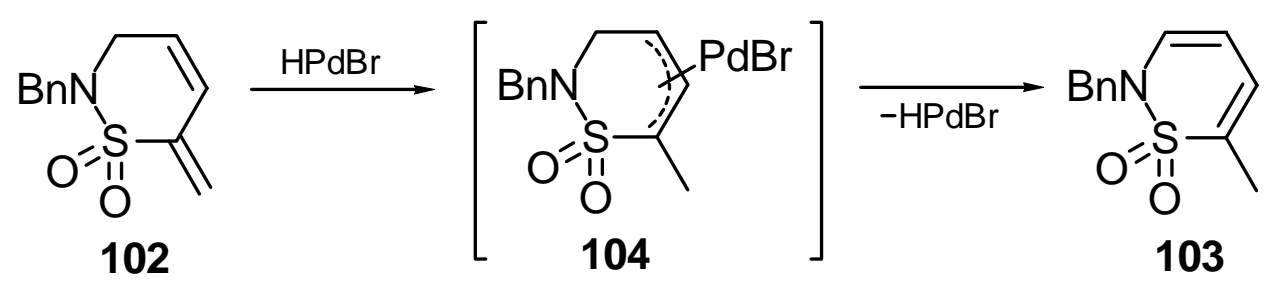

Schema 33.

Die Bildung der formalen 6-endo-Produkte 101, 102, und 103 muß aus einem anfänglichen 5-exo-trig-Ringschluß zu 105 resultieren. Ein sich unmittelbar anschließender 3-exo-trigRingschluß führt dann zum Intermediat 106 mit CyclopropylmethylpalladiumbromidSubstruktur. Nach Ringöffnung bildete sich 107, das unter Palladiumhydrid-Eliminierung zum Tetrahydrothiazin 101 reagiert (Schema 34). Dieser Reaktionsablauf ist in der HeckReaktion für $\sigma$-Alkylpalladium-Intermediate, die keine eliminierbaren Wasserstoffe in $\beta$ Position besitzen tragen, von Negishi et al. bewiesen worden.[52]

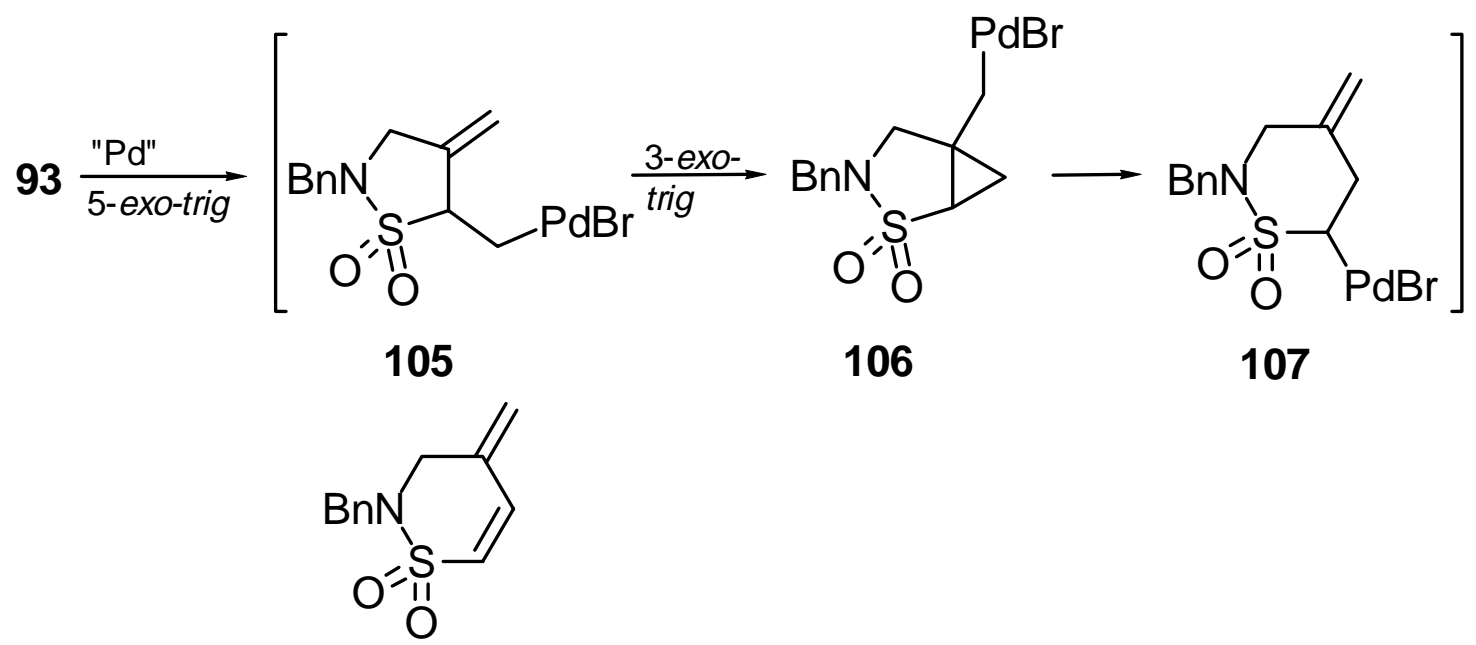

101

Schema 34.

Kürzlich berichteten Ahn et al., daß die $\beta$-Hydrideliminierung auch beim Bromdien 75-Ts gehindert ist. Als Erklärung dafür wurde eine Koordination des Palladiums der Spezies 109 mit den Sauerstoffatomen der Tosylgruppe angenommen.[53] Das Palladium-Intermediat 109 konnte in einer anschließenden Suzuki-Kupplung in guten Ausbeuten zum Methylenpyrrolidin 108 umgesetzt werden (Schema 35). 


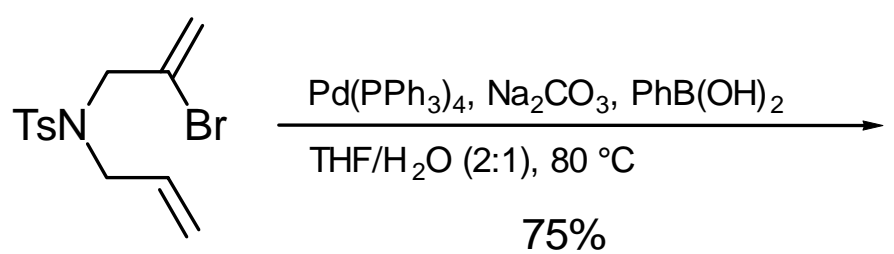

75-Ts<smiles>C=C1CN([Al])CC1Cc1ccccc1</smiles>

108

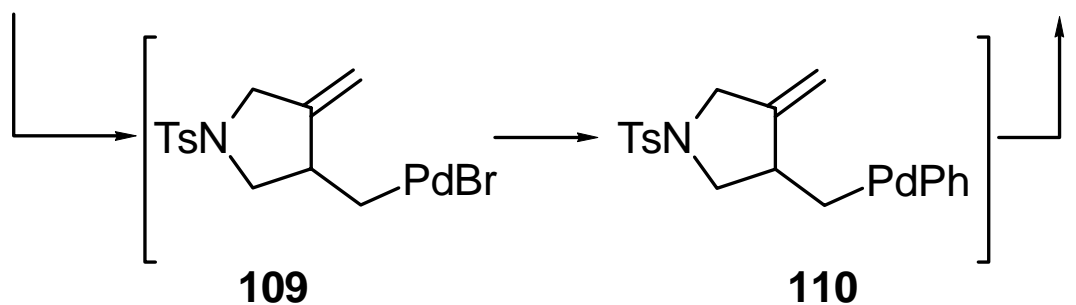

Schema 35

Der Versuch der Cyclisierung des Enins 95 schlug fehl. Sowohl bei Verwendung von $5 \mathrm{~mol} \% \mathrm{Pd}(\mathrm{OAc})_{2}$ und $10 \mathrm{~mol} \%$ BBEDA als auch von $5 \mathrm{~mol} \% \mathrm{Pd}(\mathrm{dba})_{2}, 10 \mathrm{~mol} \% \mathrm{PPh}_{3}$ und 10 mol\% Essigsäure konnte keinerlei Reaktion festgestellt werden. Auch eine Erhöhung der Temperatur auf $100{ }^{\circ} \mathrm{C}$ führte nicht zur Cyclisierung, das Edukt konnte fast vollständig zurückgewonnen werden (Schema 36).

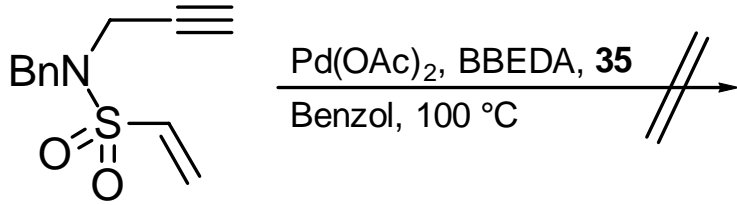

95<smiles>CC(=O)C1CCC2=C(C1)CS(=O)(=O)N2Cc1ccccc1</smiles>

$100 a, b$

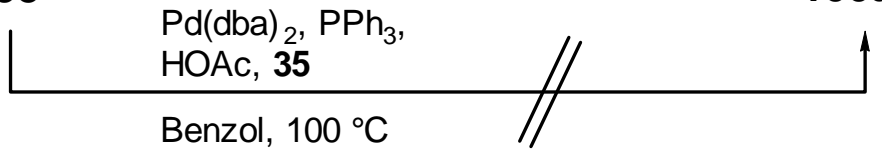

Schema 36.

Zusammenfassend läßt sich sagen, daß Enine, die Stickstoff oder Schwefel in Vinylstellung besitzen, unter den hier beschriebenen Bedingungen keine Cycloisomerisierung eingehen. Bei der Vielzahl der möglichen Palladium-Katalysatoren und Liganden ist jedoch nicht ausgeschlossen, daß Cyclisierungen von Verbindungen vom Typ 85 oder 95 realisierbar sind. 
2.3. Darstellung eines sauerstoffhaltigen Cyclisierungsvorläufers und Versuche zu dessen Cyclisierung

Außer auf stickstoffhaltige Heterocyclen sollte die Produktpalette auch auf sauerstoffhaltige Heterocyclen erweitert werden. Ringsysteme, bei denen sich der Sauerstoff an zentraler Position im Fünfringsystem befindet, wurden von Bhat und Thies eingehend untersucht.[19c,54] Die Idee, Sauerstoff an anderen Positionen im Fünfringsystem einzubauen, stößt auf das Problem, daß der Cyclisierungsvorläufer eine Enolether-Funktion enthalten müßte. Die Synthese von Enolethern erfolgt im allgemeinen am besten durch Addition des entsprechenden Alkohols in Gegenwart von Kaliumhydroxid an Acetylen.[55] Die starke Base könnte zu Nebenreaktionen an der Alkenylbromeinheit des hier einzusetzenden 3-Brombut-3-en-1-ols (112) führen. Ein weiteres Problem ist die Instabilität von Enolethern gegenüber von Säurespuren, die eine sofortige Hydrolyse zur Folge haben. Daher wurde als Ringsystem ein sauerstoffhaltiger Sechsring angestrebt.

Dazu wurde nach Otera et al. in einer Zinn-vermittelten Kupplung Formaldehyd an 2,3Dibrompropen (89) addiert.[56] Hierbei konnte der Alkohol 112 in 73\% Ausbeute isoliert werden. Anschließende Deprotonierung mit NaH und Alkylierung mit Allylbromid (113) ergab den gewünschten Ether 114 in 25\% Ausbeute (Schema 37).

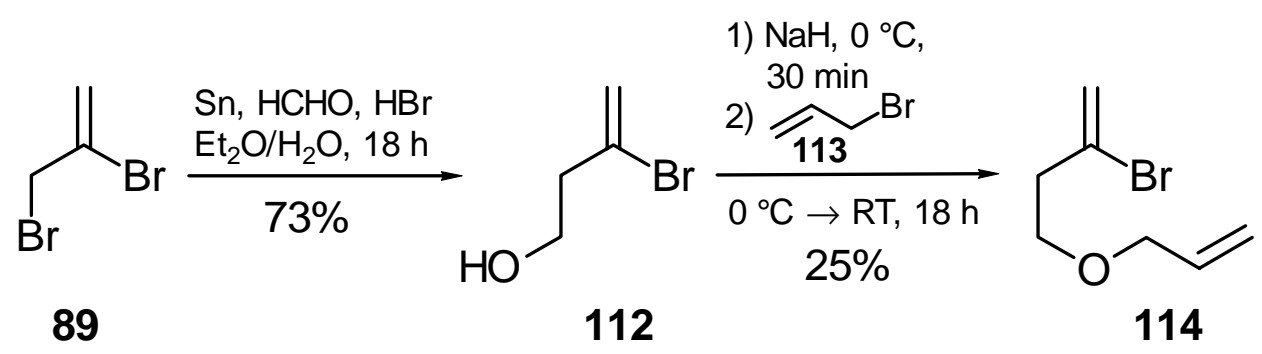

Schema 37.

In Gegenwart von $5 \mathrm{~mol} \% \mathrm{Pd}(\mathrm{OAc})_{2}, 10 \mathrm{~mol} \%$ Triphenylphosphan, einem Äquivalent Kaliumcarbonat und 3 Äquivalenten Methylacrylat (35) erhielt man neben dem erwarteten Bicyclus 115a,b (36\% Ausbeute) zusätzlich das Trienin 116 (26\% Ausbeute, Schema 38). Die Verwendung von einem Äquivalent Cäsiumcarbonat anstelle von Kaliumcarbonat unter sonst identischen Bedingungen, ergab das Hexahydroisochromen 115a,b als einziges 
Produkt in 45\% Ausbeute (Tabelle 7). Dabei lag 115a,b als Regioisomerengemisch im Verhältnis $1.1: 1$ vor. Bei Verwendung von Silbercarbonat als Base konnte überrraschenderweise nur das Diendiin 117 in 16\% Ausbeute isoliert werden.

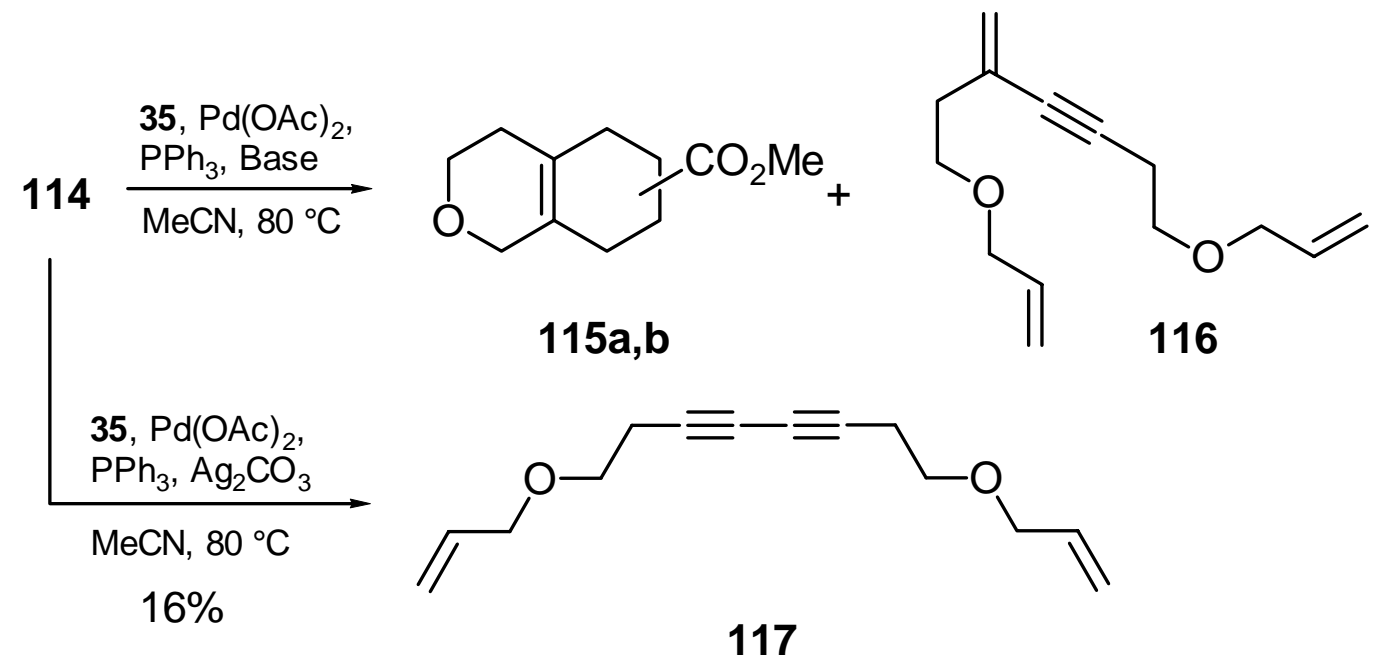

Schema 38. Details siehe Tabelle 7.

Tabelle 7. Einfluß der Base bei der Cyclisierung von 114 (Schema 38).

\begin{tabular}{cccccc}
\hline Eintrag & Base & Produkt & Ausbeute (\%) & Weiteres Produkt & Ausbeute (\%) \\
\hline 1 & $\mathrm{~K}_{2} \mathrm{CO}_{3}$ & $\mathbf{1 1 5 a , b}$ & 36 & $\mathbf{1 1 6}$ & 26 \\
2 & $\mathrm{Cs}_{2} \mathrm{CO}_{3}$ & $\mathbf{1 1 5 a , b}$ & 45 & - & - \\
\hline
\end{tabular}

Ein möglicher Mechanismus für die Bildung von 116 könnte damit beginnen, daß die zunächst gebildete Palladium-Spezies 118 nicht in einem intramolekularen 6-exo-trig Ringschluß reagiert, sondern intermolekular an der Bromvinyleinheit eines zweiten Moleküls 114 angreift. Dabei entsteht der Palladium-Komplex 119, der dann unter Abspaltung von Hydridopalladiumbromid zum Bromtetraen 120 reagiert. Anschließende Dehydrobromierung durch die zugesetzte Base ergibt 116 (Schema 39).

Eine zweite Bildungsweise beginnt mit der Dehydrobromierung von $114 \mathrm{zu} \mathrm{121}$, die in Konkurrenz zur Insertion des Palladiums in die Bromvinyleinheit steht. Somit könnten im Reaktionsgemisch sowohl 121 als auch 119 vorliegen, die dann Palladium-katalysiert aneinander zu 116 gekuppelt werden (Schema 39).

Um eine genauere Aussage über den Mechanismus treffen zu können, gab man zum Bromdien 114 Kaliumcarbonat und erwärmte in Acetonitril auf $80^{\circ} \mathrm{C}$. Dabei erhielt man 
tatsächlich ein Gemisch aus 114 und 121. Diese Beobachtung stützt den zweiten Vorschlag zum Mechanismus. Thies beobachtete bei der Palladium-katalysierten Cyclisierung von sauerstoffhaltigen Bromendiinen keine Bildung eines Produktes, das aus einer Dehydrobromierung an der Bromvinyleinheit entsteht. ${ }^{[19 c]}$ Bei den von ihr verwendeten Cyclisierungsvorläufern befand sich der Sauerstoff jedoch in Allylstellung zum Bromvinylrest, während bei $\mathbf{1 1 4}$ der Sauerstoff in Homoallylstellung steht.

Die Bildung des Diendiins 117 läßt sich durch eine Kupplung zweier Moleküle 121 erklären, die im Sinne einer Glaser-Kupplung mit dem Silbersalz als Oxidationsmittel verläuft. Bei der Glaser-Kupplung wird aus einem Acetylen durch Base ein Acetylid-Anion erzeugt, das einen Kupferkomplex bildet, der unter Übertragung eines Elektrons das Kupfer reduziert und zum symmetrischen Bisacetylen dimerisiert.[57] Die Kupfer(I)Kationen werden durch Sauerstoff zur Kupfer(II)-Verbindung oxidiert. In dem hier untersuchten Reaktionsgemisch kann das mit einem Äquivalent stöchiometrisch vorhandene Silbercarbonat die Aufgabe des Kupfers übernehmen. Dabei wird es zum metallischen Silber reduziert, was dann ausfällt.
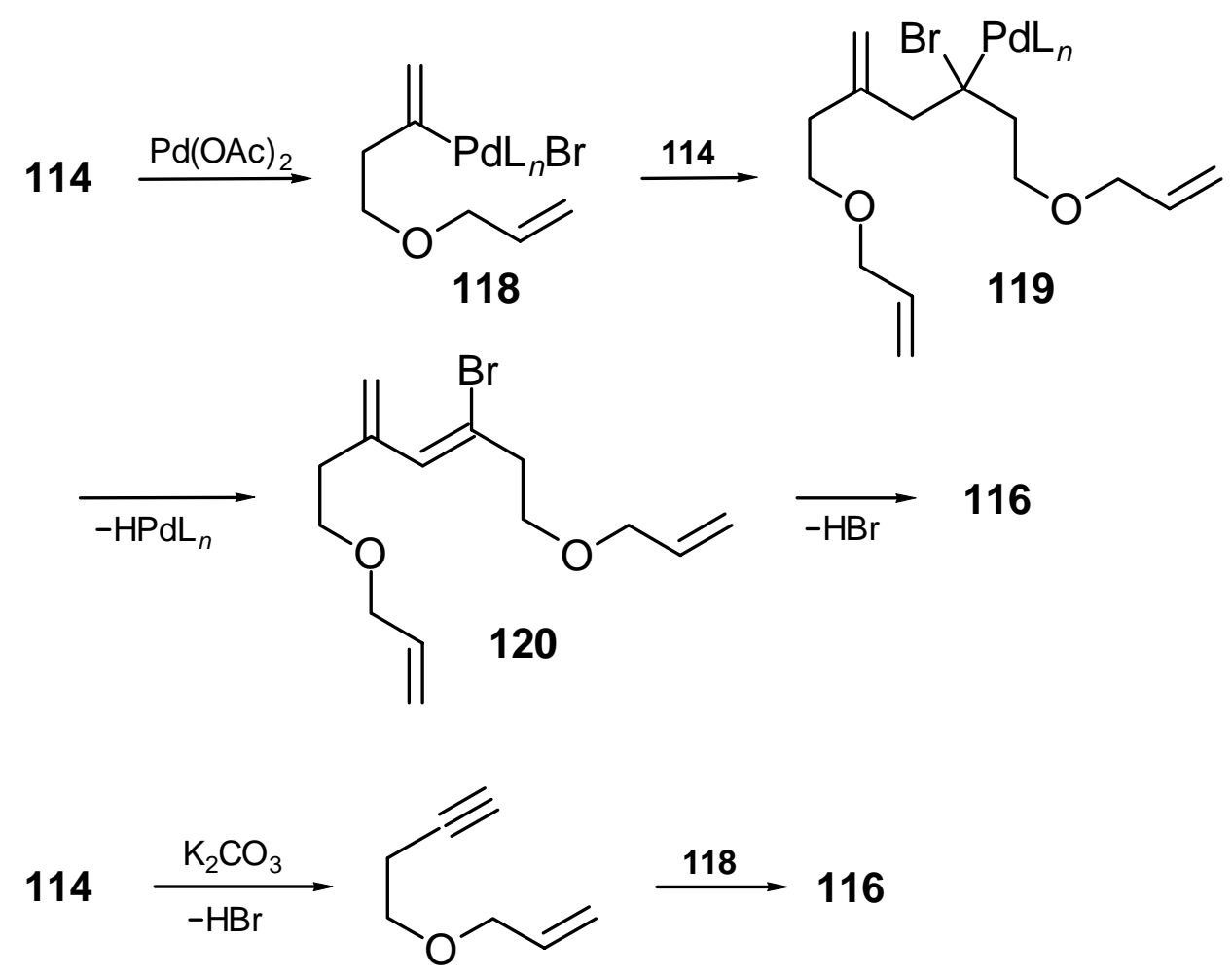

Schema 39. 


\section{Aufbau von Heterocyclen durch Kombination von Palladium-katalysierter Cyclisierung und Diels-Alder-Reaktion mit Heterodienophilen}

\subsection{Vorüberlegungen}

Die Synthese von heteroatomhaltigen cyclischen Verbindungen ist auch über Diels-AlderReaktionen möglich. Dabei können sowohl Heterodiene als auch Heterodienophile eingesetzt werden. Als Heterodiene werden meist $\alpha, \beta$-ungesättigte Carbonylverbindungen verwendet, die eine [4+2]-Cycloaddition mit inversem Elektronenbedarf eingehen, d. h. die Wechselwirkung des LUMOs vom Dien mit dem HOMO des Dienophils ist maßgeblich. Als Heterodienophile werden Aldehyde, Ketone, Imine und Nitrosoverbindungen eingesetzt,[58] dabei verläuft die Diels-Alder-Reaktion unter normalem Elektronenbedarf.

\subsubsection{Einsatz von in situ erzeugten Iminiumionen als Heterodienophile in Heck-Diels-} Alder-Reaktionssequenzen

Hetero-Diels-Alder-Reaktionen verlaufen i. a. nur dann, wenn man sehr elektronenarme Imine oder sehr elektronenreiche Diene unter Lewis-Säure Katalyse einsetzt. Grieco et al. beobachteten, daß in situ aus Formaldehyd-Lösung und Aminhydrochlorid erzeugte Iminiumionen in wäßrigem Medium als Heterodienophile ohne Zusatz von Lewis-Säure auch an unaktivierte Diene addieren.[59]

Waldmann et al. zeigten, daß entsprechende Reaktionen auch bei Verwendung von Hydrochloriden von Aminosäureestern möglich sind, und gelangte mit guten DiastereomerenÜberschüssen zu den entsprechenden Diels-Alder-Produkten (Schema 40).[60]<smiles>[C+]1C=CC=C1</smiles>

122<smiles>[R]C(N)C(OC)OCl</smiles>

123

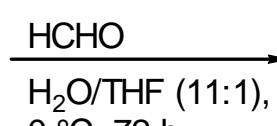
$0^{\circ} \mathrm{C}, 72 \mathrm{~h}$ $57 \%$, d. r. $93: 7$<smiles>[R][C@H]1CC2C=CC(C2)N1C(C)=O</smiles>

124

Schema 40. $\mathrm{R}=i \mathrm{Bu}$. 
Diese Hetero-Diels-Alder-Reaktion sollte sich auch auf die durch Heck-Reaktion dargestellten bisexocyclischen 1,3-Diene vom Typ 60 übertragen lassen. In vorausgegangenen Arbeiten konnte gezeigt werden, daß eine Domino-Heck-Diels-Alder-Reaktion dann die höchste Ausbeute ergibt, wenn auf die Isolierung des exocyclischen Diens 60 verzichtet wird.[21c] Da aber zur Darstellung der Iminiumionen eine Formaldehyd-Lösung zugegeben werden muß, kann die Reaktion nicht in einem einzigen Schritt durchgeführt werden. Durch Formaldehyd werden Palladium(II)salze zum Metall reduziert und fallen als sogenanntes "Palladium-Schwarz" aus. Die Reaktion wurde daher als Eintopf-ZweischrittReaktion durchgeführt.

Man setzte das Bromdien unter den üblichen Bedingungen mit $\mathrm{Pd}(\mathrm{OAc})_{2}, \mathrm{PPh}_{3}$ und einem Äquivalent Kaliumcarbonat 18 Stunden bei $80^{\circ} \mathrm{C}$ um. Nach Abkühlen auf Raumtemperatur gab man wäßrige Formaldehyd-Lösung, Glycinmethylester-Hydrochlorid (125-Gly) und Wasser zu. Nachdem man weitere 18 Stunden bei Raumtemperatur gerührt hatte, konnte nach Aufarbeitung das Hexahydropyridinderivat 126-Gly in 26\% Ausbeute isoliert werden (Schema 41, Tabelle 8). Steinig beobachtete, daß bei Verwendung von 0.5 Äquivalenten Base die Ausbeute der Heck-Hetero-Diels-Alder-Reaktion deutlich höher war. [29] Verwendete man daher nur 0.5 Äquivalente Kaliumcarbonat, konnte die Ausbeute auf $38 \%$ gesteigert werden (Eintrag 2). Weitere Variationen, wie Zugabe von Silbercarbonat als Base, Wechsel des Lösungsmittels oder Ausführung der Cycloaddition unter hohem Druck brachte keine weitere Ausbeuteerhöhung (Einträge 3-6). Unter den optimierten Bedingungen konnten verschiedene Amine zur Reaktion gebracht werden (Einträge 8-12). Setzte man Ammoniumchlorid (125-H) als Vorläufer des Dienophils ein, erhielt man nicht den Azabicyclus 126-H, sondern nur Zersetzung (Eintrag 8). Führte man die Sequenz in THF anstelle von Acetonitril durch, konnte bei Verwendung von 125-cPent neben dem Produkt 126-cPent noch das exocyclische Dien 60 in $45 \%$ Ausbeute isoliert werden (Eintrag 10). Im Gegensatz zur Reaktion von Kohlenstoffdienophilen mit 60 ergab diejenige mit in situ erzeugten Iminiumsalzen jeweils nur ein Regioisomer. 


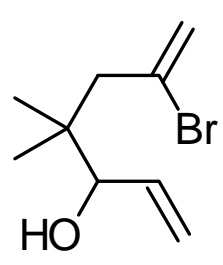

59

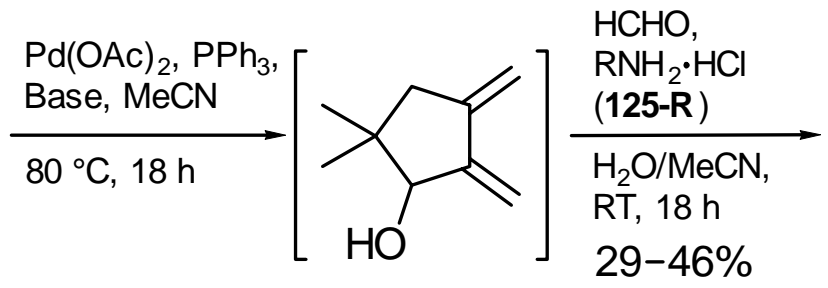

60<smiles>[R]N1CCC2=C(C1)C(O)C(C)(C)C2</smiles>

126-R

Schema 41. Details siehe Tabelle 8.

Tabelle 8. Umsetzung von $\mathbf{5 9}$ mit in situ erzeugten Iminiumsalzen als Heterodienophile 125-R (Schema 41).

\begin{tabular}{|c|c|c|c|}
\hline Eintrag & Amin & Base (Äquivalente) & Ausbeute (\%) \\
\hline 1 & 125-Gly & $\mathrm{K}_{2} \mathrm{CO}_{3}(1)$ & 26 \\
\hline 2 & 125-Gly & $\mathrm{K}_{2} \mathrm{CO}_{3}(0.5)$ & 38 \\
\hline 3 & 125-Gly & $\mathrm{Ag}_{2} \mathrm{CO}_{3}(0.5)$ & 11 \\
\hline 4 & 125-Gly & $\mathrm{K}_{2} \mathrm{CO}_{3}(0.5)$ & $4[\mathrm{a}]$ \\
\hline 5 & 125-Gly & $\mathrm{K}_{2} \mathrm{CO}_{3}(0.5)$ & $4[\mathrm{~b}]$ \\
\hline 6 & 125-Gly & $\mathrm{K}_{2} \mathrm{CO}_{3}(0.5)$ & $-[c]$ \\
\hline 7 & 125-Bn & $\mathrm{K}_{2} \mathrm{CO}_{3}(0.5)$ & 46 \\
\hline 8 & 125-H & $\mathrm{K}_{2} \mathrm{CO}_{3}(0.5)$ & - \\
\hline 9 & 125-cPr & $\mathrm{K}_{2} \mathrm{CO}_{3}(0.5)$ & 36 \\
\hline 10 & 125-cPent & $\mathrm{K}_{2} \mathrm{CO}_{3}(0.5)$ & 31 \\
\hline 11 & 125-cPent & $\mathrm{K}_{2} \mathrm{CO}_{3}(0.5)$ & $10^{[\mathrm{d}]}$ \\
\hline 12 & 125-cHex & $\mathrm{K}_{2} \mathrm{CO}_{3}(0.5)$ & 29 \\
\hline
\end{tabular}

[a] Statt Wasser wurde Methanol verwendet. - [b] Cycloaddition bei $50{ }^{\circ} \mathrm{C} .-[\mathrm{c}] 10 \mathrm{kbar}$, 3 d, Zersetzung und Polymerisation. - [d] Zusätzlich noch $45 \% 60$. 
Bei Zugabe von 3 Äquivalenten methanolischer $\mathrm{HCl}$ bildet sich aus dem Bicyclus 126-Gly durch Wasserabspaltung das Methylentetrahydropyridinderivat-Hydrochlorid 127-Gly in sehr guter Ausbeute (Schema 42).

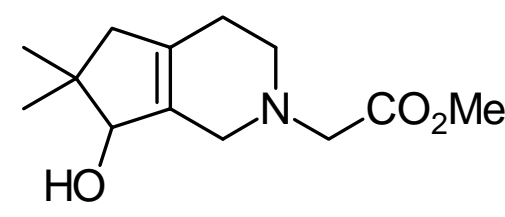

126-Gly

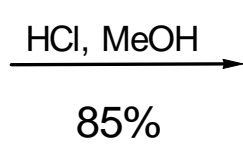

$85 \%$

Schema 42.

\subsubsection{Versuch einer Domino-Cycloisomerisierung-Hetero-Diels-Alder-Reaktion}

Entsprechend der Heck-Hetero-Diels-Alder-Sequenz wurde versucht, den fünfgliedrigen Ring durch eine Enin-Cycloisomerisierung aufzubauen und das dabei entstehende exocyclische Dien wiederum mit Heterodienophilen abzufangen.

Problematisch war jedoch die Wahl eines geeigneten Lösungsmittels. Während für die Bildung des Heterodienophils und dessen [4+2]-Cycloaddition Wasser benötigt wird, verläuft die Enin-Cycloisomerisierung am besten in Benzol. Deshalb erhielt man bei Zugabe des Prädienophils und der wäßrigen Lösung ein Zweiphasensystem, eine HeteroDiels-Alder-Reaktion fand nicht statt. Ein Versuch, die Cycloisomerisierung in Acetonitril durchzuführen, schlug ebenfalls fehl.[61]

Entfernte man vor Zugabe des Prädienophils das Benzol und löste das exocyclische Dien wieder in Acetonitril, konnte ebenfalls nicht das gewünschte Produkt erhalten werden.

Daher versuchte man den Heterocyclus 129 in zwei getrennten Stufen darzustellen. Dazu führte man im ersten Schritt die Cycloisomerisierung von $\mathbf{3 4}$ unter den üblichen Bedingungen mit $\mathrm{Pd}(\mathrm{OAc})_{2}$ und BBEDA durch und isolierte das exocyclische Dien 128 (65\% Ausbeute). Anschließend gab man zu einer Lösung von 128 in Acetonitril wäßrige Formaldehyd-Lösung, Glycinmethylester-Hydrochlorid (125-Gly) und Wasser. Nach 15 Stunden konnte weder das Dien 128 noch der Bicyclus 129 isoliert werden (Schema 43). 


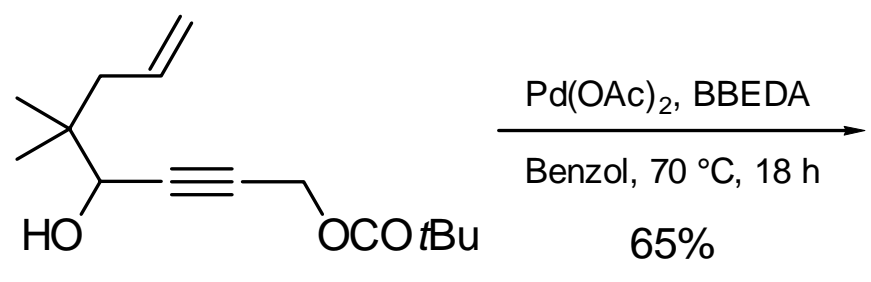

34

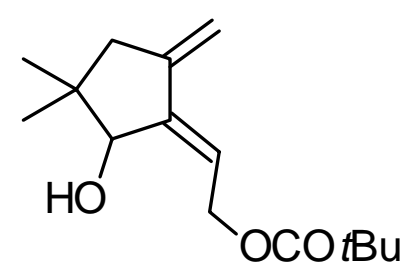

128
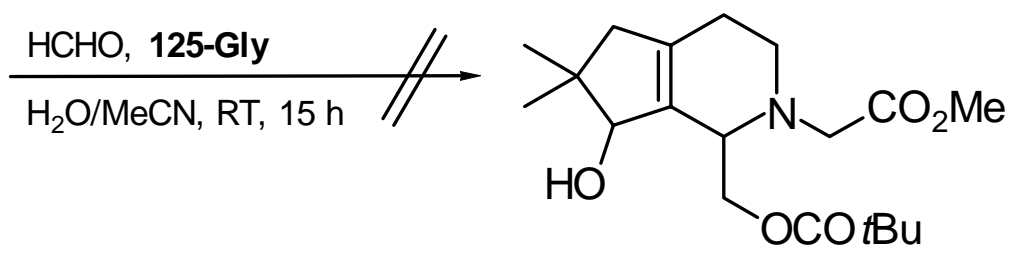

129

Schema 43.

\subsection{Versuch zur Verwendung von Trimethylsilylaldiminen als Heterodienophile}

Neben protonierten Iminen können Hetero-Diels-Alder-Reaktionen mit $N$-substituierten Iminen durchgeführt werden. Dabei werden i. a. $N$-Tosyl-, $N$-p-Methoxyphenyl-, $N$-Benzylund $\mathrm{N}$-Silylaldimine eingesetzt. Da es sich hierbei um nicht aktivierte Imine handelt, ist die Anwesenheit einer Lewis-Säure notwendig, um eine Cycloaddition zu ermöglichen. [58b] So zeigten Barluenga et al. die Zinkchlorid-katalysierte Reaktion von $N$-Silylbenzaldimin 130 zu Piperidinon 132 in guter Ausbeute und hoher Enantioselektivität (Schema 44). [62]

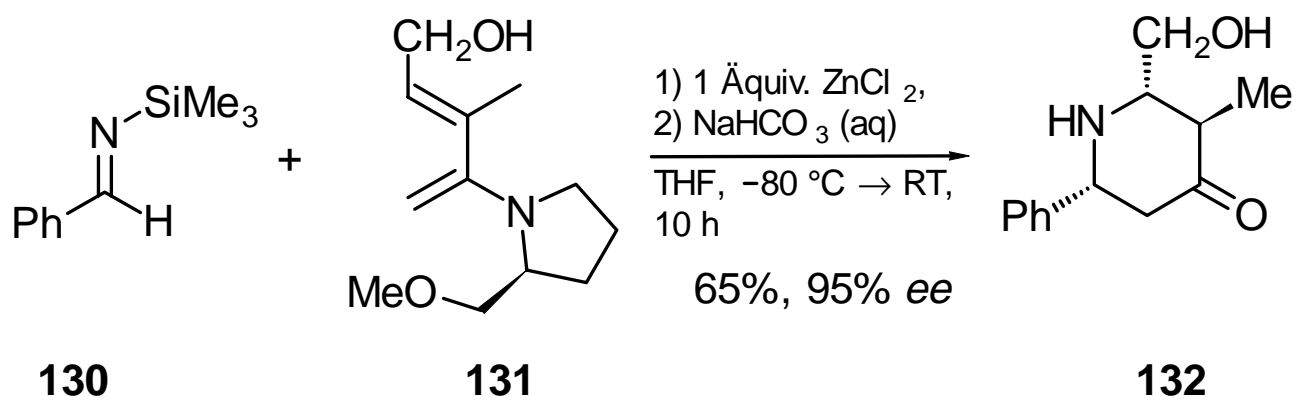

Schema 44. 
Die Verwendung von $N$-Trimethylsilylaldiminen als Heterodienophile sollte auch bei den hier beschriebenen Domino-Reaktionen möglich sein.

Ausgehend von Lithiumhexamethyldisilazan (134), was durch Lithiierung von Hexamethyldisilazan (133) hergestellt wurde, und Benzaldehyd (135) konnte in Anwesenheit von Trimethylchlorsilan $N$-Trimethylsilylbenzaldimin (130) dargestellt werden (28\% Ausbeute, Schema 45). Da sich das erhaltene Imin 130 nach wenigen Tagen bei $-26{ }^{\circ} \mathrm{C}$ bereits zersetzte, wurde es als Lösung in THF aufbewahrt und auch eingesetzt.

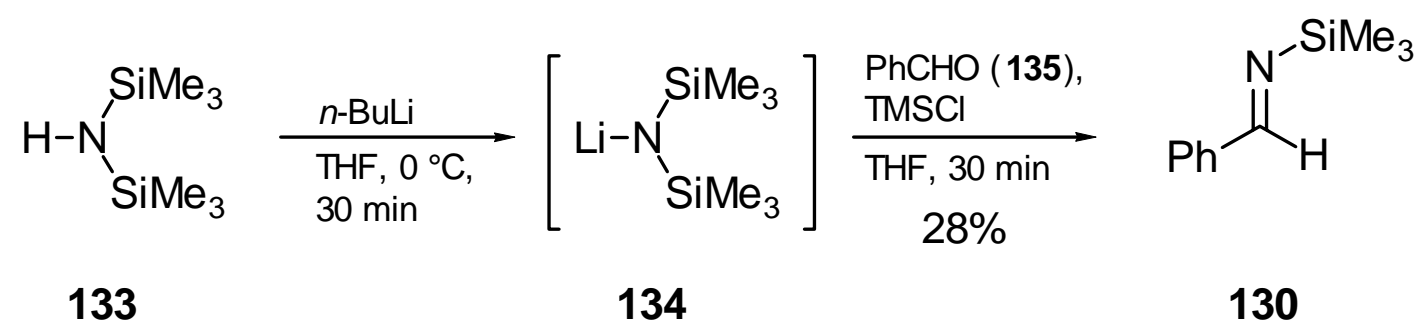

Schema 45.

In Gegenwart von $\mathrm{Pd}(\mathrm{OAc})_{2}, \mathrm{PPh}_{3}$ und $\mathrm{K}_{2} \mathrm{CO}_{3}$ konnten die Bromdiene 23 und 59 zu den entsprechenden exocyclischen Dienen 26 und 60 cyclisiert werden (Schema 46). Sodann wurde versucht, diese Diene unter verschiedenen Bedingungen mit 130 zur Reaktion zu bringen, allerdings ohne jeden Erfolg.

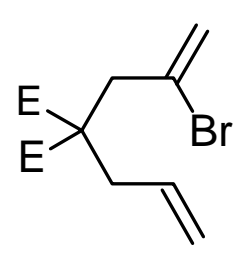

23

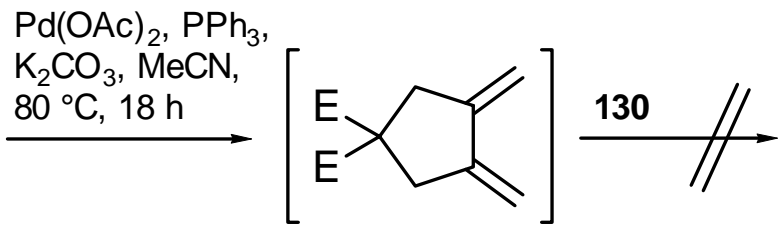

26<smiles>FC1(F)CC2=C(CC(c3ccccc3)NC2)C1</smiles>

135

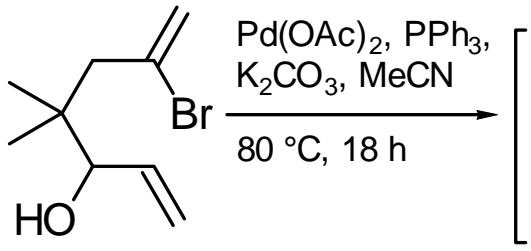

59

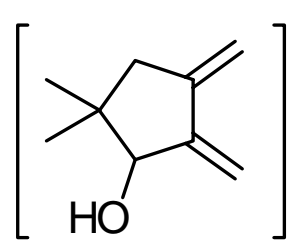

60

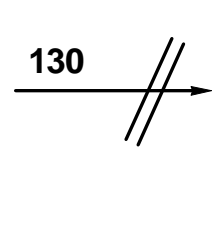<smiles>CC1(C)CC2=C(CNC(c3ccccc3)C2)C1O</smiles>

136

Schema 46. $\mathrm{E}=\mathrm{CO}_{2} \mathrm{Me}$. 
Auch bei Verwendung verschiedener Lewis-Säuren (Diethylaluminumchlorid, Zinkchlorid) und Variation von Druck und Temperatur konnten in allen Fällen nur Spuren der Diene 26 und 60 und nicht identifizierbare Zersetzungsprodukte isoliert werden (Schema 46).

\subsection{Versuch zum Aufbau von sauerstoffhaltigen Heterocyclen}

Mit Carbonylverbindungen als Dienophilen in [4+2]-Cycloadditionen, entstehen Heterocyclen mit Sauerstoff als Heteroatom. Unter "normalen" Reaktionsbedingungen sind nur Carbonylverbindungen reaktiv, die eine stark elektrophile Carbonylgruppe besitzen. Eingehend untersucht wurden Dienophile wie Formaldehyd, Chloral, Ketomalonate, Glyoxalsäureester-Derivate und verschiedene fluorierte Ketone.[58a] Bei Verwendung nichtaktivierter Carbonylverbindungen ist die Reaktion nur unter Lewis-Säure-Katalyse möglich. Auch asymmetrisch induzierende Reaktionen sind durch Anwendung von optisch aktiven Dienophilen möglich. Von Interesse ist der Einsatz von Diethylketomalonat (138) als Heterodienophil, da 138 ein Syntheseäquivalent des Kohlendioxides darstellt, das selbst nicht als Dienophil reagieren kann. Jorgensen beschrieb eine elegante Synthese eines $\gamma, \delta$ ungesätigten Lactons ausgehend von Cyclohexadien (137) und 138 (Schema 47).[58e] Die Diels-Alder-Reaktion konnte mit einer chiralen Lewis-Säure in sehr guter Ausbeute von 94\% und mit einem Enantiomerenüberschuß von 91\% durchgeführt werden. Daran schloß sich eine alkalische Verseifung und ein Curtius-Abbau zum ungesättigten Lacton 140 an. $[58 \mathrm{a}, 63]$

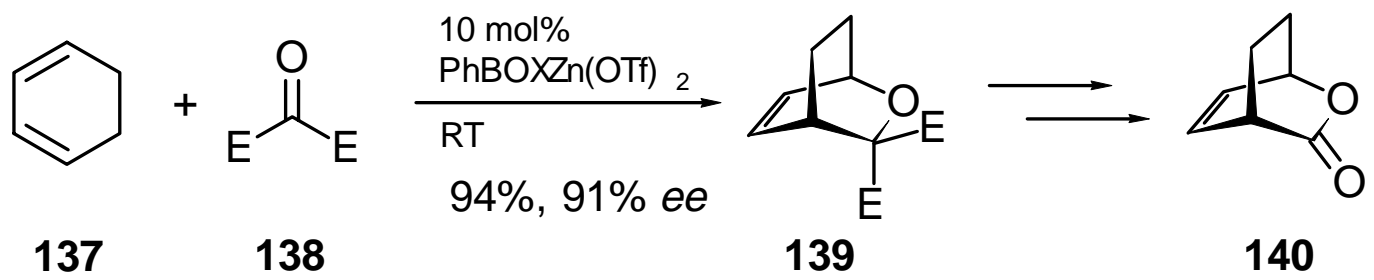

Schema 47. $\mathrm{E}=\mathrm{CO}_{2} \mathrm{Et}, \mathrm{PhBOX}=$ Phenyl-Bisoxazolin. 
Die Umsetzung mit 138 wurde auf das schon häufig benutzte Dien aus der Palladiumkatalysierten Cyclisierung des Bromdiens 59 übertragen. Dabei führte man die Reaktion als Eintopf-Zweischritt-Variante durch. Unter den bereits mehrfach beschriebenen Bedingungen, reagierte das Bromdien 59 in einer Heck-Reaktion zum exocyclischen Dien 60. Zu der Lösung des Diens gab man 2 Äquivalente Diethylketomalonat (138) und einige Kristalle Hydrochinon. Dabei fand erst bei einer Temperatur von $130{ }^{\circ} \mathrm{C}$ eine Reaktion statt, neben Zersetzung konnte nach einem Tag ein Gemisch aus den regioisomeren Produkten 141/142 und 138 im Verhältnis 1 : 1 abgetrennt werden. Die Ausbeute, bezogen auf die Bicyclen 141/142, war mit 6\% sehr niedrig (Schema 48). Aufgrund der Verunreinigung mit 138 konnte über die Regioselektivität keine Aussage getroffen werden. Durch Zusatz einer geeigneten Lewis-Säure sollte sich die Reaktion auch bei niedrigeren Temperaturen durchführen lassen. Die bei Temperaturen von $130{ }^{\circ} \mathrm{C}$ auftretende Zersetzung des Diens könnte dadurch verhindert werden.

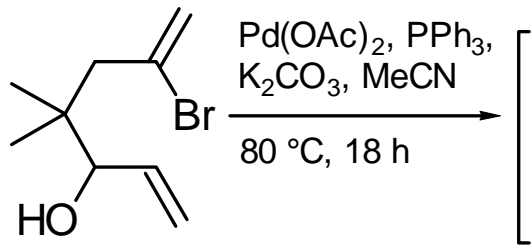

59<smiles>C=C1CC(C)(C)C1O</smiles>

60

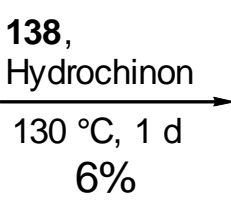

138 , $\underset{130{ }^{\circ} \mathrm{C}, 1 \mathrm{~d}}{6 \%}$
$\frac{\text { Hydrochinon }}{\longrightarrow}$

und/oder<smiles>CC1(C)CC2=C(COC(F)(F)C2)C1O</smiles>

141<smiles>CC1(C)CC2=C(C1)C(O)C(F)(F)OC2</smiles>

142

Schema 48. $\mathrm{E}=\mathrm{CO}_{2} \mathrm{Et}$. 


\section{Heck-Reaktionen an Bromeninen mit terminaler Dreifachbindung}

Bereits eingehend studiert wurden Palladium-katalysierte Kaskaden-Reaktionen von Bromdieninen des Typs 143 zu oligocyclischen Systemen.[9,18] Dabei findet zuerst ein 5-exo-dig-Ringschluß, dann ein 5-exo-trig-Ringschluß und anschließend eine Dehydropalladierung zum Trien 144 statt. Das Trien 144 kann in einer $6 \pi$-Elektrocyclisierung zum zweifach anellierten Cyclohexadien 145 reagieren, das seinerseits unter den Reaktionsbedingungen leicht zum bisanellierten Aromaten 146 dehydriert werden kann (Schema 49). Diese Reaktion wurde von Henniges, Schweizer und Thies auf verschiedene Ringgrößen und heteroatomhaltige Ringsysteme angewendet. Auch der Einfluß unterschiedlicher Substituenten wurde detailliert untersucht.[19]
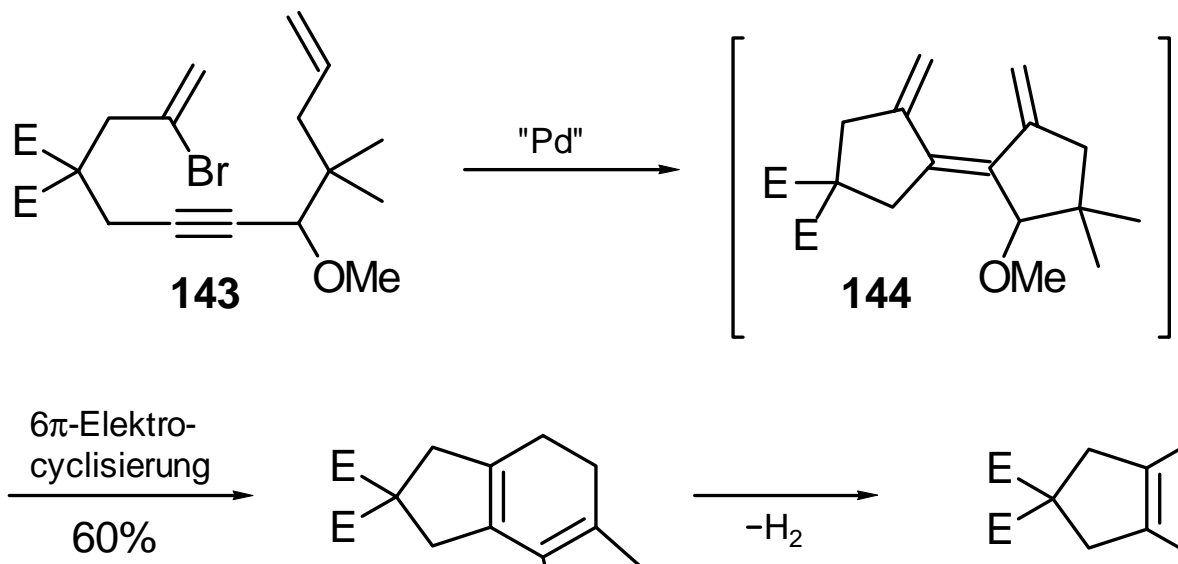

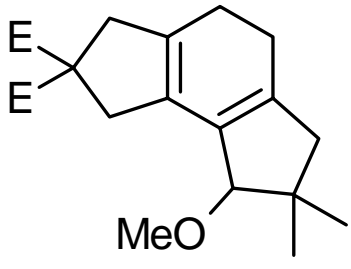

145

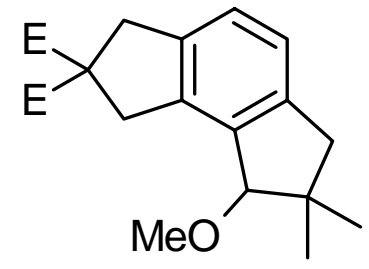

146

Schema 49. $\mathrm{E}=\mathrm{CO}_{2} \mathrm{Me}$.

Diese Reaktionskaskade sollte auf Bromenine vom Typ $\mathbf{1 5 1}$ mit terminaler Dreifachbindung übertragen werden. Dabei erwartete man in einem ersten Schritt nach der oxidativen Addition an die Palladium(0)-Spezies eine intramolekulare 5-exo-digCarbopalladierung zur Alkenyl-Palladium-Spezies 150, gefolgt von einer intermolekularen Heck-Reaktion mit Methylacrylat (35) als Alkenkomponente zu 149. Acrylate eignen sich besonders gut zum Einsatz in intermolekularen Heck-Reaktionen, da durch den elektronenziehenden Substituenten die C-C-Verknüpfung regioselektiv am sterisch weniger gehinderten Ende des Alkens erfolgt und die anschließende Dehydropalladierung 
stereoselektiv zum trans-disubstituierten Alken führt. Anschließende $6 \pi$-Elektrocyclisierung sollte das Cyclohexadien 148 ergeben, das vermutlich zu 147 aromatisieren würde (Schema 50).

Von Henniges wurden entsprechende Reaktionen von Phenyl- und Methoxycarbonylsubstituierten Bromeninen mit unterschiedlichen Alkenen $\mathrm{zu}$ anellierten Cyclohexadienderivaten und Aromaten durchgeführt.[19a]

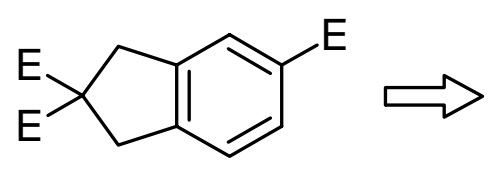

147<smiles>FC1C=CC2=C(C1)CC(F)(F)C2</smiles>

148<smiles>C=C1CC(F)(F)C/C1=C/C=C/F</smiles>

149

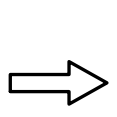

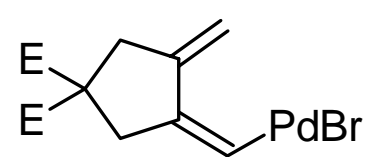

150
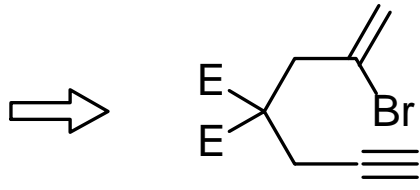

151

Schema 50. $\mathrm{E}=\mathrm{CO}_{2} \mathrm{Me}$.

Versetzte man das Bromenin 151 mit $\mathrm{Pd}(\mathrm{OAc})_{2}, \mathrm{PPh}_{3}$, Base und Methylacrylat (35) als weitere Alkenkomponente, so erhielt man ein komplexes Produktgemisch. Bei Verwendung von Kaliumcarbonat erhielt man ein Gemisch einer nicht identifizierten Verbindung 154 mit dem Tetraen 153 (5\% Ausbeute) und 12\% des achtgliedrigen Ringes 155- $\mathrm{CO}_{2} \mathrm{Me}$ (Schema 51). Die Verbindung 154 wurde immer in Gegenwart von 153 gefunden. Aus dem Massenspektrum ging hervor, das es sich dabei um ein Isomeres des Tetraens 153 handeln mußte, eine Struktur konnte jedoch nicht eindeutig ermittelt werden. Steinig konnte bei einer ähnlichen Reaktion ebenfalls eine Dimerisierung eines AlkenylPalladium-Komplexes beobachten, [29] die Bildung eines Achtringes ist jedoch bisher noch nicht festgestellt worden. Vergrößerte man den Reaktionsansatz von einem Millimol auf drei Millimol, so fand vermehrt die Bildung der Verbindungen 153 und 154 statt (18\% Ausbeute, Tabelle 9, Eintrag 2), das Cyclooctadien $155-\mathbf{C O}_{2} \mathbf{M e}$ wurde nur in 5\% Ausbeute isoliert. Die Verwendung des sterisch anspruchsvolleren tert-Butylacrylats (152) verhinderte die Bildung von 155- $\mathrm{CO}_{2} t \mathrm{Bu}$, die Ausbeute an 153 und 154 war ebenfalls geringer (9\% Ausbeute, Eintrag 4). Der Wechsel zu Cäsiumcarbonat als Base bewirkte nur eine Verschlechterung der Ausbeute (Eintrag 6), ohne Zugabe von Methylacrylat betrug die Ausbeute der Komponenten 153 und 154 15\%. Bei Verwendung von Silbercarbonat konnte 
keine Cyclisierung festgestellt werden, vielmehr fand eine Glaser-ähnliche Kupplung (s. a. Kapitel 2.3.) an der terminalen Alkineinheit zu 157 statt (Schema 51).

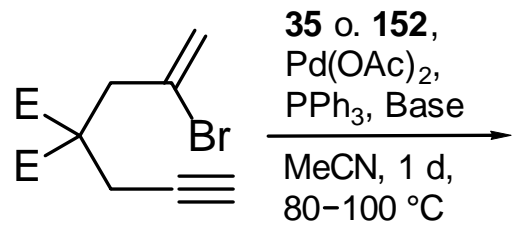

151

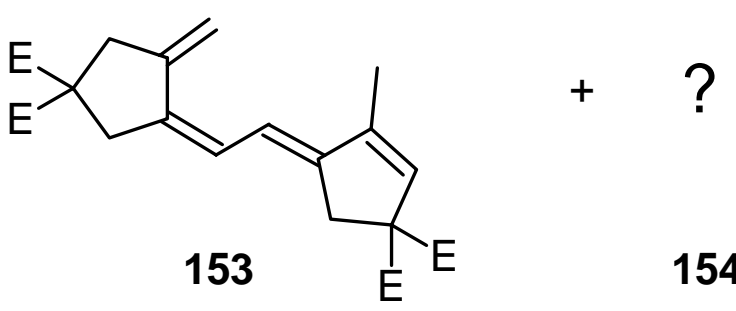<smiles>[R]C1=CC2=C(CCC([R])C1)CC(F)(F)C2</smiles>

155-R

156

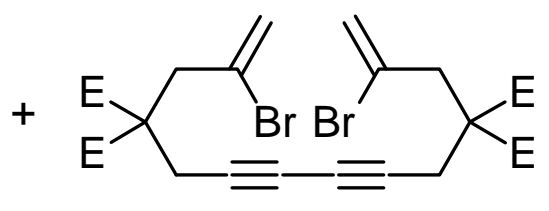

157

Schema 51. $\mathrm{E}=\mathrm{CO}_{2} \mathrm{Me}, \mathrm{R}=\mathrm{CO}_{2} \mathrm{Me}, \mathrm{CO}_{2} t \mathrm{Bu}$. Details siehe Tabelle 9.

Tabelle 9. Abhängigkeit der Produktbildung aus dem Bromenin 151 (Schema 51).

\begin{tabular}{|c|c|c|c|c|c|c|c|}
\hline Eintrag & Base & $\begin{array}{c}\text { Alken } \\
\text { (Äquiv.) }\end{array}$ & $\begin{array}{c}\text { Temp. } \\
{\left[{ }^{\circ} \mathrm{C}\right]}\end{array}$ & Produkt & $\begin{array}{c}\text { Ausbeute } \\
(\%)\end{array}$ & $\begin{array}{l}\text { Weiteres } \\
\text { Produkt }\end{array}$ & $\begin{array}{c}\text { Ausbeute } \\
(\%)\end{array}$ \\
\hline 1 & $\mathrm{~K}_{2} \mathrm{CO}_{3}$ & 35 (4) & 80 & $153 / 154$ & 5 & 155-Me & 12 \\
\hline 2 & $\mathrm{~K}_{2} \mathrm{CO}_{3}$ & $35(3)$ & 80 & $153 / 154$ & 18 & 155-Me & 5 \\
\hline 3 & $\mathrm{~K}_{2} \mathrm{CO}_{3}$ & - & 80 & $153 / 154$ & 15 & - & - \\
\hline 4 & $\mathrm{~K}_{2} \mathrm{CO}_{3}$ & $152(4)$ & 80 & $153 / 154$ & 9 & - & - \\
\hline 5 & $\mathrm{~K}_{2} \mathrm{CO}_{3}$ & $35(4)$ & 100 & $153 / 154$ & 6 & 156 & $4[\mathrm{a}]$ \\
\hline 6 & $\mathrm{Cs}_{2} \mathrm{CO}_{3}$ & $35(4)$ & 80 & $153 / 154$ & 6 & 155-Me & 4 \\
\hline 7 & $\mathrm{Ag}_{2} \mathrm{CO}_{3}$ & $35(3)$ & 80 & 157 & 43 & - & - \\
\hline
\end{tabular}

[a] zusätzlich erhielt man noch $21 \mathrm{mg}$ eines Gemisches aus 153/154 und 156. 
Die Dimerisierung der Alkenyl-Palladium-Spezies 150 zu 153 könnte wie folgt verlaufen: Im ersten Schritt findet ein 5-exo-dig-Ringschluß von 151 zu 150 statt. Diese Spezies 150 kann auf zwei denkbaren Reaktionswegen zu 153 führen:

- Durch erneute oxidative Addition des Bromenins 151 an die Alkenyl-Palladium-Spezies 150, gefolgt von einem 5-exo-dig-Ringschluß entsteht über ein Palladium(IV)-Intermediat die Bisalkenylpalladium(II)-Zwischenstufe 158. Durch reduktive Eliminierung bildet sich daraus das Tetraen 159 und eine Palladium(0)-Spezies.

- Zwischen zwei Alkenylpalladium(II)-Spezies 150 wird ein Ligand übertragen und man erhält die Palladium(II)-Spezies 158. Diese zerfällt unter reduktiver Eliminierung zum Tetraen 159 und einer Palladium(0)-Spezies.

Eine anschließende Doppelbindungsisomerisierung in 159 führt zu 153 (Schema 52).

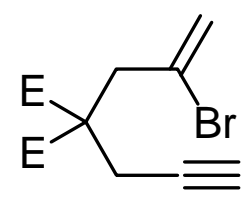

151<smiles></smiles><smiles>[3H][3H]</smiles>

150

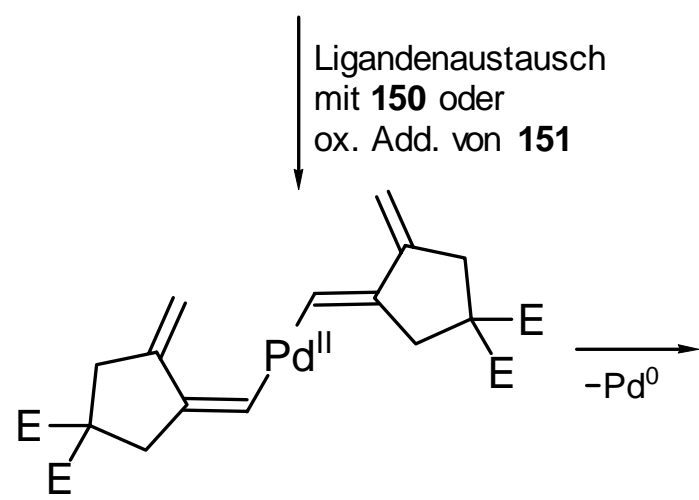

158<smiles>C=C1CC(F)(F)C/C1=C/C=C1\CC(F)(F)C=C1C</smiles>

$[1,5]-\mathrm{H} \sim$<smiles>C=C1CC(F)(F)C/C1=C/C=C1\CC(F)(F)CC1=C</smiles>

159

Schema 52. $\mathrm{E}=\mathrm{CO}_{2} \mathrm{Me}$. 
Die Bildung des achtgliedrigen Ringes 155- $\mathrm{CO}_{2} \mathrm{Me}$ läßt sich wie folgt erklären:

Im ersten Schritt findet ein 5-exo-dig-Ringschluß zur Alkenylpalladium-Spezies 150 statt, anschließend erfolgt eine intermolekulare Heck-Reaktion mit dem Acrylat 35 zur Alkylpalladium-Spezies 160. Dann folgt allerdings nicht die zu erwartende Dehydropalladierung, sondern eine weitere Insertion in die Doppelbindung eines weiteren Moleküls 35. Solche Reaktionen können im allgemeinen nur dann stattfinden, wenn die $\beta$-Hydrideliminierung retardiert ist. Da Palladium und $\beta$-Wasserstoff zur Eliminierung syn zueinander stehen müssen, ist eine Rotation um die $\sigma$-Bindung, an der sich der Palladiumrest befindet, notwendig. Ist die Rotation gehindert, so kann die Eliminierung nicht stattfinden. Beobachtet wurde dies an Systemen, bei denen sich die AlkylpalladiumSpezies an einem Ring befindet.[64,65] Betrachtet man die Verbindung 160, so läßt sich eine Beeinträchtigung der Rotation jedenfalls nicht aufgrund der Struktur erklären. Daher ist es ungewöhnlich, daß eine zweite intermolekulare Heck-Reaktion mit $\mathbf{3 5}$ zur Spezies 161 schneller abläuft als die Dehydropalladierung. Auch hier findet keine $\beta$ Hydrideliminierung statt, sondern es schließt sich eine 8-endo-trig-Carbopalladierung an. Anschließende Dehydropalladierung ergibt das Cyclooctadien 162 und Doppelbindungsisomerisierung führt zu dem Bicyclus 155- $\mathbf{C O}_{2} \mathbf{M e}$ (Schema 53).

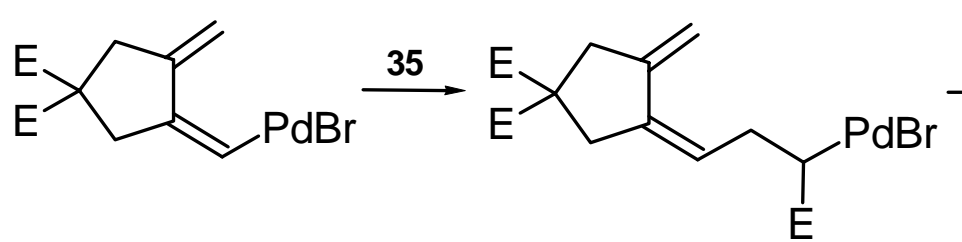

150
160

155-Me

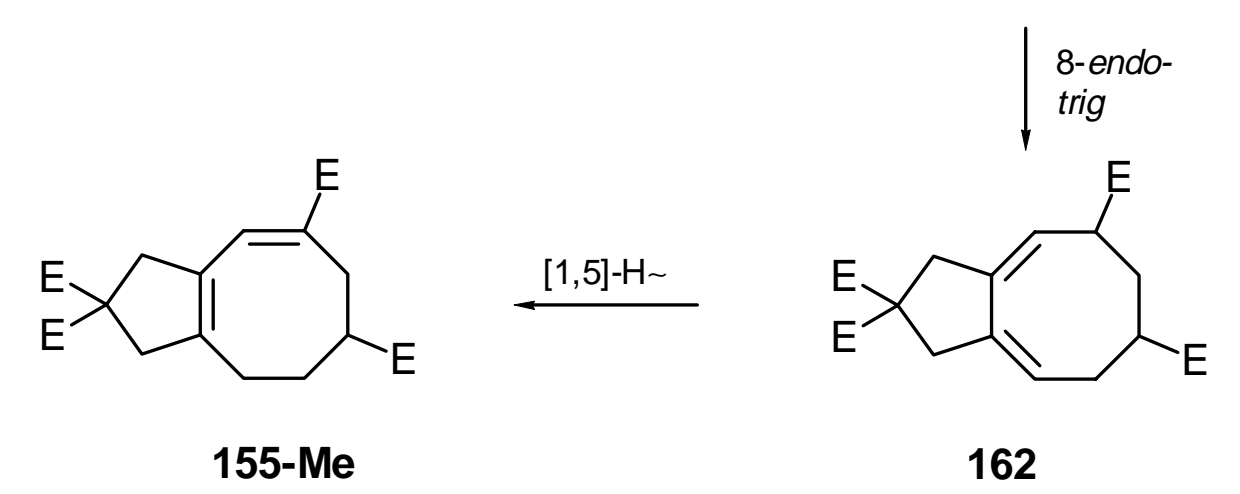<smiles>C=C1CC(F)(F)C/C1=C/CC(C)CC(C)C(=O)Br</smiles>

161

Schema 53. $\mathrm{E}=\mathrm{CO}_{2} \mathrm{Me}$. 
Zu größeren Ringen hin treten endo-trig-Cyclisierungen häufiger als exo-trig-Ringschlüsse auf. Selektive 8-endo-trig-Produkte in intramolekularen Heck-Reaktionen wurden von Rigby et al. zum Aufbau stickstoffhaltiger Achtringe genutzt.[66] Roberts et al. entwickelten zur Synthese von Tryptophan-Derivaten als Schlüsselschritt einen 8-endotrig-Ringschluß, als Nebenreaktion fand jedoch auch eine 7-exo-trig-Cyclisierung statt.[67]

Für die Bildung des Tricyclus 156 lassen sich wie für die Bildung des Tetraens 152 zwei mögliche Wege beschreiben. Ausgehend von 150 gelangt man durch eine oxidative Addition von 151, dann aber gefolgt von einem 6-endo-dig-Angriff, zu der entsprechenden Palladium(IV)-Spezies, die nach reduktiver Eliminierung das Tetraen 164 ergibt. Die Bildung von 164 kann aber auch, wie oben erwähnt, durch Ligandenaustausch von 150 und 163 stattfinden. Dabei wird 163 durch einen 6-endo-dig-Ringschluß gebildet. Während 6-endo-trig-Ringschlüsse nur formal existieren (Kapitel 2.4.), finden 6-endo-dig-Ringschlüsse häufig als Konkurrenzreaktion $\mathrm{zu}$ 5-exo-dig-Cyclisierungen statt.[68] Das erhaltene Tetraen 164 kann durch 6 $\pi$-Elektrocyclisierung zum bisanellierten Cyclohexadien 165 reagieren, was unter den Reaktionsbedingungen umgehend zum Aromaten 156 dehydriert wird (Schema 54). Die Bildung dieser Verbindung wurde erst bei einer Reaktionstemperatur von $100{ }^{\circ} \mathrm{C}$ beobachtet.<smiles></smiles>

163

156<smiles>C=C1CC(F)(F)Cc2cc3c(cc21)CC(F)(F)C3</smiles><smiles>C=C1C=C(/C=C2/CC(F)(F)CC2=C)CC(F)(F)C1</smiles>

164

$6 \pi$-Elektrocyclisierung

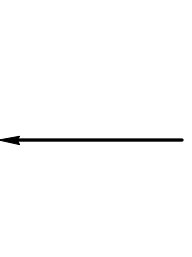<smiles>C=C1CC(F)(F)CC2=CC3=C(CC12)CC(F)(F)C3</smiles>

165

Schema 54. $\mathrm{E}=\mathrm{CO}_{2} \mathrm{Me}$. 
Bei Verwendung von Kaliumcarbonat als Base in Heck-Reaktionen können [1,5]-HVerschiebungen als Nebenreaktion stattfinden. Diese Doppelbindungsisomerisierung läßt sich vermeiden, wenn Silbercarbonat als Base eingesetzt wird. ${ }^{[9]}$ Allerdings erhielt man hier bei der Behandlung von 151 mit $\mathrm{Pd}(\mathrm{OAc})_{2}, \mathrm{PPh}_{3}, \mathrm{Ag}_{2} \mathrm{CO}_{3}$ und Methylacrylat (35) nicht die erwarteten Verbindungen 159 und 162, sondern konnte das Glaser-Kupplungsdimer 157 in $43 \%$ Ausbeute isolieren. Diese Dimerisierung verläuft entsprechend der Dimerisierung von 121 (Kapitel 2.5.) wobei das zugesetzte Silbersalz als Oxidationsmittel wirkt.

Da $\sigma$-Alkenylpalladium-Komplexe vom Typ 150 mit Hydridionen abgefangen werden können,[68d] wurde versucht, ob nicht 157 in einer weiteren Palladium-katalysierten Reaktion über das zweifache Carbopalladierungsintermediat 166 zu 159 reagieren kann, wenn Natriumformiat als Hydridquelle angeboten wird. Dazu versetzte man das Dimer 157 mit $\mathrm{Pd}(\mathrm{OAc})_{2}, \mathrm{PPh}_{3}, \mathrm{~K}_{2} \mathrm{CO}_{3}$ sowie Natriumformiat und erwärmte auf $80{ }^{\circ} \mathrm{C}$. Doch konnte man kein Cyclisierungsprodukt isolieren, sondern nur das Edukt zurückgewinnen (Schema 55).

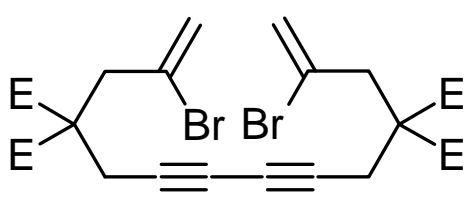

157

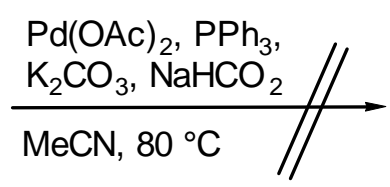

$\mathrm{MeCN}, 80^{\circ} \mathrm{C} / /$

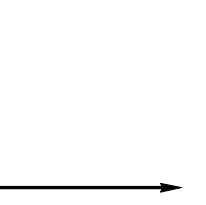

$$
\text { E }
$$

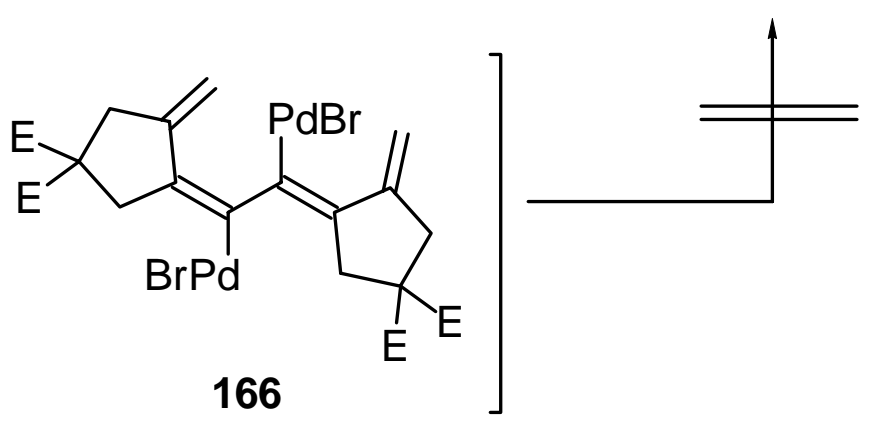

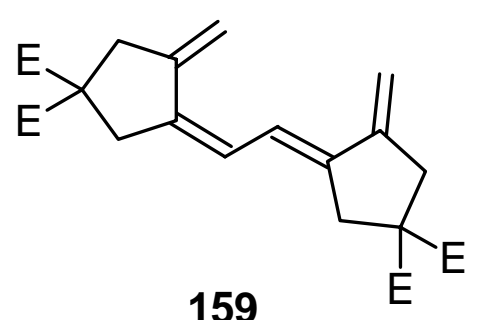

159

Schema 55. $\mathrm{E}=\mathrm{CO}_{2} \mathrm{Me}$. 


\section{Darstellung eines Cyclisierungsvorläufers zum Aufbau eines Spinosyn- Gerüstes und dessen Umsetzung}

\subsection{Vorüberlegungen}

Spinosyn A (167-H) ist ein Makrolid, das von Saccharopolyspora spinosa, einer Bodenmikrobe aus der Klasse der Actinomyceten, produziert wird. Dieses Makrolid trägt an zwei Positionen eines tetracyclischen Ringsystems einen glykosidisch verknüpften Zuckerrest (Abb. 2). Ein Ring im Tetracyclus ist ein macrocyclisches Lacton, die drei restlichen Ringe bilden ein Octahydro- $a s$-indacen Gerüst, das auch in anderen Antibiotika wie Ikarugamycin und Capsimycin vorkommt.[69]

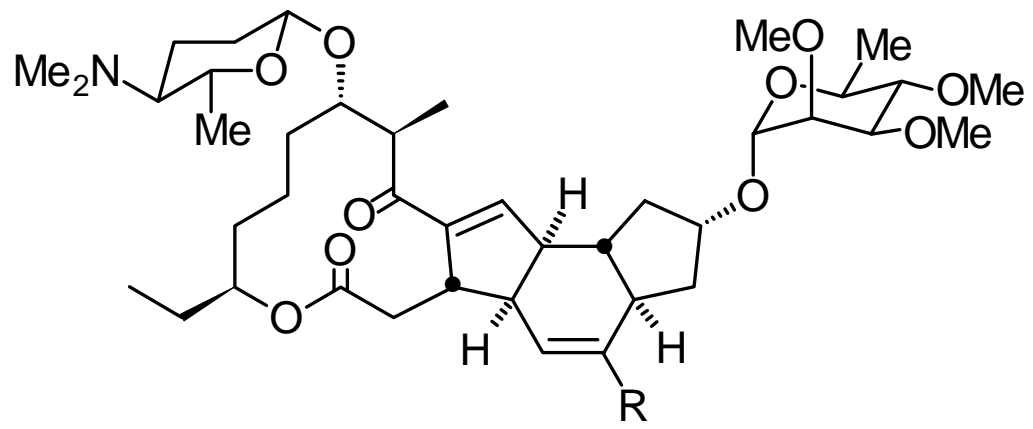

167-R

Abbildung 2. R = H: Spinosyn A, R= Me: Spinosyn D.

Spinosyn A besitzt ein großes Insektizid-Potential. Das von DowAgroScience vertriebene Insektizid Spinosad ${ }^{\circledR}$, ein Gemisch aus Spinosyn A (85\%) und D (15\%), ist das derzeit wirksamste Insektizid auf dem Markt und wird vor allem gegen Lepidopterenlarven (Schmetterlingslarven) eingesetzt.[69a] Der Wirkmechanismus besteht darin, daß Spinosyn das Nervensystem der Insekten reizt, was zu unwillkürlichen Muskelzuckungen und letztendlich zu Lähmungen führt. Neben einen Angriff am GABA-Rezeptor liegt die Hauptwirkung von 167 in einer Aktivierung des nikotinischen Acetylcholin-Rezeptors. Im Gegensatz zu anderen Insektiziden wie Pyrethroiden oder Carbamaten zeigt Spinosyn keine Kontakt- und Fraßtoxizität auf Nützlinge.[69b] 
Die zentrale Octahydro-as-indaceneinheit von 167-R wurde von Paquette et al. ausgehend von (+)-7,7-Dimethoxynorbornen-2-on (168) aufgebaut (Schema 55).[70] In Gegenwart von tert-Butyllithium und $\mathrm{CeCl}_{3}$ wurde an 168 3-Brom-1(R)-(tert-butyldimethylsilyloxy)cyclopent-3-en (169) addiert. Das Norbornenderivat 170 konnte in 76\% Ausbeute erhalten und in wenigen Schritten zum Tricyclus 171 umgelagert werden (Schema 56). Dabei mußte zunächst racemisches $( \pm)-\mathbf{1 6 8}$ in entantiomerenreines (+)-168 umgewandelt werden. Unter Verwendung von Johnson's Sulfoximin[71] gelang dies, jedoch nur in mäßigen Ausbeuten von $35 \%$ für (+)-168 und $18 \%$ für (-)-168.

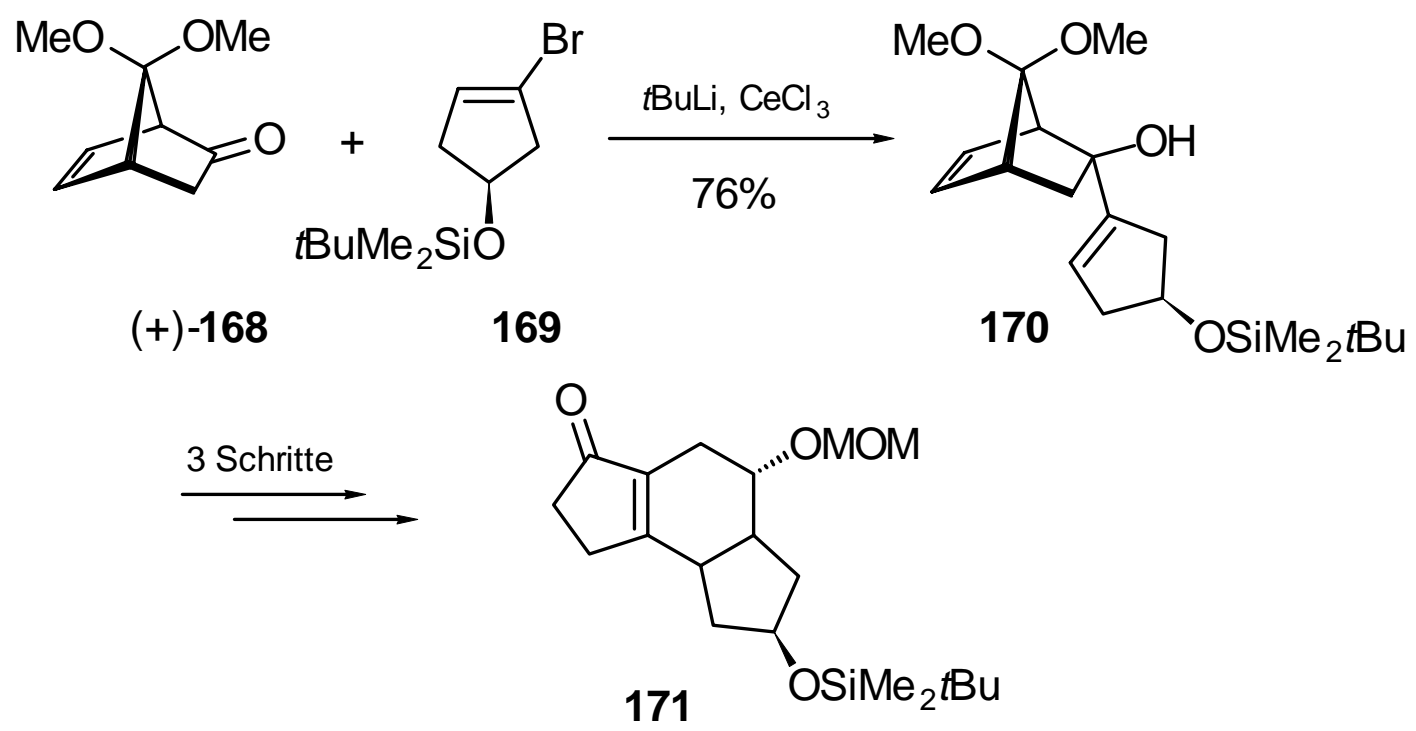

Schema 56.

Ein solcher Tricyclus könnte auch direkt aus einem Enin vom Typ 176 durch einen intraintermolekularen Prozeß aus Enin-Cycloisomerisierung und Diels-Alder-Reaktion hervorgehen (Schema 57). Ähnliche Oligohydro-as-indacene konnten durch Palladiumkatalysierte Transformationen von Thies[19c] und Schweizer[19b] dargestellt werden. Die von ihnen verwendeten Cyclisierungsvorläufer besaßen jedoch eine Alkoholfunktion in $\alpha$ Stellung zur Alkin-Einheit, weil die Darstellung durch Umsetzung eines Lithiumacetylids mit einem Aldehyd erfolgte. Diese einfache Synthese läßt sich auf die Darstellung eines Vorläufers für das Gerüst von 167-R nicht anwenden, da sich die Hydroxyfunktion in $\beta$ Stellung zur Dreifachbindung befinden muß. Für die Synthese eines Alkins vom Typ 175 mußte daher eine andere Methode gefunden werden. 
Ein Weg könnte folgendermaßen aussehen: Protonen in Propargylposition sind ausreichend acide, um mit einer starken Base, wie $n$-Butyllithium abstrahiert zu werden. Dabei ist jedoch ein Zusatz von $N, N, N^{\prime}, N^{\prime}$-Tetramethylethylendiamin (TMEDA) notwendig. Normalerweise liegen Lithiumalkyle in THF in Form von Tetrameren oder Hexameren vor. Durch den Zusatz von TMEDA wird das Lithiumion komplexiert und diese Aggregate aufgespalten.[72] Damit nimmt die Reaktivität der Organolithiumverbindung zu und die Deprotonierung in Propargylstellung wird möglich. Das so gebildete Propargylanion kann als Nucleophil an Aldehyde addieren.

Eine weitere Möglichkeit ist die Verwendung eines Propargylmagnesiumhalogenids. Ausgehend von Propargylbromid läßt sich das entsprechende Grignard-Reagenz nach einer Vorschrift von Marshall leicht darstellen.[73] Die Addition an einen Aldehyd ergibt ebenfalls Alkine vom Typ 175 (Schema 57).

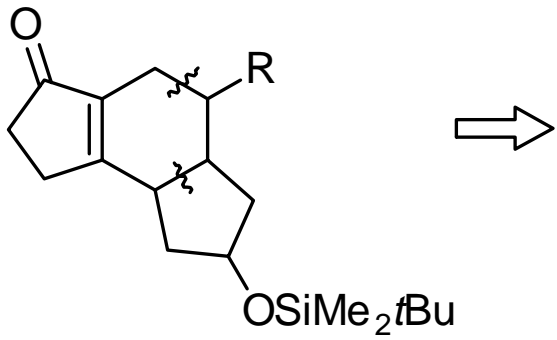

172

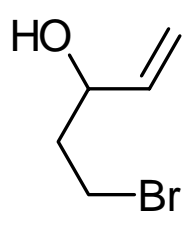

174

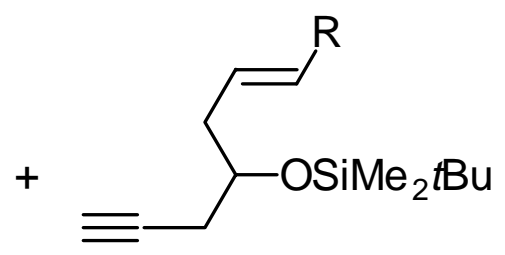

175

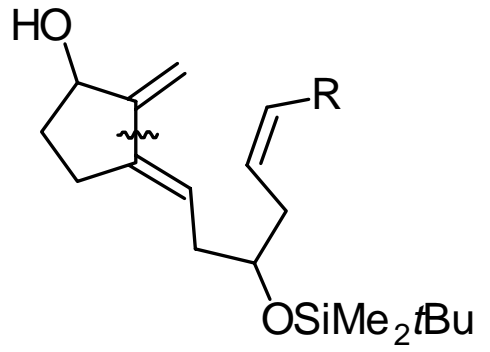

173
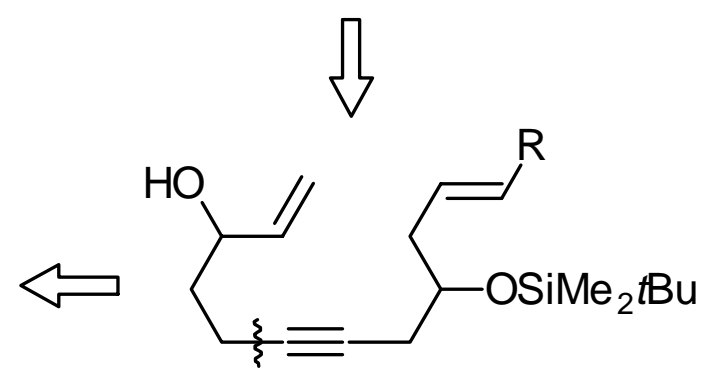

176

Schema 57. Retrosynthetische Überlegungen. 


\subsection{Versuch zur Synthese eines Vorläufers mit Silylenolether-Funktion}

In einem ersten Versuch sollte der Cyclisierungsvorläufers 176 in racemischer Form dargestellt werden. Zuerst wurde die Synthese eines Dienins mit einer Silylenoletherfunktion angestrebt. Die Verwendung von Enolethern als Dienophile in Diels-AlderReaktionen ist bekannt, ${ }^{[74]}$ allerdings würden sie nach der Cycloaddition einen Ether ergeben, dessen Spaltung recht drastische Bedingungen erfordern dürfte. Der Einsatz von Silylenolethern als Dienophil ist bislang nur wenig untersucht. Besser bekannt sind Diene vom Danishefsky-Typ, bei denen ein Silylenolether Teil der Dieneinheit ist.[3] Diese Diene sind elektronenreich und sehr reaktiv bei Umsetzungen mit elektronenarmen Dienophilen. Reissig et al. untersuchten die Verwendung von Silylenolethern als Dienophile in HeteroDiels-Alder-Reaktionen. ${ }^{[75]}$ Sie stellten fest, daß die [4+2]-Cycloaddition besonders gut mit elektronenarmen Dienen im Sinne einer Diels-Alder-Reaktion mit inversem Elektronenbedarf abläuft. Obwohl hier ein Dien mit elektronenspendenden Substituenten vorliegt, sollte dennoch eine Diels-Alder-Reaktion mit einem Silylenolether versucht werden. Der Vorteil läge darin, daß nach der Cycloaddition ein Silylether gebildet würde, der leicht zum gewünschten Alkohol zu spalten wäre.[76]

Ausgehend von 1,4-Butandiol (177) wurde durch Zugabe von 0.2 Äquivalenten Dihydropyran der einfach geschützte THP-Ether dargestellt. Eine anschließende SwernOxidation ergab den Aldehyd 178 in einer Gesamtausbeute von 56\% Ausbeute (Schema 58). Zur Synthese des Alkins 180 fügte man 1-Trimethylsilylpropin (179) zu, das mit $n$-Butyllithium und TMEDA in Propargylstellung deprotoniert wurde. Das gebildete Propargylanion konnte als Nucleophil an den Aldehyd 178 addieren. Anschließend spaltete man mit Kaliumcarbonat in Methanol die Trimethylsilylgruppe ab und isolierte das Alkin 180-H (17\% Ausbeute). Zur Schützung der Alkoholfunktion versetzte man 180-H mit tertButyldimethylsilylchlorid (181) und Imidazol und konnte 180-SiMe 2 von 69\% gewinnen. Da TBDMS-Ether unter den üblichen sauren Bedingungen einer THPEntschützung ebenfalls abgespalten werden, griff man zur selektiven Spaltung des THPEthers auf eine von Shibasaki et al. entwickelte Methode zurück.[77] Dazu versetzte man den Ether 180-SiMe $e_{2} t$ Bu mit 2 Äquivalenten einer 1 M Diethylaluminiumchlorid-Lösung, dabei konnte man den TBDMS-Ether 182 in 64\% Ausbeute isolieren. Eine Oxidation mit dem Dess-Martin-Periodinan[78] ergab den Aldehyd 183 (45\% Ausbeute). Die 
Deprotonierung von 183 mit Triethylamin in DMF bei $120^{\circ} \mathrm{C}$ nach einer Vorschrift von Effenberger et al.[79] und der Abfang des Enolats mit tert-Butyldiphenylsilylchlorid führte nicht zum gewünschten Silylenolether 184. Vielmehr konnte nur die Zersetzung des Aldehyds 183 festgestellt werden.

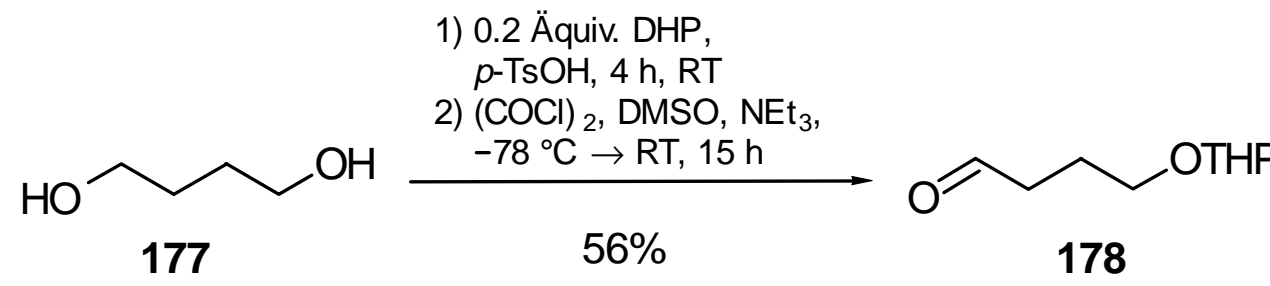

1) $n \mathrm{BuLi}$, TMEDA, $\mathrm{Me}_{3} \mathrm{Si}-\bar{\equiv}$

179

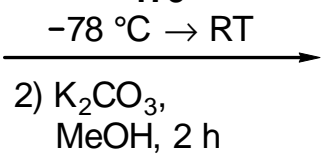

$17 \%$

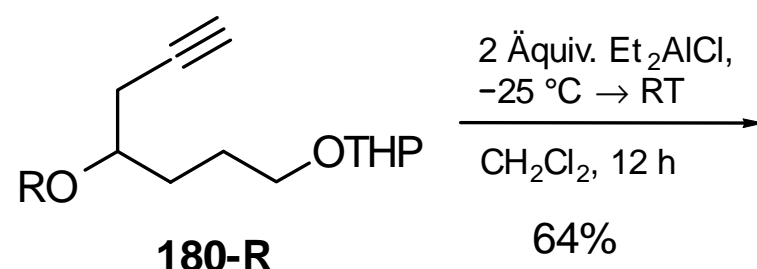

180-R<smiles>C#CCC([R]O)CCCO</smiles>

$$
\begin{aligned}
& \mathrm{R}=\mathrm{H} \quad \longrightarrow \quad \mathrm{SiMe}_{2} \mathrm{tBuCl}(181) \text {, } \\
& R=\mathrm{SiMe}_{2} t \mathrm{Bu} \longleftarrow \quad \begin{array}{r}
\text { Imidazol } \\
69 \%
\end{array}
\end{aligned}
$$

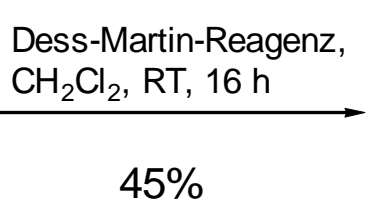

$45 \%$

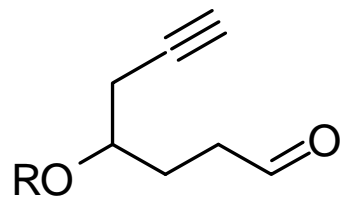

183

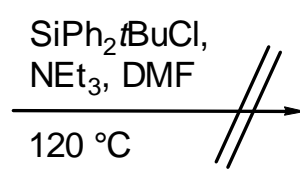

$\mathrm{RO}$<smiles>[R]C(CC#C)C/C=C/O</smiles>

184

Schema 58. $\mathrm{R}=\mathrm{SiMe}_{2} t \mathrm{Bu}, \mathrm{R}^{\prime}=\mathrm{SiPh}_{2} t \mathrm{Bu}$.

\section{3. $\quad$ Synthese eines Vorläufers mit Vinylchlorid-Terminus}

Nach der misslungenen Darstellung des Vorläufers mit einer Silylenolether-Funktion wurde nun eine Verbindung mit einem Vinylchlorid-Terminus angestrebt. Aldehyde lassen sich nach Takai in einer Wittig-ähnlichen Reaktion in Vinylchloride umwandeln.[80] Um einen entsprechenden Vorläufer darzustellen ging man von 1,3-Propandiol (185) aus. Der Schutz nur einer Hydroxygruppe von 185 ließ sich bei Verwendung eines 5-fachen Überschusses des Diols 185 in einer Ausbeute von 72\% realisieren (Schema 59). Nach 
Swern-Oxidation erhielt man den Aldehyd 187 (80\% Ausbeute). Die Alkylierung von 187 mit dem Grignard-Reagenz 188 ergab das Alkinol 189 (52\% Ausbeute). Dieser Alkohol 189 konnte anschließend in $89 \%$ Ausbeute zum TBDMS-geschützten Ether umgesetzt werden. Anschließende Entschützung mit 2 Äquivalenten $\mathrm{Et}_{2} \mathrm{AlCl}$ ergab den einfach TBDMS-geschützten Alkohol 190-H (56\% Ausbeute).

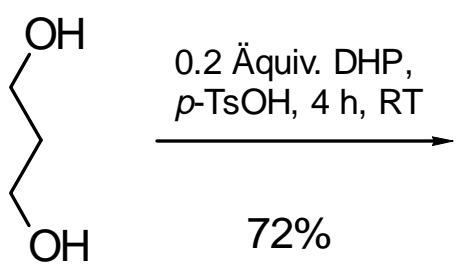

185

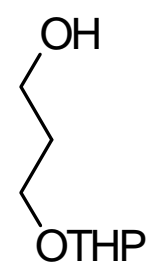

186

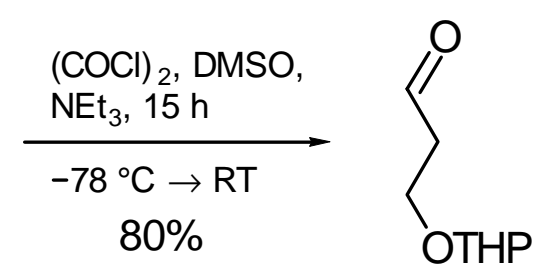

187

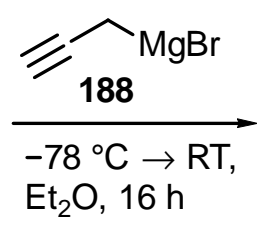

$52 \%$

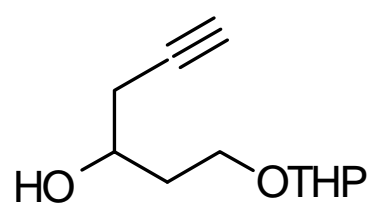

189

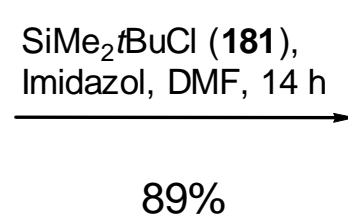

$89 \%$

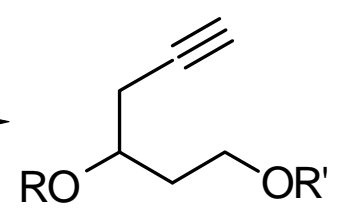

190-R'

Schema 59. $\mathrm{R}=\mathrm{SiMe}_{2} t \mathrm{Bu}$.

In einem Vorversuch zur Abschätzung des Reaktionsverhaltens eines Enins vom Typ 175 mit Vinylchlorideinheit wurde der Alkohol 190-H in einer Swern-Oxidation zum Aldehyd 191 umgesetzt (89\% Ausbeute, Schema 60). Nach der Methode von Takai et al. ${ }^{[80]}$ konnte aus 191 durch Behandlung mit Chrom(II)chlorid und Chloroform das Vinylchlorid 192 in 41\% Ausbeute mit einem E/Z-Verhältnis von 84:16 gewonnen werden. Da Alkenylchloride in Heck-Reaktionen sich relativ inert verhalten, sollte in Gegenwart von Palladium keine Nebenreaktion an der Vinylchlorid-Einheit statfinden. Der Versuch einer Enin-Cycloisomerisierung von 192 unter Zugabe von $\mathrm{Pd}(\mathrm{OAc})_{2}$ und BBEDA gelang ebenfalls nicht. Im Hinblick darauf, daß die Chlorvinyleinheit in der geplanten intramolekularen Domino-Reaktion als Dienophil-Einheit reagieren sollte, war dieses Ergebnis sogar erfreulich. 


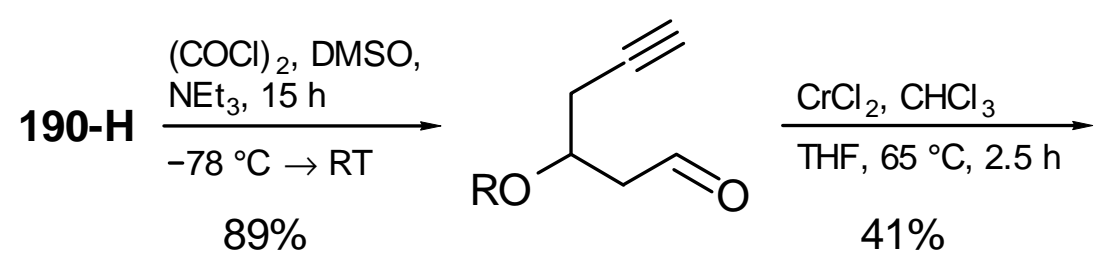

191<smiles>[R]C(CC#C)CC=CCl</smiles>

192

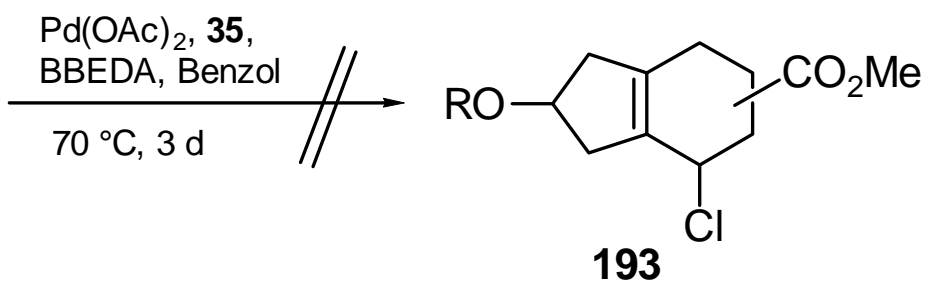

Schema $60 . \mathrm{R}=\mathrm{SiMe}_{2} t \mathrm{Bu}$.

Die Alkylierung des Alkins 190-H mit 5-Brom-1-penten (194) in Gegenwart von 2 Äquivalenten $n$-Butyllithium fand nicht statt (Schema 61).Wahrscheinlich findet durch die starke Base eine Dehydrobromierung von 194 statt. Auch der Zusatz von HMPT führte nicht zur Bildung von 195.

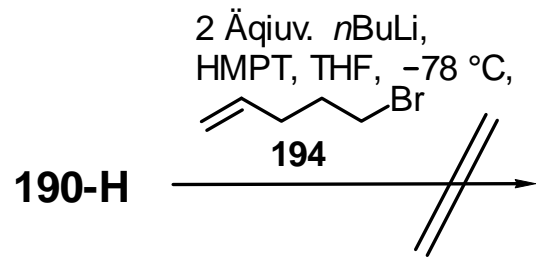

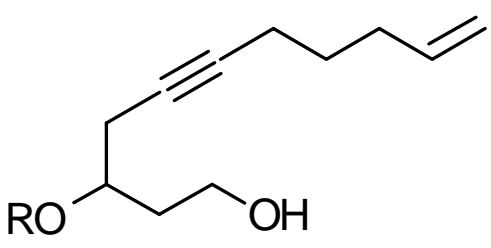

195

Schema 61. $\mathrm{R}=\mathrm{SiMe}_{2} t \mathrm{Bu}$.

Die Addition von Lithiumacetyliden an Aldehyde verläuft bei Temperaturen von $-78{ }^{\circ} \mathrm{C}$ dagegen schnell. Daher wurde das geschützte Alkin 190-THP bei $-78^{\circ} \mathrm{C}$ mit 4-Penten-1-al (196) umgesetzt und das gebildete Alkoholation mit Methyliodid zum Methylether 197 abgefangen (Schema 62). Eine nachfolgende Entschützung des THP-Ethers und DessMartin-Oxidation ergab den Aldehyd 198 in 57\% Ausbeute (über zwei Stufen). Dieser Aldehyd konnte ebenfalls durch eine Takai-Reaktion zum Vinylchlorid 199 umgesetzt werden (36\% Ausbeute). Das Dienin wurde als Gemisch mehrerer Diastereomere erhalten. Aufgrund der Überlagerung der Signale im Bereich vinylischer Protonen konnte das (E/Z)-Verhältnis nicht aus dem ${ }^{1} \mathrm{H}-\mathrm{NMR}$-Spektrum bestimmt werden. Nach Zugabe von 
$\operatorname{Pd}(\mathrm{OAc})_{2}$ und BBEDA fand keine Enin-Cycloisomerisierung statt, nach fünf Tagen bei $70{ }^{\circ} \mathrm{C}$ konnte NMR-spektroskopisch nur das Ausgangsmaterial 199 nachgewiesen werden. Ein Grund dafür, daß die Cycloisomerisierung nicht abläuft, liegt vermutlich darin, daß in dem Cyclisierungsvorläufer 199 keine geminalen Alkylgruppen oder Estergruppen vorhanden sind, die einen Thorpe-Ingold-Effekt ausüben.[30]

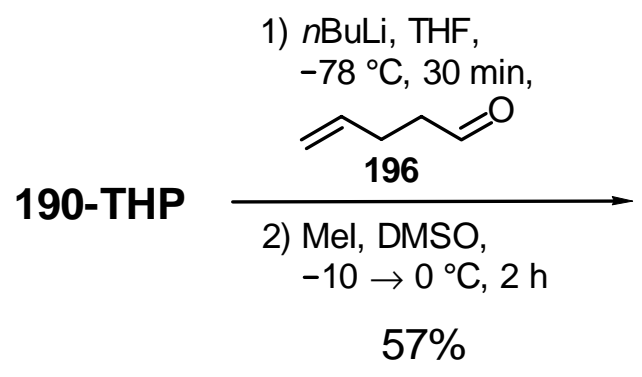

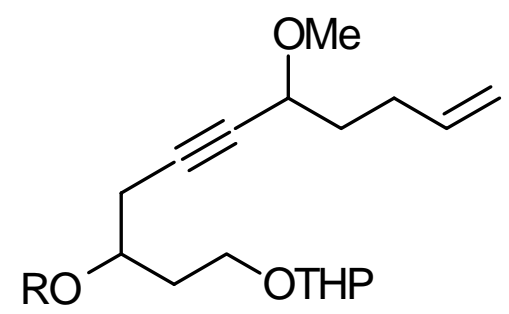

197

1) 2 Äquiv. $\mathrm{Et}_{2} \mathrm{AlCl}$, $\mathrm{CH}_{2} \mathrm{Cl}_{2},-25^{\circ} \mathrm{C} \rightarrow \mathrm{RT}$

2) Dess-Martin-Reagenz $\mathrm{CH}_{2} \mathrm{Cl}_{2}, \mathrm{RT}, 12 \mathrm{~h}$ $57 \%$<smiles>C=CCCC(C#CCC(O)OC)OC</smiles>
198

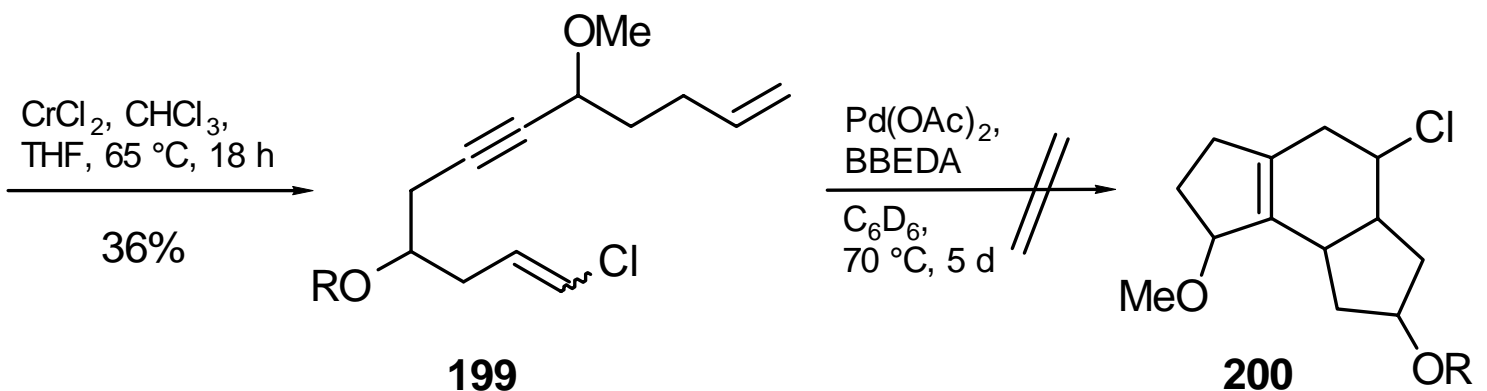

Schema 62. $\mathrm{R}=\mathrm{SiMe}_{2} t \mathrm{Bu}$. 


\section{Palladium-katalysierte Reaktionen von 1-Chlor-1-ethenylcyclopropan- Derivaten}

\subsection{Vorbemerkungen}

Neben Heck-Reaktionen und Enin-Cycloisomerisierungen sind Palladium(0)-katalysierte nucleophile allylische Substitutionen weitere wichtige $\mathrm{C}-\mathrm{C}-$ Verknüpfungsreaktionen. Zunächst findet eine oxidative Addition einer Verbindung mit ausreichend guter Abgangsgruppe in Allylposition an einen Palladium(0)-Katalysator unter Ausbildung eines $\eta^{1}$-Allylpalladium-Komplexes statt. Dieser Komplex wird unter Inversion gebildet und steht im Gleichgewicht mit einer $\eta^{3}$-Allylpalladium-Spezies. Mit Liganden im Überschuß bildet sich ein kationischer Komplex, der eine hohe Reaktivität gegenüber Nucleophilen aufweist. Der nucleophile Angriff erfolgt ebenfalls unter Inversion von der dem Metall abgewandten Seite. Als Nucleophile können stabilisierte Carbanionen verwendet werden. Durch reduktive Eliminierung von Palladium(0) wird der Katalysator zurückgebildet. Diese Substitutionen verlaufen im allgemeinen hochstereoselektiv unter Retention (zweifache Inversion). Das Nucleophil greift bevorzugt am weniger substituierten Ende des $\eta^{3}$-Komplexes an. Die dabei am häufigsten verwendeten Katalysatoren sind $\mathrm{Pd}\left(\mathrm{PPh}_{3}\right)_{4}$ und $\operatorname{Pd}(\mathrm{dba})_{2}$, als Abgangsgruppe eignen sich u. a. Tosylat, Mesylat, Chlorid und Acetat.[81]

Wie bereits untersucht wurde, können Palladium-katalysierte nucleophile Substitutionen auch an 1-Ethenylcyclopropyl-Derivaten durchgeführt werden, deren Abgangsgruppe sich am Cyclopropanring befndet.[82] Dabei bleibt der dreigliedrige Ring erhalten. Unter anderem konnte gezeigt werden, daß (2'-Trimethylsilylethenyl)cyclopropan-Derivate vom Typ 201 unter Zusatz von Natriumformiat als Hydridquelle ausschließlich das Allylsilan 206 bilden (Schema 63, 90\% Ausbeute). Verwendete man hingegen $n$-Butylzinkchlorid, wurde das Vinylsilan 207 in $85 \%$ Ausbeute erhalten. Zunächst wird die Bildung des unsymmetrischen $\eta^{3}$-Palladium-Komplexes $\mathbf{2 0 2}$ angenommen. Nach Zugabe der Nucleophile bilden sich aus diesem die entsprechenden $\sigma$-Komplexe 203 und 204, bei denen sich das Palladium am Cyclopropanring befindet. An diesem Kohlenstoffatom wird eine positive Ladung am wenigsten stabilisiert, da die Bildung eines Cyclopropylkations aufgrund der Ringspannung stark benachteiligt ist.[82b,83] Bei der Umsetzung mit Natriumformiat findet ein $\mathrm{S}_{\mathrm{N}} \mathrm{i}$-Hydrid-Transfer über einen sechsgliedrigen 
Übergangszustand statt unter reduktiver Eliminierung von Palladium(0) und $\mathrm{CO}_{2}$-Abspaltung, wobei das Allylsilan 206 resultiert. Bei Verwendung von $n$-Butylzinkchlorid erfolgt eine $\beta$-Hydrideliminierung zu 205 und ebenfalls eine reduktive Eliminierung von Palladium(0) zu dem isomeren Vinylsilan 207.[84]

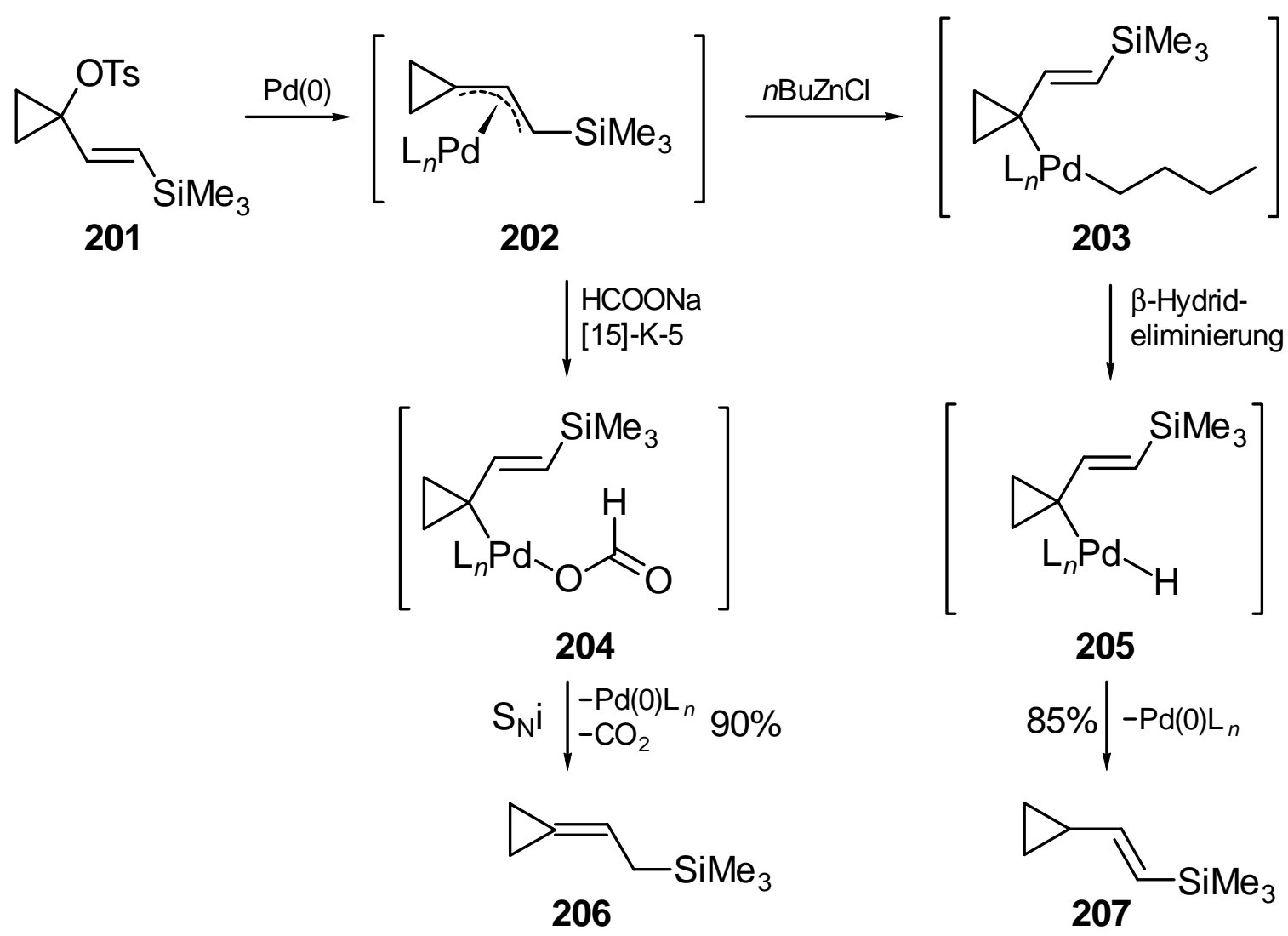

Schema 63.

Das am Dreiring Trimethylsilyl-substituierte Ethenylcyclopropylderivates 208 verhielt sich bei der Palladium-katalysierten Reaktion mit Natriumformiat genau umgekehrt (Schema 64). Dabei wird die intermediäre Bildung des $\eta^{1}$-Allylpalladium-Komplexes 212 angenommen, aus dem durch $\mathrm{S}_{\mathrm{N}} \mathrm{i}$-Reaktion unter reduktiver Eliminierung das Vinylcyclopropan (Z)-215 hervorgeht. Der Trimethylsilylrest in $\beta$-Stellung ist in der Lage, eine positive Ladung am dreigliedrigen Ring zu stabilisieren. ${ }^{[85]}$ Der $\beta$-Effekt des Siliciums kann offenbar den Ringspannungseffekt überkompensieren. [82a] Die Bildung des Endproduktes verläuft ausgehend von einem $(E, Z)$-Gemisch mit dem $E / Z$-Verhältnis $1: 2.3$ vollständig (Z)-selektiv. Setzt man $n$-Butylzinkchlorid zu, entsteht ebenfalls 215, aber als $(E, Z)$-Gemisch. Hierbei muß der $\sigma$-Komplex 211 durchlaufen werden. Der Grund dafür 
könnte in der Bildung des thermodynamisch stabileren Vinylsilans 215 liegen, würde ein 212 entsprechender Komplex 210 gebildet, müßte das gespannte Alkylidencyclopropan 216 entstehen.[84]

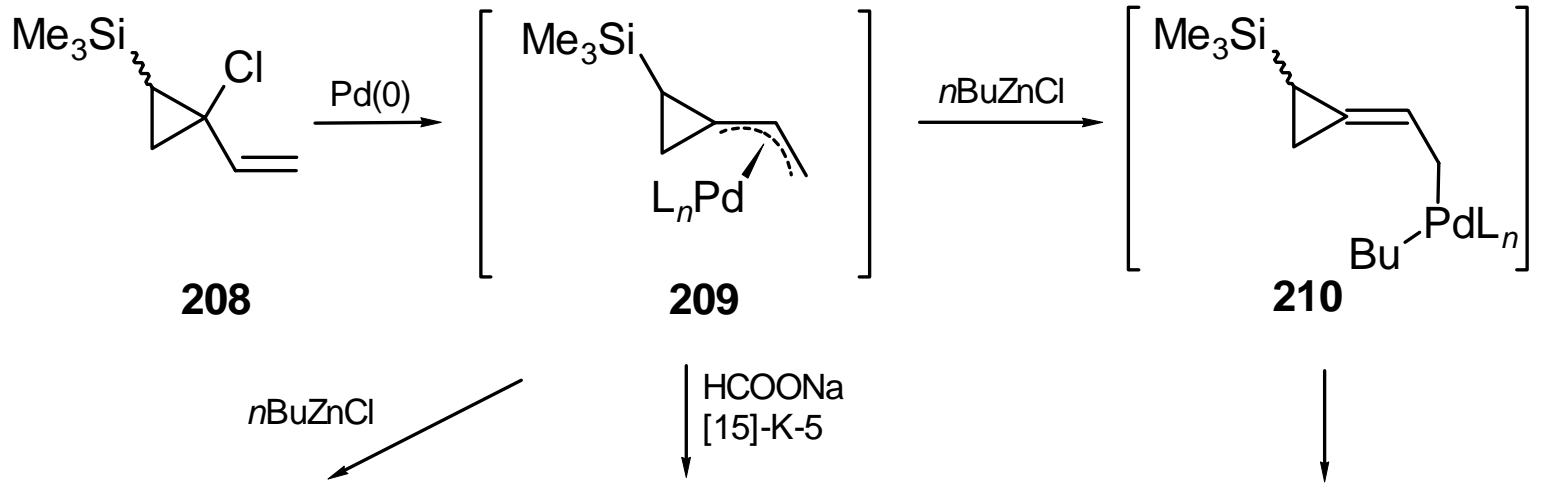

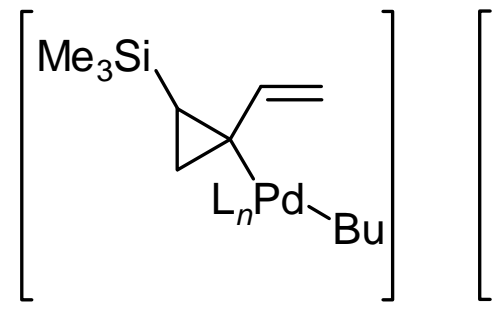

211

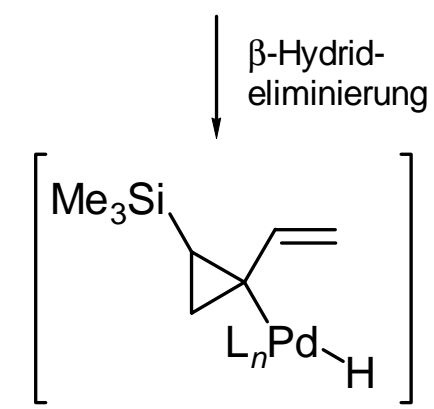

214

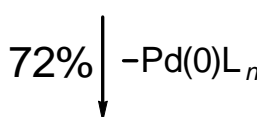<smiles>C=CC1CC1[SiH3]</smiles>

$(E / Z)-215$

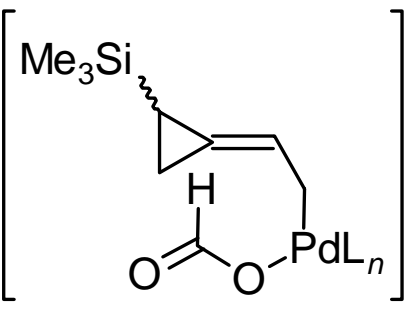

212
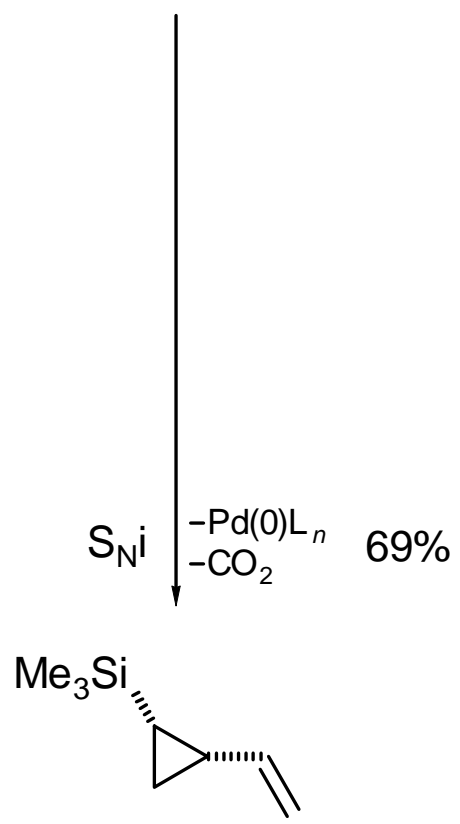

(Z)-215<smiles>C[SiH2]C1CC1=CC[PbH]</smiles>

213

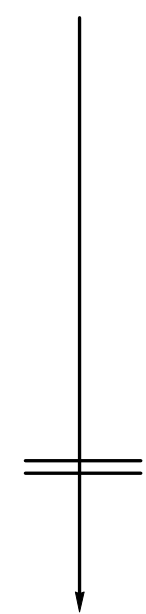<smiles>CC=C1C[C@@H]1[SiH3]</smiles>

216

Schema 64. 
Aus diesen Beobachtungen wird deutlich, daß die nucleophile allylische Substitution an 1-Ethenylcyclopropyl-Derivaten stark von dem Substitutionsmuster am Substrat und von der Natur des Nucleophils abhängt.

\subsection{Synthese der Ausgangsverbindungen}

Um über den Einfluß der Trimethylsilylgruppen an unterschiedlichen Positionen von 1-Chlor-1-ethenylcyclopropanen bei der Palladium-katalysierten Reaktion mit Natriumformiat genauere Aussagen treffen zu können, sollte das 1-Chlor-2-trimethylsilyl-1-(2'trimethylsilylethenyl)cyclopropan (217) dargestellt werden (Schema 65), da dieses Molekül zwei Gruppen mit gegenläufiger Wirkung trägt. Denn die Umsetzung von 202 und 208 lieferte in Gegenwart von Natruimformiat unterschiedliche Produkte. Daher sollte die nucleophile Substitution von 217 Informationen darüber liefern, welche der beiden Trimethylsilylgruppen eine dirigierende Funktion übernimmt, oder ob überhaupt ein Effekt überwiegt, so daß ein einheitliches Produkt gebildet werden kann. Zusätzlich sollte auch die Umsetzung mit $n$-Butylzinkchlorid durchgeführt werden.

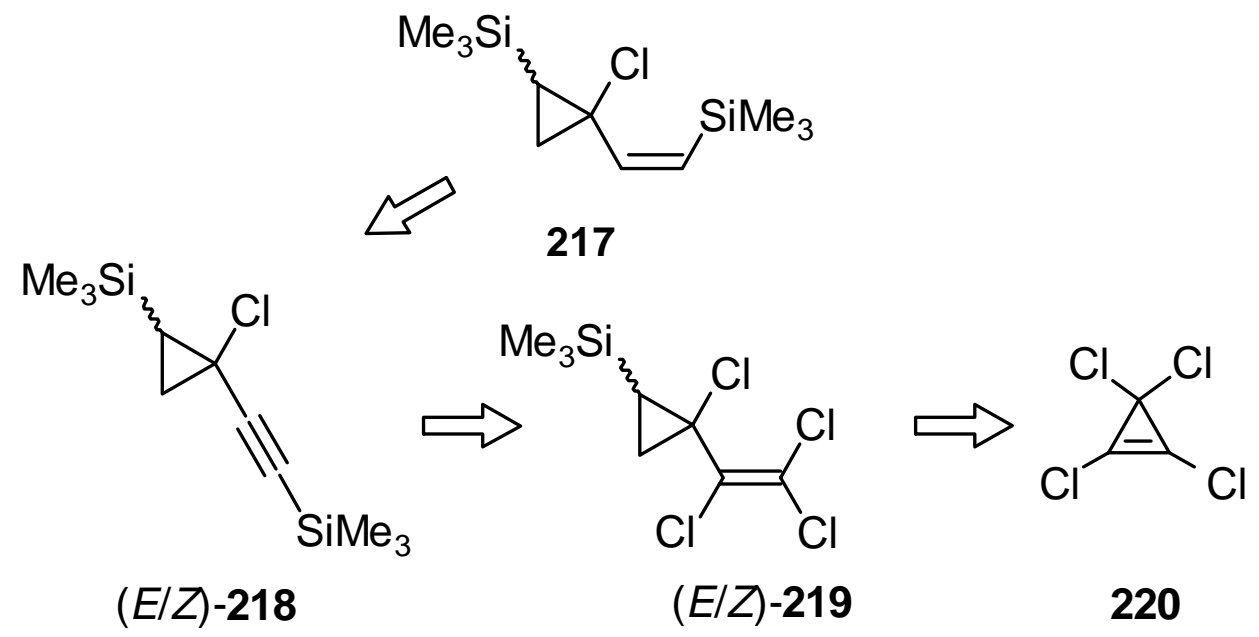

Schema 65. Retrosynthetische Überlegungen zur Darstellung von 1-Chlor-2-trimethylsilyl1-(2'-trimethylsilylethenyl)cyclopropan (217). 
Die Darstellung von 217 erfolgte nach einer von de Meijere et al. entwickelten Methode.[86] Ausgehend von Tetrachlorcyclopropen (220) und Trimethylsilylethylen (222) wurde das Trichlorethenylcyclopropanderivat (E/Z)-219 gewonnen (Schema 66). Die Addition des intermediär gebildeten Perchlorethenylcarbens (221) an das Trimethylvinylsilan (222) zum entsprechenden Cyclopropanderivat (E/Z)-219 verlief mit 80\% Ausbeute (E/Z-Verhältnis $1: 2.0$, bestimmt aus dem ${ }^{1} \mathrm{H}$-NMR-Spektrum). Durch Zugabe von 2 Äquivalenten $n$-Butyllitihium und Aufarbeitung mit Chlortrimethylsilan konnte das Alken (E/Z)-219 in einer Ausbeute von 80\% in das entsprechende Alkin (E/Z)-218 transformiert werden.[86]<smiles>ClC1=C(Cl)C1(Cl)Cl</smiles>

220

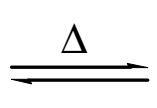<smiles>ClC(Cl)=C(Cl)Cl</smiles>

221

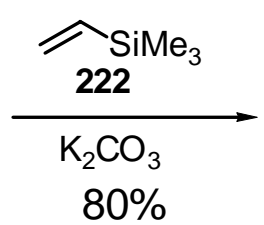

$80 \%$<smiles>C[SiH3][C@@H]1C[C@@H]1C(Cl)=C(Cl)Cl</smiles>

$(E / Z)-219$

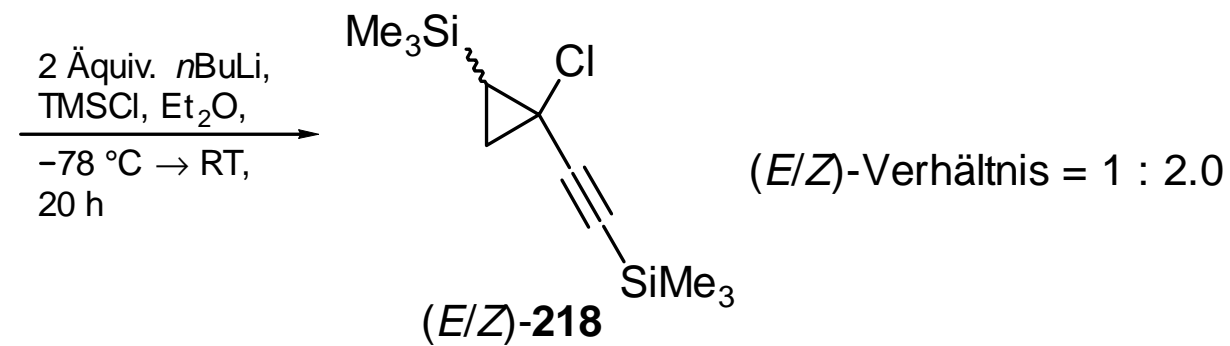

Schema 66.

Beim Versuch das Alkin (E/Z)-218 über Lindlar-Katalysator (vergiftet mit 0.2 mol\% Chinolin) in Pentan zu 217 katalytisch zu hydrieren, war nach fünf Tagen kein Verbrauch von Wasserstoff festzustellen, und 72\% des Ausgangmaterials (E/Z)-218 konnten zurückgewonnen werden (Schema 67). Eine andere Möglichkeit, Alkine zu Alkenen zu reduzieren, ist die Addition von Alkylboranen und anschließende saure Aufarbeitung. [87] Daher stellte man in situ aus Cyclohexen und dem $\mathrm{BH}_{3} \cdot \mathrm{THF}-K o m p l e x$ das Dicyclohexylboran her und addierte letzteres an das Alkin (E/Z)-218. Das gebildete Alkenylboran wurde nicht isoliert, sondern durch Zusatz von Essigsäure zum Alken 217 hydrolysiert (Schema 67). Die Reaktion fand offenbar nur mit dem (Z)-Isomer statt, das 
(E)-Isomer reagierte nicht mit dem Alkylboran. Dafür dürften sterische Gründe verantwortlich sein, das räumlich anspruchsvolle Dicyclohexylboran kann bei $(E)$-218 nicht an die Alkinyleinheit angreifen, da der Trimethylsilylrest ebenfalls auf dieser Seite steht. Eine säulenchromatographische Trennung des Alkens (Z,Z)-217 von dem unumgesetzten Edukt (E)-219 war nur schlecht möglich. Daher versetzte man das Gemisch mit Kaliumcarbonat in Methanol, um die Trimethylsilylgruppe vom Alkinterminus abzuspalten. Das dabei gebildete terminale Alkin (E)-223 war wesentlich polarer, so daß das Alken (Z,Z)-217 problemlos säulenchromatographisch abgetrennt werden konnte. Die Ausbeute an (Z,Z)-217 betrug 60\%, bezogen auf die vorhandene Menge an (Z)-Isomer im ursprünglichen Gemisch entsprach das einer Ausbeute von $90 \%$.

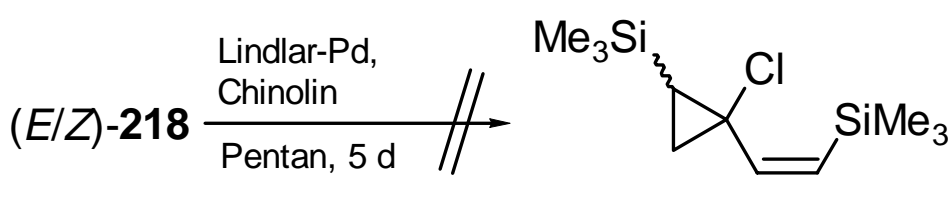

217

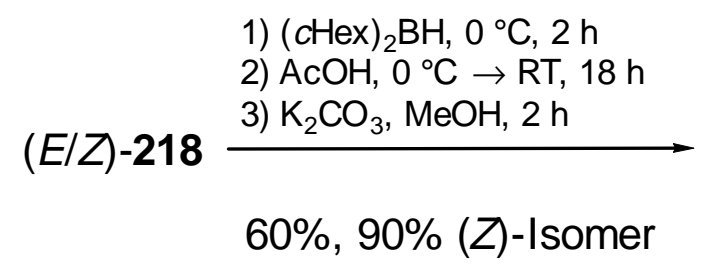

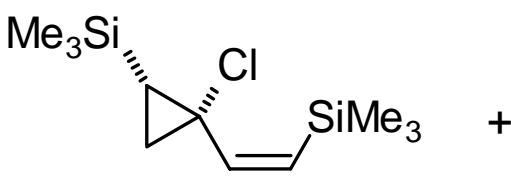

$(Z, Z)-217$

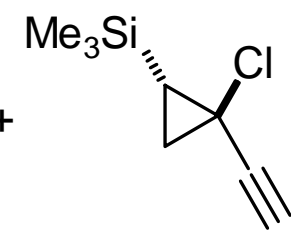

$(E)-223$

Schema 67.

\subsubsection{Verwendung von Natriumformiat als Hydridquelle}

Zur Palladium(0)-katalysierten Reduktion versetzte man das Allylchlorid (Z,Z)-217 in Acetonitril mit $5 \mathrm{~mol} \% \mathrm{Pd}(\mathrm{dba})_{2}$ und $12 \mathrm{~mol} \% \mathrm{PPh}_{3}$ in Gegenwart von [15]-Krone-5. Nach drei Tagen bei Raumtemperatur konnten nur 6\% des Allylsilans (E)-224 isoliert werden (Schema 68, Tabelle 10, Eintrag 1). Verwendete man THF anstelle von Acetonitril konnte nach vier Tagen bei Raumtemperatur nur Ausgangsmaterial detektiert werden (Eintrag 2). Führte man hingegen die Reaktion in Acetonitril bei $50{ }^{\circ} \mathrm{C}$ aus, konnte nach 36 Stunden das Allylsilan (E)-224 mit Spuren des Vinylsilans 225 (Verhältnis 
(E)-224: $225=93: 7$, nach dem ${ }^{1} \mathrm{H}-\mathrm{NMR}$-Spektrum) in 90\% Ausbeute erhalten werden. Dieses Ergebnis zeigte eindeutig, daß der Einfluß des Silylrestes an der Doppelbindung den Einfluß des Trimethylsilylrestes am dreigliedrigen Ring überwiegt, denn es wurde fast ausschließlich das Allylsilan (E)-224 gebildet.

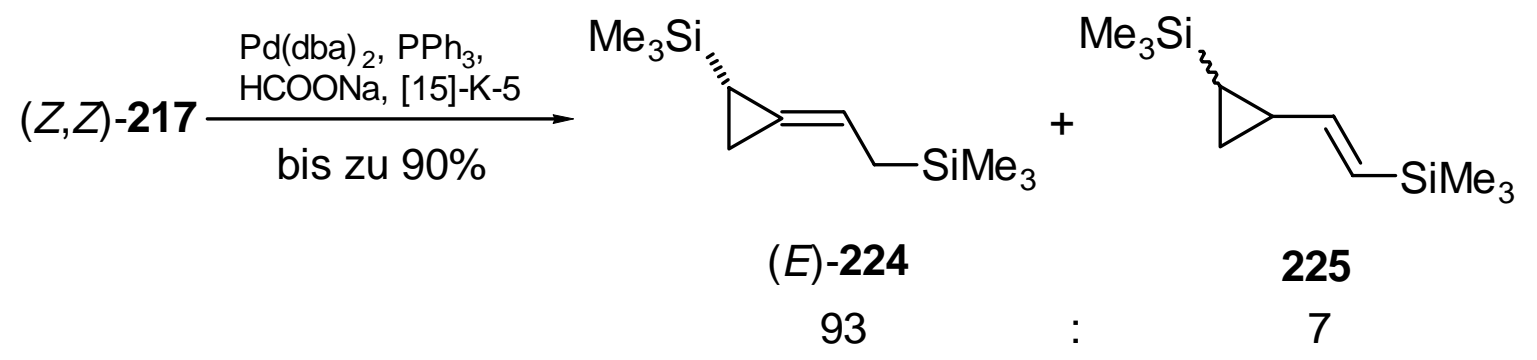

Schema 68. Details siehe Tabelle 10.

Tabelle 10. Palladium-katalysierte Substitution an (Z,Z)-217 unter verschiedenen Reaktionsbedingungen (Schema 68). [a]

\begin{tabular}{ccccc}
\hline Eintrag & Allylchlorid & Bedingungen[a] & Produkt & Ausbeute (\%) \\
\hline 1 & $(Z, Z)-\mathbf{2 1 7}$ & $\mathbf{A}$ & $(E)-\mathbf{2 2 4}$ & 6 \\
2 & $(Z, Z)-\mathbf{2 1 7}$ & $\mathbf{B}$ & - & - \\
3 & $(Z, Z)-\mathbf{2 1 7}$ & $\mathbf{C}$ & $(E)-\mathbf{2 2 4}$ & $84[\mathrm{~b}]$ \\
\hline
\end{tabular}

[a] A: MeCN, Raumtemp, 3 d; B: THF, Raumtemp. 4 d; C: MeCN, $50{ }^{\circ}$ C, 36 h. - ${ }^{[b]}$ Mit 7\% des Vinylsilans 225 verunreinigt.

\subsubsection{Verwendung von n-Butylzinkchlorid als Hydridquelle}

Entsprechend den in Kapitel 6.2.1. beschriebenen Bedingungen versetzte man das Ethenylcyclopropan $(Z, Z)-217$ in THF mit $5 \mathrm{~mol} \% \mathrm{Pd}(\mathrm{dba})_{2}$ und $12 \mathrm{~mol} \% \mathrm{PPh}_{3}$. Bei $0{ }^{\circ} \mathrm{C}$ gab man eine Lösung von $n$-Butylzinkchlorid in Ether hinzu und erwärmte auf Raumtemperatur. Nach vier Tagen konnten die Vinylsilane 225 (10\% Ausbeute) und 226 (24\% Ausbeute) neben 56\% Ausgangsmaterial (Z,Z)-217 isoliert werden (Schema 69). Führte man die Reaktion bei $50{ }^{\circ} \mathrm{C}$ aus, erhöhten sich die Ausbeuten auf $12 \% 225$ und 55\% 226. Die Bildung von 226 läßt sich durch eine Palladium-katalysierte Kreuzkupplung zwischen 
(Z,Z)-217 und $n$-Butylzinkchlorid erklären. Ähnliche Kupplungsprodukte wurden von Salaün et al. bei der Umsetzung von (E)-1-(1-Styryl)-1-(tosyloxy)cyclopropan mit $n$-Butylzinkchlorid isoliert.[82b]

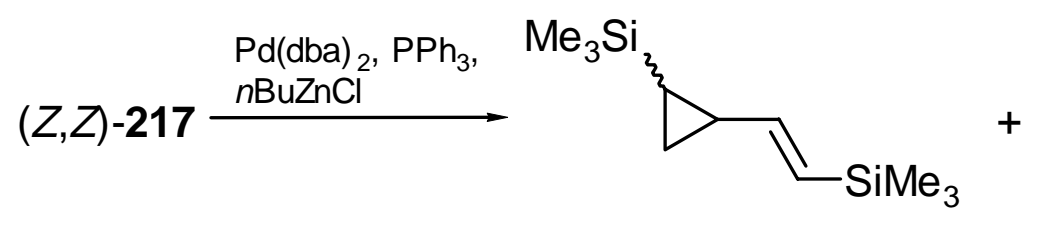

225

$55 \%$

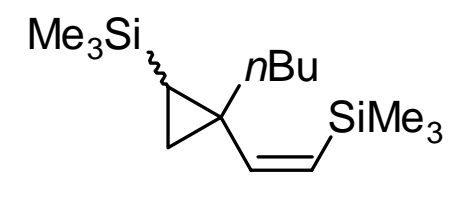

226

$12 \%$

Schema 69.

\subsection{Versuch zur Durchführung von Folgereaktionen des Allylsilans (E)-224}

Allylsilane stellen nützliche Syntheseäquivalente dar, da sie stabil sind, viele Reaktionen unbeschädigt durchlaufen können und selbst die Fähigkeit besitzen, mit Elektrophilen zu reagieren.[88] Das Allylsilan (E)-224 könnte in weiteren Reaktionen als Kohlenstoffnucleophil eingesetzt werden und damit eine Cyclopropankomponente in andere Moleküle einbringen. Cyclopropanderivate sind aufgrund ihres vielfältigen Synthesepotentials von Interesse. Der dreigliedrige Ring kann selektive Ringöffnungen, Ringerweiterungen oder Cycloadditionen eingehen und tritt als Strukturelement in vielen Naturstoffen auf. In ihrem Reaktionsverhalten ähnelt die Cyclopropaneinheit einer $\mathrm{C}=\mathrm{C}$-Doppelbindung.

\subsubsection{Versuche einer Sakurai-Reaktion}

In Gegenwart von Lewis-Säuren wie $\mathrm{TiCl}_{4}$ oder $\mathrm{BF}_{3} \cdot \mathrm{Et}_{2} \mathrm{O}$ können Carbonylverbindungen derart aktiviert werden, daß sie von Allylsilanen nucleophil angegriffen werden. Dabei bildet sich intermediär ein Carbeniumion, das den Elektronenmangel unter Abspaltung des Trimethylsilylrestes ausgleicht. Nach Hydrolyse gelangt man zu Homoallylalkoholen. ${ }^{[57,89]}$ Diese sogenannte Sakurai-Reaktion wurde mit dem Allylsilan (E)-224 getestet. Man erhoffte sich damit eine Methode zum Einführen der Cyclopropaneinheit in andere Moleküle. Dazu gab man zu einer Lösung der 
Silylverbindung (E)-224 und Benzaldehyd (135) in Dichlormethan bei $-78{ }^{\circ} \mathrm{C}$ eine Lösung von $\mathrm{TiCl}_{4}$ in Dichlormethan (Schema 70). Nach zwei Stunden bei Raumtemperatur konnte nach dünnschichtchromatographischer Analyse kein Edukt mehr detektiert werden. Allerdings konnte auch keines der erwarteten Produkte 227 und 228 isoliert werden, man erhielt nur Zersetzungsprodukte. Das ${ }^{1} \mathrm{H}-\mathrm{NMR}$-Spektrum des Rohproduktes enthielt weder Signale von cyclopropylischen noch von olefinischen Protonen. Auch die Verwendung von $\mathrm{BF}_{3} \cdot \mathrm{Et}_{2} \mathrm{O}$ führte nicht zu den isomeren Homoallylalkoholen 227 und 228.

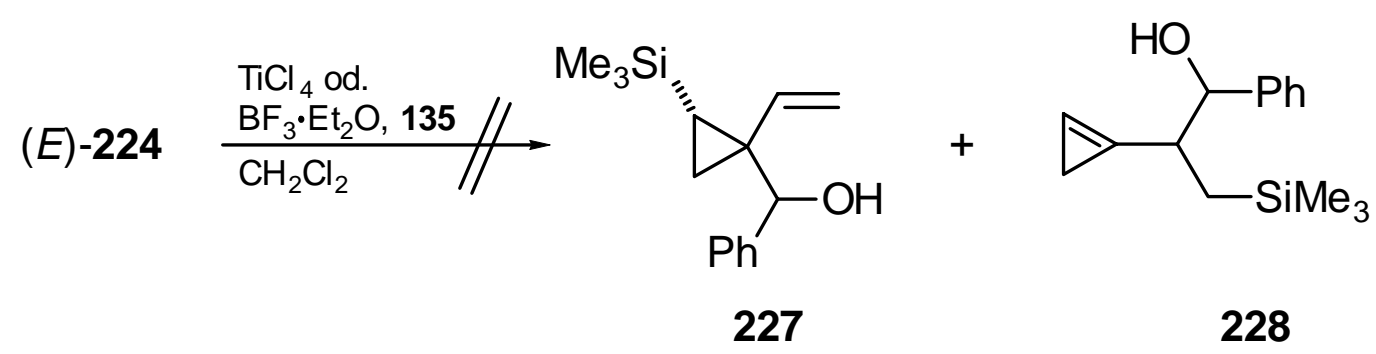

Schema 70.

\subsubsection{Deprotonierung des Allylsilans (E)-224 und Versuch der Verwendung als Nucleophil}

Neben der Stabilisierung von Carbokationen in $\beta$-Stellung (Kapitel 6.1.) ist das Silicium in der Lage, am $\alpha$-Kohlenstoffatom negative Ladungen $\mathrm{zu}$ stabilisieren ( $\alpha$-Effekt des Siliciums). Die Acidität der Wasserstoffatome an dieser Position ist groß genug, so daß Silane in $\alpha$-Position mit starken Basen deprotoniert werden können. Das dabei gebildete Carbanion kann an Elektrophile addieren. Daher wurde versucht, das Allylsilan (E)-224 mit $n$-Butyllithium zu deprotonieren und das gebildete Lithiumderivat mit Benzaldehyd (135) abzufangen. Das Silan (E)-224 besitzt zwei mögliche Positionen, an denen eine Deprotonierung stattfinden kann. Eine Deprotonierung am Cyclopropanring und anschließende elektrophile Addition würde zu C-2-funktionalisierten Cyclopropanderivaten führen.

Versetzte man (E)-224 mit n-Butyllithium und anschließend mit Benzaldehyd (135), konnte jedoch keines der erwarteten Produkte isoliert werden, vielmehr erhielt man ein komplexes Gemisch nicht identifizierter Verbindungen (Schema 71). Dabei waren im 
${ }^{1}$ H-NMR-Spektrum zwar Cyclopropylsignale zu sehen, im Bereich der Doppelbindungsprotonen befanden sich keine Signale. Auch ein Zusatz von HMPT zur Komplexierung führte nicht zum Erfolg. Salaün et al. untersuchten ähnliche Verbindungen, eine elektrophile Addition am Cyclopropanring gelang ihnen trotz vielfältiger Variationen der Base jedoch ebenfalls nicht. [84]

(E) -224

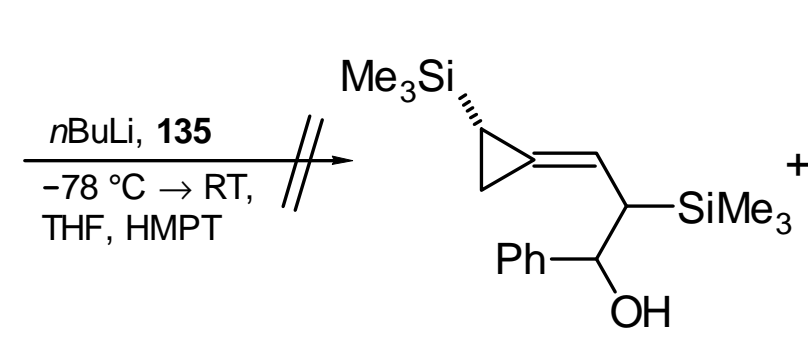

229<smiles>C[SiH2]CC=C1CC1([SiH3])c1ccccc1</smiles>

230

Schema 71.

Während die Darstellung des Allylsilans (E)-224 auf einfache Weise durchführbar war, konnten unter den hier verwendeten Reaktionsbedingungen bisher keine Folgereaktionen daran durchgeführt werden. In Anbetracht der Vielzahl der verfügbaren Lewis-Säuren und Basen sollten sich aber Bedingungen finden lassen, unter denen die Verwendung des Allylsilans (E)-224 als Nucleophil möglich ist. 


\section{Experimenteller Teil}

\section{Allgemeines}

IR: Bruker IFS 66 (FT-IR). - ${ }^{1}$ H-NMR: Bruker AC 200 (200 MHz), Bruker AM 250 (250 MHz), Bruker AMX 300 (300 MHz) oder Varian VXR $500 \mathrm{~S}$ (500 MHz); Referenzen: $\delta=7.26$ für Chloroform, 7.15 für $\left[\mathrm{D}_{5}\right]$ Benzol. Charakterisierung der Signalaufspaltungen: $\mathrm{s}=$ Singulett, $\mathrm{d}=$ Dublett, $\mathrm{dd}=$ doppeltes Dublett, $\mathrm{ddd}=$ Dublett eines doppelten Dubletts, dddd $=$ doppeltes Dublett eines doppelten Dubletts, $t=$ Triplett, $\mathrm{q}=$ Quartett, $\mathrm{m}=$ Multiplett, $\mathrm{m}_{\mathrm{c}}=$ zentrosymmetrisches Multiplett, $\mathrm{AB}=\mathrm{AB}-$ System, br $=$ breit. Spektren wurden - soweit möglich - nach erster Ordnung ausgewertet. Sämtliche Kopplungskonstanten sind als Betrag angegeben. Abkürzungen für Signalzuordnungen: $c$ Pr-H cyclopropylisch, $c$ Pent-H cyclopentylisch, $c H e x-H$ cyclohexylisch, $\mathrm{Ph}-\mathrm{H}$ phenylisch. Austauschbare Zuordnung wurden mit einem Stern gekennzeichnet. 13C-NMR: Bruker AC 200 (50.3 MHz), Bruker AM $250(62.9 \mathrm{MHz})$ Bruker AMX 300 (75.5 MHz) oder Varian VXR 500 S (125.7 MHz); $\delta=77.00$ für Deuterochloroform, 128.0 für $\left[\mathrm{D}_{6}\right]$ Benzol. Die Multiplizität der Signale wurde durch die DEPT-Aufnahmetechnik $($ DEPT $=$ Distortionless Enhancement by Polarisation Transfer $)$ oder APTAufnahmetechnik (APT $=$ attached proton test) bestimmt und ist wie folgt angegeben; DEPT: $+=$ primäre oder tertiäre (positives DEPT-Signal),$-=$ sekundäre (negatives DEPTSignal), $\mathrm{C}_{\text {quart }}=$ quartäre (DEPT-Signalintensität Null) C-Atome; APT: $+=$ primäre oder tertiäre (positives APT-Signal), $-=$ sekundäre oder quartäre (negatives APT-Signal) C-Atome. Abkürzungen für Signalzuordnungen: $c$ Pr-C cyclopropylisch, $c$ Pent-C cyclopentylisch, $c$ Hex-C cyclohexylisch, Ph-C phenylisch. Austauschbare Zuordnungen wurden mit einem Stern gekennzeichnet. - MS: Varian MAT CH 7, MAT 731. - HRMS (hochauflösende MS): Varian MAT 311; korrekte HRMS = vorgewählter Ionenpeak stimmt bei $\mathrm{R} \approx 10000$ mit einer Fehlerbreite von \pm 2 ppm mit der exakten Masse überein. Analytische Gaschromatographie: Siemens Sichromat 3 (25 m Kapillarsäule, CB-SE-54, Trägergas Helium). - Säulenchromatographie (SC): Chromatographische Trennungen erfolgten an Merck Kieselgel 60 (0.063-0.200 mm, 70-230 mesh). Die Säulendimensionen werden als "Säulendurchmesser $\times$ Höhe der Kieselgelsäule" angegeben. Alle Laufmittel wurden destilliert verwendet. - Dünnschichtchromatographie (DC): Merck-Kieselgel 60 
$\mathrm{F}_{254}$ auf Aluminiumfolien der Fa. Macherey-Nagel, Detektion unter UV-Licht bei $254 \mathrm{~nm}$ und durch Entwicklung mit Iod, p-Anisaldehyd- (1/100 p-Anisaldehyd, 10/100 konz. Essigsäure, 5/100 konz. Schwefelsäure, 84/100 Methanol) oder MolybdatophosphorsäureTauchreagenz (10proz. Lösung in Ethanol). - Schmelzpunkte: Schmelzpunktapparatur der Fa. Büchi, die gemessenen Werte sind unkorrigiert. - Elementaranalysen: Mikroanalytisches Laboratorium des Institutes für Organische Chemie der Universität Göttingen. - Reaktionen unter hohem Druck wurden in zugeschweißten Teflonröhrchen durchgeführt. Hochdruckaggregat der Fa. Andreas Hofer, Mühlheim. - Wasserfreie Lösungsmittel wurden nach üblichen Labormethoden gewonnen.[90] - Alle Umsetzungen mit metallorganischen Verbindungen und wasserfreien Lösungsmitteln wurden unter Sauerstoff- und Feuchtigkeitsausschluß in ausgeheizten Glasgeräten durchgeführt, die Palladium-katalysierten Cyclisierungen in 25 ml-Pyrexflaschen mit Schraubverschluß. Ein Teil der Substanzen fällt als Gemisch zweier oder mehrerer Regioisomere und/oder Diastereomere an, deren Signale im ${ }^{13}$ C-NMR-Spektrum aufgrund ihrer großen Ähnlichkeit nicht als vollständig getrennte Liniensätze erscheinen. - Verwendete Abkürzungen: $\quad \mathrm{DMF}=N, N$-Dimethylformamid,$\quad \mathrm{THF}=$ Tetrahydrofuran, $\mathrm{DMAP}=\mathrm{p}-\mathrm{Di}$ methylaminopyridin, DMSO $=$ Dimethylsulfoxid, $B B E A=N, N '$-Bisbenzylidenethylendiamin, $\quad$ DME = Dimethoxyethan, $\quad$ TMEDA $=N, N, N^{\prime}, N^{\prime}$-Tetramethylethylendiamin, HMPT $=$ Hexamethylphosphorsäuretriamid.

Folgende Verbindungen wurden nach Literaturangaben dargestellt:

2-Chlor-2-cyclopropylidenessigsäuremethylester (27) nach de Meijere,[91] 3-tert-Butyldimethylsilyloxy-1-propin (30) nach Logue und Teng,[92] 2,2-Dimethylpent-4-enal (31) nach Normant,[93] Isophoronoxid (38) und 4,4-Dimethylhept-6-in-2-on (39) nach Tietze und Eicher,[94] Trimethylsilylethin (43) nach Brandsma,[95] 2-Chlor-2-cyclopropylidenessigsäure-tert-butylester (49) nach de Meijere,[96] Butin-3-ol (56) nach Brandsma,[95] 6-Brom-4,4-dimethylhepta-1,6-dien-3-ol (59) nach Körbe,[21c] $N$-Allyl- $N$-(p-tosyl)amin (70) nach Hubert, [97] Propargylsäuremethylester (81) nach Baldwin, [98] 2,3-Dibromprop-1en (89) nach Johnson et al.,[99] $N$-(2-Bromprop-2-enyl)benzylamin (90) nach Ennis, ${ }^{[47]} \mathrm{N}$ Benzyl-N-prop-2-in-1-amin (94) nach Gordon,[100] $N$ - Allyl- $N$-benzylamin (98) nach Mitsch et al.,[101] Phenyl- $N$-trimethylsilylimin (130) nach Colvin et al.,[102] 2-Brom-4,4dicarbonsäuremethylester-1-hepten-6-in (151) nach Henniges,[19a] Tetrachlorcyclopropan 
(220) und (E/Z)-1-Chlor-1-(1',2',2'-trichlorethenyl)-2-trimethylsilylcyclopropan [(E/Z)-219] nach de Meijere et al.[86]

Alle nicht gesondert aufgeführten Verbindungen sind kommerziell erhältlich.

\section{Darstellung der Verbindungen}

\subsection{Synthese der Enin-Cycloisomerisierungsvorläufer}

Allgemeine Arbeitsvorschrift zur Alkylierung von Aldehyden mit terminalen Alkinen (AAV 1): Zu einer Lösung von $20.0 \mathrm{mmol}$ des Alkins in $30 \mathrm{ml}$ wasserfreiem THF tropft man bei $-78{ }^{\circ} \mathrm{C}$ die angegebene Menge einer $n$-Butyllithiumlösung in Hexan und rührt 30 min bei dieser Temperatur. Zu dieser Reaktionslösung gibt man 1.00-1.50 Äquivalente des Aldehyds und rührt erneut $1 \mathrm{~h}$ bei $-78^{\circ} \mathrm{C}$. Die Lösung wird auf Raumtemp. erwärmt und weitere $18 \mathrm{~h}$ gerührt. Man versetzt mit $50 \mathrm{ml}$ Wasser und extrahiert die wäßrige Phase mit $3 \times 30 \mathrm{ml}$ Diethylether. Die vereinigten organischen Phasen werden mit $30 \mathrm{ml}$ ges. Natriumchloridlösung gewaschen, über $\mathrm{MgSO}_{4}$ getrocknet und i. Vak. eingeengt. Das Rohprodukt wird durch Säulenchromatographie an Kieselgel gereinigt.

4-Hydroxy-5,5-dimethyloct-7-en-2-in-1-ol (33): Analog AAV 1 gab man zu $5.61 \mathrm{~g}$

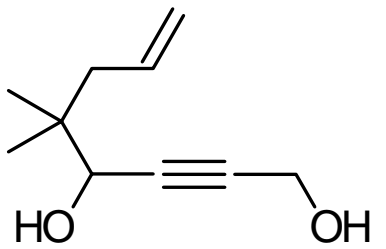
(100 mmol) Propargylalkohol in $50 \mathrm{ml}$ wasserfreiem THF bei $-78{ }^{\circ} \mathrm{C} 150 \mathrm{ml}(240 \mathrm{mmol}, 1.60 \mathrm{M}$ in Hexan) $n$-Butyllithium und rührte $30 \mathrm{~min}$. Dann versetzte man mit $11.22 \mathrm{~g}$ (100.0 mmol) 2,2Dimethylpent-4-en-1-al, ließ $1 \mathrm{~h}$ bei $-78^{\circ} \mathrm{C}$ und $18 \mathrm{~h}$ bei Raumtemp. rühren. Nach Aufarbeitung reinigte man das Rohprodukt durch Säulenchromatographie an $150 \mathrm{~g}$ Kieselgel (Säule $5.5 \times 12 \mathrm{~cm}$, Pentan/ $\mathrm{Et}_{2} \mathrm{O}=1: 1$ ). Man erhielt $8.13 \mathrm{~g}(48 \%) 33$ als farbloses, hochviskoses Öl, $R_{\mathrm{f}}\left(\right.$ Pentan/Et $\left._{2} \mathrm{O}, 1: 1\right)=0.21$. ${ }^{1} \mathrm{H}-\mathrm{NMR}\left(250 \mathrm{MHz}, \mathrm{CDCl}_{3}\right): \delta=0.95\left(\mathrm{~s}, 3 \mathrm{H}, \mathrm{CH}_{3}\right), 0.96\left(\mathrm{~s}, 3 \mathrm{H}, \mathrm{CH}_{3}\right), 2.12\left(\mathrm{~m}_{\mathrm{c}}, 2 \mathrm{H}\right.$, 
6-H), 2.77 (br s, $2 \mathrm{H}, \mathrm{OH}), 4.12(\mathrm{t}, 5 \mathrm{~J}=1.6 \mathrm{~Hz}, 1 \mathrm{H}, 4-\mathrm{H}), 4.31(\mathrm{~d}, 5 \mathrm{~J}=1.6 \mathrm{~Hz}, 2 \mathrm{H}, 1-\mathrm{H})$, 5.04-5.10 (m, 2 H, 8-H), $5.31\left(\mathrm{ddd},{ }^{3} J=7.6,{ }^{3} J=9.4 \mathrm{~Hz},{ }^{3} J=17.7 \mathrm{~Hz}, 1 \mathrm{H}, 7-\mathrm{H}\right)$. ${ }^{13} \mathrm{C}-\mathrm{NMR}\left(62.9 \mathrm{MHz}, \mathrm{CDCl}_{3}, \mathrm{DEPT}\right): \delta=22.36\left(+, \mathrm{CH}_{3}\right), 22.59\left(+, \mathrm{CH}_{3}\right), 38.55$ $\left(\mathrm{C}_{\text {quart }}, \mathrm{C}-5\right), 42.62(-, \mathrm{C}-6), 50.87$ (-, C-1), 70.06 (+, C-4), $84.32\left(\mathrm{C}_{\text {quart }}, \mathrm{C}-2^{*}\right), 85.07$ $\left(\mathrm{C}_{\text {quart }}, \mathrm{C}-3^{*}\right), 117.78(-, \mathrm{C}-8), 134.73(+, \mathrm{C}-7) .-\mathrm{MS}(\mathrm{EI}, 70 \mathrm{eV}), \mathrm{m} / z(\%): 135(8)\left[\mathrm{M}^{+}-\right.$ $\left.\mathrm{H}_{2} \mathrm{O}-\mathrm{CH}_{3}\right], 107$ (7), 83 (91), 68 (33), 55 (100), 41 (46) $\left[\mathrm{C}_{3} \mathrm{H}_{5}^{+}\right]$. - MS (CI, $\left.\mathrm{NH}_{3}, 70 \mathrm{eV}\right)$, $m / z(\%): 354(7)\left[2 \mathrm{M}+\mathrm{NH}_{4}^{+}\right], 203(17)\left[\mathrm{M}+\mathrm{NH}_{4}{ }^{+}+\mathrm{NH}_{3}\right], 186(100)\left[\mathrm{M}+\mathrm{NH}_{4}^{+}\right] .-$ $\mathrm{C}_{10} \mathrm{H}_{16} \mathrm{O}_{2}(168.2)$.

4,4-Dimethylhept-6-en-2-on (40): In eine Schüttelapparatur gab man $876 \mathrm{mg}$ (5\% Pd auf

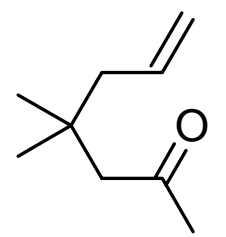
$\mathrm{CaCO}_{3}$, vergiftet mit $\left.\mathrm{Pb}\right)$ Lindlar-Katalysator, spülte die Apparatur dreimal mit Wasserstoff und gab $2 \mathrm{ml}$ Chinolin und $150 \mathrm{ml}$ Pentan zu. Man ließ bei Atmosphärendruck 15 min bis zur Wasserstoffsättigung schütteln, setzte dann $2.76 \mathrm{~g}$ (20.0 mmol) 4,4-Dimethylhept-6-in-2-on zu und hydrierte bei Raumtemp. und Atmosphärendruck. Nach 3 d waren 470 ml (19.3 mmol) Wasserstoff verbraucht. Der Katalysator wurde abfiltriert, das Lösungsmittel entfernt und das Rohprodukt umkondensiert. Man erhielt $2.51 \mathrm{~g}$ (90\%) 40, das ohne weitere Reinigung weiterverwendet wurde. Die spektroskopischen Daten stimmen mit den Daten der Literatur überein.[29]

4,4-Dimethylhept-6-en-1-in (41): Man stellte bei $0{ }^{\circ} \mathrm{C}$ aus $2.02 \mathrm{~g}$ (20.0 mmol)

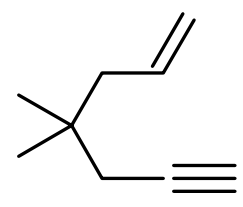

Diisopropylamin und $13 \mathrm{ml}$ (21.5 mmol, $1.65 \mathrm{M}$ in Hexan) $n$-Butyllitium in $20 \mathrm{ml}$ wasserfreiem THF eine LDA-Lösung her, ließ 30 min rühren, kühlte diese Lösung auf $-78^{\circ} \mathrm{C}$ ab und gab dann $2.38 \mathrm{~g}$ (17.0 mmol) 4,4Dimethylhept-6-en-2-on zu. Nachdem man $1 \mathrm{~h}$ bei dieser Temperatur gerührt hatte, fügte man $3.97 \mathrm{~g}(23.0 \mathrm{mmol})$ Diethylchlorophosphat zu und erwärmte langsam auf Raumtemp. Die so erhaltene Lösung gab man zu 2 Äquivalenten einer weiteren LDA-Lösung, die man aus $4.04 \mathrm{~g}$ (40 mmol) Diisopropylamin und $26 \mathrm{ml}$ (42.9 mmol, $1.65 \mathrm{M}$ in Hexan) $n$-Butyllithium in $40 \mathrm{ml}$ wasserfreiem THF hergestellt hatte. Man erwärmte innerhalb von $15 \mathrm{~h}$ langsam auf Raumtemp. Zur Aufarbeitung setzte man $100 \mathrm{ml}$ Wasser zu und extrahierte die organische Phase mit $3 \times 100 \mathrm{ml}$ Pentan. Die vereinigten organischen Phasen wurden mit $1 \mathrm{M} \mathrm{HCl}$ gewaschen, anschließend mit ges. 
$\mathrm{NaHCO}_{3}$-Lösung neutralisiert, mit Wasser gewaschen und über $\mathrm{MgSO}_{4}$ getrocknet. Nach Entfernen des Lösungsmittels reinigte man den Rückstand durch Destillation und erhielt $463 \mathrm{mg}(22 \%) 41$ als farblose Flüssigkeit, Sdp. $61^{\circ} \mathrm{C} / 100$ Torr. - ${ }^{1} \mathrm{H}-\mathrm{NMR}(250 \mathrm{MHz}$, $\left.\mathrm{CDCl}_{3}\right): \delta=0.97\left(\mathrm{~s}, 6 \mathrm{H}, 4-\mathrm{CH}_{3}\right), 1.97\left(\mathrm{t},{ }^{4} \mathrm{~J}=2.7 \mathrm{~Hz}, 1 \mathrm{H}, 1-\mathrm{H}\right), 2.04-2.08(\mathrm{~m}, 4 \mathrm{H}$, 3,5-H), 5.01-5.10 (m, 2 H, 7-H), 5.72-5.89 (m, 1 H, 6-H). - ${ }^{13} \mathrm{C}-\mathrm{NMR}\left(62.9 \mathrm{MHz}, \mathrm{CDCl}_{3}\right.$, DEPT): $\delta=26.46\left(+, 4-\mathrm{CH}_{3}\right), 31.28(-, \mathrm{C}-3), 43.83\left(\mathrm{C}_{\text {quart }}, \mathrm{C}-4\right), 45.50(-, \mathrm{C}-5), 69.87$ (+, C-1), 81.38 ( $\left.\mathrm{C}_{\text {quart }}, \mathrm{C}-2\right), 117.46$ (-, C-7), 134.91 (+, C-6). $-\mathrm{C}_{9} \mathrm{H}_{14}(122.2)$.

4,4-Dimethyl-1-trimethylsilylhept-6-en-1-in-3-ol (44-SiMe): Zu $10.0 \mathrm{ml} \quad(23.0 \mathrm{mmol}$,

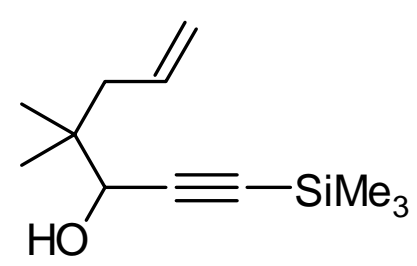
$2.30 \mathrm{M}$ in Hexan) $n$-Butyllithium in $30 \mathrm{ml}$ wasserfreiem THF gab man bei $-78{ }^{\circ} \mathrm{C} 2.00 \mathrm{~g}$ (20.4 mmol) Trimethylsilylethin und rührte $45 \mathrm{~min}$. bei dieser Temperatur. Dann versetzte man mit $2.80 \mathrm{~g}$ (25.0 mmol) 2,2-Dimethylpent-4-en-1-al, ließ $1 \mathrm{~h}$ bei $-78^{\circ} \mathrm{C}$ und $18 \mathrm{~h}$ bei Raumtemp. rühren. Nach Aufarbeitung reinigte man das Rohprodukt durch Säulenchromatographie an 70 g Kieselgel (Säule $3.5 \times 15 \mathrm{~cm}$, Pentan/ $\left.\mathrm{Et}_{2} \mathrm{O}=10: 1\right)$. Man erhielt $3.98 \mathrm{~g}(93 \%) \quad \mathbf{4 4 - \mathbf { S i M e } _ { 3 }}$ als farbloses Öl, $R_{\mathrm{f}}\left(\operatorname{Pentan} / \mathrm{Et}_{2} \mathrm{O}, 10: 1\right)=0.60 .-{ }^{1} \mathrm{H}-\mathrm{NMR}\left(250 \mathrm{MHz}, \mathrm{CDCl}_{3}\right): \delta=0.17[\mathrm{~s}$, $\left.9 \mathrm{H}, \mathrm{Si}\left(\mathrm{CH}_{3}\right)_{3}\right], 0.96$ (s, $\left.3 \mathrm{H}, 4-\mathrm{CH}_{3}\right), 0.97$ (s, 3 H, 4-CH $\left.)\right), 2.13\left(\mathrm{~m}_{\mathrm{c}}, 2 \mathrm{H}, 5-\mathrm{H}\right), 4.05$ (s, $1 \mathrm{H}, 3-\mathrm{H}), 5.03-5.12$ (m, $2 \mathrm{H}, 7-\mathrm{H}), 5.76-5.95$ (m, $1 \mathrm{H}, 6-\mathrm{H}) .-{ }^{13} \mathrm{C}-\mathrm{NMR}(62.9 \mathrm{MHz}$, $\left.\mathrm{CDCl}_{3}, \mathrm{DEPT}\right): \delta=-0.14\left[+, \mathrm{Si}\left(\mathrm{CH}_{3}\right)_{3}\right], 22.51\left(+, 4-\mathrm{CH}_{3}\right), 22.63\left(+, 4-\mathrm{CH}_{3}\right), 38.61$ $\left(\mathrm{C}_{\text {quart }}, \mathrm{C}-4\right), 42.75(-, \mathrm{C}-5), 67.96\left(\mathrm{C}_{\text {quart }}, \mathrm{C}-1 *\right), 70.54(+, \mathrm{C}-3), 105.26\left(\mathrm{C}_{\text {quart }}, \mathrm{C}-2^{*}\right)$, 117.69 (-, C-7), 134.95 (+, C-6). - MS (CI, $\left.\mathrm{NH}_{3}, 70 \mathrm{eV}\right), \mathrm{m} / z(\%): 228$ (100) $\left[\mathrm{M}+\mathrm{NH}_{4}^{+}\right]$, $211(15)\left[\mathrm{M}+\mathrm{H}^{+}\right] .-\mathrm{C}_{12} \mathrm{H}_{22} \mathrm{OSi}(210.4)$.

4,4-Dimethylhept-6-en-1-in-3-ol (44-H): $\quad \mathrm{Zu} \quad 631 \mathrm{mg} \quad$ (3.00 mmol) 4,4-Dimethyl-1-

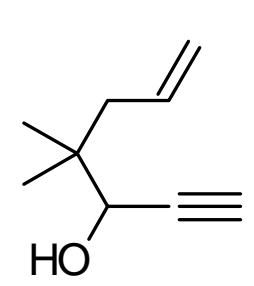
trimethylsilylhept-6-en-1-in-3-ol (44-SiMe $)$ gab $\quad$ man $2.07 \mathrm{~g}$ (15.0 mmol) $\mathrm{K}_{2} \mathrm{CO}_{3}$ in $50 \mathrm{ml}$ Methanol und $5 \mathrm{ml}$ Wasser. Nachdem man $2 \mathrm{~h}$ bei Raumtemp. gerührt hatte, versetzte man mit $50 \mathrm{ml}$ Ether und $30 \mathrm{ml}$ Wasser, extrahierte die wäßrige Phase mit $2 \times 50 \mathrm{ml}$ Ether und trocknete die vereinigten organischen Phasen über $\mathrm{Na}_{2} \mathrm{SO}_{4}$. Nach Einengen reinigte man das Rohprodukt durch Säulenchromatographie an $30 \mathrm{~g}$ Kieselgel (Säule $2.2 \times 15 \mathrm{~cm}$, Pentan/ $\mathrm{Et}_{2} \mathrm{O}=6: 1$ ) und isolierte $338 \mathrm{mg}(82 \%)$ 44-H als farbloses Öl, 
$R_{\mathrm{f}}\left(\operatorname{Pentan} / \mathrm{Et}_{2} \mathrm{O}, 6: 1\right)=0.30 .-{ }^{1} \mathrm{H}-\mathrm{NMR}\left(250 \mathrm{MHz}, \mathrm{CDCl}_{3}\right): \delta=0.97\left(\mathrm{~s}, 3 \mathrm{H}, 4-\mathrm{CH}_{3}\right)$, $0.98\left(\mathrm{~s}, 3 \mathrm{H}, 4-\mathrm{CH}_{3}\right), 1.89\left(\mathrm{~d},{ }^{3} \mathrm{~J}=5.6 \mathrm{~Hz}, 1 \mathrm{H}, \mathrm{OH}\right), 2.14\left(\mathrm{~m}_{\mathrm{c}}, 2 \mathrm{H}, 5-\mathrm{H}\right), 2.47(\mathrm{~d}$, $\left.{ }^{4} J=2.2 \mathrm{~Hz}, 1 \mathrm{H}, 1-\mathrm{H}\right), 4.08\left(\mathrm{~m}_{\mathrm{c}}, 1 \mathrm{H}, 3-\mathrm{H}\right), 5.05-5.13(\mathrm{~m}, 2 \mathrm{H}, 7-\mathrm{H}), 5.76-5.93(\mathrm{~m}, 1 \mathrm{H}$, 6-H). $-{ }^{13} \mathrm{C}-\mathrm{NMR}\left(62.9 \mathrm{MHz}, \mathrm{CDCl}_{3}, \mathrm{DEPT}\right): \delta=22.36\left(+, 4-\mathrm{CH}_{3}\right), 22.52\left(+, 4-\mathrm{CH}_{3}\right)$, 38.47 ( $\left.\mathrm{C}_{\text {quart }}, \mathrm{C}-4\right), 42.59$ (-, C-5), 69.98 (+, C-2), 74.19 ( $\left.\mathrm{C}_{\text {quart }}, \mathrm{C}-2\right), 83.24$ (+, C-3), 117.85 (-, C-7), 134.73 (+, C-6). - $\mathrm{C}_{9} \mathrm{H}_{14} \mathrm{O}(138.2)$.

4-Hydroxy-5,5-dimethyl-oct-7-en-2-inacrylsäureester (54): $\mathrm{Zu}$ einer Lösung von $841 \mathrm{mg}$

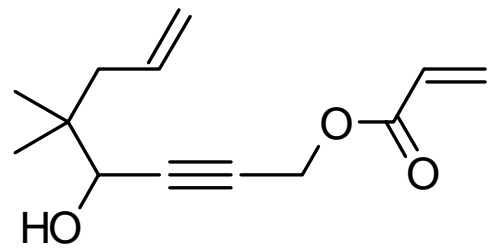
(5.00 mmol) 5,5-Dimethyloct-7-en-2-in-1,4-diol (33) und $506 \mathrm{mg}$ (5.00 mmol) Triethylamin in $20 \mathrm{ml}$ wasserfreiem $\mathrm{CH}_{2} \mathrm{Cl}_{2}$ gab man tropfenweise bei $0{ }^{\circ} \mathrm{C} 453 \mathrm{mg}$ (5.00 mmol) Acryloylchlorid zu. Man rührte $10 \mathrm{~min}$ bei $0{ }^{\circ} \mathrm{C}$ und 30 min bei Raumtemp. Anschließend extrahierte man die organische Phase mit ges. $\mathrm{NaHCO}_{3}$-Lösung $(3 \times 20 \mathrm{ml})$ und trocknete über $\mathrm{MgSO}_{4}$. Das Rohprodukt wurde durch Säulenchromatographie an $60 \mathrm{~g}$ Kieselgel (Säule $\left.2.5 \times 25 \mathrm{~cm}, \mathrm{Pentan} / \mathrm{Et}_{2} \mathrm{O}=2: 1\right)$ gereingt. Man erhielt $875 \mathrm{mg}(79 \%) 54$ als farbloses Öl, $R_{\mathrm{f}}($ Pentan/Et $2 \mathrm{O}, 2: 1)=0.47$. - IR $\left(\right.$ Film): $v=3472 \mathrm{~cm}^{-1}(\mathrm{OH}), 3075,2967,2874,1733$ $(\mathrm{C}=\mathrm{O}), 1636(\mathrm{C}=\mathrm{C}), 1408,1366,1268,1049,983,917 .-{ }^{1} \mathrm{H}-\mathrm{NMR}\left(250 \mathrm{MHz}, \mathrm{CDCl}_{3}\right)$ : $\delta=0.96\left(\mathrm{~s}, 3 \mathrm{H}, 5-\mathrm{CH}_{3}\right), 0.97\left(\mathrm{~s}, 3 \mathrm{H}, 5-\mathrm{CH}_{3}\right), 1.94(\mathrm{~s}, 1 \mathrm{H}, \mathrm{OH}), 2.13\left(\mathrm{~m}_{\mathrm{c}}, 2 \mathrm{H}, 6-\mathrm{H}\right) 4.12$ $(\mathrm{t}, 5 \mathrm{~J}=1.7 \mathrm{~Hz}, 1 \mathrm{H}, 4-\mathrm{H}), 4.80(\mathrm{~d}, 5 \mathrm{~J}=1.7 \mathrm{~Hz}, 2 \mathrm{H}, 1-\mathrm{H}), 5.04-5.11(\mathrm{~m}, 2 \mathrm{H}, 8-\mathrm{H})$, 5.74-5.91 (m, $1 \mathrm{H}, 7-\mathrm{H}), 5.86\left(\mathrm{dd},{ }^{2} J=1.5,{ }^{3} J=10.4 \mathrm{~Hz}, 1 \mathrm{H}, 3^{\prime}-\mathrm{H}\right), 6.15\left(\mathrm{dd},{ }^{3} J=10.4\right.$, $\left.{ }^{3} J=17.3 \mathrm{~Hz}, 1 \mathrm{H}, 3^{\prime}-\mathrm{H}\right), 6.46\left(\mathrm{dd},{ }^{2} J=1.5,{ }^{3} J=17.3 \mathrm{~Hz}, 1 \mathrm{H}, 3^{\prime}-\mathrm{H}\right) . \quad-{ }^{13} \mathrm{C}-\mathrm{NMR}$ (62.9 MHz, $\left.\mathrm{CDCl}_{3}, \mathrm{DEPT}\right): \delta=22.48\left(+, 5-\mathrm{CH}_{3}\right), 22.63\left(+, 5-\mathrm{CH}_{3}\right), 38.69\left(\mathrm{C}_{\text {quart }}, \mathrm{C}-5\right)$, 42.67 (-, C-6), 52.38 (-, C-1), 70.08 (+, C-4), $79.97\left(\mathrm{C}_{\text {quart }}, \mathrm{C}-2^{*}\right), 86.32\left(\mathrm{C}_{\text {quart }}, \mathrm{C}-3^{*}\right)$, $117.84(-, \mathrm{C}-8), 127.65$ (+, C-2'), 131.76 (-, C-3'), 134.72 (+, C-7), $165.36\left(\mathrm{C}_{\text {quart }}, \mathrm{C}=\mathrm{O}\right)$. MS (CI, $\left.\mathrm{NH}_{3}, 70 \mathrm{eV}\right), \mathrm{m} / z(\%): 462$ (1) $\left[2 \mathrm{M}+\mathrm{NH}_{4}^{+}\right], 240$ (100) $\left[\mathrm{M}+\mathrm{NH}_{4}^{+}\right]$. $\mathrm{C}_{13} \mathrm{H}_{18} \mathrm{O}_{3}$ (222.3): ber. C 70.24, H 8.16; gef. C 70.24, H 7.92. 
5-Hydroxy-6,6-dimethylnon-8-en-3-in-1-ol (57): Analog AAV 1 gab man bei $-78{ }^{\circ} \mathrm{C}$ zu

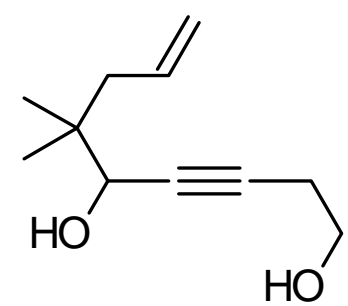
$1.20 \mathrm{~g}$ (17.1 mmol) 3-Butinol in $20 \mathrm{ml}$ wasserfreiem THF $18.0 \mathrm{ml}$ (46.8 mmol, 2.60 $\mathrm{M}$ in Hexan) $n$-Butyllithium und rührte $30 \mathrm{~min}$. Dann versetzte man mit $2.24 \mathrm{~g}$ (20.0 mmol) 2,2-Dimethylpent-4en-1-al, ließ $1 \mathrm{~h}$ bei $-78{ }^{\circ} \mathrm{C}$ und $18 \mathrm{~h}$ bei Raumtemp. rühren. Nach Aufarbeitung reinigte man das Rohprodukt durch Säulenchromatographie an $70 \mathrm{~g}$ Kieselgel (Säule $3.5 \times 15 \mathrm{~cm}, \mathrm{Et}_{2} \mathrm{O}$ ). Man erhielt $2.81 \mathrm{~g}(90 \%) 57$ als farbloses, hochviskoses Öl, $R_{\mathrm{f}}\left(\mathrm{Et}_{2} \mathrm{O}\right)=0.47$. ${ }^{1} \mathrm{H}-\mathrm{NMR}\left(250 \mathrm{MHz}, \mathrm{CDCl}_{3}\right)$ : $\delta=0.95\left(\mathrm{~s}, 3 \mathrm{H}, \mathrm{CH}_{3}\right), 0.96\left(\mathrm{~s}, 3 \mathrm{H}, \mathrm{CH}_{3}\right), 2.10-2.17(\mathrm{~m}, 2 \mathrm{H}, 7-\mathrm{H}), 2.50\left(\mathrm{dt},{ }^{3} \mathrm{~J}=6.1\right.$, $5 J=2.0 \mathrm{~Hz}, 2 \mathrm{H}, 2-\mathrm{H}), 2.78($ br s, $2 \mathrm{H}, \mathrm{OH}), 3.73\left(\mathrm{t},{ }^{3} \mathrm{~J}=6.1 \mathrm{~Hz}, 2 \mathrm{H}, 1-\mathrm{H}\right), 4.07$ (t, $5 J=2.0 \mathrm{~Hz}, 1 \mathrm{H}, 5-\mathrm{H}), 5.01-5.11(\mathrm{~m}, 2 \mathrm{H}, 9-\mathrm{H}), 5.84\left(\mathrm{ddd},{ }^{3} J=7.3,{ }^{3} J=9.3 \mathrm{~Hz}\right.$, $\left.{ }^{3} J=17.1 \mathrm{~Hz}, 1 \mathrm{H}, 8-\mathrm{H}\right) .-{ }^{13} \mathrm{C}-\mathrm{NMR}\left(62.9 \mathrm{MHz}, \mathrm{CDCl}_{3}, \mathrm{DEPT}\right): \delta=22.42\left(+, \mathrm{CH}_{3}\right)$, $22.64\left(+, \mathrm{CH}_{3}\right), 23.02$ (-, C-2), $38.58\left(\mathrm{C}_{\text {quart }}, \mathrm{C}-6\right), 42.71$ (-, C-7), 61.01 (-, C-1), 70.25 (+, C-5), $81.41\left(\mathrm{C}_{\text {quart }}, \mathrm{C}-3 *\right), 83.19\left(\mathrm{C}_{\text {quart }}, \mathrm{C}-4^{*}\right), 117.59$ (-, C-9), 134.94 (+, C-8). $\mathrm{C}_{11} \mathrm{H}_{18} \mathrm{O}_{2}(182.3)$.

\subsection{Darstellung von Hexahydroindengerüsten durch Enin-Cycloisomerisierung}

Allgemeine Arbeitsvorschrift zur Enin-Cycloisomerisierung (AAV 2): $\mathrm{Zu}$ einer Lösung von $1 \mathrm{mmol}$ Enin und $3 \mathrm{mmol}$ Dienophil in $10 \mathrm{ml}$ wasserfreiem Benzol in einer starkwandigen Pyrexflasche mit Schraubdeckel gibt man $5 \mathrm{~mol} \% \quad \mathrm{Pd}(\mathrm{OAc})_{2}$ und $10 \mathrm{~mol} \% \quad N, N^{\prime}-$ Bis(benzyliden)ethylendiamin. Man leitet 5 min Stickstoff durch das Reaktionsgemisch, verschließt die Flasche und rührt die angegebene Zeit bei $70{ }^{\circ} \mathrm{C}$. Nach Abkühlen auf Raumtemp. filtriert man über Aktivkohle und Celite, wäscht mit Diethylether nach und entfernt das Lösungsmittel i. Vak. Das verbleibende Rohprodukt wird durch Säulenchromatographie an Kieselgel gereinigt.

2,2-Dimethyl-2,3,4,5,6,7-hexahydro-1H-inden-5-carbonsäuremethylester (42): Variante A: Man gab zu einer Lösung von $123 \mathrm{mg}$ (1.01 mmol) 4,4-Dimethyl-hept-7-en-1-in und $258 \mathrm{mg}$ (3.00 mmol) Methylacrylat in $10 \mathrm{ml}$ wasserfreiem Benzol $29 \mathrm{mg}(0.050 \mathrm{mmol}$, $5.0 \mathrm{~mol} \%) \mathrm{Pd}(\mathrm{dba})_{2}, 26 \mathrm{mg}(0.10 \mathrm{mmol}, 10 \mathrm{~mol} \%) \mathrm{PPh}_{3}$ und $6.0 \mathrm{mg}$ (0.10 mmol, 
10 mol\%) Essigsäure. Man rührte $1 \mathrm{~d}$ bei $70{ }^{\circ} \mathrm{C}$. Säulenchromatographie an $18 \mathrm{~g}$ Kieselgel (Säule $2.0 \times 15 \mathrm{~cm}$, Pentan $\left./ \mathrm{Et}_{2} \mathrm{O}=10: 1\right)$ ergab $126 \mathrm{mg}(60 \%) 42$ als farbloses Öl, $R_{\mathrm{f}}$

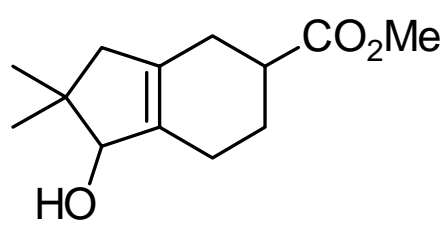
$\left(\right.$ Pentan/Et $\left.{ }_{2} \mathrm{O}, 10: 1\right)=0.57$. - IR $\left(\right.$ Film) $: v=2953 \mathrm{~cm}^{-1}$, 2837, $1739(\mathrm{C}=\mathrm{O}), 1437,1363,1311,1229,1168,1012$, 978, 912. - ${ }^{1} \mathrm{H}-\mathrm{NMR}\left(250 \mathrm{MHz}, \mathrm{CDCl}_{3}\right): \delta=1.05(\mathrm{~s}, 6 \mathrm{H}$, 2- $\left.\mathrm{CH}_{3}\right), 1.62-1.72\left(\mathrm{~m}, 1 \mathrm{H}, 4-\mathrm{H}^{*}\right), 1.92-2.18(\mathrm{~m}, 9 \mathrm{H}$, 1,3,4,6,7-H*), 2.50-2.62 (m, $1 \mathrm{H}, 5-\mathrm{H}), 3.67$ (s, $\left.3 \mathrm{H}, \mathrm{CO}_{2} \mathrm{CH}_{3}\right) .-{ }^{13} \mathrm{C}-\mathrm{NMR}(62.9 \mathrm{MHz}$, $\left.\mathrm{CDCl}_{3}, \mathrm{DEPT}\right): \delta=25.09(-, \mathrm{C}-7 *), 25.82\left(-, \mathrm{C}-4^{*}\right), 28.40(-, \mathrm{C}-6 *), 30.26\left(+, 2-\mathrm{CH}_{3}\right)$, $37.10\left(\mathrm{C}_{\text {quart }}, \mathrm{C}-2\right), 40.11(+, \mathrm{C}-5), 51.13\left(-, \mathrm{C}-1^{*}, \mathrm{C}-3^{*}\right), 51.54\left(+, \mathrm{CO}_{2} \mathrm{CH}_{3}\right), 131.09$ $\left(\mathrm{C}_{\text {quart }}, \mathrm{C}-3 \mathrm{a}^{* *}\right), 132.42\left(\mathrm{C}_{\text {quart }}, \mathrm{C}-7 \mathrm{a} * *\right), 176.55\left(\mathrm{C}_{\text {quart }}, \mathrm{CO}_{2} \mathrm{CH}_{3}\right) .-\mathrm{MS}(\mathrm{EI}, 70 \mathrm{eV}), \mathrm{m} / \mathrm{z}$ (\%): 208 (48) $\left[\mathrm{M}^{+}\right], 193$ (49) $\left[\mathrm{M}^{+}-\mathrm{CH}_{3}\right], 177$ (9) $\left[\mathrm{M}^{+}-\mathrm{OMe}\right], 161$ (10) $\left[\mathrm{M}^{+}-\mathrm{CH}_{3}-\right.$

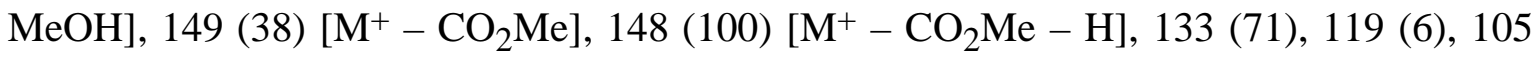
(15), 93 (25), 91 (24), 77 (11), 55 (8), 41 (11) $\left[\mathrm{C}_{3} \mathrm{H}_{5}{ }^{+}\right] .-\mathrm{C}_{13} \mathrm{H}_{20} \mathrm{O}_{2}$ (208.3): ber. C 74.96, H 9.68; gef. C 74.70, H 9.54.

Variante B: Nach AAV 2 wurden $123 \mathrm{mg}$ (1.01 mmol) 4,4-Dimethyl-hept-7-en-1-in und $258 \mathrm{mg}$ (3.00 mmol) Methylacrylat in $10 \mathrm{ml}$ wasserfreiem Benzol mit $26 \mathrm{mg}(0.050 \mathrm{mmol}$, $5.0 \mathrm{~mol} \%) \mathrm{Pd}(\mathrm{OAc})_{2}$ und $24 \mathrm{mg}(0.10 \mathrm{mmol}, 10 \mathrm{~mol} \%) \quad N, N^{\prime}$-Bis(benzyliden)ethylendiamin bei $70{ }^{\circ} \mathrm{C}$ zur Reaktion gebracht. Man erhielt nach einer Reaktionszeit von $1 \mathrm{~d}$ jedoch nur $34 \mathrm{mg}(16 \%) 42$.

4-Trimethylsilyl-3-hydroxy-2,2-dimethyl-2,3,4,5,6,7-hexahydro-1H-inden-5-carbonsäuremethylester (45a-SiMe $\mathbf{3}_{3}$ und 4-Trimethylsilyl-3-hydroxy-2,2-dimethyl-2,3,4,5,6,7hexahydro-1H-inden-6-carbonsäuremethylester (45b-SiMe $\mathbf{3})$ : Analog AAV 2 wurden $210 \mathrm{mg}(1.00 \mathrm{mmol})$ Enin 44-SiMe 3 und $258 \mathrm{mg}$ (3.00 mmol) Methylacrylat in $10 \mathrm{ml}$ wasserfreiem Benzol gelöst und mit $11 \mathrm{mg}(0.050 \mathrm{mmol}, 5.0 \mathrm{~mol} \%) \mathrm{Pd}(\mathrm{OAc})_{2}$ und $24 \mathrm{mg}$ (0.10 mmol, $10 \mathrm{~mol} \%) N, N^{\prime}$-Bis(benzyliden)ethylendiamin versetzt. Man rührte $3 \mathrm{~d}$ bei $70{ }^{\circ} \mathrm{C}$. Säulenchromatographie an $18 \mathrm{~g}$ Kieselgel (Säule $2.0 \times 15 \mathrm{~cm}, \mathrm{Pentan} / \mathrm{Et}_{2} \mathrm{O}=6: 1$ ) ergab 143 mg (48\%) eines Gemisches aus 45a-SiMe 3 und 45b-SiMe 3 im Verhältnis 1.3: 1 (aus ${ }^{1} \mathrm{H}-\mathrm{NMR}-$ Spektrum) als farbloses Öl, $R_{\mathrm{f}}\left(\mathrm{Pentan}_{\mathrm{E}} \mathrm{Et}_{2} \mathrm{O}, 6: 1\right)=0.25$. - IR (Film), Gemisch: $v=3514 \mathrm{~cm}^{-1}(\mathrm{OH}), 2951,2897,2837,1719$ (C=O), 1635, 1436, 1362, 1246, 1175, 1103, 1076, 838, 760, 686. - 45a-SiMe $:{ }^{1} \mathrm{H}-\mathrm{NMR}\left(250 \mathrm{MHz}, \mathrm{CDCl}_{3}\right): \delta=0.05$ [s, $\left.9 \mathrm{H}, \mathrm{Si}\left(\mathrm{CH}_{3}\right)_{3}\right], 1.02\left(\mathrm{~s}, 3 \mathrm{H}, 2-\mathrm{CH}_{3}\right), 1.04\left(\mathrm{~s}, 3 \mathrm{H}, 2-\mathrm{CH}_{3}\right), 1.46-2.23(\mathrm{~m}, 7 \mathrm{H}$, 
1,4,5,7-H), $2.65\left(\mathrm{~m}_{\mathrm{c}}, 1 \mathrm{H}, 6-\mathrm{H}\right), 3.67\left(\mathrm{~s}, 3 \mathrm{H}, \mathrm{CO}_{2} \mathrm{CH}_{3}\right), 3.90$

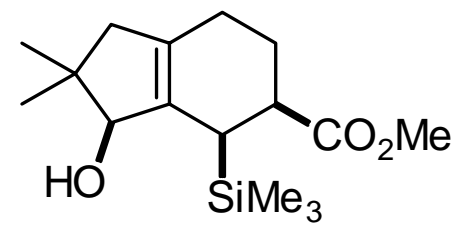

(br s, $1 \mathrm{H}, 3-\mathrm{H}) .-{ }^{13} \mathrm{C}-\mathrm{NMR}\left(62.9 \mathrm{MHz}, \mathrm{CDCl}_{3}\right.$, DEPT): $\delta=0.21\left[+, \mathrm{Si}\left(\mathrm{CH}_{3}\right)_{3}\right], 23.00(-, \mathrm{C}-7 *), 23.90\left(+, 2-\mathrm{CH}_{3}\right)$, $25.83\left(+, 2-\mathrm{CH}_{3}\right), 28.44(-, \mathrm{C}-5 *), 29.06(+, \mathrm{C}-4), 41.38(+$, C-6), $43.40\left(\mathrm{C}_{\text {quart }}, \mathrm{C}-2\right), 48.17$ (-, C-1*), $51.41 \quad(+$, $\left.\mathrm{CO}_{2} \mathrm{CH}_{3}\right), 87.39$ (+, C-3), $135.51\left(\mathrm{C}_{\text {quart }}, \mathrm{C}-3 \mathrm{a}^{* *}\right), 138.02\left(\mathrm{C}_{\text {quart }}, \mathrm{C}-7 \mathrm{a}^{* *}\right), 175.31\left(\mathrm{C}_{\text {quart }}\right.$, $\left.\mathrm{CO}_{2} \mathrm{CH}_{3}\right) .-45 \mathrm{~b}-\mathrm{SiMe}_{3}:{ }^{1} \mathrm{H}-\mathrm{NMR}\left(250 \mathrm{MHz}, \mathrm{CDCl}_{3}\right): \delta=0.08\left[\mathrm{~s}, 9 \mathrm{H}, \mathrm{Si}\left(\mathrm{CH}_{3}\right)_{3}\right], 0.94$<smiles>COC1CC2=C(C(O)C(C)(C)C2)C(S(C)(C)C)C1</smiles>
(s, $\left.3 \mathrm{H}, 2-\mathrm{CH}_{3}\right), 1.08\left(\mathrm{~s}, 3 \mathrm{H}, 2-\mathrm{CH}_{3}\right), 1.41-1.55(\mathrm{~m}, 2 \mathrm{H}$, 1-H), $1.73\left(\mathrm{~m}_{\mathrm{c}}, 1 \mathrm{H}, 4-\mathrm{H}\right), 2.08-2.77$ (m, $\left.5 \mathrm{H}, 5,6,7-\mathrm{H}\right), 3.69$ (s, $\left.3 \mathrm{H}, \mathrm{CO}_{2} \mathrm{CH}_{3}\right), 3.72($ br s, $1 \mathrm{H}, 3-\mathrm{H}) .-{ }^{13} \mathrm{C}-\mathrm{NMR}$ $\left(62.9 \mathrm{MHz}, \mathrm{CDCl}_{3}, \mathrm{DEPT}\right): \delta=-1.70\left[+, \mathrm{Si}\left(\mathrm{CH}_{3}\right)_{3}\right], 22.59$ (-, C-7*), $26.55\left(+, 2-\mathrm{CH}_{3}\right), 28.73\left(+, 2-\mathrm{CH}_{3}\right), 28.89$ (-, C-6*), 29.27 (+, C-4), $40.85(+$, C-5), $41.12\left(\mathrm{C}_{\text {quart }}, \mathrm{C}-2\right), 48.33(-, \mathrm{C}-1 *), 51.69\left(+, \mathrm{CO}_{2} \mathrm{CH}_{3}\right), 85.74(+, \mathrm{C}-3), 136.86$ $\left(\mathrm{C}_{\text {quart }}, \mathrm{C}-3 \mathrm{a}^{* *}\right), 137.48\left(\mathrm{C}_{\text {quart }}, \mathrm{C}-7 \mathrm{a}^{* *}\right), 176.84\left(\mathrm{C}_{\text {quart }}, \mathrm{CO}_{2} \mathrm{CH}_{3}\right) .-\mathrm{MS}(\mathrm{EI}, 70 \mathrm{eV}), \mathrm{m} / \mathrm{z}$

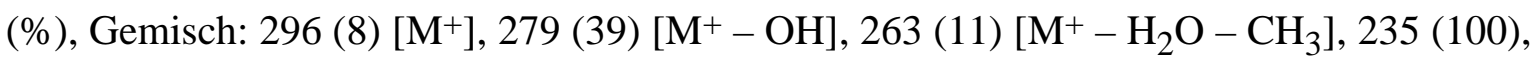
219 (17), 147 (26) $\left[\mathrm{M}^{+}-\mathrm{CO}_{2} \mathrm{Me}-\mathrm{SiMe}_{3}-\mathrm{OH}\right], 138$ (39), 129 (20), 89 (17), 73 (65) $\left[\mathrm{SiMe}_{3}+\right], 69$ (18). $-\mathrm{C}_{16} \mathrm{H}_{28} \mathrm{O}_{3} \mathrm{Si}(296.5)$.

3-Hydroxy-2,2-dimethyl-2,3,4,5,6,7-hexahydro-1H-inden-5-carbonsäuremethylester (45aH) und 3-Hydroxy-2,2-dimethyl-2,3,4,5,6,7-hexahydro-1H-inden-6-carbonsäuremethylester (45b-H): Nach AAV 2 wurden $138 \mathrm{mg}(1.00 \mathrm{mmol})$ Enin $\mathbf{4 4 - H}$ und $258 \mathrm{mg}$ (3.00 mmol) Methylacrylat in $10 \mathrm{ml}$ wasserfreiem Benzol gelöst und mit $11 \mathrm{mg}$ $(0.050 \mathrm{mmol}, \quad 5.0 \mathrm{~mol} \%) \quad \mathrm{Pd}(\mathrm{OAc})_{2} \quad$ und $\quad 24 \mathrm{mg} \quad(0.10 \mathrm{mmol}, \quad 10 \mathrm{~mol} \%) \quad N, N^{\prime}-$ Bis(benzyliden)ethylendiamin versetzt. Man rührte $1 \mathrm{~d}$ bei $70^{\circ} \mathrm{C}$. Säulenchromatographie an $18 \mathrm{~g}$ Kieselgel (Säule $2.0 \times 15 \mathrm{~cm}$, Pentan/Et $2 \mathrm{O}, 6: 1$ ) ergab $54 \mathrm{mg}$ (24\%) eines aus jeweils zwei Diastereomeren bestehendes Gemisch aus 45a-H und $\mathbf{4 5 b}-\mathbf{H}$ im Verhältnis $2.5: 1$ als farbloses Öl, $R_{\mathrm{f}}\left(\mathrm{Pentan}_{\mathrm{E}} \mathrm{Et}_{2} \mathrm{O}, 2: 1\right)=0.15$. Die spektroskopischen Daten stimmen mit den Daten in der Literatur überein.[21c]

5-Chlor-2,2-dimethyl-4-pivaloxymethyl-2,3,4,5,6,7-hexahydro-1H-inden-5-carbonsäuremethylester (47a) und 6-Chlor-2,2-dimethyl-4-pivaloxymethyl-2,3,4,5,6,7-hexahydro-1Hinden-6-carbonsäuremethylester (47b): Analog AAV 2 wurden $252 \mathrm{mg}(1.00 \mathrm{mmol})$ 
4-Hydroxy-5,5-dimethyl-oct-7-en-2-inpivaloylsäureester $\quad$ und $\quad 362 \mathrm{mg} \quad(3.00 \mathrm{mmol})$ $\alpha$-Chloracrylsäuremethylester in $10 \mathrm{ml}$ wasserfreiem Benzol gelöst und mit $11 \mathrm{mg}$ $(0.050 \mathrm{mmol}, \quad 5.0 \mathrm{~mol} \%) \quad \mathrm{Pd}(\mathrm{OAc})_{2} \quad$ und $24 \mathrm{mg} \quad(0.10 \mathrm{mmol}, \quad 10 \mathrm{~mol} \%) \quad N, N^{\prime}-$ Bis(benzyliden)ethylendiamin versetzt. Man rührte $18 \mathrm{~h}$ bei $70{ }^{\circ} \mathrm{C}$. Säulenchromatographie an 18 g Kieselgel (Säule $2.0 \times 15 \mathrm{~cm}$, Pentan/ $\mathrm{Et}_{2} \mathrm{O}=2: 1$ ) ergab $167 \mathrm{mg}(45 \%)$ 47a und 47b im Verhältnis $4.5: 1$ als farbloses Öl, $R_{\mathrm{f}}\left(\right.$ Pentan/Et $\left._{2} \mathrm{O}, 2: 1\right)=0.25$. - IR (Film), Gemisch: $v=3506 \mathrm{~cm}^{-1}(\mathrm{OH}), 2957,2839,1734(\mathrm{C}=\mathrm{O}), 1481,1464,1437,1261,1152$, 1042, 916, 769, 702. - 47a: ${ }^{1} \mathrm{H}-\mathrm{NMR}\left(250 \mathrm{MHz}, \mathrm{CDCl}_{3}\right): \delta=0.96\left(\mathrm{~s}, 3 \mathrm{H}, 2-\mathrm{CH}_{3}\right), 1.11$
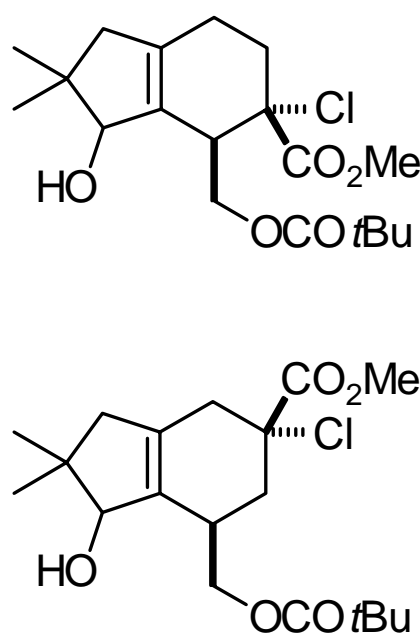
$\left(\mathrm{s}, 3 \mathrm{H}, 2-\mathrm{CH}_{3}\right), 1.23(\mathrm{~s}, 1 \mathrm{H}, \mathrm{OH}), 2.10-2.47(\mathrm{~m}, 6 \mathrm{H}$, 1,6,7-H), 3.05 (br s, $1 \mathrm{H}, 3-\mathrm{H}), 3.86$ (s, $3 \mathrm{H}, \mathrm{CO}_{2} \mathrm{CH}_{3}$ ), 3.92 $\left(\mathrm{dd},{ }^{2} J=1.7,{ }^{3} \mathrm{~J}=12.4 \mathrm{~Hz}, 1 \mathrm{H}, 4-\mathrm{CH}_{2}\right), 4.13$ (br s, $1 \mathrm{H}, 3-\mathrm{H}$ ), $4.71\left(\mathrm{dd}, \quad 2 J=4.5, \quad 3 J=12.4 \mathrm{~Hz}, \quad 1 \mathrm{H}, \quad 4-\mathrm{CH}_{2}\right) . \quad-\quad 47 \mathbf{b}$ : ${ }^{1} \mathrm{H}-\mathrm{NMR}\left(250 \mathrm{MHz}, \mathrm{CDCl}_{3}\right): \delta=1.04\left(\mathrm{~s}, 3 \mathrm{H}, 2-\mathrm{CH}_{3}\right), 1.07$ $\left(\mathrm{s}, 3 \mathrm{H}, 2-\mathrm{CH}_{3}\right), 1.23(\mathrm{~s}, 1 \mathrm{H}, \mathrm{OH}), 2.10-2.47(\mathrm{~m}, 6 \mathrm{H}$, 1,5,7-H), 3.17 (br s, $1 \mathrm{H}, 3-\mathrm{H}), 3.84\left(\mathrm{~s}, 3 \mathrm{H}, \mathrm{CO}_{2} \mathrm{CH}_{3}\right), 3.93$ $\left(\mathrm{dd},{ }^{2} J=1.7,{ }^{3} \mathrm{~J}=12.4 \mathrm{~Hz}, 1 \mathrm{H}, 4-\mathrm{CH}_{2}\right), 4.13$ (br s, $\left.1 \mathrm{H}, 3-\mathrm{H}\right)$, $4.25\left(\mathrm{dd},{ }^{2} J=4.5,{ }^{3} J=12.4 \mathrm{~Hz}, 1 \mathrm{H}, 4-\mathrm{CH}_{2}\right) .-{ }^{13} \mathrm{C}-\mathrm{NMR}$ (62.9 MHz, $\left.\mathrm{CDCl}_{3}, \mathrm{DEPT}\right)$, Gemisch: $\delta=22.90\left(+, 2-\mathrm{CH}_{3}\right)$, $23.33\left(-, \mathrm{C}-6^{*}\right), 27.03\left[+, \mathrm{C}\left(\mathrm{CH}_{3}\right)_{3}\right], 28.37\left(+, 2-\mathrm{CH}_{3}\right), 29.38\left(-, \mathrm{C}-7^{*}\right), 38.80\left(\mathrm{C}_{\text {quart }}\right.$, C-2), $41.42\left(\mathrm{C}_{\text {quart }}, \mathrm{C}-5\right), 46.31$ (+, C-4), 48.37 (-, C-1*), $53.10\left(+, \mathrm{CO}_{2} \mathrm{CH}_{3}\right), 63.81$ (-, 4- $\left.\mathrm{CH}_{2}\right), 70.09\left[\mathrm{C}_{\text {quart }}, C\left(\mathrm{CH}_{3}\right)_{3}\right], 87.71(+, \mathrm{C}-3), 129.80\left(\mathrm{C}_{\text {quart }}, \mathrm{C}-3 \mathrm{a} * *\right), 139.46\left(\mathrm{C}_{\text {quart }}\right.$, C-7a**), $170.71\left(\mathrm{C}_{\text {quart }}, \mathrm{OCO} t \mathrm{Bu}\right), 178.67\left(\mathrm{C}_{\text {quart }}, \mathrm{CO}_{2} \mathrm{CH}_{3}\right) .-\mathrm{MS}(\mathrm{EI}, 70 \mathrm{eV}), \mathrm{m} / z(\%)$, Gemisch: 372 (0.2) $\left[\mathrm{M}^{+}\right], 235$ (100), $217(10)\left[\mathrm{M}^{+}-\mathrm{Cl}-\mathrm{OCO} t \mathrm{Bu}-\mathrm{OH}\right], 201$ (5), 150 (9), 105 (9), 91 (10), 57 (27) $\left[\mathrm{C}_{4} \mathrm{H}_{9}{ }^{+}\right], 41$ (11) $\left[\mathrm{C}_{3} \mathrm{H}_{5}{ }^{+}\right] .-\mathrm{C}_{19} \mathrm{H}_{29} \mathrm{ClO}_{5}$ (372.9): ber. C 61.20, H 7.84; gef. C 61.35, H 7.68.

8-Hydroxy-7,7-dimethyl-1,3a,4,5,6,7,8,8b-octahydro-3H-indeno[4,5-c]furan-3-on (52): Variante A: Analog AAV 2 wurden $168 \mathrm{mg}$ (1.00 mmol) Enin 33 und $258 \mathrm{mg}$ (3.00 mmol) Methylacrylat in $10 \mathrm{ml}$ wasserfreiem Benzol gelöst und mit $11 \mathrm{mg}(0.050 \mathrm{mmol}$, $5.0 \mathrm{~mol} \%) \mathrm{Pd}(\mathrm{OAc})_{2}$ und $24 \mathrm{mg}(0.10 \mathrm{mmol}, 10 \mathrm{~mol} \%) \quad N, N^{\prime}$-Bis(benzyliden)ethylendiamin versetzt. Man rührte $1 \mathrm{~d}$ bei $70{ }^{\circ} \mathrm{C}$. Säulenchromatographie an $18 \mathrm{~g}$ Kieselgel (Säule $2.0 \times 15 \mathrm{~cm}$, Pentan/Et $2 \mathrm{O}=1: 2)$ ergab $83 \mathrm{mg}$ (37\%) 52 als farblose Kristalle, Schmp. 
87-88 ${ }^{\circ} \mathrm{C}, R_{\mathrm{f}}\left(\mathrm{Pentan} / \mathrm{Et}_{2} \mathrm{O}, 1: 2\right)=0.25 .-\mathrm{IR}(\mathrm{KBr}): v=3492 \mathrm{~cm}^{-1}(\mathrm{OH}), 2944,2928$, 2841, $1757(\mathrm{C}=\mathrm{O}), 1482,1365,1218,1163,1001 .-{ }^{1} \mathrm{H}-\mathrm{NMR}\left(250 \mathrm{MHz}, \mathrm{CDCl}_{3}\right)$ : $\delta=1.01\left(\mathrm{~s}, 3 \mathrm{H}, \mathrm{CH}_{3}\right), 1.03\left(\mathrm{~s}, 3 \mathrm{H}, \mathrm{CH}_{3}\right), 1.62(\mathrm{br} \mathrm{s}, 1 \mathrm{H}, \mathrm{OH}), 1.79-2.26(\mathrm{~m}, 6 \mathrm{H}$,

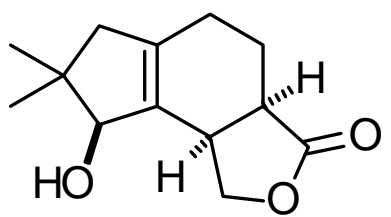
4,5,6-H), 2.73-2.80 (m, $1 \mathrm{H}, 8 \mathrm{~b}-\mathrm{H}), 3.12$ (br s, $1 \mathrm{H}, 3 \mathrm{a}-\mathrm{H}), 4.07$ (br s, $1 \mathrm{H}, 8-\mathrm{H}), 4.37\left(\mathrm{dd}, 2 J=8.9,{ }^{3} J=6.8 \mathrm{~Hz}, 1 \mathrm{H}, 1-\mathrm{H}\right), 4.54$ $\left(\mathrm{dd},{ }^{2} J=8.9,{ }^{3} J=6.8 \mathrm{~Hz}, 1 \mathrm{H}, 1-\mathrm{H}\right) .-{ }^{13} \mathrm{C}-\mathrm{NMR}(62.9 \mathrm{MHz}$, $\left.\mathrm{CDCl}_{3}, \mathrm{DEPT}\right): \delta=20.72(-, \mathrm{C}-4 *), 22.53\left(+, \mathrm{CH}_{3}\right), 23.12(-$, C-5*), $28.38\left(+, \mathrm{CH}_{3}\right), 36.27\left(+, \mathrm{C}-8 \mathrm{~b}^{* *}\right), 38.86\left(+, \mathrm{C}-3 \mathrm{a}^{* *}\right), 41.11\left(\mathrm{C}_{\text {quart }}, \mathrm{C}-7\right), 48.91(-$, C-6*), 71.42 (-, C-1), 86.81 (+, C-8), 131.85 (C quart, $\left.\mathrm{C}-8 \mathrm{a}^{* * *}\right), 142.03$ (C $\left.\mathrm{C}_{\text {quart }}, \mathrm{C}-5 \mathrm{a}^{* * *}\right)$, $179.23\left(\mathrm{C}_{\text {quart }}, \mathrm{C}-3\right)$. - MS (EI, $\left.70 \mathrm{eV}\right), \mathrm{m} / \mathrm{z}(\%): 222(38)\left[\mathrm{M}^{+}\right], 207(51)\left[\mathrm{M}^{+}-\mathrm{CH}_{3}\right], 204$ (28) $\left[\mathrm{M}^{+}-\mathrm{H}_{2} \mathrm{O}\right], 153$ (65), 149 (34), 110 (41), 107 (48), 91 (51), 43 (100). - $\mathrm{C}_{13} \mathrm{H}_{18} \mathrm{O}_{3}$ (222.3): ber. C 70.25, H 8.16; gef. C 69.95, H 8.20.

Variante B: Verwendete man $10 \mathrm{ml}$ wasserfreies Acetonitril als Lösungsmittel unter sonst identischen Bedingungen konnte kein Produkt isoliert werden. Es wurden $77 \mathrm{mg}$ (46\%) Ausgangsmaterial zurückgewonnen.

Variante C: Setzte man zusätzlich zu Variante A noch $28 \mathrm{mg}(0.10 \mathrm{mmol}, 10 \mathrm{~mol} \%)$ $\operatorname{Ti}(\mathrm{O} i \mathrm{Pr})_{4} \mathrm{zu}$, konnte nur oligomeres Material isoliert werden.

Variante D: Man löste $168 \mathrm{mg}$ (1.00 mmol) Enin 33 in $10 \mathrm{ml}$ wasserfreiem Benzol und versetzte mit $11 \mathrm{mg}(0.050 \mathrm{mmol}, 5.0 \mathrm{~mol} \%) \mathrm{Pd}(\mathrm{OAc})_{2}$ und $24 \mathrm{mg}(0.10 \mathrm{mmol}, 10 \mathrm{~mol} \%)$ $N, N^{\prime}$-Bis(benzyliden)ethylendiamin. Man erwärmte $18 \mathrm{~h}$ auf $60^{\circ} \mathrm{C}$, kühlte anschließend auf Raumtemp. und gab $258 \mathrm{mg}$ ( $3.00 \mathrm{mmol})$ Methylacrylat und $28 \mathrm{mg}(0.10 \mathrm{mmol}, 10 \mathrm{~mol} \%)$ $\mathrm{Ti}(\mathrm{O} i \mathrm{Pr})_{4}$ zu. Das Reaktionsgemisch wurde erneut auf $60^{\circ} \mathrm{C}$ erhitzt und $2 \mathrm{~d}$ gerührt. Nach Filtration über Celite und Aktivkohle erhielt man jedoch ebenfalls nur nicht identifizierbare Oligomere.

Versuch der Darstellung von 52 durch Cyclisierung von 54: Analog AAV 2 gab man zu $222 \mathrm{mg}(1.00 \mathrm{mmol}) 54$ in $10 \mathrm{ml}$ wasserfreiem Benzol $11 \mathrm{mg}(0.050 \mathrm{mmol}, 5.0 \mathrm{~mol} \%)$ $\mathrm{Pd}(\mathrm{OAc})_{2} \quad$ und $24 \mathrm{mg} \quad(0.10 \mathrm{mmol}, \quad 10 \mathrm{~mol} \%) \quad N, N^{\prime}$-Bis(benzyliden)ethylendiamin. Nachdem man $18 \mathrm{~h}$ bei $70^{\circ} \mathrm{C}$ gerührt hatte, konnte nur oligomeres Material isoliert werden. 
9-Hydroxy-8,8-dimethyl-1,4a,5,6,7,8,9,9b-octahydrocyclopenta[f] isochromen-4(2H)-on

(58): Nach AAV 2 versetzte man $182 \mathrm{mg}(1.00 \mathrm{mmol})$ Enin 57 in $10 \mathrm{ml}$ wasserfreiem<smiles>CC1(C)CC2=C(C1O)[C@H]1CCOC(=O)[C@@H]1CC2</smiles>

Benzol mit $258 \mathrm{mg} \quad(3.00 \mathrm{mmol}) \quad$ Methylacrylat, $11 \mathrm{mg}$ $(0.050 \mathrm{mmol}, 5.0 \mathrm{~mol} \%) \mathrm{Pd}(\mathrm{OAc})_{2}$ und $24 \mathrm{mg}(0.10 \mathrm{mmol}$, $10 \mathrm{~mol} \%) \quad N, N^{\prime}$-Bis(benzyliden)ethylendiamin. Man erhitzte $18 \mathrm{~h}$ auf $70{ }^{\circ} \mathrm{C}$. Säulenchromatographie an $18 \mathrm{~g}$ Kieselgel (Säule $2.0 \times 15 \mathrm{~cm}$, Pentan $\left./ \mathrm{Et}_{2} \mathrm{O}=1: 1\right)$ ergab $36 \mathrm{mg}(15 \%) \mathbf{5 8}$ als farblose Kristalle, Schmp. 128-129 ${ }^{\circ} \mathrm{C}, R_{\mathrm{f}}\left(\mathrm{Pentan} / \mathrm{Et}_{2} \mathrm{O} 1: 1\right)=0.23 .-\mathrm{IR}(\mathrm{KBr}): \mathrm{v}=3513(\mathrm{OH}), 2974,2956,2886$, 2834, 1707 (C=O), 1475, 1361, 1257, 1161, 1057, 1039. - ${ }^{1} \mathrm{H}-\mathrm{NMR}\left(250 \mathrm{MHz}, \mathrm{CDCl}_{3}\right)$ : $\delta=1.03\left(\mathrm{~s}, 3 \mathrm{H}, \mathrm{CH}_{3}\right), 1.05\left(\mathrm{~s}, 3 \mathrm{H}, \mathrm{CH}_{3}\right), 1.27\left(\mathrm{~d},{ }^{3} \mathrm{~J}=7.4 \mathrm{~Hz}, 1 \mathrm{H}, \mathrm{OH}\right), 1.92-2.28(\mathrm{~m}$, $8 \mathrm{H}, 1,5,6,7-\mathrm{H}), 2.64-2.79(\mathrm{~m}, 2 \mathrm{H}, 4 \mathrm{a}, 9 \mathrm{~b}-\mathrm{H}), 4.06\left(\mathrm{~d},{ }^{3} J=7.4 \mathrm{~Hz}, 1 \mathrm{H}, 9-\mathrm{H}\right), 4.28$ (dt, $\left.{ }^{2} J=3.6,{ }^{3} J=10.4 \mathrm{~Hz}, 1 \mathrm{H}, 2-\mathrm{H}\right), 4.38-4.46(\mathrm{~m}, 1 \mathrm{H}, 2-\mathrm{H}) .-{ }^{13} \mathrm{C}-\mathrm{NMR}(62.9 \mathrm{MHz}$, $\left.\mathrm{CDCl}_{3}, \mathrm{DEPT}\right): \delta=22.56\left(+, \mathrm{CH}_{3}\right), 24.27\left(-, \mathrm{C}-1^{*}\right), 25.04\left(-, \mathrm{C}-5^{*}\right), 26.87(-, \mathrm{C}-6 *)$, $28.43\left(+, \mathrm{CH}_{3}\right), 33.43\left(+, \mathrm{C}-9 \mathrm{~b}^{* *}\right), 40.56\left(+, \mathrm{C}-4 \mathrm{a}^{* *}\right), 41.34\left(\mathrm{C}_{\text {quart }}, \mathrm{C}-8\right), 48.40(-, \mathrm{C}-7 *)$, 69.05 (-, C-2), 87.39 (+, C-9), $134.81\left(\mathrm{C}_{\text {quart }}, \mathrm{C}-9 \mathrm{a}^{* * *}\right), 141.25$ (C $\left.\mathrm{C}_{\text {quart }}, \mathrm{C}-6 \mathrm{a}^{* * *}\right), 174.15$ $\left(\mathrm{C}_{\text {quart }}, \mathrm{C}-4\right)$. - MS (EI, $\left.70 \mathrm{eV}\right), \mathrm{m} / \mathrm{z}(\%): 236$ (9) $\left[\mathrm{M}^{+}\right], 218$ (45), 203 (100), 175 (19), 145 (33), 131 (28), 91 (36). $-\mathrm{C}_{14} \mathrm{H}_{20} \mathrm{O}_{3}$ (236.3): ber. C 71.16, H 8.53; gef. C 71.07, H 8.37.

\subsection{Heck-Diels-Alder-Reaktionen unter Zusatz von Lewis-Säure}

Allgemeine Arbeitsvorschrift zur Heck-Diels-Alder-Reaktion unter Verwendung von LewisSäuren ( $A A V$ 3): $\mathrm{Zu}$ einer Lösung von $1 \mathrm{mmol}$ des Bromdiens in $10 \mathrm{ml}$ wasserfreiem Acetonitril in einem dickwandigen Schraubdeckelgläschen gibt man $5 \mathrm{~mol} \% \mathrm{Pd}(\mathrm{OAc})_{2}$, $10 \mathrm{~mol} \%$ und $1 \mathrm{mmol} \mathrm{K}_{2} \mathrm{CO}_{3}$. Man entgast das Reaktionsgemisch 5 min, verschließt das Gläschen und erwärmt die angegebene Zeit auf $80^{\circ} \mathrm{C}$. Anschließend kühlt man auf Raumtemp. ab, filtriert über Celite und wäscht mit Acetonitril nach. Die so erhaltene Lösung versetzt man mit 3-5 mmol Dienophil und 1-3 mmol der Lewis-Säure und rührt $1 \mathrm{~d}$ bei Raumtemp. Man entfernt das Lösungsmittel und überschüssiges Dienophil i. Vak. und reinigt das Rohprodukt durch Säulenchromatographie an Kieselgel. 
2,2-Dimethyl-2,4,5,6-tetrahydro-1H-inden-5-carbonsäuremethylester (61): Analog AAV 3

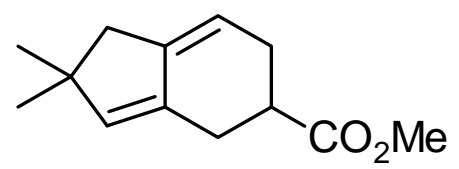

wurde eine Lösung von $219 \mathrm{mg}$ (1.00 mmol) 6-Brom-4,4dimethylhepta-1,6-dien-3-ol in $10 \mathrm{ml}$ wasserfreiem $\mathrm{MeCN}$ mit $11 \mathrm{mg} \quad(0.050 \mathrm{mmol}, \quad 5.0 \mathrm{~mol} \%) \quad \mathrm{Pd}(\mathrm{OAc})_{2}, \quad 26 \mathrm{mg}$ (0.10 mmol, $10 \mathrm{~mol} \%) \mathrm{PPh}_{3}$ und $138 \mathrm{mg} \quad(1.00 \mathrm{mmol})$ $\mathrm{K}_{2} \mathrm{CO}_{3}$ versetzt und $18 \mathrm{~h}$ auf $80^{\circ} \mathrm{C}$ erwärmt. Nach Abkühlung auf Raumtemp. gab man $258 \mathrm{mg}(3.00 \mathrm{mmol})$ Methylacrylat und $3.0 \mathrm{ml}$ (3.0 mmol, $1.0 \mathrm{M}$ in Hexan) Diethylaluminiumchlorid-Lösung $\mathrm{zu}$ und rührte $1 \mathrm{~d}$ bei Raumtemp. Man hydrolysierte überschüssiges Diethylaluminiumchlorid mit $5 \mathrm{ml}$ Wasser, extrahierte die wäßrige Phase mit $2 \times 10 \mathrm{ml}$ Ether, trocknete die vereinigten organischen Phasen über $\mathrm{MgSO}_{4}$ und entfernte das Lösungsmittel i. Vak. Das Rohprodukt wurde durch Säulenchromatographie an $18 \mathrm{~g}$ Kieselgel (Säule $2.0 \times 15 \mathrm{~cm}, \quad P e n t a n / \mathrm{Et}_{2} \mathrm{O}=4: 1$ ) gereinigt, man erhielt Fraktion I: $53 \mathrm{mg}(26 \%) \mathbf{6 1}$ als farbloses Öl, $R_{\mathrm{f}}\left(\operatorname{Pentan}_{\mathrm{Et}} \mathrm{O}\right.$, $4: 1)=0.64 .-$ IR $($ Film $): v=3055 \mathrm{~cm}^{-1}(\mathrm{OH}), 2956,1734(\mathrm{C}=\mathrm{O}), 1437,1367,1267$, 1199, 1106, 1027, 919, 737. - ${ }^{1} \mathrm{H}-\mathrm{NMR}\left(250 \mathrm{MHz}, \mathrm{CDCl}_{3}\right): \delta=1.06$ (s, $\left.6 \mathrm{H}, 2-\mathrm{CH}_{3}\right)$, 2.26-2.78 (m, $7 \mathrm{H}, 1,4,5,6-\mathrm{H}), 3.68\left(\mathrm{~s}, 3 \mathrm{H}, \mathrm{CO}_{2} \mathrm{CH}_{3}\right), 5.35-5.37\left(\mathrm{~m}, 1 \mathrm{H}, 3-\mathrm{H}^{*}\right), 5.45$ (br s, $\left.1 \mathrm{H}, 7-\mathrm{H}^{*}\right) .-{ }^{13} \mathrm{C}-\mathrm{NMR}\left(62.9 \mathrm{MHz}, \mathrm{CDCl}_{3}, \mathrm{DEPT}\right): \delta=27.49\left(-, \mathrm{C}-4^{*}\right), 27.94(-$, C-6*), $29.15\left(+, 2-\mathrm{CH}_{3}\right), 29.21\left(+, 2-\mathrm{CH}_{3}\right), 39.83(+, \mathrm{C}-5), 43.45\left(\mathrm{C}_{\text {quart }}, \mathrm{C}-2\right), 43.73(-$, $\mathrm{C}-1), 51.67\left(+, \mathrm{CO}_{2} \mathrm{CH}_{3}\right), 113.19$ (+, C-3*), 136.28 (C $\left.\mathrm{Cuart}_{\text {, }}, \mathrm{C}-3 \mathrm{a} * *\right), 139.85$ (+, C-7*), $144.82\left(\mathrm{C}_{\text {quart }}, \mathrm{C}-7 \mathrm{a} * *\right), 175.95\left(\mathrm{C}_{\text {quart }}, \mathrm{CO}_{2} \mathrm{CH}_{3}\right) .-\mathrm{MS}\left(\mathrm{CI}, \mathrm{NH}_{3}, 70 \mathrm{eV}\right), \mathrm{m} / z(\%): 224$ (96) $\left[\mathrm{M}+\mathrm{NH}_{4}^{+}\right], 207(100)\left[\mathrm{M}+\mathrm{H}^{+}\right] .-\mathrm{C}_{13} \mathrm{H}_{18} \mathrm{O}_{2}$ (206.3): ber. C 75.69, H 8.80; gef. C 75.81, H 8.98.

Fraktion II: $61 \mathrm{mg}(28 \%)$ Ausgangsmaterial.

6,6-Dimethyl-3,3a,5,6,8,8a-hexahydro-2H-s-indacen-1-on (65): Variante A: Nach AAV 3 gab man zu $219 \mathrm{mg}$ (1.00 mmol) 6-Brom-4,4-dimethylhepta-1,6-dien-3-ol in $10 \mathrm{ml}$ wasserfreiem $\mathrm{MeCN} 11 \mathrm{mg}(0.050 \mathrm{mmol}, 5.0 \mathrm{~mol} \%) \mathrm{Pd}(\mathrm{OAc})_{2}, 26 \mathrm{mg}(0.10 \mathrm{mmol}$, $10 \mathrm{~mol} \%) \mathrm{PPh}_{3}, 138 \mathrm{mg}(1.00 \mathrm{mmol}) \mathrm{K}_{2} \mathrm{CO}_{3}$. Anschließend erwärmte man $18 \mathrm{~h}$ auf $80{ }^{\circ} \mathrm{C}$, kühlte auf Raumtemp. ab und versetzte mit $410 \mathrm{mg}$ (4.99 mmol) Cyclopentenon und $94 \mathrm{mg}$ $(1.0 \mathrm{mmol}) \mathrm{LiBF}_{4}$. Man rührte $14 \mathrm{~d}$ bei Raumtemp. bis dünnschichtchromatographisch kein Ausgangsmaterial mehr nachweisbar war. Das Lösungsmittel und überschüssiges Dienophil wurde i. Vak. entfernt. Das Rohprodukt reinigte man durch 
Säulenchromatographie an $18 \mathrm{~g}$ Kieselgel (Säule $2.0 \times 15 \mathrm{~cm}, \mathrm{Pentan} / \mathrm{Et}_{2} \mathrm{O}=4: 1$ ) und erhielt $51 \mathrm{mg}(25 \%) 65$ als farbloses Öl, $R_{\mathrm{f}}\left(\operatorname{Pentan} / \mathrm{Et}_{2} \mathrm{O}, 4: 1\right)=0.46$. - IR (Film):

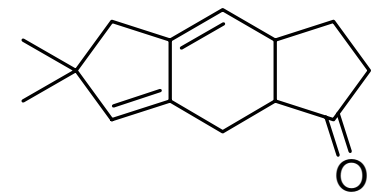
$\mathrm{v}=3058 \mathrm{~cm}^{-1}, 2961,1737(\mathrm{C}=\mathrm{O}), 1650,1434,1406,1267$, 1172, 1034, 919, 737, 703. - ${ }^{1} \mathrm{H}-\mathrm{NMR}\left(250 \mathrm{MHz}, \mathrm{CDCl}_{3}\right)$ : $\delta=0.99\left(\mathrm{~s}, 3 \mathrm{H}, 6-\mathrm{CH}_{3}\right), 1.04\left(\mathrm{~s}, 3 \mathrm{H}, 6-\mathrm{CH}_{3}\right), 1.84-1.91(\mathrm{~m}$, $1 \mathrm{H}, \quad 3-\mathrm{H}), \quad 2.01-2.40 \quad(\mathrm{~m}, \quad 7 \mathrm{H}, \quad 2,3,5,8,8 \mathrm{a}-\mathrm{H}), 2.83 \quad(\mathrm{~d}$, $\left.{ }^{3} J=12.8 \mathrm{~Hz}, 1 \mathrm{H}, 8-\mathrm{H}\right), 3.07$ (br s, $\left.1 \mathrm{H}, 3 \mathrm{a}-\mathrm{H}\right), 5.19$ (br s, $\left.1 \mathrm{H}, 4-\mathrm{H}\right), 5.51$ (br s, $1 \mathrm{H}, 7-\mathrm{H}$ ). - ${ }^{13} \mathrm{C}-\mathrm{NMR}\left(62.9 \mathrm{MHz}, \mathrm{CDCl}_{3}, \mathrm{DEPT}\right): \delta=21.76(-, \mathrm{C}-8), 27.52(-, \mathrm{C}-3), 29.01(+$, 6- $\left.\mathrm{CH}_{3}\right), 29.04\left(+, 6-\mathrm{CH}_{3}\right), 34.82$ (-, C-2), $43.02\left(\mathrm{C}_{\text {quart }}, \mathrm{C}-6\right), 44.18$ (-, C-5), 47.98 (+, C-8a), 116.99 (+, C-4), 133.76 (C quart, $\mathrm{C}-4 \mathrm{a}), 141.32$ (+, C-7), 147.44 (C $\left.\mathrm{C}_{\text {quart }}, \mathrm{C}-7 \mathrm{a}\right)$, $219.80\left(\mathrm{C}_{\text {quart }}, \mathrm{C}-1\right)$. - MS (EI, $\left.70 \mathrm{eV}\right), \mathrm{m} / \mathrm{z}(\%): 202$ (46) [M+], $187(52)\left[\mathrm{M}^{+}-\mathrm{CH}_{3}\right], 143$ (100), 131 (37), 115 (16), 91 (23), 77 (9), 41 (10). - $\mathrm{C}_{14} \mathrm{H}_{18} \mathrm{O}$ (202.3).

Variante B: Nachdem man die Heck-Reaktion unter den in AAV 3 beschriebenen Bedingungen durchgeführt hatte, versetzte man das so erhaltene exocyclische Dien mit einer Lösung aus $410 \mathrm{mg}(4.99 \mathrm{mmol})$ Cyclopentenon und $3.0 \mathrm{ml}(3.0 \mathrm{mmol}, 1.0 \mathrm{M}$ in Hexan) Diethylaluminiumchloridlösung, die man vorher 10-15 min gerührt hatte. Man ließ bei Raumtemp. $18 \mathrm{~h}$ rühren, hydrolysierte mit $15 \mathrm{ml}$ Wasser und extrahierte die wäßrige Phase mit $3 \times 20 \mathrm{ml}$ Ether. Nach Trocknung über $\mathrm{MgSO}_{4}$ entfernte man das Lösungsmittel i. Vak. und reinigte das Rohprodukt durch Säulenchromatographie an 18 g Kieselgel (Säule $2.0 \times 15 \mathrm{~cm}$, Pentan/ $\left./ \mathrm{Et}_{2} \mathrm{O}=4: 1\right)$. Man erhielt $45 \mathrm{mg}(22 \%) 65$ als farbloses Öl, $R_{\mathrm{f}}$ $\left(\right.$ Pentan $\left./ \mathrm{Et}_{2} \mathrm{O}, 4: 1\right)=0.46$

Variante $C$ : Verwendete man als Lewis-Säure $1 \mathrm{ml} \mathrm{BF}_{3} \cdot \mathrm{Et}_{2} \mathrm{O}$, so erhielt man $18 \mathrm{mg}(13 \%)$ 2,3,5,5-Tetramethylcyclopent-2-en-1-on. - ${ }^{1} \mathrm{H}-\mathrm{NMR}\left(250 \mathrm{MHz}, \mathrm{CDCl}_{3}\right): \delta=1.07$ (s, $6 \mathrm{H}, 5-\mathrm{CH}_{3}$ ), 1.68 (br s, $3 \mathrm{H}, 3-\mathrm{CH}_{3}$ ), 2.00 (br s, $3 \mathrm{H}, 2-\mathrm{CH}_{3}$ ), 2.35 (br s, $2 \mathrm{H}, 4-\mathrm{H}$ ). ${ }^{13} \mathrm{C}-\mathrm{NMR}\left(62.9 \mathrm{MHz}, \mathrm{CDCl}_{3}\right.$, DEPT): $\delta=8.14\left(+, 3-\mathrm{CH}_{3} *\right), 16.98\left(+, 2-\mathrm{CH}_{3} *\right), 25.13(+$, 5- $\left.\mathrm{CH}_{3}\right), 42.71\left(\mathrm{C}_{\text {quart }}, \mathrm{C}-5\right), 48.68(-, \mathrm{C}-4), 133.40\left(\mathrm{C}_{\text {quart }}, \mathrm{C}-3 * *\right), 166.58\left(\mathrm{C}_{\text {quart }}, \mathrm{C}-2^{* *}\right)$, $214.07\left(\mathrm{C}_{\text {quart }}, \mathrm{C}-1\right) .-\mathrm{MS}(\mathrm{EI}, 70 \mathrm{eV}), \mathrm{m} / \mathrm{z}(\%): 138(18)\left[\mathrm{M}^{+}\right], 123(59)\left[\mathrm{M}^{+}-\mathrm{CH}_{3}\right], 83$ (46), 49 (100). $-\mathrm{C}_{9} \mathrm{H}_{14} \mathrm{O}$ (138.2).

Variante D: Verwendete man anstelle von Diethylaluminiumchlorid-Lösung $1.50 \mathrm{ml}$ (3.00 mmol, $2 \mathrm{M}$ in Toluol) Trimethylaluminium-Lösung, fand keine Diels-Alder-Reaktion statt. 
Variante E: Der Einsatz von $853 \mathrm{mg}(3.00 \mathrm{mmol}) \mathrm{Ti}(\mathrm{O} i \mathrm{Pr})_{4}$ als Lewis-Säure ergab nur Zersetzungsprodukte.

Variante F: Verwendete man nur $1.5 \mathrm{ml}$ (1.5 mmol, $1.0 \mathrm{M}$ in Hexan) Diethylaluminiumchloridlösung, so konnte man 13 mg (6\%) 65 und 24 mg (17\%) 60 isolieren.

\subsection{Darstellung heteroatomhaltiger Cyclisierungsvorläufer}

Allgemeine Arbeitsvorschrift zur Alkylierung von Benzylamin (AAV 4): Variante A: Man versetzt $100 \mathrm{mmol}$ Benzylamin in $50 \mathrm{ml}$ Ether mit $50 \mathrm{mmol}$ Triethylamin und $50 \mathrm{mmol}$ des Alkylbromids. Es wird die angegebene Zeit bei Raumtemp. gerührt und zur Aufarbeitung mit $30 \mathrm{ml}$ Wasser versetzt. Die wäßrige Phase wird mit $3 \times 30 \mathrm{ml}$ Ether extrahiert und die vereinigten organischen Phasen über $\mathrm{Na}_{2} \mathrm{SO}_{4}$ getrocknet. Nach Entfernen des Lösungsmittels i. Vak. wird das Rohprodukt säulenchromatographisch an Kieselgel gereinigt.

Variante B: Eine Lösung von $35 \mathrm{mmol}$ Benzylamin in $100 \mathrm{ml}$ DMF wird mit $70 \mathrm{mmol} \mathrm{NaI}$, 70 mmol $\mathrm{K}_{2} \mathrm{CO}_{3}$ und $35 \mathrm{mmol}$ des Alkylbromids versetzt. Anschließend erhitzt man die angegebene Zeit auf $100{ }^{\circ} \mathrm{C}$. Nach Abkühlen auf Raumtemp. gibt man $100 \mathrm{ml}$ Wasser zu und extrahiert die wäßrige Phase mit $3 \times 80 \mathrm{ml}$ Ether. Man trocknet die vereinigten organischen Phasen über $\mathrm{MgSO}_{4}$. Nach Entfernen des Lösungsmittels i. Vak. wird das Rohprodukt säulenchromatographisch an Kieselgel gereinigt.

$N$-Allyl-N-prop-2-inyl-N-(p-tosyl)amin (73) und N-Allenyl-N-allyl-N-(p-tosyl)amin (72): Zu

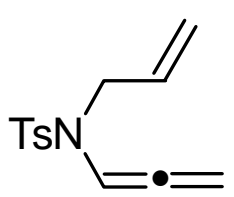
einer Suspension von $0.601 \mathrm{~g}$ (15.0 mmol, 60proz. in Mineralöl) $\mathrm{NaH}$ in $20 \mathrm{ml}$ wasserfreiem DME gab man bei $0{ }^{\circ} \mathrm{C}$ tropfenweise $1.40 \mathrm{~g}$ (11.8 mmol) Propargylbromid und $2.11 \mathrm{~g}$ (9.99 mmol) $N$-Allyl- $N-(p-$ tosyl)amin. Nach Erwärmen auf Raumtemp. rührte man 18 h. Zur Aufarbeitung gab man $20 \mathrm{ml}$ Wasser und $20 \mathrm{ml}$ Ether zu und extrahierte die wäßrige Phase mit $3 \times 20 \mathrm{ml}$ Ether. Man trocknete über $\mathrm{Na}_{2} \mathrm{SO}_{4}$ und engte i. Vak. ein. Das Rohprodukt wurde durch Säulenchromatographie an $30 \mathrm{~g}$ Kieselgel (Säule $2.0 \times 20 \mathrm{~cm}$, Pentan $/ \mathrm{CH}_{2} \mathrm{Cl}_{2}=4: 1$, nach Durchlaufen der 1. Fraktion Wechsel zu $1: 1$ ) gereingt. Man isolierte Fraktion I: $478 \mathrm{mg}(19 \%) 72$ als farbloses Öl, $R_{\mathrm{f}}\left(\mathrm{CH}_{2} \mathrm{Cl}_{2}\right)=0.73 .-{ }^{1} \mathrm{H}-\mathrm{NMR}$ 
$\left(250 \mathrm{MHz}, \mathrm{CDCl}_{3}\right): \delta=2.43\left(\mathrm{~s}, 3 \mathrm{H}, \mathrm{CH}_{3}\right), 3.79\left(\mathrm{~d},{ }^{3} \mathrm{~J}=6.4 \mathrm{~Hz}, 2 \mathrm{H}, 1-\mathrm{H}\right), 5.09-5.22(\mathrm{~m}$, $2 \mathrm{H}, 3-\mathrm{H}), 5.29\left(\mathrm{~d},{ }^{4} \mathrm{~J}=6.8 \mathrm{~Hz}, 2 \mathrm{H}, 3^{\prime}-\mathrm{H}\right), 5.77\left(\mathrm{~m}_{\mathrm{c}}, 1 \mathrm{H}, 2-\mathrm{H}\right), 6.81\left(\mathrm{t},{ }^{4} \mathrm{~J}=6.8 \mathrm{~Hz}, 1 \mathrm{H}\right.$, $\left.1^{\prime}-\mathrm{H}\right), 7.31\left(\mathrm{~d},{ }^{3} \mathrm{~J}=8.3 \mathrm{~Hz}, 2 \mathrm{H}, \mathrm{Ph}-\mathrm{H}\right), 7.75\left(\mathrm{~d},{ }^{3} \mathrm{~J}=8.3 \mathrm{~Hz}, 2 \mathrm{H}, \mathrm{Ph}-\mathrm{H}\right) .-{ }^{13} \mathrm{C}-\mathrm{NMR}$ (62.9 MHz, $\left.\mathrm{CDCl}_{3}, \mathrm{DEPT}\right): \delta=21.55\left(+, \mathrm{CH}_{3}\right), 48.79$ (-, C-1), 87.89 (-, C-3'), $99.88(+$, C-1'), 118.13 (-, C-3), 127.20 (+, Ph-C), 129.68 (+, Ph-C), 132.31 ( $\mathrm{C}_{\text {quart }}$, Ph-C), 135.45 (+, C-2), $143.73\left(\mathrm{C}_{\text {quart }}, \mathrm{Ph}-\mathrm{C}\right)$. - MS (EI, $\left.70 \mathrm{eV}\right), \mathrm{m} / z(\%): 249$ (3) $\left[\mathrm{M}^{+}\right], 221$ (4), 184 (12) $\left[\mathrm{M}^{+}-\mathrm{C}_{5} \mathrm{H}_{5}\right], 155$ (13) $\left[\mathrm{C}_{7} \mathrm{H}_{7} \mathrm{SO}_{2}^{+}\right], 139$ (35) $\left[\mathrm{C}_{7} \mathrm{H}_{7} \mathrm{SO}^{+}\right], 105$ (26) $\left[\mathrm{C}_{8} \mathrm{H}_{9}{ }^{+}\right], 94$ (100) $\left[\mathrm{M}^{+}-\mathrm{C}_{7} \mathrm{H}_{7} \mathrm{SO}_{2}\right], 91(79)\left[\mathrm{C}_{7} \mathrm{H}_{7}{ }^{+}\right], 67$ (56), 65 (26) $\left[\mathrm{C}_{5} \mathrm{H}_{5}^{+}\right], 41(39)\left[\mathrm{C}_{3} \mathrm{H}_{5}{ }^{+}\right]$.

Fraktion II: $1.21 \mathrm{~g}(49 \%) 73$ als farbloses Öl, $R_{\mathrm{f}}\left(\mathrm{CH}_{2} \mathrm{Cl}_{2}\right)=0.73 .-{ }^{1} \mathrm{H}-\mathrm{NMR}(250 \mathrm{MHz}$, $\left.\mathrm{CDCl}_{3}\right): \delta=2.00\left(\mathrm{t},{ }^{4} \mathrm{~J}=2.4 \mathrm{~Hz}, 1 \mathrm{H}, 3-\mathrm{H}\right), 2.43\left(\mathrm{~s}, 3 \mathrm{H}, \mathrm{CH}_{3}\right), 3.82$

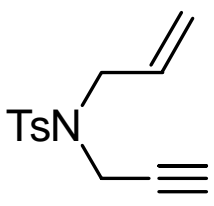
$\left(\mathrm{d},{ }^{3} J=6.5 \mathrm{~Hz}, 2 \mathrm{H}, 1^{\prime}-\mathrm{H}\right), 4.09\left(\mathrm{~d},{ }^{4} J=2.4 \mathrm{~Hz}, 2 \mathrm{H}, 1-\mathrm{H}\right), 5.22-5.33$ (m, 2 H, 3'-H), 5.73 (m, 1 H, 2'-H), 7.30 (d, $\left.{ }^{3} J=8.0 \mathrm{~Hz}, 2 \mathrm{H}, \mathrm{Ph}-\mathrm{H}\right)$, $7.73\left(\mathrm{~d},{ }^{3} \mathrm{~J}=8.0 \mathrm{~Hz}, 2 \mathrm{H}, \mathrm{Ph}-\mathrm{H}\right) .-{ }^{13} \mathrm{C}-\mathrm{NMR}\left(62.9 \mathrm{MHz}, \mathrm{CDCl}_{3}\right.$, DEPT): $\delta=21.49\left(+, \mathrm{CH}_{3}\right), 35.66(-, \mathrm{C}-1), 48.79\left(-, \mathrm{C}-1{ }^{\prime}\right), 73.70(+$,

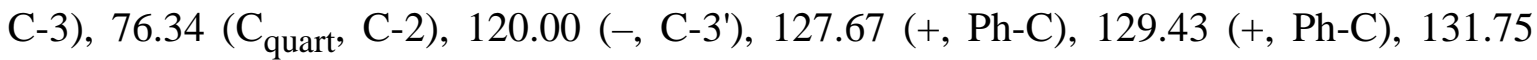
$\left(\mathrm{C}_{\text {quart }}, \mathrm{Ph}-\mathrm{C}\right), 135.82$ (+, C-2'), $143.54\left(\mathrm{C}_{\text {quart }}, \mathrm{Ph}-\mathrm{C}\right) .-\mathrm{MS}$ (EI, $\left.70 \mathrm{eV}\right), \mathrm{m} / \mathrm{z}(\%): 249$ (7) $\left[\mathrm{M}^{+}\right], 222(6), 184(8)\left[\mathrm{M}^{+}-\mathrm{C}_{5} \mathrm{H}_{5}\right], 155(28)\left[\mathrm{C}_{7} \mathrm{H}_{7} \mathrm{SO}_{2}{ }^{+}\right], 139(7)\left[\mathrm{C}_{7} \mathrm{H}_{7} \mathrm{SO}^{+}\right], 94$ (100) $\left[\mathrm{M}^{+}-\mathrm{C}_{7} \mathrm{H}_{7} \mathrm{SO}_{2}\right], 91$ (83) $\left[\mathrm{C}_{7} \mathrm{H}_{7}^{+}\right], 67$ (4), 65 (5) $\left[\mathrm{C}_{5} \mathrm{H}_{5}^{+}\right], 41$ (2) $\left[\mathrm{C}_{3} \mathrm{H}_{5}^{+}\right]$. $\mathrm{C}_{13} \mathrm{H}_{15} \mathrm{NO}_{2} \mathrm{~S}(249.3)$.

N-Benzyl-N-(3-brombut-3-enyl)amin (80): Nach AAV 4, Variante B gab man zu $2.68 \mathrm{~g}$ $(25.0 \mathrm{mmol})$ Benzylamin in $50 \mathrm{ml}$ DMF $7.49 \mathrm{~g}(50.0 \mathrm{mmol}) \mathrm{NaI}, 6.91 \mathrm{~g}(50.0 \mathrm{mmol})$ $\mathrm{K}_{2} \mathrm{CO}_{3}$ und $5.24 \mathrm{~g}$ (24.5 mmol) 2,4-Dibrombut-1-en. Nachdem man $18 \mathrm{~h}$ auf $100{ }^{\circ} \mathrm{C}$ erhitzte, arbeitete man auf und reinigte das Rohprodukt durch Säulenchromatographie an 50 g Kieselgel (Säule $2.5 \times 20 \mathrm{~cm}$, Pentan/ $\mathrm{Et}_{2} \mathrm{O}=4: 1$, nach Durchlaufen der 1. Fraktion Wechsel zu Et ${ }_{2} \mathrm{O}$ ). Man erhielt Fraktion I: 237 mg einer nicht identifizierten Substanz. Fraktion II: $2.44 \mathrm{~g}(42 \%) \mathbf{8 0}$ als farbloses Öl, $R_{\mathrm{f}}\left(\mathrm{Et}_{2} \mathrm{O}\right)=0.45 .-{ }^{1} \mathrm{H}-\mathrm{NMR}(250 \mathrm{MHz}$, $\left.\mathrm{CDCl}_{3}\right): \delta=2.64\left(\mathrm{t},{ }^{3} \mathrm{~J}=6.3 \mathrm{~Hz}, 2 \mathrm{H}, 2-\mathrm{H}\right), 2.85\left(\mathrm{t},{ }^{3} \mathrm{~J}=6.3 \mathrm{~Hz}\right.$,<smiles>C=C(Br)CCNc1ccccc1</smiles>
$2 \mathrm{H}, 1-\mathrm{H}), 3.82\left(\mathrm{~s}, 2 \mathrm{H}, \mathrm{PhCH}_{2}\right), 5.48(\mathrm{~d}, 2 \mathrm{~J}=1.5 \mathrm{~Hz}, 1 \mathrm{H}, 4-\mathrm{H})$, 5.66 (br s, $1 \mathrm{H}, 4-\mathrm{H}), 7.27-7.34(\mathrm{~m}, 5 \mathrm{H}, \mathrm{Ph}-\mathrm{H}) .-{ }^{13} \mathrm{C}-\mathrm{NMR}$ $\left(62.9 \mathrm{MHz}, \mathrm{CDCl}_{3}, \mathrm{DEPT}\right): \delta=41.65(-, \mathrm{C}-2), 46.68(-, \mathrm{C}-1)$, $53.70\left(-, \mathrm{PhCH}_{2}\right), 118.34$ (-, C-4), 126.96 (+, Ph-C), 128.06 (+, 
Ph-C), 128.39 (+, Ph-C), 132.10 ( $\left.\mathrm{C}_{\text {quart }}, \mathrm{C}-3\right), 140.04\left(\mathrm{C}_{\text {quart }}, \mathrm{Ph}-\mathrm{C}^{*}\right) .-\mathrm{C}_{11} \mathrm{H}_{14} \mathrm{BrN}$ (240.1).

N-Benzyl-N-(3'-brombut-3'-en)prop-1-en-2-carbonsäuremethylester-1-amin $\quad(\mathbf{8 2})$ : $\quad \mathrm{Zu}$

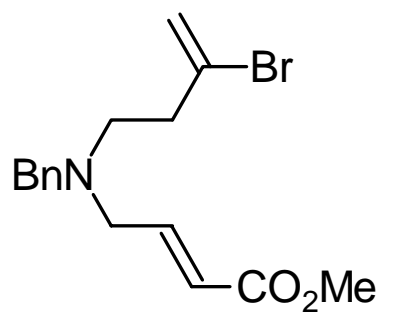

$2.30 \mathrm{~g}$ (9.58 mmol) $N$-Benzyl- $N$-(3-brombut-3-enyl)amin in $50 \mathrm{ml}$ wasserfreiem $\mathrm{Et}_{2} \mathrm{O}$ gab man unter Eiskühlung langsam $1.26 \mathrm{~g}$ (15.0 mmol) Propiolsäuremethylester und rührte $2 \mathrm{~d}$ bei Raumtemp. Anschließend entfernte man das Lösungsmittel i. Vak. und reinigte das Rohprodukt durch Säulenchromatographie an $25 \mathrm{~g}$ Kieselgel (Säule $2.5 \times 10 \mathrm{~cm}, \mathrm{Et}_{2} \mathrm{O}$ ). Man erhielt $2.93 \mathrm{~g}$ $(94 \%) 82$ als farbloses Öl, $R_{\mathrm{f}}\left(\mathrm{Et}_{2} \mathrm{O}\right)=0.76$. - IR (Film): v = $3298 \mathrm{~cm}^{-1}, 3029,2946,1689$, 1611 1454, 1421, 1355, 1300, 1256, 1151, 1042, 977, 896, 792, 723, 697. - ${ }^{1} \mathrm{H}-\mathrm{NMR}$ $\left(250 \mathrm{MHz}, \mathrm{CDCl}_{3}\right): \delta=2.61\left(\mathrm{t},{ }^{3} \mathrm{~J}=6.8 \mathrm{~Hz}, 2 \mathrm{H}, 2^{\prime}-\mathrm{H}\right), 3.34-3.39\left(\mathrm{~m}, 2 \mathrm{H}, 1^{\prime}-\mathrm{H}\right), 3.67(\mathrm{~s}$, $\left.3 \mathrm{H}, \mathrm{CO}_{2} \mathrm{CH}_{3}\right), 4.36\left(\mathrm{~s}, 2 \mathrm{H}, \mathrm{PhCH}_{2}\right), 4.69(\mathrm{~d}, 3 J=13.2 \mathrm{~Hz}, 1 \mathrm{H}, 2-\mathrm{H}), 5.47(\mathrm{~d}$, $\left.2 J=1.8 \mathrm{~Hz}, 1 \mathrm{H}, 4^{\prime}-\mathrm{H}\right), 5.60\left(\mathrm{~m}_{\mathrm{c}}, 1 \mathrm{H}, 4{ }^{\prime}-\mathrm{H}\right), 7.18-7.22$ (m, $\left.2 \mathrm{H}, \mathrm{Ph}-\mathrm{H}\right), 7.29-7.37$ (m, $3 \mathrm{H}$, $\mathrm{Ph}-\mathrm{H}), 7.62\left(\mathrm{~d},{ }^{3} \mathrm{~J}=13.2 \mathrm{~Hz}, 1 \mathrm{H}, 1-\mathrm{H}\right) .-{ }^{13} \mathrm{C}-\mathrm{NMR}\left(50.3 \mathrm{MHz}, \mathrm{C}_{6} \mathrm{D}_{6}, \mathrm{APT}, 70{ }^{\circ} \mathrm{C}\right)$ : $\delta=39.17\left(-, \mathrm{C}-2^{\prime}\right), 49.37\left(-, \mathrm{PhCH}_{2}^{*}\right), 50.12\left(+, \mathrm{CO}_{2} \mathrm{CH}_{3}\right), 56.68$ (-, C-1'*), 86.88 (+, C-2), 118.90 (-, C-4'), 127.67 (+, Ph-C), 127.86 (+, Ph-C), 128.86 (+, Ph-C), 130.71 (-, C-3'), 137.00 (-, Ph-C), 151.49 (+, C-1), 169.15 (-, $\left.\mathrm{CO}_{2} \mathrm{CH}_{3}\right)$. - MS (EI, $\left.70 \mathrm{eV}\right), \mathrm{m} / z(\%)$ :

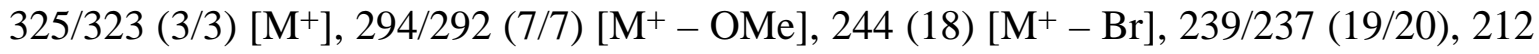
(8) $\left[\mathrm{M}^{+}-\mathrm{MeOH}-\mathrm{Br}\right], 204$ (15), 184 (7) $\left[\mathrm{M}^{+}-\mathrm{CO}_{2} \mathrm{Me}-\mathrm{HBr}\right], 91$ (100) $\left[\mathrm{C}_{7} \mathrm{H}_{7}{ }^{+}\right]$. $\mathrm{C}_{15} \mathrm{H}_{18} \mathrm{BrNO}_{2}$ (324.2).

N-Benzyl-N-(but-3'-in)prop-1-en-2-carbonsäuremethylester-1-amin (85): Man löste $1.27 \mathrm{~g}$

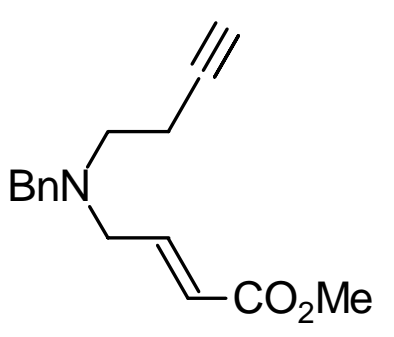

(7.98 mmol) $\quad N$-Benzyl- $N$-but-3-inylamin $\quad$ in $50 \mathrm{ml}$ wasserfreiem $\mathrm{Et}_{2} \mathrm{O}$ und gab unter Eiskühlung langsam $1.26 \mathrm{~g} \quad(15.0 \mathrm{mmol}) \quad$ Propiolsäuremethylester $\mathrm{zu}$. Anschließend rührte man $2 \mathrm{~d}$ bei Raumtemp., entfernte das Lösungsmittel i. Vak. und reinigte das Rohprodukt durch Säulenchromatographie an $25 \mathrm{~g}$ Kieselgel (Säule $2.5 \times 10 \mathrm{~cm}, \mathrm{Et}_{2} \mathrm{O}$ ). Man erhielt $1.81 \mathrm{~g}$ (93\%) 85 als gelben Feststoff, Schmp. 53-54 ${ }^{\circ} \mathrm{C}, R_{\mathrm{f}}\left(\mathrm{Et}_{2} \mathrm{O}\right)=0.60$. $-\mathrm{IR}(\mathrm{KBr})$ : $v=3234 \mathrm{~cm}^{-1}, 2948,1678,1605,1499,1454,1418,1362,1317,1286,1263,1148,1078$, 
1040, 973, 946, 841, 789, 745, 700. - ${ }^{1} \mathrm{H}-\mathrm{NMR}\left(250 \mathrm{MHz}, \mathrm{CDCl}_{3}\right): \delta=2.01(\mathrm{t}$, $\left.{ }^{4} J=2.6 \mathrm{~Hz}, 1 \mathrm{H}, 4^{\prime}-\mathrm{H}\right), 2.40\left(\mathrm{dt},{ }^{3} J=7.1,{ }^{4} J=2.6 \mathrm{~Hz}, 2 \mathrm{H}, 2^{\prime}-\mathrm{H}\right), 3.32\left(\mathrm{br} \mathrm{t},{ }^{3} J=7.1 \mathrm{~Hz}\right.$, $\left.2 \mathrm{H}, 1^{\prime}-\mathrm{H}\right), 3.67$ (s, $\left.3 \mathrm{H}, \mathrm{CO}_{2} \mathrm{CH}_{3}\right), 4.42\left(\mathrm{~s}, 2 \mathrm{H}, \mathrm{PhCH}_{2}\right), 4.70$ (d, $\left.{ }^{3} \mathrm{~J}=13.2 \mathrm{~Hz}, 1 \mathrm{H}, 2-\mathrm{H}\right)$, 7.18-7.22 (m, 2 H, Ph-H), 7.29-7.37 (m, 3 H, Ph-H), 7.63 (d, ${ }^{3} J=13.2$ Hz, 1 H, 1-H). ${ }^{13} \mathrm{C}-\mathrm{NMR}\left(50.3 \mathrm{MHz}, \mathrm{C}_{6} \mathrm{D}_{6}, \mathrm{APT}, 70{ }^{\circ} \mathrm{C}\right): \delta=17.51\left(-, \mathrm{C}-2^{\prime}\right), 50.10\left(+, \mathrm{CO}_{2} \mathrm{CH}_{3}\right), 50.20$ $\left(-, \mathrm{PhCH}_{2}{ }^{*}\right), 55.87$ (-, C-1'*), 70.65 (+, C-4'), 81.16 (-, C-3'), 86.96 (+, C-2), 127.75 (+, Ph-C), 128.29 (+, Ph-C), 128.86 (+, Ph-C), 136.93 (-, Ph-C), 151.55 (+, C-1), 169.15 (-, $\mathrm{CO}_{2} \mathrm{CH}_{3}$ ). - MS (EI, $\left.70 \mathrm{eV}\right), \mathrm{m} / z(\%): 243$ (24) [M+], 212 (13), 204 (14) [ $\left.\mathrm{M}^{+}-\mathrm{C}_{3} \mathrm{H}_{3}\right], 184$ (8) $\left[\mathrm{M}^{+}-\mathrm{CO}_{2} \mathrm{Me}\right], 132$ (6), 91 (100) $\left[\mathrm{C}_{7} \mathrm{H}_{7}{ }^{+}\right], 82$ (5), 65 (7). $-\mathrm{C}_{15} \mathrm{H}_{17} \mathrm{NO}_{2}$ (243.3): ber. C 74.05, H 7.04, N 5.76; C 73.87, H 6.83, N 5.71.

N-Benzyl-N-but-3-inylamin (88): Nach AAV 4, Variante B löste man 3.75 g (35.0 mmol) Benzylamin in $100 \mathrm{ml}$ DMF und versetzte mit $10.5 \mathrm{~g}$ (70.0 mmol)

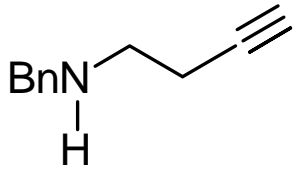
NaI, $9.67 \mathrm{~g}(70.0 \mathrm{mmol}) \mathrm{K}_{2} \mathrm{CO}_{3}$ und $4.66 \mathrm{~g}$ (35.0 mmol) 4Brombutin. Nachdem man $18 \mathrm{~h}$ auf $100{ }^{\circ} \mathrm{C}$ erhitzte, arbeitete man auf und reinigte das Rohprodukt durch Säulenchromatographie an $25 \mathrm{~g}$ Kieselgel (Säule $2.5 \times 10 \mathrm{~cm}, \mathrm{Et}_{2} \mathrm{O}$ ). Man erhielt $1.39 \mathrm{~g}(25 \%) \mathbf{8 8}$ als farbloses Öl, $R_{\mathrm{f}}$ $\left(\mathrm{Et}_{2} \mathrm{O}\right)=0.41 .-{ }^{1} \mathrm{H}-\mathrm{NMR}\left(250 \mathrm{MHz}, \mathrm{CDCl}_{3}\right): \delta=1.63$ (br s, $\left.1 \mathrm{H}, \mathrm{NH}\right), 2.00$ (t, $\left.{ }^{4} J=2.7 \mathrm{~Hz}, 1 \mathrm{H}, 4-\mathrm{H}\right), 2.44\left(\mathrm{dt},{ }^{3} \mathrm{~J}=6.6,{ }^{4} \mathrm{~J}=2.7 \mathrm{~Hz}, 2 \mathrm{H}, 2-\mathrm{H}\right), 2.81\left(\mathrm{t},{ }^{3} \mathrm{~J}=6.6 \mathrm{~Hz}, 2 \mathrm{H}\right.$, 1-H), 3.83 (s, $2 \mathrm{H}, \mathrm{PhCH}_{2}$ ), 7.25-7.34 (m, $\left.5 \mathrm{H}, \mathrm{Ph}-\mathrm{H}\right) .-{ }^{13} \mathrm{C}-\mathrm{NMR}\left(62.9 \mathrm{MHz}, \mathrm{CDCl}_{3}\right.$, DEPT): $\delta=19.50(-, \mathrm{C}-2), 47.25(-, \mathrm{C}-1), 53.32\left(-, \mathrm{PhCH}_{2}\right), 69.50(+, \mathrm{C}-3), 82.43\left(\mathrm{C}_{\text {quart }}\right.$, C-4), 126.94 (+, Ph-C), 128.06 (+, Ph-C), 128.39 (+, Ph-C), 140.04 ( $\left.\mathrm{C}_{\text {quart }}, \mathrm{Ph}-\mathrm{C}\right) .-\mathrm{MS}$ (EI, $70 \mathrm{eV}), m / z(\%): 159$ (1) $\left[\mathrm{M}^{+}\right], 120(87), 91(100)\left[\mathrm{C}_{7} \mathrm{H}_{7}^{+}\right], 65(5) .-\mathrm{C}_{11} \mathrm{H}_{13} \mathrm{~N}$ (159.2).

N-Benzyl-N-2-bromallylamin (90): Analog AAV 4, Variante A gab man zu $21.4 \mathrm{~g}$ ${ }_{\mathrm{Br}}^{\mathrm{BnN}}$ (200 mmol) Benzylamin in $80 \mathrm{ml}$ Ether $10.1 \mathrm{~g}(99.8 \mathrm{mmol})$ Triethylamin und $20.0 \mathrm{~g}$ (100 mmol) Dibrompropen. Nachdem man bei Raumtemp. $15 \mathrm{~h}$ rühren ließ, arbeitete man wäßrig auf. Das Rohprodukt wurde säulenchromatographisch an $100 \mathrm{~g}$ Kieselgel (Säule $4.0 \times 15 \mathrm{~cm}$, 
Pentan/Et $2 \mathrm{O}=5: 1)$ gereinigt. Man isolierte $16.3 \mathrm{~g}(72 \%)$ des Produktes 90 als farbloses Öl, $R_{\mathrm{f}}\left(\mathrm{Pentan}_{\mathrm{Et}} \mathrm{O}, 5: 1\right)=0.23$. Die spektroskopischen Daten stimmen mit der Literatur überein.[47]

N-Benzyl-2-brom-N-vinylsulfonylprop-2-en-1-amin (93): $\mathrm{Zu}$ einer Lösung von $3.26 \mathrm{~g}$

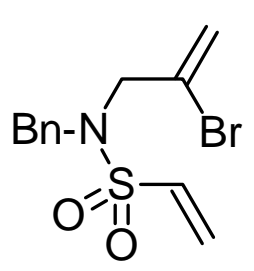
(20.0 mmol) Chlorethylsulfonylchlorid in $30 \mathrm{ml}$ wasserfreiem $\mathrm{CH}_{2} \mathrm{Cl}_{2}$ gab man bei $-60{ }^{\circ} \mathrm{C} 2.02 \mathrm{~g}(20.0 \mathrm{mmol})$ Triethylamin und ließ $30 \mathrm{~min}$ bei dieser Temperatur und $45 \mathrm{~min}$ bei $0{ }^{\circ} \mathrm{C}$ rühren. Man kühlte erneut auf $-60{ }^{\circ} \mathrm{C}$ und versetzte mit $4.52 \mathrm{~g}$ (20.0 mmol) $N$-Benzyl- $N-2-$ bromallylamin und $2.02 \mathrm{~g}(20.0 \mathrm{mmol})$ Triethylamin. Man rührte $1 \mathrm{~h}$ bei $-60{ }^{\circ} \mathrm{C}$, erwärmte auf Raumtemp. und ließ weitere $18 \mathrm{~h}$ bei dieser Temperatur rühren. Zur Aufarbeitung versetzte man mit $0.1 \mathrm{M} \mathrm{HCl}(3 \times 30 \mathrm{ml})$, trocknete die organische Phase über $\mathrm{MgSO}_{4}$ und entfernte das Lösungsmittel i. Vak. Das Rohprodukt wurde durch Säulenchromatographie an $50 \mathrm{~g}$ Kieselgel (Säule $2.5 \times 20 \mathrm{~cm}, \quad$ Pentan/Et $2 \mathrm{O}=5: 1$ ) gereinigt. Man erhielt $5.52 \mathrm{~g}(87 \%) 93$ als farbloses Öl, $R_{\mathrm{f}}\left(\operatorname{Pentan}_{\mathrm{Et}} \mathrm{O} \mathrm{O}, 5: 1\right)=0.26$. $-\mathrm{IR}$ (Film): $v=3063 \mathrm{~cm}^{-1}, 3031,2923,1629,1496,1456,1340,1254,1205,1149,1063,970$, 899, 792, 749, 701. - ${ }^{1} \mathrm{H}-\mathrm{NMR}\left(250 \mathrm{MHz}, \mathrm{CDCl}_{3}\right): \delta=3.99$ (s, $\left.2 \mathrm{H}, 1-\mathrm{H}^{*}\right), 4.41(\mathrm{~s}, 2 \mathrm{H}$, $\left.\mathrm{PhCH}_{2}{ }^{*}\right), 5.66(\mathrm{~d}, 2 J=2.1 \mathrm{~Hz}, 1 \mathrm{H}, 3-\mathrm{H}), 5.79\left(\mathrm{~m}_{\mathrm{c}}, 1 \mathrm{H}, 3-\mathrm{H}\right), 5.94\left(\mathrm{~d},{ }^{3} \mathrm{~J}=9.8 \mathrm{~Hz}, 1 \mathrm{H}\right.$, 2'-H), $6.28\left(\mathrm{~d}, 3 J=16.5 \mathrm{~Hz}, 1 \mathrm{H}, 2^{\prime}-\mathrm{H}\right), 6.57\left(\mathrm{dd}, 3^{3} J=9.8,3^{3} J=16.5 \mathrm{~Hz}, 1 \mathrm{H}, 1^{\prime}-\mathrm{H}\right)$, 7.31-7.36 (m, $5 \mathrm{H}, \mathrm{Ph}-\mathrm{H}) .-{ }^{13} \mathrm{C}-\mathrm{NMR}\left(62.9 \mathrm{MHz}, \mathrm{CDCl}_{3}, \mathrm{DEPT}\right): \delta=49.93\left(-, \mathrm{C}-1^{*}\right)$, $53.47\left(-, \mathrm{PhCH}_{2}\right), 120.90$ (-, C-3), 126.64 (-, C-2'), 127.59 ( $\left.\mathrm{C}_{\text {quart }}, \mathrm{C}-2\right), 128.12$ (+, Ph-C), 128.64 (+, Ph-C), 128.72 (+, Ph-C), 135.07 (C $\left.\mathrm{C}_{\text {quart }}, \mathrm{Ph}-\mathrm{C}\right), 135.97$ (+, C-1'). - MS (EI, $70 \mathrm{eV}), \mathrm{m} / \mathrm{z}(\%): 317 / 315(0.2 / 0.2)\left[\mathrm{M}^{+}\right], 236(53)\left[\mathrm{M}^{+}-\mathrm{Br}\right], 226 / 224(18 / 22)\left[\mathrm{M}^{+}{ }_{-}\right.$ $\left.\mathrm{C}_{7} \mathrm{H}_{7}\right], 144$ (25), 104 (24), 91 (100) $\left[\mathrm{C}_{7} \mathrm{H}_{7}{ }^{+}\right] .-\mathrm{C}_{12} \mathrm{H}_{14} \mathrm{BrNO}_{2} \mathrm{~S}$ (316.2): ber. C 45.58, H 4.46; N 4.43, gef. C 45.61, H 4.56, N 4.24.

N-Benzyl-N-prop-2-in-1-amin (94): Analog AAV 4, Variante A gab man zu $10.7 \mathrm{~g}$

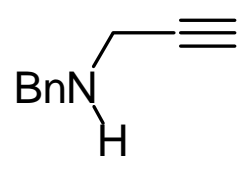
(99.9 mmol) Benzylamin in $50 \mathrm{ml}$ Ether $5.10 \mathrm{~g}(50.4 \mathrm{mmol})$ Triethylamin und $5.95 \mathrm{~g}$ (50.0 mmol) Propargylbromid. Nachdem man bei Raumtemp. $15 \mathrm{~h}$ rühren ließ, arbeitete man wäßrig auf. Das Rohprodukt wurde 
säulenchromatographisch an $50 \mathrm{~g}$ Kieselgel (Säule $3.0 \times 15 \mathrm{~cm}$, Pentan $/ \mathrm{Et}_{2} \mathrm{O}=5: 1$, nach Durchlaufen der 1. Fraktion Wechsel $\mathrm{zu} \mathrm{Et}_{2} \mathrm{O}$ ) gereinigt. Man isolierte Fraktion I: $517 \mathrm{mg}$ $(11 \%)$ dialkyliertes Benzylamin als farbloses Öl, $R_{\mathrm{f}}\left(\operatorname{Pentan}_{\mathrm{Et}} \mathrm{O}, 5: 1\right)=0.50$.

Fraktion II: $3.36 \mathrm{~g}(46 \%)$ des Produktes 94 als farbloses Öl, $R_{\mathrm{f}}\left(\mathrm{Et}_{2} \mathrm{O}\right)=0.56$. Die spektroskopischen Daten stimmen mit der Literatur überein.[100]

N-Benzyl-N-vinylsulfonylprop-2-in-1-amin (95): Zu 3.26 g (20.0 mmol) Chlorethyl-

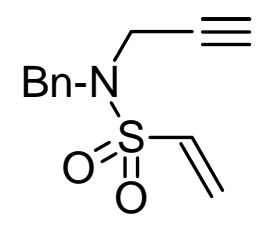
sulfonylchlorid in $30 \mathrm{ml}$ wasserfreiem $\mathrm{CH}_{2} \mathrm{Cl}_{2}$ gab man bei $-60{ }^{\circ} \mathrm{C}$ $2.02 \mathrm{~g}$ (20.0 mmol) Triethylamin. Man ließ $30 \mathrm{~min}$ bei dieser Temperatur und 45 min bei $0{ }^{\circ} \mathrm{C}$ rühren, kühlte erneut auf $-60{ }^{\circ} \mathrm{C}$ und versetzte mit $2.90 \mathrm{~g}$ (20.0 mmol) $N$-Benzyl- $N$-prop-2-inylamin und $2.02 \mathrm{~g}$ (20.0 mmol) Triethylamin. Man rührte $1 \mathrm{~h}$ bei $-60{ }^{\circ} \mathrm{C}$, erwärmte auf Raumtemp. und ließ weitere $18 \mathrm{~h}$ bei dieser Temperatur rühren. Zur Aufarbeitung versetzte man mit $0.1 \mathrm{M} \mathrm{HCl}(3 \times 30 \mathrm{ml})$, trocknete die organische Phase über $\mathrm{MgSO}_{4}$ und entfernte das Lösungsmittel i. Vak. Das Rohprodukt wurde durch Säulenchromatographie an $50 \mathrm{~g}$ Kieselgel (Säule $2.5 \times 20 \mathrm{~cm}, \mathrm{Et}_{2} \mathrm{O}$ ) gereinigt. Man erhielt $3.88 \mathrm{~g}(82 \%) 95$ als farbloses Öl, $R_{\mathrm{f}}\left(\mathrm{Et}_{2} \mathrm{O}\right)=0.74$. - IR (Film): v = $3282 \mathrm{~cm}^{-1}, 3063,3032,2923,1456,1349,1254$, $1152,1061,971,896,779,748,700,650,606 .-{ }^{1} \mathrm{H}-\mathrm{NMR}\left(250 \mathrm{MHz}, \mathrm{CDCl}_{3}\right): \delta=2.39$ $\left(\mathrm{t},{ }^{4} J=2.5 \mathrm{~Hz}, 1 \mathrm{H}, 3-\mathrm{H}\right), 3.91\left(\mathrm{~d},{ }^{4} J=2.5 \mathrm{~Hz}, 2 \mathrm{H}, 1-\mathrm{H}\right), 4.34\left(\mathrm{~s}, 2 \mathrm{H}, \mathrm{PhCH}_{2}\right), 6.04(\mathrm{~d}$, $\left.{ }^{3} J=9.8 \mathrm{~Hz}, 1 \mathrm{H}, 2^{\prime}-\mathrm{H}\right), 6.33\left(\mathrm{~d},{ }^{3} J=16.5 \mathrm{~Hz}, 1 \mathrm{H}, 2^{\prime}-\mathrm{H}\right), 6.64\left(\mathrm{dd},{ }^{3} J=9.8,{ }^{3} J=16.5 \mathrm{~Hz}\right.$, $\left.1 \mathrm{H}, 1^{\prime}-\mathrm{H}\right), 7.33-7.39$ (m, $\left.5 \mathrm{H}, \mathrm{Ph}-\mathrm{H}\right) .-{ }^{13} \mathrm{C}-\mathrm{NMR}\left(62.9 \mathrm{MHz}, \mathrm{CDCl}_{3}, \mathrm{DEPT}\right): \delta=35.41$ (-, C-1), 49.63 (-, $\left.\mathrm{PhCH}_{2}\right), 74.29$ (C $\left.\mathrm{C}_{\text {quart }}, \mathrm{C}-2\right), 77.18$ (+, C-3), 127.70 (-, C-2'), 128.22 (+, Ph-C), 128.73 (+, Ph-C), 128.75 (+, Ph-C), 134.69 (C quart $\left._{\text {, }} \mathrm{Ph}-\mathrm{C}\right), 134.74$ (+, C-1'). - MS

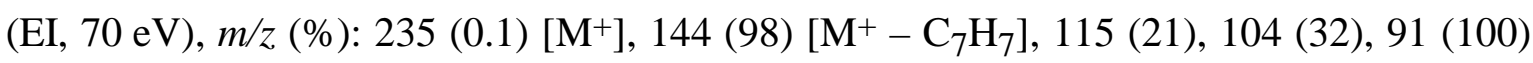
$\left[\mathrm{C}_{7} \mathrm{H}_{7}{ }^{+}\right], 77$ (13), 65 (18), 51 (10). $-\mathrm{C}_{12} \mathrm{H}_{13} \mathrm{NO}_{2} \mathrm{~S}$ (235.3): ber. C 61.25, H 5.57, N 5.95; gef. C 61.51, H 5.61, N 5.82.

N-Benzyl-N-(1'-brom)vinylsulfonylprop-2-en-1-amin (99): $\mathrm{Zu}$ einer Lösung von $3.26 \mathrm{~g}$<smiles>C=CCN(Cc1ccccc1)S(=O)(=O)C(=C)Br</smiles>
(20.0 mmol) Chlorethylsulfonylchlorid in $30 \mathrm{ml}$ wasserfreiem $\mathrm{CH}_{2} \mathrm{Cl}_{2}$ gab man bei $-60{ }^{\circ} \mathrm{C} 2.02 \mathrm{~g}(20.0 \mathrm{mmol})$ Triethylamin und ließ $30 \mathrm{~min}$ bei dieser Temperatur und anschließend $45 \mathrm{~min}$ bei $0{ }^{\circ} \mathrm{C}$ rühren. Dann gab man bei $0{ }^{\circ} \mathrm{C} 3.19 \mathrm{~g}$ (20.0 mmol) Brom 
tropfenweise zu, erwärmte auf Raumtemp. und ließ weitere $18 \mathrm{~h}$ bei dieser Temperatur rühren. Die so erhaltene Dibromsulfonylchlorid-Lösung kühlte man erneut auf $-60{ }^{\circ} \mathrm{C}$, versetzte mit $2.02 \mathrm{~g}(20.0 \mathrm{mmol})$ Triethylamin und rührte $30 \mathrm{~min}$ bei $-60{ }^{\circ} \mathrm{C}$ und $45 \mathrm{~min}$ bei $0{ }^{\circ} \mathrm{C}$. Anschließend kühlte man wieder auf $-60{ }^{\circ} \mathrm{C}$ und fügte $2.94 \mathrm{~g}(20.0 \mathrm{mmol}) \mathrm{N}$ Allyl- $N$-benzylamin und $2.02 \mathrm{~g}(20.0 \mathrm{mmol})$ Triethylamin zu. Nachdem man auf Raumtemp. erwärmte und $18 \mathrm{~h}$ rührte, versetzte man zur Aufarbeitung mit $0.1 \mathrm{M} \mathrm{HCl}$ $(3 \times 30 \mathrm{ml})$, trocknete die organische Phase über $\mathrm{MgSO}_{4}$ und entfernte das Lösungsmittel i. Vak. Das Rohprodukt wurde durch Säulenchromatographie an $50 \mathrm{~g}$ Kieselgel (Säule $2.5 \times 20 \mathrm{~cm}$, Pentan/Et $2 \mathrm{O}=4: 1)$ gereinigt. Man erhielt $1.74 \mathrm{~g}(28 \%) 99$ als farbloses Öl, $R_{\mathrm{f}}\left(\right.$ Pentan/Et $\left.{ }_{2} \mathrm{O}, 4: 1\right)=0.53$. - IR (Film): $\mathrm{v}=3032 \mathrm{~cm}^{-1}, 2923,1602,1496,1455,1344$, 1162, 1095, 1051, 928, 795, 737, 700. - ${ }^{1} \mathrm{H}-\mathrm{NMR}\left(250 \mathrm{MHz}, \mathrm{CDCl}_{3}\right): \delta=3.81(\mathrm{~d}$, $\left.{ }^{3} J=6.6 \mathrm{~Hz}, 2 \mathrm{H}, 1-\mathrm{H}\right), 4.49\left(\mathrm{~s}, 2 \mathrm{H}, \mathrm{PhCH}_{2}\right), 5.09-5.24(\mathrm{~m}, 2 \mathrm{H}, 3-\mathrm{H}), 5.69-5.85(\mathrm{~m}, 1 \mathrm{H}$, 2-H), $6.18\left(\mathrm{~d},{ }^{2} J=2.7 \mathrm{~Hz}, 1 \mathrm{H}, 2^{\prime}-\mathrm{H}\right), 6.86\left(\mathrm{~d},{ }^{2} J=2.7 \mathrm{~Hz}, 1 \mathrm{H}, 2^{\prime}-\mathrm{H}\right), 7.28-7.39$ (m, $5 \mathrm{H}$, $\mathrm{Ph}-\mathrm{H}) .{ }^{13} \mathrm{C}-\mathrm{NMR}\left(62.9 \mathrm{MHz}, \mathrm{CDCl}_{3}, \mathrm{DEPT}\right): \delta=49.80(-, \mathrm{C}-1 *), 51.31\left(-, \mathrm{PhCH}_{2}{ }^{*}\right)$, 120.00 (-, C-3), 127.96 (+, Ph-C), 128.01 (-, C-2'), 128.14 (C $\left.\mathrm{C}_{\text {quart }}, \mathrm{C}^{\prime} \mathbf{1}^{\prime}\right), 128.52$ (+, Ph-C), 128.62 (+, Ph-C), 131.62 (+, C-2), 135.49 (C quart $_{1}$ Ph-C). - MS (EI, $\left.70 \mathrm{eV}\right), \mathrm{m} / \mathrm{z}(\%)$ : $317 / 315(3 / 3)\left[\mathrm{M}^{+}\right], 226 / 224(4 / 4)\left[\mathrm{M}^{+}-\mathrm{C}_{7} \mathrm{H}_{7}\right], 194$ (3), 144 (37), 117 (8), 104 (7), 91 (100) $\left[\mathrm{C}_{7} \mathrm{H}_{7}^{+}\right], 65$ (8), 41 (12). $-\mathrm{C}_{12} \mathrm{H}_{14} \mathrm{BrNO}_{2} \mathrm{~S}$ (316.2).

1-Allyloxy-3-brom-but-3-en (114): $\mathrm{Zu}$ einer Suspension von $1.00 \mathrm{~g}$ (25.0 mmol, 60proz. in<smiles>C=CCOCCC(=C)Br</smiles>
Mineralöl) $\mathrm{NaH}$ in $40 \mathrm{ml}$ wasserfreiem $\mathrm{Et}_{2} \mathrm{O}$ tropfte man bei $0{ }^{\circ} \mathrm{C} 3.02 \mathrm{~g}$ (20.0 mmol) 3-Brombut-3-en-1-ol langsam zu und rührte $45 \mathrm{~min}$ bei dieser Temperatur, bis die Gasentwicklung beendet war. Dann gab man $2.42 \mathrm{~g}$ (20.0 mmol) Allylbromid zu, ließ auf Raumtemp. erwärmen und rührte $18 \mathrm{~h}$ bei der Temperatur. Zur Aufarbeitung versetzte man mit $20 \mathrm{ml}$ Wasser, extrahierte die wäßrige Phase mit $3 \times 20 \mathrm{ml}$ Ether und trocknete die vereinigten organischen Phasen über $\mathrm{MgSO}_{4}$. Das Lösungsmittel wurde i. Vak. entfernt und das Rohprodukt durch Säulenchromatographie an $50 \mathrm{~g}$ Kieselgel (Säule $2.5 \times 20 \mathrm{~cm}$, Pentan/Et $2 \mathrm{O}=20: 1$, nach Durchlaufen der 1. Fraktion Wechsel zu Pentan/Et $2 \mathrm{O}=1: 1$ ) gereinigt. Man erhielt $0.97 \mathrm{~g}(25 \%) 114$ als farbloses Öl, $R_{\mathrm{f}}\left(\mathrm{Pentan}_{\mathrm{EEt}} \mathrm{O}, 20: 1\right)=0.43$. Als 2. Fraktion erhielt man $0.71 \mathrm{~g}$ Edukt und Butinol im Verhältnis $2: 1, R_{\mathrm{f}}\left(\right.$ Pentan/ $_{\mathrm{Et}} \mathrm{O}_{2} \mathrm{O}$, $1: 1)=0.41-$ IR (Film): $v=3304 \mathrm{~cm}^{-1}, 3081,2864,1734,1632,1420,1347,1264,1144$, 
1101, 991, 927, 891, 735, 639. - ${ }^{1} \mathrm{H}-\mathrm{NMR}\left(250 \mathrm{MHz}, \mathrm{CDCl}_{3}\right): \delta=2.70\left(\mathrm{t},{ }^{3} \mathrm{~J}=6.4 \mathrm{~Hz}\right.$, $2 \mathrm{H}, 2-\mathrm{H}), 3.62\left(\mathrm{t},{ }^{3} \mathrm{~J}=6.4 \mathrm{~Hz}, 2 \mathrm{H}, 1-\mathrm{H}\right), 4.01\left(\mathrm{dt},{ }^{3} J=5.7,{ }^{4} J=1.4 \mathrm{~Hz}, 2 \mathrm{H}, 1^{\prime}-\mathrm{H}\right)$, 5.16-5.33 (m, 2 H, 3'-H), 5.48 (d, $\left.{ }^{2} J=1.6 \mathrm{~Hz}, 1 \mathrm{H}, 4-\mathrm{H}\right), 5.67$ (d, $\left.2 J=1.6 \mathrm{~Hz}, 1 \mathrm{H}, 4-\mathrm{H}\right)$, $5.91\left(\mathrm{ddt},{ }^{3} J=5.7,{ }^{3} J=10.4,{ }^{3} J=16.0 \mathrm{~Hz}, 1 \mathrm{H}, 2^{\prime}-\mathrm{H}\right) .-{ }^{13} \mathrm{C}-\mathrm{NMR}\left(62.9 \mathrm{MHz}, \mathrm{CDCl}_{3}\right.$, DEPT): $\delta=41.69$ (-, C-2), 67.59 (-, C-1*), $71.95\left(-, \mathrm{C}^{\prime} 1^{\prime *}\right), 117.15\left(-, \mathrm{C}-3^{\prime * *}\right), 118.39(-$, C-4**), 130.64 (C $\left.\mathrm{C}_{\text {quart }}, \mathrm{C}-3\right), 134.58$ (+, C-2'). - MS (EI, $\left.70 \mathrm{eV}\right), \mathrm{m} / \mathrm{z}(\%):$ 136/134 (7/8) $\left[\mathrm{M}^{+}\right], 111(20)\left[\mathrm{M}^{+}-\mathrm{Br}\right], 81(26)\left[\mathrm{Br}^{+}\right], 71(59), 53$ (22), $41(100)\left[\mathrm{C}_{3} \mathrm{H}_{5}{ }^{+}\right] .-\mathrm{MS}(\mathrm{CI}$, $\left.\mathrm{NH}_{3}, 70 \mathrm{eV}\right), \mathrm{m} / \mathrm{z}(\%): 402 / 400 / 398(52 / 100 / 54)\left[2 \mathrm{M}+\mathrm{NH}_{4}^{+}\right], 227 / 225(36 / 37)[\mathrm{M}+$ $\left.\mathrm{NH}_{4}{ }^{+}+\mathrm{NH}_{3}\right], 210 / 208(36 / 37)\left[\mathrm{M}+\mathrm{NH}_{4}{ }^{+}\right] .-\mathrm{C}_{7} \mathrm{H}_{11} \mathrm{BrO}$ (191.1): ber. C 44.00, H 5.80; gef. C 44.28, H 5.60.

\subsection{Cyclisierung der heteroatomhaltigen Vorläufer}

Allgemeine Arbeitsvorschrift für die Heck-Diels-Alder-Reaktion mit Heteroatomhaltigen Cyclisierungsvorläufern nach dem Eintopfverfahren (AAV 5): In einer starkwandigen Pyrexflasche mit Schraubdeckel gibt man zu einer Lösung von 1 mmol Bromdien und $3 \mathrm{mmol}$ Dienophil in $10 \mathrm{ml}$ wasserfreiem Acetonitril $5 \mathrm{~mol} \% \mathrm{Pd}(\mathrm{OAc})_{2}, 10 \mathrm{~mol} \% \mathrm{PPh}_{3}$ und die angegebenen Menge Base. Man leitet 5 min Stickstoff durch das Reaktionsgemisch, verschließt die Flasche und rührt die angegebene Zeit bei $80-100{ }^{\circ} \mathrm{C}$. Nach Abkühlen auf Raumtemp. filtriert man über Aktivkohle und Celite, wäscht mit Diethylether nach und entfernt das Lösungsmittel i. Vak. Das verbleibende Rohprodukt wird durch Säulenchromatographie an Kieselgel gereinigt.

N-(p-Tosyl)-2,3,4,5,6,7-hexahydro-1H-isoindol-5-carbonsäuremethylester (74): Analog AAV 2 erhitzte man $249 \mathrm{mg}(1.00 \mathrm{mmol})$ Enin 73 mit<smiles>CC(=O)C1CCC2=C(C1)CN([As])C2</smiles>
$258 \mathrm{mg}$ Methylacrylat $(3.00 \mathrm{mmol}), 11 \mathrm{mg}(0.050 \mathrm{mmol}$, $5 \mathrm{~mol} \%) \mathrm{Pd}(\mathrm{OAc})_{2}$ und $24 \mathrm{mg}(0.10 \mathrm{mmol}, 10 \mathrm{~mol} \%) N, N^{\prime}-$ Bis(benzyliden)ethylendiamin in $10 \mathrm{ml}$ wasserfreiem Benzol $1 \mathrm{~d}$ bei $80^{\circ} \mathrm{C}$. Säulenchromatographie an $18 \mathrm{~g}$ Kieselgel (Säule $2.0 \times 15 \mathrm{~cm}, \mathrm{CH}_{2} \mathrm{Cl}_{2}$ ) ergab $186 \mathrm{mg}(55 \%)$ der Verbindung 74 als farblose Kristalle, Schmp. $86-88{ }^{\circ} \mathrm{C}, R_{\mathrm{f}}$ $\left(\mathrm{CH}_{2} \mathrm{Cl}_{2}\right)=0.18 .-\mathrm{IR}(\mathrm{KBr}): \mathrm{v}=2951 \mathrm{~cm}^{-1}, 2845,1731(\mathrm{C}=\mathrm{O}), 1598,1493,1438,1343$, 
1165, 1102, 816, 720, 664, 594. - ${ }^{1} \mathrm{H}-\mathrm{NMR}\left(250 \mathrm{MHz}, \mathrm{CDCl}_{3}\right): \delta=1.61-1.73(\mathrm{~m}, 2 \mathrm{H}$, 7-H), 2.02-2.06 (m, 3 H, 5,6-H), 2.15 (d, $\left.{ }^{3} J=7.7 \mathrm{~Hz}, 2 \mathrm{H}, 4-\mathrm{H}\right), 2.42\left(\mathrm{~s}, 3 \mathrm{H}, \mathrm{CH}_{3}\right), 3.66$ (s, $\left.3 \mathrm{H}, \mathrm{CO}_{2} \mathrm{CH}_{3}\right), 3.98\left(\mathrm{~m}_{\mathrm{c}}, 4 \mathrm{H}, 1,3-\mathrm{H}\right), 7.33\left(\mathrm{~d},{ }^{3} \mathrm{~J}=8.4 \mathrm{~Hz}, 2 \mathrm{H}, \mathrm{Ph}-\mathrm{H}\right), 7.71(\mathrm{~d}$, $\left.{ }^{3} J=8.4 \mathrm{~Hz}, 2 \mathrm{H}, \mathrm{Ph}-\mathrm{H}\right) .-{ }^{13} \mathrm{C}-\mathrm{NMR}\left(62.9 \mathrm{MHz}, \mathrm{CDCl}_{3}, \mathrm{DEPT}\right): \delta=21.51\left(+, \mathrm{CH}_{3}\right)$, $22.13\left(-, \mathrm{C}-7^{*}\right), 24.82\left(-, \mathrm{C}-6^{*}\right), 25.27\left(-, \mathrm{C}-4^{*}\right), 39.00(+, \mathrm{C}-5), 51.80\left(+, \mathrm{CO}_{2} \mathrm{CH}_{3}\right)$, 56.79 (-, C-1, C-3), 127.42 (+, Ph-C), 128.42 (C quart, $\left.\mathrm{C}-3 \mathrm{a}^{* *}\right), 129.58\left(\mathrm{C}_{\text {quart }}, \mathrm{C}-7 \mathrm{a}^{* *}\right)$, 129.69 (+, Ph-C), 134.25 ( $\left.\mathrm{C}_{\text {quart }}, \mathrm{Ph}-\mathrm{C}\right), 143.31$ ( $\left.\mathrm{C}_{\text {quart }}, \mathrm{Ph}-\mathrm{C}\right), 175.27\left(\mathrm{C}_{\text {quart }}, \mathrm{CO}_{2} \mathrm{CH}_{3}\right)$. - MS (EI, $70 \mathrm{eV}), m / z(\%): 335$ (33) $\left[\mathrm{M}^{+}\right], 274$ (10), 249 (48), 180 (51) $\left[\mathrm{M}^{+}-\mathrm{C}_{7} \mathrm{H}_{7} \mathrm{SO}_{2}\right]$, 155 (34) $\left[\mathrm{C}_{7} \mathrm{H}_{7} \mathrm{SO}_{2}^{+}\right], 120$ (90), 94 (81), 91 (100) $\left[\mathrm{C}_{7} \mathrm{H}_{7}{ }^{+}\right], 65$ (18). $-\mathrm{C}_{17} \mathrm{H}_{21} \mathrm{NO}_{4} \mathrm{~S}$ (335.4): ber. C 60.87, H 6.31, N 4.18; gef. C 60.56, H 6.29, N 4.04.

Variante B: Versetzte man $249 \mathrm{mg}(1.00 \mathrm{mmol})$ Enin 73 in $10 \mathrm{ml}$ wasserfreiem Benzol mit $258 \mathrm{mg}$ Methylacrylat (3.00 mmol), $29 \mathrm{mg}(0.050 \mathrm{mmol}, 5 \mathrm{~mol} \%) \mathrm{Pd}(\mathrm{dba})_{2}, 26 \mathrm{mg}$ (0.10 mmol, $10 \mathrm{~mol} \%) \mathrm{PPh}_{3}$ und $6.0 \mathrm{mg}(0.10 \mathrm{mmol}, 10 \mathrm{~mol} \%)$ Essigsäure und erhitzte $1 \mathrm{~d}$ auf $80{ }^{\circ} \mathrm{C}$, erhielt man $202 \mathrm{mg}(60 \%)$ der Verbindung 74.

Variante C: Bei Verwendung von $11 \mathrm{mg}(0.050 \mathrm{mmol}, 5.0 \mathrm{~mol} \%) \mathrm{Pd}(\mathrm{OAc})_{2}$ und $26 \mathrm{mg}$ (0.10 mmol, $10 \mathrm{~mol} \%) \mathrm{PPh}_{3}$ als Katalysatorgemisch unter sonst identischen Bedingungen wie unter Variante A beschrieben, konnten 200 mg (60\%) der gewünschten Verbindung isoliert werden.

Versuch der Cyclisierung von 85: Analog AAV 2 gab man zu einer Lösung von 243 mg $(1.00 \mathrm{mmol}) 85$ und $258 \mathrm{mg}(3.00 \mathrm{mmol})$ Methylacrylat in $10 \mathrm{ml}$ wasserfreiem Benzol $11 \mathrm{mg}(0.050 \mathrm{mmol}, 5.0 \mathrm{~mol} \%) \mathrm{Pd}(\mathrm{OAc})_{2}$ und $24 \mathrm{mg}(0.10 \mathrm{mmol}, 10 \mathrm{~mol} \%)$ BBEDA. Nachdem man $3 \mathrm{~d}$ bei $60{ }^{\circ} \mathrm{C}$ gerührt hatte, war nach dünnschichtchromatographischer Analyse nur Ausgangsmaterial festzustellen. Anschließend erhöhte man die Temperatur auf $100{ }^{\circ} \mathrm{C}$. Nach weiteren $2 \mathrm{~d}$ waren im ${ }^{1} \mathrm{H}-\mathrm{NMR}$-Spektrum des Rohproduktes neben Edukt nur Zersetzungsprodukte zu erkennen.

N,N'-Benzyl-1,13-dicarbonsäuremethylester-8-methylentrideca-1,12-dien-6-in

(86):

Entsprechend AAV 5 gab man zu $324 \mathrm{mg}(0.999 \mathrm{mmol}) 82$ in $10 \mathrm{ml}$ wasserfreiem MeCN $11 \mathrm{mg}(0.050 \mathrm{mmol}, 5.0 \mathrm{~mol} \%) \mathrm{Pd}(\mathrm{OAc})_{2}, 26 \mathrm{mg}(0.10 \mathrm{mmol}, 10 \mathrm{~mol} \%) \mathrm{PPh}_{3}, 138 \mathrm{mg}$ $(1.00 \mathrm{mmol}) \mathrm{K}_{2} \mathrm{CO}_{3}$ und $258 \mathrm{mg}(3.00 \mathrm{mmol})$ Methylacrylat. Man rührte $18 \mathrm{~h}$ bei $80{ }^{\circ} \mathrm{C}$. Säulenchromatographie des Rohprodukts an $18 \mathrm{~g}$ Kieselgel (Säule $2.0 \times 15 \mathrm{~cm}$, 
Pentan/Et $2 \mathrm{O}=1: 1$, nach Durchlaufen der 1. Fraktion Wechsel zu Et ${ }_{2} \mathrm{O}$ ) ergab Fraktion I: 39 mg (16\%) 86 als Rotamere im Verhältnis 1.9 : 1 (aus ${ }^{1} \mathrm{H}-\mathrm{NMR}-$ Spektrum) als farbloses

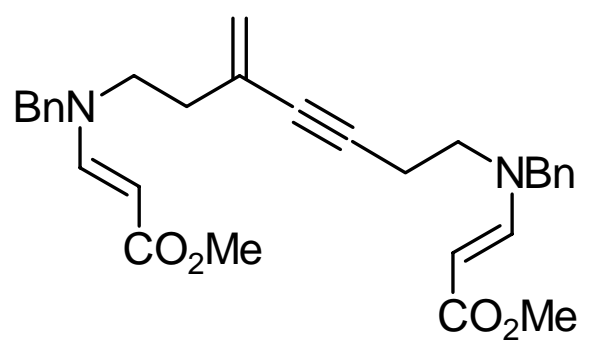

Öl, $R_{\mathrm{f}}\left(\mathrm{Et}_{2} \mathrm{O}\right)=0.54$. - IR (Film): $v=3353 \mathrm{~cm}^{-1}$, $3055,2925,2854,1684,1611,1496,1455,1357$, $1265,1152,1079,1040,978,900,739,702 .-$ ${ }^{1} \mathrm{H}-\mathrm{NMR} \quad\left(250 \mathrm{MHz}, \quad \mathrm{CDCl}_{3}\right): \delta=2.31 \quad(\mathrm{t}$, $\left.3 J=7.2 \mathrm{~Hz}, 2 \mathrm{H}, 5-\mathrm{H}^{*}\right), 2.51(\mathrm{t}, 3 \mathrm{~J}=7.0 \mathrm{~Hz}, 2 \mathrm{H}$, 9-H*), 3.11-3.39 (m, $4 \mathrm{H}, 4,10-\mathrm{H}), 3.64$ (s, $3 \mathrm{H}, \mathrm{CO}_{2} \mathrm{CH}_{3}$ ), 3.65 (s, $3 \mathrm{H}, \mathrm{CO}_{2} \mathrm{CH}_{3}$ ), 4.28$4.40\left(\mathrm{~m}, 4 \mathrm{H}, \mathrm{PhCH}_{2}\right), 4.64\left(\mathrm{~d},{ }^{3} \mathrm{~J}=11.2 \mathrm{~Hz}, 1 \mathrm{H}, 1-\mathrm{H}^{* *}\right), 4.70\left(\mathrm{~d},{ }^{3} J=11.2 \mathrm{~Hz}, 1 \mathrm{H}\right.$, 13-H**), 4.94, 5.19 (s, $\left.1 \mathrm{H}, 8-\mathrm{CH}_{2}\right), 5.01,5.30$ (s, $\left.1 \mathrm{H}, 8-\mathrm{CH}_{2}\right), 7.16-7.26$ (m, 4 H, Ph-H), 7.28-7.36 (m, $6 \mathrm{H}, \mathrm{Ph}-\mathrm{H}), 7.58-7.64$ (m, $2 \mathrm{H}, 2,12-\mathrm{H}) .-{ }^{13} \mathrm{C}-\mathrm{NMR}\left(62.9 \mathrm{MHz}, \mathrm{CDCl}_{3}\right.$, DEPT): $\delta=24.62$ (-, C-5), 27.35 (-, C-9), $50.56\left(+, \mathrm{CO}_{2} \mathrm{CH}_{3}\right), 51.89$ (-, C-4*), 52.13 (-, $\left.\mathrm{C}-10^{*}\right), 65.83\left(-, \mathrm{PhCH}_{2}\right), 84.68(+, 2 \mathrm{C}, \mathrm{C}-1, \mathrm{C}-13), 85.44\left(\mathrm{C}_{\text {quart }}, \mathrm{C}-6 * *\right), 87.21\left(\mathrm{C}_{\text {quart }}\right.$, C-7**), $114.76\left(-, 8-\mathrm{CH}_{2}\right), 123.29$ (-, 8- $\left.\mathrm{CH}_{2}\right), 127.23$ (+, Ph-C), 127.31 (+, Ph-C), 127.74 (+, Ph-C), 127.84 (+, Ph-C), 128.75 (+, Ph-C), 128.80 (+, Ph-C), 136.13 (C $\left.\mathrm{C}_{\text {quart }}, \mathrm{Ph}-\mathrm{C}\right)$, $136.23\left(\mathrm{C}_{\text {quart }}, \mathrm{Ph}-\mathrm{C}\right), 143.01\left(\mathrm{C}_{\text {quart }}, \mathrm{C}-8\right), 152.04$ (+, $\left.2 \mathrm{C}, \mathrm{C}-2, \mathrm{C}-12\right), 169.86\left(\mathrm{C}_{\text {quart }}\right.$, $\left.\mathrm{CO}_{2} \mathrm{CH}_{3}\right), 170.07\left(\mathrm{C}_{\text {quart }}, \mathrm{CO}_{2} \mathrm{CH}_{3}\right) .-\mathrm{MS}(\mathrm{EI}, 70 \mathrm{eV}), \mathrm{m} / \mathrm{z}(\%): 486$ (6) [M+], 455 (10) [M+ - OMe], 415 (77), 384 (40), 342 (94), 262 (33), 243 (13), 204 (100), 183 (12), 91 (57) $\left[\mathrm{C}_{7} \mathrm{H}_{7}{ }^{+}\right] .-\mathrm{C}_{30} \mathrm{H}_{34} \mathrm{~N}_{2} \mathrm{O}_{4}$ (486.6).

Fraktion II: 102 mg eines Gemisches aus $\mathbf{8 6}$ und einer nicht identifizierten Verbindung.

Versuch der Cyclisierung von 95: Variante A: Nach AAV 2 versetzte man $235 \mathrm{mg}$ (1.00 mmol) 95 und $258 \mathrm{mg}$ (3.00 mmol) Methylacrylat in $10 \mathrm{ml}$ wasserfreiem Benzol mit $11 \mathrm{mg}(0.050 \mathrm{mmol}, 5.0 \mathrm{~mol} \%) \mathrm{Pd}(\mathrm{OAc})_{2}$ und $24 \mathrm{mg}(0.10 \mathrm{mmol}, 10 \mathrm{~mol} \%)$ BBEDA. Man rührte $1 \mathrm{~d}$ bei $60{ }^{\circ} \mathrm{C}$. Das ${ }^{1} \mathrm{H}-\mathrm{NMR}$-Spektrum des Rohproduktes zeigte nur Ausgangsmaterial.

Variante B: Eine Lösung von $235 \mathrm{mg}(1.00 \mathrm{mmol}) 95$ und $258 \mathrm{mg}$ (3.00 mmol) Methylacrylat in $10 \mathrm{ml}$ wasserfreiem Benzol wurde mit $29 \mathrm{mg}(0.050 \mathrm{mmol}, 5.0 \mathrm{~mol} \%)$ $\mathrm{Pd}(\mathrm{dba})_{2}, \quad 26 \mathrm{mg} \quad(0.10 \mathrm{mmol}, \quad 10 \mathrm{~mol} \%) \mathrm{PPh}_{3}$ und $6.0 \mathrm{mg} \quad(0.10 \mathrm{mmol}, 10 \mathrm{~mol} \%)$ Essigsäure versetzt und bei $60{ }^{\circ} \mathrm{C}$ Badtemperatur $4 \mathrm{~d}$ gerührt. Im ${ }^{1} \mathrm{H}-\mathrm{NMR}$-Spektrum des Rohproduktes war ebenfalls nur Edukt zu erkennen. 
Variante $C$ : Erhöhte man die Temperatur unter den in AAV 2 beschriebenen Bedingungen auf $100{ }^{\circ} \mathrm{C}$, so konnten nur Zersetzungsprodukte detektiert werden.

N-Benzyl-2,3,4,5,6,7-hexahydro-benzo[d] isothiazol-5-carbonsäuremethylester (100a) und N-Benzyl-2,3,4,5,6,7-hexahydro-benzo[d] isothiazol-6-carbonsäuremethylester

(100b):

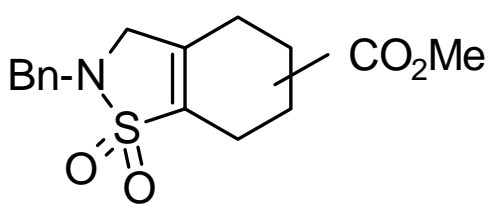

Analog AAV 5 versetzte man eine Lösung von 316 mg (0.999 mmol) $\quad \mathbf{9 3}, \quad 11 \mathrm{mg} \quad(0.050 \mathrm{mmol}, \quad 5.0 \mathrm{~mol} \%)$ $\mathrm{Pd}(\mathrm{OAc})_{2}, 26 \mathrm{mg}(0.10 \mathrm{mmol}, 10 \mathrm{~mol} \%) \mathrm{PPh}_{3}, 326 \mathrm{mg}$ $(1.00 \mathrm{mmol}) \quad \mathrm{Cs}_{2} \mathrm{CO}_{3}$ und $258 \mathrm{mg} \quad(3.00 \mathrm{mmol})$

Methylacrylat in $10 \mathrm{ml}$ wasserfreiem $\mathrm{MeCN}$ und erwärmte $3 \mathrm{~d}$ auf $100{ }^{\circ} \mathrm{C}$. Säulenchromatographie des Rohproduktes an $18 \mathrm{~g}$ Kieselgel (Säule $2.0 \times 15 \mathrm{~cm}$, $\left.\mathrm{CH}_{2} \mathrm{Cl}_{2} / \mathrm{MeOH}=20: 1\right)$ ergab neben $145 \mathrm{mg}$ nicht identifizierten oligomeren Materials 92 mg (29\%) eines Regioisomerengemisches 100a,b im Verhältnis 1.9 : 1 als farbloses Öl, $R_{\mathrm{f}}\left(\mathrm{CH}_{2} \mathrm{Cl}_{2} / \mathrm{MeOH}, 20: 1\right)=0.19$. - IR (Film), Gemisch: $v=3616 \mathrm{~cm}^{-1}, 3063,3030$, 2950, 2849, 1730 (C=O), 1684, 1604, 1532, 1496, 1454, 1286, 1161, 1029, 946, 778, 737, 700. - ${ }^{1} \mathrm{H}-\mathrm{NMR}\left(250 \mathrm{MHz}, \mathrm{CDCl}_{3}\right)$, Gemisch: $\delta=1.81-2.91(\mathrm{~m}, 7 \mathrm{H}, 4,5,6,7-\mathrm{H})$, 3.53-3.73 (m, 5 H, 3-H, $\mathrm{CO}_{2} \mathrm{CH}_{3}$ ), 4.30-4.34 (m, $2 \mathrm{H}, \mathrm{PhCH}_{2}$ ), 7.30-7.37 (m, 5 H, Ph-H). - Hauptmengenisomer: ${ }^{13} \mathrm{C}-\mathrm{NMR}\left(62.9 \mathrm{MHz}, \mathrm{CDCl}_{3}, \mathrm{DEPT}\right): \delta=18.11$ (-, C-4*), 24.13 (-, C-5*), $26.84\left(-\right.$, C-7*), 38.08 (+, C-6), 47.90 (-, C-3), $52.18\left(+, \mathrm{CO}_{2} \mathrm{CH}_{3}\right), 52.62$ (-, $\left.\mathrm{PhCH}_{2}\right), 128.55$ (+, Ph-C), 128.61 (+, Ph-C), 128.77 (+, Ph-C), 131.63 (C $\left.\mathrm{C}_{\text {quart }}, \mathrm{C}-3 \mathrm{a}^{* *}\right)$, $135.15\left(\mathrm{C}_{\text {quart }}, \mathrm{C}-7 \mathrm{a}^{* *}\right), 139.63\left(\mathrm{C}_{\text {quart }}, \mathrm{Ph}-\mathrm{C}^{* *}\right), 174.00\left(\mathrm{C}_{\text {quart }}, \mathrm{CO}_{2} \mathrm{CH}_{3}\right)$. Mindermengenisomer: ${ }^{13} \mathrm{C}-\mathrm{NMR}\left(62.9 \mathrm{MHz}, \mathrm{CDCl}_{3}, \mathrm{DEPT}\right): \delta=21.23\left(-, \mathrm{C}-4^{*}\right), 23.86$ (-, C-6*), $23.95\left(-, \mathrm{C}-7^{*}\right), 38.08$ (+, C-5), 51.58 (-, C-3), $52.57\left(+, \mathrm{CO}_{2} \mathrm{CH}_{3}\right), 52.62$ (-, $\mathrm{PhCH}_{2}$ ), 128.58 (+, Ph-C), 128.72 (+, Ph-C), 128.77 (+, Ph-C), 135.51 ( $\left.\mathrm{C}_{\text {quart }}, \mathrm{C}-3 \mathrm{a}^{* *}\right)$, $138.94\left(\mathrm{C}_{\text {quart }}, \mathrm{C}-7 \mathrm{a} * *\right), 140.65\left(\mathrm{C}_{\text {quart }}, \mathrm{Ph}-\mathrm{C}^{* *}\right), 173.89\left(\mathrm{C}_{\text {quart }}, \mathrm{CO}_{2} \mathrm{CH}_{3}\right) .-\mathrm{MS}(\mathrm{EI}$, $70 \mathrm{eV}), \mathrm{m} / z(\%)$, Gemisch: $321(5)\left[\mathrm{M}^{+}\right], 257(38)\left[\mathrm{M}^{+}-\mathrm{SO}_{2}\right], 198$ (16) $\left[\mathrm{M}^{+}-\mathrm{SO}_{2}-\right.$ $\left.\mathrm{CO}_{2} \mathrm{Me}\right], 170(8), 120(8), 91(100)\left[\mathrm{C}_{7} \mathrm{H}_{7}{ }^{+}\right] .-\mathrm{C}_{16} \mathrm{H}_{19} \mathrm{NO}_{4} \mathrm{~S}$ (321.4).

N-Benzyl-4-methylen-3,4-dihydro-2H[1,2]thiazin-1,1-dioxid (101): Variante A: Nach<smiles>C=C1C=CS(=O)(=O)N(Cc2ccccc2)C1</smiles>
AAV 5 löste man $316 \mathrm{mg}(0.999 \mathrm{mmol})$ 93, $11 \mathrm{mg}(0.050 \mathrm{mmol}$, $5.0 \mathrm{~mol} \%) \quad \mathrm{Pd}(\mathrm{OAc})_{2}, \quad 26 \mathrm{mg} \quad\left(0.10 \mathrm{mmol}, 10 \mathrm{~mol}_{\%}\right) \quad \mathrm{PPh}_{3}, 276 \mathrm{mg}$ $(2.00 \mathrm{mmol}) \mathrm{K}_{2} \mathrm{CO}_{3}$ und $258 \mathrm{mg}(3.00 \mathrm{mmol})$ Methylacrylat in $10 \mathrm{ml}$ wasserfreiem $\mathrm{MeCN}$ und erwärmte $3 \mathrm{~d}$ auf $100{ }^{\circ} \mathrm{C}$. 
Säulenchromatographie des Rohproduktes an $18 \mathrm{~g}$ Kieselgel (Säule $2.0 \times 15 \mathrm{~cm}$, Pentan/Et $2 \mathrm{O}=3: 1$ ) ergab neben $273 \mathrm{mg}$ oligomeren Materials $12 \mathrm{mg}$ (5\%) 101 als farbloses Öl, $R_{\mathrm{f}}\left(\operatorname{Pentan} / \mathrm{Et}_{2} \mathrm{O}, 3: 1\right)=0.19 .-{ }^{1} \mathrm{H}-\mathrm{NMR}\left(250 \mathrm{MHz}, \mathrm{CDCl}_{3}\right): \delta=4.11(\mathrm{~s}$, $2 \mathrm{H}, 3-\mathrm{H}), 4.18\left(\mathrm{~s}, 2 \mathrm{H}, \mathrm{PhCH}_{2}\right), 5.27\left(\mathrm{~s}, 1 \mathrm{H}, 4-\mathrm{CH}_{2}\right), 5.48\left(\mathrm{~s}, 1 \mathrm{H}, 4-\mathrm{CH}_{2}\right), 6.49$ (d, $3 J=10.6 \mathrm{~Hz}, 1 \mathrm{H}, 5-\mathrm{H}), 6.71(\mathrm{~d}, 3 J=10.6 \mathrm{~Hz}, 1 \mathrm{H}, 6-\mathrm{H}), 7.32-7.37(\mathrm{~m}, 5 \mathrm{H}, \mathrm{Ph}-\mathrm{H})$. ${ }^{13} \mathrm{C}-\mathrm{NMR}\left(75.5 \mathrm{MHz}, \mathrm{CDCl}_{3}, \mathrm{APT}\right): \delta=49.75(-, \mathrm{C}-3), 51.96\left(-, \mathrm{PhCH}_{2}\right), 123.82(-$, 4- $\mathrm{CH}_{2}$ ), 125.28 (+, C-5), 128.12 (+, Ph-C), 128.70 (+, Ph-C), 128.85 (+, Ph-C), 132.12 (-, Ph-C*), 134.88 (-, C-4*), 136.36 (+, C-6). - MS (EI, 70 eV), m/z (\%): 235 (6) [M+], 171 (45) $\left[\mathrm{M}^{+}-\mathrm{SO}_{2}\right], 170$ (74), $156(4), 144$ (8) $\left[\mathrm{M}^{+}-\mathrm{C}_{7} \mathrm{H}_{7}\right], 104$ (5), 91 (100) $\left[\mathrm{C}_{7} \mathrm{H}_{7}{ }^{+}\right], 77$ (6), 65 (15), 51 (11). $-\mathrm{C}_{12} \mathrm{H}_{13} \mathrm{NO}_{2} \mathrm{~S}$ (235.3).

Variante B: Erwärmte man nur auf $80^{\circ} \mathrm{C}$, so war nach $3 \mathrm{~d}$ dünnschichtchromatographisch nur Ausgangsmaterial feststellbar.

Variante $C$ : Verwendete man anstelle von $\mathrm{Pd}(\mathrm{OAc})_{2} 46 \mathrm{mg}(0.050 \mathrm{mmol}, 5.0 \mathrm{~mol} \%)$ $\operatorname{Pd}(\text { dppe })_{2}$ und erwärmte $3 \mathrm{~d}$ bei $100{ }^{\circ} \mathrm{C}$, so konnte ebenfalls nur Edukt detektiert werden. Variante D: Setzte man als Palladiumspezies $47 \mathrm{mg}(0.050 \mathrm{mmol}, 5.0 \mathrm{~mol} \%)$ des BellerKatalysators [103] ein, so erhielt man 42 mg 101, jedoch verunreingt durch Tri(o-toloyl)phosphan, was sich säulenchromatographisch nicht abtrennen ließ.

Variante E: Verzichtete man auf die Zugabe von Methylacrylat unter den unter Variante A beschriebenen Bedingungen, so erhöhte sich die Ausbeute von 101 auf 20\%

N-Benzyl-6-methylen-3,6-dihydro-2H[1,2]thiazin-1,1-dioxid (102): Analog AAV 5 gab man zu einer Lösung von $316 \mathrm{mg}(0.999 \mathrm{mmol}) 99$ in $10 \mathrm{ml}$

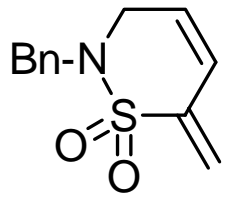
wasserfreiem MeCN $11 \mathrm{mg}(0.050 \mathrm{mmol}, 5.0 \mathrm{~mol} \%) \operatorname{Pd}(\mathrm{OAc})_{2}, 26 \mathrm{mg}$ (0.10 mmol, $10 \mathrm{~mol} \%) \mathrm{PPh}_{3}, 276 \mathrm{mg}(2.00 \mathrm{mmol}) \mathrm{K}_{2} \mathrm{CO}_{3}, 258 \mathrm{mg}$ $(3.00 \mathrm{mmol})$ Methylacrylat und rührte $18 \mathrm{~h}$ bei $80^{\circ} \mathrm{C}$. Säulenchromatographie des Rohprodukts an $18 \mathrm{~g}$ Kieselgel (Säule $\left.2.0 \times 15 \mathrm{~cm}, \mathrm{CH}_{2} \mathrm{Cl}_{2}\right)$ ergab $47 \mathrm{mg}(20 \%)$ eines Gemisches aus $102(18 \%)$ und $103(2 \%)$ als farblosen Feststoff, Schmp. $63-64^{\circ} \mathrm{C}, R_{\mathrm{f}}\left(\mathrm{CH}_{2} \mathrm{Cl}_{2}\right)=0.58$. - 102: IR (unterkühlte Schmelze): $v=3058 \mathrm{~cm}^{-1}, 1705,1497,1456,1336,1266,1144,1060,1029,896,737$, 701. $-{ }^{1} \mathrm{H}-\mathrm{NMR}\left(250 \mathrm{MHz}, \mathrm{CDCl}_{3}\right): \delta=3.90$ (br s, $\left.2 \mathrm{H}, 3-\mathrm{H}\right), 4.17$ (s, $2 \mathrm{H}, \mathrm{PhCH}_{2}$ ), 5.62 (s, $\left.1 \mathrm{H}, 6-\mathrm{CH}_{2}\right), 5.77-5.85(\mathrm{~m}, 1 \mathrm{H}, 4-\mathrm{H}), 6.12\left(\mathrm{~s}, 1 \mathrm{H}, 6-\mathrm{CH}_{2}\right), 6.34\left(\mathrm{~d},{ }^{3} \mathrm{~J}=10.3 \mathrm{~Hz}, 1 \mathrm{H}\right.$, 5-H), 7.29-7.40 (m, $5 \mathrm{H}, \mathrm{Ph}-\mathrm{H}) .-{ }^{13} \mathrm{C}-\mathrm{NMR}\left(62.9 \mathrm{MHz}, \mathrm{CDCl}_{3}, \mathrm{DEPT}\right): \delta=49.61(-$, 
C-3), 51.08 (-, $\left.\mathrm{PhCH}_{2}\right), 118.99$ (-, 6-CH 2$), 124.10$ (+, C-4*), 126.21 (+, C-5*), 128.08 (+, Ph-C), 128.55 (+, Ph-C), 128.72 (+, Ph-C), 135.21 (C $\left.\mathrm{C}_{\text {quart }}, \mathrm{Ph}-\mathrm{C}\right), 140.09$ (C $\left.\mathrm{C}_{\text {quart }}, \mathrm{C}-6\right) .-$ MS (EI, $70 \mathrm{eV}), m / z(\%): 235$ (28) $\left[\mathrm{M}^{+}\right], 171$ (12) $\left[\mathrm{M}^{+}-\mathrm{SO}_{2}\right], 170$ (13), 144 (4) $\left[\mathrm{M}^{+}-\right.$ $\left.\mathrm{C}_{7} \mathrm{H}_{7}\right], 104$ (3), 91 (100) $\left[\mathrm{C}_{7} \mathrm{H}_{7}+\right], 77$ (5), 65 (17), 53 (9), 41 (5). - $\mathrm{C}_{12} \mathrm{H}_{13} \mathrm{NO}_{2} \mathrm{~S}$ (235.3): ber. 235.0667 (HRMS korrekt).

N-Benzyl-6-methyl-2H[1,2]thiazin-1,1-dioxid (103): Nach AAV 5 löste man $316 \mathrm{mg}$

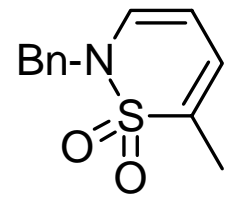
(0.999 mmol) 99, $11 \mathrm{mg}(0.050 \mathrm{mmol}, 5.0 \mathrm{~mol} \%) \mathrm{Pd}(\mathrm{OAc})_{2}, 26 \mathrm{mg}$ (0.10 mmol, $10 \mathrm{~mol} \%) \mathrm{PPh}_{3}, 326 \mathrm{mg}(1.00 \mathrm{mmol}) \mathrm{Cs}_{2} \mathrm{CO}_{3}$ und $258 \mathrm{mg}$ (3.00 mmol) Methylacrylat in $10 \mathrm{ml}$ wasserfreiem MeCN und erwärmte $3 \mathrm{~d}$ auf $100^{\circ} \mathrm{C}$. Säulenchromatographie des Rohproduktes an $18 \mathrm{~g}$ Kieselgel (Säule $2.0 \times 15 \mathrm{~cm}$, Pentan $/ \mathrm{Et}_{2} \mathrm{O}=3: 1$ ) ergab $36 \mathrm{mg}$ (15\%) eines Gemisches aus $103(12 \%)$ und $102(3 \%)$ als farblosen Feststoff, Schmp. $42-43{ }^{\circ} \mathrm{C}, R_{\mathrm{f}}$ $\left(\mathrm{CH}_{2} \mathrm{Cl}_{2}\right)=$ 0.64. - 103: IR (unterkühlte Schmelze): $v=3063 \mathrm{~cm}^{-1}, 3030,2924,2809$, 1455, 1334, 1266, 1158, 1143, 981, 923, 773, 736, 700, 668. - 1H-NMR (250 MHz, $\left.\mathrm{CDCl}_{3}\right): \delta=2.30\left(\mathrm{~s}, 3 \mathrm{H}, 6-\mathrm{CH}_{3}\right), 4.86\left(\mathrm{~s}, 2 \mathrm{H}, \mathrm{PhCH}_{2}\right), 5.59\left(\mathrm{t},{ }^{3} \mathrm{~J}=7.2 \mathrm{~Hz}, 1 \mathrm{H}, 3-\mathrm{H}^{*}\right)$, $6.51\left(\mathrm{~d},{ }^{3} \mathrm{~J}=7.2 \mathrm{~Hz}, 2 \mathrm{H}, 4,5-\mathrm{H}^{*}\right), 7.31-7.36$ (m, $\left.5 \mathrm{H}, \mathrm{Ph}-\mathrm{H}\right) .-{ }^{13} \mathrm{C}-\mathrm{NMR}(62.9 \mathrm{MHz}$, $\left.\mathrm{CDCl}_{3}, \mathrm{DEPT}\right): \delta=14.83\left(+, 6-\mathrm{CH}_{3}\right), 50.21\left(-, \mathrm{PhCH}_{2}\right), 103.35(+, \mathrm{C}-3), 128.00(+$, $\mathrm{Ph}-\mathrm{C}), 128.07$ ( $\left.\mathrm{C}_{\text {quart }}, \mathrm{C}-6\right), 128.71$ (+, C-4*), 128.88 (+, Ph-C), 129.56 (+, Ph-C), 133.31 $\left(+, \mathrm{C}-5^{*}\right), 135.44\left(\mathrm{C}_{\text {quart }}, \mathrm{Ph}-\mathrm{C}\right) .-\mathrm{MS}(\mathrm{EI}, 70 \mathrm{eV}), \mathrm{m} / \mathrm{z}(\%): 235$ (29) $\left[\mathrm{M}^{+}\right], 171$ (2) $\left[\mathrm{M}^{+}-\right.$ $\left.\mathrm{SO}_{2}\right], 170$ (2), 144 (2) $\left[\mathrm{M}^{+}-\mathrm{C}_{7} \mathrm{H}_{7}\right], 91$ (100) $\left[\mathrm{C}_{7} \mathrm{H}_{7}{ }^{+}\right], 65$ (7). - $\mathrm{C}_{12} \mathrm{H}_{13} \mathrm{NO}_{2} \mathrm{~S}$ (235.3): ber. 235.0667 (HRMS korrekt).

1,7-Diallyloxy-5-methylenhept-3-in （116), 3,4,5,6,7,8-Hexahydro-1H-isochromen-6carbonsäuremethylester (115a) und 3,4,5,6,7,8-Hexahydro-1H-isochromen-7-carbonsäuremethylester (115b): Variante A: Analog AAV 5 gab man zu einer Lösung von<smiles>C=CCOCCC#CC(=C)CCOCC=C</smiles>
$191 \mathrm{mg}$ (1.00 mmol) 1-Allyloxy-3-brom-but-3-en (114) in $10 \mathrm{ml}$ wasserfreiem MeCN $11 \mathrm{mg}(0.050 \mathrm{mmol}, 5.0 \mathrm{~mol} \%)$ $\mathrm{Pd}(\mathrm{OAc})_{2}, 26 \mathrm{mg}(0.10 \mathrm{mmol}, 10 \mathrm{~mol} \%) \mathrm{PPh}_{3}, 138 \mathrm{mg}$ $(1.00 \mathrm{mmol}) \quad \mathrm{K}_{2} \mathrm{CO}_{3} \quad$ und $258 \mathrm{mg} \quad(3.00 \mathrm{mmol})$ Methylacrylat. Man rührte $18 \mathrm{~h}$ bei $80^{\circ} \mathrm{C}$. Säulenchromatographie des Rohprodukts an $18 \mathrm{~g}$ Kieselgel 
(Säule $2.0 \times 15 \mathrm{~cm}$, Pentan/Et $2 \mathrm{O}=5: 1$ ) ergab Fraktion I: $58 \mathrm{mg}(26 \%) 116$ als farbloses Öl, $R_{\mathrm{f}}\left(\right.$ Pentan/Et $\left.{ }_{2} \mathrm{O}, 5: 1\right)=0.69$. - IR (Film): v = $3402 \mathrm{~cm}^{-1}, 3060,2924,2214,1724$, 1674, 1410, 1267, 1174, 1095, 1057, 992, 812, 736, 703. - ${ }^{1} \mathrm{H}-\mathrm{NMR}\left(250 \mathrm{MHz}, \mathrm{CDCl}_{3}\right)$ : $\delta=2.41(\mathrm{t}, 3 J=7.0 \mathrm{~Hz}, 2 \mathrm{H}, 2-\mathrm{H}), 2.56(\mathrm{t}, 3 J=7.0 \mathrm{~Hz}, 2 \mathrm{H}, 6-\mathrm{H}), 3.58(2 \times \mathrm{t}$, $\left.3 J=7.0 \mathrm{~Hz}, 4 \mathrm{H}, 1,7-\mathrm{H}^{*}\right), 4.00\left(\mathrm{~m}_{\mathrm{c}}, 4 \mathrm{H}, 1^{\prime}, 1^{\prime \prime}-\mathrm{H}^{*}\right), 5.18\left(\mathrm{~m}_{\mathrm{c}}, 2 \mathrm{H}, 5-\mathrm{CH}_{2} * *\right), 5.24\left(\mathrm{~m}_{\mathrm{c}}\right.$, $\left.2 \mathrm{H}, 3^{\prime}, 3^{\prime \prime}-\mathrm{H}^{* *}\right), 5.31\left(\mathrm{~m}_{\mathrm{c}}, 2 \mathrm{H}, 3^{\prime}, 3^{\prime \prime}-\mathrm{H}^{* *}\right), 5.91\left(\mathrm{ddd},{ }^{3} \mathrm{~J}=5.5,3^{3} \mathrm{~J}=10.7,{ }^{3} \mathrm{~J}=15.7 \mathrm{~Hz}\right.$, $\left.2 \mathrm{H}, 2^{\prime}, 2^{\prime \prime}-\mathrm{H}\right) .-{ }^{13} \mathrm{C}-\mathrm{NMR}\left(62.9 \mathrm{MHz}, \mathrm{CDCl}_{3}, \mathrm{DEPT}\right): \delta=20.63$ (-, C-2), 37.68 (-, C-6), $68.32\left(-, \mathrm{C}-1^{*}\right), 68.38(-, \mathrm{C}-7 *), 71.82\left(-, 2 \mathrm{C}, \mathrm{C}-1^{\prime *} \mathrm{C}-1^{\prime *}\right), 81.31\left(\mathrm{C}_{\mathrm{quart}}, \mathrm{C}-3^{* *}\right), 86.77$ $\left(\mathrm{C}_{\text {quart }}, \mathrm{C}-4^{* *}\right), 116.80\left(-, \mathrm{C}-3^{\prime * * *}\right), 117.06$ (-, C-3"***), $121.90\left(-, 5-\mathrm{CH}_{2}^{* * *}\right), 128.36$ $\left(\mathrm{C}_{\text {quart }}, \mathrm{C}-5\right), 134.52\left(+, \mathrm{C}-2^{\prime * * * *}\right), 134.84(+, \mathrm{C}-2$ "****). - MS (EI, $70 \mathrm{eV}), \mathrm{m} / \mathrm{z}(\%): 179$ (5) $\left[\mathrm{M}^{+}-\mathrm{C}_{3} \mathrm{H}_{5}\right], 161$ (4), 149 (17), 135 (9), 121 (5), 105 (10), 91 (25), 79 (20), 71 (26), 53 (13), 41 (100) $\left[\mathrm{C}_{3} \mathrm{H}_{5}^{+}\right] .-\mathrm{C}_{14} \mathrm{H}_{20} \mathrm{O}_{2}$ (220.3).

Fraktion II: $71 \mathrm{mg}$ (36\%) 115a und 115b als Regioisomerengemisch im Verhältnis $1.1: 1$ (aus $\left.{ }^{1} \mathrm{H}-\mathrm{NMR}-\mathrm{Spektrum}\right)$ als farbloses Öl, $R_{\mathrm{f}}\left(\mathrm{Pentan}_{\mathrm{Et}} \mathrm{E}_{2} \mathrm{O}\right.$,

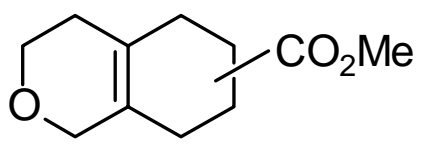
$5: 1)=0.27$. - IR (Film), Gemisch: $v=3409 \mathrm{~cm}^{-1}, 2952$, $1729(\mathrm{C}=\mathrm{O}), 1438,1380,1172,1070,1014,735,702$. 115a: ${ }^{1} \mathrm{H}-\mathrm{NMR}\left(250 \mathrm{MHz}, \mathrm{CDCl}_{3}\right): \delta=1.63-2.17(\mathrm{~m}$, $\left.8 \mathrm{H}, \mathrm{CH}_{2}\right), 2.53-2.64\left(\mathrm{~m}, 1 \mathrm{H}, \mathrm{CH}_{2}\right), 3.61-3.73\left(\mathrm{~m}, 1 \mathrm{H}, \mathrm{OCH}_{2}\right), 3.68\left(\mathrm{~s}, 3 \mathrm{H}, \mathrm{CO}_{2} \mathrm{Me}\right)$, 3.82-3.91 (m, $\left.3 \mathrm{H}, \mathrm{OCH}_{2}\right)$ - - 115b: ${ }^{1} \mathrm{H}-\mathrm{NMR}\left(250 \mathrm{MHz}, \mathrm{CDCl}_{3}\right): \delta=1.63-2.17(\mathrm{~m}, 8 \mathrm{H}$, $\mathrm{CH}_{2}$ ), 2.53-2.64 (m, $\left.1 \mathrm{H}, \mathrm{CH}_{2}\right), 3.61-3.73$ (m, $\left.1 \mathrm{H}, \mathrm{OCH}_{2}\right), 3.69$ (s, $\left.3 \mathrm{H}, \mathrm{CO}_{2} \mathrm{Me}\right), 3.82-$ $3.91\left(\mathrm{~m}, 3 \mathrm{H}, \mathrm{OCH}_{2}\right) .-{ }^{13} \mathrm{C}-\mathrm{NMR}\left(62.9 \mathrm{MHz}, \mathrm{CDCl}_{3}, \mathrm{DEPT}\right)$, Gemisch: $\delta=24.61(-$, $\left.\mathrm{CH}_{2}\right), 24.97,25.19\left(-, \mathrm{CH}_{2}\right), 27.49,28.75\left(-, \mathrm{CH}_{2}\right), 29.18,29.27\left(-, \mathrm{CH}_{2}\right), 39.25,39.44(+$, $\left.\mathrm{CHCO}_{2} \mathrm{Me}\right), 51.62\left(+, \mathrm{CO}_{2} \mathrm{CH}_{3}\right), 64.64\left(-, \mathrm{OCH}_{2}\right), 64.70\left(-, \mathrm{OCH}_{2}\right), 124.40,125.65$ $\left(\mathrm{C}_{\text {quart }}, \mathrm{C}-4 \mathrm{a}^{*}\right), 125.68,126.98\left(\mathrm{C}_{\text {quart }}, \mathrm{C}-8 \mathrm{a}^{*}\right), 175.85,175.94\left(\mathrm{C}_{\text {quart }}, \mathrm{CO}_{2} \mathrm{CH}_{3}\right) .-\mathrm{MS}$

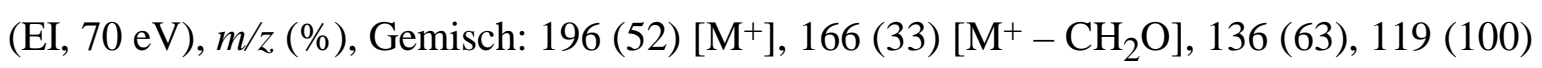
$\left[\mathrm{M}^{+}-\mathrm{CO}_{2} \mathrm{Me}-\mathrm{H}_{2} \mathrm{O}\right], 107(75)\left[\mathrm{M}^{+}-\mathrm{CH}_{2} \mathrm{O}-\mathrm{CO}_{2} \mathrm{Me}\right], 91$ (46), 79 (46), 67 (16), 43 (25). $-\mathrm{C}_{11} \mathrm{H}_{16} \mathrm{O}_{3}$ (196.2).

Variante B: Führte man die Reaktion unter identischen Bedingungen wie unter Variante A beschrieben durch, setzte jedoch kein Dienophil zu, so erhielt man nach säulenchromatographischer Reinigung Fraktion I: $9 \mathrm{mg} \quad(8 \%)$ 3,4Dimethylentetrahydropyran als farbloses Ö1, $R_{\mathrm{f}}\left(\operatorname{Pentan}_{\mathrm{Et}} \mathrm{O}, 10: 1\right)=0.53$. $-{ }^{1} \mathrm{H}-\mathrm{NMR}$ $\left(250 \mathrm{MHz}, \mathrm{CDCl}_{3}\right): \delta=2.42\left(\mathrm{t},{ }^{3} \mathrm{~J}=5.8 \mathrm{~Hz}, 2 \mathrm{H}, \mathrm{CH}_{2}\right), 3.78\left(\mathrm{t},{ }^{3} \mathrm{~J}=5.8 \mathrm{~Hz}, 2 \mathrm{H}, \mathrm{OCH}_{2}\right)$, 
4.14 (br s, $2 \mathrm{H}, \mathrm{OCH}_{2}$ ), 4.77 (br s, $2 \mathrm{H},=\mathrm{CH}_{2}$ ), 5.10 (br s, $1 \mathrm{H},=\mathrm{CH}_{2}$ ), 5.13 (br s, $1 \mathrm{H}$, $\left.=\mathrm{CH}_{2}\right) \cdot-\mathrm{C}_{7} \mathrm{H}_{10} \mathrm{O}(110.2)$.

Fraktion II: $63 \mathrm{mg}(29 \%) 116$ als farbloses Öl, $R_{\mathrm{f}}\left(\operatorname{Pentan} / \mathrm{Et}_{2} \mathrm{O}, 10: 1\right)=0.32$.

Variante C: Nach AAV 5 gab man zu $191 \mathrm{mg}$ (1.00 mmol) 1-Allyloxy-3-brom-but-3-en (114) in $10 \mathrm{ml}$ wasserfreiem $\mathrm{MeCN} 11 \mathrm{mg}(0.050 \mathrm{mmol}, 5.0 \mathrm{~mol} \%) \mathrm{Pd}(\mathrm{OAc})_{2}, 26 \mathrm{mg}$ (0.10 mmol, $10 \mathrm{~mol} \%) \mathrm{PPh}_{3}, 326 \mathrm{mg}(1.00 \mathrm{mmol}) \mathrm{Cs}_{2} \mathrm{CO}_{3}$ und $258 \mathrm{mg}$ (3.00 mmol) Methylacrylat. Nachdem man $20 \mathrm{~h}$ bei $80{ }^{\circ} \mathrm{C}$ rührte, reinigte man das Rohprodukt durch Säulenchromatographie an $18 \mathrm{~g}$ Kieselgel (Säule $2.0 \times 15 \mathrm{~cm}$, Pentan/Et ${ }_{2} \mathrm{O}=5: 1$ ). Man erhielt als einziges Produkt $88 \mathrm{mg}(45 \%)$ 115a,b als farbloses Öl, $R_{\mathrm{f}}\left(\operatorname{Pentan}_{\mathrm{Et}} \mathrm{\textrm {O }}\right.$, $5: 1)=0.25$.

1,8-Diallyloxyocta-3,5-diin (117): Analog AAV 5 gab man zu einer Lösung von $191 \mathrm{mg}$

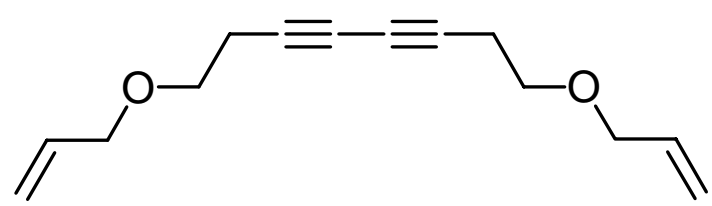

(1.00 mmol) 1-Allyloxy-3-brom-but3-en (114) in $10 \mathrm{ml}$ wasserfreiem $\mathrm{MeCN} \quad 11 \mathrm{mg} \quad(0.050 \mathrm{mmol}$, $5.0 \mathrm{~mol} \%) \quad \mathrm{Pd}(\mathrm{OAc})_{2}, \quad 26 \mathrm{mg}$ (0.10 mmol, $10 \mathrm{~mol} \%) \mathrm{PPh}_{3}, 276 \mathrm{mg}$ $(1.00 \mathrm{mmol}) \mathrm{Ag}_{2} \mathrm{CO}_{3}$ und $258 \mathrm{mg}(3.00 \mathrm{mmol})$ Methylacrylat. Man rührte $18 \mathrm{~h}$ bei $80{ }^{\circ} \mathrm{C}$. Säulenchromatographie des Rohprodukts an $18 \mathrm{~g}$ Kieselgel (Säule $2.0 \times 15 \mathrm{~cm}$, Pentan/Et $2 \mathrm{O}=5: 1)$ ergab $17 \mathrm{mg}(16 \%) 117$ als farbloses Öl, $R_{\mathrm{f}}$ (Pentan/Et ${ }_{2} \mathrm{O}$, $5: 1)=0.53$ sowie nicht weiter identifizierte Verunreinigungen. - IR (Film): $v=3055 \mathrm{~cm}^{-1}, 2923,1730,1265,1164,896,737,705 .-{ }^{1} \mathrm{H}-\mathrm{NMR}\left(250 \mathrm{MHz}, \mathrm{CDCl}_{3}\right)$ : $\delta=2.54\left(\mathrm{t},{ }^{3} J=7.0 \mathrm{~Hz}, 4 \mathrm{H}, 2,7-\mathrm{H}\right), 3.55\left(\mathrm{t},{ }^{3} J=7.0 \mathrm{~Hz}, 4 \mathrm{H}, 1,8-\mathrm{H}\right), 4.00\left(\mathrm{dt},{ }^{3} J=5.7\right.$, $\left.{ }^{4} J=1.4 \mathrm{~Hz}, 4 \mathrm{H}, 1^{\prime}, 1^{\prime \prime}-\mathrm{H}\right), 5.16-5.32$ (m, $\left.4 \mathrm{H}, 33^{\prime}, 3 "-\mathrm{H}\right), 5.82-5.95$ (m, $\left.2 \mathrm{H}, 2^{\prime}, 2^{\prime \prime}-\mathrm{H}\right)$. ${ }^{13} \mathrm{C}-\mathrm{NMR}\left(62.9 \mathrm{MHz}, \mathrm{CDCl}_{3}, \mathrm{DEPT}\right): \delta=20.61(-, 2 \mathrm{C}, \mathrm{C}-2, \mathrm{C}-7), 66.13\left(\mathrm{C}_{\text {quart }}, 2 \mathrm{C}\right.$, C-4, C-5), 67.82 (-, 2 C, C-1*, C-8*), 71.93 (-, 2 C, C-1'*, C-1"*), 74.36 (C quart 2 C, C-3, C-6), 117.29 (-, 2 C, C-3', C-3"), 134.40 (+, 2 C, C-2', C-2"). - MS (EI, 70 eV), m/z (\%): 162 (6), 131 (13), 111 (14), 103 (8), 91 (10), 81 (18), 71 (36), 53 (21), 41 (100) $\left[\mathrm{C}_{3} \mathrm{H}_{5}^{+}\right]$. MS (CI, $\left.\mathrm{NH}_{3}, 70 \mathrm{eV}\right), \mathrm{m} / \mathrm{z}(\%): 454$ (1) $\left[2 \mathrm{M}+\mathrm{NH}_{4}^{+}\right], 236(100)\left[\mathrm{M}+\mathrm{NH}_{4}^{+}\right] .-$ $\mathrm{C}_{14} \mathrm{H}_{18} \mathrm{O}_{2}$ (218.3). 


\subsection{Verwendung von stickstoffhaltigen Heterodienophilen}

Allgemeine Arbeitsvorschrift für Heck-Hetero-Diels-Alder-Reaktionen nach dem Zweischritt-Verfahren ( $A A V$ 6): In einer starkwandigen Pyrexflasche mit Schraubdeckel gibt man zu einer Lösung von $1 \mathrm{mmol}$ Bromdien und $3 \mathrm{mmol}$ Dienophil in $10 \mathrm{ml}$ wasserfreiem Acetonitril $5 \mathrm{~mol} \% \mathrm{Pd}(\mathrm{OAc})_{2}, 10 \mathrm{~mol}_{0} \mathrm{PPh}_{3}$ und 0.5 Äquivalente $\mathrm{K}_{2} \mathrm{CO}_{3}$. Man leitet 5 min Stickstoff durch das Reaktionsgemisch, verschließt die Flasche und rührt die angegebene Zeit bei $80{ }^{\circ} \mathrm{C}$. Nach Abkühlen auf Raumtemp. versetzt man mit $1 \mathrm{ml}$ HCHO-Lösung ( $37 \%$ in $\mathrm{H}_{2} \mathrm{O}$ ), 2 mmol Amin-Hydrochlorid und $8 \mathrm{ml}$ Wasser und rührt die angegebene Zeit bei Raumtemp. Anschließend filtriert man über Celite und wäscht mit $10 \mathrm{ml}$ Wasser nach. Die vereinigten Filtrate werden mit $20 \mathrm{ml}$ Diethylether extrahiert, die wäßrige Phase mit $2 \mathrm{M} \mathrm{NaOH}$ auf etwa $\mathrm{pH} 13$ gebracht und erneut mit $3 \times 20 \mathrm{ml}$ Diethylether extrahiert. Die vereingten organischen Phasen werden mit $20 \mathrm{ml}$ ges. NaClLösung gewaschen, über $\mathrm{Na}_{2} \mathrm{SO}_{4}$ getrocknet und das Lösungsmittel i. Vak. entfernt. Das verbleibende Rohprodukt wird durch Säulenchromatographie an Kieselgel gereinigt.

(7-Hydroxy-6,6-dimethyl-1,3,4,5,6,7-hexahydro-2H-cyclopenta[c]pyridin-2-yl)essigsäuremethylester (126-Gly): Variante A: Nach AAV 6 gab man zu 219 mg (1.00 mmol) 6-Brom4,4-dimethylhepta-1,6-dien-3-ol in $10 \mathrm{ml}$ wasserfreiem MeCN $11 \mathrm{mg}$ (0.050 mmol, $5.0 \mathrm{~mol} \%) \mathrm{Pd}(\mathrm{OAc})_{2}, 26 \mathrm{mg}(0.10 \mathrm{mmol}, 10 \mathrm{~mol} \%) \mathrm{PPh}_{3}$ und $69 \mathrm{mg}(0.50 \mathrm{mmol}) \mathrm{K}_{2} \mathrm{CO}_{3}$ und rührte $18 \mathrm{~h}$ bei $80^{\circ} \mathrm{C}$. Nach Abkühlen auf Raumtemp. versetzte man mit $1.0 \mathrm{ml}$ (12 mmol, 37\% in $\left.\mathrm{H}_{2} \mathrm{O}\right)$ HCHO-Lösung, $251 \mathrm{mg}$ (2.00 mmol) GlycinmethylesterHydrochlorid und $8 \mathrm{ml} \mathrm{H}_{2} \mathrm{O}$ und ließ weitere $18 \mathrm{~h}$ bei Raumtemp. rühren. Nach Aufarbeitung wie unter AAV 6 beschrieben wurde das so erhaltene Rohprodukt durch Säulenchromatographie an $18 \mathrm{~g}$ Kieselgel (Säule $2.0 \times 15 \mathrm{~cm}, \mathrm{CH}_{2} \mathrm{Cl}_{2} / \mathrm{MeOH}=20: 1$, nach Durchlaufen der Verunreinigungen Wechsel zu $10: 1$ ) gereinigt. Man erhielt $92 \mathrm{mg}$ (38\%) 126-Gly als farbloses Öl, $R_{\mathrm{f}}\left(\mathrm{CH}_{2} \mathrm{Cl}_{2} / \mathrm{MeOH}, 10: 1\right)=0.21$. - IR (Film): $v=3340 \mathrm{~cm}^{-1}(\mathrm{OH}), 2955,1749(\mathrm{C}=\mathrm{O}), 1646,1437$,

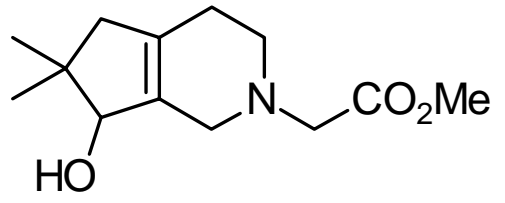
1364, 1205, 1122, 1004, 701, 677, 669. - ${ }^{1} \mathrm{H}-\mathrm{NMR}$ $\left(250 \mathrm{MHz}, \mathrm{CDCl}_{3}\right): \delta=1.03\left(\mathrm{~s}, 3 \mathrm{H}, 6-\mathrm{CH}_{3}\right), 1.06(\mathrm{~s}$, $\left.3 \mathrm{H}, 6-\mathrm{CH}_{3}\right), 1.95-2.21(\mathrm{~m}, 4 \mathrm{H}, 4,5-\mathrm{H}), 2.71\left(\mathrm{~m}_{\mathrm{c}}, 2 \mathrm{H}\right.$, 1-H*), 3.14-3.22 (m, $2 \mathrm{H}, 3-\mathrm{H}), 3.37\left(\mathrm{~s}, 2 \mathrm{H}, \mathrm{NCH}_{2}\right) 3.73\left(\mathrm{~s}, 3 \mathrm{H}, \mathrm{CO}_{2} \mathrm{CH}_{3}\right.$ ), 4.03 (br s, 
$1 \mathrm{H}, 7-\mathrm{H}) .-{ }^{13} \mathrm{C}-\mathrm{NMR}\left(62.9 \mathrm{MHz}, \mathrm{CDCl}_{3}, \mathrm{DEPT}\right): \delta=22.96\left(+, 6-\mathrm{CH}_{3}\right), 26.50(-, \mathrm{C}-4 *)$, $28.81\left(+, 6-\mathrm{CH}_{3}\right), 41.42\left(\mathrm{C}_{\text {quart }}, \mathrm{C}-6\right), 48.65\left(-, \mathrm{C}-5^{*}\right), 49.89(-, \mathrm{C}-3 * *), 50.87\left(-, \mathrm{C}-1^{* *}\right)$, $51.72\left(+, \mathrm{CO}_{2} \mathrm{CH}_{3}\right), 58.84\left(-, \mathrm{CH}_{2} \mathrm{CO}_{2} \mathrm{Me}^{*}\right), 85.68$ (+, C-7), $133.50\left(\mathrm{C}_{\text {quart }}, \mathrm{C}-4 \mathrm{a}^{* * *}\right)$, $136.74\left(\mathrm{C}_{\text {quart }}, \mathrm{C}-7 \mathrm{a} * * *\right), 170.95\left(\mathrm{C}_{\text {quart }}, \mathrm{CO}_{2} \mathrm{CH}_{3}\right) .-\mathrm{MS}(\mathrm{EI}, 70 \mathrm{eV}), \mathrm{m} / z(\%): 239$ (2)

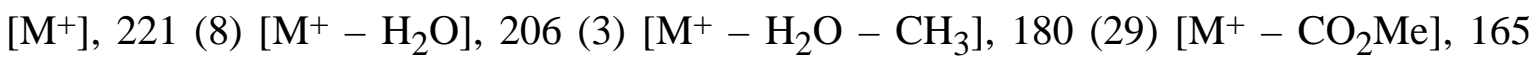
(100) $\left[\mathrm{M}^{+}-\mathrm{CO}_{2} \mathrm{Me}-\mathrm{CH}_{3}\right], 150$ (6) $\left[\mathrm{M}^{+}-\mathrm{CO}_{2} \mathrm{Me}-2 \mathrm{CH}_{3}\right], 132$ (5) $\left[\mathrm{M}^{+}-\mathrm{CO}_{2} \mathrm{Me}-\right.$ $2 \mathrm{CH}_{3}-\mathrm{H}_{2} \mathrm{O}$ ], 107 (7), 91 (4), 77 (5), 42 (10). $-\mathrm{C}_{13} \mathrm{H}_{21} \mathrm{NO}_{3}$ (239.3): ber. 239.1521 (HRMS korrekt).

Variante B: Bei Verwendung von $138 \mathrm{mg}(1.00 \mathrm{mmol}) \mathrm{K}_{2} \mathrm{CO}_{3}$ verringerte sich die Ausbeute auf $63 \mathrm{mg}(26 \%)$.

Variante $C$ : Bei Verwendung von $138 \mathrm{mg}(0.500 \mathrm{mmol}) \mathrm{Ag}_{2} \mathrm{CO}_{3}$ als Base konnten $27 \mathrm{mg}$ $(11 \%)$ isoliert werden.

Variante D: Verwendete man statt $8 \mathrm{ml}$ Wasser die gleiche Menge Methanol, sank die Ausbeute auf $10 \mathrm{mg}(4 \%)$.

Variante E: Führte man die Hetero-Diels-Alder-Reaktion bei $10 \mathrm{kbar}$ unter sonst identischen Bedingungen wie unter Variante A beschrieben durch, konnten nur Zersetzungsprodukte und oligomeres Material isoliert werden.

Variante F: Erhöhung der Temperatur bei der Diels-Alder-Reaktion auf $50{ }^{\circ} \mathrm{C}$ führte zur Bildung von nicht identifizierten Nebenprodukten, die Ausbeute von 126-Gly verringerte sich auf $9 \mathrm{mg}(4 \%)$

(6,6-Dimethyl-1,3,5,6-tetrahydro-2H-cyclopenta[c]pyridin-2-yl)essigsäuremethylesterHydrochlorid (127-Gly): Man löste $88.0 \mathrm{mg}$ (0.368 mmol) 126-Gly in $3 \mathrm{ml}$ Methanol und

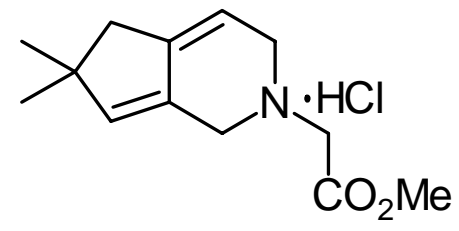
gab vorsichtig einige Tropfen konz. $\mathrm{HCl}$ zu. Anschließend ließ man 30 min rühren und entfernte das Lösungsmittel i. Vak. Man erhielt $81 \mathrm{mg}(85 \%)$ 127-Gly als farblosen Feststoff, Zers. $168-169{ }^{\circ} \mathrm{C} .-{ }^{1} \mathrm{H}-\mathrm{NMR}\left(250 \mathrm{MHz}, \mathrm{CDCl}_{3}\right)$ : $\delta=1.16\left(\mathrm{~s}, 6 \mathrm{H}, 6-\mathrm{CH}_{3}\right), 2.44($ br s, $2 \mathrm{H}, 5-\mathrm{H}), 3.82\left(\mathrm{~s}, 3 \mathrm{H}, \mathrm{CO}_{2} \mathrm{CH}_{3}\right), 3.91$ (br s, $2 \mathrm{H}$, $\mathrm{NCH}_{2}$ ), 4.03 (br s, $2 \mathrm{H}, \mathrm{NCH}_{2}$ ), 4.21 (br s, $2 \mathrm{H}, \mathrm{NCH}_{2}$ ), 5.34 (br s, $1 \mathrm{H}, 4-\mathrm{H}^{*}$ ), 5.98 (br s, $\left.1 \mathrm{H}, 7-\mathrm{H}^{*}\right) .-{ }^{13} \mathrm{C}-\mathrm{NMR}\left(62.9 \mathrm{MHz}, \mathrm{CDCl}_{3}, \mathrm{DEPT}\right): \delta=28.59\left(+, 6-\mathrm{CH}_{3}\right), 43.03\left(\mathrm{C}_{\text {quart }}\right.$, C-6), 44.97 (-, C-5), $50.04\left(-, \mathrm{NCH}_{2}\right), 50.07\left(-, \mathrm{NCH}_{2}\right), 52.01\left(-, \mathrm{NCH}_{2}\right), 53.13(+$, $\left.\mathrm{CO}_{2} \mathrm{CH}_{3}\right), 105.51(+, \mathrm{C}-4 *), 126.91\left(+, \mathrm{C}-7^{*}\right), 144.76\left(\mathrm{C}_{\text {quart }}, \mathrm{C}-4 \mathrm{a}^{*}\right), 147.00\left(\mathrm{C}_{\text {quart }}\right.$, 
C-7a*), $165.36\left(\mathrm{C}_{\text {quart }}, \mathrm{CO}_{2} \mathrm{CH}_{3}\right) .-\mathrm{MS}(\mathrm{EI}, 70 \mathrm{eV}), \mathrm{m} / \mathrm{z}(\%): 221(43)\left[\mathrm{M}^{+}-\mathrm{HCl}\right], 206$ (76) $\left[\mathrm{M}^{+}-\mathrm{HCl}-\mathrm{CH}_{3}\right], 192$ (37) $\left[\mathrm{M}^{+}-\mathrm{Cl}-\mathrm{CH}_{3}\right], 162$ (89) $\left[\mathrm{M}^{+}-\mathrm{HCl}-\mathrm{CO}_{2} \mathrm{Me}\right], 148$ (100) $\left[\mathrm{M}^{+}-\mathrm{Cl}-\mathrm{CO}_{2} \mathrm{Me}-\mathrm{CH}_{3}\right], 133(48)\left[\mathrm{M}^{+}-\mathrm{Cl}-\mathrm{CO}_{2} \mathrm{Me}-2 \mathrm{CH}_{3}\right], 119$ (22), 107 (42), 91 (50), 77 (25), 42 (38). $-\mathrm{C}_{13} \mathrm{H}_{20} \mathrm{ClNO}_{2}$ (257.8).

N-Benzyl-(7-hydroxy-6,6-dimethyl-1,3,4,5,6,7-hexahydro-2H-cyclopenta[c]-2-pyridin) (126-Bn): Nach AAV 6 versetzte man $219 \mathrm{mg}$ (1.00 mmol) 6-Brom-4,4-dimethylhepta-

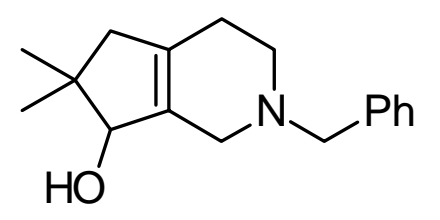
1,6-dien-3-ol in $10 \mathrm{ml}$ wasserfreiem $\mathrm{MeCN}$ mit $11 \mathrm{mg}$ $(0.050 \mathrm{mmol}, \quad 5.0 \mathrm{~mol} \%) \quad \mathrm{Pd}(\mathrm{OAc})_{2}, \quad 26 \mathrm{mg} \quad(0.10 \mathrm{mmol}$, $10 \mathrm{~mol} \%) \mathrm{PPh}_{3}$ und $69 \mathrm{mg}(0.50 \mathrm{mmol}) \mathrm{K}_{2} \mathrm{CO}_{3}$ und rührte $18 \mathrm{~h}$ bei $80{ }^{\circ} \mathrm{C}$. Nach Abkühlen auf Raumtemp. gab man $1.0 \mathrm{ml}$ (12 mmol, 37\% in $\mathrm{H}_{2} \mathrm{O}$ ) HCHO-Lösung, $287 \mathrm{mg}$ (2 mmol) Benzylamin-Hydrochlorid und $8 \mathrm{ml} \mathrm{H}_{2} \mathrm{O}$ zu und ließ weitere $18 \mathrm{~h}$ bei Raumtemp. rühren. Das Rohprodukt wurde durch Säulenchromatographie an $18 \mathrm{~g}$ Kieselgel (Säule $2.0 \times 15 \mathrm{~cm}, \mathrm{CH}_{2} \mathrm{Cl}_{2} / \mathrm{MeOH}=20: 1$ ) gereinigt. Man erhielt $118 \mathrm{mg}(46 \%)$ 126-Bn als farbloses Öl, $R_{\mathrm{f}}\left(\mathrm{CH}_{2} \mathrm{Cl}_{2} / \mathrm{MeOH}\right.$, $20: 1)=0.15$. - IR (Film): $v=3358 \mathrm{~cm}^{-1}(\mathrm{OH}), 3062,3028,2953,2900,2838,1667$, 1602, 1454, 1362, 1265, 1199, 1065, 1028, 1004, 738, 699. - 1H-NMR (250 MHz, $\left.\mathrm{CDCl}_{3}\right): \delta=1.04\left(\mathrm{~s}, 3 \mathrm{H}, 6-\mathrm{CH}_{3}\right), 1.06\left(\mathrm{~s}, 3 \mathrm{H}, 6-\mathrm{CH}_{3}\right), 1.95-2.22(\mathrm{~m}, 4 \mathrm{H}, 4,5-\mathrm{H}), 2.60$ (m $\left.\mathrm{c}_{\mathrm{c}}, 2 \mathrm{H}, 1-\mathrm{H}\right), 2.92-3.02(\mathrm{~m}, 1 \mathrm{H}, 3-\mathrm{H}) 3.12-3.19(\mathrm{~m}, 1 \mathrm{H}, 3-\mathrm{H}), 3.65\left(\mathrm{~s}, 1 \mathrm{H}, \mathrm{PhCH}_{2}\right)$, $3.66\left(\mathrm{~s}, 1 \mathrm{H}, \mathrm{PhCH}_{2}\right.$ ), 4.02 (br s, $\left.1 \mathrm{H}, 7-\mathrm{H}\right), 7.24-7.37$ (m, $\left.5 \mathrm{H}, \mathrm{Ph}-\mathrm{H}\right) .-{ }^{13} \mathrm{C}-\mathrm{NMR}$ $\left(62.9 \mathrm{MHz}, \mathrm{CDCl}_{3}, \mathrm{DEPT}\right): \delta=23.04\left(+, 6-\mathrm{CH}_{3}\right), 26.57(-, \mathrm{C}-4 *), 28.89\left(+, 6-\mathrm{CH}_{3}\right)$, $41.44\left(\mathrm{C}_{\text {quart }}, \mathrm{C}-6\right), 48.68\left(-, \mathrm{C}-5^{*}\right), 49.74\left(-, \mathrm{C}-3^{* *}\right), 51.37\left(-, \mathrm{C}-1^{* *}\right), 62.70\left(-, \mathrm{CH}_{2} \mathrm{Ph}\right)$, 85.70 (+, C-7), 127.19 (+, Ph-C), 128.25 (+, Ph-C), $129.30\left(\mathrm{C}_{\mathrm{quart}}, \mathrm{C}-4 \mathrm{a}^{* * *}\right), 129.44$ (+, $\mathrm{Ph}-\mathrm{C}), 133.84\left(\mathrm{C}_{\text {quart }}, \mathrm{Ph}-\mathrm{C}^{* * *}\right), 136.74\left(\mathrm{C}_{\text {quart }}, \mathrm{C}-7 \mathrm{a} * * *\right), 170.95\left(\mathrm{C}_{\text {quart }}, \mathrm{CO}_{2} \mathrm{CH}_{3}\right)$. MS (EI, $70 \mathrm{eV}), m / z(\%): 257$ (7) $\left[\mathrm{M}^{+}\right], 240$ (9) $\left[\mathrm{M}^{+}-\mathrm{OH}\right], 224(12)\left[\mathrm{M}^{+}-\mathrm{H}_{2} \mathrm{O}-\mathrm{CH}_{3}\right]$, 201 (21), 185 (35), 172 (4), 132 (3), 120 (4), 91 (4) $\left[\mathrm{C}_{7} \mathrm{H}_{7}{ }^{+}\right], 65$ (6), 41 (3). - $\mathrm{C}_{17} \mathrm{H}_{23} \mathrm{NO}$ (257.4).

Versuch der Darstellung von 7-Hydroxy-6,6-dimethyl-1,3,4,5,6,7-hexahydro-2Hcyclopenta[c]-2-pyridin (126-H): Nach AAV 6 gab man zu 219 mg (1.00 mmol) 6-Brom4,4-dimethylhepta-1,6-dien-3-ol in $10 \mathrm{ml}$ wasserfreiem $\mathrm{MeCN} 11 \mathrm{mg}$ (0.050 mmol, $5.0 \mathrm{~mol} \%) \mathrm{Pd}(\mathrm{OAc})_{2}, 26 \mathrm{mg}(0.10 \mathrm{mmol}, 10 \mathrm{~mol} \%) \mathrm{PPh}_{3}$ und $69 \mathrm{mg}(0.50 \mathrm{mmol}) \mathrm{K}_{2} \mathrm{CO}_{3}$ 
und rührte $18 \mathrm{~h}$ bei $80^{\circ} \mathrm{C}$. Nach Abkühlen auf Raumtemp. versetzte man mit $1.0 \mathrm{ml}$ (12 mmol, 37\% in $\mathrm{H}_{2} \mathrm{O}$ ) HCHO-Lösung, $107 \mathrm{mg}$ (2.00 mmol) Ammoniumchlorid und $8 \mathrm{ml}$ $\mathrm{H}_{2} \mathrm{O}$ und ließ weitere $18 \mathrm{~h}$ bei Raumtemp. rühren. Das ${ }^{1} \mathrm{H}-\mathrm{NMR}-$ Spektrum des Rohproduktes zeigte keinerlei Signale des erwarteten Produktes 126-H.

N-Cyclopropyl-(7-hydroxy-6,6-dimethyl-1,3,4,5,6,7-hexahydro-2H-cyclopenta[c]-2pyridin) (126-cPr): Nach AAV 6 gab man zu $219 \mathrm{mg}$ (1.00 mmol) 6-Brom-4,4-

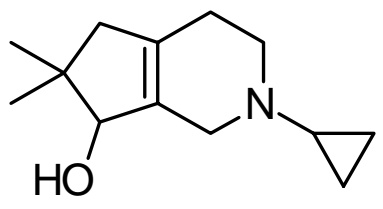
dimethylhepta-1,6-dien-3-ol in $10 \mathrm{ml}$ wasserfreiem $\mathrm{MeCN}$ $11 \mathrm{mg}(0.050 \mathrm{mmol}, 5.0 \mathrm{~mol} \%) \mathrm{Pd}(\mathrm{OAc})_{2}, 26 \mathrm{mg}(0.10 \mathrm{mmol}$, $10 \mathrm{~mol} \%) \mathrm{PPh}_{3}$ und $69 \mathrm{mg}(0.50 \mathrm{mmol}) \mathrm{K}_{2} \mathrm{CO}_{3}$ und rührte $18 \mathrm{~h}$ bei $80{ }^{\circ} \mathrm{C}$. Nach Abkühlen auf Raumtemp. versetzte man mit $1.0 \mathrm{ml}$ (12 mmol, 37\% in $\mathrm{H}_{2} \mathrm{O}$ ) HCHO-Lösung, $187 \mathrm{mg}$ (2.00 mmol) CyclopropylaminHydrochlorid und $8 \mathrm{ml} \mathrm{H}_{2} \mathrm{O}$ und ließ weitere $18 \mathrm{~h}$ bei Raumtemp. rühren. Nach Aufarbeitung wie unter AAV 6 beschrieben, wurde das so erhaltene Rohprodukt durch Säulenchromatographie an $18 \mathrm{~g}$ Kieselgel (Säule $2.0 \times 15 \mathrm{~cm}, \mathrm{CH}_{2} \mathrm{Cl}_{2} / \mathrm{MeOH}=10: 1$ ) gereinigt. Man erhielt $74 \mathrm{mg}(36 \%)$ 126-c $\mathbf{P r}$ als farbloses Öl, $R_{\mathrm{f}}\left(\mathrm{CH}_{2} \mathrm{Cl}_{2} / \mathrm{MeOH}\right.$, $10: 1)=0.31$. - IR (Film): $v=3368 \mathrm{~cm}^{-1}(\mathrm{OH}), 3087,2953,2908,2839,1669,1459$, 1362, 1265, 1219, 1057, 1018, 1002, 869, 826, 737, 702. - ${ }^{1} \mathrm{H}-\mathrm{NMR}\left(250 \mathrm{MHz}, \mathrm{CDCl}_{3}\right)$ : $\delta=0.46-0.50(\mathrm{~m}, 4 \mathrm{H}, c \mathrm{Pr}-\mathrm{H}), 1.02\left(\mathrm{~s}, 3 \mathrm{H}, 6-\mathrm{CH}_{3}\right), 1.06\left(\mathrm{~s}, 3 \mathrm{H}, 6-\mathrm{CH}_{3}\right), 1.69-1.77(\mathrm{~m}$, $1 \mathrm{H}, c \mathrm{Pr}-\mathrm{H}), 1.93-2.20$ (m, $4 \mathrm{H}, 4,5-\mathrm{H}), 2.75$ (m, 2 H, 1-H*), 3.06-3.25 (m, $\left.2 \mathrm{H}, 3-\mathrm{H}^{*}\right)$, 4.03 (br s, $1 \mathrm{H}, 7-\mathrm{H}) .-{ }^{13} \mathrm{C}-\mathrm{NMR}\left(75.5 \mathrm{MHz}, \mathrm{CDCl}_{3}, \mathrm{APT}\right): \delta=5.94$ (-, cPr-C), $23.03(+$, 6- $\left.\mathrm{CH}_{3}\right), 26.65$ (-, C-4*), $28.88\left(+, 6-\mathrm{CH}_{3}\right), 38.17$ (+, cPr-C), 41.44 (-, C-6), 48.76 (-, C-5*), 50.51 (-, C-3**), 51.31 (-, C-1**), 85.75 (+, C-7), 134.12 (-, C-4a***), 136.38 (-,

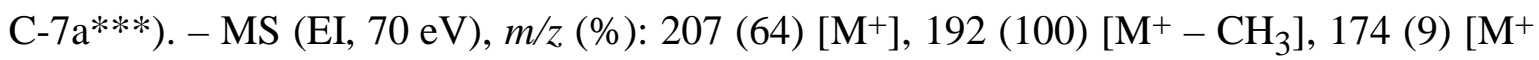
$\left.-\mathrm{H}_{2} \mathrm{O}-\mathrm{CH}_{3}\right], 151$ (4) $\left[\mathrm{M}^{+}-\mathrm{CH}_{3}-\mathrm{C}_{3} \mathrm{H}_{5}\right], 135$ (10), 121 (6), 107 (5), 91 (6), 84 (26), 70 (14), 56 (15), 41 (22) $\left[\mathrm{C}_{3} \mathrm{H}_{5}^{+}\right] .-\mathrm{C}_{13} \mathrm{H}_{21} \mathrm{NO}$ (207.3).

N-Cyclopentyl-(7-hydroxy-6,6-dimethyl-1,3,4,5,6,7-hexahydro-2H-cyclopenta[c]-2pyridin) (126-cPent): Variante A: Analog AAV 6 löste man 219 mg (1.00 mmol) 6-Brom4,4-dimethylhepta-1,6-dien-3-ol in $10 \mathrm{ml}$ wasserfreiem $\mathrm{MeCN}$ und fügte $11 \mathrm{mg}$ $(0.050 \mathrm{mmol}, 5.0 \mathrm{~mol} \%) \mathrm{Pd}(\mathrm{OAc})_{2}, 26 \mathrm{mg}(0.10 \mathrm{mmol}, 10 \mathrm{~mol} \%) \mathrm{PPh}_{3}$ und $69 \mathrm{mg}$ (0.50 mmol) $\mathrm{K}_{2} \mathrm{CO}_{3}$ hinzu. Man rührte $18 \mathrm{~h}$ bei $80{ }^{\circ} \mathrm{C}$, ließ auf Raumtemp. abkühlen und 
versetzte mit $1.0 \mathrm{ml}\left(12 \mathrm{mmol}, 37 \%\right.$ in $\left.\mathrm{H}_{2} \mathrm{O}\right)$ HCHO-Lösung, $243 \mathrm{mg}$ (2.00 mmol) Cyclopentylamin-Hydrochlorid und $8 \mathrm{ml} \mathrm{H}_{2} \mathrm{O}$. Man rührte weitere $18 \mathrm{~h}$ bei Raumtemp. Nach Aufarbeitung wurde das erhaltene Rohprodukt durch Säulenchromatographie an $18 \mathrm{~g}$ Kieselgel (Säule $\left.2.0 \times 15 \mathrm{~cm}, \mathrm{CH}_{2} \mathrm{Cl}_{2} / \mathrm{MeOH}=10: 1\right)$ gereinigt. Man erhielt $72 \mathrm{mg}(31 \%)$ 126-cPent als farbloses Öl, $R_{\mathrm{f}}\left(\mathrm{CH}_{2} \mathrm{Cl}_{2} / \mathrm{MeOH}, 10: 1\right)=0.13$. - IR (Film): $v=3437 \mathrm{~cm}^{-1}$

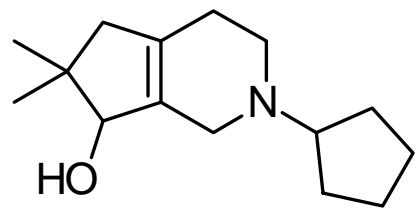
$(\mathrm{OH}), 3053,2956,2864,2306,1696,1464,1381,1361,1265$, 1053, 894, 868, 736, 703. - ${ }^{1} \mathrm{H}-\mathrm{NMR}\left(250 \mathrm{MHz}, \mathrm{CDCl}_{3}\right)$ : $\delta=1.02\left(\mathrm{~s}, 3 \mathrm{H}, 6-\mathrm{CH}_{3}\right), 1.06\left(\mathrm{~s}, 3 \mathrm{H}, 6-\mathrm{CH}_{3}\right), 1.48-1.76$ (m, $7 \mathrm{H}, c$ Pent-H* $\left.{ }^{*} \mathrm{OH}\right), 1.88-2.07$ (m, $3 \mathrm{H}, c$ Pent,4,5-H*), 2.15-2.27 (m, $3 \mathrm{H}, c$ Pent,4,5-H*), 2.58-2.82 (m, $3 \mathrm{H}, c$ Pent,1-H**), 2.97-3.13 (m, $1 \mathrm{H}$, 3-H**), 3.22-3.38 (m, $\left.1 \mathrm{H}, 3-\mathrm{H}^{* *}\right), 4.00$ (br s, $\left.1 \mathrm{H}, 7-\mathrm{H}\right) .-{ }^{13} \mathrm{C}-\mathrm{NMR}\left(62.9 \mathrm{MHz}, \mathrm{CDCl}_{3}\right.$, DEPT): $\delta=23.00\left(+, 6-\mathrm{CH}_{3}\right), 24.02\left(-, c\right.$ Pent-C*), $26.13(-, \mathrm{C}-4 *), 28.86\left(+, 6-\mathrm{CH}_{3}\right)$, 30.23 (-, cPent-C*), $41.46\left(\mathrm{C}_{\text {quart }}, \mathrm{C}-6\right), 48.50$ (-, C-5*), 49.08 (-, C-1**), 50.24 (-, C-3**), 67.12 (+, cPent-C), 85.48 (+, C-7), 133.04 (-, C-4a***), 136.97 (-, C-7a***). MS (EI, $70 \mathrm{eV}), \mathrm{m} / \mathrm{z}(\%): 235(62)\left[\mathrm{M}^{+}\right], 218(15)\left[\mathrm{M}^{+}-\mathrm{OH}\right], 206(70), 192(28)\left[\mathrm{M}^{+}{ }_{-}\right.$ $\left.\mathrm{C}_{2} \mathrm{H}_{5} \mathrm{~N}\right], 179(45)\left[\mathrm{M}^{+}-\mathrm{C}_{3} \mathrm{H}_{6} \mathrm{~N}\right], 163(100)\left[\mathrm{M}^{+}-\mathrm{C}_{4} \mathrm{H}_{10} \mathrm{~N}\right], 150$ (6), 123 (7), 98 (10), 84 (8), 68 (14), 41 (22) $\left[\mathrm{C}_{3} \mathrm{H}_{5}{ }^{+}\right] .-\mathrm{C}_{15} \mathrm{H}_{25} \mathrm{NO}$ (235.4).

Variante B: Führte man die Reaktion in $10 \mathrm{ml}$ wasserfreiem THF anstelle von MeCN durch so erhielt man Fraktion I: 62 mg (45\%) 1,2-Dimethylen-4,4-dimethylcyclopentan-3-ol (60)

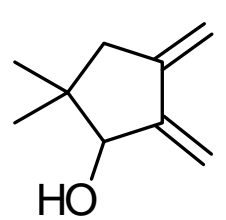
als farbloses Öl, $R_{\mathrm{f}}\left(\operatorname{Pentan} / \mathrm{Et}_{2} \mathrm{O}, 4: 1\right)=0.45 .-{ }^{1} \mathrm{H}-\mathrm{NMR}(250 \mathrm{MHz}$, $\left.\mathrm{CDCl}_{3}\right): \delta=0.82\left(\mathrm{~s}, 3 \mathrm{H}, 4-\mathrm{CH}_{3}\right), 1.07\left(\mathrm{~s}, 3 \mathrm{H}, 4-\mathrm{CH}_{3}\right), 1.49$ (d, $3 J=7.9 \mathrm{~Hz}, 1 \mathrm{H}, \mathrm{OH}), 2.22\left(\mathrm{~m}_{\mathrm{c}}, 2 \mathrm{H}, 5-\mathrm{H}\right), 4.06\left(\mathrm{ddd},{ }^{3} \mathrm{~J}=7.9,{ }^{4} \mathrm{~J}=2.3\right.$, $\left.{ }^{4} J=2.3 \mathrm{~Hz}, 1 \mathrm{H}, 3-\mathrm{H}\right), 4.90\left(\mathrm{~m}_{\mathrm{c}}, 1 \mathrm{H}, 1-\mathrm{CH}_{2}{ }^{*}\right), 5.10\left(\mathrm{~d},{ }^{2} J=2.2 \mathrm{~Hz}, 1 \mathrm{H}\right.$, 2- $\left.\mathrm{CH}_{2}{ }^{*}\right), 5.43\left(\mathrm{~m}_{\mathrm{c}}, 1 \mathrm{H}, 1-\mathrm{CH}_{2}{ }^{*}\right), 5.48\left(\mathrm{~d},{ }^{2} \mathrm{~J}=2.2 \mathrm{~Hz}, 1 \mathrm{H}, 2-\mathrm{CH}_{2}{ }^{*}\right) .-{ }^{13} \mathrm{C}-\mathrm{NMR}$ $\left(62.9 \mathrm{MHz}, \mathrm{CDCl}_{3}, \mathrm{DEPT}\right): \delta=19.61\left(+, 4-\mathrm{CH}_{3}\right), 25.65\left(+, 4-\mathrm{CH}_{3}\right), 39.75\left(\mathrm{C}_{\text {quart }}, \mathrm{C}-4\right)$, 44.33 (-, C-5), 82.57 (+, C-3), $105.44\left(-, 1-\mathrm{CH}_{2}{ }^{*}\right), 105.90\left(-, 2-\mathrm{CH}_{2}{ }^{*}\right), 143.95\left(\mathrm{C}_{\text {quart }}\right.$, C-1**), 150.89 (C quart, $\left.\mathrm{C}-2^{* *}\right) .-\mathrm{MS}(\mathrm{EI}, 70 \mathrm{eV}), \mathrm{m} / \mathrm{z}(\%): 138(33)\left[\mathrm{M}^{+}\right], 123(100)\left[\mathrm{M}^{+}\right.$ $\left.-\mathrm{CH}_{3}\right], 109$ (12), 105 (20) [M+ $\left.-\mathrm{CH}_{3}-\mathrm{H}_{2} \mathrm{O}\right], 95$ (73), 91 (11), 79 (16), 67 (30), 55 (16), 43 (16), 41 (13) $\left[\mathrm{C}_{3} \mathrm{H}_{5}^{+}\right] .-\mathrm{C}_{9} \mathrm{H}_{14} \mathrm{O}$ (235.4).

Fraktion II: 23 mg (10\%) 126-cPent. 
N-Cyclohexyl-(7-hydroxy-6,6-dimethyl-1,3,4,5,6,7-hexahydro-2H-cyclopenta[c]-2-pyridin)

(126-cHex): Nach AAV 6 versetzte man 219 mg (1.00 mmol) 6-Brom-4,4-dimethylhepta-

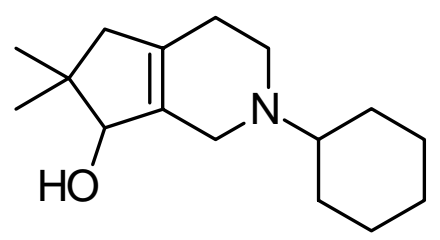

1,6-dien-3-ol in $10 \mathrm{ml}$ wasserfreiem $\mathrm{MeCN}$ mit $11 \mathrm{mg}$ $(0.050 \mathrm{mmol}, \quad 5.0 \mathrm{~mol} \%) \quad \mathrm{Pd}(\mathrm{OAc})_{2}, \quad 26 \mathrm{mg} \quad(0.10 \mathrm{mmol}$, $10 \mathrm{~mol} \%) \mathrm{PPh}_{3}$ und $69 \mathrm{mg}(0.50 \mathrm{mmol}) \mathrm{K}_{2} \mathrm{CO}_{3}$ und rührte $18 \mathrm{~h}$ bei $80^{\circ} \mathrm{C}$. Nach Abkühlen auf Raumtemp. gab man $1.0 \mathrm{ml}$ (12 mmol, 37\% in $\left.\mathrm{H}_{2} \mathrm{O}\right)$ HCHO-Lösung, $271 \mathrm{mg}$ ( $2.00 \mathrm{mmol}$ ) Cyclohexylamin-Hydrochlorid und $8 \mathrm{ml} \mathrm{H}_{2} \mathrm{O} \mathrm{zu}$ und ließ weitere $18 \mathrm{~h}$ bei Raumtemp. rühren. Das Rohprodukt wurde durch Säulenchromatographie an $18 \mathrm{~g}$ Kieselgel (Säule $\left.2.0 \times 15 \mathrm{~cm}, \mathrm{CH}_{2} \mathrm{Cl}_{2} / \mathrm{MeOH}=10: 1\right)$ gereinigt. Man erhielt $73 \mathrm{mg}(29 \%)$ 126-cHex als farbloses Öl, $R_{\mathrm{f}}\left(\mathrm{CH}_{2} \mathrm{Cl}_{2} / \mathrm{MeOH}, 10: 1\right)=0.23$. - IR (Film): $v=3352 \mathrm{~cm}^{-1}$ (OH), 2930, 2856, 1668, 1451, 1380, 1264, 1205, 1135, 1069, 1036, 1003, 894, 737, 702. ${ }^{1} \mathrm{H}-\mathrm{NMR}\left(250 \mathrm{MHz}, \mathrm{CDCl}_{3}\right): \delta=1.03\left(\mathrm{~s}, 3 \mathrm{H}, 6-\mathrm{CH}_{3}\right), 1.06\left(\mathrm{~s}, 3 \mathrm{H}, 6-\mathrm{CH}_{3}\right), 1.14-1.36$ (m, $\left.6 \mathrm{H}, c \mathrm{Hex}-\mathrm{H}^{*}\right), 1.62-2.21\left(\mathrm{~m}, 9 \mathrm{H}, c \mathrm{Hex}, 4,5-\mathrm{H}^{*}, \mathrm{OH}\right), 2.41-2.48(\mathrm{~m}, 1 \mathrm{H}, c \mathrm{Hex}-\mathrm{H})$, $2.69\left(\mathrm{~m}_{\mathrm{c}}, 2 \mathrm{H}, 1-\mathrm{H}^{* *}\right), 3.06-3.34\left(\mathrm{~m}, 2 \mathrm{H}, 3-\mathrm{H}^{* *}\right), 4.02$ (br s, $\left.1 \mathrm{H}, 7-\mathrm{H}\right) .-{ }^{13} \mathrm{C}-\mathrm{NMR}$ $\left(75.5 \mathrm{MHz}, \mathrm{CDCl}_{3}, \mathrm{APT}\right): \delta=23.08\left(+, 6-\mathrm{CH}_{3}\right), 25.95(-, c \mathrm{Hex}-\mathrm{C} *), 26.25\left(-, \mathrm{C}-4^{*}\right)$, 27.08 (-, cHex-C*), 28.80 (-, cHex-C*), 28.92 (+, 6- $\left.\mathrm{CH}_{3}\right), 41.48$ (-, C-6), 46.17 (-, C-5*), 46.86 (-, C-1**), 48.70 (-, C-3**), 63.44 (+, cHex-C), 85.85 (+, C-7), 134.14 (-, C-4a***), 137.08 (-, C-7a***). - MS (EI, $70 \mathrm{eV}), \mathrm{m} / \mathrm{z}(\%): 249(33)\left[\mathrm{M}^{+}\right], 206(100)\left[\mathrm{M}^{+}\right.$ $\left.-\mathrm{C}_{2} \mathrm{H}_{5} \mathrm{~N}\right], 193(18)\left[\mathrm{M}^{+}-\mathrm{C}_{3} \mathrm{H}_{6} \mathrm{~N}\right], 177(28)\left[\mathrm{M}^{+}-\mathrm{C}_{4} \mathrm{H}_{10} \mathrm{~N}\right], 165$ (4) $\left[\mathrm{M}^{+}-\mathrm{C}_{6} \mathrm{H}_{12} \mathrm{~N}\right]$, 133 (5), 126 (6), 84 (13), 68 (11), 53 (14), 49 (18), 41 (15) [ $\left.\mathrm{C}_{3} \mathrm{H}_{5}{ }^{+}\right] .-\mathrm{C}_{16} \mathrm{H}_{27} \mathrm{NO}$ (249.4).

3-Hydroxy-4,4-dimethyl-1-methylen-2-methylenpivaloxymethylcyclopentan (128): Nach

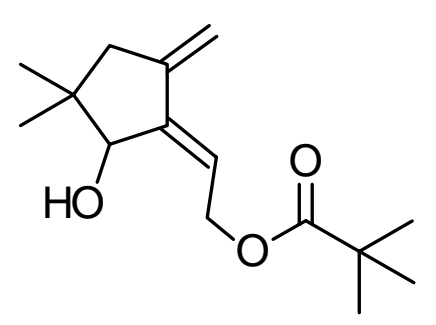
AAV 2 gab man zu einer Lösung von $252 \mathrm{mg}(1.00 \mathrm{mmol})$ 4-Hydroxy-5,5-dimethyl-oct-7-en-2-inpivaloylsäureester in $10 \mathrm{ml}$ wasserfreiem Benzol $11 \mathrm{mg}(0.050 \mathrm{mmol}, 5.0 \mathrm{~mol} \%)$ $\mathrm{Pd}(\mathrm{OAc})_{2} \quad$ und $24 \mathrm{mg} \quad(0.10 \mathrm{mmol}, \quad 10 \mathrm{~mol} \%) \quad N, N^{\prime}-$ Bis(benzyliden)ethylendiamin. Man rührte $3 \mathrm{~d}$ bei $70^{\circ} \mathrm{C}$. Säulenchromatographie an $18 \mathrm{~g}$ Kieselgel (Säule $2.0 \times 15 \mathrm{~cm}$, Pentan/Et $\left.\mathrm{Et}_{2} \mathrm{O}=3: 1\right)$ ergab $165 \mathrm{mg}(65 \%) 128$ als farbloses Öl, $R_{\mathrm{f}}\left(\operatorname{Pentan}_{2} / \mathrm{Et}_{2} \mathrm{O}\right.$, $3: 1)=0.52 .-$ IR (Film): $v=3450 \mathrm{~cm}^{-1}(\mathrm{OH}), 2972,2872,1729(\mathrm{C}=\mathrm{O}), 1481,1462$, 1398, 1366, 1284, 1153, 1055, 997, 739. - ${ }^{1} \mathrm{H}-\mathrm{NMR}\left(250 \mathrm{MHz}, \mathrm{CDCl}_{3}\right): \delta=0.87(\mathrm{~s}$, 
$\left.3 \mathrm{H}, 4-\mathrm{CH}_{3}\right), 1.06\left(\mathrm{~s}, 3 \mathrm{H}, 4-\mathrm{CH}_{3}\right), 1.18$ [s, $\left.9 \mathrm{H}, \mathrm{C}\left(\mathrm{CH}_{3}\right)_{3}\right], 1.21(\mathrm{~s}, 1 \mathrm{H}, \mathrm{OH}), 2.08$ (d, $\left.J_{\mathrm{AB}}=15.4 \mathrm{~Hz}, 1 \mathrm{H}, 5-\mathrm{H}\right), 2.48\left(\mathrm{~d}, J_{\mathrm{AB}}=15.4 \mathrm{~Hz}, 1 \mathrm{H}, 5-\mathrm{H}\right), 4.27$ (br s, $\left.1 \mathrm{H}, 3-\mathrm{H}\right), 4.56$ $\left(\mathrm{dd}, 2 J=12.5,3 J=5.9 \mathrm{~Hz}, 1 \mathrm{H}, \mathrm{CH}_{2} \mathrm{OCO} t \mathrm{Bu}\right), 4.92\left(\right.$ br s, $\left.1 \mathrm{H}, 1-\mathrm{CH}_{2}\right), 5.10(\mathrm{dd}$, $\left.2 J=12.5,3 J=9.3 \mathrm{~Hz}, 1 \mathrm{H}, \mathrm{CH}_{2} \mathrm{OCO} t \mathrm{Bu}\right), 5.37\left(\right.$ br s, $\left.1 \mathrm{H}, 1-\mathrm{CH}_{2}\right), 5.94\left(\mathrm{dd},{ }^{3} J=5.9\right.$, $\left.{ }^{3} J=9.3 \mathrm{~Hz}, 1 \mathrm{H},=\mathrm{CHR}\right) .-{ }^{13} \mathrm{C}-\mathrm{NMR}\left(62.9 \mathrm{MHz}, \mathrm{CDCl}_{3}, \mathrm{DEPT}\right): \delta=21.10\left(+, 4-\mathrm{CH}_{3}\right)$, $25.89\left(+, 4-\mathrm{CH}_{3}\right), 27.11\left[+, \mathrm{C}\left(\mathrm{CH}_{3}\right)_{3}\right], 38.86\left(\mathrm{C}_{\text {quart }}, \mathrm{C}-4 *\right), 39.99\left[\mathrm{C}_{\text {quart }}, C\left(\mathrm{CH}_{3}\right)_{3}\right], 44.51$ (-, C-5), 62.10 (-, $\left.\mathrm{CH}_{2} \mathrm{OCO} t \mathrm{Bu}\right), 79.33$ (+, C-3), $106.02\left(-, 1-\mathrm{CH}_{2}\right), 117.12$ (+, =CHR), $145.96\left(\mathrm{C}_{\text {quart }}, \mathrm{C}-1^{* *}\right), 147.44\left(\mathrm{C}_{\mathrm{quart}}, \mathrm{C}-2^{* *}\right), 179.26\left(\mathrm{C}_{\mathrm{quart}}, \mathrm{OCO} t \mathrm{Bu}\right) .-\mathrm{MS}(\mathrm{EI}$, $70 \mathrm{eV}$ ), $m / z$ (\%): 250 (4), 225 (4), 180 (7) [ $\left.\mathrm{M}^{+}-t \mathrm{Bu}-\mathrm{CH}_{3}\right], 169$ (14), 152 (13), 137 (9), 109 (12), 85 (19), 77 (5), 57 (100) $\left[\mathrm{C}_{4} \mathrm{H}_{9}{ }^{+}\right], 41$ (18). $-\mathrm{C}_{15} \mathrm{H}_{24} \mathrm{O}_{3}(252.4)$.

Versuch der Darstellung von 6,6-Dicarbonsäuremethylester-3-phenyl-1,3,4,5,6,7hexahydro-2H-cyclopenta[c]-2-pyridin (135): Variante A: Eine Lösung von $291 \mathrm{mg}$ (1.00 mmol) 2-Brom-4,4-dicarbonsäuremethylester-1,6-dien in $10 \mathrm{ml}$ wasserfreiem Acetonitril wurde mit $11 \mathrm{mg}(0.050 \mathrm{mmol}, 5.0 \mathrm{~mol} \%) \mathrm{Pd}(\mathrm{OAc})_{2}, 26 \mathrm{mg}(0.10 \mathrm{mmol}$, $10 \mathrm{~mol} \%) \mathrm{PPh}_{3}$ und $138 \mathrm{mg}(1.00 \mathrm{mmol}) \mathrm{K}_{2} \mathrm{CO}_{3}$ versetzt. Nachdem man $18 \mathrm{~h}$ bei $80{ }^{\circ} \mathrm{C}$ gerührt hatte, kühlte man auf Raumtemp. und gab 532 mg (3.00 mmol) Benzyltrimethylsilylimin zu. Anschließend rührte man $7 \mathrm{~d}$ bei Raumtemp. Das ${ }^{1} \mathrm{H}-\mathrm{NMR}$-Spektrum des Rohproduktes zeigte Signale des exocyclischen Diens und nicht identifizierte Produkte.

Variante B: Man versetzte zusätzlich zu unter den unter Variante A beschriebenen Bedingungen noch mit $3.0 \mathrm{ml}$ (3.0 mmol, $1.0 \mathrm{M}$ in Hexan) Diethylaluminiumchloridlösung als Lewis-Säure und rührte $2 \mathrm{~d}$ bei Raumtemp. Das ${ }^{1} \mathrm{H}-\mathrm{NMR}$-Spektrum des Rohproduktes zeigte nur Signale des exocyclischen Diens und Zersetzung.

Variante $C$ : Verwendete man $2.21 \mathrm{~g}$ (3.00 mmol) $\mathrm{ZnCl}_{2}$ als Lewis-Säure unter sonst identischen Bedingungen, konnten nach $3 \mathrm{~d}$ bei Raumtemp. ebenfalls nur exocyclisches Dien und nicht identifizierte Produkte nachgewiesen werden.

Versuch der Darstellung von 7-Hydroxy-6,6-dimethyl-3-phenyl-1,3,4,5,6,7-hexahydro-2Hcyclopenta[c]-2-pyridin (136): Variante A: Man erwärmte $219 \mathrm{mg}$ (1.00 mmol) 6-Brom4,4-dimethylhepta-1,6-dien-3-ol in $10 \mathrm{ml}$ wasserfreiem Acetonitril mit $11 \mathrm{mg}(0.050 \mathrm{mmol}$, $5.0 \mathrm{~mol} \%) \mathrm{Pd}(\mathrm{OAc})_{2}, 26 \mathrm{mg}(0.10 \mathrm{mmol}, 10 \mathrm{~mol} \%) \mathrm{PPh}_{3}$ und $415 \mathrm{mg}$ (3.00 mmol) $\mathrm{K}_{2} \mathrm{CO}_{3} 1 \mathrm{~d}$ auf $80{ }^{\circ} \mathrm{C}$. Anschließend kühlte man auf Raumtemp. und gab eine Lösung von $532 \mathrm{mg}$ (3.00 mmol) Phenyltrimethylsilylimin in $5 \mathrm{ml}$ wasserfreiem THF zu. Die 
Reaktionslösung wurde erneut auf $70{ }^{\circ} \mathrm{C}$ erwärmt und $7 \mathrm{~d}$ gerührt. Das ${ }^{1} \mathrm{H}-\mathrm{NMR}-$ Spektrum des Rohproduktes zeigte neben $\mathbf{6 0}$ nur nicht identifizierte Produktgemische.

Variante B: Man versetzte zusätzlich $\mathrm{zu}$ unter den unter Variante A beschriebenen Bedingungen noch mit $3.0 \mathrm{ml}$ (3.0 mmol, $1.0 \mathrm{M}$ in Hexan) Diethylaluminiumchloridlösung und rührte $2 \mathrm{~d}$ bei Raumtemp. Das ${ }^{1} \mathrm{H}-\mathrm{NMR}-$ Spektrum des Rohproduktes zeigte ein Gemisch nicht identifizierter Verbindungen.

3,3-Dicarbonsäureethylester-5-hydroxy-6,6-dimethyl-1,3,4,5,6,7-hexahydrocyclopenta[c]pyran (141) und 3,3-Dicarbonsäureethylester-7-hydroxy-6,6-dimethyl-1,3,4,5,6,7hexahydrocyclopenta[c]pyran (142): Zu einer Lösung von 219 mg (1.00 mmol) 6-Brom-

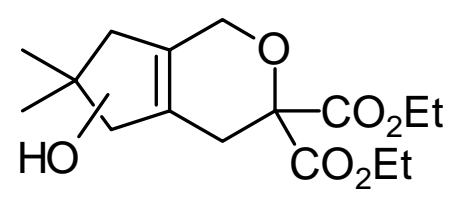
4,4-dimethylhepta-1,6-dien-3-ol in $10 \mathrm{ml}$ wasserfreiem Acetonitril gab man $11 \mathrm{mg} \quad(0.050 \mathrm{mmol}, 5.0 \mathrm{~mol} \%)$ $\mathrm{Pd}(\mathrm{OAc})_{2}, 26 \mathrm{mg}(0.10 \mathrm{mmol}, 10 \mathrm{~mol} \%) \mathrm{PPh}_{3}$ und $138 \mathrm{mg}$ (1.00 mmol) $\mathrm{K}_{2} \mathrm{CO}_{3}$. Nachdem man $18 \mathrm{~h}$ bei $80{ }^{\circ} \mathrm{C}$ gerührt hatte, fügte man $348 \mathrm{mg}$ (2.00 mmol) Diethylketomalonat (138) und eine Spatelspitze Hydrochinon zu und erwärmte für $1 \mathrm{~d}$ auf $130{ }^{\circ} \mathrm{C}$. Nach Aufarbeitung analog AAV 6 reinigte man das Rohprodukt durch Säulenchromatographie an $18 \mathrm{~g}$ Kieselgel (Säule $2.0 \times 15 \mathrm{~cm}$, Pent/Et ${ }_{2} \mathrm{O}=1: 1$ ) gereinigt. Man erhielt $28 \mathrm{mg}$ eines Gemisches aus 138 und 141/142 $(6 \%)$ im Verhältnis $1: 1$ als farbloses Öl, $R_{\mathrm{f}}\left(\right.$ Pent/Et $\left._{2} \mathrm{O}, 1: 1\right)=0.19$. - IR (Film): $v=3482 \mathrm{~cm}^{-1}(\mathrm{OH}), 2982,2907,2871,1743(\mathrm{C}=\mathrm{O}), 1467,1447,1369,1273,1153,1098$, 1061, 1029, 860, 735, 703. - ${ }^{1} \mathrm{H}-\mathrm{NMR}\left(250 \mathrm{MHz}, \mathrm{CDCl}_{3}\right): \delta=1.00-1.40(\mathrm{~m}, 13 \mathrm{H}$, 6- $\left.\mathrm{CH}_{3}, \mathrm{OCH}_{2} \mathrm{CH}_{3}, \mathrm{OH}\right), 2.02\left(\mathrm{~m}_{\mathrm{c}}, 2 \mathrm{H}, 7(5)-\mathrm{H}^{*}\right), 2.74\left(\mathrm{~m}_{\mathrm{c}}, 2 \mathrm{H}, 4-\mathrm{H}^{*}\right), 3.69\left(\mathrm{~m}_{\mathrm{c}}, 1 \mathrm{H}\right.$, 1-H), $3.86\left(\mathrm{~m}_{\mathrm{c}}, 1 \mathrm{H}, 1-\mathrm{H}\right), 4.06$ (br s, $\left.1 \mathrm{H}, 5(7)-\mathrm{H}\right), 4.21-4.37$ (m, $\left.4 \mathrm{H}, \mathrm{OCH}_{2} \mathrm{CH}_{3}\right)$. ${ }^{13} \mathrm{C}-\mathrm{NMR}\left(62.9 \mathrm{MHz}, \mathrm{CDCl}_{3}, \mathrm{DEPT}\right): \delta=13.93\left(+, \mathrm{OCH}_{2} \mathrm{CH}_{3}\right), 14.03\left(+, \mathrm{OCH}_{2} \mathrm{CH}_{3}\right)$, $15.29(-, \mathrm{C}-4 *), 17.88\left(-, \mathrm{C}-7(5)^{*}\right), 22.56\left(+, 6-\mathrm{CH}_{3}\right), 28.27\left(+, 6-\mathrm{CH}_{3}\right), 44.93\left(\mathrm{C}_{\text {quart }}\right.$, C-6), $62.18\left(-, \mathrm{OCH}_{2} \mathrm{CH}_{3}\right), 63.41\left(\mathrm{C}_{\text {quart }}, \mathrm{C}-3\right), 63.81$ (-, $\left.\mathrm{OCH}_{2} \mathrm{CH}_{3}\right), 64.83$ (-, C-1), $85.70(+, \mathrm{C}-5(7)), 123.83\left(\mathrm{C}_{\text {quart }}, \mathrm{C}-4 \mathrm{a}^{* *}\right), 131.32\left(\mathrm{C}_{\text {quart }}, \mathrm{C}-7 \mathrm{a}^{* *}\right), 158.76\left(\mathrm{C}_{\text {quart }}\right.$, $\left.\mathrm{CO}_{2} \mathrm{Et}\right) .-\mathrm{C}_{16} \mathrm{H}_{24} \mathrm{O}_{6}(312.4)$. 


\subsection{Heck-Reaktionen an Bromeninen}

3,3,4",4"-Tetracarbonsäuremethylester-1-methyl-5-[2'-(2"-methylencyclopentyliden)ethyliden]-cyclopent-1-en (153) und 2,2,5,7-Tetracarbonsäuremethylester-2,3,4,5,6,7hexahydro-1H-cyclopentacycloocten $\left(\mathbf{1 5 5}-\mathbf{C O}_{2} \mathbf{M e}\right)$ : Variante A: Entsprechend AAV 5 versetzte man eine Lösung von $289 \mathrm{mg}(1.00 \mathrm{mmol})$ Bromenin in $10 \mathrm{ml}$ wasserfreiem $\mathrm{MeCN}$ mit $11 \mathrm{mg}(0.050 \mathrm{mmol}, 5.0 \mathrm{~mol} \%) \mathrm{Pd}(\mathrm{OAc})_{2}, 26 \mathrm{mg}(0.10 \mathrm{mmol}, 10 \mathrm{~mol} \%) \mathrm{PPh}_{3}$, $138 \mathrm{mg}$ (1.00 mmol) $\mathrm{K}_{2} \mathrm{CO}_{3}$ und $344 \mathrm{mg}(4.00 \mathrm{mmol})$ Methylacrylat. Man erwärmte $3 \mathrm{~d}$ auf $80{ }^{\circ} \mathrm{C}$ und reinigte anschließend das Rohprodukt durch Säulenchromatographie an $18 \mathrm{~g}$ Kieselgel (Säule $2.0 \times 15 \mathrm{~cm}$, Pentan/ $\mathrm{Et}_{2} \mathrm{O}=2: 1$, nach Durchlaufen der 1. Fraktion Wechsel zu $1: 1$ ). Man erhielt Fraktion I: 21 mg (5\%) 153 mit einer nicht identifizierten

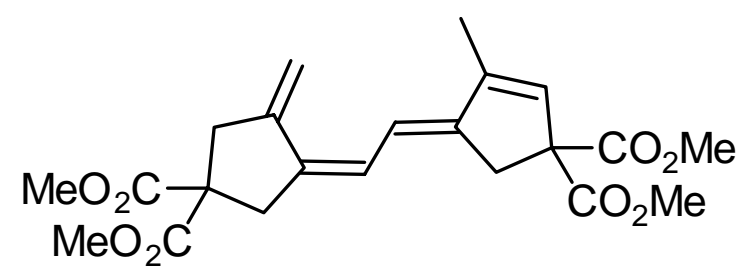

Verbindung 154 als farbloses Öl, $R_{\mathrm{f}}$ $($ Pentan/Et $2 \mathrm{O}, 2: 1)=0.26$. - IR $($ Film $)$, Gemisch: $v=3435 \mathrm{~cm}^{-1}, 2955,2848,1746$, 1712, 1643, 1440, 1421, 1276, 1168, 1074, 873, 737, 702. - 153: ${ }^{1} \mathrm{H}-\mathrm{NMR}$ (250 MHz,

$\left.\mathrm{CDCl}_{3}\right): \delta=1.72\left(\right.$ br s, $3 \mathrm{H}, 1-\mathrm{CH}_{3}$ ), 2.93-2.97 (m, $\left.4 \mathrm{H}, 3 ", 5 "-\mathrm{H}\right), 3.21$ (br t, ${ }^{4} \mathrm{~J}=2.0 \mathrm{~Hz}$, $2 \mathrm{H}, 4-\mathrm{H}), 3.71-3.74\left(\mathrm{~m}, 12 \mathrm{H}, \mathrm{CO}_{2} \mathrm{CH}_{3}\right), 4.91\left(\mathrm{~m}_{\mathrm{c}}, 1 \mathrm{H}, 2 "-\mathrm{CH}_{2}\right), 4.96\left(\mathrm{~m}_{\mathrm{c}}, 1 \mathrm{H}, 2\right.$ "- $\left.\mathrm{CH}_{2}\right)$, $5.85\left(\mathrm{~d},{ }^{3} \mathrm{~J}=12.3 \mathrm{~Hz}, 1 \mathrm{H}, 2^{\prime *}-\mathrm{H}\right), 5.96\left(\right.$ br s, $\left.1 \mathrm{H}, 2-\mathrm{H}^{*}\right), 6.33\left(\mathrm{~d},{ }^{3} \mathrm{~J}=12.3 \mathrm{~Hz}, 1 \mathrm{H}, 1^{\prime}-\mathrm{H}\right)$. - ${ }^{13} \mathrm{C}-\mathrm{NMR}\left(50.3 \mathrm{MHz}, \mathrm{CDCl}_{3}, \mathrm{APT}\right): \delta=14.11\left(+, 1-\mathrm{CH}_{3}\right), 37.70$ (-, C-3"*), 42.19 (-, C-5"*), 52.69 (+, $\left.4 \mathrm{C}, \mathrm{CO}_{2} \mathrm{CH}_{3}\right), 57.71$ (-, C-4**), 63.96 (-, $\left.2 \mathrm{C}, \mathrm{C}-3,4 * * *\right), 105.61$ (-, 2"- $\left.\mathrm{CH}_{2}\right), 120.08$ (+, C-2'***), 127.13 (+, C-1'***), 130.22 (-, C-1****), 136.60 (+, C-2***), 137.99 (-, C-5****), 144.94 (-, C-1"****), 150.25 (-, C-2"****), 170.74 (-, 4 C, $\left.\mathrm{CO}_{2} \mathrm{CH}_{3}\right)$. - MS (EI, $\left.70 \mathrm{eV}\right), \mathrm{m} / z(\%)$, Gemisch: 418 (56) $\left[\mathrm{M}^{+}\right], 358(65)\left[\mathrm{M}^{+}-\mathrm{CO}_{2} \mathrm{Me}-\right.$

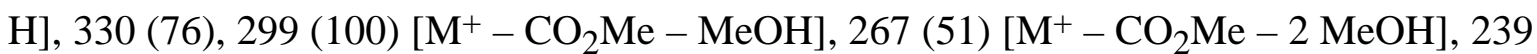
(98) $\left[\mathrm{M}^{+}-2 \mathrm{CO}_{2} \mathrm{Me}-\mathrm{H}-\mathrm{MeOH}\right], 207(33)\left[\mathrm{M}^{+}-2 \mathrm{CO}_{2} \mathrm{Me}-2 \mathrm{MeOH}-\mathrm{H}\right], 179$ (86), 165 (57), 91 (69), 57 (49). - MS (CI, $\left.\mathrm{NH}_{3}, 70 \mathrm{eV}\right), \mathrm{m} / z$ (\%), Gemisch: 854 (1) [2 M + $\left.\mathrm{NH}_{4}^{+}\right], 436$ (100) $\left[\mathrm{M}+\mathrm{NH}_{4}^{+}\right] .-\mathrm{C}_{25} \mathrm{H}_{36} \mathrm{O}_{8}$ (464.6).

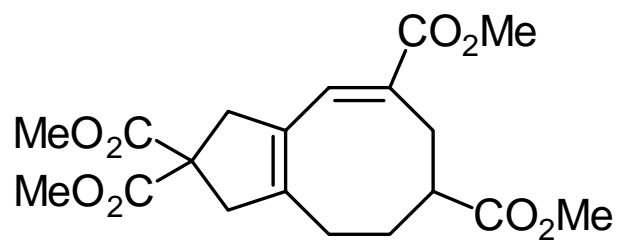

Fraktion II: $44 \mathrm{mg} \quad(12 \%) \quad \mathbf{1 5 5}-\mathbf{C O}_{\mathbf{2}} \mathbf{M e}$ als farbloses Öl, $R_{\mathrm{f}}\left(\right.$ Pentan$\left._{/ E_{2}} \mathrm{O}, 1: 1\right)=0.32$. - IR (Film): $v=3435 \mathrm{~cm}^{-1}, 2955,2848,1746,1712$, $1643,1440,1421,1276,1168,1074,873,737$, 
702. $-{ }^{1} \mathrm{H}-\mathrm{NMR}\left(250 \mathrm{MHz}, \mathrm{CDCl}_{3}\right): \delta=2.95-3.27(\mathrm{~m}, 10 \mathrm{H}, 1,3,6,8,9-\mathrm{H}), 3.58-3.75$ (m, $\left.13 \mathrm{H}, \mathrm{CO}_{2} \mathrm{CH}_{3}, 7-\mathrm{H}\right), 5.54$ (t, $\left.{ }^{4} \mathrm{~J}=7.2 \mathrm{~Hz}, 1 \mathrm{H}, 4-\mathrm{H}\right) .-{ }^{13} \mathrm{C}-\mathrm{NMR}\left(62.9 \mathrm{MHz}, \mathrm{CDCl}_{3}\right.$, DEPT): $\delta=22.55(-$, C-1*), $24.80(-$, C-3*), $33.41(-$, C-6*), $39.66(+$, C-7), 40.09 (-, C-8*), $44.55(-, \mathrm{C}-9 *), 51.88,52.82,52.87$ (+, $\left.4 \mathrm{C}, \mathrm{CO}_{2} \mathrm{CH}_{3}\right), 57.14\left(\mathrm{C}_{\text {quart }}, \mathrm{C}-2\right), 118.26$ (+, C-4), $130.69\left(\mathrm{C}_{\text {quart }}, \mathrm{C}-5^{* *}\right), 132.38\left(\mathrm{C}_{\text {quart }}, \mathrm{C}-3 \mathrm{a}^{* *}\right), 136.89\left(\mathrm{C}_{\text {quart }}, \mathrm{C}-9 \mathrm{a} * *\right), 171.93$, 172.31, 172.67, $172.92\left(\mathrm{C}_{\text {quart }}, 4 \mathrm{C}, \mathrm{CO}_{2} \mathrm{CH}_{3}\right)$. - MS (EI, $\left.70 \mathrm{eV}\right), m / z(\%): 380(15)\left[\mathrm{M}^{+}\right]$,

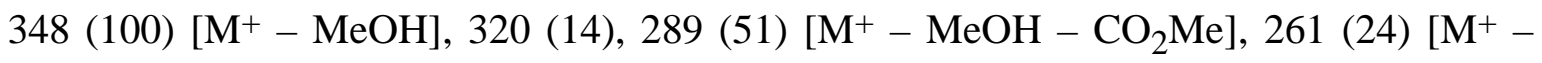
$\left.2 \mathrm{CO}_{2} \mathrm{Me}-\mathrm{H}\right], 235$ (49), 229 (53), 201 (20), 175 (23), 141 (17), 115 (22), 91 (11), 59 (21) $\left[\mathrm{CO}_{2} \mathrm{Me}^{+}\right] .-\mathrm{C}_{19} \mathrm{H}_{24} \mathrm{O}_{8}$ (380.4): ber. 380.1471 (HRMS korrekt).

Variante B: Führte man die Reaktion bei $40^{\circ} \mathrm{C}$ unter sonst identischen Bedingungen wie unter Variante A beschrieben durch, so erhielt man Fraktion I: $21 \mathrm{mg}$ der nicht identifizierten Verbindung 154 als farbloses Öl, $R_{\mathrm{f}}\left(\operatorname{Pentan}_{/} \mathrm{Et}_{2} \mathrm{O}, 2: 1\right)=0.26$. $-{ }^{1} \mathrm{H}-\mathrm{NMR}$ $\left(250 \mathrm{MHz}, \mathrm{CDCl}_{3}\right): \delta=2.28$ (br s, $\left.2 \mathrm{H}\right), 3.04$ (br s, $\left.2 \mathrm{H}\right), 3.08$ (br s, $\left.2 \mathrm{H}\right), 3.72(\mathrm{~s}, 12 \mathrm{H})$, 5.67 (br s, $1 \mathrm{H}) .-{ }^{13} \mathrm{C}-\mathrm{NMR}\left(62.9 \mathrm{MHz}, \mathrm{CDCl}_{3}, \mathrm{DEPT}\right): \delta=27.91(-), 45.79(-), 46.23$ $(-), 52.83(+), 57.14\left(\mathrm{C}_{\text {quart }}\right), 124.49(+), 129.95\left(\mathrm{C}_{\text {quart }}\right), 138.81\left(\mathrm{C}_{\text {quart }}\right), 172.52\left(\mathrm{C}_{\text {quart }}\right)$. Fraktion II: $40 \mathrm{mg}(11 \%)$ 155-CO $\mathbf{C O}_{\mathbf{2}} \mathbf{M e}$.

Variante C: Der unter Variante A beschriebene Ansatz wurde auf $867 \mathrm{mg}(3.00 \mathrm{mmol})$ Bromenin, $860 \mathrm{mg}(10 \mathrm{mmol})$ Methylacrylat, $33 \mathrm{mg}(0.15 \mathrm{mmol}, 5.0 \mathrm{~mol} \%) \mathrm{Pd}(\mathrm{OAc})_{2}$, $78 \mathrm{mg}$ (0.30 mmol, $10 \mathrm{~mol} \%) \mathrm{PPh}_{3}$ und $414 \mathrm{mg}$ (3.00 mmol) $\mathrm{K}_{2} \mathrm{CO}_{3}$ vergrößert. Man erwärmte $2 \mathrm{~d}$ auf $80^{\circ} \mathrm{C}$ und reinigte anschließend das Rohprodukt durch Säulenchromatographie an $50 \mathrm{~g}$ Kieselgel (Säule $2.5 \times 20 \mathrm{~cm}$, Pentan/Et ${ }_{2} \mathrm{O}=1: 1$ ). Man erhielt $113 \mathrm{mg}$ (18\%) eines Gemisches aus 153 und 154 und $58 \mathrm{mg}(5 \%)$ 155-CO $\mathbf{C O}_{2}$ Me.

Variante D: Führte man die Reaktion analog AAV 5 durch, setzte jedoch kein Methylacrylat zu, konnten 32 mg (15\%) des Gemisches aus 153 und 154 isoliert werden.

Variante E: Verwendete man tert-Butylacrylat als Dienophil, so wurde kein Achtringprodukt isoliert, sondern nur $19 \mathrm{mg}(9 \%)$ des Dimers 153, verunreinigt mit 154.

Variante F: Benutzte man als Base $326 \mathrm{mg}(1.00 \mathrm{mmol}) \mathrm{Cs}_{2} \mathrm{CO}_{3}$, so erhielt man $14 \mathrm{mg}$ (6\%) eines Gemisches aus 153 und 154 und $15 \mathrm{mg}(4 \%)$ 155-CO $\mathbf{C O}_{\mathbf{2}} \mathbf{M e}$.

\section{2,13-Brom-4,4,11,11-tetracarbonsäuremethylester-tetradeca-1,13-dien-6,8-diin}

(157):

Nach AAV 5 versetzte man eine Lösung von $289 \mathrm{mg}$ (1.00 mmol) Bromenin und $258 \mathrm{mg}$ (3.00 mmol) Methylacrylat in $10 \mathrm{ml}$ wasserfreiem $\mathrm{MeCN}$ mit $11 \mathrm{mg}$ (0.050 mmol, $5.0 \mathrm{~mol} \%) \mathrm{Pd}(\mathrm{OAc})_{2}, 26 \mathrm{mg}\left(0.10 \mathrm{mmol}, 10 \mathrm{~mol}^{2}\right) \mathrm{PPh}_{3}$ und $276 \mathrm{mg}(1.00 \mathrm{mmol})$ 
$\mathrm{Ag}_{2} \mathrm{CO}_{3}$. Man rührte $18 \mathrm{~h}$ bei $80^{\circ} \mathrm{C}$ und reinigte anschließend das Rohprodukt durch Säulenchromatographie an $18 \mathrm{~g}$ Kieselgel (Säule $2.0 \times 15 \mathrm{~cm}, \operatorname{Pentan} / \mathrm{Et}_{2} \mathrm{O}=1: 1$ ). Man erhielt $123 \mathrm{mg}$ (43\%) 157 als farblosen Feststoff, Schmp. $136-137^{\circ} \mathrm{C}, R_{\mathrm{f}}\left(\mathrm{Pentan}_{\mathrm{Et}} \mathrm{O}\right.$,

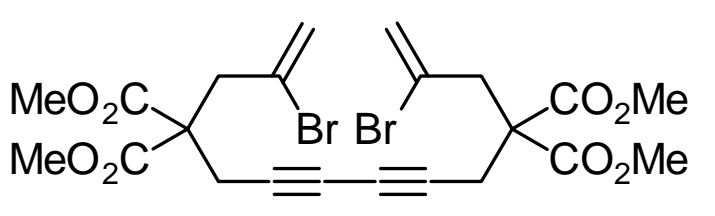
$1: 1)=0.54$. - IR (Film): $v=3104 \mathrm{~cm}^{-1}$, 3003, 2953, $1736(\mathrm{C}=\mathrm{O}), 1621,1435,1326$, 1301, 1230, 1205, 1149, 1056, 971, 922, 875, 856, 769, 713. - ${ }^{1} \mathrm{H}-\mathrm{NMR}\left(250 \mathrm{MHz}, \mathrm{CDCl}_{3}\right): \delta=3.00(\mathrm{~s}, 4 \mathrm{H}, 5,10-\mathrm{H}), 3.27$ (s, $4 \mathrm{H}, 3,12-\mathrm{H}), 3.76\left(\mathrm{~s}, 6 \mathrm{H}, \mathrm{CO}_{2} \mathrm{CH}_{3}\right), 5.63\left(\mathrm{~d},{ }^{2} \mathrm{~J}=0.7 \mathrm{~Hz}, 2 \mathrm{H}, 1,14-\mathrm{H}\right), 5.80(\mathrm{~m}, 2 \mathrm{H}$, 1,14-H). - ${ }^{13} \mathrm{C}-\mathrm{NMR}\left(62.9 \mathrm{MHz}, \mathrm{CDCl}_{3}, \mathrm{DEPT}\right): \delta=23.07$ (-, $\left.2 \mathrm{C}, \mathrm{C}-5, \mathrm{C}-10\right), 43.10(-$, $2 \mathrm{C}, \mathrm{C}-3, \mathrm{C}-12), 53.19\left(+, \mathrm{CO}_{2} \mathrm{CH}_{3}\right), 55.95\left(\mathrm{C}_{\text {quart }}, 2 \mathrm{C}, \mathrm{C}-4, \mathrm{C}-11\right), 68.22\left(\mathrm{C}_{\text {quart }}, 2 \mathrm{C}\right.$, C-6*, C-9*), $72.46\left(\mathrm{C}_{\text {quart }}, \mathrm{C}-7^{*}, \mathrm{C}-8^{*}\right), 123.08$ (-, $\left.2 \mathrm{C}, \mathrm{C}-1, \mathrm{C}-14\right), 125.87\left(\mathrm{C}_{\text {quart }}, 2 \mathrm{C}\right.$, C-2, C-13), $169.21\left(\mathrm{C}_{\text {quart }}, \mathrm{CO}_{2} \mathrm{CH}_{3}\right)$. - MS (EI, $\left.70 \mathrm{eV}\right), \mathrm{m} / \mathrm{z}(\%): 437 / 435(60 / 59)\left[\mathrm{M}^{+}-\right.$ $\left.\mathrm{Br}-\mathrm{CO}_{2} \mathrm{Me}-\mathrm{H}\right], 415$ (69), $377 / 375$ (48/47), 355 (66), 295 (100), 237 (63), 178 (51), 127 (34), 115 (25), 59 (66). - MS (CI, $\left.\mathrm{NH}_{3}, 70 \mathrm{eV}\right), \mathrm{m} / \mathrm{z}$ (\%): 596/594/592 (52/100/49) [M + $\mathrm{NH}_{4}^{+}$]. $-\mathrm{C}_{22} \mathrm{H}_{24} \mathrm{Br}_{2} \mathrm{O}_{8}$ (576.2): ber. C 45.86, H 4.20; gef. C 45.62, H 4.05.

Versuch der Cyclisierung von 157: Analog AAV 5 gab man zu 58 mg (0.10 mmol) 157 in $5 \mathrm{ml}$ wasserfreiem $\mathrm{MeCN} 2.2 \mathrm{mg}(10 \mu \mathrm{mol}, 10 \mathrm{~mol} \%) \mathrm{Pd}(\mathrm{OAc})_{2}, 5.2 \mathrm{mg}(20 \mathrm{mmol}$, $20 \mathrm{~mol} \%) \mathrm{PPh}_{3}, 28 \mathrm{mg}(0.20 \mathrm{mmol}) \mathrm{K}_{2} \mathrm{CO}_{3}$ und $14 \mathrm{mg}(0.21 \mathrm{mmol}) \mathrm{HCOONa}$. Nachdem man $3 \mathrm{~d}$ auf $80^{\circ} \mathrm{C}$ erwärmt hatte, konnte $43 \mathrm{mg}$ (74\%) 157 zurückgewonnen werden

\section{2,2,7,7-Tetracarbonsäuremethylester-5-methylen-1,2,3,6,7,8-hexahydrocyclo-}

penta[b]naphthalin (156): Entsprechend AAV 5 versetzte man eine Lösung von $289 \mathrm{mg}$ (1.00 mmol) Bromenin in $10 \mathrm{ml}$ wasserfreiem MeCN mit $11 \mathrm{mg}(0.050 \mathrm{mmol}, 5.0 \mathrm{~mol} \%)$ $\mathrm{Pd}(\mathrm{OAc})_{2}, 26 \mathrm{mg}(0.10 \mathrm{mmol}, 10 \mathrm{~mol} \%) \mathrm{PPh}_{3}$ und $138 \mathrm{mg}(1.00 \mathrm{mmol}) \mathrm{K}_{2} \mathrm{CO}_{3}$. Man erwärmte $2 \mathrm{~h}$ auf $100^{\circ} \mathrm{C}$ und reinigte anschließend das Rohprodukt durch

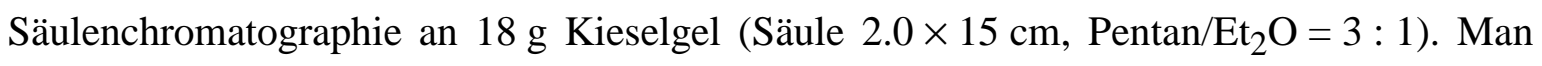
erhielt Fraktion I: 13 mg (6\%) 153 mit der nicht identifizierten Verunreinigung 154 als farbloses Öl, $R_{\mathrm{f}}($ Pentan/Et $2 \mathrm{O}, 3: 1)=0.14$.

Fraktion II: $21 \mathrm{mg}$ einer Mischfraktion aus Fraktion I und III. 
Fraktion III: $8 \mathrm{mg}$ (4\%) 156 als farbloses Öl, $R_{\mathrm{f}}\left(\mathrm{Pentan}_{\mathrm{EEt}} \mathrm{O}, 3: 1\right)=0.08$. - IR (Film):

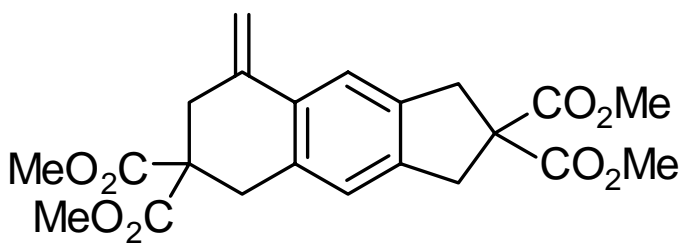
$v=3470 \mathrm{~cm}^{-1}, 2957, \quad 1754 \quad(\mathrm{C}=\mathrm{O}), 1721$, $1620,1434,1280,1051,893,823,736,703 .-$ ${ }^{1} \mathrm{H}-\mathrm{NMR}\left(250 \mathrm{MHz}, \mathrm{CDCl}_{3}\right): \delta=3.02(\mathrm{~s}$, $\left.2 \mathrm{H}, 6-\mathrm{H}^{*}\right), 3.29$ (s, $\left.2 \mathrm{H}, 8-\mathrm{H}^{*}\right), 3.54$ (s, $4 \mathrm{H}$, 1,3-H), $3.70\left(\mathrm{~s}, 6 \mathrm{H}, \mathrm{CO}_{2} \mathrm{CH}_{3}\right), 3.74(\mathrm{~s}, 6 \mathrm{H}$, $\left.\mathrm{CO}_{2} \mathrm{CH}_{3}\right), 5.02\left(\mathrm{~s}, 1 \mathrm{H}, 5-\mathrm{CH}_{2}\right), 5.52\left(\mathrm{~s}, 1 \mathrm{H}, 5-\mathrm{CH}_{2}\right), 6.97$ (s, $\left.1 \mathrm{H}, 4-\mathrm{H}^{* *}\right), 7.42(\mathrm{~s}, 1 \mathrm{H}$, 9-H**). - ${ }^{13} \mathrm{C}-\mathrm{NMR}\left(62.9 \mathrm{MHz}, \mathrm{CDCl}_{3}, \mathrm{DEPT}\right): \delta=35.57\left(-, \mathrm{CH}_{2}\right), 38.00\left(-, \mathrm{CH}_{2}\right)$, $40.21\left(-, \mathrm{CH}_{2}\right), 40.23\left(-, \mathrm{CH}_{2}\right), 52.81\left(+, \mathrm{CO}_{2} \mathrm{CH}_{3}\right), 52.98\left(+, \mathrm{CO}_{2} \mathrm{CH}_{3}\right), 54.43\left(\mathrm{C}_{\text {quart }}\right.$, C-2*), $60.38\left(\mathrm{C}_{\text {quart }}, \mathrm{C}-7 *\right), 110.58\left(-, 5-\mathrm{CH}_{2}\right), 119.54$ (+, Ph-C), 124.48 (+, Ph-C), 132.06 $\left(\mathrm{C}_{\text {quart }}, \mathrm{C}-3 \mathrm{a}^{* *}\right), 132.42\left(\mathrm{C}_{\text {quart }}, \mathrm{C}-4 \mathrm{a}^{* *}\right), 138.41\left(\mathrm{C}_{\text {quart }}, \mathrm{C}-8 \mathrm{a}^{* *}\right), 138.89\left(\mathrm{C}_{\text {quart }}\right.$, C-9a**), $140.36\left(\mathrm{C}_{\text {quart }}, \mathrm{C}-5^{* *}\right), 171.13\left(\mathrm{C}_{\text {quart }}, \mathrm{CO}_{2} \mathrm{CH}_{3}\right), 172.00\left(\mathrm{C}_{\text {quart }}, \mathrm{CO}_{2} \mathrm{CH}_{3}\right)$. MS (EI, $70 \mathrm{eV}), \mathrm{m} / z(\%): 416$ (37) $\left[\mathrm{M}^{+}\right], 378,(26), 357$ (100) $\left[\mathrm{M}^{+}-\mathrm{CO}_{2} \mathrm{Me}\right], 318$ (41) $\left[\mathrm{M}^{+}-\mathrm{CO}_{2} \mathrm{Me}-\mathrm{C}_{3} \mathrm{H}_{3}\right], 297(82)\left[\mathrm{M}^{+}-2 \mathrm{CO}_{2} \mathrm{Me}-\mathrm{H}\right], 258(24)\left[\mathrm{M}^{+}-2 \mathrm{CO}_{2} \mathrm{Me}-\mathrm{C}_{3} \mathrm{H}_{3}\right.$ - H], 246 (44), 237 (40), 187 (29), 179 (34), 107 (11), 59 (12) $\left[\mathrm{CO}_{2} \mathrm{Me}^{+}\right]-\mathrm{C}_{22} \mathrm{H}_{24} \mathrm{O}_{8}$ (416.4).

\subsection{Synthese eines Spinosyn-Vorläufers}

Allgemeine Arbeitsvorschrift zur einseitigen Schützung von Diolen als Tetrahydropyranyloxyethern ( $A A V 7)$ : Man versetzt 5 Äquivalente des zu schützenden Diols mit 1 Äquivalent Dihydropyran und gibt $200 \mathrm{mg} p$-Toluolsulfonsäure zu. Anschließend rührt man $4 \mathrm{~h}$ bei Raumtemp., fügt $50 \mathrm{ml}$ Wasser zu und extrahiert die wäßrige Phase mit $3 \times 50 \mathrm{ml} \mathrm{CH}_{2} \mathrm{Cl}_{2}$. Die vereingten organischen Phasen werden mit ges. $\mathrm{NaCl}$-Lösung gewaschen, über $\mathrm{MgSO}_{4}$ getrocknet und das Lösungsmittel i. Vak. entfernt. Das Rohprodukt wird durch Säulenchromatographie an Kieselgel gereingt.

Allgemeine Arbeitsvorschrift zur Oxidation von Alkoholen zu Aldehyden nach Swern ( $A A V$ 8): $\mathrm{Zu}$ einer auf $-60^{\circ} \mathrm{C}$ gekühlten Lösung von $7.5 \mathrm{ml}(85 \mathrm{mmol})$ Oxalylchlorid in $100 \mathrm{ml}$ Dichlormethan tropft man $12.8 \mathrm{ml}(180 \mathrm{mmol})$ DMSO und rührt $30 \mathrm{~min}$ bei dieser Temperatur. Anschließend fügt man $75 \mathrm{mmol}$ des Alkohols langsam zu und rührt weitere $1.5 \mathrm{~h}$ bei $-60^{\circ} \mathrm{C}$. Danach versetzt man mit $53.0 \mathrm{ml}$ (375 mmol) Triethylamin, erwärmt auf 
Raumtemp. und läßt weitere $16 \mathrm{~h}$ bei Raumtemp. rühren. Zur Aufarbeitung versetzt man mit $100 \mathrm{ml}$ Wasser und extrahiert die wäßrige Phase mit $3 \times 100 \mathrm{ml} \mathrm{CH}_{2} \mathrm{Cl}_{2}$. Die vereingten organischen Phasen werden nacheinander mit je $50 \mathrm{ml}$ ges. AmmoniumchloridLösung und $\mathrm{NaCl}$-Lösung gewaschen und über $\mathrm{MgSO}_{4}$ getrocknet. Nach Entfernung des Lösungsmittels i. Vak. reinigt man das Rohprodukt durch Säulenchromatographie an Kieselgel.

Allgemeine Arbeitsvorschrift zur Schützung von Alkoholen als TBDMS-Ether (AAV 9): Zu 9 mmol des Alkohols in $20 \mathrm{ml}$ DMF gibt man $11 \mathrm{mmol}$ TBDMSCl und $23 \mathrm{mmol}$ Imidazol und läßt $14 \mathrm{~h}$ bei Raumtemp. rühren. Zur Aufarbeitung fügt man $30 \mathrm{ml}$ Wasser und $30 \mathrm{ml}$ Pentan zu, wäscht die organische Phase mit $3 \times 20 \mathrm{ml}$ Wasser und trocknet über $\mathrm{MgSO}_{4}$. Nach Entfernung des Lösungsmittels i. Vak. wird das Rohprodukt durch Säulenchromatographie an Kieselgel gereinigt.

Allgemeine Arbeitsvorschrift zur Entschützung von THP-Ethern in Gegenwart von TBDMS-Ethern (AAV 10): Zu einer Lösung von $5 \mathrm{mmol}$ des THP-Ethers in $20 \mathrm{ml}$ wasserfreiem Dichlormethan gibt man bei $-25^{\circ} \mathrm{C} \quad 10 \mathrm{ml} \quad(10 \mathrm{mmol})$ Diethylaluminiumchlorid-Lösung und erwärmt auf Raumtemp. Man rührt das Reaktionsgemisch für den angegebenen Zeitraum bis dünnschichtchromatographisch kein Ausgangsmaterial mehr $\mathrm{zu}$ detektieren ist und hydrolysiert überschüssiges Diethylaluminiumchlorid unter Eiskühlung mit ges. $\mathrm{KHCO}_{3}$-Lösung. Die wäßrige Phase wird mit $3 \times 20 \mathrm{ml}$ Ether extrahiert, die vereingten organischen Phasen über $\mathrm{MgSO}_{4}$ getrocknet und das Lösungsmittel i. Vak. entfernt. Anschließend wird das Rohprodukt durch Säulenchromatographie an Kieselgel gereinigt.

Allgemeine Arbeitsvorschrift zur Überführung von Aldehyden in Vinylchloride nach Takai (AAV 11): $\mathrm{Zu}$ einer Suspension von $9 \mathrm{mmol} \mathrm{CrCl}_{2}$ in $10 \mathrm{ml}$ wasserfreiem THF gibt man bei Raumtemp. $1.5 \mathrm{mmol}$ des Aldehyds und $3 \mathrm{mmol} \mathrm{CHCl}_{3}$ in $5 \mathrm{ml}$ wasserfreiem THF. Man erwärmt auf $65^{\circ} \mathrm{C}$ bis die Farbe der Suspension von grün nach violett umschlägt. Zur Aufarbeitung versetzt man mit $15 \mathrm{ml}$ Wasser und extrahiert die wäßrige Phase mit $3 \times 20 \mathrm{ml}$ Ether. Anschließend wäscht man die vereingten organischen Phasen mit ges. 
$\mathrm{NaCl}-\mathrm{Lösung}$, trocknet über $\mathrm{MgSO}_{4}$ und engt i. Vak. ein. Das Rohprodukt wird durch Säulenchromatographie an Kieselgel gereinigt.

4-Tetrahydropyranyloxybutan-1-ol: Analog AAV 7 gab man zu 45.1 g (0.500 mol) Butandiol $8.40 \mathrm{~g}$ (100 mmol) 3,4-Dihydro-2H-pyran und THPO $200 \mathrm{mg}(1.16 \mathrm{mmol}$,) $p$-Toluolsulfonsäure. Nachdem man $4 \mathrm{~h}$ rührte, arbeitete man wäßrig auf, trocknete und engte i. Vak. ein. Säulenchromatographie des Rohproduktes an $250 \mathrm{~g}$ Kieselgel (Säule $8.0 \times 20 \mathrm{~cm}$, $\left.\mathrm{Et}_{2} \mathrm{O}\right)$ ergab $12.5 \mathrm{~g}(72 \%)$ des Alkohols als farbloses Öl, $R_{\mathrm{f}}\left(\mathrm{Et}_{2} \mathrm{O}\right)=0.44 .-{ }^{1} \mathrm{H}-\mathrm{NMR}$ $\left(250 \mathrm{MHz}, \mathrm{CDCl}_{3}\right): \delta=1.48-1.85\left(\mathrm{~m}, 10 \mathrm{H}, 2,3,2^{\prime}, 3^{\prime}, 4^{\prime}-\mathrm{H}\right), 2.28$ (br s, $\left.1 \mathrm{H}, \mathrm{OH}\right)$, 3.38-3.54 (m, $\left.2 \mathrm{H}, 4-\mathrm{H}^{*}\right), 3.66\left(\mathrm{t},{ }^{3} J=5.9 \mathrm{~Hz}, 2 \mathrm{H}, 1-\mathrm{H}^{*}\right), 3.75-3.89$ (m, $\left.2 \mathrm{H}, 5^{\prime}-\mathrm{H}^{*}\right), 4.60$ $\left(\mathrm{m}_{\mathrm{c}}, 1 \mathrm{H}, 1^{\prime}-\mathrm{H}\right) .-{ }^{13} \mathrm{C}-\mathrm{NMR}\left(62.9 \mathrm{MHz}, \mathrm{CDCl}_{3}, \mathrm{DEPT}\right): \delta=19.52\left(-, \mathrm{C}-3^{\prime *}\right), 25.35(-$, C-4'*), 26.54 (-, C-2*), 30.11 (-, C-3), 30.58 (-, C-2*), 62.32 (-, C-4**), 62.70 (-, $\left.\mathrm{C}-1^{* *}\right), 67.51\left(-, \mathrm{C}-5^{\prime * *}\right), 98.86\left(+, \mathrm{C}-1^{\prime}\right)$.

4-Tetrahydropyranyloxybutan-1-al (178): Nach AAV 8 versetzte man eine Lösung aus

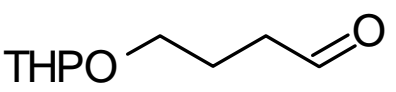
$7.5 \mathrm{ml}$ (85 mmol) Oxalylchlorid und $12.8 \mathrm{ml} \quad(180 \mathrm{mmol})$ DMSO mit $13.1 \mathrm{~g}$ (75.2 mmol) 4-Tetrahydropyranyloxybutan1-ol in $250 \mathrm{ml} \mathrm{CH}_{2} \mathrm{Cl}_{2}$ und rührte $30 \mathrm{~min}$. Danach fügte man $53.0 \mathrm{ml}(375 \mathrm{mmol})$ Triethylamin zu und rührte weitere $16 \mathrm{~h}$. Nach wäßriger Aufarbeitung reinigte man das Rohprodukt durch Säulenchromatographie an $150 \mathrm{~g}$ Kieselgel (Säule $5.0 \times 15 \mathrm{~cm}$, Pentan $\left./ \mathrm{Et}_{2} \mathrm{O}=1: 2\right)$. Man erhielt $10.1 \mathrm{~g}(78 \%) 178$ als farbloses Öl, $R_{\mathrm{f}}$ $\left(\mathrm{Pentan}_{\mathrm{Et}} \mathrm{O}\right)=0.56 .-{ }^{1} \mathrm{H}-\mathrm{NMR}\left(250 \mathrm{MHz}, \mathrm{CDCl}_{3}\right): \delta=1.48-1.90\left(\mathrm{~m}, 6 \mathrm{H}, 2^{\prime}, 3^{\prime}, 4^{\prime}-\mathrm{H}\right)$, 1.92 (quint, $\left.{ }^{3} J=6.8 \mathrm{~Hz}, 2 \mathrm{H}, 3-\mathrm{H}\right), 2.52\left(\mathrm{dt},{ }^{3} J=1.6,{ }^{3} \mathrm{~J}=6.8 \mathrm{~Hz}, 2 \mathrm{H}, 2-\mathrm{H}\right), 3.35-3.52$ (m, $\left.2 \mathrm{H}, 4-\mathrm{H}^{*}\right), 3.71-3.84\left(\mathrm{~m}, 2 \mathrm{H}, 5^{\prime}-\mathrm{H}^{*}\right), 4.54\left(\mathrm{~m}_{\mathrm{c}}, 1 \mathrm{H}, 1^{\prime}-\mathrm{H}\right), 9.76\left(\mathrm{t},{ }^{3} \mathrm{~J}=1.6 \mathrm{~Hz}, 1 \mathrm{H}\right.$, 1-H). $-{ }^{13} \mathrm{C}-\mathrm{NMR}\left(62.9 \mathrm{MHz}, \mathrm{CDCl}_{3}, \mathrm{DEPT}\right): \delta=19.41\left(-, \mathrm{C}-3^{\prime *}\right), 22.57$ (-, C-4'*), 25.34 $(-$, C-3*), $30.50(-$, C-2'*), 41.05 (-, C-2), 62.21 (-, C-4**), 66.32 (-, C-5'**), 98.77 (+, C-1'), 202.34 (+, C-1). 
1-Tetrahydropyranyloxyhept-6-in-4-ol (180-H): Zu einer Lösung von $7.30 \mathrm{~g}$ (65.0 mmol)

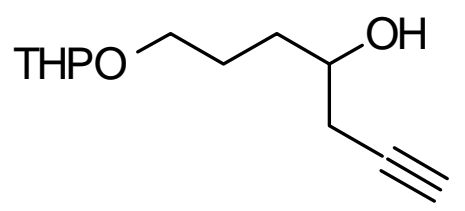

Trimethylsilylpropin und $7.88 \mathrm{~g}(71.5 \mathrm{mmol})$ TMEDA in $100 \mathrm{ml}$ wasserfreiem THF gab man bei $-78^{\circ} \mathrm{C} 26 \mathrm{ml}$ (65 mmol, $2.5 \mathrm{M}$ in Hexan) $n$-Butyllitiumlösung und erwärmte innerhalb von $12 \mathrm{~h}$ langsam auf Raumtemp. Man

rührte weitere $6 \mathrm{~h}$ bei Raumtemp., kühlte erneut auf $-78^{\circ} \mathrm{C}$ und fügte $10.05 \mathrm{~g}$ (58.35 mmol) $178 \mathrm{zu}$. Nachdem man $16 \mathrm{~h}$ bei Raumtemp. gerührt hatte, versetzte man mit $50 \mathrm{ml}$ Wasser, um noch vorhandene $n$-Butyllithiumlösung zu hydrolysieren. Anschließend gab man $10.0 \mathrm{~g}$ (72.4 mmol) $\mathrm{K}_{2} \mathrm{CO}_{3}$ und $80 \mathrm{ml}$ Methanol $\mathrm{zu}$ und rührte erneut $2 \mathrm{~h}$ bei Raumtemp. Danach wurden $100 \mathrm{ml}$ Wasser und $50 \mathrm{ml}$ Ether zugegeben, die organische Phase abgetrennt und die wäßrige Phase mit $3 \times 50 \mathrm{ml}$ Ether extrahiert. Nach Trocknen über $\mathrm{Na}_{2} \mathrm{SO}_{4}$ und Entfernen des Lösungsmittels i. Vak. reinigte man das Rohprodukt durch Säulenchromatographie an $50 \mathrm{~g}$ Kieselgel (Säule $2.5 \times 20 \mathrm{~cm}, P \operatorname{Pentan} / \mathrm{Et}_{2} \mathrm{O}=1: 1$ ). Es konnten $2.05 \mathrm{~g}(17 \%)$ der Verbindung 180-H als farbloses Öl, $R_{\mathrm{f}}\left(\right.$ Pentan/Et $_{2} \mathrm{O}$, $1: 1)=0.28$ isoliert werden. - IR (Film): $v=3414 \mathrm{~cm}^{-1}(\mathrm{OH}), 2944,2871,1653,1442$, 1354, 1261, 1201, 1138, 1120, 1076, 1025, 906, 867, 810. - ${ }^{1} \mathrm{H}-\mathrm{NMR}\left(250 \mathrm{MHz}, \mathrm{CDCl}_{3}\right)$ : $\delta=1.52-1.83\left(\mathrm{~m}, 10 \mathrm{H}, 2,3,2^{\prime}, 3^{\prime}, 4^{\prime}-\mathrm{H}\right), 2.04\left(\mathrm{t},{ }^{4} \mathrm{~J}=2.7 \mathrm{~Hz}, 1 \mathrm{H}, 7-\mathrm{H}\right), 2.39\left(\mathrm{~m}_{\mathrm{c}}, 2 \mathrm{H}\right.$, 5-H), 3.38-3.54 (m, 3 H, 1,4-H*), 3.77-3.85 (m, $\left.2 \mathrm{H}, 5{ }^{\prime}-\mathrm{H}^{*}\right), 4.59$ (m $\left., 1 \mathrm{H}, 1^{\prime}-\mathrm{H}\right)$. ${ }^{13} \mathrm{C}-\mathrm{NMR}\left(62.9 \mathrm{MHz}, \mathrm{CDCl}_{3}, \mathrm{DEPT}\right): \delta=19.49,19.56\left(-, \mathrm{C}-3^{\prime *}\right), 25.34\left(-, \mathrm{C}-4^{\prime *}\right), 25.98$, $26.16\left(-, \mathrm{C}^{*}\right), 27.23,27.26\left(-, \mathrm{C}-3^{*}\right), 30.54,30.58\left(-, \mathrm{C}-2^{* *}\right), 33.59$ (-, C-2*), 62.32, $62.39\left(-, \mathrm{C}^{\prime * *}\right), 67.52,67.60\left(-, \mathrm{C}-1^{* *}\right), 69.70,69.86(+, \mathrm{C}-4), 70.50(+, \mathrm{C}-7), 81.06$

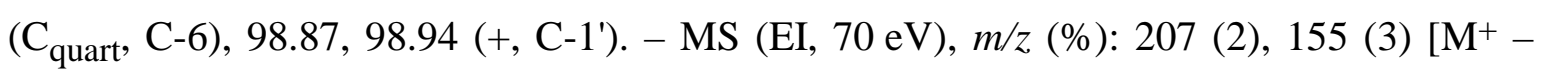
$\left.\mathrm{C}_{3} \mathrm{H}_{3}-\mathrm{H}_{2} \mathrm{O}\right], 127$ (4) $\left[\mathrm{M}^{+}-\mathrm{C}_{5} \mathrm{H}_{9} \mathrm{O}\right], 111$ (9), 101 (18), 85 (100) $\left[\mathrm{C}_{5} \mathrm{H}_{9} \mathrm{O}^{+}\right], 71$ (45), 56 (9), 41 (19). - MS (CI, $\left.\mathrm{NH}_{3}, 70 \mathrm{eV}\right), m / z$ (\%): 230 (29) [M + $\left.\mathrm{NH}_{4}^{+}\right] .-\mathrm{C}_{12} \mathrm{H}_{20} \mathrm{O}_{3}$ (212.3).

4-tert-Butyldimethylsilyloxy-1-tetrahydropyranyloxyhept-6-in $\quad\left(\mathbf{1 8 0 - S i M e} \mathbf{S}_{2}\right.$ tBu): $\quad \mathrm{Nach}$

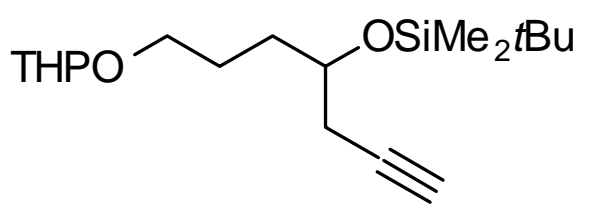

AAV 9 wurde eine Lösung von $1.80 \mathrm{~g}(8.48 \mathrm{mmol})$ 180-H in $20 \mathrm{ml}$ DMF mit $1.66 \mathrm{~g}(11.0 \mathrm{mmol})$ tert-Butyldimethylsilylchlorid und $\quad 1.56 \mathrm{~g}$ (23.0 mmol) Imidazol umgesetzt. Säulenchromatographie des Rohproduktes an $50 \mathrm{~g}$ Kieselgel (Säule $2.5 \times 20 \mathrm{~cm}, \mathrm{Pentan}_{2} / \mathrm{Et}_{2} \mathrm{O}=10: 1$ ) ergab 1.91 g (65\%) 180-SiMe 2 tBu als farbloses Öl, $R_{\mathrm{f}}\left(\right.$ Pentan/Et $\left._{2} \mathrm{O}, 10: 1\right)=0.37$. - IR 
(Film): $v=3314 \mathrm{~cm}^{-1}, 2952,2857,2121,1473,1362,1323,1257,1121,1035,869,837$, 776, 637. - ${ }^{1} \mathrm{H}-\mathrm{NMR}\left(250 \mathrm{MHz}, \mathrm{CDCl}_{3}\right): \delta=0.06\left(\mathrm{~s}, 3 \mathrm{H}, \mathrm{SiCH}_{3}\right), 0.07\left(\mathrm{~s}, 3 \mathrm{H}, \mathrm{SiCH}_{3}\right)$, 0.88 [s, $\left.9 \mathrm{H}, \mathrm{C}\left(\mathrm{CH}_{3}\right)_{3}\right], 1.43-1.82\left(\mathrm{~m}, 10 \mathrm{H}, 2,3,2^{\prime}, 3^{\prime}, 4^{\prime}-\mathrm{H}\right), 1.96$ (t, $\left.{ }^{4} \mathrm{~J}=2.6 \mathrm{~Hz}, 1 \mathrm{H}, 7-\mathrm{H}\right)$, 2.30-2.39 (m, 2 H, 5-H), 3.36-3.54 (m, 2 H, 5'-H*), 3.68-3.93 (m, 3 H, 1,4-H*), 4.58 (br t, $3 J=3.0 \mathrm{~Hz}, 1 \mathrm{H}, 1 '-\mathrm{H}) .-{ }^{13} \mathrm{C}-\mathrm{NMR}\left(62.9 \mathrm{MHz}, \mathrm{CDCl}_{3}, \mathrm{DEPT}\right): \delta=-4.68\left(+, \mathrm{SiCH}_{3}\right)$, $-4.52\left(+, \mathrm{SiCH}_{3}\right), 18.06\left[\mathrm{C}_{\text {quart }}, C\left(\mathrm{CH}_{3}\right)_{3}\right], 19.57\left(-, \mathrm{C}-3^{\prime *}\right), 25.30\left(-, \mathrm{C}-4^{\prime *}\right), 25.47$ (-, C-5*), $25.81\left[+, \mathrm{C}\left(\mathrm{CH}_{3}\right)_{3}\right], 27.27$ (-, C-3*), 30.71 (-, C-2*), 33.17 (-, C-2*), 62.21 (-, C-5'**), 67.45, 67.49 (-, C-1**), 69.88 (+, C-7), 70.63, 70.69 (+, C-4), $81.63\left(\mathrm{C}_{\text {quart }}, \mathrm{C}-6\right)$, 98.69, 98.72 (+, C-1'). - MS (EI, $70 \mathrm{eV}), m / z(\%): 241$ (3) [ $\left.\mathrm{M}^{+}-\mathrm{C}_{5} \mathrm{H}_{9} \mathrm{O}\right], 225$ (8), 203 (6), 185 (2), 159 (7), 145 (5), 85 (100) [ $\left.\mathrm{C}_{5} \mathrm{H}_{9} \mathrm{O}^{+}\right], 73$ (9), 41 (4). - MS (CI, $\left.\mathrm{NH}_{3}, 70 \mathrm{eV}\right), \mathrm{m} / z$ (\%): 344 (100) $\left[\mathrm{M}+\mathrm{NH}_{4}^{+}\right] .-\mathrm{C}_{18} \mathrm{H}_{34} \mathrm{O}_{3} \mathrm{Si}$ (326.5): ber. C 66.21, $\mathrm{H}$ 10.49; gef. C 66.07, H 10.23.

4-tert-Butyldimethylsilyloxyhept-6-in-1-ol (182): Nach AAV 10 versetze man $1.79 \mathrm{~g}$

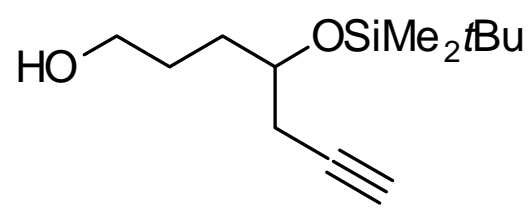
$(5.48 \mathrm{mmol}) \mathbf{1 8 0}-\mathrm{SiMe}_{2} t \mathrm{Bu}$ in $20 \mathrm{ml}$ wasserfreiem $\mathrm{CH}_{2} \mathrm{Cl}_{2}$ bei $-25^{\circ} \mathrm{C}$ mit $11 \mathrm{ml}(11 \mathrm{mmol}, 1.0 \mathrm{M}$ in Hexan) Diethylaluminiumchloridlösung und erwärmte auf Raumtemp. Man rührte $12 \mathrm{~h}$ bei dieser Temperatur und reinigte das erhaltene Rohprodukt durch Säulenchromatographie an $25 \mathrm{~g}$ Kieselgel (Säule $2.2 \times 15 \mathrm{~cm}, \mathrm{Pentan} \mathrm{Et}_{2} \mathrm{O}=1: 1$ ). Man isolierte $0.85 \mathrm{~g}(64 \%) 182$ als farbloses Öl, $R_{\mathrm{f}}\left(\operatorname{Pentan} / \mathrm{Et}_{2} \mathrm{O}, 1: 1\right)=0.41$. - IR (Film): $v=3314 \mathrm{~cm}^{-1}, 2930,2858,2122,1473,1362,1257,1103,1006,838,810,776,635$. ${ }^{1} \mathrm{H}-\mathrm{NMR}\left(250 \mathrm{MHz}, \mathrm{CDCl}_{3}\right): \delta=0.07\left(\mathrm{~s}, 3 \mathrm{H}, \mathrm{SiCH}_{3}\right), 0.09$ (s, $\left.3 \mathrm{H}, \mathrm{SiCH}_{3}\right), 0.89$ [s, $\left.9 \mathrm{H}, \mathrm{C}\left(\mathrm{CH}_{3}\right)_{3}\right], 1.65-1.72(\mathrm{~m}, 4 \mathrm{H}, 2,3-\mathrm{H}), 1.84(\mathrm{~s}, 1 \mathrm{H}, \mathrm{OH}), 1.98\left(\mathrm{t},{ }^{4} \mathrm{~J}=2.7 \mathrm{~Hz}, 1 \mathrm{H}\right.$, 7-H), 2.34-2.38 (m, 2 H, 5-H), 3.62-3.69 (m, 2 H, 1-H), 3.83-3.95 (m, $1 \mathrm{H}, 4-\mathrm{H})$. 13C-NMR (62.9 MHz, $\left.\mathrm{CDCl}_{3}, \mathrm{DEPT}\right): \delta=-4.73\left(+, \mathrm{SiCH}_{3}\right),-4.57\left(+, \mathrm{SiCH}_{3}\right), 18.05$ $\left[\mathrm{C}_{\text {quart }}, \mathrm{C}\left(\mathrm{CH}_{3}\right)_{3}\right], 25.78\left[+, \mathrm{C}\left(\mathrm{CH}_{3}\right)_{3}\right], 26.90\left(-, \mathrm{C}-2^{*}\right), 28.08\left(-, \mathrm{C}-3^{*}\right), 32.83\left(-, \mathrm{C}-5^{*}\right)$, 63.00 (-, C-1), 70.07 (+, C-7), 70.64 (+, C-4), 81.36 (C quart, $\mathrm{C}-6)$. - MS (CI, NH, $70 \mathrm{eV})$, $m / z(\%): 260$ (80) $\left[\mathrm{M}+\mathrm{NH}_{4}^{+}\right], 243$ (100) $\left[\mathrm{M}+\mathrm{H}^{+}\right] .-\mathrm{C}_{13} \mathrm{H}_{26} \mathrm{O}_{2} \mathrm{Si}$ (242.4): ber. C 64.41, H 10.81; gef. C 64.64, H 10.83 . 
4-tert-Butyldimethylsilyloxyhept-6-in-1-al (183): Man gab zu 2.13 g (5.00 mmol) Dess-

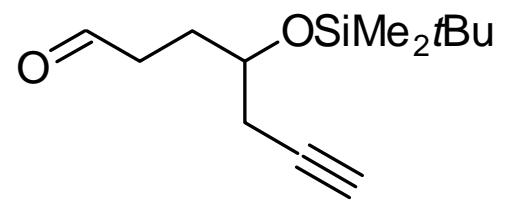

Martin-Reagenz in $25 \mathrm{ml}$ wasserfreiem $\mathrm{CH}_{2} \mathrm{Cl}_{2} 815 \mathrm{mg}$ (3.36 mmol) 182 und rührte $16 \mathrm{~h}$ bei Raumtemp. Zur

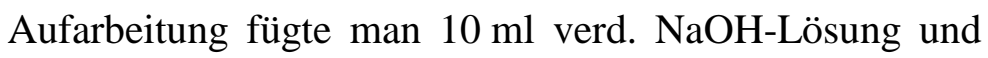
$10 \mathrm{ml}$ Ether $\mathrm{zu}$, wusch die organische Phase mit je $10 \mathrm{ml}$ verd. $\mathrm{NaOH}-\mathrm{Lösung}$ und Wasser und trocknete über $\mathrm{MgSO}_{4}$. Nach Entfernen des Lösungsmittel i. Vak. reinigte man das Rohprodukt durch

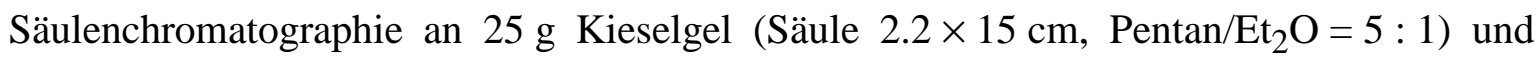

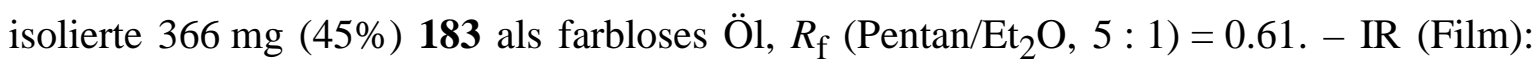
$v=3313 \mathrm{~cm}^{-1}, 2959,2858,1712(\mathrm{C}=\mathrm{O}), 1473,1414,1259,1104,1007,836,778,637$. ${ }^{1} \mathrm{H}-\mathrm{NMR}\left(250 \mathrm{MHz}, \mathrm{CDCl}_{3}\right): \delta=0.05\left(\mathrm{~s}, 3 \mathrm{H}, \mathrm{SiCH}_{3}\right), 0.08\left(\mathrm{~s}, 3 \mathrm{H}, \mathrm{SiCH}_{3}\right), 0.88$ [s, $\left.9 \mathrm{H}, \mathrm{C}\left(\mathrm{CH}_{3}\right)_{3}\right], 1.88\left(\mathrm{~m}_{\mathrm{c}}, 1 \mathrm{H}, 3-\mathrm{H}\right), 2.00\left(\mathrm{t},{ }^{4} \mathrm{~J}=2.7 \mathrm{~Hz}, 1 \mathrm{H}, 7-\mathrm{H}\right), 1.99-2.10(\mathrm{~m}, 1 \mathrm{H}$, 3-H), 2.30-2.36 (m, 2 H, 5-H), 2.49-2.55 (m, 2 H, 2-H), 3.89 (m, 1 H, 4-H), 9.79 (t, $3 J=1.6 \mathrm{~Hz}, 1 \mathrm{H}, 1-\mathrm{H}) .-{ }^{13} \mathrm{C}-\mathrm{NMR}\left(62.9 \mathrm{MHz}^{3} \mathrm{CDCl}_{3}, \mathrm{DEPT}\right): \delta=-4.78\left(+, \mathrm{SiCH}_{3}\right)$, $-4.56\left(+, \mathrm{SiCH}_{3}\right), 17.98\left[\mathrm{C}_{\text {quart }}, \mathrm{C}\left(\mathrm{CH}_{3}\right)_{3}\right], 25.74\left[+, \mathrm{C}\left(\mathrm{CH}_{3}\right)_{3}\right], 27.11(-, \mathrm{C}-2 *), 28.56(-$, C-3*), $\left.39.46\left(-, \mathrm{C}^{*}\right)^{*}\right), 69.68$ (+, C-7), 70.48 (+, C-4), $80.76\left(\mathrm{C}_{\text {quart }}, \mathrm{C}-6\right), 202.34$ (+, C-1). - MS (CI, $\left.\mathrm{NH}_{3}, 70 \mathrm{eV}\right), \mathrm{m} / z(\%): 275(18)\left[\mathrm{M}+\mathrm{NH}_{4}{ }^{+}+\mathrm{NH}_{3}\right], 258$ (27) $\left[\mathrm{M}+\mathrm{NH}_{4}{ }^{+}\right], 241$ (14) $\left[\mathrm{M}+\mathrm{H}^{+}\right] .-\mathrm{C}_{13} \mathrm{H}_{24} \mathrm{O}_{2} \mathrm{Si}(240.4)$.

Versuch der Darstellung eines Silylenolethers aus 183: Zu einer Lösung von $347 \mathrm{mg}$ $(1.44 \mathrm{mmol})$ in $20 \mathrm{ml}$ DMF gab man $146 \mathrm{mg}(1.44 \mathrm{mmol})$ Triethylamin und $396 \mathrm{mg}$ (1.44 mmol) tert-Butyldiphenylsilylchlorid. Man erwärmte $16 \mathrm{~h}$ auf $120^{\circ} \mathrm{C}$. Nach Abkühlen auf Raumtemp. fügte man $20 \mathrm{ml}$ Wasser und $20 \mathrm{ml}$ Pentan zu und extrahierte die wäßrige Phase mit $3 \times 20 \mathrm{ml}$ Pentan. Man trocknete über $\mathrm{Na}_{2} \mathrm{SO}_{4}$ und entfernte das Lösungsmittel i. Vak. Das ${ }^{1} \mathrm{H}-\mathrm{NMR}-$ Spektrum des Rohproduktes zeigte nur Signale von tert-Butyldiphenylsilylchlorid und Zersetzung.

3-Tetrahydropyranyloxypropan-1-ol (186): Analog AAV 7 gab man zu 38.1 g (0.501 mol) Propandiol $8.40 \mathrm{~g} \quad(99.9 \mathrm{mmol}) \quad 3,4$-Dihydro-2H-pyran und THPO $ح$ OH $200 \mathrm{mg}(1.16 \mathrm{mmol})$ p-Toluolsulfonsäure. Nachdem man $4 \mathrm{~h}$ rührte, arbeitete man wäßrig auf, trocknete und engte i. Vak. ein. Säulenchromatographie des Rohproduktes an $250 \mathrm{~g}$ Kieselgel (Säule $8.0 \times 20 \mathrm{~cm}, \mathrm{Et}_{2} \mathrm{O}$ ) 
ergab $11.6 \mathrm{~g}(72 \%) 186$ als farbloses Öl, $R_{\mathrm{f}}\left(\mathrm{Et}_{2} \mathrm{O}\right)=0.44 .-{ }^{1} \mathrm{H}-\mathrm{NMR}\left(250 \mathrm{MHz}, \mathrm{CDCl}_{3}\right)$ : $\delta=1.51-1.89\left(\mathrm{~m}, 8 \mathrm{H}, 2,2^{\prime}, 3^{\prime}, 4^{\prime}-\mathrm{H}\right), 2.39$ (br s, $\left.1 \mathrm{H}, \mathrm{OH}\right), 3.47-3.62\left(\mathrm{~m}, 2 \mathrm{H}, 3-\mathrm{H}^{*}\right), 3.78$ $\left(\mathrm{t}, 3 \mathrm{~J}=5.6 \mathrm{~Hz}, 2 \mathrm{H}, 1-\mathrm{H}^{*}\right), 3.81-3.97\left(\mathrm{~m}, 2 \mathrm{H}, 5^{\prime}-\mathrm{H}^{*}\right), 4.57-4.59$ (m, 1 H, 1'-H). ${ }^{13} \mathrm{C}-\mathrm{NMR}\left(62.9 \mathrm{MHz}, \mathrm{CDCl}_{3}, \mathrm{DEPT}\right): \delta=19.63\left(-, \mathrm{C}-3^{\prime *}\right), 25.28$ (-, C-4'*), 30.61 (-, C-2), $31.97(-$, C-2*), 61.58 (-, C-3**), 62.54 (-, C-1**), 66.33 (-, C-5'**), 99.13 (+, C-1'). $-\mathrm{C}_{8} \mathrm{H}_{16} \mathrm{O}(160.2)$.

3-Tetrahydropyranyloxypropan-1-al (187): Nach AAV 8 versetzte man eine Lösung aus $7.5 \mathrm{ml}$ (85 mmol) Oxalylchlorid und $12.8 \mathrm{ml}$ (180 mmol) DMSO

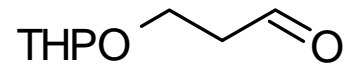
mit $11.6 \mathrm{~g}$ (72.4 mmol) des Alkohols 186 in $250 \mathrm{ml} \mathrm{CH}_{2} \mathrm{Cl}_{2}$ und rührte30 min. Anschließend fügte man $53.0 \mathrm{ml}$ (375 mmol) Triethylamin zu und rührte weitere $16 \mathrm{~h}$. Nach wäßriger Aufarbeitung reinigte man das Rohprodukt durch Säulenchromatographie an $150 \mathrm{~g}$ Kieselgel (Säule $5.0 \times 15 \mathrm{~cm}$, Pentan/ $\left.\mathrm{Et}_{2} \mathrm{O}=1: 2\right)$. Man erhielt $9.16 \mathrm{~g} \quad(80 \%) \quad 187$ als farbloses Öl, $R_{\mathrm{f}}$ $\left(\mathrm{Pentan}_{\mathrm{Et}} \mathrm{O}\right)=0.56 .-{ }^{1} \mathrm{H}-\mathrm{NMR}\left(250 \mathrm{MHz}, \mathrm{CDCl}_{3}\right): \delta=1.46-1.82\left(\mathrm{~m}, 6 \mathrm{H}, 2^{\prime}, 3^{\prime}, 4^{\prime}-\mathrm{H}\right)$, $2.69\left(\mathrm{dt},{ }^{3} \mathrm{~J}=1.8,{ }^{3} \mathrm{~J}=6.1 \mathrm{~Hz}, 2 \mathrm{H}, 2-\mathrm{H}\right), 3.46-3.55\left(\mathrm{~m}, 1 \mathrm{H}, 5^{\prime}-\mathrm{H}^{*}\right), 3.70-3.88(\mathrm{~m}, 2 \mathrm{H}$, 3,5'-H*), $4.08\left(\mathrm{~m}_{\mathrm{c}}, 1 \mathrm{H}, 3-\mathrm{H}^{*}\right), 4.62\left(\mathrm{~m}_{\mathrm{c}}, 1 \mathrm{H}, 1^{\prime}-\mathrm{H}\right), 9.81\left(\mathrm{t},{ }^{3} \mathrm{~J}=1.8 \mathrm{~Hz}, 1 \mathrm{H}, 1-\mathrm{H}\right)$. $\mathrm{C}_{8} \mathrm{H}_{14} \mathrm{O}_{3}$ (158.2).

1-Tetrahydropyranyloxyhex-5-in-3-ol (189): Zunächst tropfte man etwa ein Zehntel von

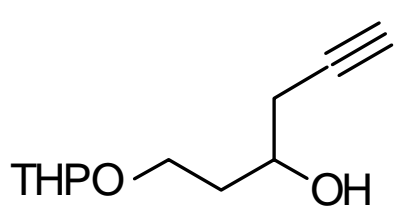
$9.67 \mathrm{~g}$ (65.0 mmol, 80\% in Toluol) Propargylbromid zu $1.59 \mathrm{~g}$ (65.0 mmol) Magnesium, das mit einer Spatelspitze $\mathrm{HgCl}_{2}$ angeätzt worden war. Man wartete 5-10 min, bis die Reaktion einsetzte und gab dann bei $0{ }^{\circ} \mathrm{C}$ das restliche Propargylbromid und $60 \mathrm{ml}$ Ether so zu, daß die Lösung leicht siedete. Man ließ $1 \mathrm{~h}$ bei $0{ }^{\circ} \mathrm{C}$ rühren. Das so erhaltene Grignard-Reagenz wurde dann bei $-78^{\circ} \mathrm{C} \mathrm{zu}$ einer Lösung von $9.16 \mathrm{~g}$ (57.9 mmol) 187 in $150 \mathrm{ml}$ Ether getropft. Man erwärmte langsam auf Raumtemp. und rührte $16 \mathrm{~h}$ bei dieser Temperatur. Anschließend hydrolysierte man mit $50 \mathrm{ml}$ ges. $\mathrm{NH}_{4} \mathrm{Cl}$ Lösung, wusch die organische Phase mit je $50 \mathrm{ml}$ Wasser und ges. NaCl-Lösung, trocknete über $\mathrm{Na}_{2} \mathrm{SO}_{4}$ und entfernte das Lösungsmittel i. Vak. Säulenchromatographie des Rohproduktes an $70 \mathrm{~g}$ Kieselgel (Säule $3.5 \times 15 \mathrm{~cm}$, Pentan/ $\mathrm{Et}_{2} \mathrm{O}=1: 2$ ) ergab 5.96 (52\%)

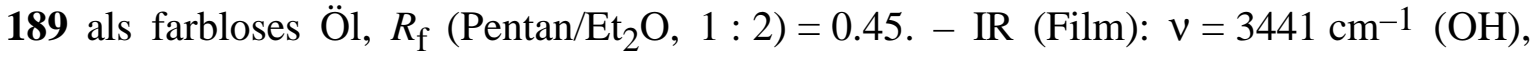


3296, 2944, 1442, 1353, 1261, 1138, 1120, 1035, 978, 905, 869, 812, 635. - ${ }^{1} \mathrm{H}-\mathrm{NMR}$ $\left(250 \mathrm{MHz}, \mathrm{CDCl}_{3}\right): \delta=1.48-1.92\left(\mathrm{~m}, 8 \mathrm{H}, 2,2^{\prime}, 3^{\prime}, 4^{\prime}-\mathrm{H}\right), 2.03\left(\mathrm{dt},{ }^{4} \mathrm{~J}=2.7,{ }^{5} \mathrm{~J}=0.8 \mathrm{~Hz}\right.$, $1 \mathrm{H}, 6-\mathrm{H}), 2.41\left(\mathrm{dd},{ }^{3} \mathrm{~J}=6.1,{ }^{4} \mathrm{~J}=2.7 \mathrm{~Hz}, 2 \mathrm{H}, 4-\mathrm{H}\right), 3.14($ br s, $1 \mathrm{H}, \mathrm{OH}), 3.48-3.69$ (m, $\left.2 \mathrm{H}, 1-\mathrm{H}^{*}\right), 3.79-4.04\left(\mathrm{~m}, 3 \mathrm{H}, 3,5^{\prime}-\mathrm{H}^{*}\right), 4.58-4.61\left(\mathrm{~m}, 1 \mathrm{H}, 1^{\prime}-\mathrm{H}\right) . \quad-{ }^{13} \mathrm{C}-\mathrm{NMR}$ (62.9 MHz, $\left.\mathrm{CDCl}_{3}, \mathrm{DEPT}\right): \delta=19.35,19.61$ (-, C-3'*), $25.23(-, \mathrm{C}-2 *), 26.98,27.05$ (-, C-4'*), 30.41, 30.61 (-, C-2'*), 35.20, 35.24 (-, C-4*), 62.20, 62.67 (-, C-1**), 65.62, $65.82\left(-, \mathrm{C}^{\prime * * *}\right), 69.04,69.62$ (+, C-3), 70.30, 70.38 (+, C-6), 80.94, 81.01 (C quart, $\left.\mathrm{C}-5\right)$, 98.89, 99.16 (+, C-1'). - MS (EI, $70 \mathrm{eV}), \mathrm{m} / z$ (\%): 101 (25), 85 (100), 67 (8), 57 (18), 41 (25). - MS (CI, $\left.\mathrm{NH}_{3}, 70 \mathrm{eV}\right), m / z(\%): 612$ (1) $\left[3 \mathrm{M}+\mathrm{NH}_{4}^{+}\right], 414$ (100) $\left[2 \mathrm{M}+\mathrm{NH}_{4}^{+}\right]$, 216 (51) $\left[\mathrm{M}+\mathrm{NH}_{4}^{+}\right], 199$ (3) $\left[\mathrm{M}+\mathrm{H}^{+}\right] .-\mathrm{C}_{11} \mathrm{H}_{18} \mathrm{O}_{3}$ (198.3): ber. C 66.64, H 9.15; gef. C 66.45, H 9.00.

3-tert-Butyldimethylsilyloxy-1-tetrahydropyranyloxyhex-5-in (190-THP): Analog AAV 9

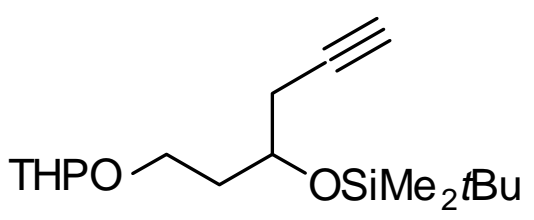
versetzte man eine Lösung von $5.70 \mathrm{~g}(28.8 \mathrm{mmol}) \mathbf{1 8 9}$ in $\quad 100 \mathrm{ml} \quad$ DMF mit $5.21 \mathrm{~g} \quad(34.5 \mathrm{mmol})$ tert-Butyldimethylsilylchlorid und $4.88 \mathrm{~g}(72.0 \mathrm{mmol})$ Imidazol. Säulenchromatographie des Rohproduktes an $100 \mathrm{~g}$ Kieselgel (Säule $3.5 \times 20 \mathrm{~cm}$, Pentan/ $\mathrm{Et}_{2} \mathrm{O}=10: 1$ ) ergab $7.97 \mathrm{~g}(89 \%)$ 190-THP als farbloses Öl, $R_{\mathrm{f}}\left(\right.$ Pentan$\left./ \mathrm{Et}_{2} \mathrm{O}, 10: 1\right)=0.44$. - IR (Film): $v=3314 \mathrm{~cm}^{-1}, 2953,2857$, 2121, 1473, 1441, 1361, 1323, 1257, 1201, 1184, 1121, 1070, 1035, 986, 870, 837, 815, 776, 737, 637. - ${ }^{1} \mathrm{H}-\mathrm{NMR}\left(250 \mathrm{MHz}, \mathrm{CDCl}_{3}\right): \delta=0.08$ (br s, $\left.6 \mathrm{H}, \mathrm{SiCH}_{3}\right), 0.88[\mathrm{~s}, 9 \mathrm{H}$, $\left.\mathrm{C}\left(\mathrm{CH}_{3}\right)_{3}\right], 1.44-1.95\left(\mathrm{~m}, 8 \mathrm{H}, 2,2^{\prime}, 3^{\prime}, 4^{\prime}-\mathrm{H}\right), 1.97\left(\mathrm{t},{ }^{4} \mathrm{~J}=2.7 \mathrm{~Hz}, 1 \mathrm{H}, 6-\mathrm{H}\right), 2.36\left(\mathrm{~m}_{\mathrm{c}}, 2 \mathrm{H}\right.$,

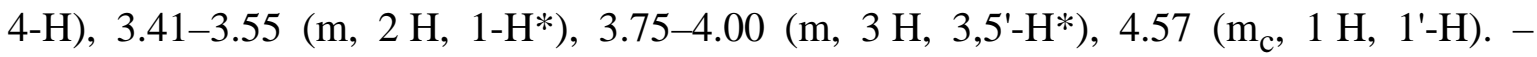
${ }^{13} \mathrm{C}-\mathrm{NMR}\left(62.9 \mathrm{MHz}, \mathrm{CDCl}_{3}, \mathrm{DEPT}\right): \delta=-4.85,-4.79\left(+, \mathrm{SiCH}_{3}\right),-4.53,-4.51(+$, $\left.\mathrm{SiCH}_{3}\right), 18.04\left[\mathrm{C}_{\text {quart }}, C\left(\mathrm{CH}_{3}\right)_{3}\right], 19.59\left(-, \mathrm{C}-3^{\prime *}\right), 25.46\left(-, \mathrm{C}-2^{*}\right), 25.79\left[+, \mathrm{C}\left(\mathrm{CH}_{3}\right)_{3}\right]$, 27.58, 27.65 (-, C-4'*), 30.68, 30.74 (-, C-2'*), 36.52 (-, C-4*), 62.27 (-, C-5'**), 63.54, $63.87\left(-, \mathrm{C}-1^{* *}\right), 67.79,68.39$ (+, C-3), 69.98, $70.04(+, \mathrm{C}-6), 81.42,81.50\left(\mathrm{C}_{\text {quart }}, \mathrm{C}-5\right)$, 98.63, 98.84 (+, C-1'). - MS (CI, $\left.\mathrm{NH}_{3}, 70 \mathrm{eV}\right), \mathrm{m} / \mathrm{z}(\%): 330$ (53) $\left[\mathrm{M}+\mathrm{NH}_{4}^{+}\right], 313$ (13) $\left[\mathrm{M}+\mathrm{H}^{+}\right] .-\mathrm{C}_{17} \mathrm{H}_{32} \mathrm{O}_{3} \mathrm{Si}$ (312.5): ber. C 65.34, H 10.32; gef. C 65.23, H 10.10. 
Versuch der Alkylierung von 190-H mit Brompenten: Variante A: $\mathrm{Zu}$ einer Lösung von $1.60 \mathrm{~g}$ (7.00 mmol) des Alkins 190-H gab man bei $-78{ }^{\circ} \mathrm{C} 6.8 \mathrm{ml}(17 \mathrm{mmol}, 2.5 \mathrm{M}$ in Hexan) n-Butyllithium und rührte $30 \mathrm{~min}$ bei dieser Temp. Dann gab man $1.08 \mathrm{~g}$ (7.25 mmol) Brompenten zu und ließ auf Raumtemp. erwärmen. Nachdem man $18 \mathrm{~h}$ bei dieser Temp. gerührt hatte, arbeitete man wäßrig auf. Das ${ }^{1} \mathrm{H}-\mathrm{NMR}-$ Spektrum des Rohproduktes zeigte nur Signale der Ausgangsverbindungen.

Variante B: Nach Zugabe von 2 ml HMPT unter sonst identischen Bedingungen konnte ebenfalls kein Produkt isoliert werden.

3-tert-Butyldimethylsilyloxyhex-5-in-1-ol (190-H): Analog AAV 10 löste man $7.81 \mathrm{~g}$

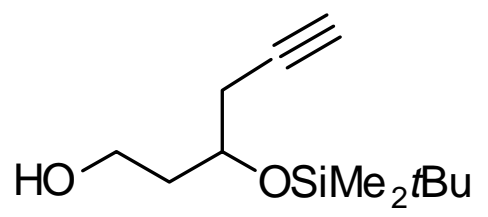

(25.0 mmol) 190-THP in $100 \mathrm{ml}$ wasserfreiem $\mathrm{CH}_{2} \mathrm{Cl}_{2}$, versetzte bei $-25{ }^{\circ} \mathrm{C}$ mit $50 \mathrm{ml}(50 \mathrm{mmol}, 1.0 \mathrm{M}$ in Hexan)

Diethylaluminiumchloridlösung und erwärmte auf Raumtemp. Man rührte $12 \mathrm{~h}$ bei dieser Temperatur und reinigte das erhaltene Rohprodukt durch Säulenchromatographie an 50 g Kieselgel (Säule $2.5 \times 20 \mathrm{~cm}$, Pentan/Et $2 \mathrm{O}=1: 1)$. Man isolierte $3.18 \mathrm{~g}(56 \%) \mathbf{1 9 0}-\mathbf{H}$ als farbloses Öl, $R_{\mathrm{f}}$ $\left(\right.$ Pentan $\left./ \mathrm{Et}_{2} \mathrm{O}, 1: 1\right)=0.54$. - IR $\left(\right.$ Film) $: v=3314 \mathrm{~cm}^{-1}, 2930,2858,1473,1464,1362$, 1258, 1102, 1066, 1023, 837, 777, 636. ${ }^{1} \mathrm{H}-\mathrm{NMR}\left(250 \mathrm{MHz}, \mathrm{CDCl}_{3}\right): \delta=0.10(\mathrm{~s}, 6 \mathrm{H}$, $\left.\mathrm{SiCH}_{3}\right), 0.89$ [s, $\left.9 \mathrm{H}, \mathrm{C}\left(\mathrm{CH}_{3}\right)_{3}\right], 1.75-1.97(\mathrm{~m}, 2 \mathrm{H}, 2-\mathrm{H}), 2.00\left(\mathrm{t},{ }^{4} \mathrm{~J}=2.7 \mathrm{~Hz}, 1 \mathrm{H}, 6-\mathrm{H}\right)$, $2.16(\mathrm{~s}, 1 \mathrm{H}, \mathrm{OH}), 2.40\left(\mathrm{dd},{ }^{3} \mathrm{~J}=6.5,{ }^{4} \mathrm{~J}=2.7 \mathrm{~Hz}, 2 \mathrm{H}, 4-\mathrm{H}\right), 3.79\left(\mathrm{~m}_{\mathrm{c}}, 2 \mathrm{H}, 1-\mathrm{H}\right), 4.06\left(\mathrm{~m}_{\mathrm{c}}\right.$, $1 \mathrm{H}, 3-\mathrm{H}) .-{ }^{13} \mathrm{C}-\mathrm{NMR}\left(62.9 \mathrm{MHz}, \mathrm{CDCl}_{3}, \mathrm{DEPT}\right): \delta=-4.94\left(+, \mathrm{SiCH}_{3}\right),-4.58(+$, $\left.\mathrm{SiCH}_{3}\right), 17.92\left[\mathrm{C}_{\text {quart }}, \mathrm{C}\left(\mathrm{CH}_{3}\right)_{3}\right], 25.72\left[+, \mathrm{C}\left(\mathrm{CH}_{3}\right)_{3}\right], 27.00(-, \mathrm{C}-2 *), 37.70\left(-, \mathrm{C}-4^{*}\right)$, $59.80(-, \mathrm{C}-1), 69.93$ (+, C-3), 70.43 (+, C-6), 80.90 (C quart, $\mathrm{C}-6)$. - MS (CI, NH $3,70 \mathrm{eV})$, $m / z(\%): 263$ (3) $\left[\mathrm{M}+\mathrm{NH}_{4}^{+}+\mathrm{NH}_{3}\right], 246(100)\left[\mathrm{M}+\mathrm{NH}_{4}^{+}\right], 229$ (44) $\left[\mathrm{M}+\mathrm{H}^{+}\right] .-$ $\mathrm{C}_{12} \mathrm{H}_{24} \mathrm{O}_{2} \mathrm{Si}$ (228.4): ber. C 63.10, H 10.59; gef. C 63.33, H 10.30 .

3-tert-Butyldimethylsilyloxyhex-5-in-1-al (191): Entsprechend AAV 8 wurden $1.41 \mathrm{~g}$

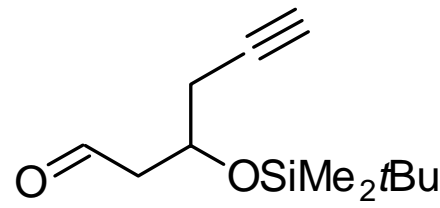
(6.17 mmol) $\mathbf{1 9 0}-\mathbf{H}$ mit $0.70 \mathrm{ml}(7.0 \mathrm{mmol})$ Oxalylchlorid, $1.1 \mathrm{ml} \quad(14 \mathrm{mmol}) \quad$ DMSO und $3.14 \mathrm{~g} \quad(31.0 \mathrm{mmol})$ Triethylamin in $50 \mathrm{ml} \mathrm{CH}_{2} \mathrm{Cl}_{2}$ bei $-60{ }^{\circ} \mathrm{C}$ oxidiert. Nach wäßriger Aufarbeitung, Trocknung über $\mathrm{MgSO}_{4}$ und Entfernen des Lösungsmittels i. Vak. wurde das erhaltene Rohprodukt ohne weitere 
Reinigung weiterverwendet. Man erhielt $1.24 \mathrm{~g}$ (89\%) als farbloses Öl. - IR (Film): $v=3312 \mathrm{~cm}^{-1}, 2930,2857,1727(\mathrm{C}=\mathrm{O}), 1473,1464,1362,1257,1106,837,772,631$. ${ }^{1} \mathrm{H}-\mathrm{NMR}\left(250 \mathrm{MHz}, \mathrm{CDCl}_{3}\right): \delta=0.07$ (s, $\left.3 \mathrm{H}, \mathrm{SiCH}_{3}\right), 0.10$ (s, $\left.3 \mathrm{H}, \mathrm{SiCH}_{3}\right), 0.86$ [s, $\left.9 \mathrm{H}, \mathrm{C}\left(\mathrm{CH}_{3}\right)_{3}\right], 2.03\left(\mathrm{t},{ }^{4} \mathrm{~J}=2.7 \mathrm{~Hz}, 1 \mathrm{H}, 6-\mathrm{H}\right), 2.39-2.45(\mathrm{~m}, 2 \mathrm{H}, 4-\mathrm{H}), 2.71$ (ddd, $\left.2 J=4.7,{ }^{3} J=1.8,{ }^{3} J=6.6 \mathrm{~Hz}, 2 \mathrm{H}, 2-\mathrm{H}\right), 4.35\left(\mathrm{~m}_{\mathrm{c}}, 1 \mathrm{H}, 3-\mathrm{H}\right), 9.81\left(\mathrm{t},{ }^{3} \mathrm{~J}=1.8 \mathrm{~Hz}, 1 \mathrm{H}\right.$, 1-H). $-{ }^{13} \mathrm{C}-\mathrm{NMR}\left(62.9 \mathrm{MHz}, \mathrm{CDCl}_{3}, \mathrm{DEPT}\right): \delta=-4.92\left(+, \mathrm{SiCH}_{3}\right),-4.56\left(+, \mathrm{SiCH}_{3}\right)$, $17.91\left[\mathrm{C}_{\text {quart }}, C\left(\mathrm{CH}_{3}\right)_{3}\right], 24.61\left[+, \mathrm{C}\left(\mathrm{CH}_{3}\right)_{3}\right], 27.64(-, \mathrm{C}-4), 50.19$ (-, C-2), 66.65 (+, C-3), 71.11 (+, C-6), 80.17 (C quart $\left._{1}, \mathrm{C}-5\right), 201.28$ (+, C-1). - MS (EI, $\left.70 \mathrm{eV}\right), \mathrm{m} / z(\%): 187$ (24) $\left[\mathrm{M}^{+}-\mathrm{C}_{3} \mathrm{H}_{3}\right], 169$ (74) $\left[\mathrm{M}^{+}-\mathrm{C}_{4} \mathrm{H}_{9}\right], 131$ (43), 125 (25), 101 (76), 95 (31), 75 (100), 59 (35). - MS (CI, $\left.\mathrm{NH}_{3}, 70 \mathrm{eV}\right), m / z(\%): 261$ (44) $\left[\mathrm{M}+\mathrm{NH}_{4}{ }^{+}+\mathrm{NH}_{3}\right], 244$ (100) $[\mathrm{M}+$ $\mathrm{NH}_{4}^{+}$]. $-\mathrm{C}_{12} \mathrm{H}_{22} \mathrm{O}_{2} \mathrm{Si}(226.4)$.

4-tert-Butyldimethylsilyloxy-1-chlorhept-6-in-1-en (192): Nach AAV 11 gab man zu einer

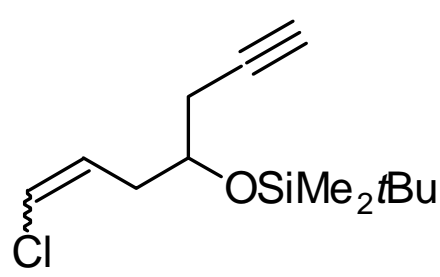

Suspension von $1.11 \mathrm{~g}$ (9.03 mmol) $\mathrm{CrCl}_{2}$ in $10 \mathrm{ml} \mathrm{THF}$ $340 \mathrm{mg}(1.50 \mathrm{mmol}) 191 \mathrm{und} 254 \mathrm{mg}(2.13 \mathrm{mmol}) \mathrm{CHCl}_{3}$ in $5 \mathrm{ml}$ THF. Nach $2.5 \mathrm{~h}$ arbeitete man die Reaktion auf, trocknete über $\mathrm{MgSO}_{4}$ und entfernte das Lösungsmittel i. Vak. Säulenchromatographie an $20 \mathrm{~g}$ Kieselgel $(2.0 \times 18 \mathrm{~cm}$,

Pentan/Et $2 \mathrm{O}=40: 1)$ ergab $159 \mathrm{mg}(41 \%) 192$ als farbloses Öl als E/Z-Gemisch, Verhältnis 84:16 (aus $\left.{ }^{1} \mathrm{H}-\mathrm{NMR}-S p e k t r u m\right), R_{\mathrm{f}}\left(\mathrm{Pentan}_{\mathrm{Et}} \mathrm{O}, 40: 1\right)=0.77+0.69$. - IR (Film): $v=3312 \mathrm{~cm}^{-1}, 2956,2930,2858,1635,1473,1362,1259,1100,940,837,810$, 777, 640. - ${ }^{1} \mathrm{H}-\mathrm{NMR}\left(250 \mathrm{MHz}, \mathrm{CDCl}_{3}\right): \delta=0.06$ (s, $\left.3 \mathrm{H}, \mathrm{SiCH}_{3}\right), 0.07$ (s, $\left.3 \mathrm{H}, \mathrm{SiCH}_{3}\right)$, 0.89 [s, $9 \mathrm{H}, \mathrm{C}\left(\mathrm{CH}_{3}\right)_{3}$ ], $2.00\left(\mathrm{t},{ }^{4} \mathrm{~J}=2.7 \mathrm{~Hz}, 1 \mathrm{H}, 7-\mathrm{H}\right), 2.21-2.53(\mathrm{~m}, 4 \mathrm{H}, 3,5-\mathrm{H}), 3.84$ $\left(\mathrm{m}_{\mathrm{c}}, 1 \mathrm{H}, 4-\mathrm{H}\right)$, 5.84-6.04 (m, $\left.2 \mathrm{H}, 1,2-\mathrm{H}\right) .-{ }^{13} \mathrm{C}-\mathrm{NMR}\left(62.9 \mathrm{MHz}, \mathrm{CDCl}_{3}, \mathrm{DEPT}\right)$ : $\delta=-4.75\left(+, \mathrm{SiCH}_{3}\right),-4.66\left(+, \mathrm{SiCH}_{3}\right), 18.03\left[\mathrm{C}_{\text {quart }}, \mathrm{C}\left(\mathrm{CH}_{3}\right)_{3}\right], 25.73\left[+, \mathrm{C}\left(\mathrm{CH}_{3}\right)_{3}\right]$, $26.89\left(-, \mathrm{C}^{*}\right), 37.70\left(-, \mathrm{C}-3^{*}\right), 70.06\left(+, \mathrm{C}-7^{* *}\right), 70.40\left(+, \mathrm{C}-4^{* *}\right), 80.95\left(\mathrm{C}_{\text {quart }}, \mathrm{C}-6\right)$, $119.21(+, \mathrm{C}-1 * * *), 129.70$ (+, C-2***). - MS (CI, $\left.\mathrm{NH}_{3}, 70 \mathrm{eV}\right), \mathrm{m} / z(\%): 293$ (7) [M + $\left.\mathrm{NH}_{4}{ }^{+}+\mathrm{NH}_{3}\right], 276(100)\left[\mathrm{M}+\mathrm{NH}_{4}^{+}\right], 259$ (2) $\left[\mathrm{M}+\mathrm{H}^{+}\right] .-\mathrm{C}_{13} \mathrm{H}_{23} \mathrm{ClOSi}$ (258.9). 
Versuch der Enin-Cycloisomerisierung von 192: Analog AAV 2 löste man $129 \mathrm{mg}$ $(0.500 \mathrm{mmol})$ 192, $5.6 \mathrm{mg}(0.025 \mathrm{mmol}, 5.0 \mathrm{~mol} \%) \mathrm{Pd}(\mathrm{OAc})_{2}, 12 \mathrm{mg}$ (0.051 mmol, $10 \mathrm{~mol} \%)$ BBEDA und $129 \mathrm{mg}(1.50 \mathrm{mmol})$ Methylacrylat in $5 \mathrm{ml}$ wasserfreiem Benzol. Man erwärmte auf $70^{\circ} \mathrm{C}$. Nach $2 \mathrm{~d}$ konnte dünnschichtchromatographisch nur Ausgangsmaterial detektiert werden.

3-tert-Butyldimethylsilyloxy-7-methoxy-1-tetrahydropyranyloxyundec-5-in-10-en (197): $\mathrm{Zu}$

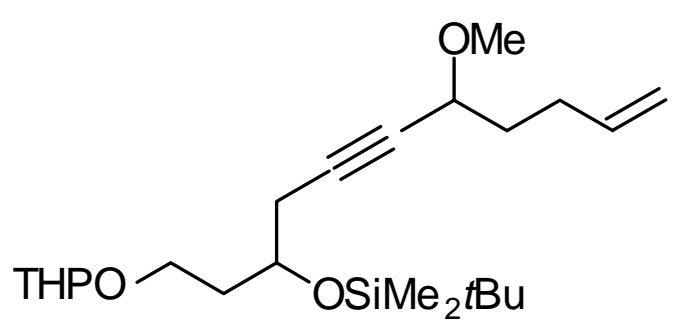
$312 \mathrm{mg}(0.998 \mathrm{mmol})$ 190-THP in $10 \mathrm{ml}$ THF gab man bei $-78{ }^{\circ} \mathrm{C} 0.44 \mathrm{ml}(1.1 \mathrm{mmol}, 2.5 \mathrm{M}$ in Hexan) $n$-Butyllithiumlösung und rührte $30 \mathrm{~min}$ bei dieser Temperatur. Dann tropfte man $88.0 \mathrm{mg}$ (1.05 mmol) Pentenal zu und rührte weitere $30 \mathrm{~min}$. Man erwärmte auf etwa $-10{ }^{\circ} \mathrm{C}$ und gab $156 \mathrm{mg}(1.10 \mathrm{mmol})$ Methyliodid und $10 \mathrm{ml}$ DMSO zu. Die Reaktionsmischung wurde $2 \mathrm{~h}$ bei $0{ }^{\circ} \mathrm{C}$ gerührt, dann auf $20 \mathrm{ml}$ Wasser gegeben und mit $2 \times 30 \mathrm{ml}$ Ether extrahiert. Die vereingten organischen Phasen wurden mit $20 \mathrm{ml}$ ges. NaCl-Lösung gewaschen, über $\mathrm{MgSO}_{4}$ getrocknet und i. Vak. eingeengt. Das Rohprodukt wurde säulenchromatographisch an $20 \mathrm{~g}$ Kieselgel $(2.0 \times 20 \mathrm{~cm}$, Pentan/Et $2 \mathrm{O}=5: 1)$ gereinigt. Man erhielt $234 \mathrm{mg}(57 \%) 197$ als farbloses Öl, $R_{\mathrm{f}}($ Pentan/Et $2 \mathrm{O}, 5: 1)=0.53$. - IR (Film): v = $3315 \mathrm{~cm}^{-1}, 2951,2857,1739,1473$, 1361, 1257, 1119, 1035, 908, 837, 777. - ${ }^{1} \mathrm{H}-\mathrm{NMR}\left(250 \mathrm{MHz}, \mathrm{CDCl}_{3}\right): \delta=0.07(\mathrm{~s}, 6 \mathrm{H}$, $\mathrm{SiCH}_{3}$ ), 0.88 [s, $\left.9 \mathrm{H}, \mathrm{C}\left(\mathrm{CH}_{3}\right)_{3}\right], 1.50-1.97$ (m, $\left.10 \mathrm{H}, 2,8,2^{\prime}, 3^{\prime}, 4^{\prime}-\mathrm{H}\right), 2.20$ (q, ${ }^{3} \mathrm{~J}=7.5 \mathrm{~Hz}$, $2 \mathrm{H}, 9-\mathrm{H}), 2.40(\mathrm{~d}, 3 \mathrm{~J}=5.8 \mathrm{~Hz}, 2 \mathrm{H}, 4-\mathrm{H}), 3.37\left(\mathrm{~s}, 3 \mathrm{H}, \mathrm{OCH}_{3}\right), 3.40-3.55(\mathrm{~m}, 2 \mathrm{H}$,

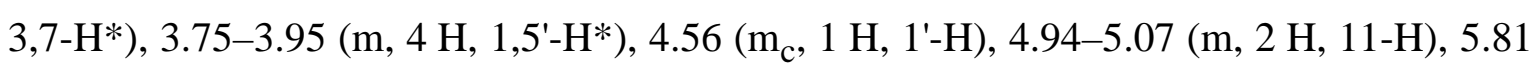
$\left(\mathrm{ddt},{ }^{3} J=7.5,{ }^{3} J=10.3,{ }^{3} J=17.1 \mathrm{~Hz}, 1 \mathrm{H}, 10-\mathrm{H}\right) . \quad{ }^{13} \mathrm{C}-\mathrm{NMR}\left(62.9 \mathrm{MHz}, \mathrm{CDCl}_{3}\right.$, DEPT): $\delta=-4.88,-4.80\left(+, \mathrm{SiCH}_{3}\right),-4.54\left(+, \mathrm{SiCH}_{3}\right), 17.99\left[\mathrm{C}_{\text {quart }}, C\left(\mathrm{CH}_{3}\right)_{3}\right], 19.57(-$, C-3'*), $25.46\left(-, \mathrm{C}-4^{\prime *}\right), 25.76\left[+, \mathrm{C}\left(\mathrm{CH}_{3}\right)_{3}\right], 27.85,27.90\left(-, \mathrm{C}-2^{*}\right), 29.44\left(-, \mathrm{C}-4^{*}\right)$, 30.67, $30.74\left(-, \mathrm{C}-2^{\prime *}\right), 34.91\left(-, \mathrm{C}-8^{*}\right), 36.57(-, \mathrm{C}-9 *), 56.23\left(+, \mathrm{OCH}_{3}\right), 62.20,62.25(-$, C-5**), 63.56, 63.94 (-, C-1**), 67.87 (+, C-7***), 68.47 (+, C-3***), $80.36\left(\mathrm{C}_{\text {quart }}\right.$, C-5****), $83.34\left(\mathrm{C}_{\mathrm{quart}}, \mathrm{C}-6^{* * * * *}\right), 98.63,98.81\left(\mathrm{C}-1^{\prime}\right), 114.92(-, \mathrm{C}-11), 137.84$ (+, C-10). - MS (CI, $\left.\mathrm{NH}_{3}, 70 \mathrm{eV}\right), \mathrm{m} / z(\%): 428$ (100) $\left[\mathrm{M}+\mathrm{NH}_{4}{ }^{+}\right] .-\mathrm{C}_{23} \mathrm{H}_{42} \mathrm{O}_{4} \mathrm{Si}(410.7)$. 
3-tert-Butyldimethylsilyloxy-7-methoxyundec-5-in-10-en-1-al (198): Entsprechend AAV 10

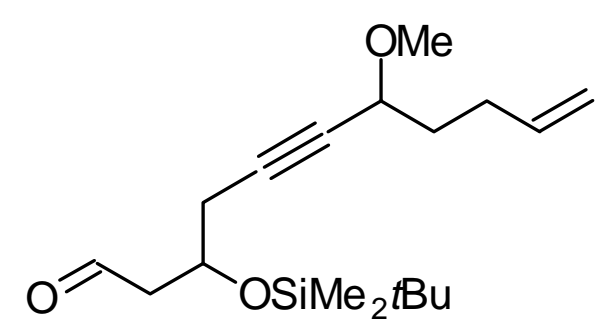
gab man zu einer Lösung von 206 g (0.502 mmol) 197 in $10 \mathrm{ml}$ wasserfreiem $\mathrm{CH}_{2} \mathrm{Cl}_{2}$, bei $-25^{\circ} \mathrm{C}$ $1.0 \mathrm{ml} \quad(1.0 \mathrm{mmol}, \quad 1.0 \quad \mathrm{M} \quad$ in Hexan $)$ Diethylaluminiumchloridlösung und erwärmte auf Raumtemp. Nachdem man 2 h bei dieser Temperatur gerührt hatte, arbeitete man wäßrig auf und engte i.

Vak. nicht bis zur vollständigen Trockne ein. Die so erhaltene Lösung gab man zu einer Lösung von $400 \mathrm{mg}$ (0.939 mmol) Dess-Martin-Reagenz in $3 \mathrm{ml} \mathrm{CH}_{2} \mathrm{Cl}_{2}$. Man rührte $12 \mathrm{~h}$ bei Raumtemp. Zur Aufarbeitung fügte man $10 \mathrm{ml}$ verd. NaOH-Lösung und $10 \mathrm{ml}$ Ether $\mathrm{zu}$, wusch die organische Phase mit je $10 \mathrm{ml}$ verd. NaOH-Lösung und Wasser und trocknete über $\mathrm{MgSO}_{4}$. Nach Entfernen des Lösungsmittel i. Vak. reinigte man das Rohprodukt durch Säulenchromatographie an $18 \mathrm{~g}$ Kieselgel (Säule $2.0 \times 15 \mathrm{~cm}$, Pentan/ $\left./ \mathrm{Et}_{2} \mathrm{O}=5: 1\right)$. Man isolierte $93 \mathrm{mg}$ (57\%) 198 als farbloses Öl, $R_{\mathrm{f}}\left(\right.$ Pentan/Et $_{2} \mathrm{O}$, $5: 1)=0.42$. - IR (Film): $v=2956 \mathrm{~cm}^{-1}, 2930,2857,2242,1715(\mathrm{C}=\mathrm{O}), 1472,1464$, 1410, 1390, 1362, 1258, 1160, 1102, 1006, 974, 837, 811, 779, 739, 703. - ${ }^{1} \mathrm{H}-\mathrm{NMR}$ $\left(250 \mathrm{MHz}, \mathrm{CDCl}_{3}\right): \delta=0.07\left(\mathrm{~s}, 3 \mathrm{H}, \mathrm{SiCH}_{3}\right), 0.10\left(\mathrm{~s}, 3 \mathrm{H}, \mathrm{SiCH}_{3}\right), 0.86[\mathrm{~s}, 9 \mathrm{H}$, $\left.\mathrm{C}\left(\mathrm{CH}_{3}\right)_{3}\right], 1.76$ (br quint, ${ }^{3} \mathrm{~J}=6.6 \mathrm{~Hz}, 2 \mathrm{H}, 9-\mathrm{H}^{*}$ ), 2.19 (br q, ${ }^{3} J=7.5 \mathrm{~Hz}, 2 \mathrm{H}, 8-\mathrm{H}^{*}$ ), 2.40-2.49 (m, 2 H, 2,4-H), 2.60-2.71 (m, 2 H, 2,4-H), 3.37 (s, 3 H, OCH 3 ), $3.92\left(\mathrm{~m}_{\mathrm{c}}, 1 \mathrm{H}\right.$, $\left.3-\mathrm{H}^{* *}\right), 4.34\left(\mathrm{~m}_{\mathrm{c}}, 1 \mathrm{H}, 7-\mathrm{H}^{* *}\right), 4.96-5.07$ (m, $\left.2 \mathrm{H}, 11-\mathrm{H}\right), 5.81$ (ddt, ${ }^{3} J=6.6,3 \mathrm{~J}=10.2$, $\left.{ }^{3} J=17.0 \mathrm{~Hz}, 1 \mathrm{H}, 10-\mathrm{H}\right), 9.82\left(\mathrm{t},{ }^{3} \mathrm{~J}=2.1 \mathrm{~Hz}, 1 \mathrm{H}, 1-\mathrm{H}\right) .-{ }^{13} \mathrm{C}-\mathrm{NMR}\left(62.9 \mathrm{MHz}, \mathrm{CDCl}_{3}\right.$, DEPT $): \delta=-4.92\left(+, \mathrm{SiCH}_{3}\right),-4.55\left(+, \mathrm{SiCH}_{3}\right), 17.89\left[\mathrm{C}_{\text {quart }}, C\left(\mathrm{CH}_{3}\right)_{3}\right], 25.63[+$, $\left.\mathrm{C}\left(\mathrm{CH}_{3}\right)_{3}\right], 27.95\left(-, \mathrm{C}-8^{*}\right), 29.42\left(-, \mathrm{C}-2^{*}\right), 34.84$ (-, C-4*), 50.20, 50.29 (-, C-9), 56.36 $\left(+, \mathrm{OCH}_{3}\right), 66.80(+, \mathrm{C}-3 * *), 70.66(+, \mathrm{C}-7 * *), 81.57\left(\mathrm{C}_{\text {quart }}, \mathrm{C}-5 * * *\right), 81.99\left(\mathrm{C}_{\text {quart }}\right.$, C-6***), 115.08 (-, C-11), 137.70 (+, C-10), 201.35 (+, C-1). - MS (CI, $\left.\mathrm{NH}_{3}, 70 \mathrm{eV}\right), \mathrm{m} / z$ (\%): 342 (91) $\left[\mathrm{M}+\mathrm{NH}_{4}^{+}\right] .-\mathrm{C}_{18} \mathrm{H}_{32} \mathrm{O}_{3} \mathrm{Si}(324.5)$.

4-tert-Butyldimethylsilyloxy-1-chlor-8-methoxydodeca-1,11-dien-6-in (199): Nach AAV 11 gab man zu einer Suspension von $105 \mathrm{mg}(0.854 \mathrm{mmol}) \mathrm{CrCl}_{2}$ in $5 \mathrm{ml}$ THF $80 \mathrm{mg}$ $(0.25 \mathrm{mmol}) 198$ und $60 \mathrm{mg}(0.50 \mathrm{mmol}) \mathrm{CHCl}_{3}$ in $3 \mathrm{ml}$ THF. Nach $18 \mathrm{~h}$ wurde die Reaktion aufgearbeitet, über $\mathrm{MgSO}_{4}$ getrocknet und das Lösungsmittel i. Vak. entfernt. Säulenchromatographie an $20 \mathrm{~g}$ Kieselgel $\left(2.0 \times 18 \mathrm{~cm}, \quad \mathrm{Pentan} \mathrm{Et}_{2} \mathrm{O}=40: 1\right)$ ergab 
Fraktion I: $32 \mathrm{mg}(36 \%) 199$ als farbloses Öl als Diastereomerengemisch im Verhältnis

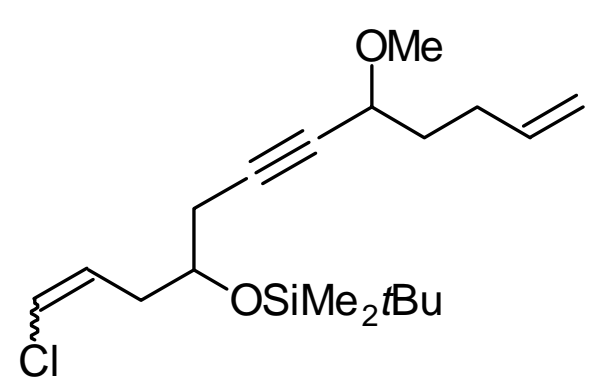
$1.23: 1, R_{\mathrm{f}}($ Pentan/Et $2 \mathrm{O}, 5: 1)=0.63+0.53$. - IR (Film): $v=3310 \mathrm{~cm}^{-1}, 2931,2858,1718,1473$, 1362, 1258, 1099, 838, 779, 739. - ${ }^{1} \mathrm{H}-\mathrm{NMR}$ (250 $\left.\mathrm{MHz}, \quad \mathrm{CDCl}_{3}\right)$ : Hauptmengenisomer: $\delta=0.03\left(\mathrm{~s}, 3 \mathrm{H}, \mathrm{SiCH}_{3}\right), 0.07\left(\mathrm{~s}, 3 \mathrm{H}, \mathrm{SiCH}_{3}\right)$, $1.00\left[\mathrm{~s}, \quad 9 \mathrm{H}, \quad \mathrm{C}\left(\mathrm{CH}_{3}\right)_{3}\right], \quad 1.87-2.45(\mathrm{~m}, \quad 7 \mathrm{H}$, 3,5,9,10-H), 2.72-2.81 (m, $1 \mathrm{H}, 10-\mathrm{H}), 3.38\left(\mathrm{~s}, 3 \mathrm{H}, \mathrm{OCH}_{3}\right), 3.93-4.09$ (m, $\left.2 \mathrm{H}, 4,8-\mathrm{H}\right)$, 5.00-5.17 (m, 3 H, 2,12-H*), 5.77-5.92 (m, $\left.2 \mathrm{H}, 1,11-\mathrm{H}^{*}\right)$. - Mindermengenisomer: $\delta=0.10\left(\mathrm{~s}, 3 \mathrm{H}, \mathrm{SiCH}_{3}\right), 0.15\left(\mathrm{~s}, 3 \mathrm{H}, \mathrm{SiCH}_{3}\right), 0.97$ [s, $\left.9 \mathrm{H}, \mathrm{C}\left(\mathrm{CH}_{3}\right)_{3}\right], 1.82-2.45(\mathrm{~m}$, $7 \mathrm{H}, 3,5,9,10-\mathrm{H}), 2.72-2.81(\mathrm{~m}, 1 \mathrm{H}, 10-\mathrm{H}), 3.43\left(\mathrm{~s}, 3 \mathrm{H}, \mathrm{OCH}_{3}\right), 3.93-4.09(\mathrm{~m}, 2 \mathrm{H}$, 4,8-H), 5.00-5.17 (m, 2 H, 12-H), 5.32-5.35 (m, $\left.1 \mathrm{H}, 2-\mathrm{H}^{*}\right), 5.77-5.92$ (m, $\left.1 \mathrm{H}, 11-\mathrm{H}\right)$, 6.39-6.57 (m, $\left.1 \mathrm{H}, 1-\mathrm{H}^{*}\right) .-{ }^{13} \mathrm{C}-\mathrm{NMR}\left(62.9 \mathrm{MHz}, \mathrm{CDCl}_{3}\right.$, DEPT): Gemisch: $\delta=-4.85,-$ $4.60\left(+, \mathrm{SiCH}_{3}\right),-4.54,-4.47\left(+, \mathrm{SiCH}_{3}\right), 18.17,18.33\left[\mathrm{C}_{\text {quart }}, C\left(\mathrm{CH}_{3}\right)_{3}\right], 25.88,26.00[+$, $\left.\mathrm{C}\left(\mathrm{CH}_{3}\right)_{3}\right], 28.14,28.28$ (-, C-3*), 29.92, 29.96 (-, C-5*), 35.63, 35.67 (-, C-9*), 50.37, 50.54 (-, C-10), 56.25, $56.31\left(+, \mathrm{OCH}_{3}\right), 66.69$ (+, C-4**), 70.92, 70.95 (+, C-8**), 81.25, $81.32\left(\mathrm{C}_{\text {quart }}, \mathrm{C}-6 * * *\right), 82.20,82.55\left(\mathrm{C}_{\mathrm{quart}}, \mathrm{C}-7 * * *\right), 115.10,115.15(-, \mathrm{C}-12), 127.83(+$, $2 \mathrm{C}, \mathrm{C}-2, \mathrm{C}-11), 138.08(+, \mathrm{C}-1)$. - MS (CI, NH $3,70 \mathrm{eV}), \mathrm{m} / \mathrm{z}(\%): 374(16)\left[\mathrm{M}+\mathrm{NH}_{4}^{+}\right] .-$ $\mathrm{C}_{19} \mathrm{H}_{33} \mathrm{ClO}_{2} \mathrm{Si}$ (357.0).

Fraktion II: 14 mg einer nicht identifizierten Verbindung, $R_{\mathrm{f}}\left(\mathrm{Pentan} / \mathrm{Et}_{2} \mathrm{O}, 5: 1\right)=0.12$.

Versuch der Cyclisierung von 4-tert-Butyldimethylsilyloxy-1-chlor-8-methoxydodeca-1,11dien-6-in (199): In einem NMR-Röhrchen erwärmte man $32 \mathrm{mg}(90 \mu \mathrm{mol}) 199$ mit $1.00 \mathrm{mg}(4.48 \mu \mathrm{mol}, 5.00 \mathrm{~mol} \%) \mathrm{Pd}(\mathrm{OAc})_{2}$ und $2.12 \mathrm{mg}(8.96 \mu \mathrm{mol}, 10.0 \mathrm{~mol} \%) N, N^{\prime}-$ Bis(benzyliden)ethylendiamin in $0.5 \mathrm{ml} \mathrm{C}_{6} \mathrm{D}_{6}$ auf $70^{\circ} \mathrm{C}$. Nach $7 \mathrm{~d}$ konnte im ${ }^{1}$ H-NMR-Spektrum nur Ausgangsmaterial nachgewiesen werden. 


\subsection{Palladium-katalysierte nucleophile Substitutionen}

(E/Z)-1-Chlor-1-(2'-trimethylsilylethinyl)-2-trimethylsilylcyclopropan [(E/Z)-218]: Zu einer

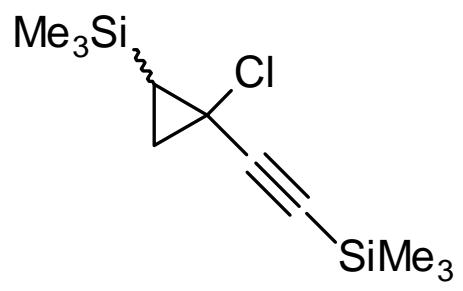

Lösung von $23.6 \mathrm{~g} \quad(85.0 \mathrm{mmol}) \quad$ 1-Chlor-1-(1',2',2'trichlorethenyl-2-trimethylsilyl-cyclopropan $[(E / Z)-219]$ in $200 \mathrm{ml}$ Ether gab man tropfenweise bei $-78{ }^{\circ} \mathrm{C} 120 \mathrm{ml}$ (190 mmol, $1.58 \mathrm{M}$ in Hexan) $n$-Butyllithiumlösung. Man erwärmte auf Raumtemp. und rührte $1 \mathrm{~h}$ bei dieser

Temperatur. Dann versetzte man bei $0{ }^{\circ} \mathrm{C}$ mit $15.0 \mathrm{~g}(138 \mathrm{mmol})$ Trichlormethylsilan (222) und rührte weitere $15 \mathrm{~h}$ bei Raumtemp. Zur Aufarbeitung gab man $200 \mathrm{ml}$ ges. $\mathrm{NH}_{4} \mathrm{Cl}$ Lösung zu und extrahierte die wäßrige Phase mit $3 \times 150 \mathrm{ml}$ Ether. Die vereingten organischen Phasen trocknete man über $\mathrm{MgSO}_{4}$. Nach Entfernen des Lösungsmittels i. Vak. wurde das Rohprodukt durch Destillation (Sdp.: $65^{\circ} \mathrm{C}, 10 \mathrm{mbar}$ ) gereingt. Man erhielt $16.6 \mathrm{~g}(80 \%)(E / Z)-218$ als farblose Flüssigkeit, E/Z-Verhältnis 1:2.0 (aus ${ }^{1} \mathrm{H}-\mathrm{NMR}-$ Spektrum). $-{ }^{1} \mathrm{H}-\mathrm{NMR}\left(250 \mathrm{MHz}, \mathrm{CDCl}_{3}\right.$ ): $\delta=0.09$ (s, $\left.9 \mathrm{H}, E-\mathrm{SiMe}_{3}\right), 0.10$ (s, $9 \mathrm{H}, E-\mathrm{SiMe}_{3}$ ), 0.13 (s, $9 \mathrm{H}, \mathrm{Z}-\mathrm{SiMe}_{3}$ ), 0.16 (s, $9 \mathrm{H}, \mathrm{Z}-\mathrm{SiMe}_{3}$ ), 0.62 (dd, ${ }^{3} J=10.1$, $3 J=11.8 \mathrm{~Hz}, 1 \mathrm{H}, Z-2-\mathrm{H}), 0.65\left(\mathrm{dd},{ }^{3} J=10.1,{ }^{3} J=11.8 \mathrm{~Hz}, 1 \mathrm{H}, E-2-\mathrm{H}\right), 0.88\left(\mathrm{~m}_{\mathrm{c}}, 1 \mathrm{H}\right.$, $E-3-\mathrm{H}), 1.21\left(\mathrm{dd}, 2 J=4.6,{ }^{3} J=10.1 \mathrm{~Hz}, 1 \mathrm{H}, Z-3-\mathrm{H}\right), 1.53\left(\mathrm{dd},{ }^{2} J=4.8,{ }^{3} J=11.8 \mathrm{~Hz}, 1 \mathrm{H}\right.$, $E-3-\mathrm{H}), 1.57\left(\mathrm{dd},{ }^{2} J=4.6,{ }^{3} J=11.8 \mathrm{~Hz}, 1 \mathrm{H}, Z-3-\mathrm{H}\right) .-{ }^{13} \mathrm{C}-\mathrm{NMR}(50.3 \mathrm{MHz}, \mathrm{DEPT}$, $\left.\mathrm{CDCl}_{3}\right): \delta=-1.92\left(+, E-\mathrm{SiMe}_{3}\right),-1.07\left(+, Z-\mathrm{SiMe}_{3}\right),-0.03\left(+, E-\mathrm{SiMe}_{3}\right),-0.01$ (+, Z-SiMe 3 ), 16.46 (-, E-C-3), 19.66 (-, Z-C-3), 20.64 (+, E-C-2), 23.58 (+, Z-C-2), 32.88 $\left(\mathrm{C}_{\text {quart }}, E-\mathrm{C}-1\right), 35.63\left(\mathrm{C}_{\text {quart }}, Z-\mathrm{C}-1\right), 84.97\left(\mathrm{C}_{\text {quart }}, \mathrm{C}-2^{\prime *}\right), 105.31\left(\mathrm{C}_{\text {quart }}, E-\mathrm{C}-1^{\prime *}\right)$, 107.25 ( $\left.\mathrm{C}_{\text {quart }}, Z-\mathrm{C}-1^{\prime *}\right) .-\mathrm{C}_{11} \mathrm{H}_{21} \mathrm{ClSi}_{2}$ (244.9).

(Z)-1-Chlor-1-(2'-trimethylsilylethenyl)-2-trimethylsilylcyclopropan [(Z,Z)-217]: Variante A: Man gab zu $2.46 \mathrm{~g}(29.2 \mathrm{mmol})$ Cyclohexan $15.0 \mathrm{ml}\left(15.0 \mathrm{mmol}, 1.0 \mathrm{M}\right.$ in THF) $\mathrm{BH}_{3}$

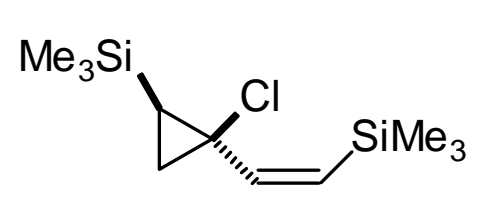
bei $0{ }^{\circ} \mathrm{C}$ und rührte $2 \mathrm{~h}$ bei dieser Temperatur. Dann fügte man $\quad 3.67 \mathrm{~g} \quad(15.0 \mathrm{mmol}) \quad(E / Z)-1-C h l o r-1-\left(2^{\prime}\right.$-trimethylsilylethinyl)-2-trimethylsilylcyclopropan $\quad[(E / Z)-218]$ bei $-15^{\circ} \mathrm{C}$ zu und rührte weitere $2 \mathrm{~h}$ bei $0{ }^{\circ} \mathrm{C}$. Nach Zugabe von $15 \mathrm{ml}$ Essigsäure erwärmte man auf Raumtemp. und rührte $18 \mathrm{~h}$. Anschließend versetzte man zur Aufarbeitung mit $150 \mathrm{ml}$ Wasser und extrahierte die wäßrige Phase mit $3 \times 100 \mathrm{ml}$ Pentan. Die vereinigten 
organischen Phasen wurden mit ges. $\mathrm{NaHCO}_{3}$-Lösung neutralisiert und mit $5 \mathrm{~g} \mathrm{~K}_{2} \mathrm{CO}_{3}$ und $80 \mathrm{ml}$ Methanol versetzt. Nachdem man $2 \mathrm{~h}$ bei Raumtemp. rührte, filtrierte man und versetzte mit $100 \mathrm{ml}$ Wasser und $100 \mathrm{ml}$ Ether. Die organische Phase wurde über $\mathrm{Na}_{2} \mathrm{SO}_{4}$ getrocknet und eingeengt. Das Rohprodukt wurde durch Säulenchromatographie an $80 \mathrm{~g}$ Kieselgel (Säule $3.5 \times 20 \mathrm{~cm}$, Pentan) gereingt. Man erhielt $2.23 \mathrm{~g}[60 \%, 90 \%$ bezogen auf das (Z)-Isomer] $(Z, Z)-217$ als farblose Flüssigkeit, $R_{\mathrm{f}}($ Pentan $)=0.66$. - IR $\left(\mathrm{CDCl}_{3}\right)$ : $v=2957 \mathrm{~cm}^{-1}, 1604,1409,1249,1136,1062$, 980, 942, 939, 936. - ${ }^{1} \mathrm{H}-\mathrm{NMR}$ $\left(250 \mathrm{MHz}, \mathrm{CDCl}_{3}\right): \delta=0.15\left(\mathrm{~s}, 9 \mathrm{H}, \mathrm{SiMe}_{3}\right), 0.16-0.18(\mathrm{~m}, 1 \mathrm{H}, 2-\mathrm{H}), 0.20(\mathrm{~s}, 9 \mathrm{H}$, $\left.\mathrm{SiMe}_{3}\right), 1.21\left(\mathrm{dd}, 2 J=4.8,{ }^{3} \mathrm{~J}=9.4 \mathrm{~Hz}, 1 \mathrm{H}, 3-\mathrm{H}\right), 1.34\left(\mathrm{dd},{ }^{2} J=4.8,{ }^{3} \mathrm{~J}=11.7 \mathrm{~Hz}, 1 \mathrm{H}\right.$, 3-H), $5.56\left(\mathrm{~d},{ }^{3} \mathrm{~J}=14.3 \mathrm{~Hz}, 1 \mathrm{H}, 1^{\prime}-\mathrm{H}^{*}\right), 6.31\left(\mathrm{~d},{ }^{3} \mathrm{~J}=14.3 \mathrm{~Hz}, 1 \mathrm{H}, 2^{\prime}-\mathrm{H}^{*}\right) .-{ }^{13} \mathrm{C}-\mathrm{NMR}$ (62.9 MHz, DEPT, $\left.\mathrm{CDCl}_{3}\right): \delta=-0.83\left(+, \mathrm{SiMe}_{3}\right), 0.83\left(+, \mathrm{SiMe}_{3}\right), 15.91(+, \mathrm{C}-2), 22.28$ (-, C-3), 48.24 ( $\left.\mathrm{C}_{\text {quart }}, \mathrm{C}-1\right), 130.96$ (+, C-1'*), 149.38 (+, C-2**). - MS (EI, $\left.70 \mathrm{eV}\right)$, m/z (\%): 123 (11) $\left[\mathrm{M}^{+}-\mathrm{Cl}-\mathrm{SiMe}_{3}-\mathrm{CH}_{3}\right], 95$ (7), 93 (11), 73 (100) [SiMe $], 59$ (7), 45 (16). - MS (CI, $\left.\mathrm{NH}_{3}\right), m / z(\%): 266 / 264(0.7 / 1.2)\left[\mathrm{M}+\mathrm{NH}_{4}^{+}\right], 249 / 247(8 / 19)\left[\mathrm{M}+\mathrm{H}^{+}\right] .-$ $\mathrm{C}_{11} \mathrm{H}_{23} \mathrm{ClSi}_{2}$ (246.9).

Variante $\quad B:$ Eine Lösung von $7.34 \mathrm{~g} \quad$ (30.0 mmol) (E/Z)-1-Chlor-1-(2'trimethylsilylethinyl)-2-trimethylsilylcyclopropan [(E/Z)-218)] in $150 \mathrm{ml}$ Pentan, $734 \mathrm{mg}$ (5\% auf $\mathrm{CaCO}_{3}$, vergiftet mit Blei, $\left.10 \mathrm{Gew} \%\right)$ Palladium und $8 \mathrm{mg}(0.06 \mathrm{mmol}, 0.2 \mathrm{~mol} \%)$ Chinolin wurde unter Wasserstoffatmosphäre kräftig geschüttelt. Nachdem nach $5 \mathrm{~d}$ kein Wasserstoff verbraucht worden war, wurde die Reaktion abgebrochen. Es konnten $5.28 \mathrm{~g}$ (72\%) des Ausgangsmaterials zurückgewonnen werden.

1-(2'-Trimethylsilylethyliden)-2-trimethylsilylcyclopropan [(E)-224]: Variante A: Unter

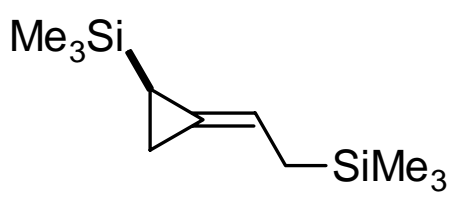
vermindertem Druck bei 0.1 mbar rührte man $70.8 \mathrm{mg}$ $(0.123 \mathrm{mmol}, \quad 5.00 \mathrm{~mol} \%) \quad \mathrm{Pd}(\mathrm{dba})_{2} \quad$ und $\quad 77.4 \mathrm{mg}$ (0.295 mmol, $12.0 \mathrm{~mol} \%) \mathrm{PPh}_{3} 2 \mathrm{~h}$ bei Raumtemp. Anschließend gab man $10 \mathrm{ml} \mathrm{MeCN}$ zu und rührte die Suspension weitere $10 \mathrm{~min}$. Eine Lösung von $600 \mathrm{mg}(2.43 \mathrm{mmol})(Z, Z)-217$ in $15 \mathrm{ml}$ $\mathrm{MeCN}$ wurde zu der Suspension gegeben. Nachdem man weitere 15 min. gerührt hat, wurde die Lösung zu $502 \mathrm{mg}(7.38 \mathrm{mmol})$ Natriumformiat und $54.0 \mathrm{mg}(0.245 \mathrm{mmol})$ [15]-Krone-5-Ether in $10 \mathrm{ml} \mathrm{MeCN}$ gegeben. Man erwärmte auf $50{ }^{\circ} \mathrm{C}$ und rührte $36 \mathrm{~h}$. Anschließend gab man zur Aufarbeitung $20 \mathrm{ml}$ Wasser und $50 \mathrm{ml}$ Ether zu. Die organische 
Phase wurde mit $2 \times 20 \mathrm{ml}$ Wasser gewaschen. Die vereinigten wäßrigen Phasen wurden mit $2 \times 50 \mathrm{ml}$ Ether extrahiert. Die vereinigten organischen Phasen wurden über $\mathrm{MgSO}_{4}$ getrocknet und das Lösungsmittel i. Vak. entfernt. Reinigung des Rohproduktes durch Säulenchromatographie an $25 \mathrm{~g}$ Kieselgel (Säule $2 \times 15 \mathrm{~cm}$, Pentan) ergab $465 \mathrm{mg}(90 \%)$ (E)-224 als farbloses Öl, $R_{\mathrm{f}}($ Pentan) $=0.70$, verunreinigt mit Vinylsilan 225 (Verhältnis (E)-224: $225=93: 7$, berechnet aus ${ }^{1} \mathrm{H}-\mathrm{NMR}-$ Spektrum) - - IR $\left(\mathrm{CDCl}_{3}\right): \mathrm{v}=3020 \mathrm{~cm}^{-1}$, 2401, 1525, 1430, 1216, 1027, 948, 944, 940, 938, 936. - ${ }^{1} \mathrm{H}-\mathrm{NMR}\left(250 \mathrm{MHz}, \mathrm{CDCl}_{3}\right)$ : $\delta=-0.03\left(\mathrm{~s}, 9 \mathrm{H}, \mathrm{SiMe}_{3}\right), 0.00\left(\mathrm{~s}, 9 \mathrm{H}, \mathrm{SiMe}_{3}\right), 0.58\left(\mathrm{~m}_{\mathrm{c}}, 1 \mathrm{H}, c \operatorname{Pr}-\mathrm{H}\right), 0.78\left(\mathrm{~m}_{\mathrm{c}}, 1 \mathrm{H}\right.$, $c \operatorname{Pr}-\mathrm{H}), 1.13\left(\mathrm{~m}_{\mathrm{c}}, 1 \mathrm{H}, c \operatorname{Pr}-\mathrm{H}\right), 1.57\left(\mathrm{~m}_{\mathrm{c}}, 2 \mathrm{H}, 2^{\prime}-\mathrm{H}\right), 5.54\left(\mathrm{dt},{ }^{3} \mathrm{~J}=8.3,{ }^{4} \mathrm{~J}=2.0 \mathrm{~Hz}, 1 \mathrm{H}\right.$, 1'-H). $-{ }^{13} \mathrm{C}-\mathrm{NMR}\left(50.3 \mathrm{MHz}, \mathrm{DEPT}, \mathrm{CDCl}_{3}\right): \delta=-2.40\left(+, \mathrm{SiMe}_{3}\right),-1.70\left(+, \mathrm{SiMe}_{3}\right)$, $4.24(+, \mathrm{C}-2), 4.70\left(-, \mathrm{C}-3^{*}\right), 21.77\left(-, \mathrm{C}-2^{\prime *}\right), 111.21$ (+, C-1'), $122.53\left(\mathrm{C}_{\text {quart }}, \mathrm{C}-1\right) .-\mathrm{MS}$ (EI, $70 \mathrm{eV}), m / z(\%): 212(1)\left[\mathrm{M}^{+}\right], 124$ (9) $\left[\mathrm{M}^{+}-\mathrm{SiMe}_{3}-\mathrm{CH}_{3}\right], 109$ (12) $\left[\mathrm{M}^{+}-\mathrm{SiMe}_{3}-\right.$ $2 \mathrm{CH}_{3}$ ], 73 (100) [ $\mathrm{SiMe}_{3}$ ], 72 (26), 59 (9), 45 (19). $-\mathrm{C}_{11} \mathrm{H}_{24} \mathrm{Si}_{2}$ (212.5): ber.: 212.1416, gef.: 212.1410 .

Variante B: Führte man die Reaktion bei Raumtemp. unter sonst identischen Bedingungen durch und rührte $3 \mathrm{~d}$, konnten nur $12 \mathrm{mg}(6 \%)(E)$-224 neben $73 \mathrm{mg}$ eines Gemisches aus (E)-224 und (Z,Z)-217 isoliert werden.

Variante C: Verwendete man THF als Lösungsmittel, so konnte nur Ausgangsmaterial zurückisoliert werden.

1-n-Butyl-1-(2'-trimethylsilylethenyl)-2-trimethylsilylcyclopropan (226) und 1-(2'Trimethylsilylethenyl)-2-trimethylsilylcyclopropan (225): Variante A: Unter vermindertem Druck bei 0.1 mbar rührte man $12 \mathrm{mg}(0.021 \mathrm{mmol}, 5 \mathrm{~mol} \%) \mathrm{Pd}(\mathrm{dba})_{2}$ und $13 \mathrm{mg}$ (0.049 mmol, $12 \mathrm{~mol} \%) \mathrm{PPh}_{3} 2 \mathrm{~h}$ bei Raumtemp. Anschließend gab man $3 \mathrm{ml} \mathrm{THF} \mathrm{zu} \mathrm{und}$ rührte die Suspension weitere $10 \mathrm{~min}$. Eine Lösung von $100 \mathrm{mg}$ (0.410 mmol) (Z)-1-Chlor1-(2'-trimethylsilyl)ethenyl-2-trimethylsilylcyclopropan [(Z,Z)-217] in $3 \mathrm{ml}$ THF wurde zu der Suspension gegeben. Nachdem man weitere 15 min. gerührt hat, wurde die Lösung bei $0{ }^{\circ} \mathrm{C} \mathrm{zu} 0.38 \mathrm{ml}(0.62 \mathrm{mmol}, 1.6 \mathrm{M}$ in Hexan) $n$-Butyllithiumlösung und $0.62 \mathrm{ml}$ (0.62 mmol, $1.0 \mathrm{M}$ in Ether) Zinkchlorid in $3 \mathrm{ml}$ THF gegeben. Nachdem man auf Raumtemp. erwärmt und $4 \mathrm{~d}$ gerührt hatte, gab man $5 \mathrm{ml}$ Wasser und $20 \mathrm{ml}$ Ether zu der Reaktionsmischung. Die organische Phase wurde mit $2 \times 10 \mathrm{ml}$ Wasser gewaschen und die vereinigten wäßrigen Phasen mit $2 \times 20 \mathrm{ml}$ Ether extrahiert. Die organischen Phasen 
trocknete man über MgSO4 und entfernte das Lösungsmittel. Reinigung des Rohproduktes an 15 g Kieselgel (Säule $1.5 \times 15 \mathrm{~cm}$, Pentan) ergab Fraktion I: $11 \mathrm{mg}$ (10\%) 226 als

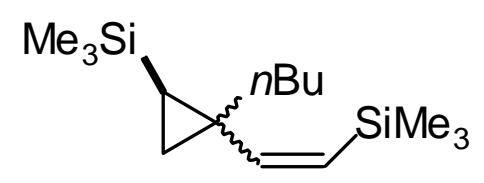
farbloses Öl, $R_{\mathrm{f}} \quad($ Pentan $)=0.77$. $\quad-$ IR $\quad\left(\mathrm{CDCl}_{3}\right)$ : $v=3020 \mathrm{~cm}^{-1}, 2937,2928,2856,1711,1600,1467,1364$, 1247, 1216, 1045，942，938，934，931. - 1H-NMR $\left(250 \mathrm{MHz}, \mathrm{CDCl}_{3}\right): \delta=-0.42(\mathrm{dd}, 3 J=7.5,3 J=9.7 \mathrm{~Hz}$,

$1 \mathrm{H}, 2-\mathrm{H}),-0.04$ (s, $\left.9 \mathrm{H}, \mathrm{SiMe}_{3}\right), 0.14$ (s, $9 \mathrm{H}, \mathrm{SiMe}_{3}$ ), 0.73-0.81 (m, $\left.2 \mathrm{H}, 3-\mathrm{H}^{*}\right), 0.83-$ 0.91 (t, $\left.{ }^{3} J=6.8 \mathrm{~Hz}, 3 \mathrm{H}, 4 "-\mathrm{H}\right), 0.93-1.07$ (m, $\left.1 \mathrm{H}, 3 "-\mathrm{H}\right), 1.22-1.37$ (m, 4 H, 1", 2"-H*), 1.63-1.74 (m, $\left.1 \mathrm{H}, 3^{\prime \prime}-\mathrm{H}^{*}\right), 5.51$ (d, $\left.{ }^{3} \mathrm{~J}=14.3 \mathrm{~Hz}, 1 \mathrm{H}, 1^{\prime}-\mathrm{H}^{* *}\right), 6.59$ (d, ${ }^{3} \mathrm{~J}=14.3 \mathrm{~Hz}, 1 \mathrm{H}$, 2'-H**). - ${ }^{13} \mathrm{C}-\mathrm{NMR}\left(62.9 \mathrm{MHz}, \mathrm{DEPT}, \mathrm{CDCl}_{3}\right): \delta=-0.14\left(+, \mathrm{SiMe}_{3}\right), 0.79\left(+, \mathrm{SiMe}_{3}\right)$, 11.71 (+, C-2), 14.49 (+, C-4"), 19.03 (-, C-3), 23.47 (-, C-1"*), 29.13 (C quart, C-1), 29.88 (-, C-2"*), 43.15 (-, C-3"*), 131.47 (+, C-1'**), 150.87 (+, C-2'**). - MS (EI, 70 eV), m/z (\%): 268 (2) $\left[\mathrm{M}^{+}\right], 165$ (11), 137 (7), 123 (6) [ $\left.\mathrm{M}^{+}-\mathrm{SiMe}_{3}-\mathrm{CH}_{3}-\mathrm{C}_{4} \mathrm{H}_{9}\right], 73$ (100) [SiMe $]$ ], 72 (60), 59 (15), 45 (8). $-\mathrm{C}_{15} \mathrm{H}_{32} \mathrm{Si}_{2}$ (268.6) - HRMS: ber.: 268.2042, gef.: 268.2049 .

Fraktion II: $21 \mathrm{mg}(24 \%) 225$ als farbloses Öl, $R_{\mathrm{f}}($ Pentan $)=0.70$. - IR $\left(\mathrm{CDCl}_{3}\right)$ :

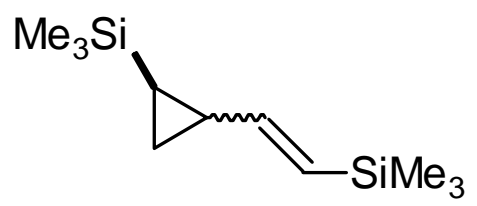
$v=3060 \mathrm{~cm}^{-1}, 2957,2856,2246,1711,1610,1404$, 1356, 1247, 1217, 1156, 1099, 1033, 983, 948, 941, 937, 933. $-{ }^{1} \mathrm{H}-\mathrm{NMR}\left(250 \mathrm{MHz}, \mathrm{CDCl}_{3}\right): \delta=-0.03$ (s, $9 \mathrm{H}$, $\mathrm{SiMe}_{3}$ ), 0.04 (s, $9 \mathrm{H}, \mathrm{SiMe}_{3}$ ), 0.63-0.69 (m, $\left.2 \mathrm{H}, 3-\mathrm{H}^{*}\right)$, $0.88\left(\mathrm{~m}_{\mathrm{c}}, 1 \mathrm{H}, 2-\mathrm{H}^{*}\right), 1.33-1.37\left(\mathrm{~m}, 1 \mathrm{H}, 1-\mathrm{H}^{*}\right), 5.43\left(\mathrm{dd},{ }^{3} \mathrm{~J}=8.4,{ }^{3} \mathrm{~J}=18.3 \mathrm{~Hz}, 1 \mathrm{H}\right.$, $\left.1^{\prime}-\mathrm{H}\right), 5.68\left(\mathrm{~d},{ }^{3} J=18.3 \mathrm{~Hz}, 1 \mathrm{H}, 2^{\prime}-\mathrm{H}\right) .-{ }^{13} \mathrm{C}-\mathrm{NMR}\left(62.9 \mathrm{MHz}, \mathrm{DEPT}, \mathrm{CDCl}_{3}\right): \delta=-2.37$ (+, $\left.\mathrm{SiMe}_{3}\right),-1.10\left(+, \mathrm{SiMe}_{3}\right), 7.32$ (+, C-1*), 11.14 (+, C-2*), 21.88 (-, C-3), 125.67 (+, C-1'**), $151.72\left(+, \mathrm{C}-2^{\prime * *}\right) .-\mathrm{MS}(\mathrm{EI}, 70 \mathrm{eV}), m / z(\%): 212(2)\left[\mathrm{M}^{+}\right], 124(16)\left[\mathrm{M}^{+}{ }_{-}\right.$ $\left.\mathrm{SiMe}_{3}-\mathrm{CH}_{3}\right], 123\left[\mathrm{M}^{+}-\mathrm{SiMe}_{3}-\mathrm{CH}_{3}-\mathrm{H}\right], 109$ (28) $\left[\mathrm{M}^{+}-\mathrm{SiMe}_{3}-2 \mathrm{CH}_{3}\right], 108$ (14), 73 (100) [ $\mathrm{SiMe}_{3}$ ], 72 (76), 59 (15), 58 (10), 45 (10). $-\mathrm{C}_{11} \mathrm{H}_{24} \mathrm{Si}_{2}$ (212.5): ber.: 212.416, gef.: 212.1413 .

Fraktion III: 56 mg (56\%) Ausgangsmaterial.

Variante B: Führte man die Reaktion $18 \mathrm{~h}$ bei $50{ }^{\circ} \mathrm{C}$ durch, so erhöhte sich die Ausbeute auf $12 \% 226$ und $55 \% 225$. 
Versuch einer Sakurai-Reaktion mit (E)-224: Variante A: Man versetzte $100 \mathrm{mg}$ (0.470 mmol) (E)-224 mit $62 \mathrm{mg}(0.58 \mathrm{mmol})$ Benzaldehyd in $5 \mathrm{ml} \mathrm{CH}_{2} \mathrm{Cl}_{2}$. Bei $-78{ }^{\circ} \mathrm{C}$ gab man eine Lösung von $98 \mathrm{mg}(0.52 \mathrm{mmol}) \mathrm{TiCl}_{4}$ in $5 \mathrm{ml} \mathrm{CH}_{2} \mathrm{Cl}_{2} \mathrm{zu}$ und rührte $2 \mathrm{~h}$ bei dieser Temperatur. Nachdem man auf Raumtemp. erwärmt hatte, hydrolysierte man das Reaktionsgemisch mit $10 \mathrm{ml}$ ges. $\mathrm{NaHCO}_{3}$-Lösung. Die wäßrige Phase wurde mit $3 \times 10 \mathrm{ml}$ Ether extrahiert und die vereinigten organischen Phasen über $\mathrm{MgSO}_{4}$ getrocknet. Man entfernte das Lösungsmittel i. Vak. und reinigte durch Säulenchromatographie an 15 g Kieselgel (Säule $1.5 \times 15 \mathrm{~cm}$, Pentan/ $\mathrm{Et}_{2} \mathrm{O}=10: 1$ ). Man erhielt $78 \mathrm{mg}$ eines Gemisches, das nicht identifiziert werden konnte.

Variante $B$ : Verwendete man $74 \mathrm{mg}(0.52 \mathrm{mmol}) \mathrm{BF}_{3} \cdot \mathrm{Et}_{2} \mathrm{O}$ als Lewis-Säure unter sonst identischen Bedingungen, konnte ebenfalls nur Zersetzungsprodukt isoliert werden.

Versuch der Deprotonierung von (E)-224: Variante A: $\mathrm{Zu} 0.20 \mathrm{ml}(0.50 \mathrm{mmol}, 2.5 \mathrm{M}$ in Hexan) einer $n$-Butyllithiumlösung in $10 \mathrm{ml}$ wasserfreiem THF gab man bei $0{ }^{\circ} \mathrm{C} 106 \mathrm{mg}$ (0.500 mmol) $224 \mathrm{zu}$ und ließ $45 \mathrm{~min}$ bei dieser Temperatur rühren. Anschließend fügte man $106 \mathrm{mg}$ (1.00 mmol) Benzaldehyd zu, erwärmte auf Raumtemp. und rührte weitere $15 \mathrm{~h}$. Zur Aufarbeitung versetzte man mit $20 \mathrm{ml}$ Wasser und $10 \mathrm{ml} \mathrm{Et}_{2} \mathrm{O}$ und extrahierte die wäßrige Phase mit $3 \times 15 \mathrm{ml} \mathrm{Et}_{2} \mathrm{O}$. Die vereinigten organischen Phasen wurden mit ges. NaCl-Lösung gewaschen und über $\mathrm{Na}_{2} \mathrm{SO}_{4}$ getrocknet. Das Rohprodukt wurde durch Säulenchromatographie an $15 \mathrm{~g}$ Kieselgel (Säule $1.5 \times 15 \mathrm{~cm}, \quad$ Pentan/ $\mathrm{Et}_{2} \mathrm{O}=4: 1$ ) gereinigt. Man erhielt $63 \mathrm{mg}$ eines nicht identifizierten Gemisches, $R_{\mathrm{f}}$ $\left(\right.$ Pentan $\left./ \mathrm{Et}_{2} \mathrm{O}\right)=0.67$.

Variante B: Führte man die Reaktion analog Variante A durch, gab jedoch zu der n-Butyllithiumlösung noch $2 \mathrm{ml} \mathrm{HMPT} \mathrm{zu,} \mathrm{konnte} \mathrm{ebenfalls} \mathrm{kein} \mathrm{Produkt} \mathrm{isoliert} \mathrm{werden.}$ 


\section{Zusammenfassung}

Im Rahmen dieser Arbeit wurden Domino-Prozesse aus Palladium-katalysierten Kreuzkupplungen - Heck-Reaktionen und Enin-Cycloisomerisierungen - mit anschließenden Diels-Alder-Reaktionen untersucht. Ein Schwerpunkt lag dabei auf einer Ausweitung auf heterocyclische Systeme durch Verwendung von heteroatomhaltigen Cyclisierungsvorläufern und Heterodienophilen.

Die in den Cyclisierungsreaktionen gebildeten bis(exocyclischen) 1,3-Diene lassen sich gut als Diene in Diels-Alder-Reaktionen einsetzen, wie auch schon in früheren eigenen Arbeiten gezeigt werden konnte.

Zunächst wurde der Einfluß des Substituenten R des Vorläufers 44 auf die Diastereo- und Regioselektivität der Diels-Alder-Reaktion zu den Bicyclen 45-R untersucht. Ein Substituent an dieser Position beeinflußt eine anschließende [4 + 2]-Cycloaddition deutlich. Man erhielt in einem Domino-Prozeß aus Enin-Cycloisomerisierung und Diels-Alder-Reaktion mit Methylacrylat unter Verwendung von 44-H ein Gemisch aus Diastereomeren und Regioisomeren. Verwendete man dagegen die Vorläufer 32, 34, oder 44-SiMe 3 , so isolierte man nur Regioisomerengemische, die jeweils als all-cis-Diastereomere vorlagen. Dabei erhielt man bei 34 die größte Regioselektivität von 2.9 : 1. Auch die Ausbeuten waren bei diesem Vorläufer am besten.

In weiteren Untersuchungen wurde der Einfluß der Dienophile auf die Regioselektivität ermittelt. Führte man die Reaktion in Gegenwart von $\alpha$-Chlormethylacrylat als Dienophil bei der Cyclisierung von 34 zu 47 durch, so erhöhte sich die Regioselektivität auf 4.5 : 1. Der Einsatz von Chlorcyclopropylidenacetat (27) ergab sogar nur ein einziges Regioisomer.

Tricyclische Lactone konnten durch Cyclisierung von $\mathbf{3 3}$ und 57 erhalten werden. Die Ausbeuten des Sechsringlactons 58 war mit 15\% unbefriedigend, das Fünfringlacton 52 konnte in einer ebenfalls nur mäßigen Ausbeute von 37\% dargestellt werden. Daher ist keine eindeutige Aussage über die Regioselektivität möglich. Führte man die Reaktion mit 54 durch, so konnte nur oligomeres Material isoliert werden.

Eine Heck-Diels-Alder-Reaktion des Bromdiens 59 mit Cyclopentenon als Dienophil zu 65 war nur in Gegenwart von Lewis-Säure möglich. Dabei bewirkte die Lewis-Säure sowohl 
eine Steuerung der Regioselektivität als auch eine Eliminierung von Wasser zum konjugierten Dien. Die Ausbeuten überstiegen jedoch 25\% nicht. Zum einen trat die Polymerisationsneigung des exocyclischen Diens 60 bei Verwendung einer schwachen Lewis-Säure als Konkurrenzreaktion zur Diels-Alder-Reaktion in den Vordergrund. Zum anderen konnte durch die stärkere Lewis-Säure Diethylaluminiumchlorid zwar die Reaktionsdauer verkürzt werden, aber sie bewirkte auch Zersetzung. Trotz vielfältiger Variation der verwendeten Lewis-Säure konnten keine Bedingungen gefunden werden, die zu einer deutlichen Ausbeuteverbesserung führten.

Der Aufbau des stickstoffhaltigen Bicyclus $\mathbf{7 4}$ gelang durch eine Kaskade aus EninCycloisomerisierung und Diels-Alder-Reaktion erstmals ohne gleichzeitige Bildung des formalen 6-endo-Produktes. Der Aufbau von Indolgerüsten gelang weder durch EninCycloisomerisierung noch durch Heck-Reaktion und anschließende [4 + 2]-Cycloaddition. Die Verwendung des Vorläufers 82 führte zur Bildung eines interessanten Trienins 86. Neu daran ist, daß zu dessen Bildung eine HBr-Eliminierung an der Bromvinyleinheit von 82 stattgefunden haben muß, wobei anschließend eine Palladium-katalysierte Kupplung an noch vorhandenes $\mathbf{8 2}$ folgte. Bei den bislang untersuchten Cyclisierungen befand sich das Heteroatom immer in Allylstellung zur Bromalkenyleinheit. Dabei wurde keine $\mathrm{HBr}$ Eliminierung und Bildung eines entsprechenden Trienins beobachtet.

Verwendete man das Sulfonamid 93, konnte nur bei Zugabe von Cäsiumcarbonat als Base das Isothiazol 100 als Regioisomerengemisch isoliert werden. Setzte man dagegen Kaliumcarbonat ein, erhielt man nur das Thiazin 101, das aus einem formalen 6-endo-Ringschluß resultiert. Das Sulfonamid 99 reagierte nicht zu 100, unabhängig von der verwendeten Base. Man erhielt die beiden isomeren Diene 102 und 103.

Eine interessante Abhängigkeit der Produktbildung von der verwendeten Base beobachtete man bei der Umsetzung des sauerstoffhaltigen Bromdiens 114. Bei Verwendung von Kaliumcarbonat erhielt man neben dem Produkt 115 das Trienin 116. Der Einsatz von Cäsiumcarbonat als Base ergab als einziges Produkt 115 als Regioisomerengemisch. In Gegenwart von Silbercarbonat isolierte man ausschließlich das Diendiin 117.

Eine weitere Methode zum Aufbau heterocyclischer Systeme war durch die Verwendung von in situ erzeugten Iminiumionen als Dienophile möglich. Man erhielt die entsprechenden Azabicyclen 126 in Ausbeuten zwischen 29 und 46\%; deren Bildung verlief regioselektiv. 
Bei Versuchen zur Heck-Reaktion an 151 erhielt man nicht den erwarteten Bicyclus 148, sondern die sehr interessanten Moleküle 153, 155, 156 und 157. Für die Entstehung dieser Verbindungen lassen sich plausible Mechanismen formulieren, durch Variation der Reaktionsbedingungen konnte man einzelne Produkte bevorzugt darstellen. Die Bildung des Cyclooctadiens 155 ist insofern interessant, als daß eine vierfache Heck-Reaktion stattfindet, obwohl in den Alkylpalladium-Zwischenstufen Wasserstoffatome für eine $\beta$-Hydrideliminierung zur Verfügung stehen.

Die Anwendung einer Cyclisierungskaskade auf die Synthese eines Spinosyn-Gerüstes über den Cycliserungsvorläufer 199 gelang nicht.

In einem weiteren Teil der Arbeit wurden Palladium-katalysierte allylische Substitutionen an (Z,Z)-217 durchgeführt. Als Nucleophil wurden dabei die Hydriddonoren Natriumformiat und $n$-Butylzinkchlorid verwendet. Dabei konnte das Substitutionsprodukt $(E)$-224 in sehr guten Ausbeuten und diastereoselektiv erhalten werden, das Produkt 225 wurde in guten Ausbeuten gebildet, wobei man auch noch eine geringe Menge des Nebenproduktes 226 isolieren konnte. Die Verwendung von $(E)$-224 in Folgereaktionen als Kohlenstoffnucleophil führte nicht zu eindeutigen Ergebnissen. 


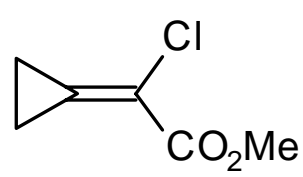

27

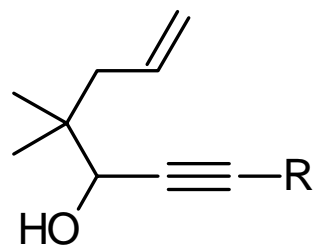

44-R

$\mathrm{R}=\mathrm{H}, \mathrm{SiMe}_{3}$

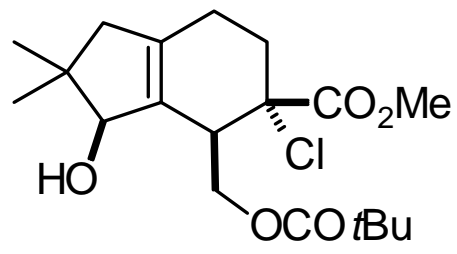

$47 a$

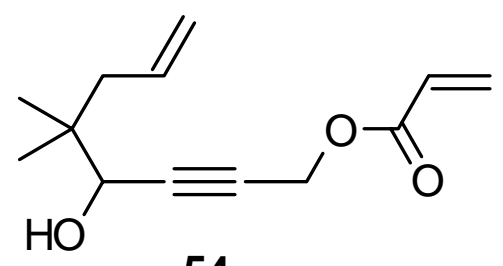

54

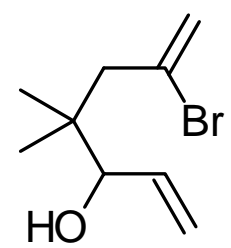

59

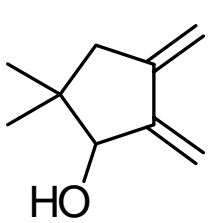

60

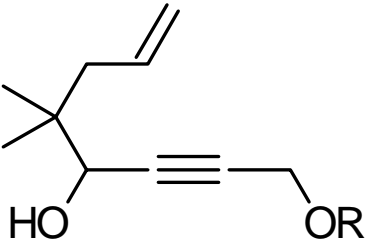

$32 \mathrm{R}=\mathrm{SiMe}_{2} \mathrm{tBu}$

$33 \mathrm{R}=\mathrm{H}$

$34 \mathrm{R}=\mathrm{COtBu}$

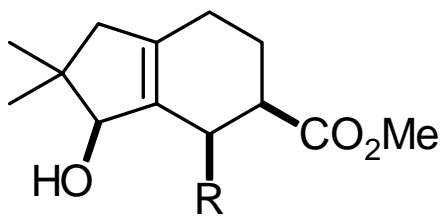

45a-R

$\mathrm{R}=\mathrm{H}, \mathrm{SiMe}_{3}$<smiles>CCOCC1C[C@@](Cl)(C(C)=O)CC2=C1C(O)C(C)(C)C2</smiles>

47b

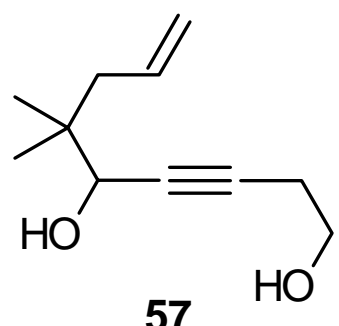

57

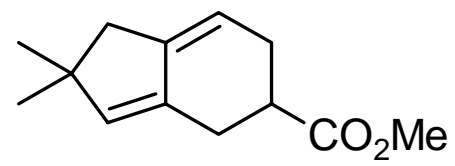

61<smiles>CC(=O)C1CCC2=C(C1)CC(C)(C)C2</smiles>

42<smiles>[R]C1CC(C(C)=O)CC2=C1C(O)C(C)(C)C2</smiles>

45b-R

$\mathrm{R}=\mathrm{H}, \mathrm{SiMe}_{3}$

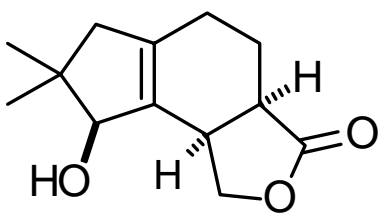

52

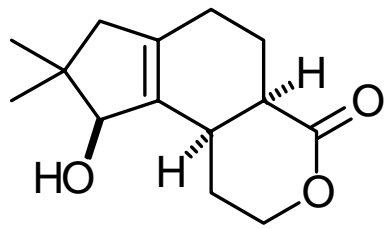

58

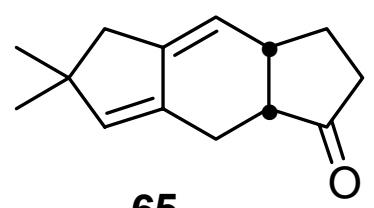

65<smiles>C=C(Br)C=CN(CCC(=C)Br)Cc1ccccc1</smiles>

74

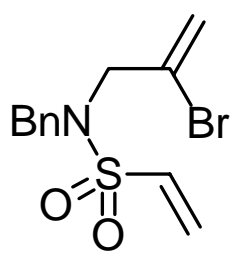

93

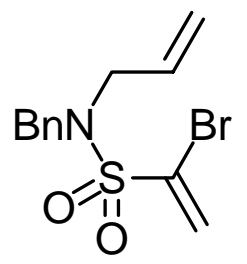

99

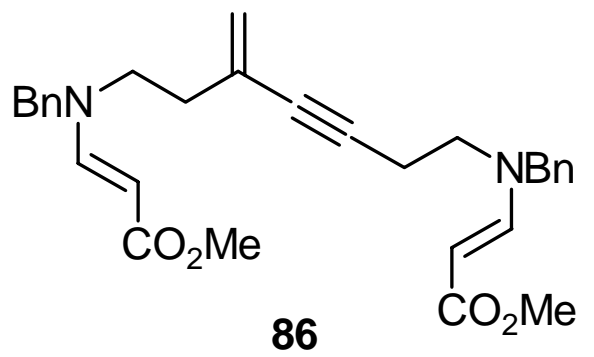

86<smiles>CC(=O)C1CCC2=C(C1)CN(Cc1ccccc1)S2(=O)=O</smiles>

100a,b 


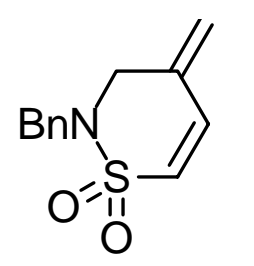

101

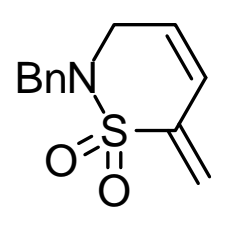

102

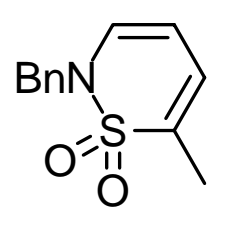

103<smiles>C=CCOCCC(=C)Br</smiles>

114<smiles>COC(=O)C1CCC2=C(CCOC2)C1</smiles>

115a,b<smiles>C=CCOCCC(=C)C</smiles><smiles>C#CCCOCCC#CC#CCCOCC=C</smiles>

117<smiles>CC(=O)CN1CCC2=C(C1)C(O)C(C)(C)C2</smiles>

126-Gly<smiles>CC1(C)CC2=C(CN(Cc3ccccc3)CC2)C1O</smiles>

126-Bn<smiles>CC1(C)CC2=C(CN(C3CC3)CC2)C1O</smiles>

126- $c \mathrm{Pr}$<smiles>CC1(C)CC2=C(CN(C3CCCC3)CC2)C1O</smiles>

126-cPent<smiles>C#CCC(F)(F)CC(=C)Br</smiles>

151<smiles>C=C1CC(F)(F)Cc2cc3c(cc21)CC(F)(F)C3</smiles>

156<smiles>CC1(C)CC2=C(C1)C(O)C(C)(C)C2</smiles>

126- $c$ Hex

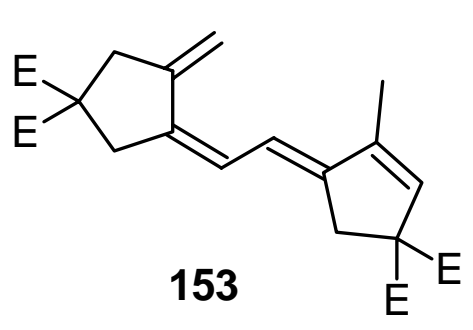<smiles>FC1C=CC2=C(C1)CC(F)(F)C2</smiles>

148<smiles>CO[C@H](F)C1CCC2=C(/C=C(/F)C1)CC(F)(F)C2</smiles>

$155-\mathrm{CO}_{2} \mathrm{Me}$
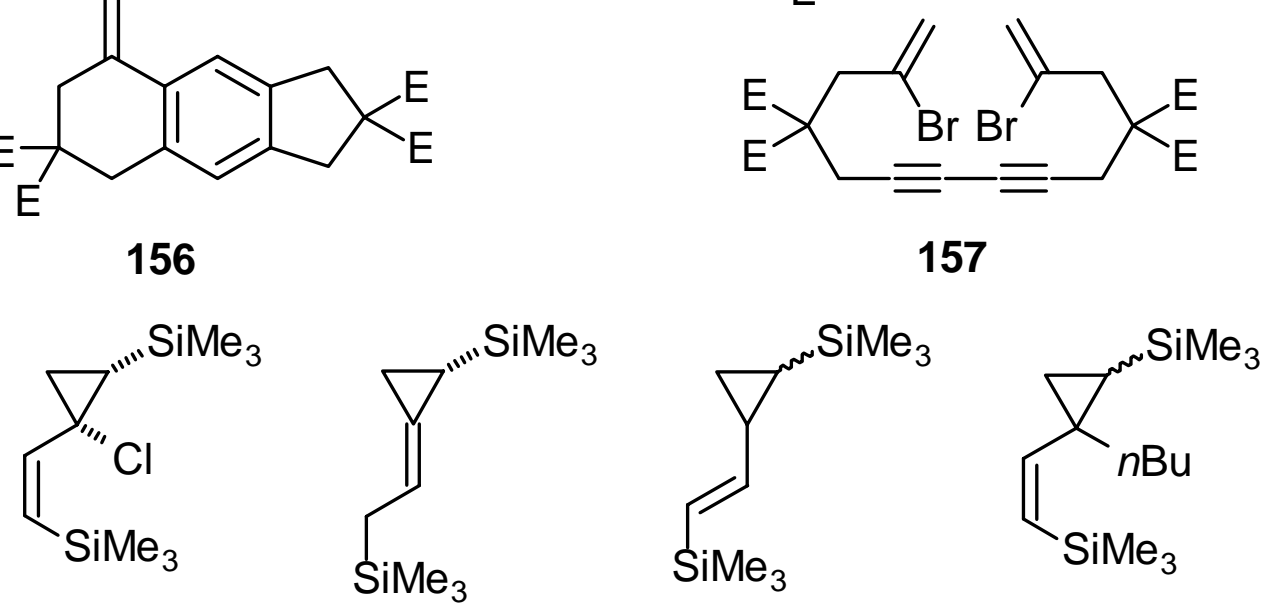

(Z,Z)-217

(E)-224

225 


\section{E. Literatur und Anmerkungen}

[1] O. Krätz, 7000 Jahre Chemie, Callwey Verlag, München, 1990.

[2] B. M. Trost, Angew. Chem. 1995, 107, 285-307; Angew. Chem. Int. Ed. Engl. 1995, $34,259-281$.

[3] [3a] O. Diels, K. Alder, Liebigs Ann. Chem. 1928, 98-122. - [3b] H. B. Kagan, O. Riant, Chem. Rev. 1992, 92, 1007-1019. - [3c] C. P. Dell, J. Chem. Soc., Perkin Trans. 1 1998, 3873-3905. - [3d] K. A. Jorgenson, Angew. Chem. 2000, 112, 37023733; Angew. Chem. Int. Ed. 2000, 39, 3558-3588. - [3e] V. Gevorgyan, Y. Yamamoto, J. Organomet. Chem. 1999, 576, 232-247. - [3f] J. Barluenga, A. Suarez-Sobrino, L. A. Lopez, Aldrichim. Acta 1999, 32, 4-15. - [3g] A. Ichihara, H. Oikawa, Curr. Org. Chem. 1998, 2, 365-394.

[4] Übersichten zur Pauson-Khand-Reaktion: [4a] P. L. Pauson, Tetrahedron 1985, 41, 5855-5860. - [4b] N. E. Schore, Comprehensive Organic Synthesis 1991, 5, 10371064. - [4c] N. E. Schore, Org. React. 1991, 40, 1-91. - [4d] D. C. Billington, P. L. Pauson, Organometallics 1982, 1, 1560-1561. - [4e] N. Jeong, B. Y. Lee, S. M. Lee, K. Chung, S.-G. Lee, Tetrahedron Lett. 1993, 34, 4023-4026. - [4f] A. J. Fletcher, S. D. R. Christie, J. Chem. Soc., Perkin Trans. 1 2000, 1657-1668. - [4g] K. M. Brummond, J. L. Kent, Tetrahedron 2000, 56, 3263-3283.

[5] [5a] L. Haughton, J. M. J. Williams, J. Chem. Soc., Perkin Trans. 1 2000, 33353349. - [5b] M. E. Krafft, Y.-Y. Cheung, C. A. Juliano-Capucao, Synthesis 2000, 1020-1026.

[6] A. de Meijere, H. Schirmer, M. Duetsch, Angew. Chem. 2000, 112, 4124-4162; Angew. Chem. Int. Ed. Engl. 2000, 39, 3964-4002.

[7] O. G. Kulinkovich, A. de Meijere, Chem. Rev. 2000, 100, 2789-2834.

[8] [8a] J. Tsuji, Organic Synthesis with Palladium Compounds, Springer, Berlin, 1980, 4-80. - [8b] J. Tsuji, Comprehensive Organic Synthesis (Hrsg.: B. M. Trost, I. Fleming), Vol. 7, Pergamon Press, Oxford, 1991, 449-468.

[9] [9a] R. F. Heck, J. Am. Chem. Soc. 1968, 90, 5518-5526. - [9b] H. A. Dieck, R. F. Heck, J. Am. Chem. Soc. 1974, 96, 1133-1136. - [9c] T. Mizoroki, K. Mori, A. Ozaki, Bull. Chem. Soc. Jpn. 1971, 44, 581. - Übersichten [9d] S. Bräse, A. de Meijere, Metal-catalyzed Cross-coupling Reactions (Hrsg.: F. Diederich, 
P. J. Stang), Wiley-VCH, Weinheim, 1997, 99-166. - [9e] A. de Meijere, F. E. Meyer, Angew. Chem. 1994, 106, 2473-2506; Angew. Chem. Int. Ed. Engl. 1994, 33, 2379-2411. - [9f] R. F. Heck, Comprehensive Organic Synthesis (Hrsg.: B. M. Trost, I. Fleming), Vol. 4, Pergamon Press, Oxford, 1991, 833-863. - [9g] R. F. Heck, Palladium Reagents in Organic Synthesis, Academic Press, New York, 1985. - [9h] A. de Meijere, S. Bräse, Transition Metal Catalyzed Reactions, IUPAC Monographs "Chemistry for the $21^{\text {st }}$ Century" (Hrsg.: S. G. Davies, S.-i. Murahashi), Blackwell Science, Oxford, 1999, 99-131. - [9i] A. de Meijere, S. Bräse, J. Organomet. Chem. 1999, 576, 88-110. - [9j] I. P. Beletskaya, A. V. Cheprakov, Chem. Rev. 2000, 100, 3009-3066. - [9k] S. Bräse, A. de Meijere, Handbook of Organopalladium Chemistry, (Hrsg.: E.-i. Negishi), Wiley, New York, 2001, in press. - [91] C. Amatore, A. Jutand, Acc. Chem. Res. 2000, 33, $314-321$.

[10] L. F. Tietze, K. Thede, Synlett 2000, 1470-1472.

[11] [11a] V. Farina, V. Krishnamurty, W. J. Scott, Org. React. 1997, 50, 1-65. - [11b] B. M. Trost, Comprehensive Organic Synthesis, Vol. 1, Pergamon Press, Oxford, 1991, 438-445. - [11c] J. K. Stille, Angew. Chem. 1986, 98, 504-519; Angew. Chem. Int. Ed. Engl. 1986, 25, 508-524. - T. N. Mitchell, Metal-catalyzed Crosscoupling Reactions (Hrsg.: F. Diederich, P. J. Stang), Wiley-VCH, Weinheim, 1998, S. 167-202.

[12] [12a] A. Suzuki, Metal-catalyzed Cross-coupling Reactions (Hrsg.: F. Diederich, P. J. Stang), Wiley-VCH, Weinheim, 1998, 49-97. - [12b] A. Suzuki, N. Miyaura, Chem. Rev. 1995, 95, 2457-2483. - [12c] A. Suzuki, Acc. Chem. Res. 1982, 15, 178-184. - [12d] B. M. Trost, I. Fleming, Comprehensive Organic Synthesis, Vol. 3, Pergamon Press, Oxford, 1991, S. 489-491.

[13] [13a] E.-i. Negishi, F. Liu, Metal-Catalyzed Cross Coupling Reactions (Hrsg.: P. J. Stang, F. Diederich), Wiley-VCH, Weinheim, 1998, S. 1-48. - [13b] M. Kumada, Pure Appl. Chem. 1980, 52, 669-679. - [13c] T. Y. Luh, Rev. Heteroatom Chem. 1996, 15, 61-82. - [13d] J. K. Huang, S. P. Nolan, J. Am. Chem. Soc. 1999, 121, 9889-9890.

[14] [14a] K. Sonogashira, Comprehensive Organic Synthesis (Hrsg.: B. M. Trost, I. Fleming), Vol. 3, Pergamon Press, Oxford, 1991, S. 521-549. - [14b] K. 
Sonogashira, Metal-catalyzed Cross-coupling Reactions (Hrsg.: F. Diederich, P. J. Stang), Wiley-VCH, Weinheim, 1998, S. 203-230.

[15] [15a] L. F. Tietze, U. Beifuß, Angew. Chem. 1993, 105, 137-170; Angew. Chem. Int. Ed. Engl. 1993, 32, 131-164. - [15b] L. F. Tietze, Nachr. Chem. Tech. Lab. 1997, 45, 1181-1187. - [15c] G. H. Posner, Chem. Rev. 1986, 86, 831-844. - [15d] L. F. Tietze in Selectivity - A Goal for Synthetic Efficiency (Hrsg.: W. Bartmann, B. M. Trost), VCH, Weinheim, 1984, S. 299.

[16] R. Grigg, D. Brown, V. Sridharan, V. Tambyrajah, M. Thornton-Pett, Tetrahedron 1998, 54, 2595-2606.

[17] [17a] E.-i. Negishi, C. Coperet, S. Ma, S. Y. Liou, F. Liu, Chem. Rev. 1996, 96, 365-393. - [17b] T. Sugihara, C. Coperet, Z. Owczarczyk, L. S. Harring, E.-i. Negishi, J. Am. Chem. Soc. 1994, 116, 7923-7924.

[18] F. E. Meyer, Dissertation, Universität Göttingen 1993.

[19] [19a] H. Henniges, Dissertation, Universität Göttingen 1994. - [19b] S. Schweizer, Dissertation, Universität Göttingen 1998. - [19c] C. Thies, Dissertation, Universität Göttingen 1999.

[20] [20a] J. E. Baldwin, J. Chem. Soc. Chem. Comm. 1976, 734-736. - [20b] J. E. Baldwin, J. Chem. Soc. Chem. Comm. 1976, 736-738.

[21] [21a] K. H. Ang, S. Bräse, A. G. Steinig, F. E. Meyer, A. Llebaria, K. Voigt, A. de Meijere, Tetrahedron 1996, 52, 11503-11529.- [21b] L. J. van Boxtel, S. Körbe, A. de Meijere, M. Noltemeyer, Eur. J. Org. Chem. 2001, 2283-2292. [21c] S. Körbe, Diplomarbeit, Universität Göttingen 1998.

[22] R. Grigg, S. Brown, V. Sridharan, M. D. Uttley, Tetrahedron Lett. 1998, 39, $3247-$ 3250 .

[23] [23a] B. M. Trost, Acc. Chem. Res. 1990, 23, 34-42. - [23b] B. M. Trost, M. J. Krische, Synlett 1998, 1-16. - [23c] B. M. Trost, Y. Li, J. Am. Chem. Soc. 1996, 118, 6625-6633. - [23d] B. M. Trost, D. L. Romero, F. Rise, J. Am. Chem. Soc. 1994, 116, 4268-4278. - [23e] B. M. Trost, G. J. Tanoury, M. Lautens, C. Chan, D. T. MacPherson, J. Am. Chem. Soc. 1994, 116, 4255-4267. - [23f] B. M. Trost, Y. Shi, J. Am. Chem. Soc. 1991, 113, 701-703. - [23g] B. M. Trost, Y. Shi, J. Am. Chem. Soc. 1993, 115, 9421-9438. - [23h] B. M. Trost, P. A. Hipskind, J. Y. L. Chung, C. Chan, Angew. Chem. 1989, 101, 1559-1561; 
Angew. Chem. Int. Ed. Engl. 1989, 28, 1502-1504. - [23i] B. M. Trost, S.-F. Chen, J. Am. Chem. Soc. 1986, 108, 6053-6054. - [23j] B. M. Trost, M. Lautens, J. Am. Chem. Soc. 1985, 107, 1781-1783.

[24] B. M. Trost, M. J. Krische, J. Am. Chem. Soc. 1999, 121, 6131-6141.

[25] L. Bhat, A. Steinig, R. Applebe, A de Meijere, Eur. J. Org. Chem. 2001, 1673-1680.

[26] [26a] S. J. Danishefsky, J. J. Masters, J. T. Link, L. B. Snyder, W. B. Young, Angew. Chem. 1995, 107, 1886-1888; Angew. Chem. Int. Ed. Engl. 1995, 34, 1723-1726. [26b] S. J. Danishefsky, J. J. Masters, J. T. Link, L. B. Snyder, W. B. Young, T. V. Magee, D. K. Jung, C. A. Isaacs, W. G. Bornmann, C. A. Alaimo, C. A. Coburn, M. J. DiGrandi, J. Am. Chem. Soc. 1996, 118, 2843-2859. - [26c] J. J. Masters, D. K. Jung, W. G. Bornmann, S. J. Danishefsky, Tetrahedron Lett. 1993, 34, 7253-7256. - [26d] J. T. Link, L. E. Overman, Metal-catalyzed Cross-coupling Reactions (Hrsg.: F. Diederich, P. J. Stang), Wiley-VCH, Weinheim, 1998, 231-269.

[27] S. Ma, E.-i. Negishi, J. Am. Chem. Soc. 1995, 117, 6345-6357.

[28] X. Han, R. C. Larock, Synlett 1998, 748-750.

[29] A. Steinig, Dissertation, Universität Göttingen 1998.

[30] [30a] E. L. Eliel, Stereochemistry of Carbon Compounds, Mc-Graw-Hill, New York, 1962, 106-202. - [30b] D. F. Detar, N. P. Luthra, J. Am. Chem. Soc. 1980, 102, 4505-4512.

[31] [31a] L. F. Tietze, T. Eicher, Reaktionen und Synthesen im organisch-chemischen Praktikum und Forschungslaboratorium, Thieme, Stuttgart, 1991. - [31b] D. Felix, J. Schreiber, G. Ohloff, A. Eschenmoser, Helv. Chim. Acta 1971, 54, 2896-2912.

[32] [32a] E.-i. Negishi, A. O. King, W. L. Klima, J. Org. Chem. 1980, 45, 2526-2528. [32b] E.-i. Negishi, A. O. King, J. M. Tour, Org. Synth. 1985, 64, 44-49.

[33] [33a] R. Woodward, R. Hoffmann, Angew. Chem. 1969, 81, 797-870; Angew. Chem. Int. Ed. Engl. 1969, 8, 781-854. - [33b] R. B. Woodward, R. Hoffmann, The Conservation of Orbital Symmetry, VCH, Weinheim, 1970. - [33c] K. N. Houk, J. Am. Chem. Soc. 1973, 95, 4092-4094. - [33d] K. Fukui, Acc. Chem. Res. 1971, 4, $57-64$.

[34] I. Fleming, Grenzorbitale und Reaktionen organischer Verbindungen, VCH, Weinheim, 1990, S. 137-142, 146-149. 
[35] I. Fleming, Grenzorbitale und Reaktionen organischer Verbindungen, VCH, Weinheim, 1990, S. 133-137, 143-145.

[36] [36a] T. C. McMorris, M. Anchel, J. Am. Chem. Soc. 1965, 87, 1594-1598. - [36b] M. J. Kelner, T. C. McMorris, W. T. Beck, G. M. Zamora, R. Taetle, Cancer Res. 1987, 47, 3186-3189. - [36c] T. C. McMorris, M. J. Kelner, W. Wang, L. A. Estes, M. A. Montoya, R. Taetle, J. Org. Chem. 1992, 57, 6876-6883. - [36d] M. Hara, M. Yoshida, M. Morimoto, H. Nakano, J. Antibiot. 1987, XL, 1643-1646.

[37] R. Imwinkelried, M. Schiess, D. Seebach, Org. Synth. 1987, 65, 230-232.

[38] [38a] W. R. Roush, H. R. Gillis, A. I. Ko, J. Am. Chem. Soc. 1982, 104, 2269-2283. - [38b] G. Stork, G. Clark, C. S. Shiner, J. Am. Chem. Soc. 1981, 103, 4948-4949. [38c] B. B. Snider, J. V. Duncia, J. Org. Chem. 1980, 45, 3461-3464. - [38d] W. R. Roush, H. R. Gillis, A. P. Essenfeld, J. Org. Chem. 1984, 49, 4674-4682. - [38e] P. G. Gassman, D. A. Singleton, J. Am. Chem. Soc. 1984, 106, 6085-6086. - [38f] U. Pindur, G. Lutz, C. Otto, Chem. Rev. 1993, 93, 741-761.

[39] I. Fleming, Grenzorbitale und Reaktionen organischer Verbindungen, VCH, Weinheim, 1990, S. 188-192.

[40] F. Fringuelli, L. Minuti, L. Radics, A. Taticchi, E. Wenkert, J. Org. Chem. 1988, $53,4607-4610$.

[41] [41a] J. W. Barton, A. Coop, J. W. Lewis, Tetrahedron Lett. 1993, 34, 6777-6778. [41b] D. A. Smith, Tetrahedron Lett. 1991, 32, 1549-1552.

[42] J. Falbe, M. Regitz (Hrsg.), Römpp kompakt, Band 2, Thieme, Stuttgart, 1998, S. 1073-1074.

[43] Mein Dank gilt Lonneke van Boxtel für die Überlassung einer Probe von 73.

[44] [44a] E. Lorthiois, I. Marek, J.-F. Normant, Bull. Soc. Chim. Fr. 1997, 134, 333341. - [44b] A. J. Peat, S. L. Buchwald, J. Am. Chem. Soc. 1996, 118, 1028-1030.

[45] [45a] J. Falbe, M. Regitz (Hrsg.), Römpp kompakt, Band 2, Thieme, Stuttgart, 1998, S. 1140-1141. - [45b] J. Falbe, M. Regitz (Hrsg.), Römpp kompakt, Band 4, Thieme, Stuttgart, 1999, S. 2652-2653.

[46] J. Falbe, M. Regitz (Hrsg.), Römpp kompakt, Band 4, Thieme, Stuttgart, 1999, S. 2285 .

[47] D. S. Ennis, J. Ince, S. Rahman, M. Shipman, J. Chem. Soc. Perkin Trans. 1, 2000, 13, 2047-2054.

[48] Lonneke J. van Boxtel, Dissertation, Universität Göttingen 2000. 
[49] C. R. Rondestvedt, J. Am. Chem. Soc. 1954, 76, 1926-1929.

[50] B. M. Trost, G. J. Tanoury, J. Am. Chem. Soc. 1988, 110, 1636-1638, Fußnote 10.

[51] I. Fleming, Grenzorbitale und Reaktionen organischer Verbindungen, VCH, Weinheim, 1990, S. 113-119.

[52] Z. Owczarczyk, F. Lamaty, E. J. Vawter, E.-i. Negishi, J. Am. Chem. Soc. 1992, 114, 10091-10092.

[53] C.-W- Lee, K. S. Oh, K. S. Kim, K. H. Ahn, Org. Letters 2000, 2, 1213-1216.

[54] L. Bhat, Arbeitsbericht, Universität Göttingen, 1996-1997.

[55] Autorenkollektiv, Organikum, Deutscher Verlag der Wissenschaften, Leipzig, Berlin, 1993, S. 283.

[56] T. Mandai, J. Nokami, T. Yano, J. Otera, J. Org. Chem. 1984, 49, 172-174.

[57] T. Laue, A. Plagens, Namen- und Schlagwort-Reaktionen der Organischen Chemie, Teubner, Stuttgart, 1995.

[58] [58a] S. Weinreb, R. R. Staib, Tetrahedron 1982, 38, 3087-3128. - [58b] H. Waldmann, Synthesis 1994, 535-551. - [58c] J. F. Kerwin, S. Danishefsky, Tetrahedron Lett. 1982, 23, 3739-3742. - [58d] J. Streith, A. Defoin, Synthesis 1994, 1107-1117. - [58e] F. Reichel, X. M. Fang, S. L. Yao, M. Ricci, K. A. Jorgensen, Chem. Comm. 1999, 1505-1506.

[59] S. D. Larsen, P. A. Grieco, J. Am. Chem. Soc. 1985, 107, 1768-1769.

[60] K. Waldmann, Liebigs Ann. Chem. 1989, 231-238.

[61] B. M. Trost, Merck-Schuchhardt-Lectureship, Göttingen 1999.

[62] [62a] J. Barluenga, F. Aznar, C. Valdez, A. Martin, S. Garcia-Granda, E. Martin, J. Am. Chem. Soc. 1993, 115, 4403-4404. - [62b] J. Barluenga, F. Aznar, C. Ribas, C. Valdez, M. Fernandez, M.-P. Cabal, J. Trujillo, Chem. Eur. J. 1996, 2, 805-811. [62c] J. Barluenga, F. Aznar, C. Valdez, C. Ribas, J. Org. Chem. 1998, 63, 39183924.

[63] R. A. Ruden, R. Bonjouklian, J. Am. Chem. Soc. 1975, $976892-6893$.

[64] A. Albeniz, P. Espinet, Y.-S. Lin, J. Am. Chem. Soc. 1996, 118, 7145-7152.

[65] H. Oppolzer, R. J. De Vita, J. Org. Chem. 1991, 56, 6256-6257.

[66] J. H. Rigby, R. C. Hughes, M. J. Heeg, J. Am. Chem. Soc. 1995, 117, 7834-7835.

[67] D. C. Horwell, P. D. Nichols, G. S. Ratcliffe, E. Roberts, J. Org. Chem. 1994, 59, 4418-4423. 
[68] [68a] H. Garcia, S. Iborra, J. Primo, M. A. Miranda, J. Org. Chem. 1986, 51, 44324436. - [68b] C. M. Brennan, C. D. Johnson, P. D. McDonnell, J. Chem. Soc., Perkin Trans. 2 1989, 957-961. - [68c] M. D. Weingarten, A. Pawda, Tetrahedron Lett. 1995, 36, 4717-4720. - [68d] B. Burns, R. Grigg, V. Santhakumar, V. Sridharan, P. Stevenson, T. Worakun, Tetrahedron 1992, 48, 7297-7320.

[69] [69a] J. Falbe, M. Regitz (Hrsg.), Römpp kompakt, Band 4, Thieme, Stuttgart, 1999, S. 2460. - [69b] http:///www.dowagro.com, 16.01.2001.

[70] [70a] L. A. Paquette, Z. L. Gao, Z. J. Ni, G. F. Smith, J. Am. Chem. Soc. 1998, 120, 2543-2552. - [70b] L A. Paquette, I. Collado, M. Purdie, J. Am. Chem. Soc. 1998, 120, 2553-2562. - [70c] L. A. Paquette, Z. L. Gao, Z. J. Ni, G. F. Smith, Tetrahedron Lett. 1997, 38, 1271-1274.

[71] C. R. Johnson, J. R. Zeller, J. Am. Chem. Soc. 1982, 104, 4021-4023.

[72] F. A. Carey, R. J. Sundberg, Organische Chemie, VCH, Weinheim, 1995.

[73] J. A. Marshall, E. D. Robinson, J. Lebreton, J. Org. Chem. 1990, 55, 227-239.

[74] O. Arjona, R. Medel, J. Pumet, Tetrahedron Lett. 1999, 40, 8431-8433.

[75] [75a] R. Zimmer, J. Angermann, U. Hain, F. Hiller, H. U. Reissig, Synthesis 1997, 1467-1474. - [75b] H. U. Reissig, C. Hippeli, T. Arnold, Chem. Ber. 1990, 123, 2403-2411.

[76] P. J. Kocienski (Hrsg.: D. Enders, R. Noyori, B. M. Trost), Protecting Groups, Thieme, Stuttgart, 1994.

[77] Y. Ogawa, M. Shibasaki, Tetrahedron Lett. 1984, 25, 663-664.

[78] D. B. Dess, J. C. Martin, J. Org. Chem. 1983, 48, 4156-4158.

[79] F. Effenberger, T. Ziegler, K.-H. Schönwälder, T. Kesmarszky, B. Bauer, Chem. Ber. 1986, 119, 3394-3404.

[80] K. Takai, K. Nitta, K. Utimoto, J. Am. Chem. Soc. 1986, 108, 7408-7410.

[81] L. S. Hegedus, Organische Synthese mit Übergangsmetallen, VCH, Weinheim, 1995, S. 239-245.

[82] [82a] J. Ollivier, P. Dorizon, P. P. Piras, J. Salaün, A. de Meijere, Inorg. Chim. Acta 1994, 222, 37-49. - [82b] A. Stolle, J. Ollivier, P. P. Piras, J. Salaün, A. de Meijere, J. Am. Chem. Soc. 1992, 114, 4051-4067. - [82c] G. McGaffin, S. Michalski, A. Stolle, S. Bräse, J. Salaün, A. de Meijere, Synlett 1992, 558-560.

[83] [83a] K. Estieu, R. Paugam, J. Ollivier, J. Salaün, F. M. Cordero, A. Goti, A. Brandi, J. Org. Chem. 1997, 62, 8276-8277. - [83b] M. Ferrara, F. M. Cordero, A. Goti, A. 
Brandi, K. Estieu, R. Paugam, J. Ollivier, J. Salaün, Eur. J. Org. Chem. 1999, 2725-2739.

[84] P. Menningen, C. Harcken, B. Stecker, S. Körbe, A. de Meijere, M. Rodrigues Lopez, J. Ollivier, J. Salaün, Synlett 1999, 1534-1538.

[85] S. E. Gibson, Bor- und Siliciumverbindungen in der Organischen Synthese, VCH, Weinheim, 1996, S. 46-47.

[86] [86a] T. Liese, A. de Meijere, Angew. Chem. 1982, 94, 65-66; Angew. Chem. Int. Ed. Engl. 1982, 21, 65-66. - [86b] T. Liese, A. de Meijere, Chem. Ber. 1986, 119, 2955-3026. - [86c] W. Weber, A. de Meijere, Angew. Chem. 1980, 92, 135-136; Angew. Chem. Int. Ed. Engl. 1980, 19, 138-140. - [86d] T. Liese, G. Splettstößer, A. de Meijere, Tetrahedron Lett. 1982, 23, 3341-3344. - [86e] W. Weber, A. de Meijere, Chem. Ber. 1985, 118, 2450-2471.

[87] [87a] M. Hoshi, Y. Masuda, A. Arase, Chem. Lett. 1991, 251-254. - [87b] M. Hoshi, Y. Masuda, A. Arase, Bull. Chem. Soc. Jpn. 1992, 65, 685-691.

[88] [88a] I. Fleming, I. Patterson, Synthesis 1979, 446-448. - [88b] I. Fleming, J. Dunogues, R. Smithers, Org. React. 1989, 37, 57.

[89] [89a] A. Hosomi, H. Sakurai, Tetrahedron Lett. 1976, 16, 1295-1298. - [89b] E. Langkopf, D. Schinzer, Chem. Rev. 1995, 95, 1375-1408. - [89c] M. G. Organ, V. Dragan, M. Miller, R. D. J. Froese, J. D. Goddart, J. Org. Chem. 2000, 65, $3666-3678$.

[90] D. Perrin, W. L. F. Armarengo, Purification of Laboratory Chemicals, Pergamon Press, Oxford, 1987.

[91] T. Liese, F. Seyed-Mahdavi, A. de Meijere, Org. Synth. 1990, 69, 148-153.

[92] M. W. Logue, K. Teng, J. Org. Chem. 1982, 47, 2549-2553.

[93] T. Cuvigny, H. Normant, Bull. Soc. Chim. Fr. 1970, 37, 3976-3980.

[94] L. F. Tietze, T. Eicher, Reaktionen und Synthesen im organisch-chemischen Praktikum und Forschungslaboratorium, Thieme, Stuttgart, 1991, S. 66.

[95] L. Brandsma, Preparative Acetylenic Chemistry, Elsevier, Amsterdam, 1971.

[96] A. de Meijere, S. Teichmann, D. Yu, J. Kopf, M. Oly, N. von Thienen, Tetrahedron 1989, 45, 2957-2968.

[97] A. J. Hubert, J. Chem. Soc., Perkin Trans. 2 1977, 11-14.

[98] J. E. Baldwin, K. A. Black, J. Am. Chem. Soc. 1984, 106, 1029-1040. 
[99] [99a] J. R. Johnson, W. L. McEwen, Org. Synth. Coll. Vol. I 1941, 507-508. - [99b] R. Lespieau, M. Bourguel, Org. Synth. Coll. Vol. I 1941, 203-205.

[100] G. J. Gordon, T. Luker, M. W. Tuckett, R. J. Witby, Tetrahedron 2000, 56, 21132130.

[101] R. A. Mitsch, N. H. Cromwell, J. Org. Chem. 1960, 25, 1719-1722.

[102] E. W. Colvin, D. G. McGarry, J. Chem. Soc. Chem. Commun. 1985, 9, 539-540.

[103] [103a] W. A. Herrmann, C. Broßmer, K. Öfele, C.-P. Reisinger, T. Priermeier, M. Beller, H. Fischer, Angew. Chem. 1995, 107, 1989-1992; Angew. Chem. Int. Ed. Engl. 1995, 34, 1844-1848. - [103b] M. Beller, H. Fischer, W. A. Herrmann, K. Öfele, C. Broßmer, Angew. Chem. 1995, 107, 1992-1993; Angew. Chem. Int. Ed. Engl. 1995, 34, 1848-1849. - [103c] M. Beller, T. H. Riermeier, Tetrahedron Lett. 1996, 37, 6535-6538. - [103d] M. Beller, Chem. Eur. J. 1998, 4, 935-941. 


\section{F. Spektrenanhang}

1. ${ }^{1} \mathrm{H}-\mathrm{NMR}-$ Sperktren 146

2. ${ }^{13}$ C-NMR-Spektren 157

3. 2D-NMR-Spektren 168 


\section{1. ${ }^{1}$ H-NMR-Spektren}
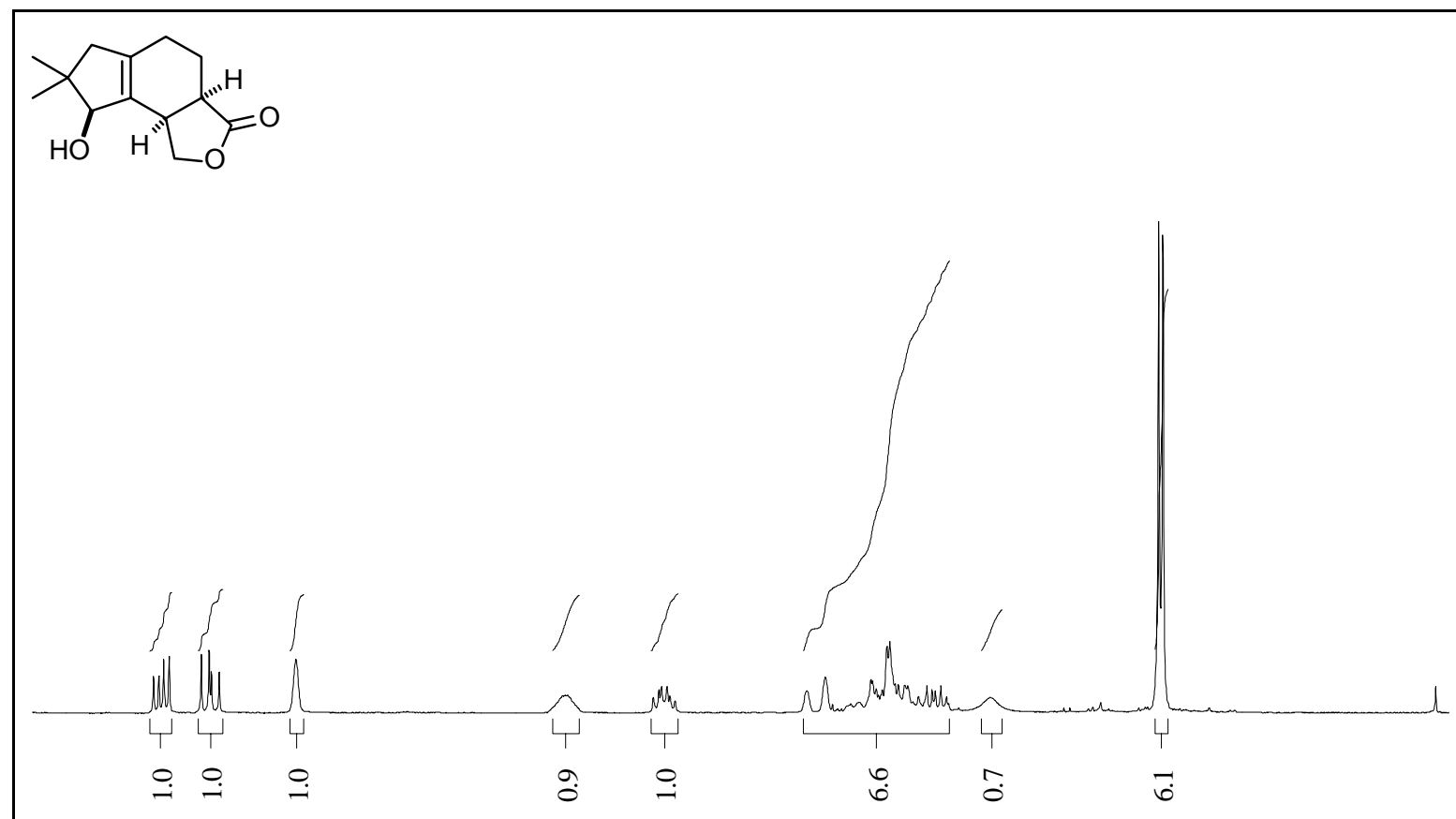

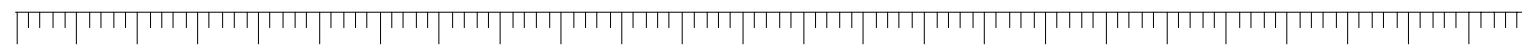

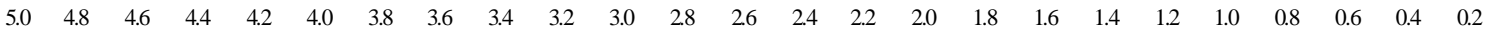

8-Hydroxy-7,7-dimethyl-1,3a,4,5,6,7,8,8b-octahydro-3H-indeno[4,5-c]furan-3-on (52)

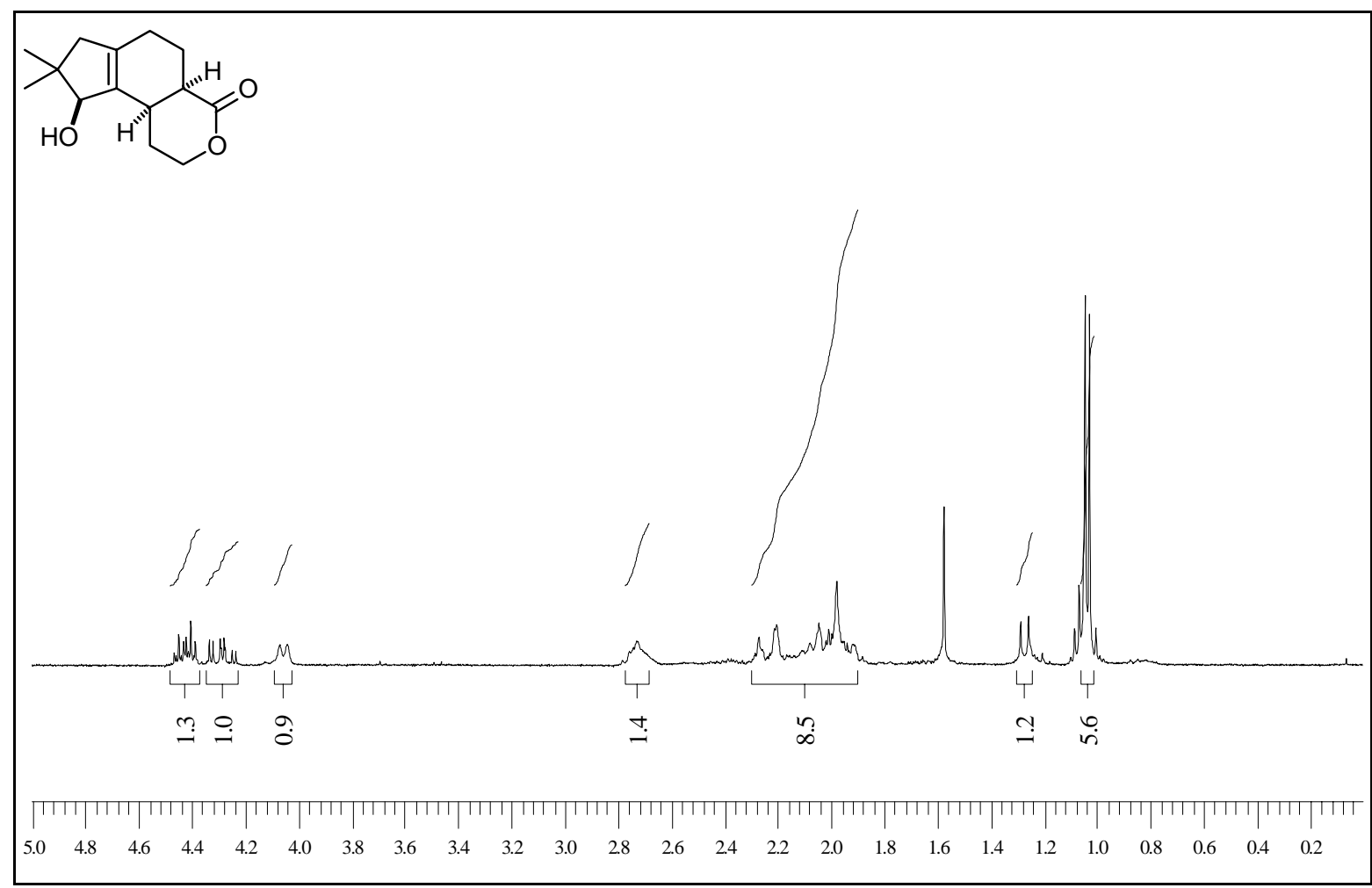

9-Hydroxy-8,8-dimethyl-1,4a,5,6,7,8,9,9b-octahydrocyclopenta[f]isochromen-4(2H)-on (58) 


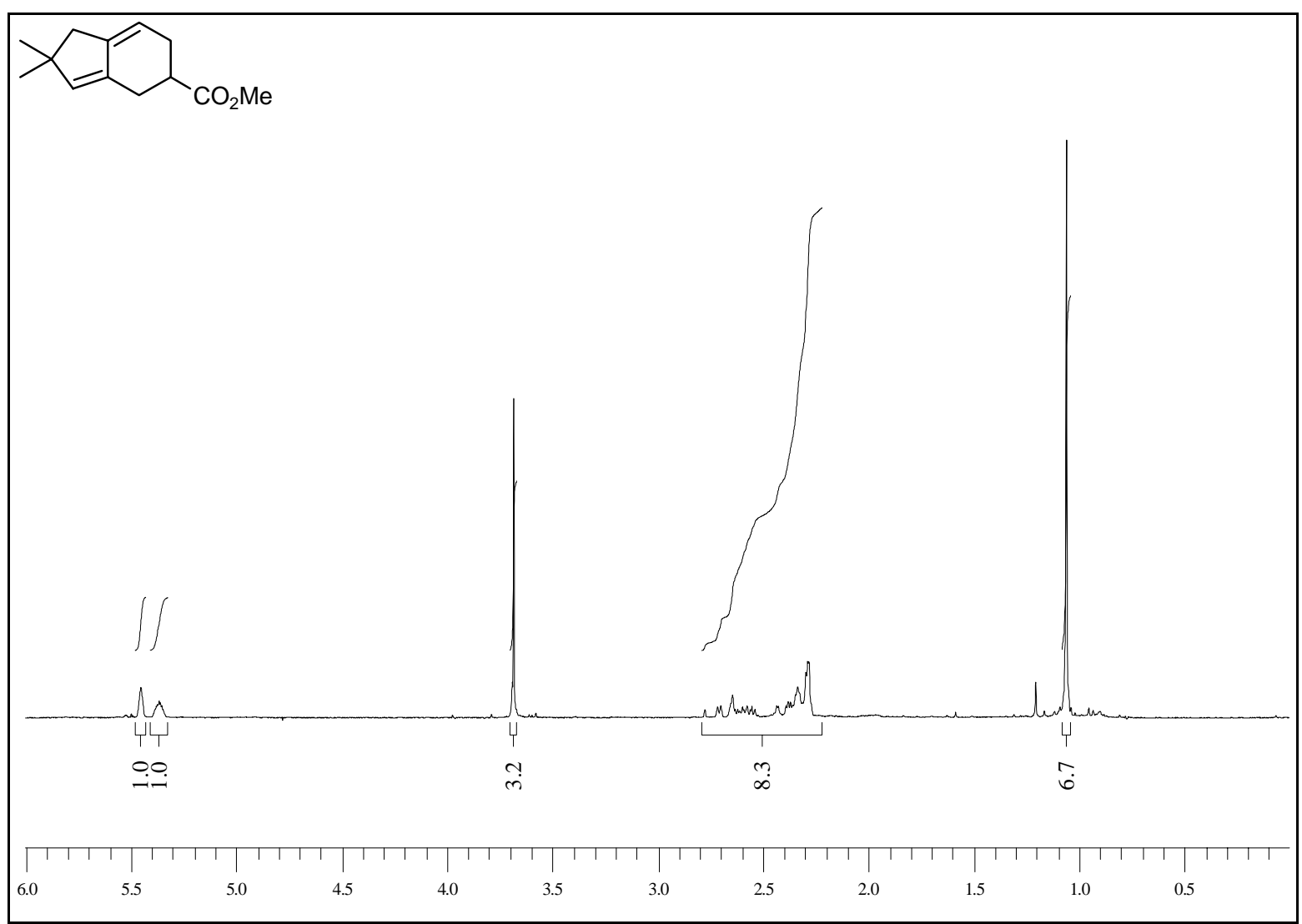

2,2-Dimethyl-2,4,5,6-tetrahydro-1 $H$-inden-5-carbonsäuremethylester (61)

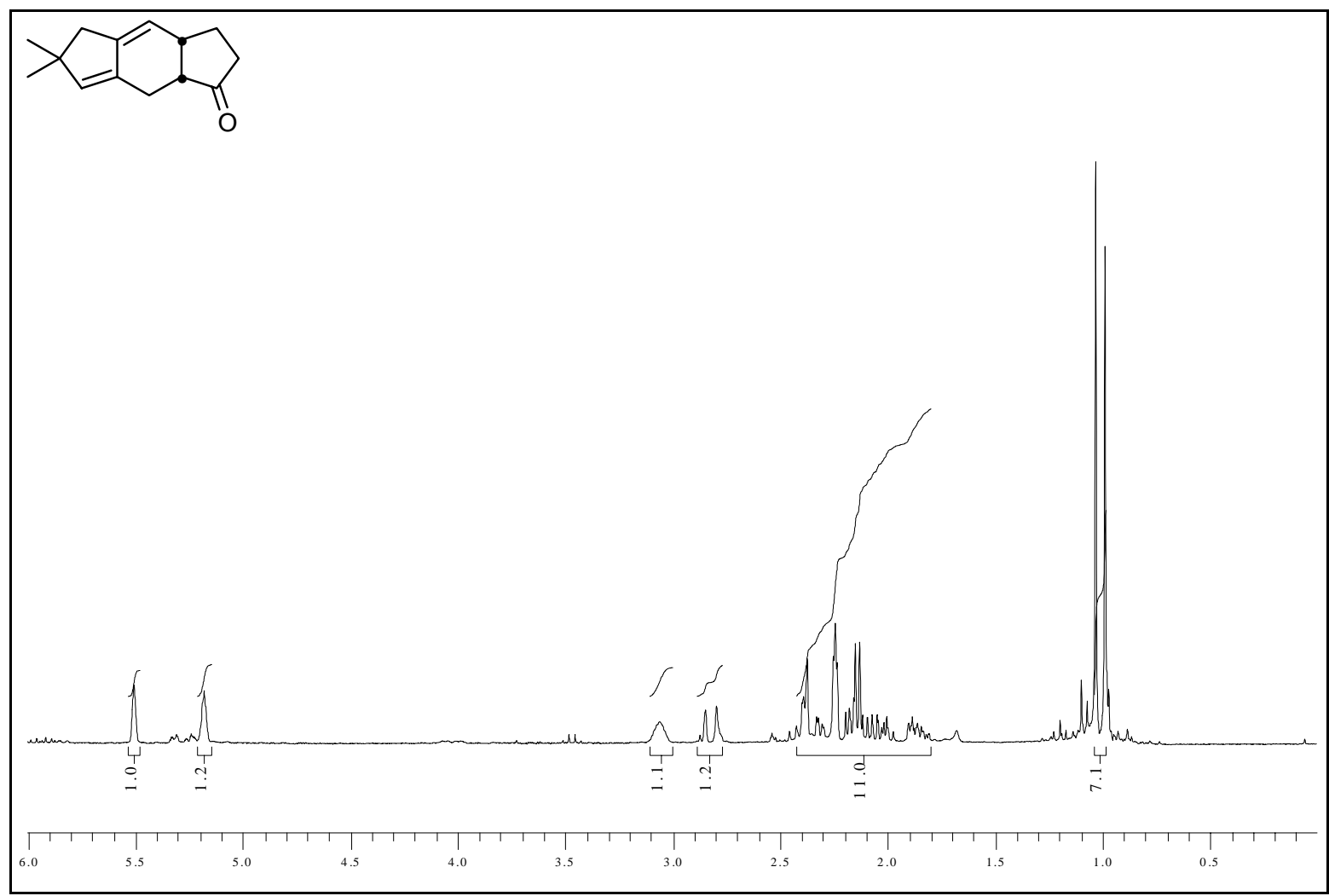

6,6-Dimethyl-3,3a,5,6,8,8a-hexahydro-2H-s-indacen-1-on (65) 


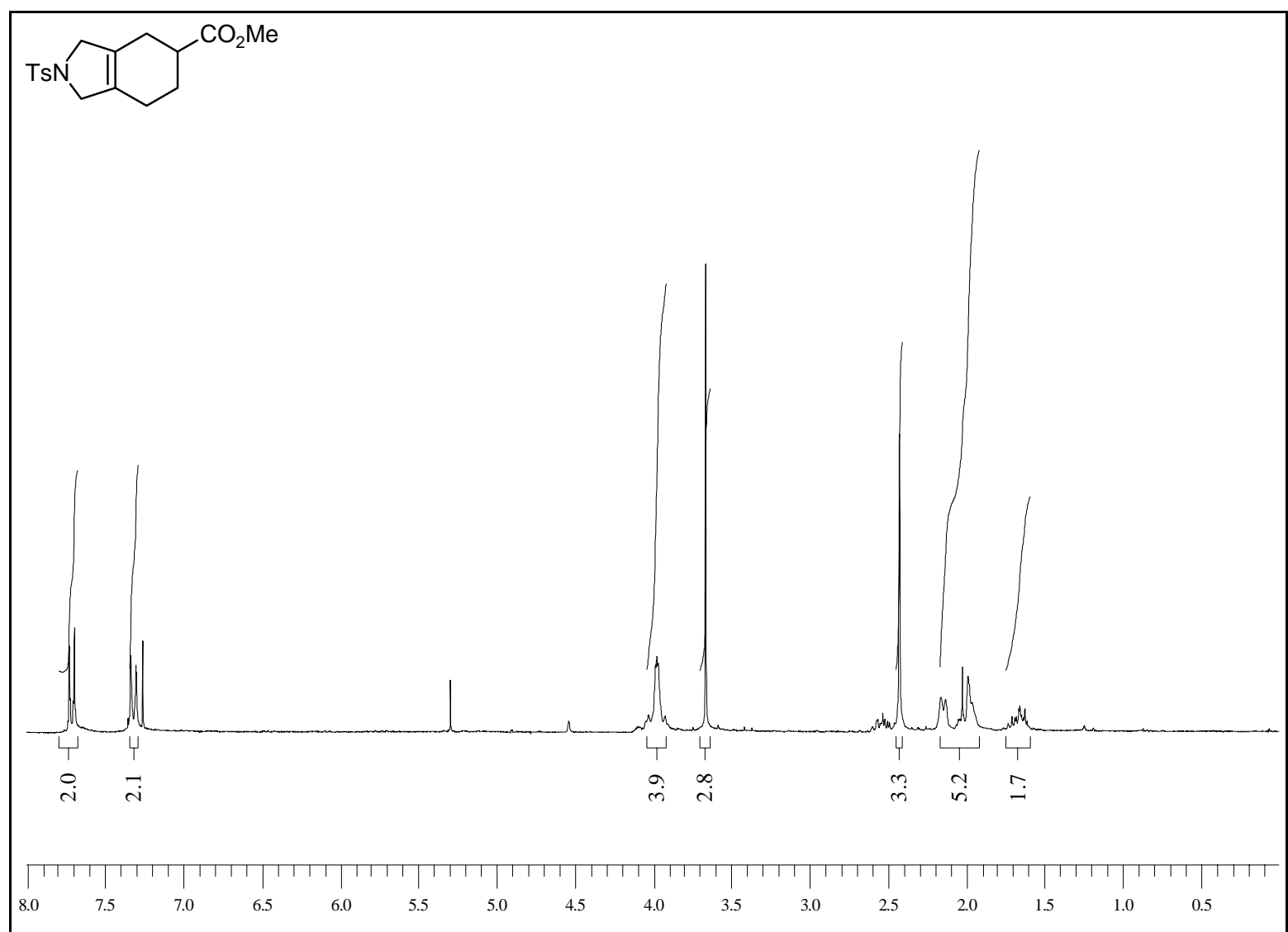

$N$-(p-Tosyl)-2,3,4,5,6,7-hexahydro-1H-isoindol-5-carbonsäuremethylester (74)

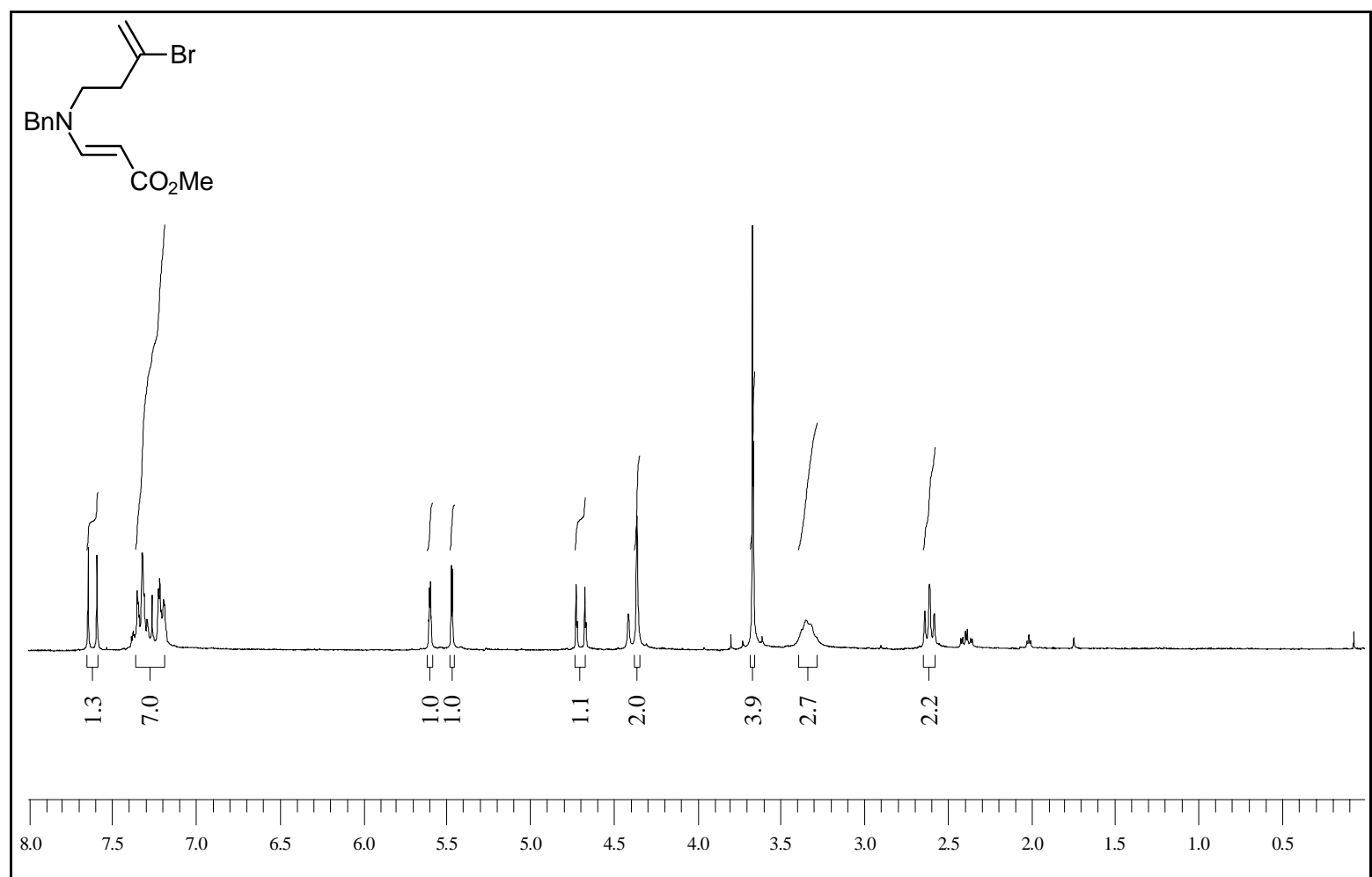

$N$-Benzyl- $N$-(3'-brombut-3'-en)prop-1-en-2-carbonsäuremethylester-1-amin (82) 


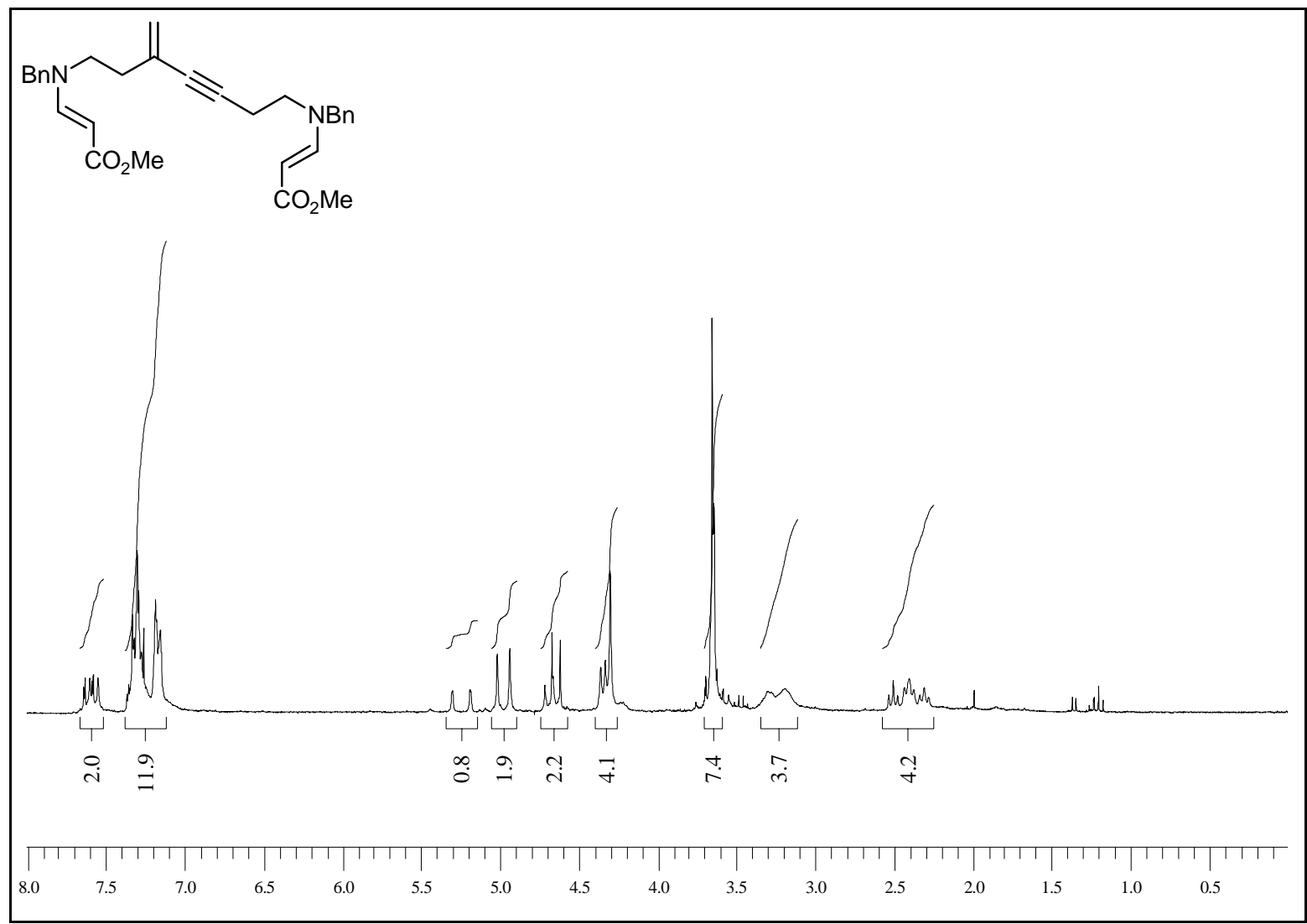

$N, N$-Benzyl-1,13-dicarbonsäuremethylester-8-methylentrideca-1,12-dien-6-in (86)

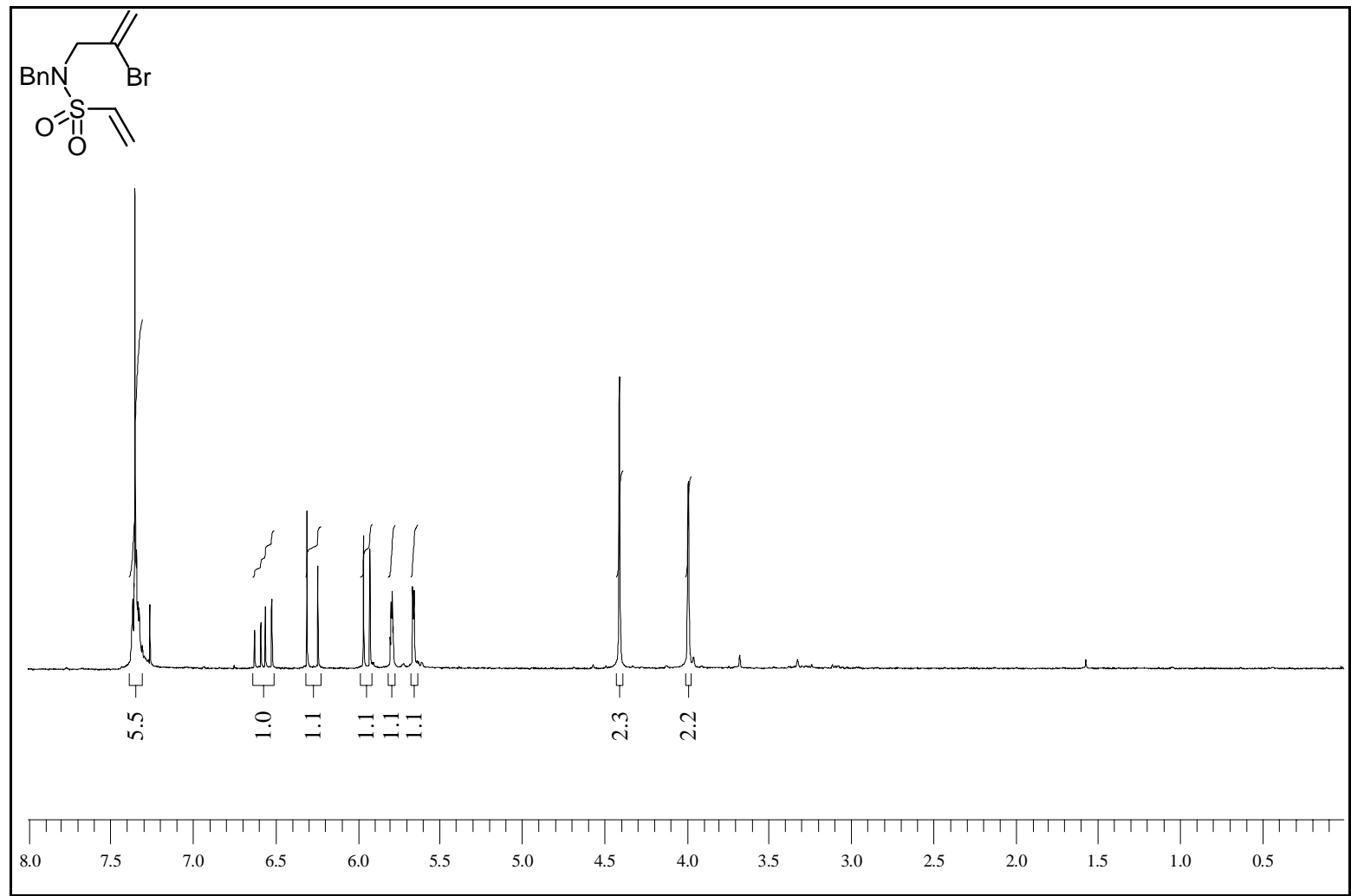

$N$-Benzyl-2-brom- $N$-vinylsulfonylprop-2-en-1-amin (93) 


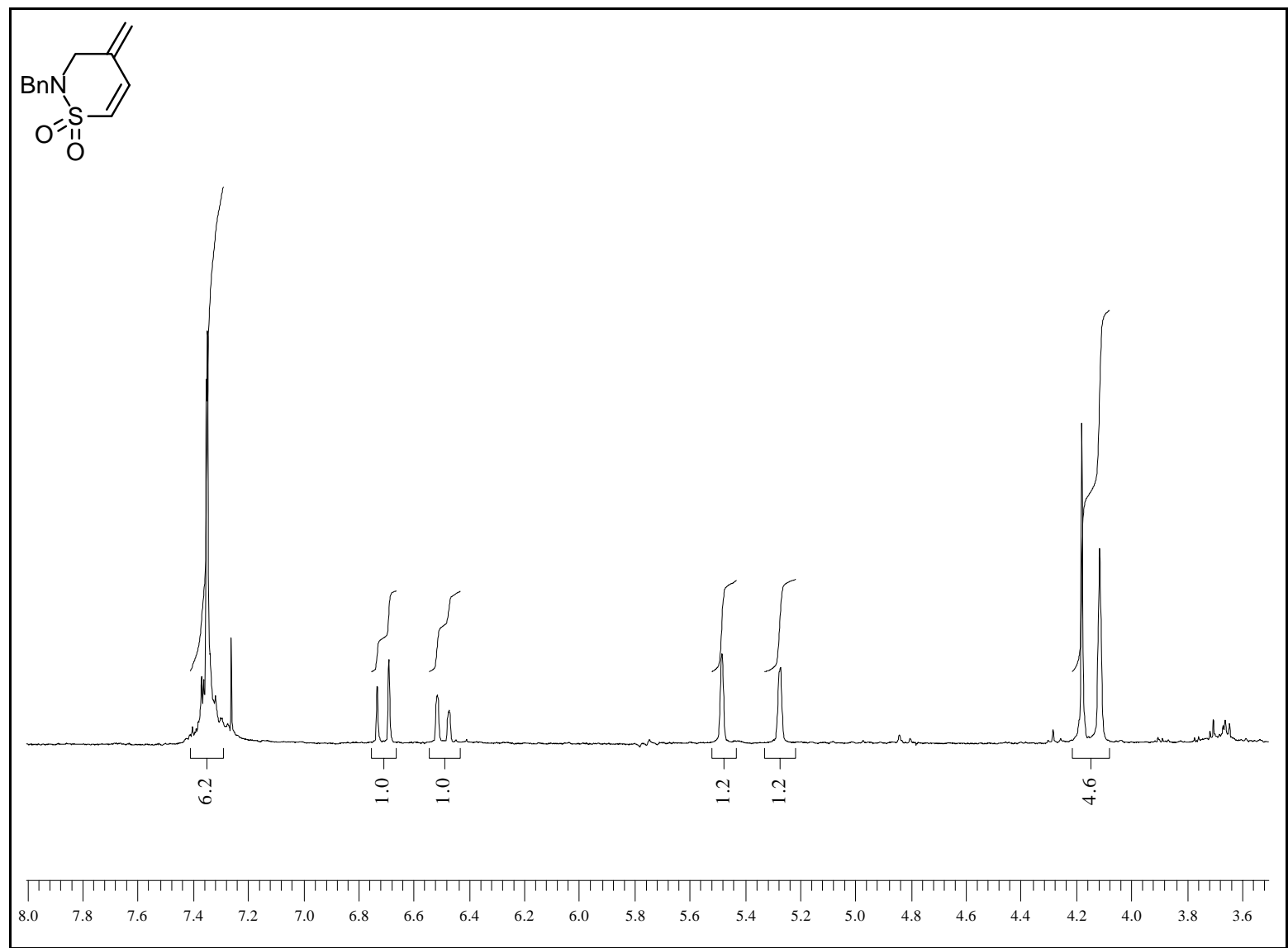

$N$-Benzyl-4-methylen-3,4-dihydro-2H[1,2]thiazin-1,1-dioxid (101)

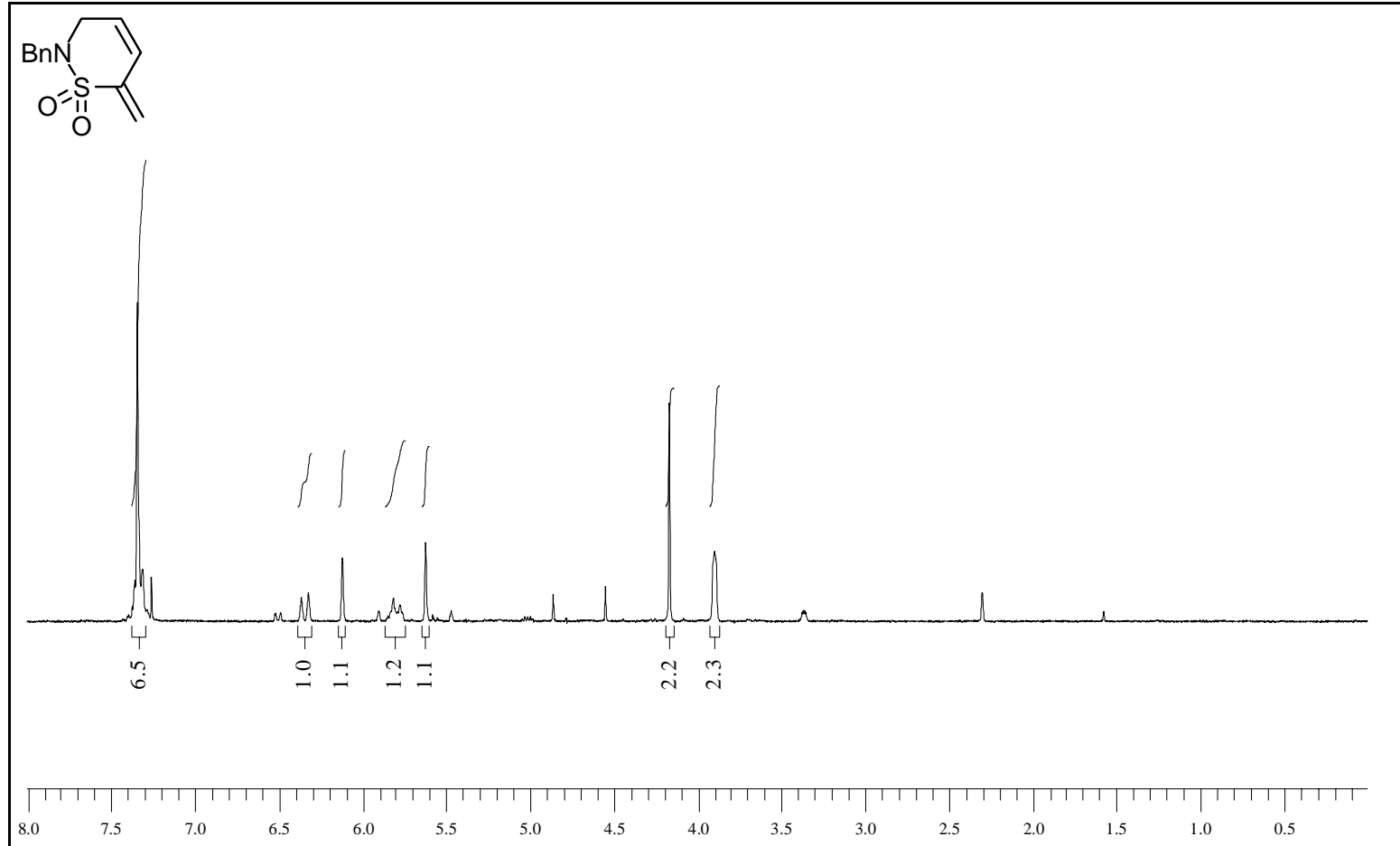

$N$-Benzyl-6-methylen-3,6-dihydro-2H[1,2]thiazin-1,1-dioxid (102) 


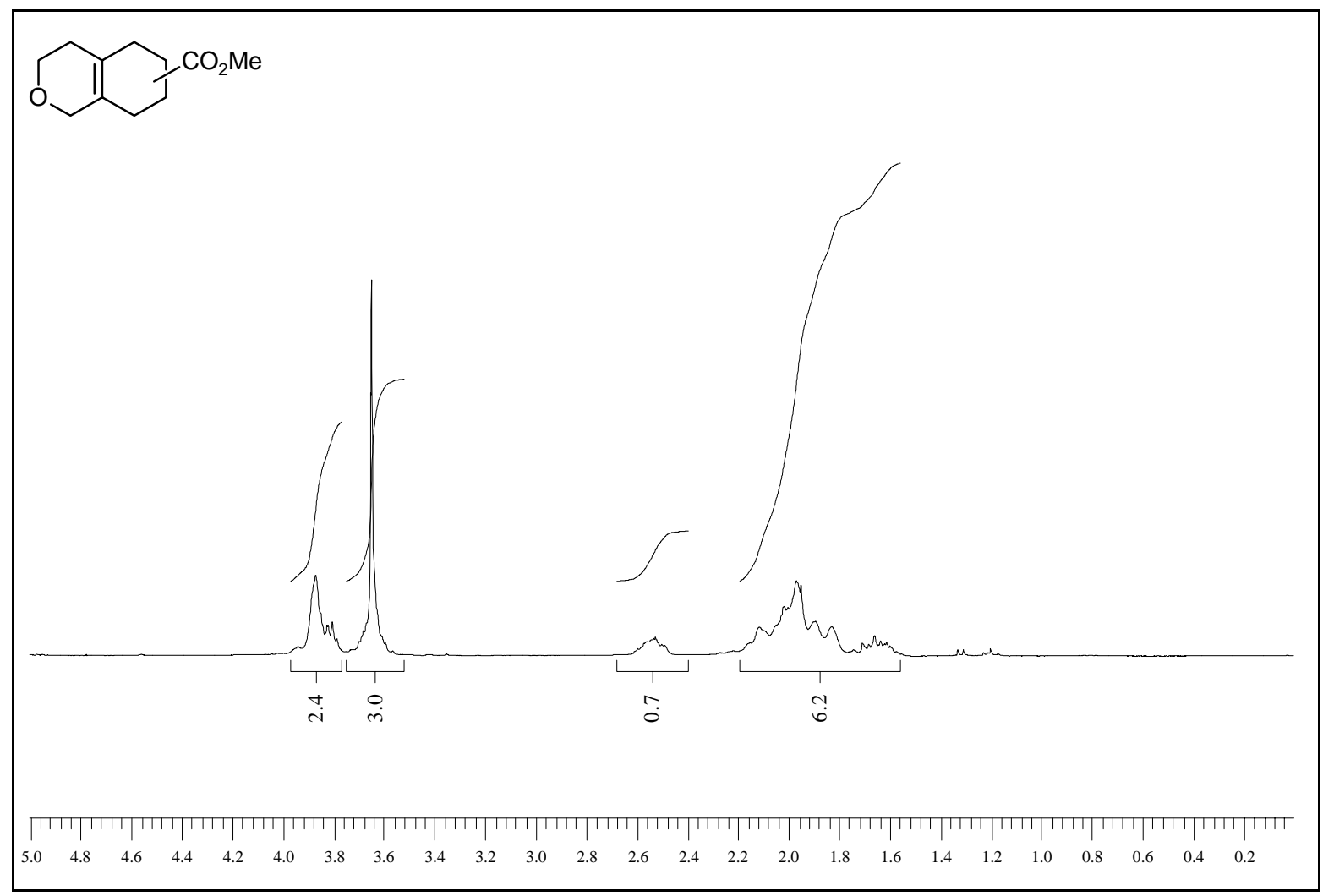

3,4,5,6,7,8-Hexahydro-1H-isochromen-6-carbonsäuremethylester (115a) und 3,4,5,6,7,8Hexahydro- $1 H$-isochromen-7-carbonsäuremethylester (115b)

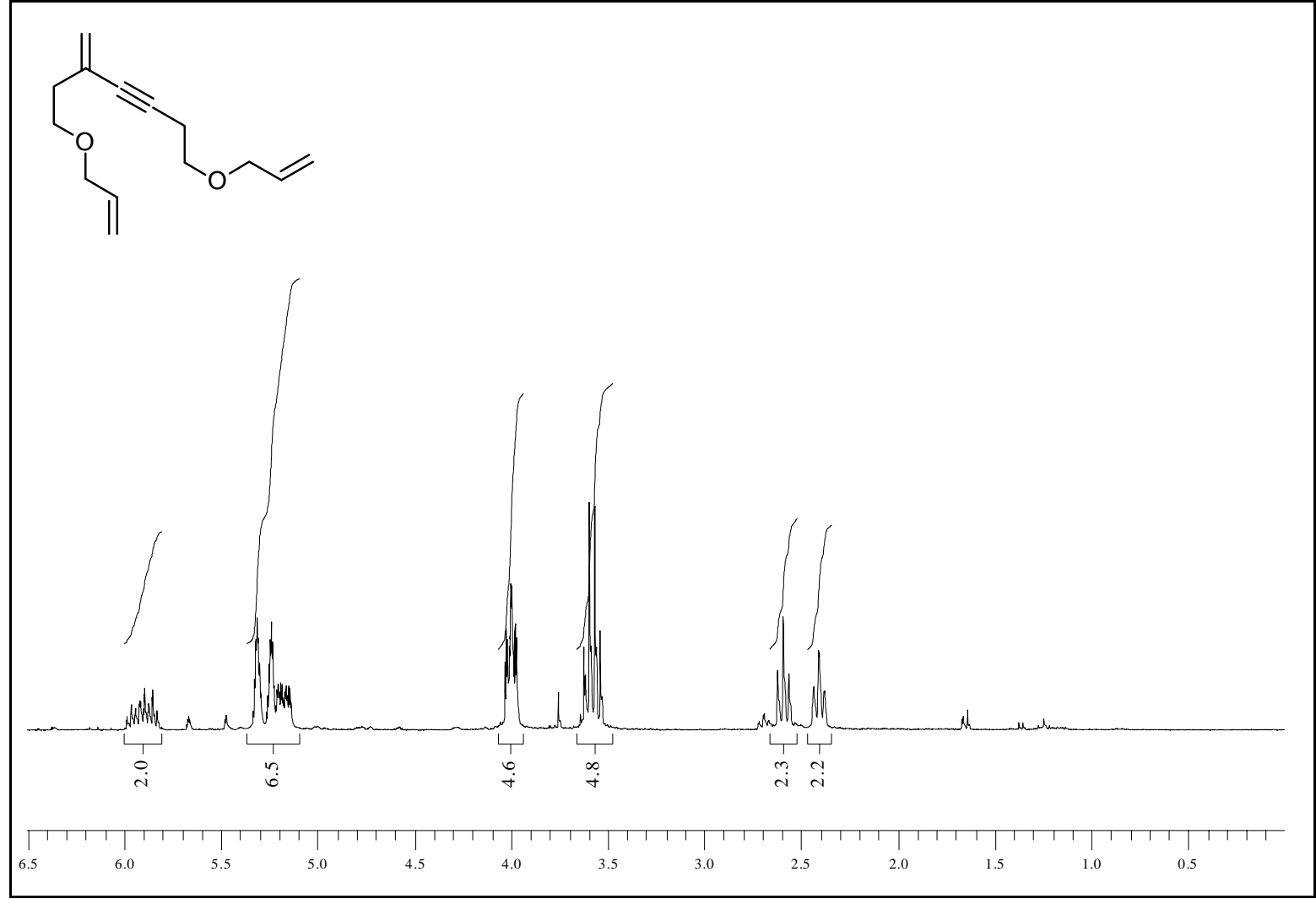

1,7-Diallyloxy-5-methylenhept-3-in (116) 


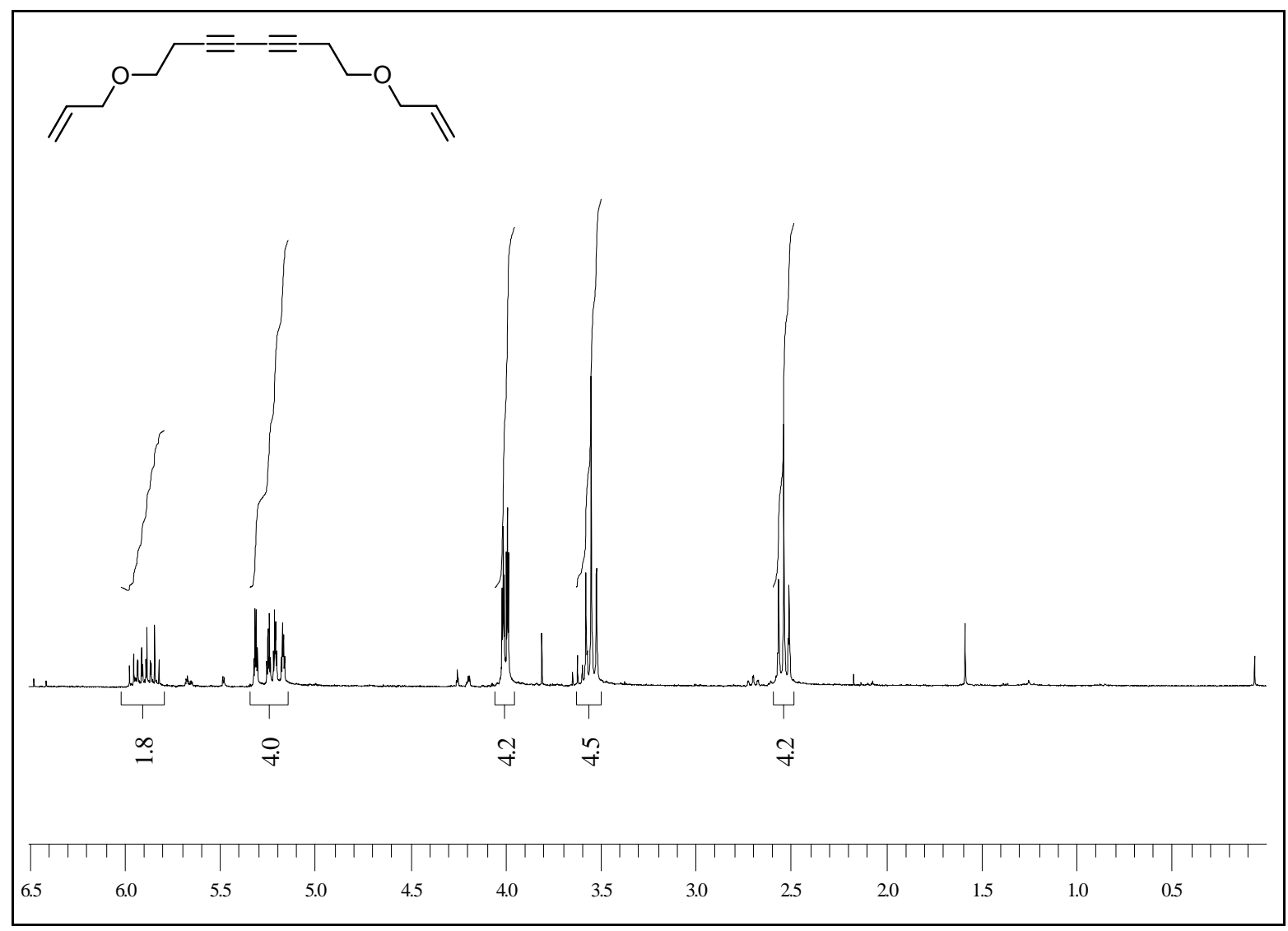

1,8-Diallyloxyocta-3,5-diin (117)

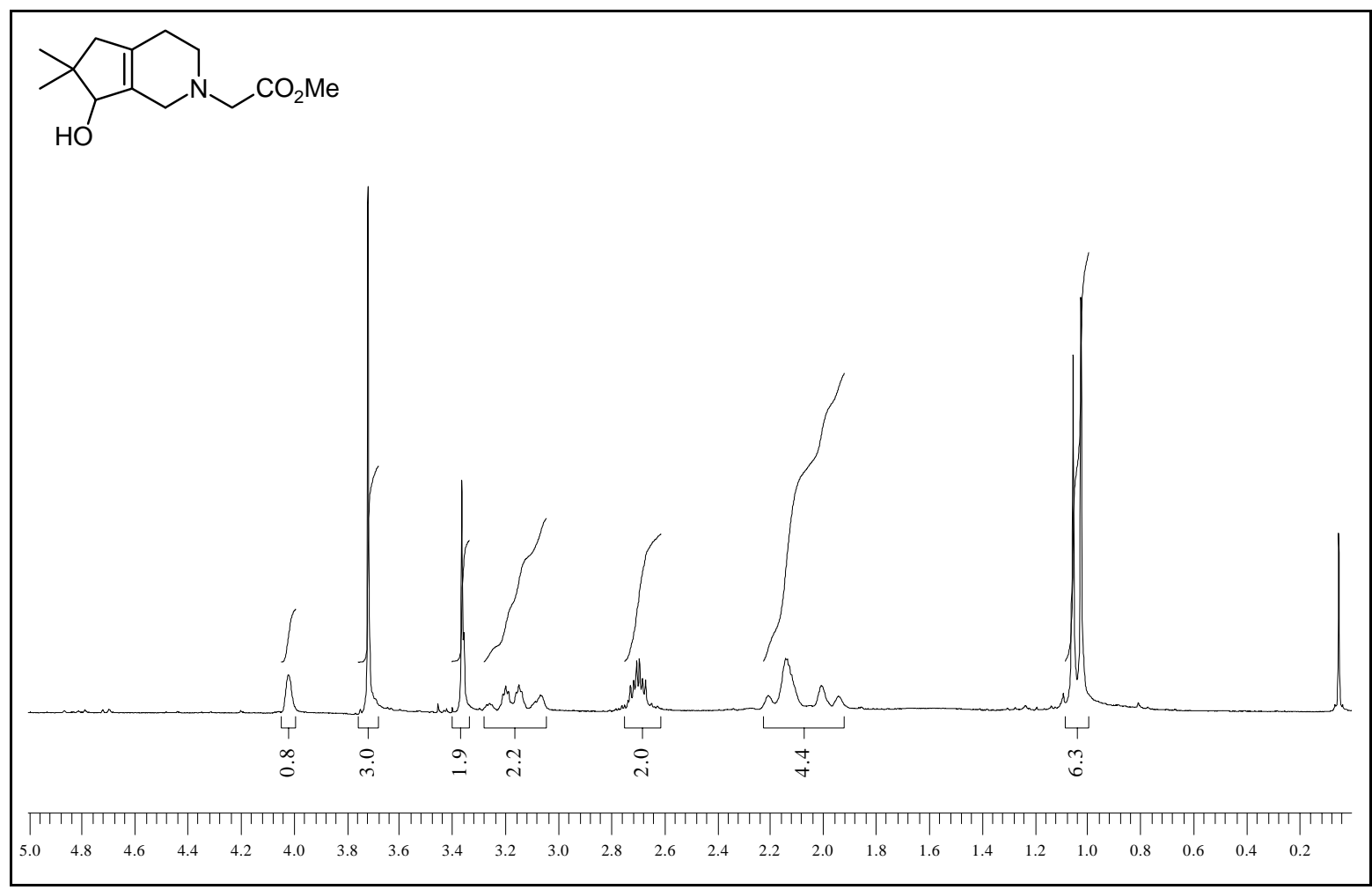

(7-Hydroxy-6,6-dimethyl-1,3,4,5,6,7-hexahydro-2H-cyclopenta[c]pyridin-2-yl)essigsäuremethylester (126-Gly) 


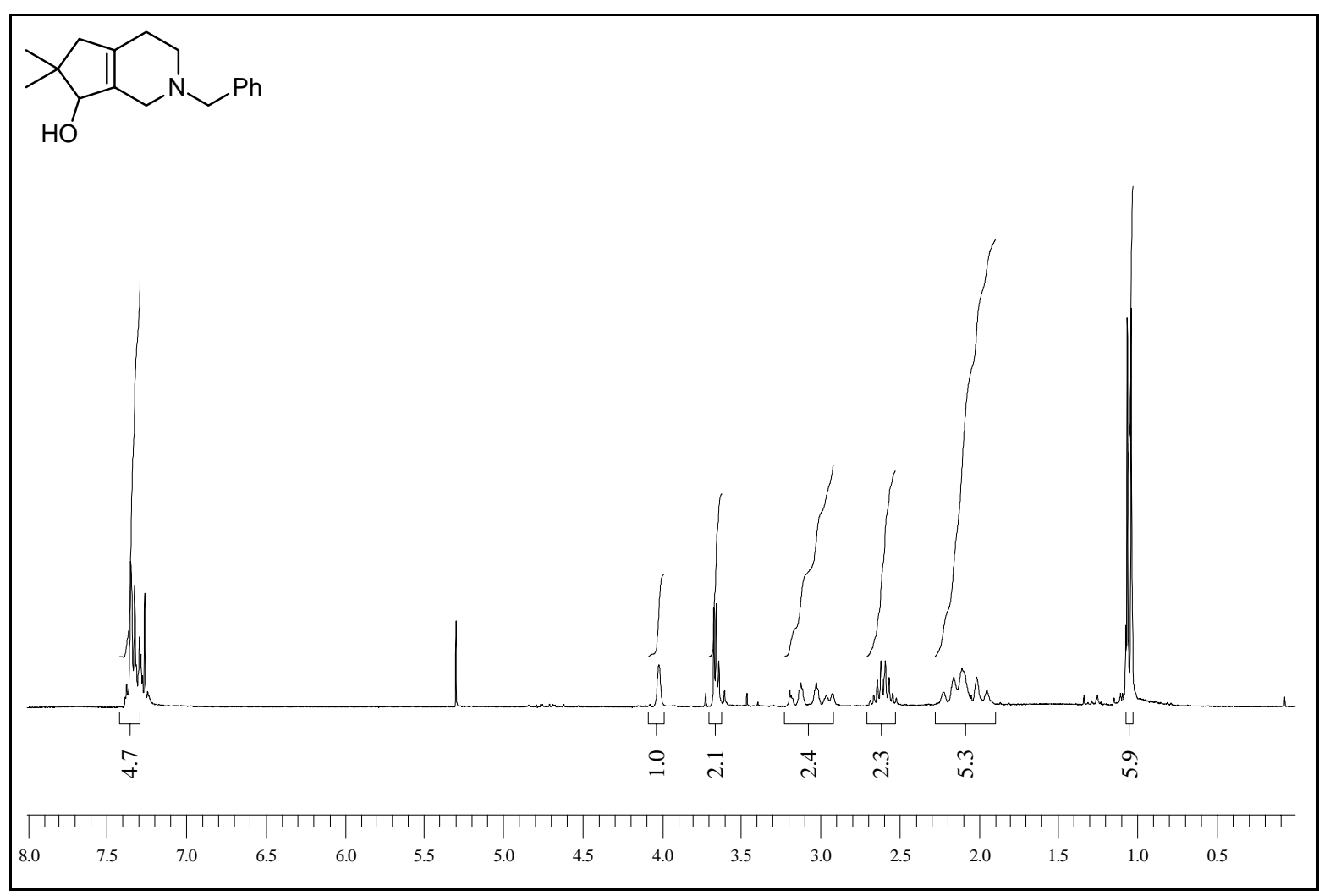

$\mathrm{N}$-Benzyl-(7-hydroxy-6,6-dimethyl-1,3,4,5,6,7-hexahydro-2H-cyclopenta[c]-2-pyridin) (126-Bn)

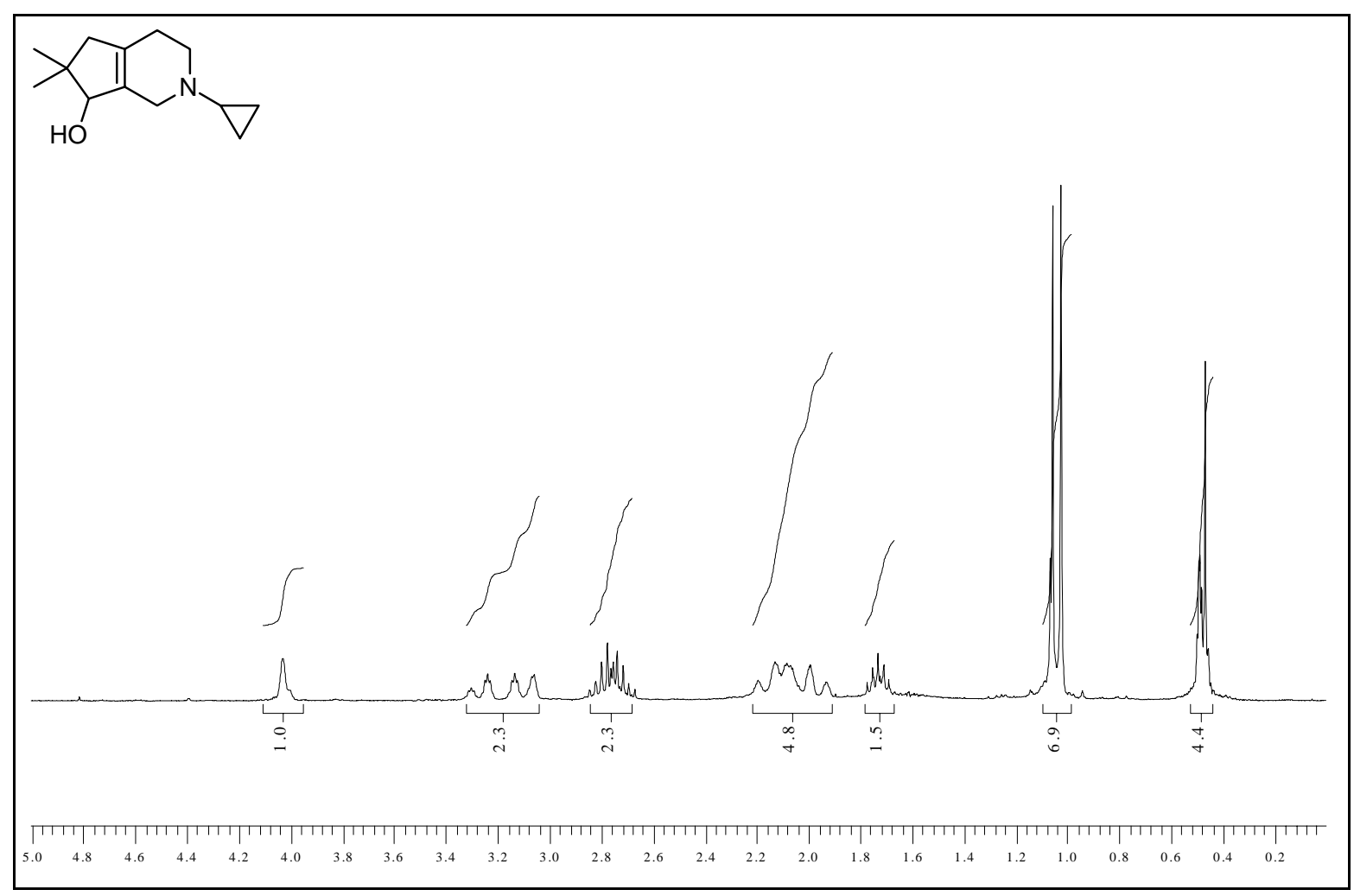

$\mathrm{N}$-Cyclopropyl-(7-hydroxy-6,6-dimethyl-1,3,4,5,6,7-hexahydro-2H-cyclopenta[c]-2pyridin) (126-cPr) 


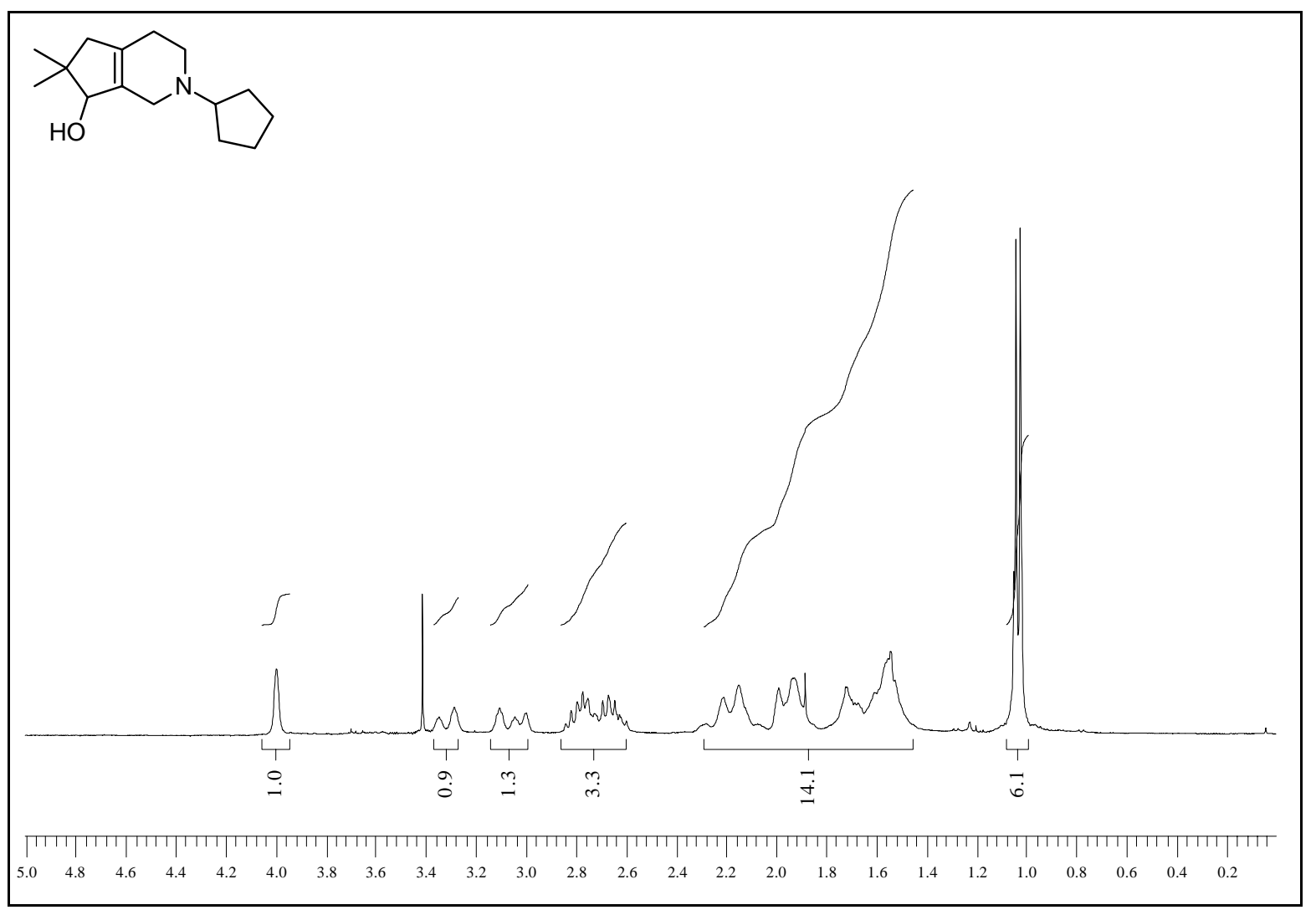

$N$-Cyclopentyl-(7-hydroxy-6,6-dimethyl-1,3,4,5,6,7-hexahydro-2H-cyclopenta[c]-2pyridin) (126-cPent)

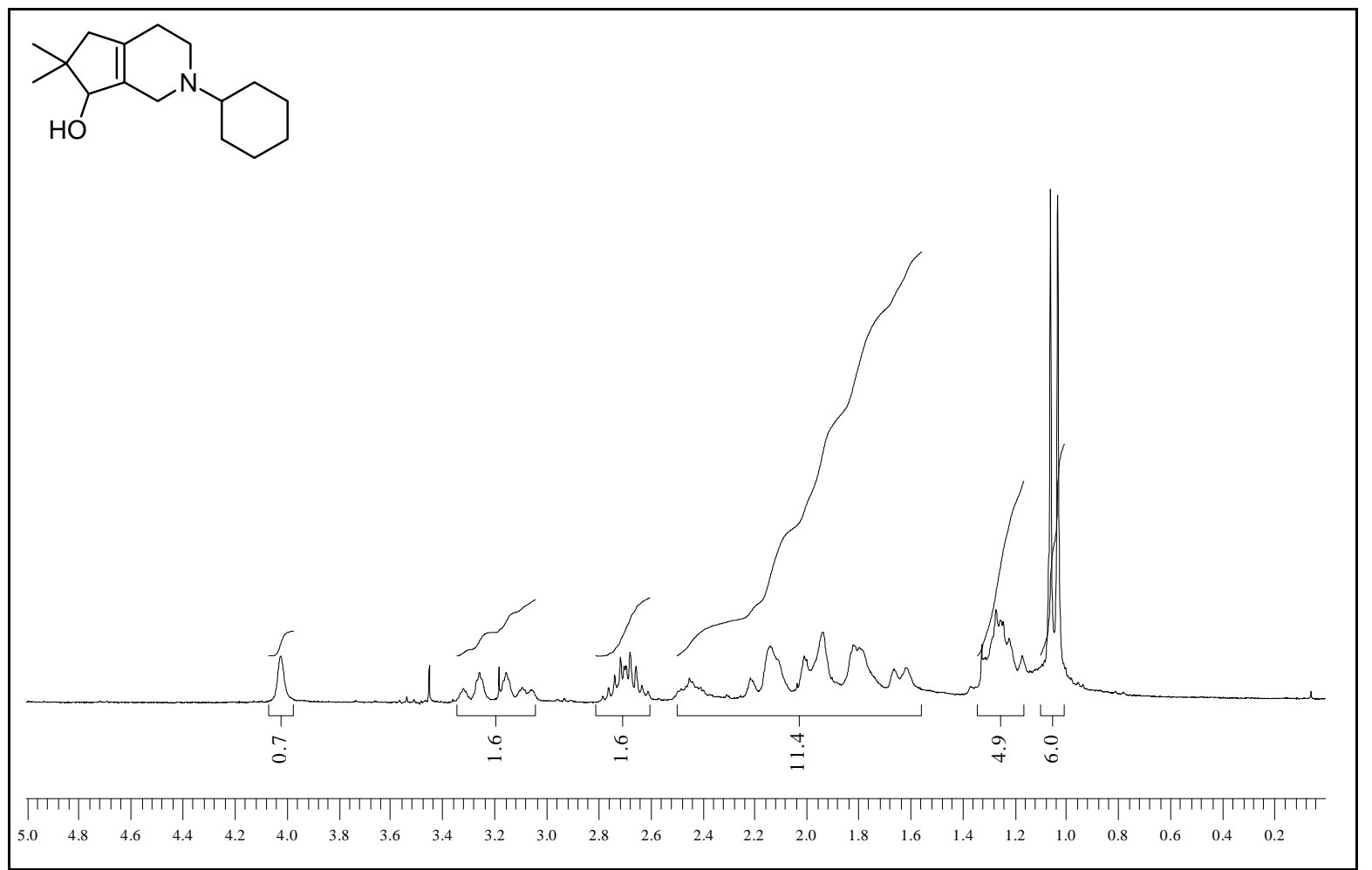

$\mathrm{N}$-Cyclohexyl-(7-hydroxy-6,6-dimethyl-1,3,4,5,6,7-hexahydro- $2 \mathrm{H}$-cyclopenta[c]-2-pyridin) (126-cHex) 


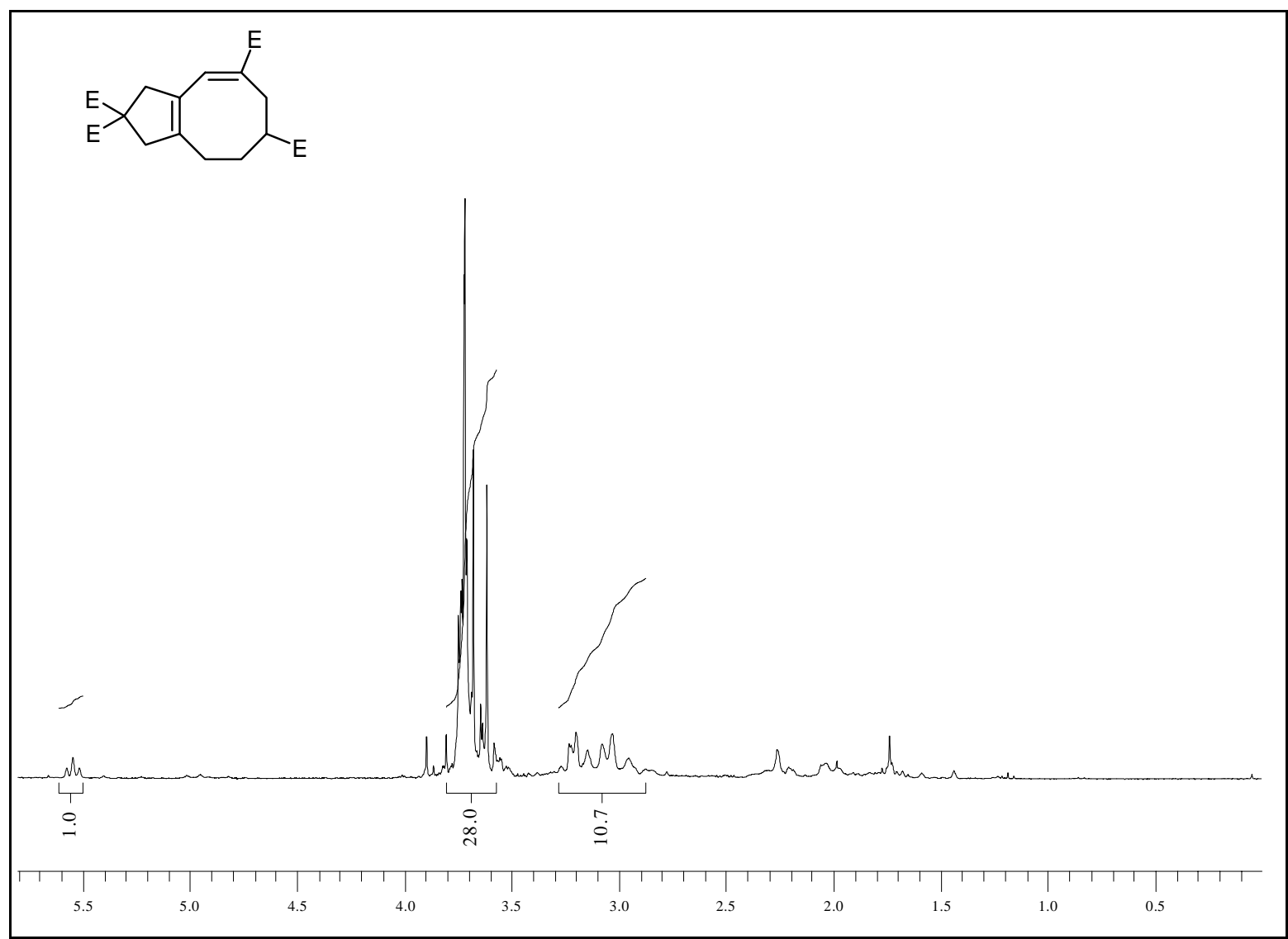

2,2,5,7-Tetracarbonsäuremethylester-2,3,4,5,6,7-hexahydro- $1 H$-cyclopentacycloocten $\left(155-\mathrm{CO}_{2} \mathrm{Me}\right)$

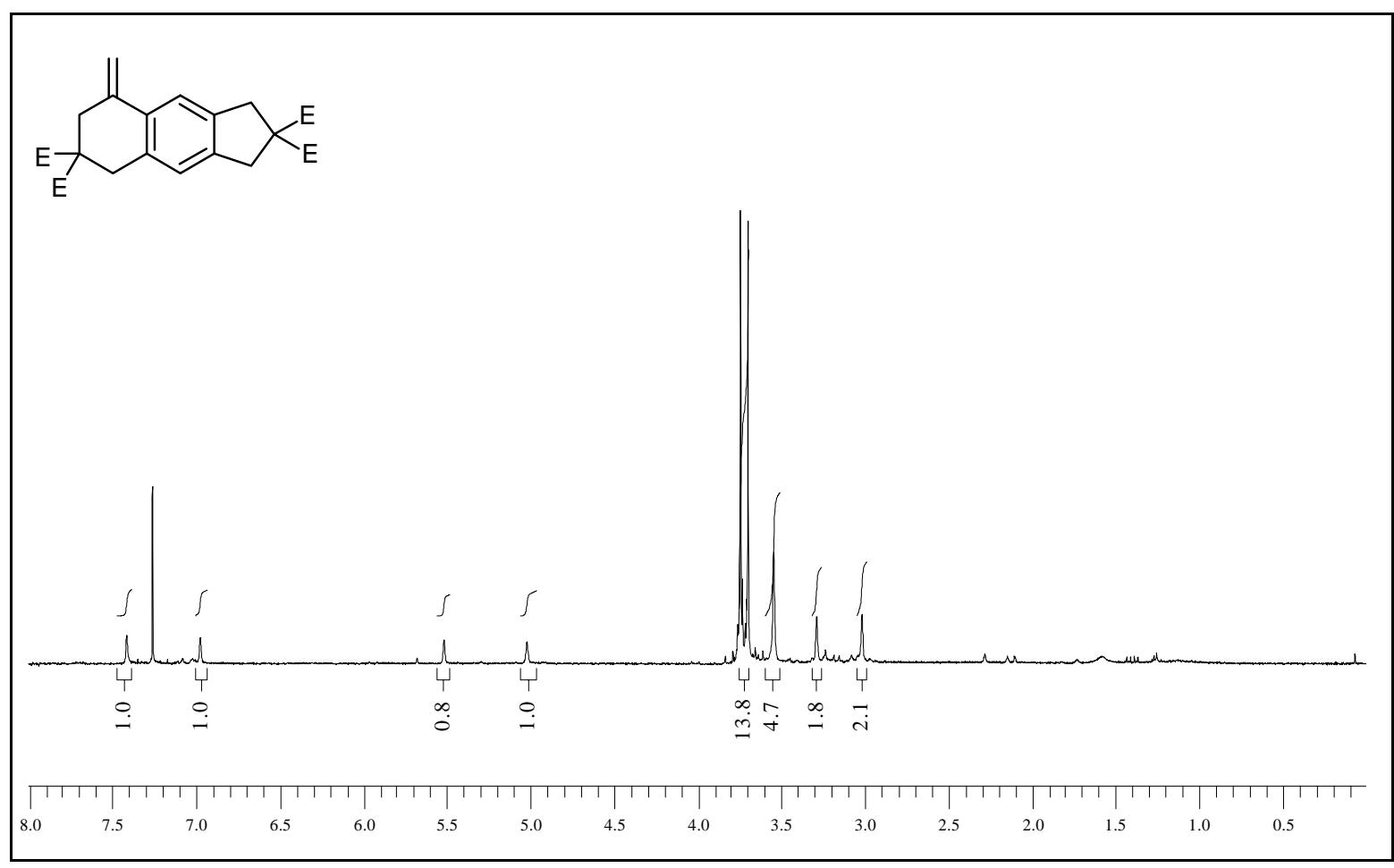

2,2,7,7-Tetracarbonsäuremethylester-5-methylen-1,2,3,6,7,8-hexahydrocyclopenta[b]naphthalin (156) 


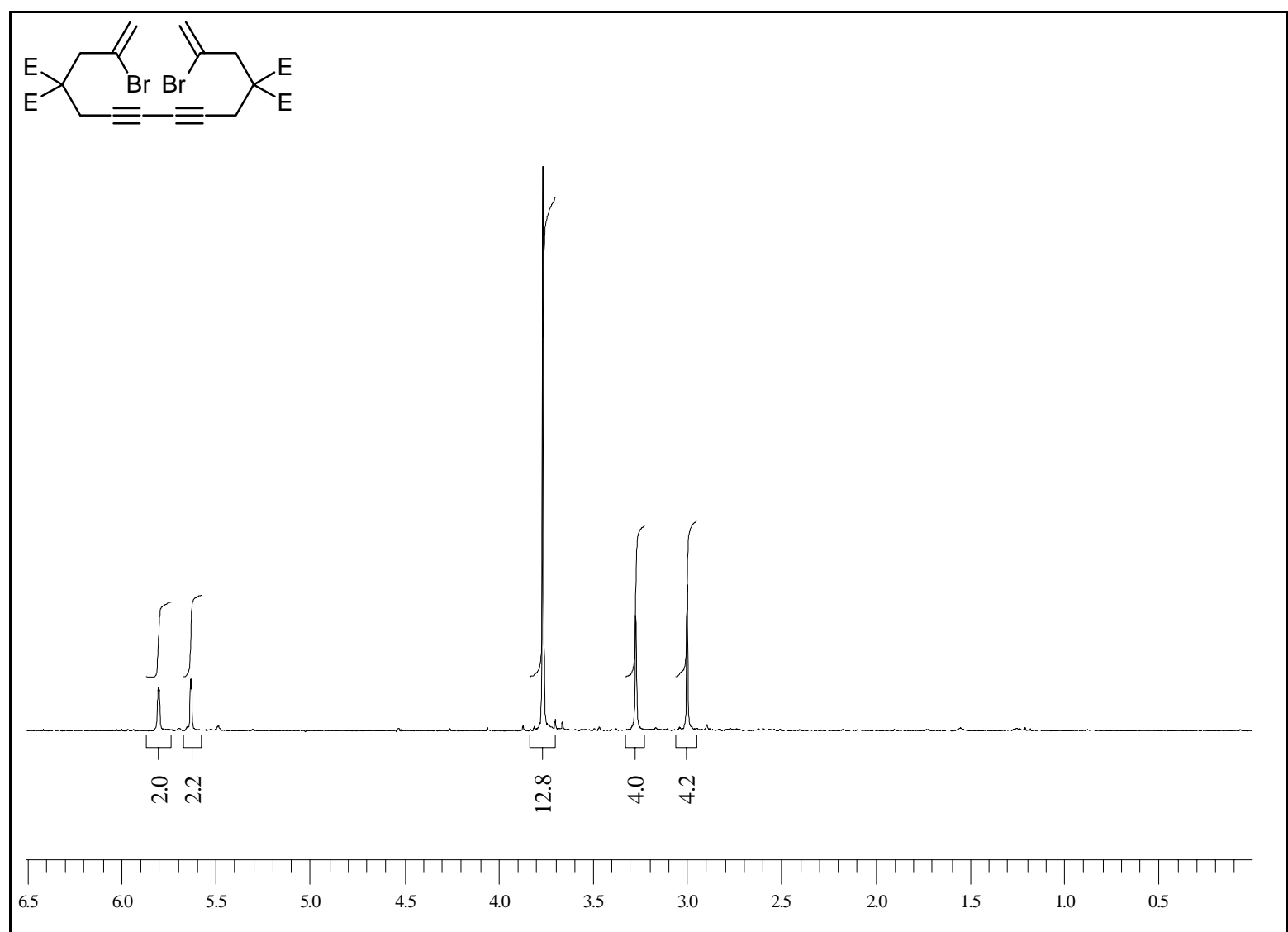

2,13-Brom-4,4,11,11-tetracarbonsäuremethylester-tetradeca-1,13-dien-6,8-diin (157)

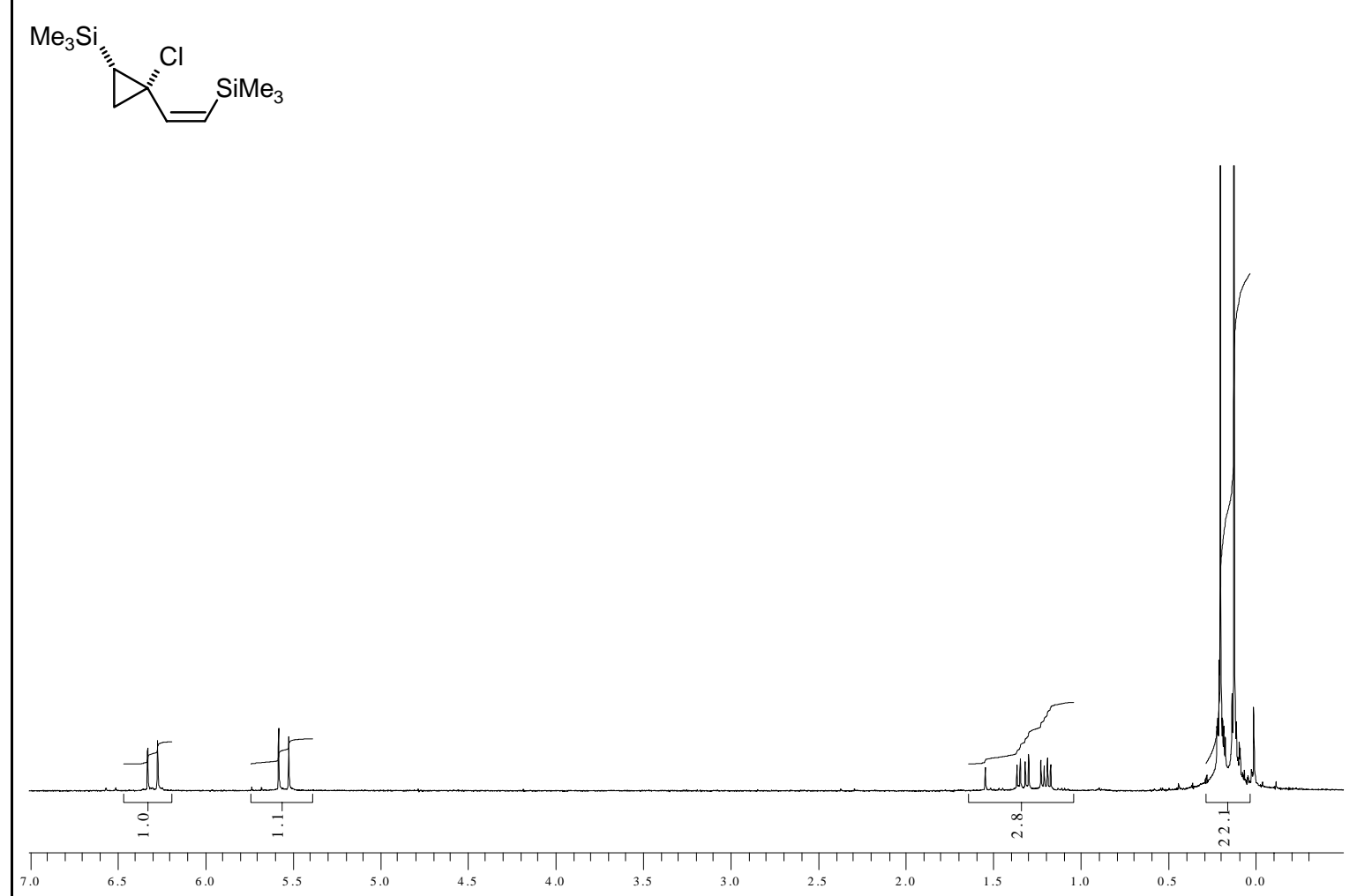

(Z)-1-Chlor-1-(2'-trimethylsilylethenyl)-2-trimethylsilylcyclopropan $[(Z, Z)-217]$ 
2. ${ }^{13} \mathrm{C}-\mathrm{NMR}-$ Spektren

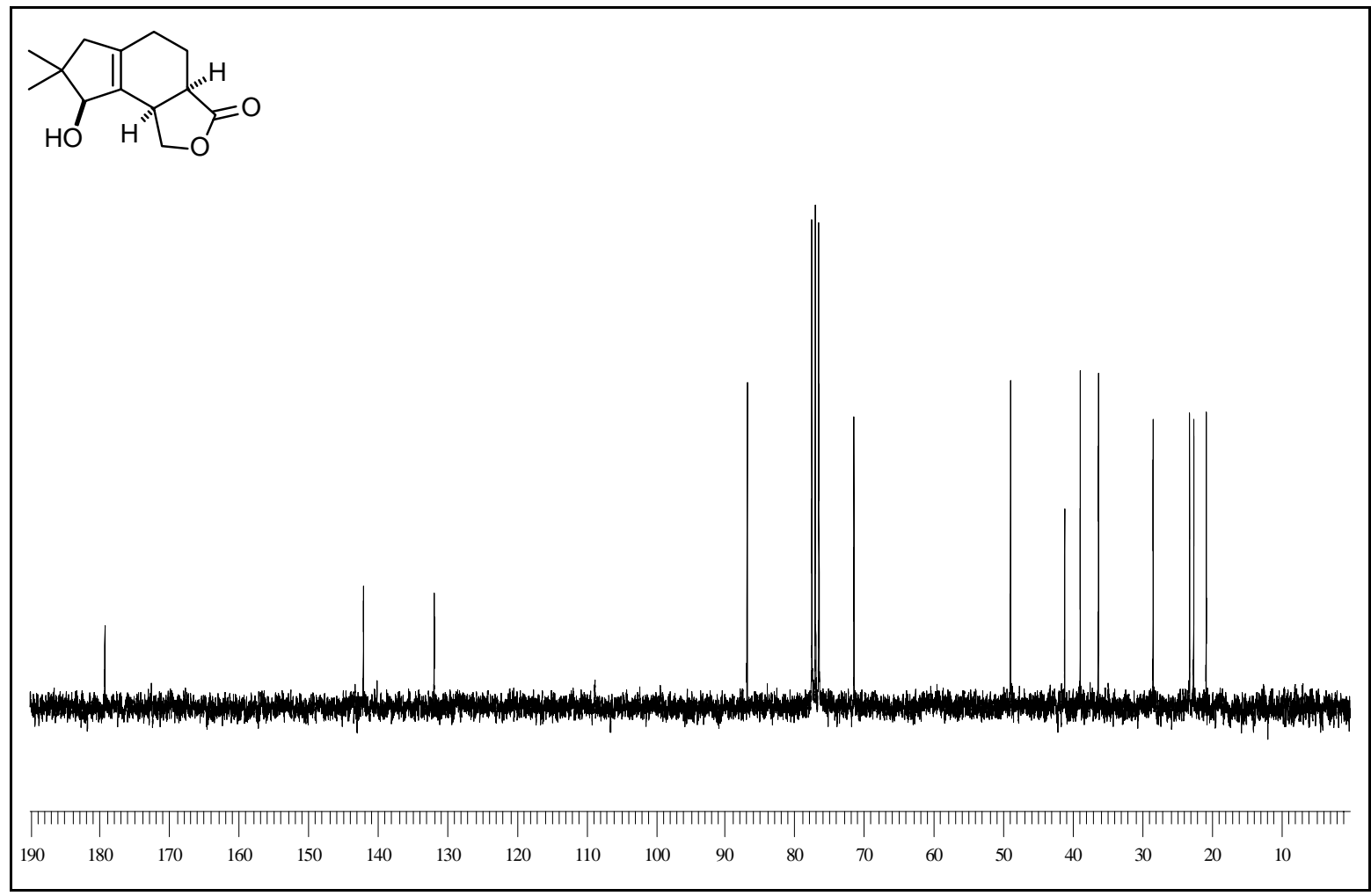

8-Hydroxy-7,7-dimethyl-1,3a,4,5,6,7,8,8b-octahydro-3H-indeno[4,5-c]furan-3-on (52)

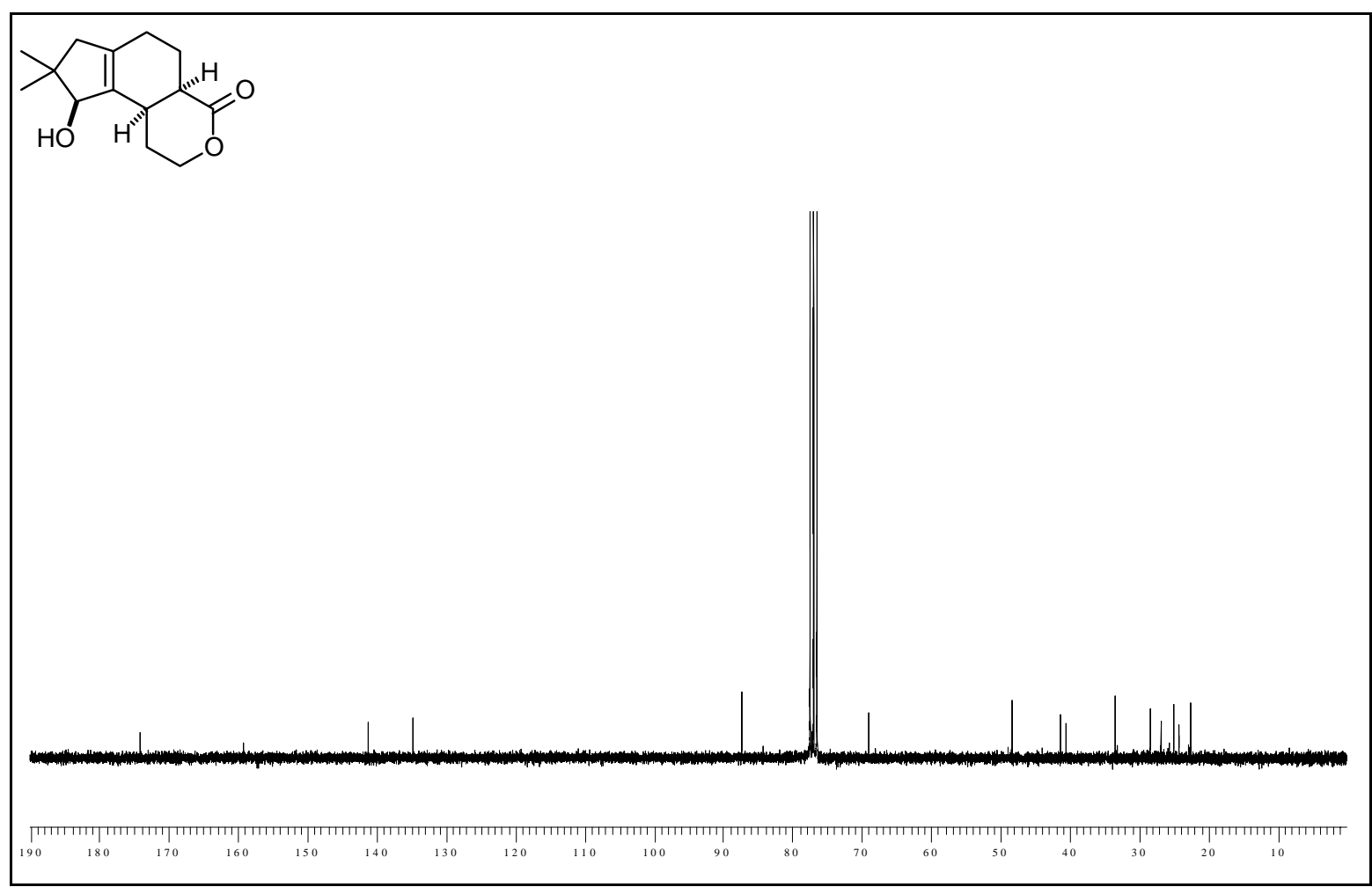

9-Hydroxy-8,8-dimethyl-1,4a,5,6,7,8,9,9b-octahydrocyclopenta[f]isochromen-4(2H)-on (58) 


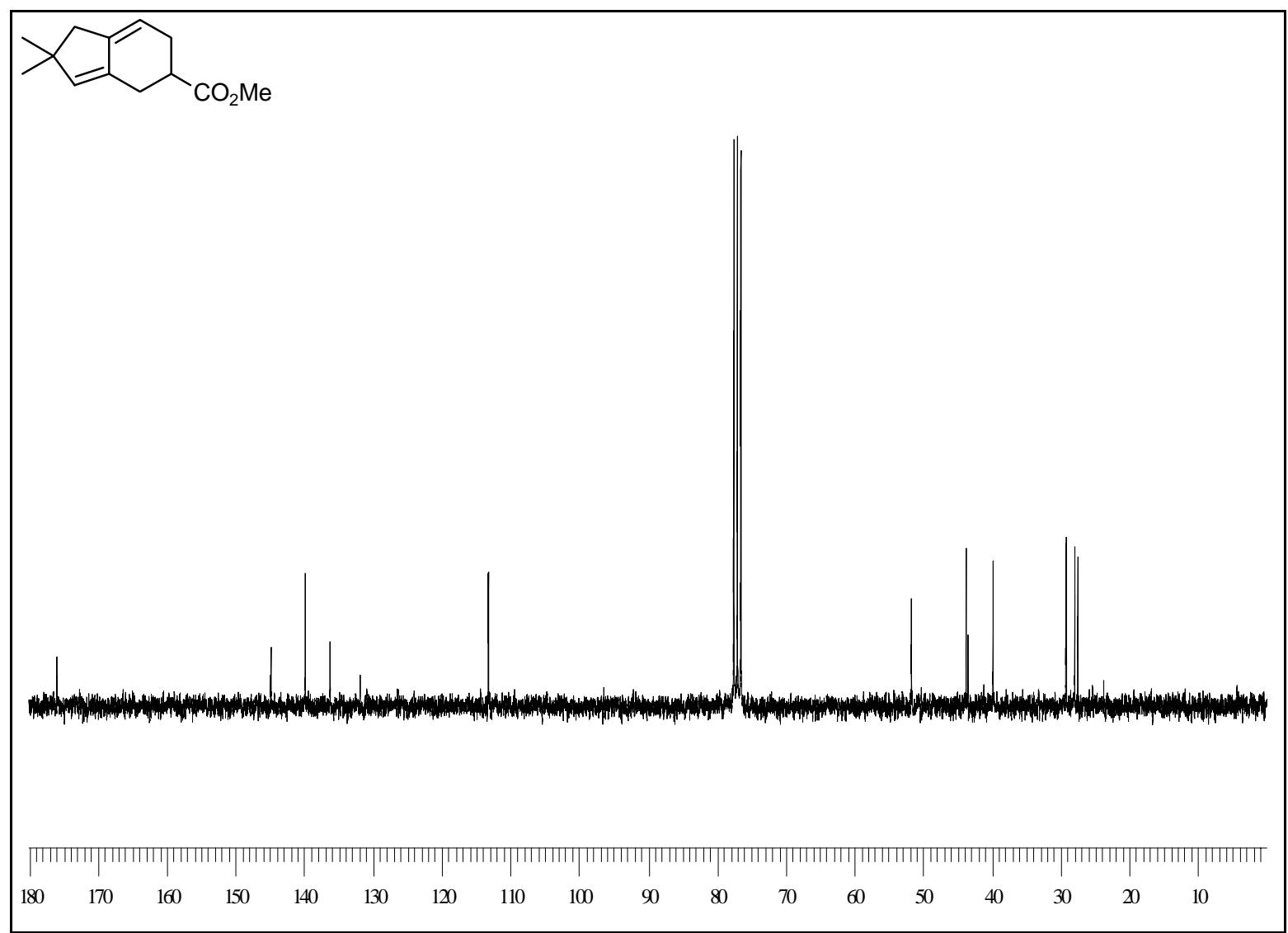

2,2-Dimethyl-2,4,5,6-tetrahydro-1 $H$-inden-5-carbonsäuremethylester (61)

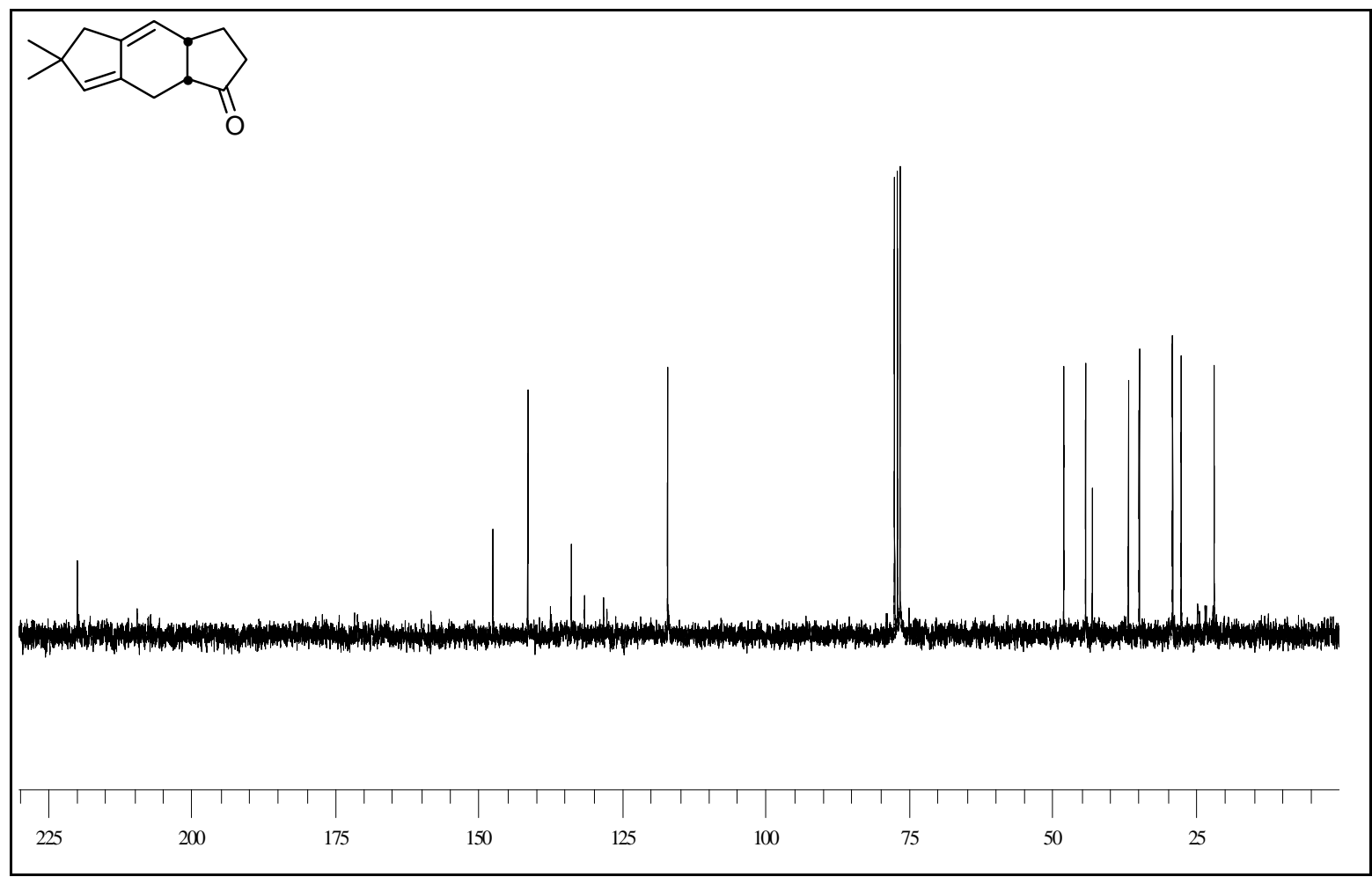

6,6-Dimethyl-3,3a,5,6,8,8a-hexahydro-2H-s-indacen-1-on (65) 


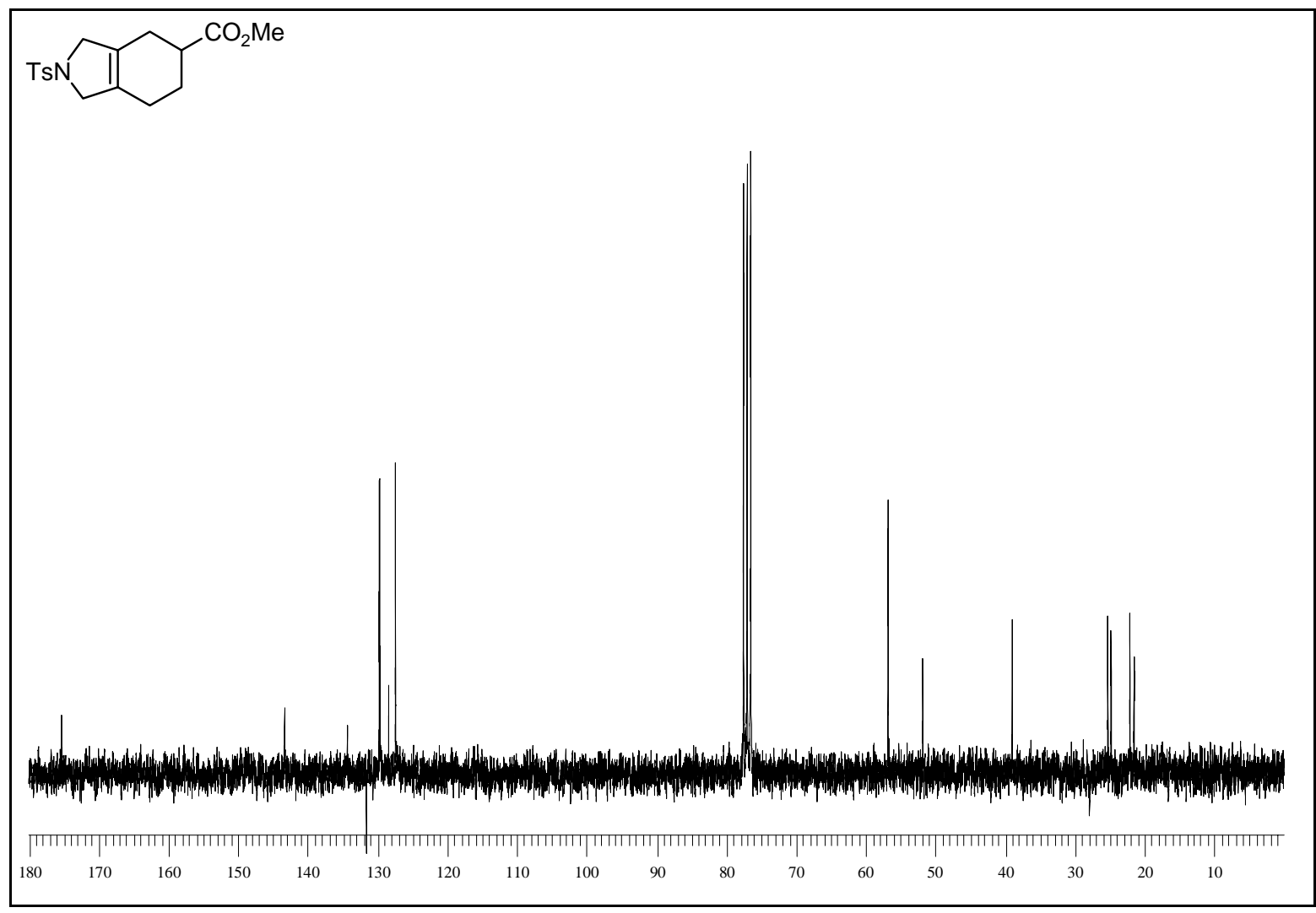

$N$-(p-Tosyl)-2,3,4,5,6,7-hexahydro-1H-isoindol-5-carbonsäuremethylester (74)

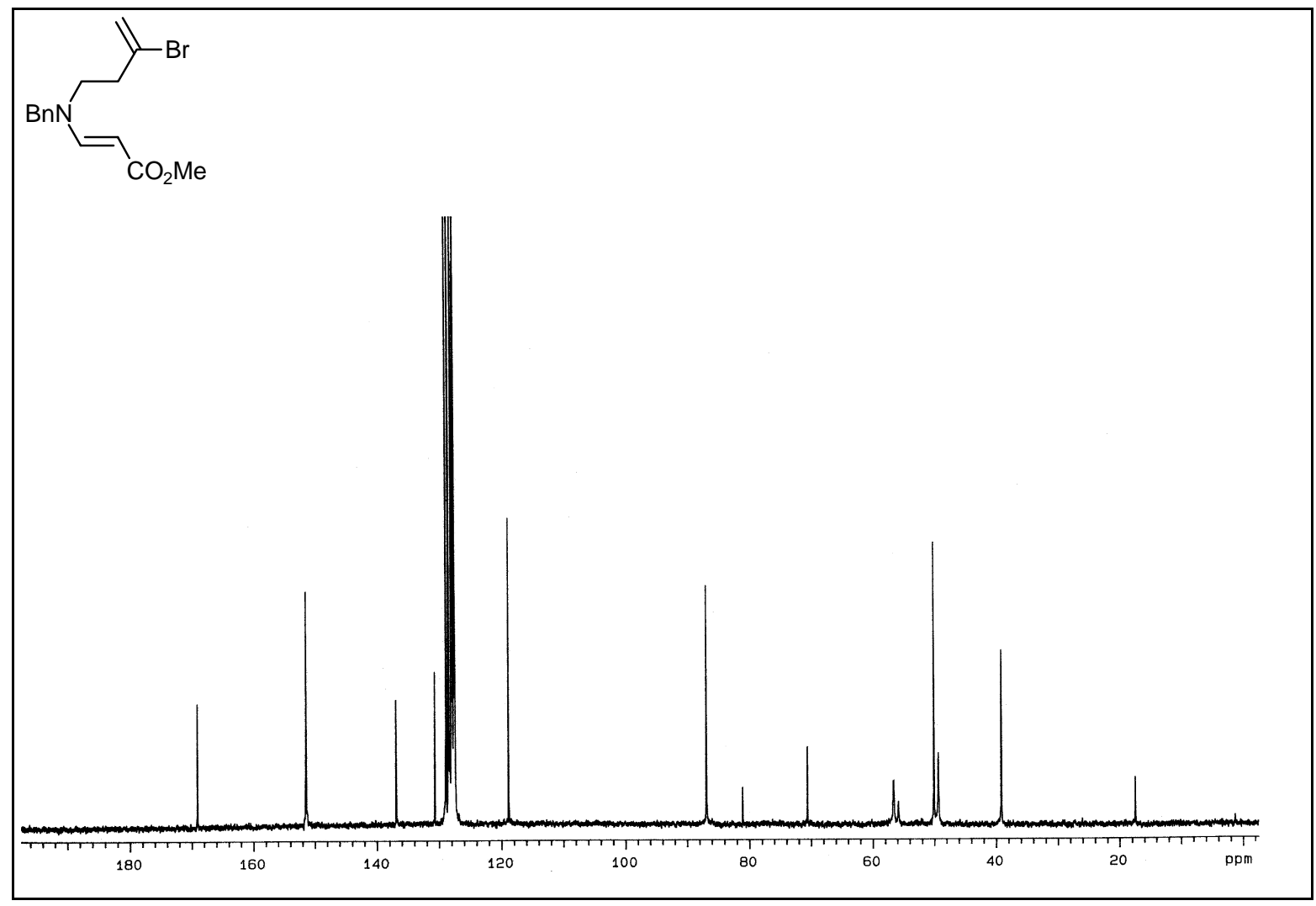

$N$-Benzyl- $N$-(3'-brombut-3'-en)prop-1-en-2-carbonsäuremethylester-1-amin (82) 


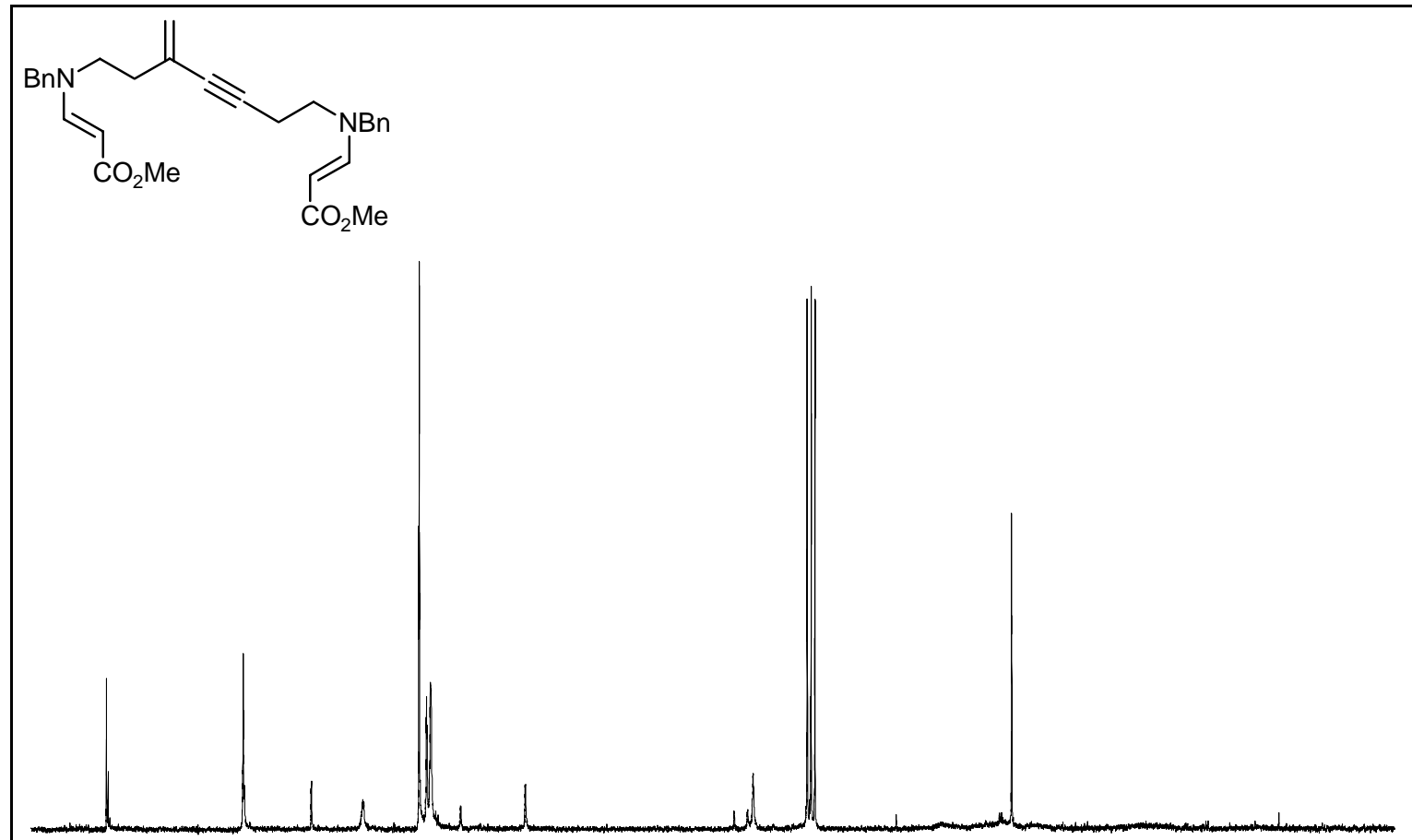

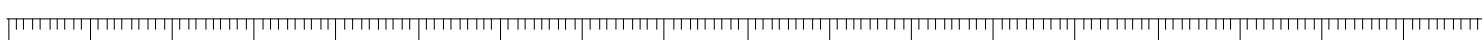

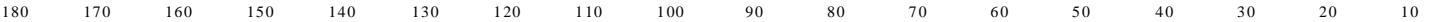

$N, N$-Benzyl-1,13-dicarbonsäuremethylester-8-methylentrideca-1,12-dien-6-in (86)

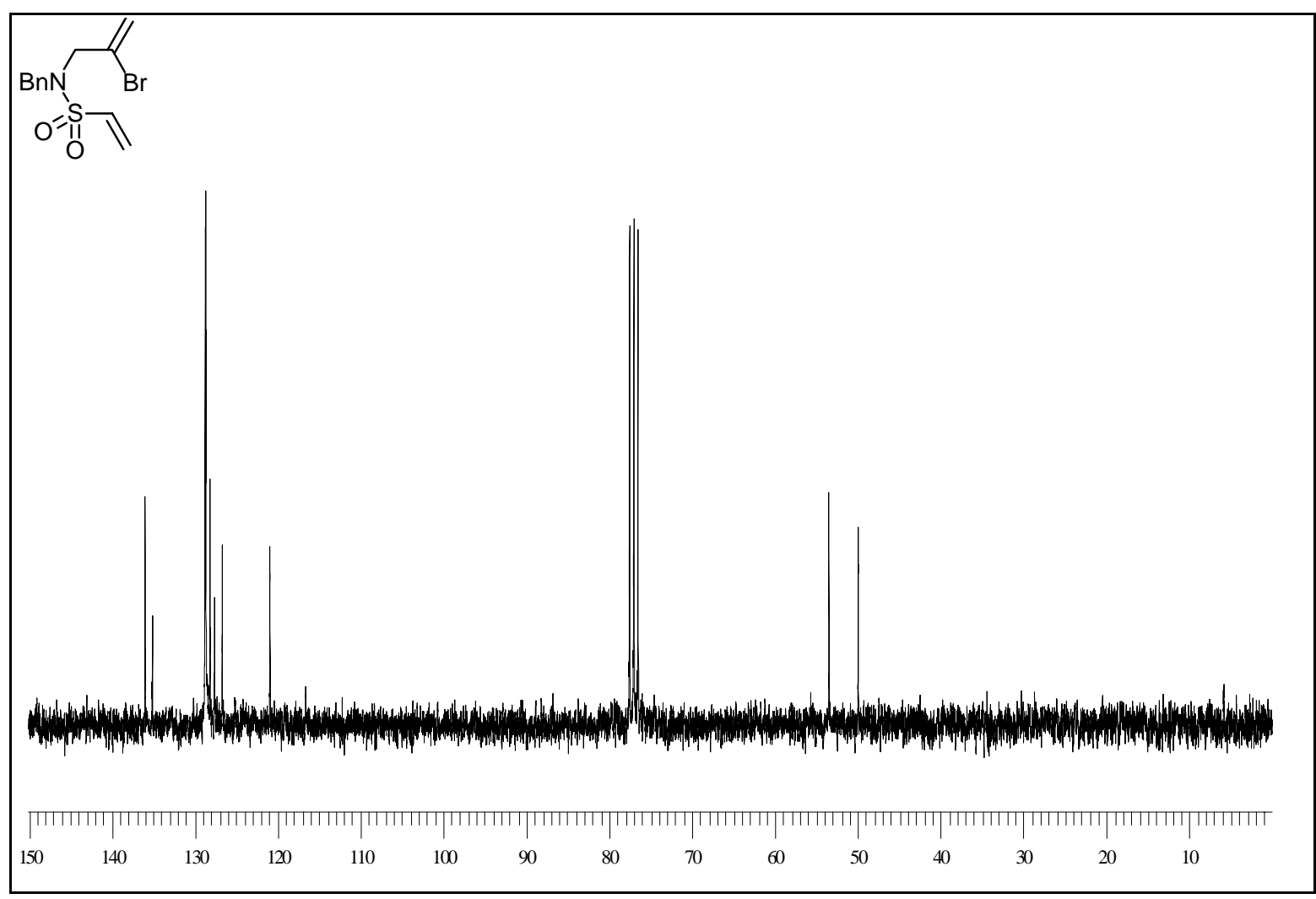

$N$-Benzyl-2-brom- $N$-vinylsulfonylprop-2-en-1-amin (93) 
161

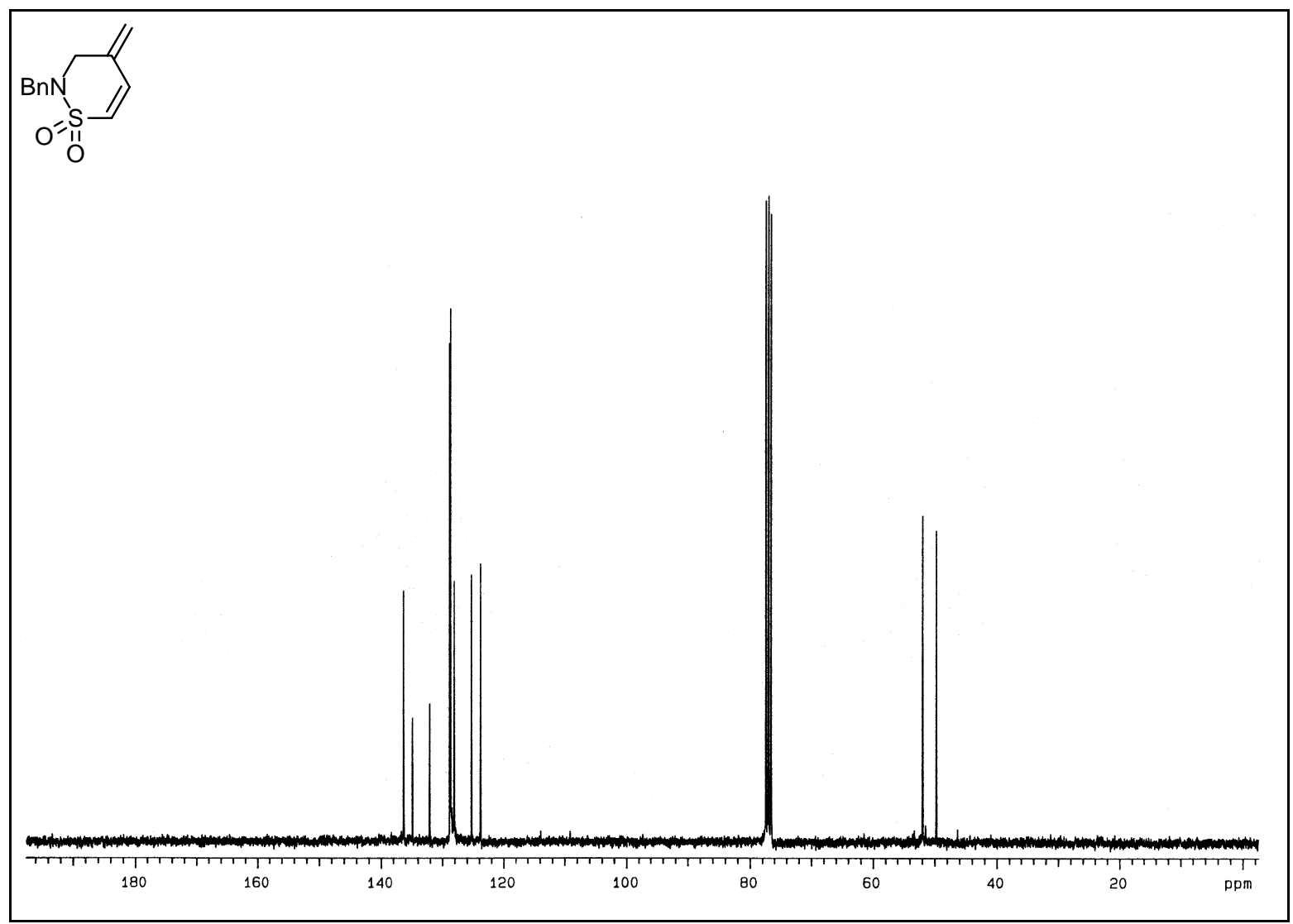

$N$-Benzyl-4-methylen-3,4-dihydro-2H[1,2]thiazin-1,1-dioxid (101)

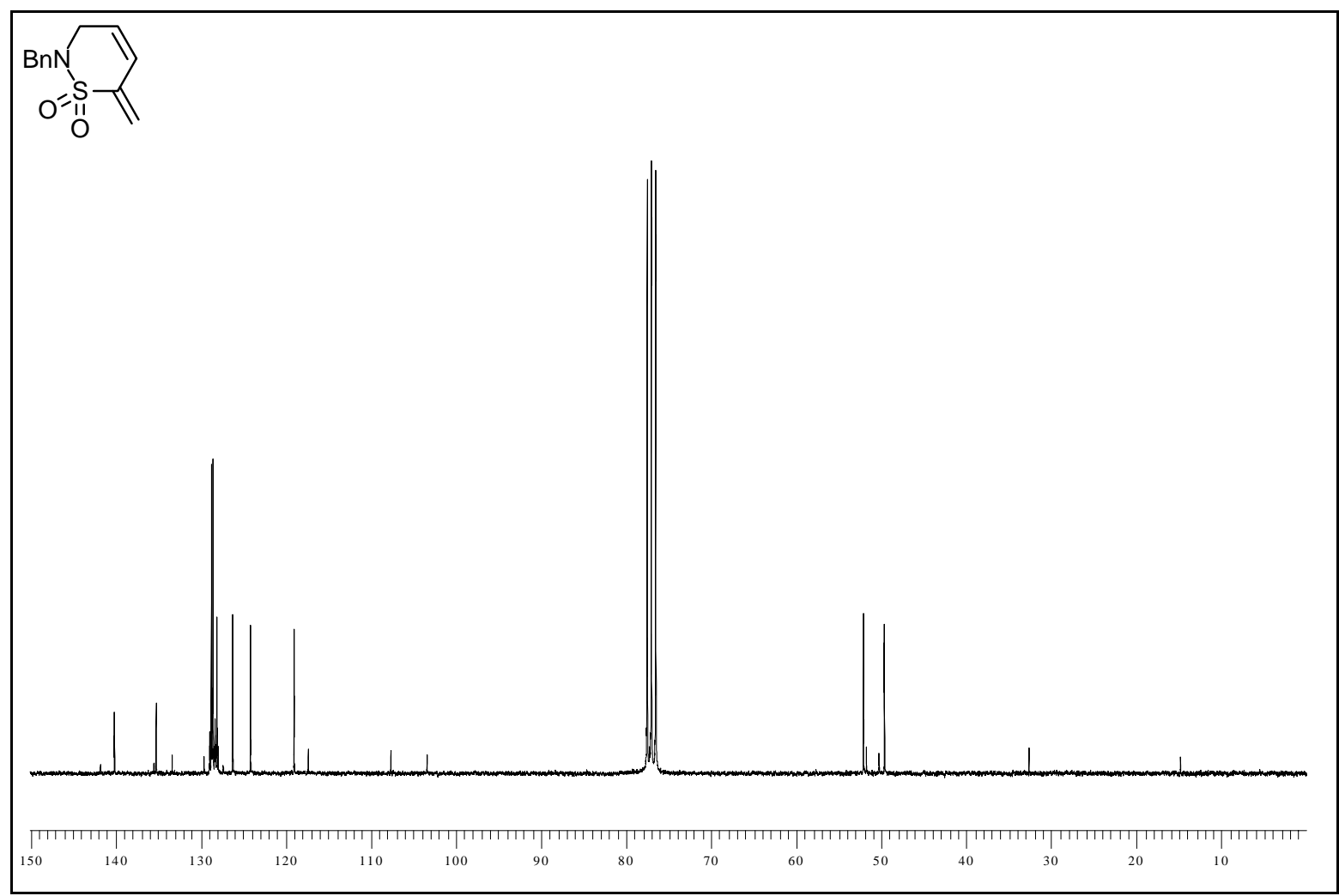

$N$-Benzyl-6-methylen-3,6-dihydro-2 $H[1,2]$ thiazin-1,1-dioxid (102) 


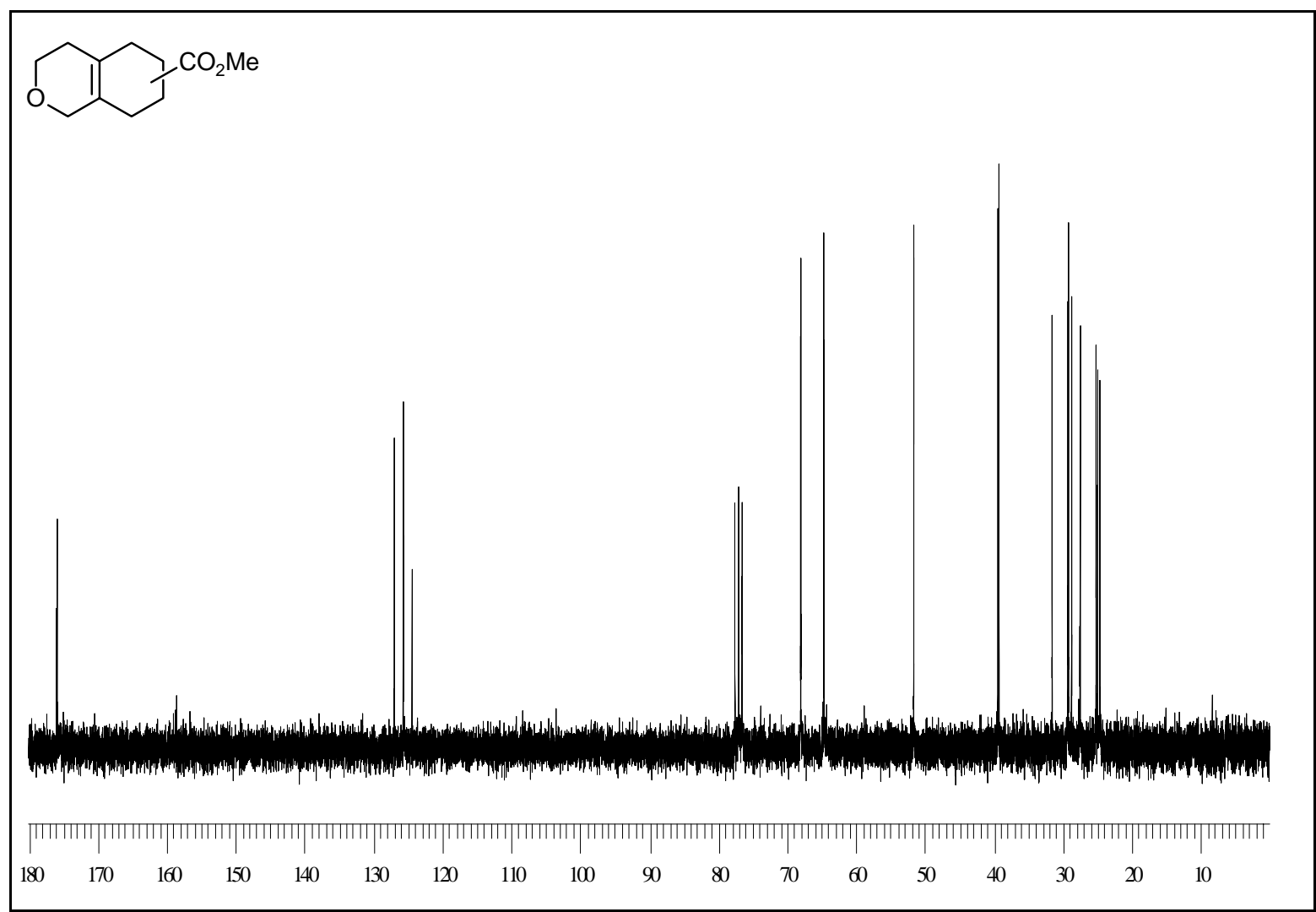

3,4,5,6,7,8-Hexahydro-1H-isochromen-6-carbonsäuremethylester (115a) und 3,4,5,6,7,8Hexahydro-1 $H$-isochromen-7-carbonsäuremethylester (115b)

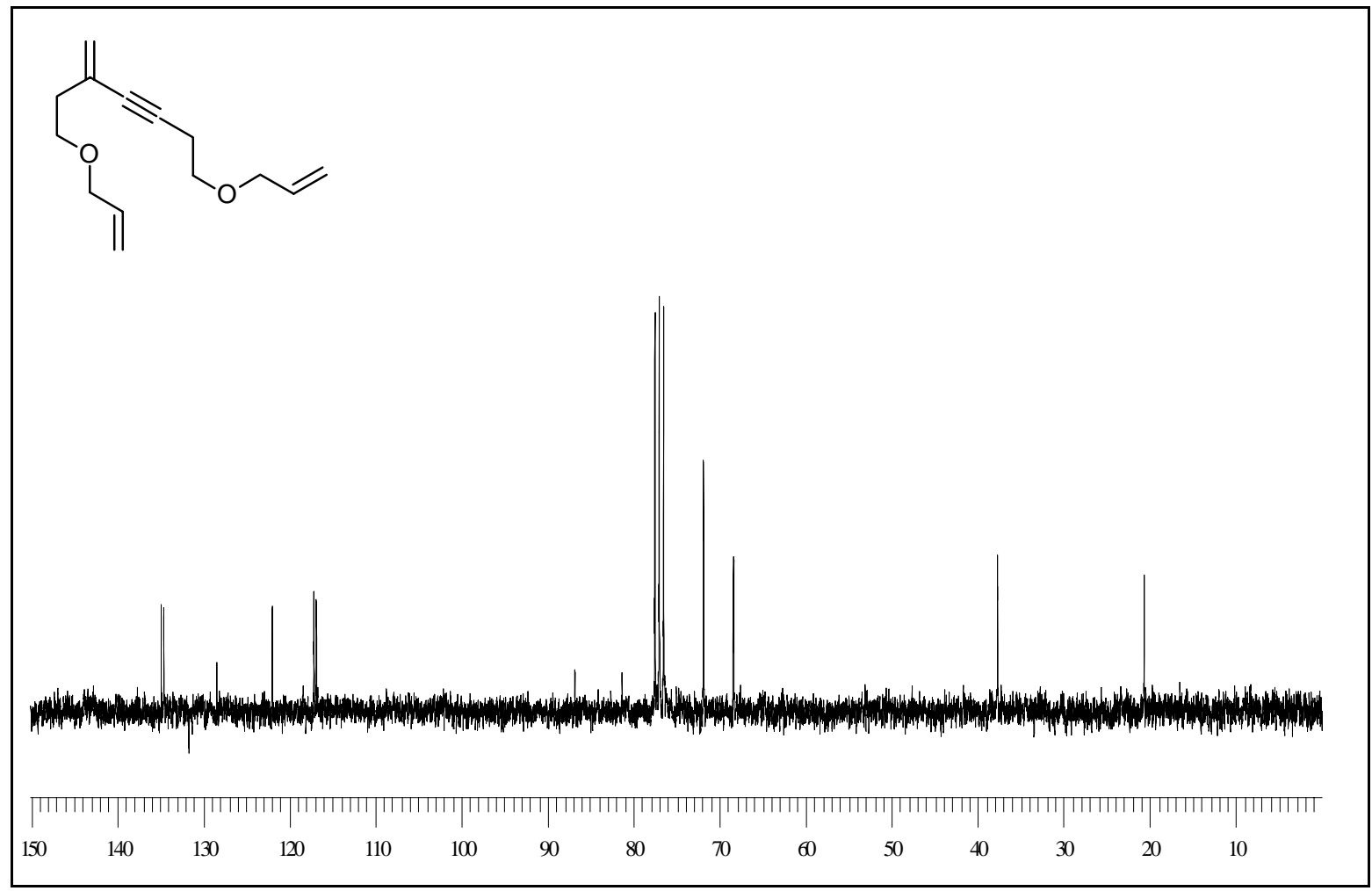

1,7-Diallyloxy-5-methylenhept-3-in (116) 


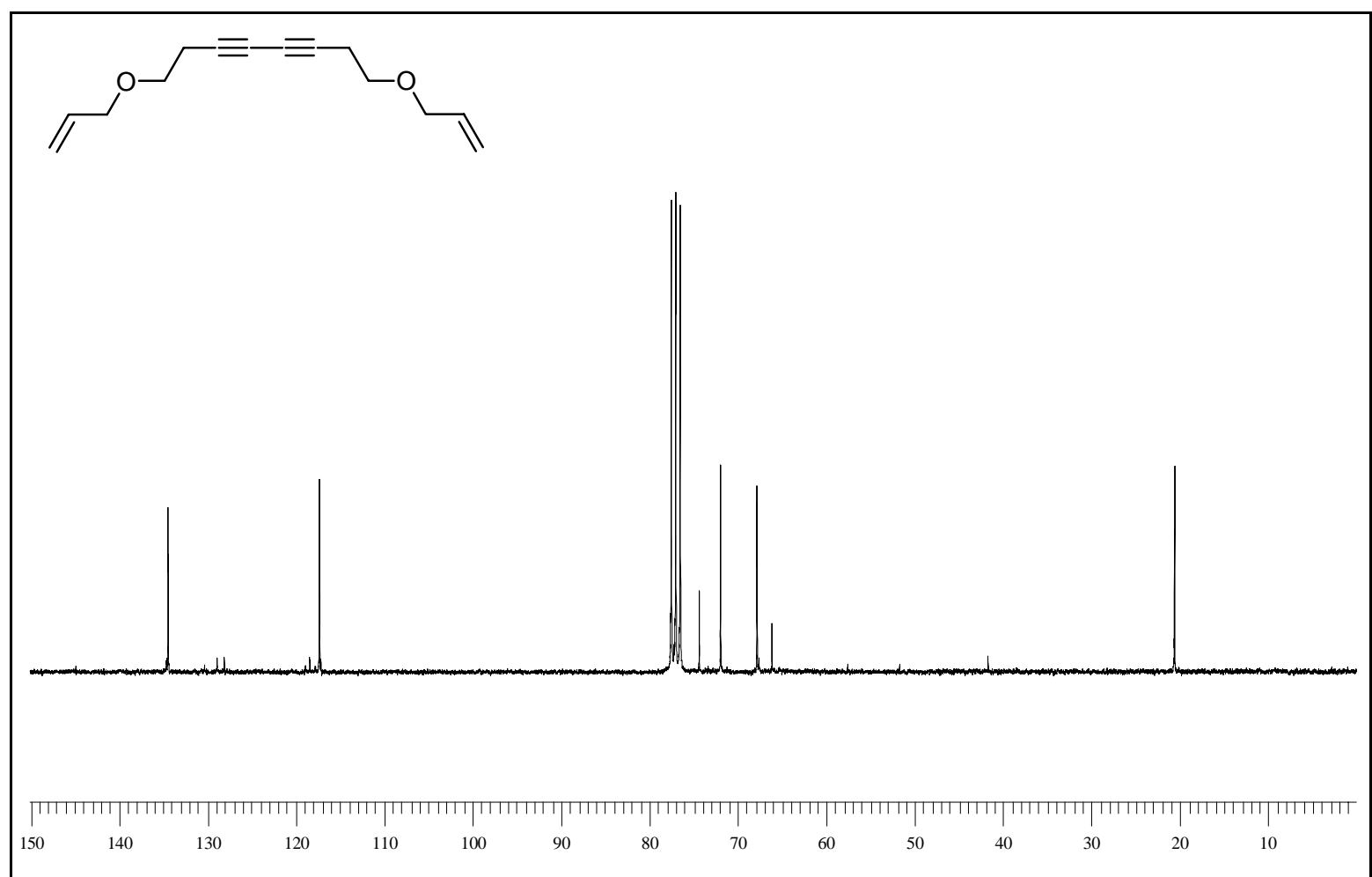

1,8-Diallyloxyocta-3,5-diin (117)

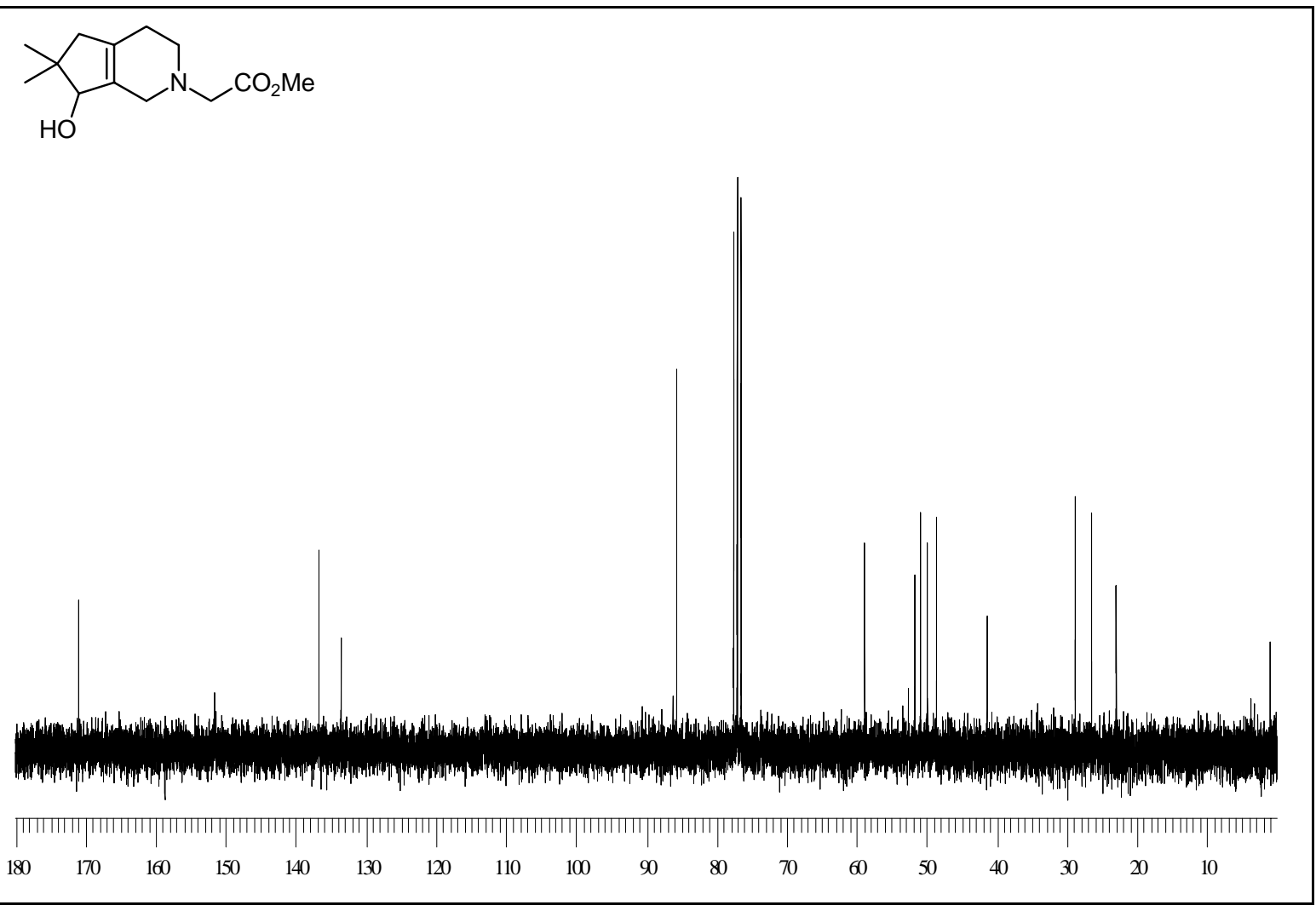

(7-Hydroxy-6,6-dimethyl-1,3,4,5,6,7-hexahydro-2H-cyclopenta[c]pyridin-2-yl)essigsäuremethylester (126-Gly) 


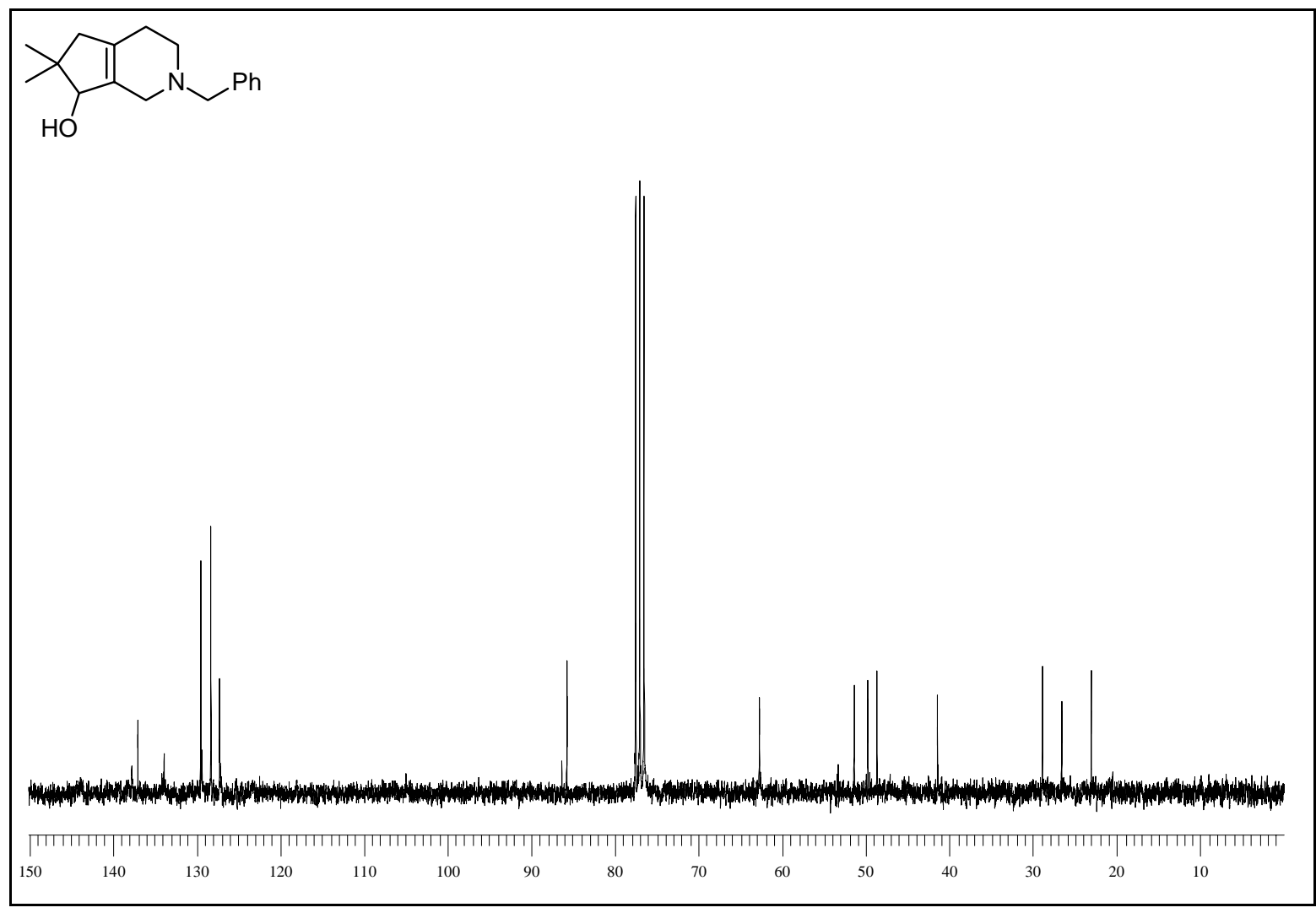

$\mathrm{N}$-Benzyl-(7-hydroxy-6,6-dimethyl-1,3,4,5,6,7-hexahydro-2H-cyclopenta[c]-2-pyridin) (126-Bn)

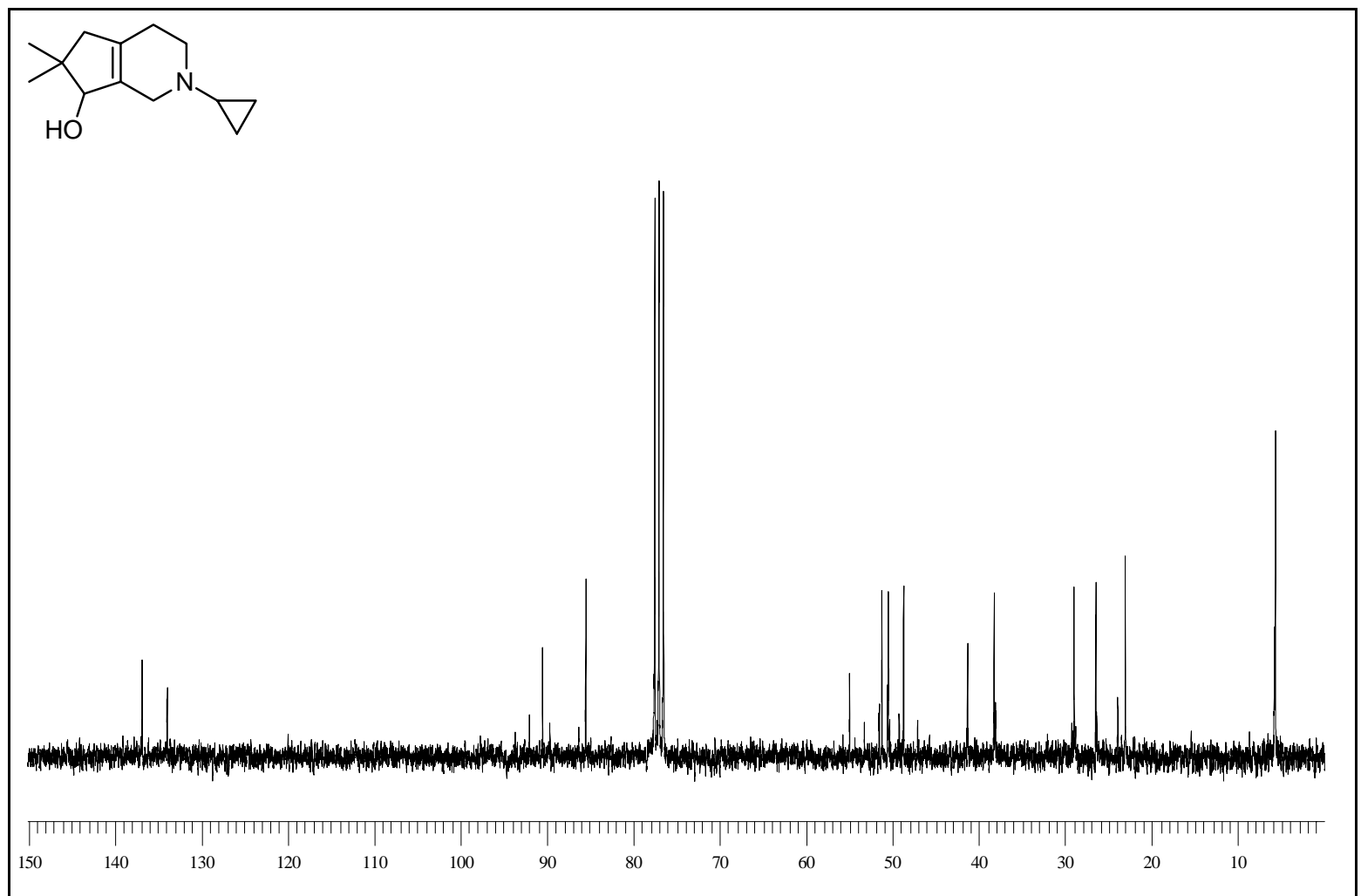

$\mathrm{N}$-Cyclopropyl-(7-hydroxy-6,6-dimethyl-1,3,4,5,6,7-hexahydro-2H-cyclopenta[c]-2pyridin) (126-cPr) 
165

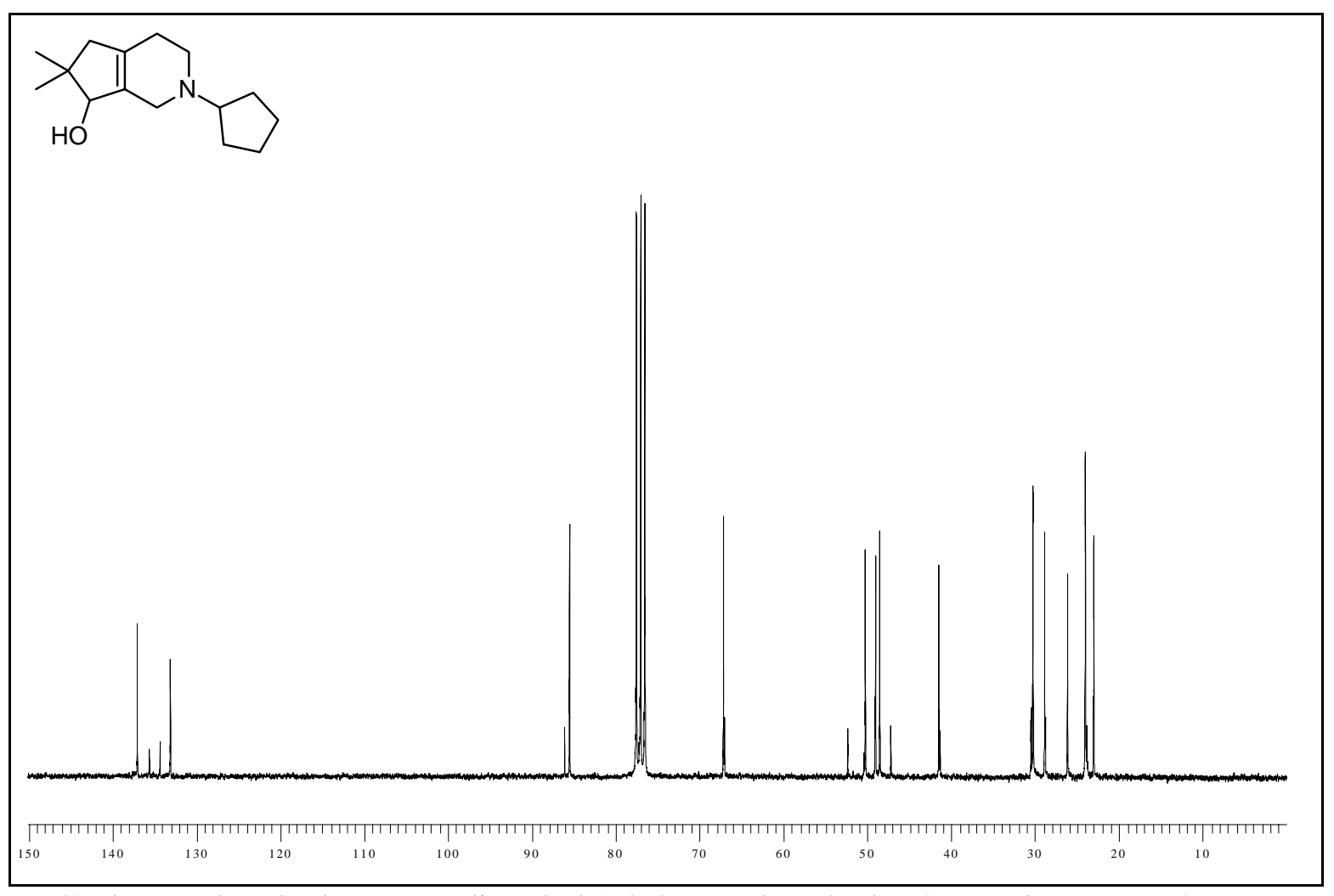

$N$-Cyclopentyl-(7-hydroxy-6,6-dimethyl-1,3,4,5,6,7-hexahydro- $2 H$-cyclopenta[c]-2pyridin) (126-cPent)
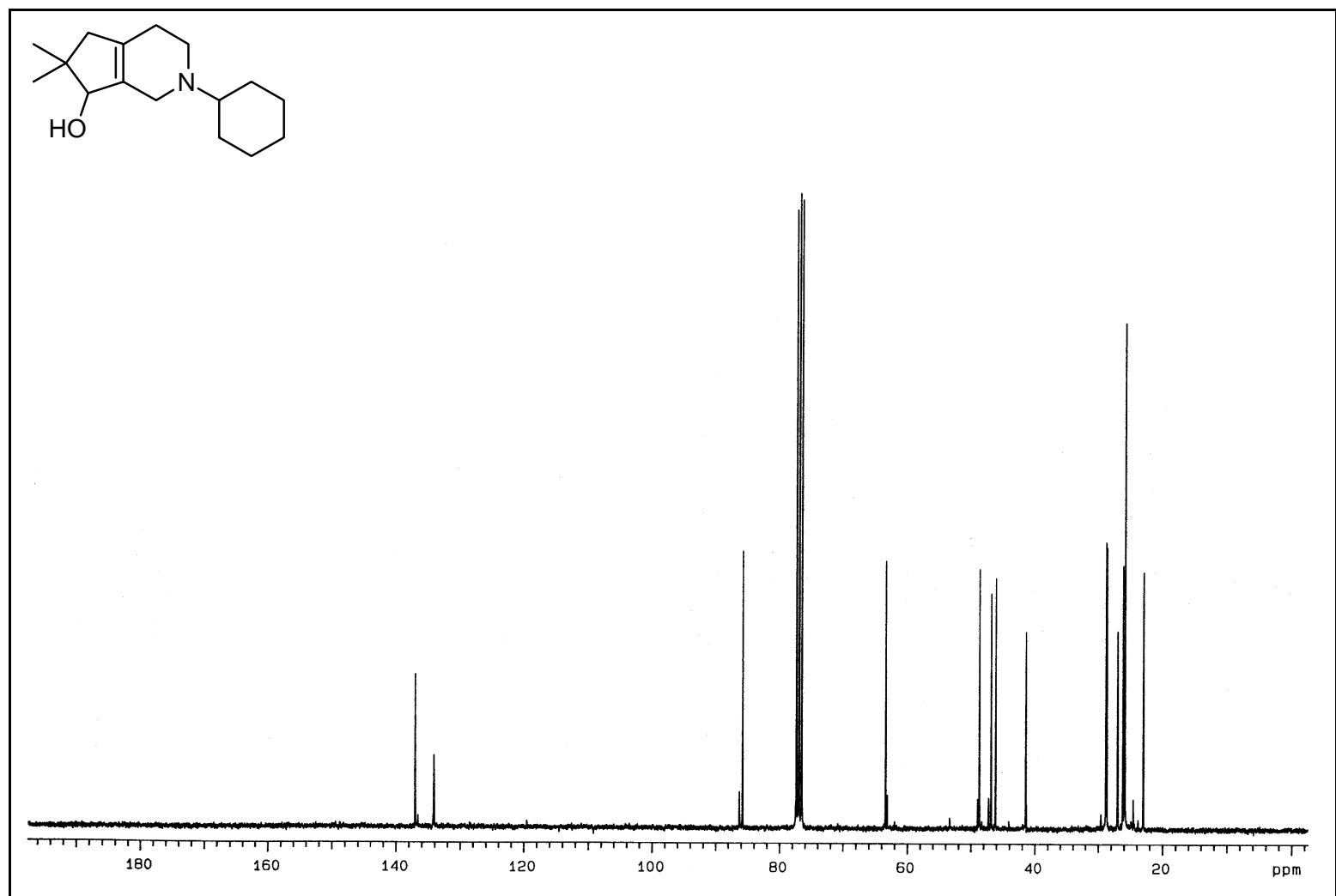

$N$-Cyclohexyl-(7-hydroxy-6,6-dimethyl-1,3,4,5,6,7-hexahydro-2H-cyclopenta[c]-2-pyridin) (126-cHex) 

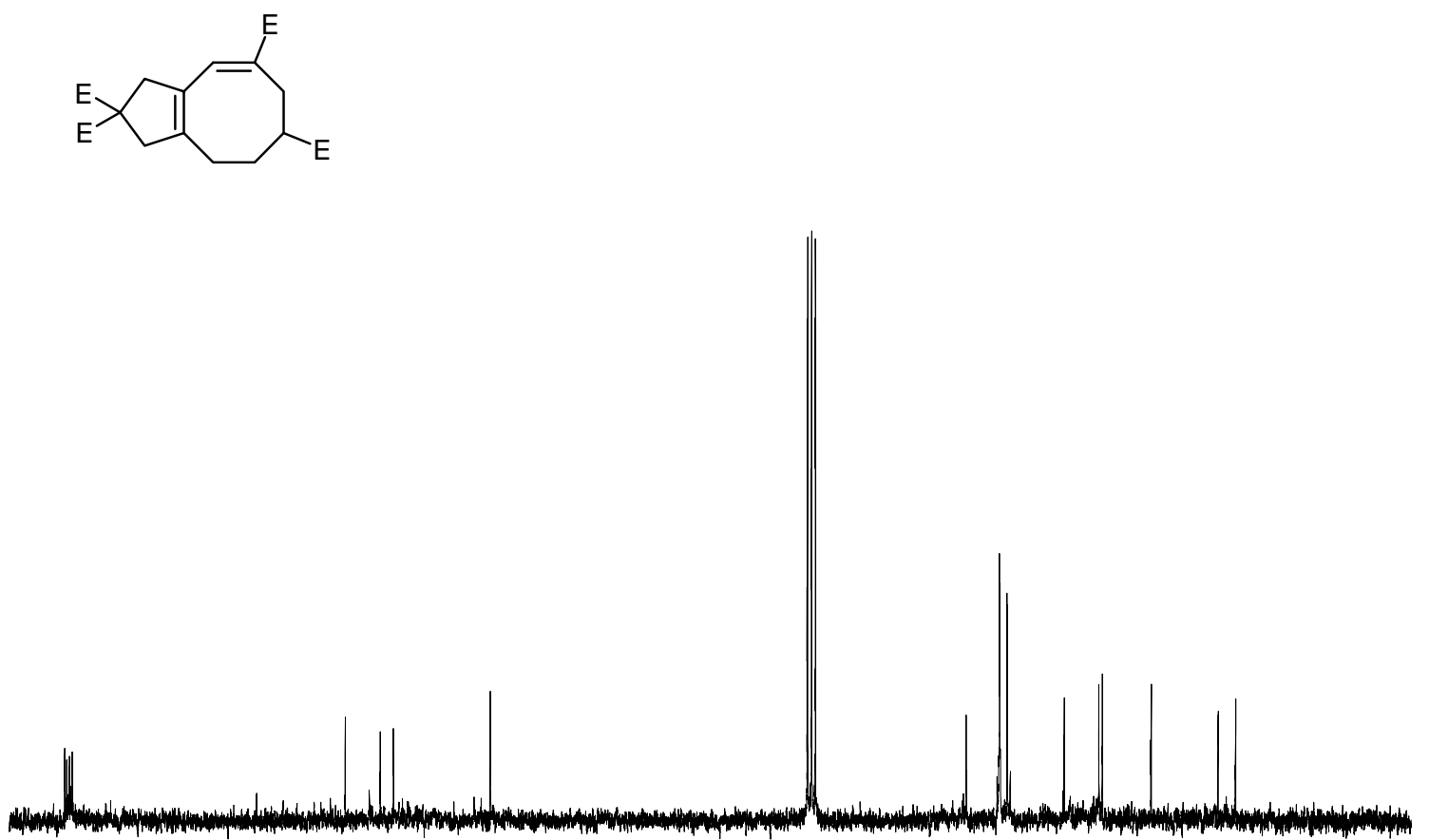

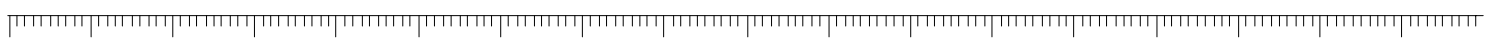
2,2,5,7-Tetracarbonsäuremethylester-2,3,4,5,6,7-hexahydro- $1 H$-cyclopentacycloocten $\left(155-\mathrm{CO}_{2} \mathrm{Me}\right)$<smiles>C=C1CC(F)(F)Cc2cc3c(cc21)CC(F)(F)C3</smiles>
thalin (156) 


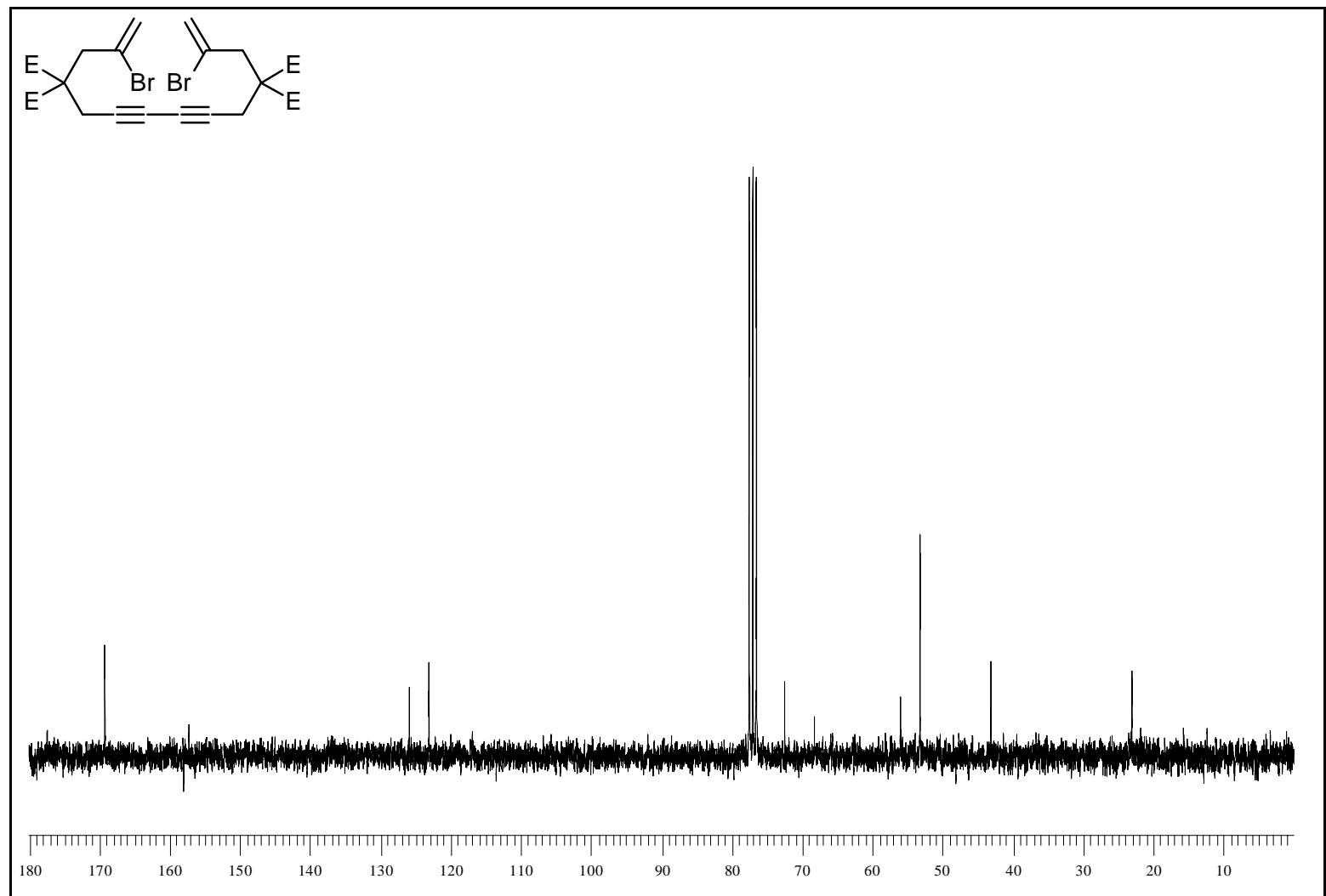

2,13-Brom-4,4,11,11-tetracarbonsäuremethylester-tetradeca-1,13-dien-6,8-diin (157)

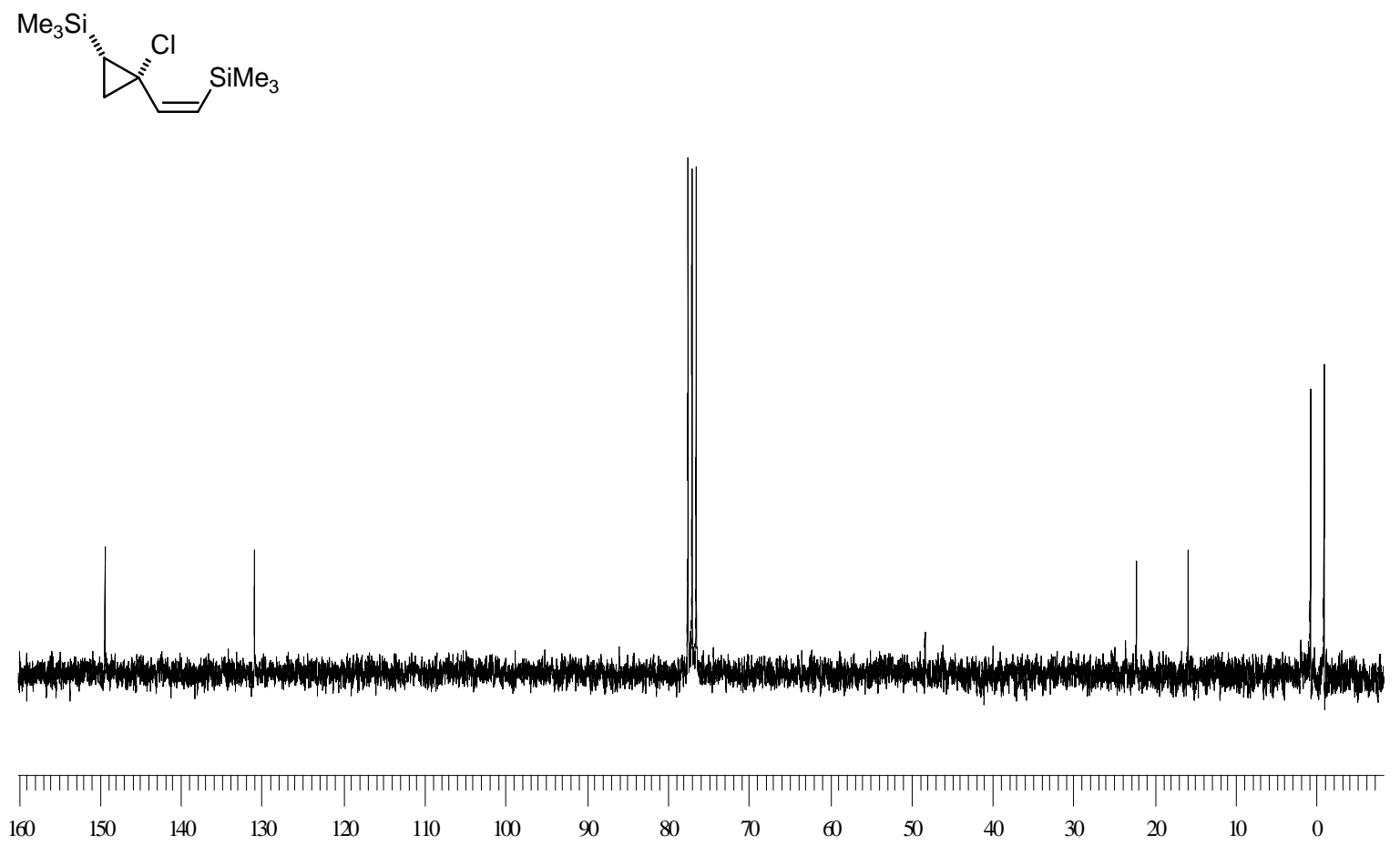

(Z)-1-Chlor-1-(2'-trimethylsilylethenyl)-2-trimethylsilylcyclopropan $[(Z, Z)-217]$ 
3. 2D-NMR-Spektren

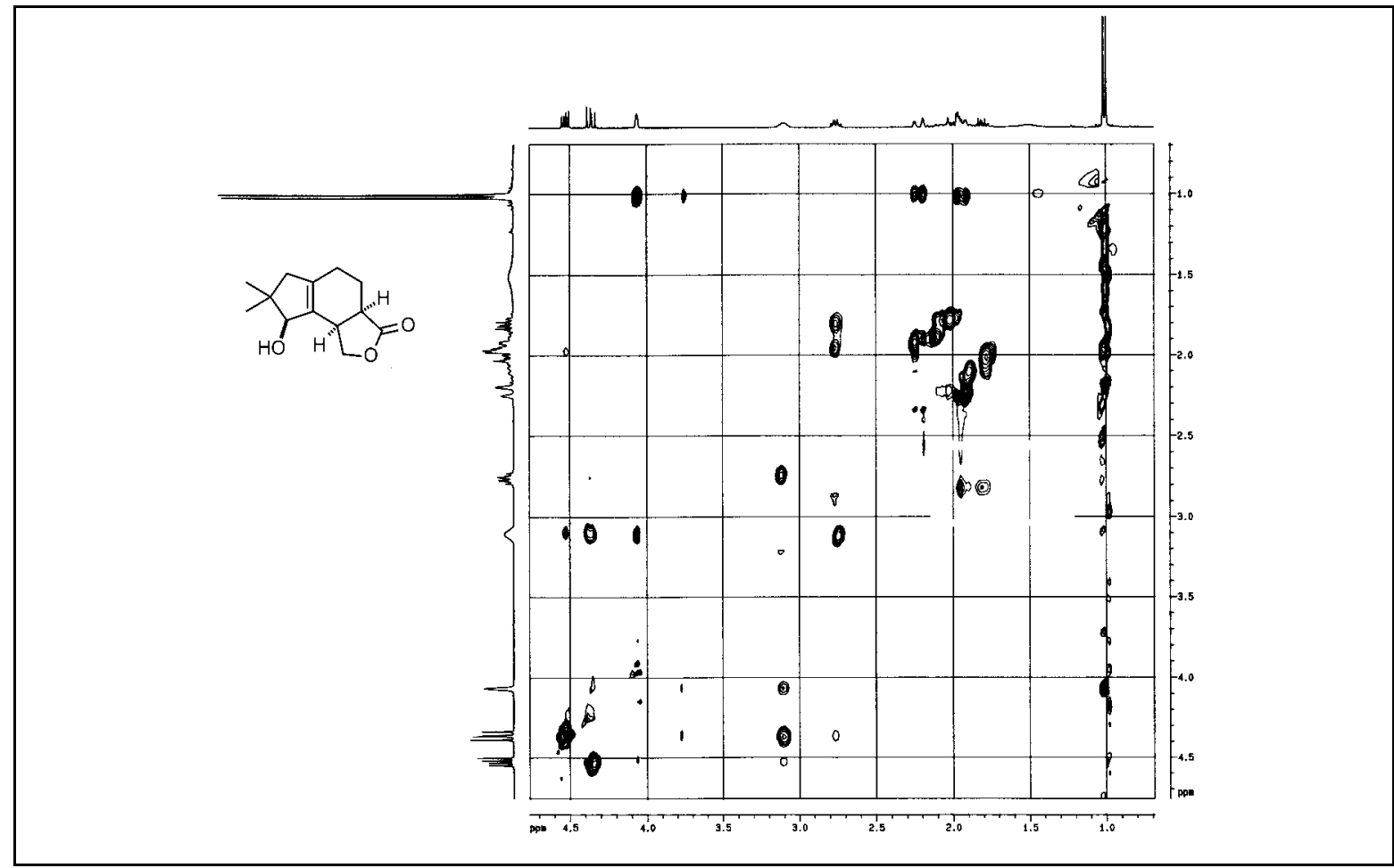

NOESY von 8-Hydroxy-7,7-dimethyl-1,3a,4,5,6,7,8,8b-octahydro-3H-indeno[4,5-c]furan3-on (52)

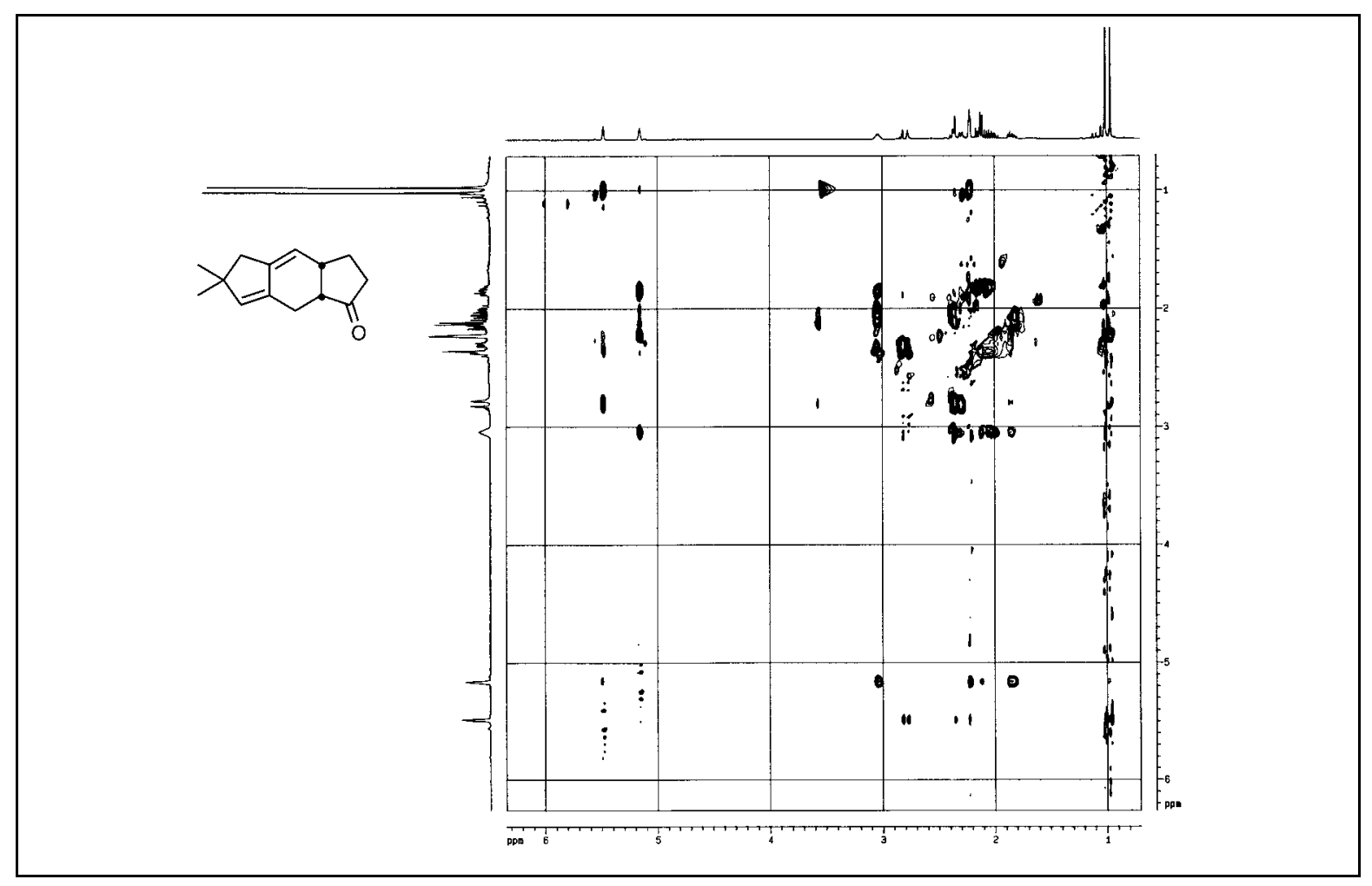

NOESY von 6,6-Dimethyl-3,3a,5,6,8,8a-hexahydro-2H-s-indacen-1-on (65) 


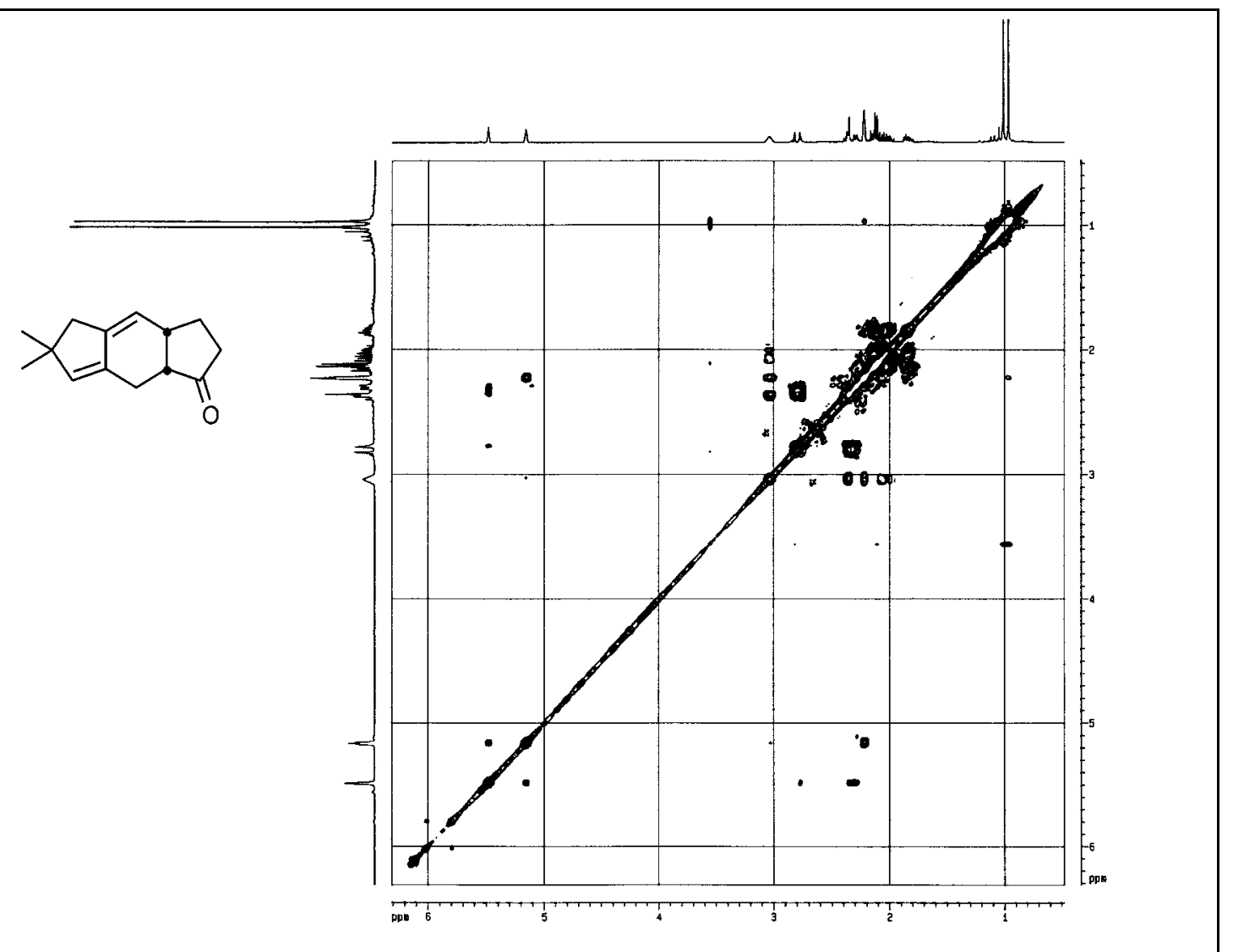

Standard COSY von 6,6-Dimethyl-3,3a,5,6,8,8a-hexahydro-2 $H$-s-indacen-1-on (65)

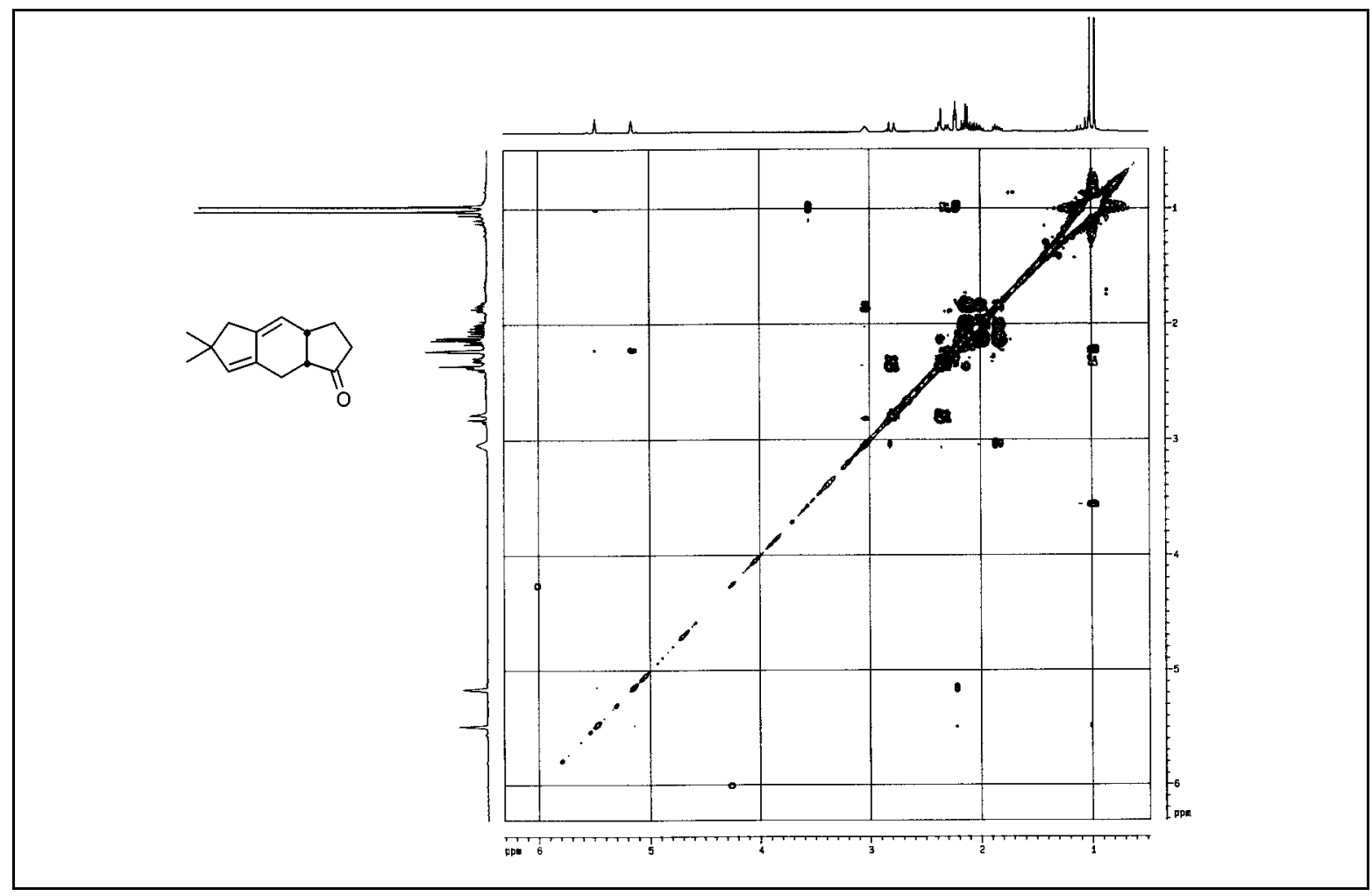

Long-Range COSY von 6,6-Dimethyl-3,3a,5,6,8,8a-hexahydro-2H-s-indacen-1-on (65) 


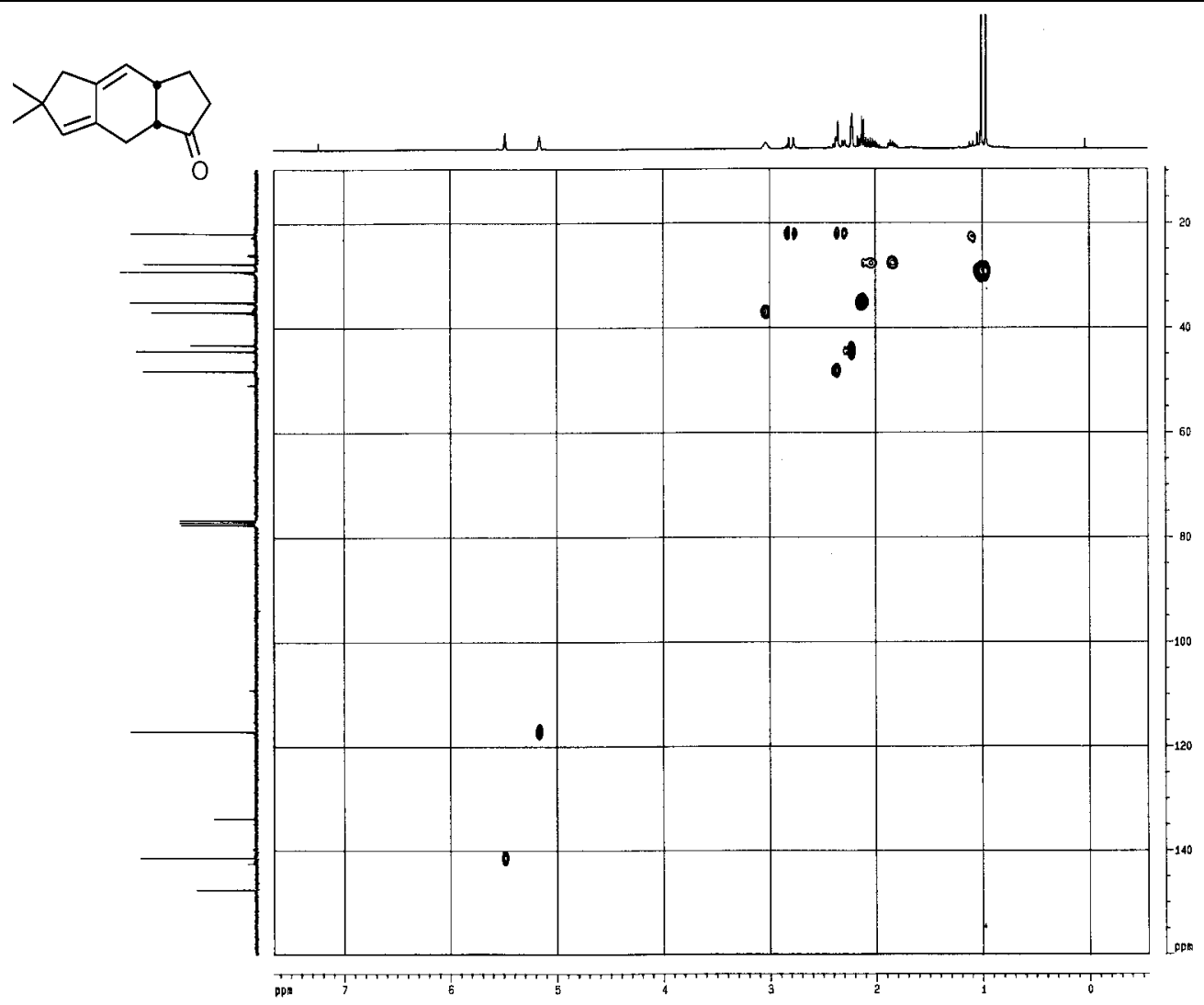

HMQC von 6,6-Dimethyl-3,3a,5,6,8,8a-hexahydro-2H-s-indacen-1-on (65)

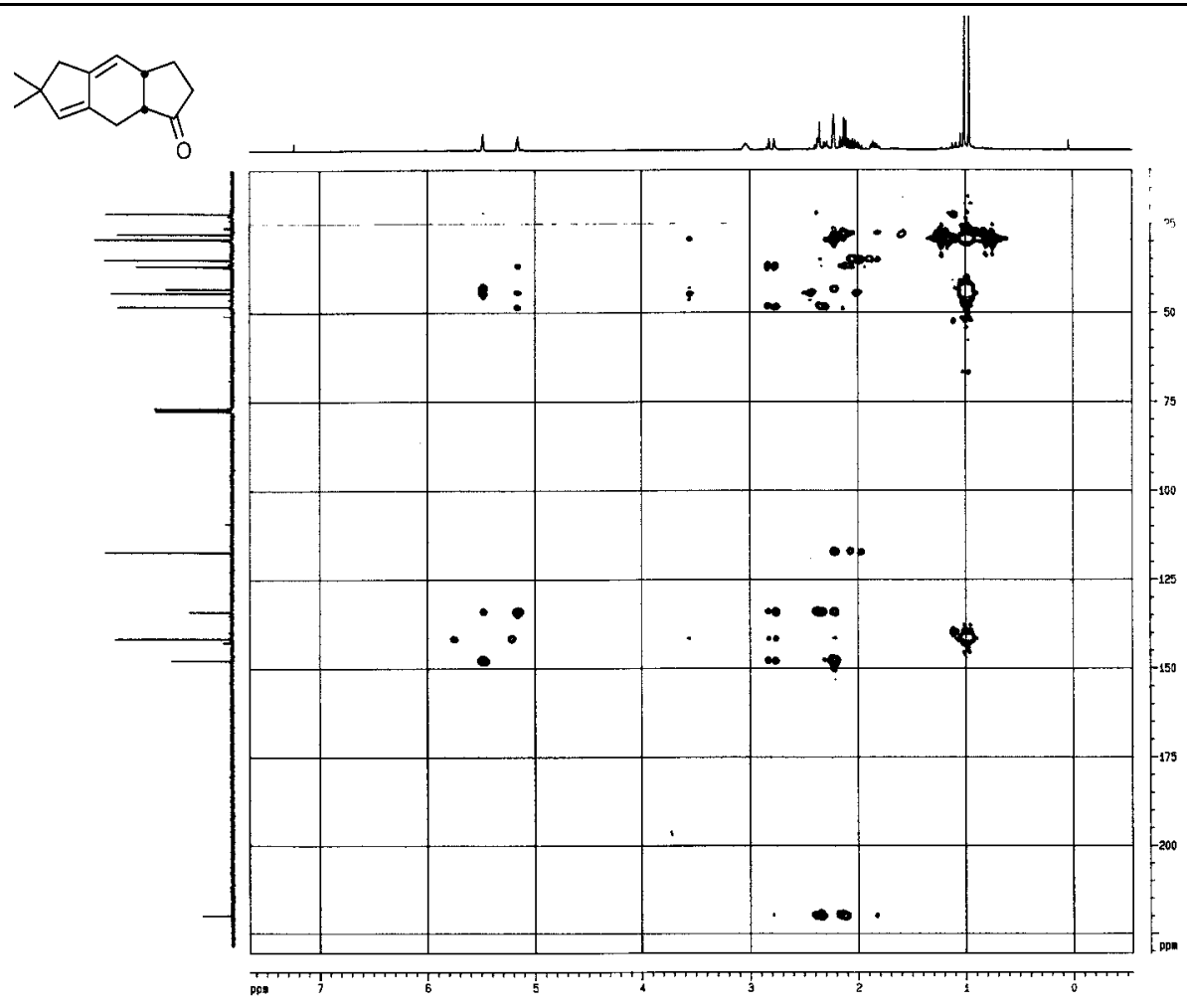

HMBC von 6,6-Dimethyl-3,3a,5,6,8,8a-hexahydro- $2 \mathrm{H}$-s-indacen-1-on (65) 


\section{Publikationen}

[1] S. Körbe, A. G. Steinig, A. de Meijere, "Synthesis of Bicyclo[4.3.0]nonenes by Using Palladium-Catalyzed Cyclizations", VSL 18, $10^{\text {th }}$ IUPAC Symposium on Organo-Metallic Chemistry Directed Towards Organic Synthesis (OMCOS 10), Versailles 1999.

[2] P. Menningen, C. Harcken, B. Stecker, S. Körbe, A. de Meijere, M. Rodrigues Lopez, J. Ollivier, J. Salaün, "Silyl Effect on the Regioselective Synthesis of Silylated Alkylidenecyclopropanes", Synlett 1999, 1534-1538.

[3] S. Körbe, A. de Meijere, " Formation of Bicyclic Systems by a Domino Process of Palladium Catalyzed Cyclization and Diels-Alder Reaction", $4^{\text {th }}$ International Symposium Transition Metals in Organic Synthesis, Leeds 2000.

[4] L. J. van Boxtel, S. Körbe, A. de Meijere, " Facile Synthesis of Bi- and Tricyclic Skeletons by Cycloisomerizations of Hept-1-en-6-ynes and 4,9-Diheterododeca1,11-dien-6-ynes Followed by [4+2] Cycloadditions", Eur. J. Org. Chem. 2001, 2283-2292. 


\section{Danksagungen}

Für den wissenschaftlichen Unterricht an der Universität Göttingen und Orsay danke ich den Herren Professoren und Dozenten J. Salaün (Orsay, Frankreich), U. Klingebiel, G. F. Kahl, H. Laatsch, K. Luther, A. de Meijere, A. Meller, O. Reiser, E. Puschmann, H. W. Roesky, C. Schneider, E. Schwarzmann, G. M. Sheldrick, L.-F. Tietze und H. Gg. Wagner (Göttingen).

Herrn R. Machinek danke ich für die Aufnahme von NMR-Spektren und seine hilfreiche Unterstützung bei der Auswertung der Spektren. Herrn Dr. Remberg und Herrn Dr. Frauendorf danke ich für die Aufnahme der Massenspektren.

Für das sorgfältige Korrekturlesen, auch des Experimentellen Teiles danke ich Bettina Kracke, Dorit Nelke, Regina von Essen, Björn Stecker, Stefan Wiedemann und Baldur Stulgies. Stefan Beußhausen und Michael Schelper sei für die Hilfe bei ComputerProblemen gedankt, Karsten Rauch danke ich für die immer gute Versorgung mit Verbrauchsmaterialien. Torsten danke ich für das Scannen der Spektren und die Hilfe beim Weiterbearbeiten.

Bei meinen Laborkollegen der letzten vier Jahre aus den Laboren 308 und 309 bedanke ich mich für die gute Zusammenarbeit und die hilfreichen Diskussionen. Für viel Spaß innerhalb des Labores danke ich Baldur, Jelena, Mario, Stefan W., Michael, Björn und Karsten, sowie Heiko, Jörg H., Ingo, Michael, Regina, Sandra, Stefan R., Stefan B. und Lonneke.

Für noch viel mehr Spaß außerhalb des Labores möchte ich mich besonders bei Torsten, Dorit, Jörg, Bettina, Bianca und Regina bedanken.

Der allergrößte Dank geht jedoch an meine Eltern und meine Großmutter, ohne deren Unterstützung diese Arbeit nicht möglich gewesen wäre. 


\section{Lebenslauf}

Ich wurde am 25. Mai 1974 als erstes Kind des Bahnbeamten Albert Körbe und seiner Ehefrau Anita, geb. Bortfeld, in Bad Hersfeld geboren.

Von August 1980 bis Juli 1984 besuchte ich die Grundschule in Bebra-Breitenbach, Hessen. Danach wechselte ich auf das Wigbert-Gymnasium, Hünfeld, Hessen, an dem ich im Juni 1993 das Abitur bestand.

Zum Wintersemester 1993/1994 begann ich das Studium der Chemie an der Georg-AugustUniversität Göttingen und legte dort im Februar 1996 das Vordiplom ab.

Meine Diplomarbeit mit dem Titel "Synthese von Bicyclo[4.3.0]nonen-Gerüsten durch einen Domino-Prozeß aus intramolekularer Heck-Reaktion oder Enin-Cycloisomerisierung und Diels-Alder-Reaktion" fertigte ich im Arbeitskreis von Prof. Dr. A. de Meijere an. Am

8. Juli 1998 bestand ich meine Diplomhauptprüfung im Fach Chemie an der GeorgAugust-Universität Göttingen. Seitdem arbeite ich an meiner Dissertation mit dem Titel "Palladium-katalysierte Domino-Reaktionen zum Aufbau bi- und tricyclischer Systeme" im Arbeitskreis von Prof. Dr. A. de Meijere.

Ein dreimonatiger Forschungsaufenthalt führte mich im Oktober 1998 an das Laboratoire des Carbocycles, Institut de Chimie Moléculaire d'Orsay der Université de Paris-Sud in Orsay, Frankreich, in den Arbeitskreis von Prof. Dr. Salaün.

Von September 1998 bis April 2001 war ich wissenschaftliche Angestellte am Institut für Organische Chemie der Georg-August-Universität und mit der Betreuung des Chemischen Praktikums für Mediziner und Zahnmediziner beschäftigt.

Von Oktober bis November 2000 nahm ich erfolgreich an einem Kurs zur allgemeinen Pharmakologie und Toxikologie teil. 